\title{
APT Blanket System Loss-of-Helium-Gas Accident Based on Initial Conceptual Design - Helium Supply Rupture into Blanket Module
}

by

L. L. Hamm

Westinghouse Savannah River Company

Savannah River Site

Aiken, South Carolina 29808

S. Y. Lee

M. A. Shadday

F. G. Smith, III

A document prepared for SENDING TO LANL at , , from - .

DOE Contract No. DE-AC09-96SR18500

This paper was prepared in connection with work done under the above contract number with the U.S. Department of Energy. By acceptance of this paper, the publisher and/or recipient acknowledges the U.S. Government's right to retain a nonexclusive, royalty-free license in and to any copyright covering this paper, along with the right to reproduce and to authorize others to reproduce all or part of the copyrighted paper. 


\section{DISCLAIMER}

Portions of this document may be illegible in electronic image products. Images are produced from the best available original document. 


\section{APT BLANKET SYSTEM LOSS-OF-HELIUM-GAS ACCIDENT (LOHGA) BASED ON INITIAL CONCEPTUAL DESIGN -}

\section{Helium Supply Rupture into Blanket Module}

Si Young Lee

Frank G Smith, III

L. Larry Hamm

Westinghouse Savannah River Company Savannah River Site Aiken, SC 29808

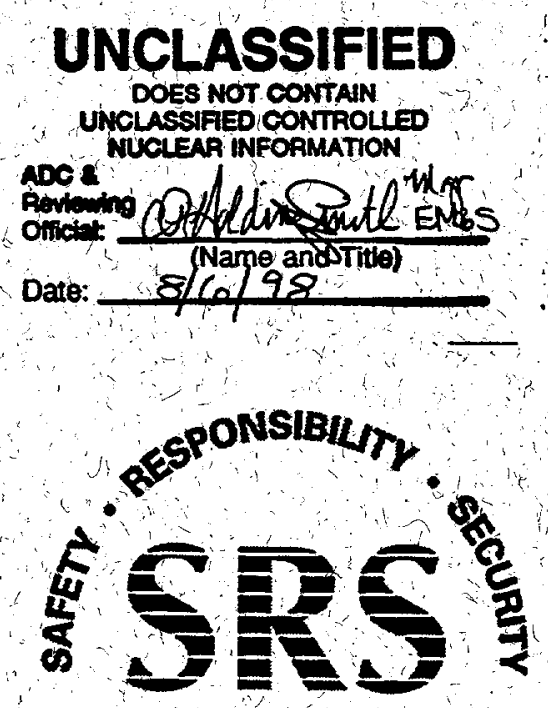

SAYANNAH RIVER SITE 


\section{DISCLAIMER}

This report was prepared as an account of work sponsored by an agency of the United States Government. Neither the United States Government nor any agency thereof, nor any of their employees, makes any warranty, express or implied, or assumes any legal liability or responsibility for the accuracy, completeness, or usefulness of any information, apparatus, product, or process disclosed, or represents that its use would not infringe privately owned rights. Reference herein to any specific commercial product, process, or service by trade name, trademark, manufacturer, or otherwise does not necessarily constitute or imply its endorsement, recommendation, or favoring by the United States Government or any agency thereof. The views and opinions of authors expressed herein do not necessarily state or reflect those of the United States Government or any agency thereof.

This report has been reproduced directly from the best available copy.

Available to DOE and DOE contractors from the Office of Scientific and Technical Information, P.O. Box 62, Oak Ridge, TN 37831; prices available from (615) 576-8401.

Available to the public from the National Technical Information Service, U.S. Department of Commerce, 5285 Port Royal Road, Springfield, VA 22161. 
KEYWORDS:

Accelerator Production of Tritium Blanket System

Conceptual Design TRAC Code

FLOWTRAN-TF Code

System Model

Detailed Bin Model

Safety Analysis

RETENTION - Permanent

\section{APT BLANKET SYSTEM LOSS-OF-HELIUM-GAS ACCIDENT (LOHGA) BASED ON INITIAL CONCEPTUAL DESIGN -}

\section{Helium Supply Rupture into Blanket Module}

\section{SAVANNAH RIVER TECHNOLOGY CENTER}

Si Young Lee

Frank G. Smith, III

L. Larry Hamm

Publication Date: July 1998

Westinghouse Savannah River Company

Savannah River Site

Aiken, SC 29808

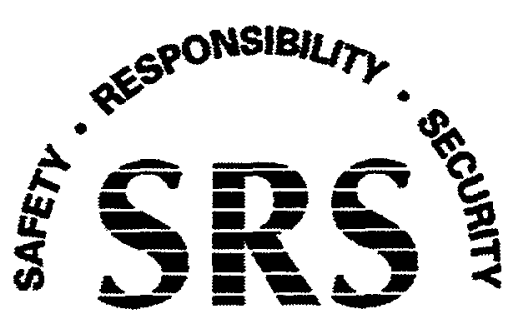

Prepared for the U.S. Department of Energy under Contract No. DE-AC09-96SR18500 
DOCUMENT: WSRC-TR-98-00177

TITLE: $\quad$ APT BLANKET SYSTEM LOSS-OF-HELIUM-GAS ACCIDENT (LOHGA) BASED ON INITIAL CONCEPTUAL DESIGN - Helium Supply Rupture into Blanket Module

APPROVALS

Si Young Lee, co-author (EM\&S

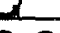

Date:

$7-20-98$

Si Young Lee, Co-author (EM\&S Group/SRTC)

Taank is lomet4

Frank G. Smith, III, Co-author (EM\&S Group/SRTC)

Date: $2 / 20 / 98$

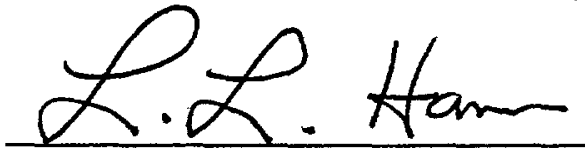

L. Larry Hamm, Co-author (PC\&C Group/SRTC)

Date: $7-16-98$

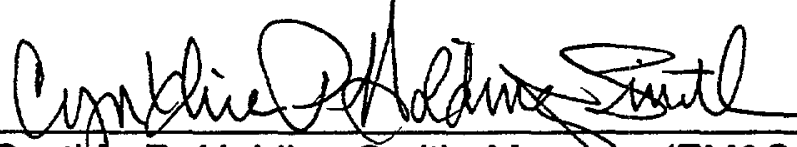

Cynthiva P. Holding-Smith, Manager (EM\&S Group/SRTC)

Date: $7 / 29 / 98$

Mastla q. Qfia

Date: $49 \operatorname{lng} 98$

Martha A. Ebra, Manager (EDS/SRTC)

Date: $2 / 21 / 98$

Mel R. Buckner, Technical \& Regulatory Lead (APT OPO)

The internal technical review function is being performed at the APT project level and is coordinated through LANL. 


\section{Table of Contents}

1 Introduction 1

2 TRAC 1-D System Model 3

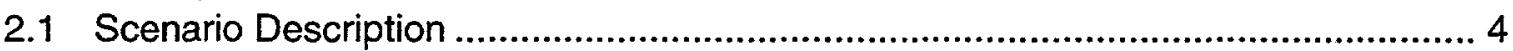

2.2 Model Upgrades ...................................................................................... 4

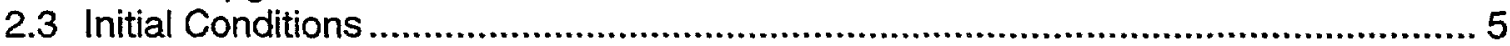

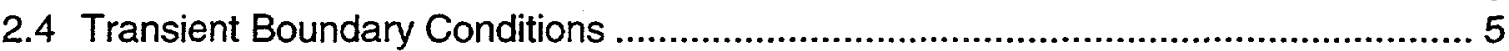

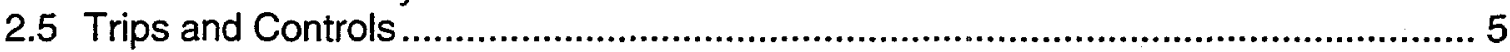

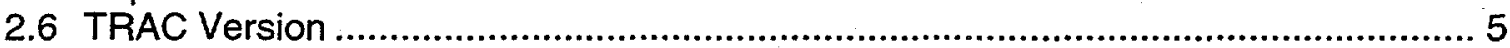

3 TRAC System Model Results $\quad 7$

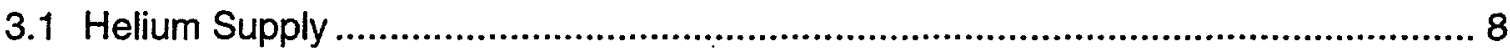

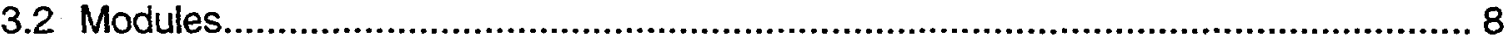

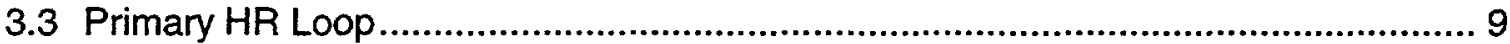

4 FLOWTRAN-TF Detailed Bin Model 10

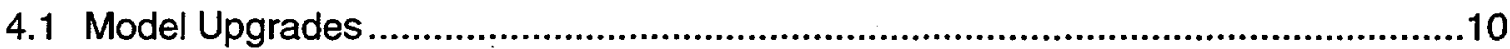

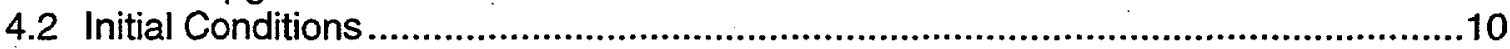

4.3 Transient Boundary Conditions ..............................................................................11

5 FLOWTRAN-TF Detailed Bin Model Results $\quad 14$

5.1 FLOWTRAN-TF Results for Component 350 Rupture.........................................14

5.2 FLOWTRAN-TF Results for Component 370 Rupture..............................................19

6 Conclusions 24

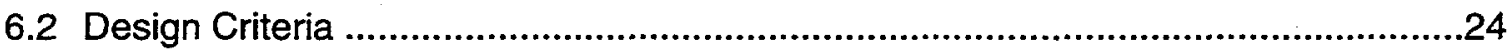

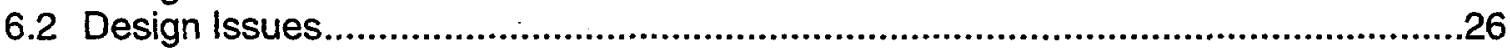

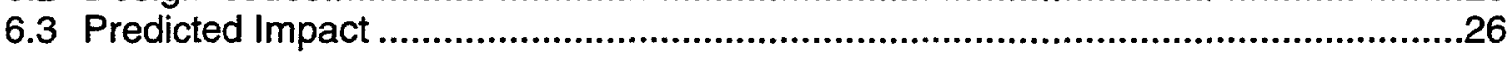

7 References $\quad 27$

Appendix A: TRAC Model Component Nomenclature

Appendix B: LOHGA (Case 1) TRAC Results

Appendix C: LOHGA (Case 2) TRAC Results

Appendix D: TRAC Standard Input File for LOHGA

Appendix E: TRAC Graphics Input File for LOHGA

Appendix F: FLOWTRAN-TF Input File for LOHGA 


\section{List of Figures}

Figure 1-1 Break location for the helium tube rupture accident (Case 1: Break at lateral blanket Decoupler inlet plenum).

Figure 1-2 Break location for the helium tube rupture accident (Case 2: Break at lateral blanket Decoupler/ Row-1 middle plenum).

Figure 2-1 Top cross-sectional facemap of 6 lumped blanket system modules.........7

Figure 4-1

Finite element mesh of APT reference 1 lateral row 1 blanket plate.

Figure 4.3-1 Inlet boundary conditions for helium tube rupture at TRAC component 350 out to 300 seconds.

Figure 4.3-2 Inlet boundary conditions for helium tube rupture at TRAC component 350 out to 60 seconds.

Figure 4.3-3 Inlet boundary conditions for helium tube rupture at TRAC component 370 out to 300 seconds.

Figure 4.3-4 Inlet boundary conditions for helium tube rupture at TRAC component 370 out to 60 seconds.

Figure 5.1-1 Maximum metal temperatures for helium tube rupture at TRAC component 350 out to 60 seconds.

Figure 5.1-2 Surface heat fluxes for helium tube rupture at TRAC component 350 out to 60 seconds, axial level 10, channels $1-4$.

Figure 5.1-3 Surface heat fluxes for helium tube rupture at TRAC component 350 out to 60 seconds, axial level 10, channels 5-8.

Figure 5.1-4 Surface heat fluxes for helium tube rupture at TRAC component 350 out to 60 seconds, axial level 10, channels $9-12$.

Figure 5.2-1 Maximum metal temperatures for helium tube rupture at TRAC component 370 out to 60 seconds.

Figure 5.2-2 Surface heat fluxes for helium tube rupture at TRAC component 370 out to 60 seconds, axial level 10, channels 1-4.

Figure 5.2-3 Surface heat fluxes for helium tube rupture at TRAC component 370 out to 60 seconds, axial level 10, channels 5-8.

Figure 5.2-4 Surface heat fluxes for helium tube rupture at TRAC component 370 out to 60 seconds, axial level 10, channels $9-12$.

\section{List of Tables}

Table 2-1 6 lumped blanket module system model used for the present PSAR analysis. 6

Table 4.2-1 Initial conditions for FLOWTRAN-TF LOHGA analysis

Table 6.1-1 FLOWTRAN-TF model results under helium tube rupture conditions. 


\section{Introduction}

The APT blanket system has about $57 \mathrm{MW}$ of thermal energy deposited within the blanket region under normal operating conditions from the release of neutrons and the interaction of the high energy particles with the blanket materials. This corresponds to about $48 \%$ of total thermal energy deposited in the APT target/blanket system [1]. The deposited thermal energy under normal operation (NO) conditions is an important input parameter used in thermal-hydraulic design and accident analyses. The thermal deposited power of the blanket system corresponds to the steady-state power of the $1700 \mathrm{Mev}$ APT design with 13 tungsten ladders.

The hazard analysis (HA) performed for the blanket primary heat removal (HR) systems identified the loss-of-helium gas accident (LOHGA), due to a compromise in the integrity of the helium gas supply system inside the Target/Blanket building, as a design basis accident. There are two types of Target/Blanket building LOHGAs:

1. Those internal to a blanket module (i.e., internal break (IB) LOHGA) where helium gas enters the blanket system's primary HR system coolant; and

2. Those external to a module (i.e., external break (EB) LOHGA) where helium gas does not enter the blanket system's primary HR system coolant.

LOHGAs vary from slow pin-hole leaks (Small Break LOHGA)-to a catastrophic failure of a helium tube resulting in the sudden release of a large volume of gas (Large Break LOHGA). In addition, two scenarios can be considered:

1. LOHGAs with the pressurizer relief valve remaining closed during the event; and

2. LOHGAs with the pressurizer relief valve opening and remaining open during the event.

When the relief valve remains closed, the helium/tritium gas mixture is contained within the blanket HR system. This gas can subsequently be recovered without release to the environment. If the relief valve opens, helium/tritium gas released into the blanket HR system could be vented to the environment. Therefore, this accident scenario would have onsite and possibly off-site consequences. In this report, two Large Break LOHGA accidents internal to the blanket module with the pressurizer relief valve remaining closed are analyzed. Future analyses addressing the varous other scenario options will be performed.

The helium gas supply system, manifolding, and blanket gas tubes have been designed to operate up to about 200 psia and down to a partial vacuum, which is required to evacuate the $\mathrm{He}^{3}$ gas. Two configurations for the operation of the Tritium Extraction Facility are being considered: (1) a continuous extraction of carrier gas and (2) an online batching process. Under this situation, the potential for wear during operation may cause a helium-3 gas leak from the high pressure gas system into the lower blanket coolant. Helium supply plenum break accidents were simulated to investigate the transient thermal-hydraulic response of the blanket system to helium gas release into the blanket primary coolant system. The helium gas leak accident from the pressurized gas system into the blanket coolant is simulated to occur under NO conditions. Based on LANL information [2], initial conditions for the helium reservoir are assumed to be 1 $\mathrm{m}^{3}$ helium gas volume, $200 \mathrm{psia}$, and $40 \mathrm{C}$ initial temperature. Break locations are 
simulated near the inlet and outlet plenums of the decoupler in a lateral Row-1 blanket module. In this report, Case 1, as the first of two cases of LOHGA presented, simulated a plenum break of the helium gas supply system near the inlet of the lateral module 1 decoupler using the one-dimensional TRAC system model with 6 lumped modules [3]. The break location for Case 1 is shown in Fig.1-1. In Case 2 the break in the helium gas supply was placed at the decoupler outlet of module 1. The break location for Case 2 is shown in Fig.1-2. The blanket system is completely enveloped within the enclosed system boundary to ensure that any hydrogen or tritium gas egress is contained and recovered even after the leak accident into the coolant.

The model results are used to determine if beam power shutdown is necessary (or not) as a result of the LOHGA accident to maintain the blanket system well below any of the thermal-hydraulic constraints imposed on the design. The results also provide boundary conditions to the detailed bin model to study the detailed temperature response of the hot blanket module structure. The results for these two cases are documented in this report.

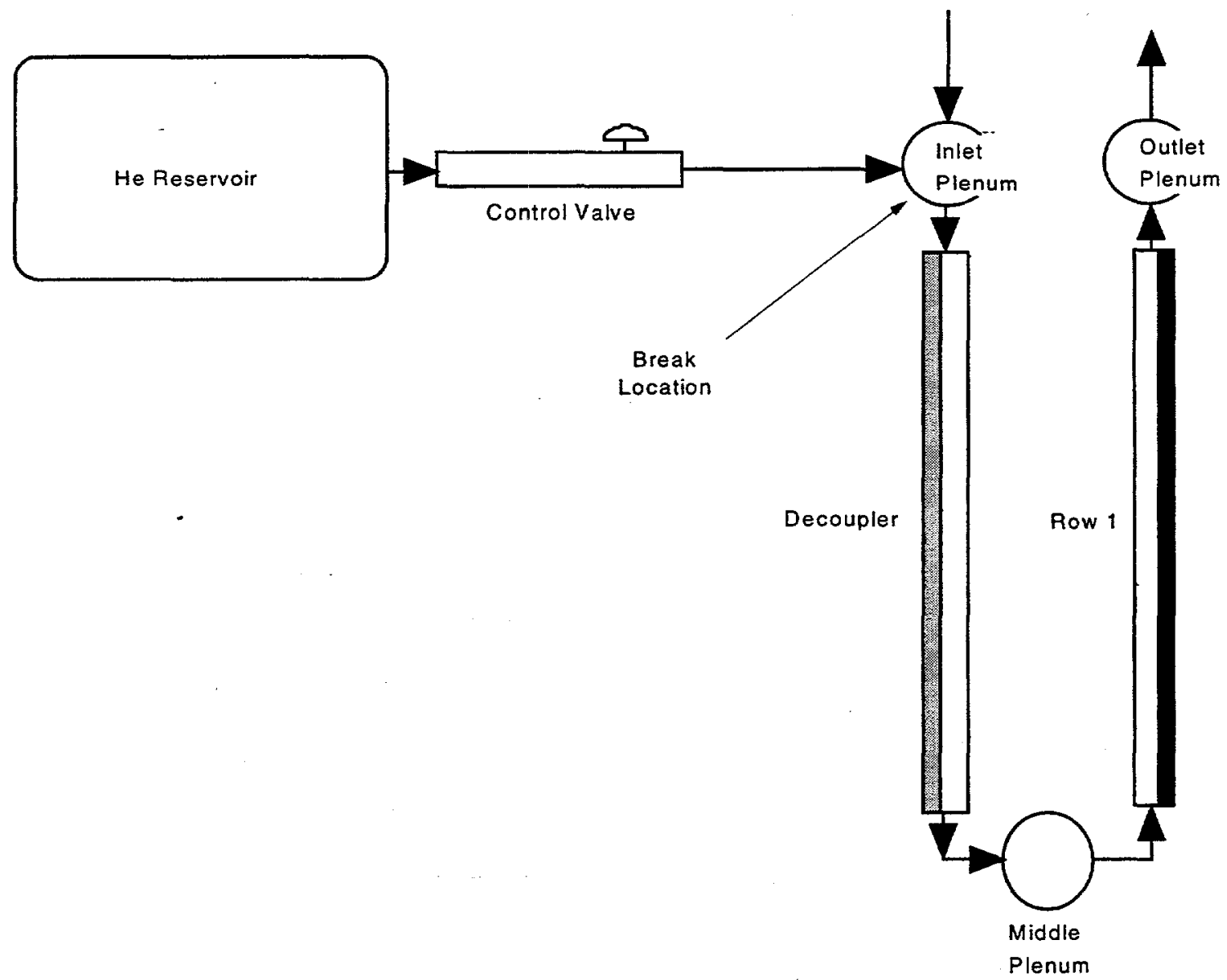

Figure 1-1 Break location for the helium tube rupture accident (Case 1: Break at lateral blanket Decoupler inlet plenum). 


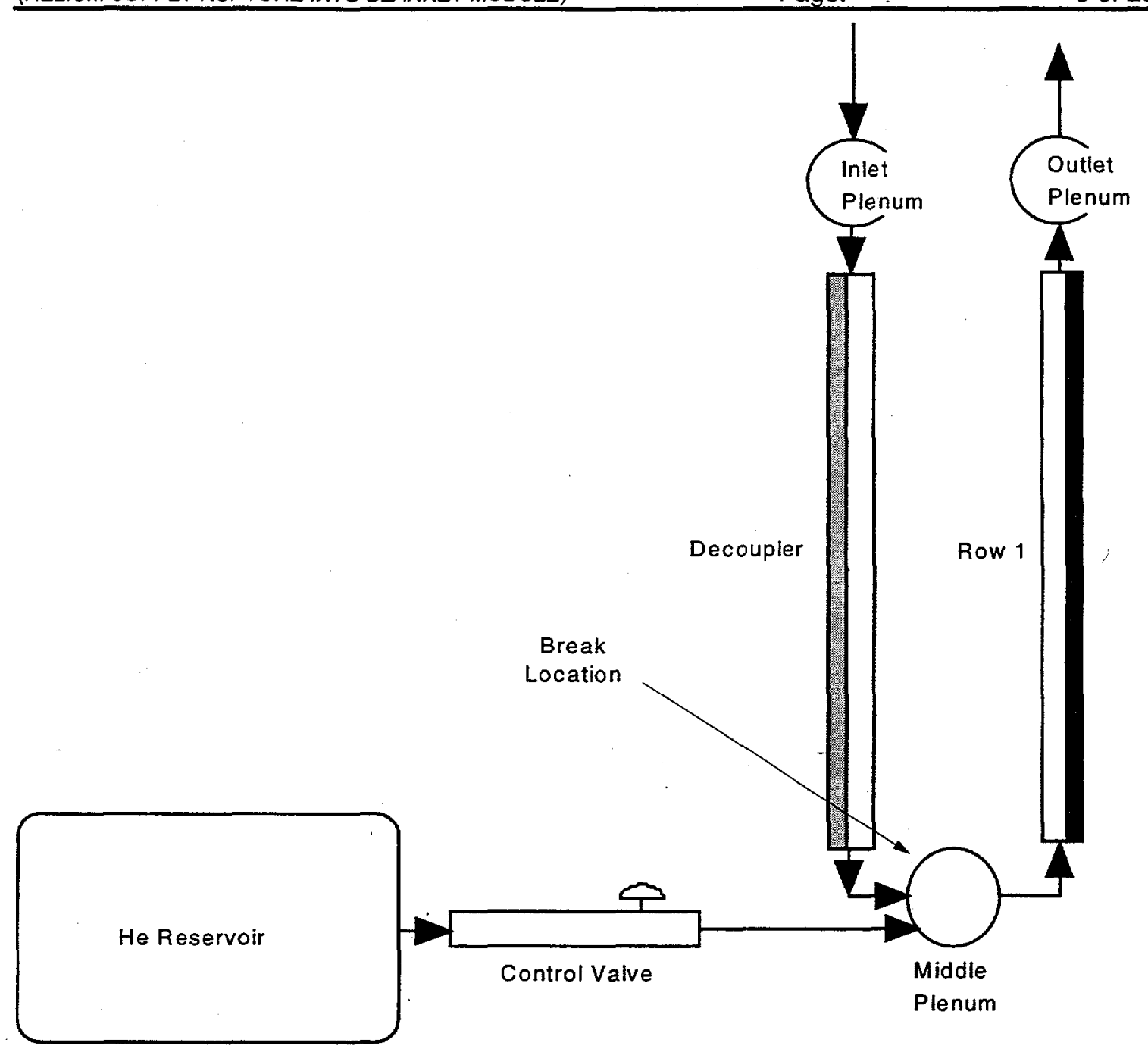

Figure 1-2 Break location for the helium tube rupture accident (Case 2: Break at lateral blanket Decoupler/ Row-1 middle plenum).

\section{TRAC 1-D System Model}

The current blanket system consists of 16 unique blanket modules based on the existing cruciform-type design components (the necessary design specifications required to develop a plate-type set of modules are currently not available). Each module is separately connected to the two fixed coolant headers. A lumping strategy was developed based on module similarity, deposited power levels, and locations that resulted in a total of six separate lumped modules and significantly reduced computational effort requirements. The six lumped modules are:

1. First-row lateral modules;

2. Second-row lateral modules;

3. Decoupler / Row 1 downstream modules;

4. Row 1 / Row 2 downstream modules; 
5. Row 2 / Row 2 downstream modules; and

6. Remaining low-power modules, which consist of the upstream module, the four bottom modules, and the two top modules.

Figure 2-1 shows a cross-sectional facemap for the six module blanket system. Table 2-1 summarizes module description, thermal deposited power, and connection pipe size of each of the six blanket modules as modeled in the one-dimensional lumped approach. Figure A-1 shows the locations of all six modules and the internal piping connections as included in the 1-D TRAC system model [3]. The TRAC components and piping connections for the HR, pressurizer, RHR blanket coolant systems, and cavity vessel flood system are also shown in Figs. A-2 to A-6. Table A-1 lists all the blanket system components, number of cells for the components, and the component descriptions for the present one-dimensional lumped blanket system model. The present six lumped module blanket system model includes 170 components and 152 junctions with 10 trip control signals for analyzing the transient LOHGA accident simulations.

\subsection{Scenario Description}

As design basis accidents, helium gas supply line breaks at the Decoupler inlet and outlet of the lateral module 1 shown in Fig. 2-1 were simulated by restarting steady-state normal operating results for the one-dimensional six module blanket system model in a transient mode. Normal operation conditions for the key blanket system parameters are shown in Table A-2. A detailed description of the lumped six module TRAC system model is provided in Ref. [3].

A Loss-of-Helium-Gas Accident (LOHGA) for Case 1 and Case 2 was simulated as a helium gas pipe break near the inlet and outlet of the Decoupler in the module 1, respectively. The break location for accident simulation of Case 1 is component number 370 shown in Fig. A-1. When helium gas is leaked into the blanket coolant system through the PLENUM component 370 (under NO conditions for the Case 1 simulation), the system rapidly pressurizes and the blanket coolant temperature increases shortly after the accident. Case 2 was simulated at PLENUM component 350, middle plenum of the lateral module 1 under the same accident conditions as those of Case 1. Normal system operation conditions are still maintained without any trip of beam power or heat removal pumps during the entire accident simulation of the LOHGA. Detailed control signals for the key component operations are provided later.

\subsection{Model Upgrades}

PLENUM and VALVE components were added to the original TRAC system model [3] for the LOHGA accident simulations. The plenum component was used for the simulation of the helium gas reservoir, and the valve component was used for transient simulation of the helium gas leakage. Since the details associated with the helium supply system is still under development, a simple plenum reservoir was deemed to be adequate for the present set of analyses. The control valve for the helium gas release into the blanket coolant system via the inlet/outlet Decoupler of the blanket module 1 was opened completely within 0.1 seconds. The valve is actuated within $10^{-4}$ seconds after the accident. The system is maintained under the normal operating conditions 
shown in Fig. A-2 during the accident simulation. Constrained steady-state (CSS) TRAC options were used to establish the NO prior to the transient runs.

\subsection{Initial Conditions}

Before simulating the accident condition, steady-state results under NO conditions are required to provide initial input to the transient simulation model of the LOHGA (see Ref. [4]). Based on personal communication with Jack N. Edwards at LANL [2], the initial volume and pressure of the helium reservoir system were specified to be $1 \mathrm{~m}^{3}$ and 200 psia, respecitvely. The initial temperature for the helium reservoir components is assumed to be $40 \mathrm{C}$, which is the initial condition of the pressurizer, RHR system, and $\mathrm{HR}$ heat exchangers. $40 \mathrm{C}$ corresponds to the maximum expected building air temperature during operating conditions. Table A-2 shows the initial steady-state values for the key system parameters of the blanket system.

\subsection{Transient Boundary Conditions}

The TRAC system model is documented in the report for the steady state NO calculations $[3,4]$. The NO TRAC run was restarted in transient mode to initiate the LOHGA simulations of Case 1 and Case 2. The helium gas reservoir component was a PLENUM component (component 430) connected to the inlet and outlet plenums of the module 1 Decoupler using the VALVE component (component 440). The helium gas line break location was selected to be close to the cold-leg downflow region where the primary coolant is distributed into the parallel multi-channel region of the blanket module system. For the LOHGA initiation, the VALVE component 440 was actuated within $10^{-4}$ seconds using the control signal logic to simulate a helium gas release into the primary. coolant system. The VALVE component was set to change from closed to complete open within 0.1 seconds after initiation of the accident. The valve size was assumed to be 3 inch in diameter. The other blanket system conditions during the entire period of the accident simulation (600 seconds after the accident) remained the same as the NO conditions.

\subsection{Trips and Controls}

Normal operating power and system conditions were maintained during the entire transient simulation period beyond the initiation of the LOHGA accident. Normal operation trips to the beam power and HR pumps were assumed to occur. The helium release valve on the discharge side of the helium reservoir was actuated in 0.1 seconds after the initiation of the LOHGA accident. We note that pressurizer relief valve settings have not been determined and that this analysis needs to be performed. The analysis presented here assumes that the relief valve remain closed throughout the entire simulation period.

\subsection{TRAC Version}

This transient model was run using TRAC-PF1/MOD2 version 5.4.28a [10]. A modified version of TRAC to generate graphics files was employed [9]. 
Table 2-1 6 lumped blanket module system model used for the present PSAR analysis.

\begin{tabular}{|c|c|c|c|}
\hline $\begin{array}{l}\text { Stix } \\
\text { Lumped } \\
\text { Modules }\end{array}$ & $\begin{array}{l}\text { Prototypic } 16 \text { Full Blanket } \\
\text { Modules }\end{array}$ & $\begin{array}{c}\text { Thermal Deposited } \\
\text { Power } \\
\text { Downflow / Upflow } 1 \\
\text { Total Power }\end{array}$ & $\begin{array}{l}\left(\mathrm{plpe}^{2}\right. \\
(\mathrm{Size}) \\
(\mathrm{inch})\end{array}$ \\
\hline Module 1 & $\begin{array}{l}\text { Front } 1^{\text {st }} \text { Lateral Decoupler / Row } \\
1 \text { Module } \\
\text { Back } 1^{\text {st }} \text { Lateral Decoupler / Row } \\
1 \text { Module }\end{array}$ & $\begin{array}{l}8.222 \mathrm{MW} / \\
15.768 \mathrm{MW} / \\
23.990 \mathrm{MW}\end{array}$ & 7.500 \\
\hline Module 2 & $\begin{array}{c}\text { Front } 2^{\text {nd }} \text { Lateral Row } 2 / \text { Row } 3 \\
\text { Modules } \\
\text { Back } 2^{\text {nd }} \text { Lateral Row } 2 / \text { Row } 3 \\
\text { Modules }\end{array}$ & $\begin{array}{l}3.060 \mathrm{MW} / \\
7.660 \mathrm{MW} / \\
10.720 \mathrm{MW}\end{array}$ & 4.750 \\
\hline Module 3 & $\begin{array}{c}1^{\text {st }} \text { Downstream Dec. / Row } 1 \\
\text { Module }\end{array}$ & $\begin{array}{c}0.744 \mathrm{MW} / 2.812 \mathrm{MW} / \\
3.556 \mathrm{MW} \\
\end{array}$ & 3.750 \\
\hline Module 4 & $\begin{array}{l}2^{\text {nd }} \text { Downstream Row } 1 / \text { Row } 2 \\
\text { Module }\end{array}$ & $\begin{array}{c}3.924 \mathrm{MW} / 5.412 \mathrm{MW} / \\
9.336 \mathrm{MW}\end{array}$ & 5.375 \\
\hline Module 5 & $\begin{array}{l}3^{\text {rd }} \text { Downstream Row } 2 / \text { Row } 2 \\
\text { Module }\end{array}$ & $\begin{array}{c}1.355 \mathrm{MW} / 1.811 \mathrm{MW} / \\
3.167 \mathrm{MW}\end{array}$ & 6.000 \\
\hline Module 6 & $\begin{array}{c}\text { Low Power Modules } \\
\text { Blanket Upstream Decoupler / } \\
\text { Row } 2 \text { Module } \\
\text { Lower Front Decoupler / Row } 2 \\
\text { Module } \\
\text { Lower Front Row } 2 \text { / Row } 2 \\
\text { Module } \\
\text { Lower Back Decoupler / Row } 2 \\
\text { Module } \\
\text { Lower Back Row } 2 \text { / Row } 2 \\
\text { Module } \\
\text { Upper Front Row } 2 \text { / Row } 2 \\
\text { Module } \\
\text { Upper Back Row } 2 \text { / Row } 2 \\
\text { Module }\end{array}$ & $\begin{array}{c}\text { (Horizontal Flow) } \\
0 \mathrm{MW} / \\
5.712 \mathrm{MW} / \\
5.712 \mathrm{MW}\end{array}$ & 3.875 \\
\hline $\begin{array}{c}\text { Total } \\
\text { Deposited } \\
\text { Power }\end{array}$ & & $\begin{array}{l}17.305 \mathrm{MW} / 39.175 \\
\mathrm{MW} / 56.480 \mathrm{MW}\end{array}$ & \\
\hline
\end{tabular}




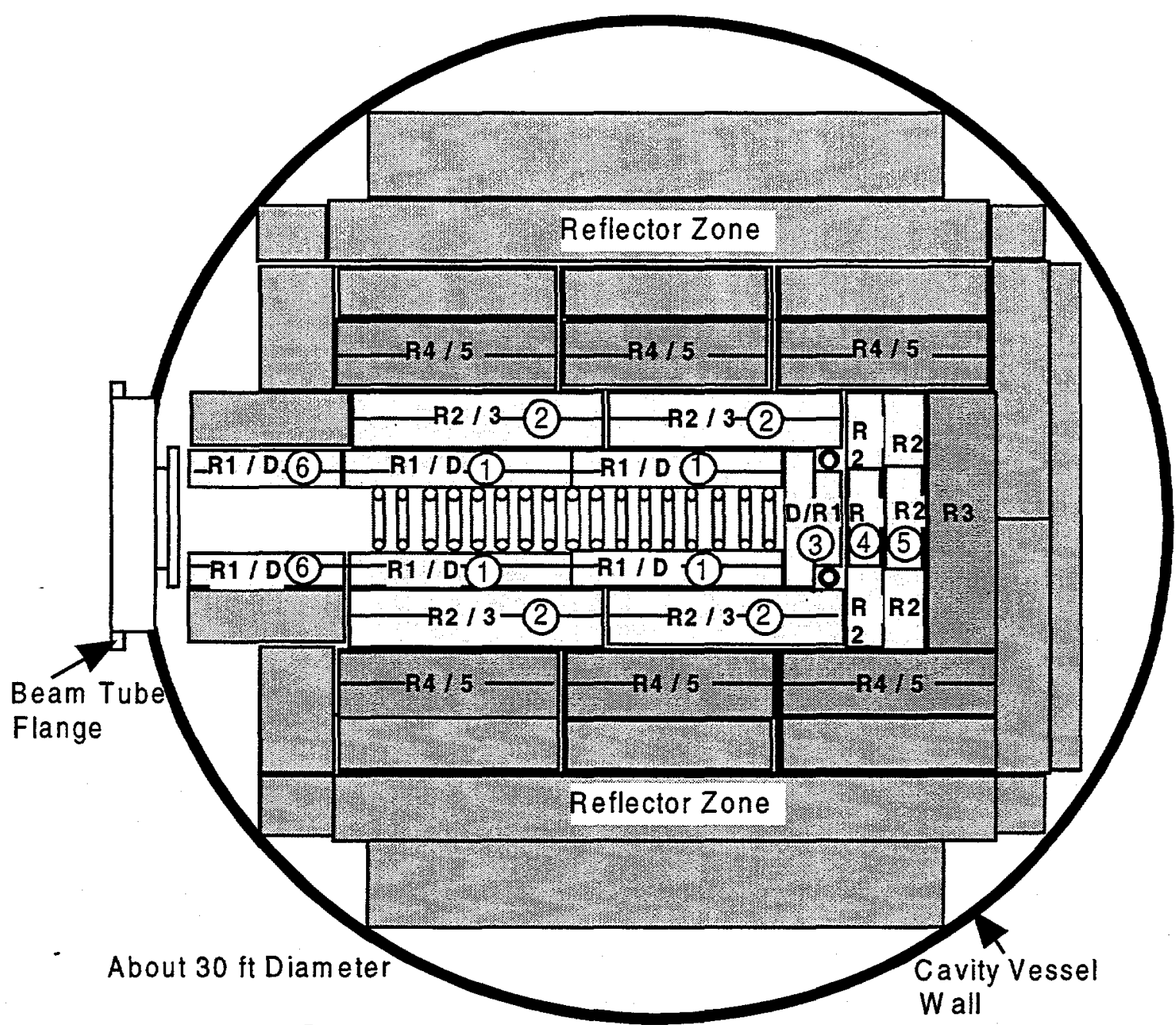

(6) Snout, 4 Bottom Modules, and 2 Top Modules

Figure 2-1 Top cross-sectional facemap of 6 lumped blanket system modules.

\section{TRAC System Model Results}

The TRAC simulation model for the rupture of a helium tube was run for 600 seconds after initiation of the accident. Simulation results show that the APT system can withstand the release of $1.0 \mathrm{~m}^{3}$ of helium into the blanket module without any damage to the blanket module system and structure. No safety systems need to be activated to mitigate this event. The following subsections provide (in more detail) descriptions of the transient phenomena for the key blanket component systems observed in the 
accident simulation. Since none of the safety systems are activated during this transient, the RHR loop, cavity vessel and flood system remain in their normal operating states throughout the accident and will not be discussed.

Results are presented in graphical form in Appendix B from the TRAC system model calculations for a helium tube rupture in PLENUM component 370 (decoupler inlet). Section B1 of Appendix B shows output for the TRAC plenum components and Section B2 of Appendix B contains the output for pipe, pump and valve components. Similar results from the TRAC system model calculations for a helium tube rupture in PLENUM component 350 (decoupler outlet) are presented in graphical form in Appendix C. A listing of the TRAC input file used for the calculations of the system response to the helium rupture is provided in Appendix $D$ and a listing of the graphics input file used to create the graphical output is given in Appendix $\mathrm{E}$.

Results from the two helium tube rupture cases are very similar. The primary difference with respect to the impact on the blanket module is that channel voiding occurs immediately with a tube rupture at the decoupler outlet while there is about a two second delay in channel voiding when the gas is released into the decoupler inlet. Reduction in coolant flow, increase in void fraction, and increase in system pressure are similar for the two cases. The following sections of this report give a brief discussion of the TRAC results for the case of a helium tube rupture at PLENUM component 350 (decoupler outlet).

\subsection{Helium Supply}

The helium supply is assumed to be a $1 \mathrm{~m}^{3}$ tank at 200 psia. Figure $\mathrm{C}-1$ a shows the transient pressure in the helium gas supply system. The valve between the helium supply and the break location is opened within 0.1 second. Since the blanket system pressure is 100 psia, the helium in the supply system quickly expands to a volume of 2 $\mathrm{m}^{3}$ as the pressures equilibrate at slightly above 100 psia releasing $1 \mathrm{~m}^{3}$ into the blanket module. As shown in Fig. C-1d, following this initial release, there is a slow replacement of the helium in the supply system from water in-leakage and another $0.5 \mathrm{~m}^{3}$ of helium is released into the blanket module over the next 500 seconds.

\subsection{Modules}

Figure A-1 shows the component layout of the internal blanket module system used in the TRAC system model. Transient results for coolant hydraulic parameters through the six blanket upward flow regions from the simulation of a helium tube rupture in TRAC component 350 are graphically shown in Figs. C-3a through C-11d. The results show a rapid transient behavior in the flow channels of all six blanket modules continuing out to about 60 seconds after initiation of the accident. The most significant part of the transient behavior is confined to the first few seconds of the accident when there is a rapid pressurization of the HR system as the helium tube ruptures. However, as shown in Fig C-3a for module 1, the pressure recovers to nearly the initial operating value after about 10 seconds followed by some small oscillations about that point. Pressure responses in the other modules are similar to that seen in module 1. 
Figure $\mathrm{C}-3 \mathrm{~b}$ shows that the fluid temperature at the inlet to the blanket modules increases by only $1.0 \mathrm{C}$ at the start of the transient. Figure C-3d shows channel void fractions at the top, middle and bottom plenums in module 1 . Since the helium tube rupture occurs at PLENUM component 350 , the void fraction at this location almost immediately increases to $16 \%$ and returns to nearly zero after a few seconds. Void fractions in the other modules not directly connected to the gas release are very small. As shown in Fig. C-21d, fluid flow in module 1 is momentarily decreased for a few seconds at the start of the transient to about $80 \%$ of its normal operating value and quickly recovers. Fluid temperature, void fraction, and coolant flow responses in the other modules are significantly smaller than that observed in module 1.

As seen in Figs. C-23a through C-24e, similar patterns are observed in all of the modules for the operating temperature and pressure transients. The release of the pressurized helium into the blanket modules produces an almost instantaneous increase in pressure. Only module 1, where the helium is released, experiences a significant increase in void fraction and corresponding decrease in coolant flow. The initial transient response is short lived with recovery of all system parameters to near normal operating values within a few seconds of the gas release. After the first 10 seconds, during which the significant transient response occurs, there is a period of about 50 seconds where the operating values oscillate about their steady-state conditions. Beyond 60 seconds, all of the module parameters have essentially returned to the normal operating values.

\subsection{Primary HR Loop}

Figure A-4 shows the HR system component layout used for the one-dimensional TRAC system model. With the initiation of a helium tube rupture, the system pressure in the HR loop increases rapidly. Figure $\mathrm{C}-2 \mathrm{a}$ shows fixed header fluid pressures for the simulation of a helium tube rupture near the decoupler outlet. Pressures in the hot and cold legs increase steeply following initiation of the accident. The results show that the most significant transient response in the fixed header pressures occurs within about 20 seconds. Pressures of both fixed headers are stabilized at the normal operating values about 60 seconds after the accident occurs as shown in Fig. C-2a. As shown in Fig. C$2 d$, the void fraction in the fixed header downstream of the gas release reaches a maximum value of just over $6 \%$ within a few seconds of the tube rupture and is less than $1 \%$ beyond about 30 seconds.

Similar responses are shown throughout the primary HR piping (Figs. C-13a through C$13 e$ and $\mathrm{C}-16 \mathrm{a}$ through $\mathrm{C}-19 \mathrm{e}$ ) and in the coolant pumps (Figs. C-14a through $\mathrm{C}-15 \mathrm{e}$ ). All of the systems experience a sudden pressure increase, relatively small temperature increases and void fractions of $12 \%$ or less. One exception is the primary HR heat exchanger outlet piping void fractions shown in Fig. $\mathrm{C}-18 \mathrm{e}$. This is the highest elevation in the system and is the point where the released helium gas accumulates. The void fraction at this location increases from zero to about $12 \%$ at 20 seconds when the released gas reaches that component. Thereafter, the void increases steadily to over $25 \%$ at 500 seconds as helium is slowly displaced from the source volume by water. As shown in Fig. C-20d, a brief flow transient is induced in the pressurizer and surge line by the pressure fluctuations occurring when the helium tube ruptures. 


\section{FLOWTRAN-TF Detailed Bin Model}

The basic FLOWTRAN-TF model of a single APT blanket plate is described in Refs. $[6,7]$ and was used in this version for the helium tube rupture analyses. The FLOWTRAN-TF model was developed to simulate the thermal-hydraulic conditions in a lateral Row 1 blanket module using the Reference 1 plate-type design [5]. The crosssectional mesh of the plate model is shown in Figure 4-1 along with the location and indexing used for the 12 discrete flow channels.

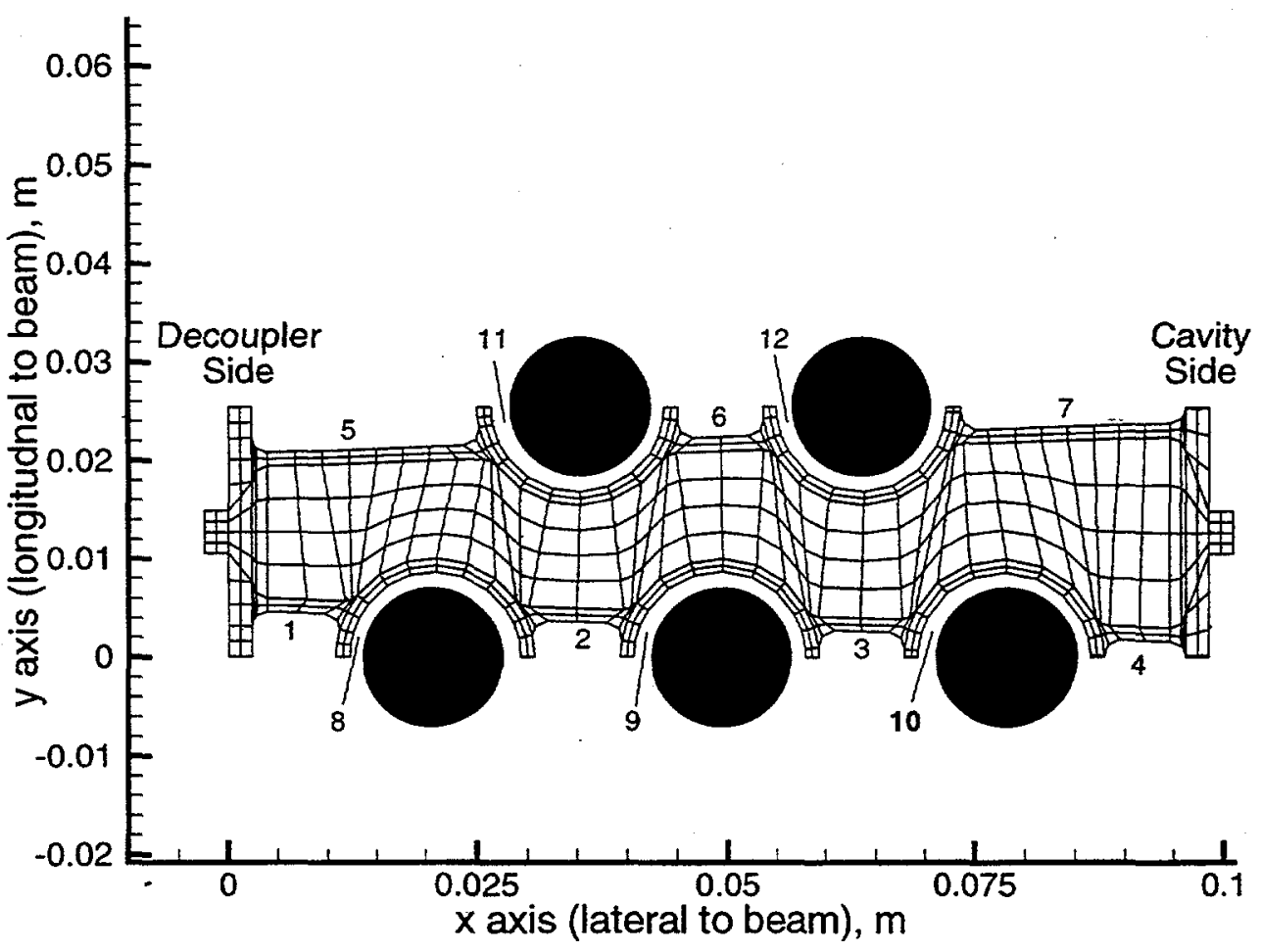

Figure 4-1 Finite element mesh of APT reference 1 lateral row 1 blanket plate.

\subsection{Model Upgrades}

No modifications to the original plate model were required for the helium tube rupture analyses.

\subsection{Initial Conditions}

Initial conditions for all of the flow transients are the normal operating conditions reported in Ref. [4]. The TRAC supplied initial conditions used in the FLOWTRAN-TF calculations are listed in Table 4.2-1. 
Table 4.2-1 Initial conditions for FLOWTRAN-TF LOHGA analysis.

\begin{tabular}{|c|c|c|c|}
\hline & Pressure (MPa) & Temperature (G) & Void \\
\hline Channel Inlet & 0.686 & 53.03 & 0 \\
Channel Outlet & 0.584 & - & - \\
\hline
\end{tabular}

\subsection{Transient Boundary Conditions}

Results from the TRAC LOHGA transient were used to supply boundary conditions to the FLOWTRAN-TF code for detailed calculations of the thermal-hydraulic behavior of a single Row 1 blanket plate. The TRAC transient results used in these calculations were:

1. Total coolant flow to the lateral Row 1 blanket module taken from TRAC PIPE component 454.

2. Inlet cooling water temperature, pressure and void fraction taken from TRAC PLENUM component 350. The calculations used inlet flow as an applied boundary condition and inlet pressure was used only for physical property evaluations.

3. Outlet pressure taken from TRAC PLENUM component 330.

Inlet conditions derived from the TRAC system analysis are shown in Figs. 4.3-1 and 4.3-2 for a helium tube rupture in TRAC PLENUM component 350 and in Figs. 4.3-3 and 4.3-4 for a helium tube rupture in TRAC PLENUM component 370. The boundary conditions are plotted as transient values relative to the pre-incident conditions. The pre-incident flow to a single blanket plate is taken to be $1.488 \mathrm{~kg} / \mathrm{s}$. Since void fraction naturally falis between zero and one, absolute values of void fraction are plotted in the figure.

The FLOWTRAN-TF transient calculations were run for 300 seconds using the boundary conditions shown in Figs. 4.3-1 and 4.3-3. For the helium tube rupture cases, a significant amount of void fraction appears at the inlet to the coolant flow channels within the first 10 seconds of the transient. The pressure and coolant flow respond to the introduction of pressurized gas during the early period of the transient and then return to close to pre-incident operating conditions. To better show the significant boundary conditions that were used in the detailed bin calculations, the first 60 seconds of the two accident scenarios are plotted in Figs. 4.3-2 and 4.3-4. 
WESTINGHOUSE SAVANNAH RIVER COMPANY

APT BLANKET SYSTEM FOR LOHGA

(HELIUM SUPPLY RUPTURE INTO BLANKET MODULE)
Report:

Section:

Date:

Page:

WSRC-TR-98-00177
1
$07 / 20 / 98$
12 of 28

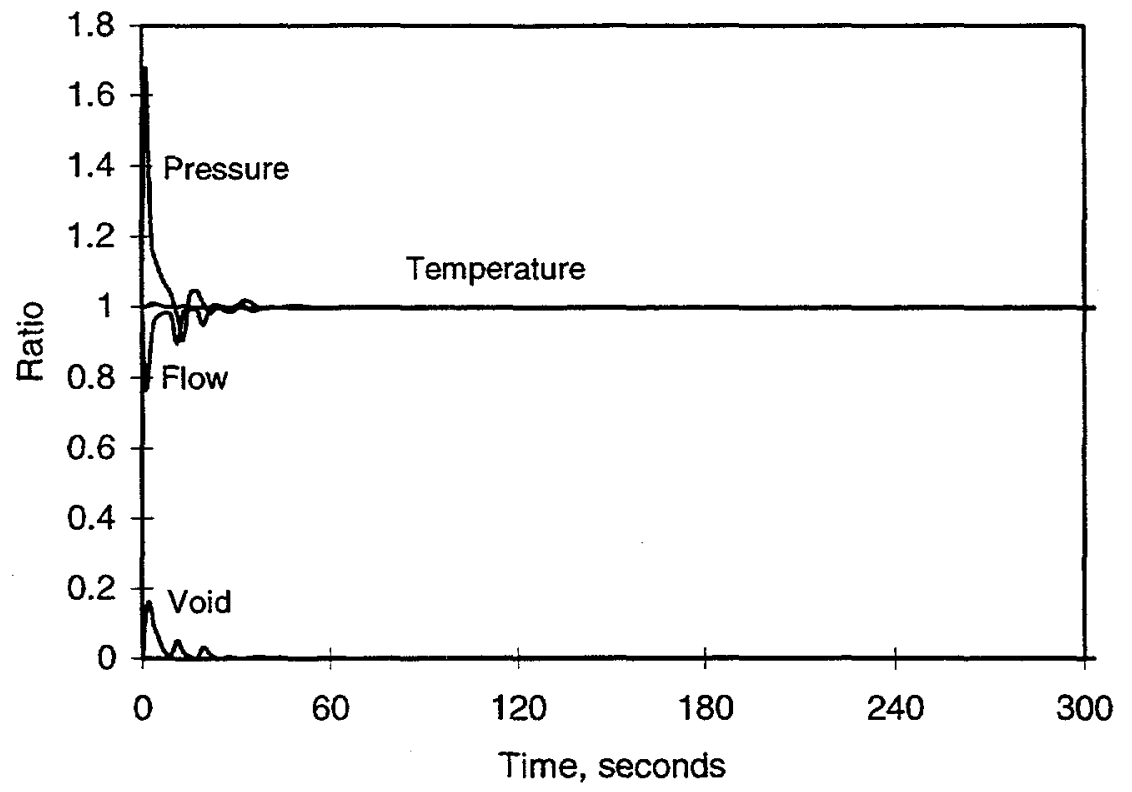

Figure 4.3-1 inlet boundary conditions for helium tube rupture at TRAC component 350 out to 300 seconds.

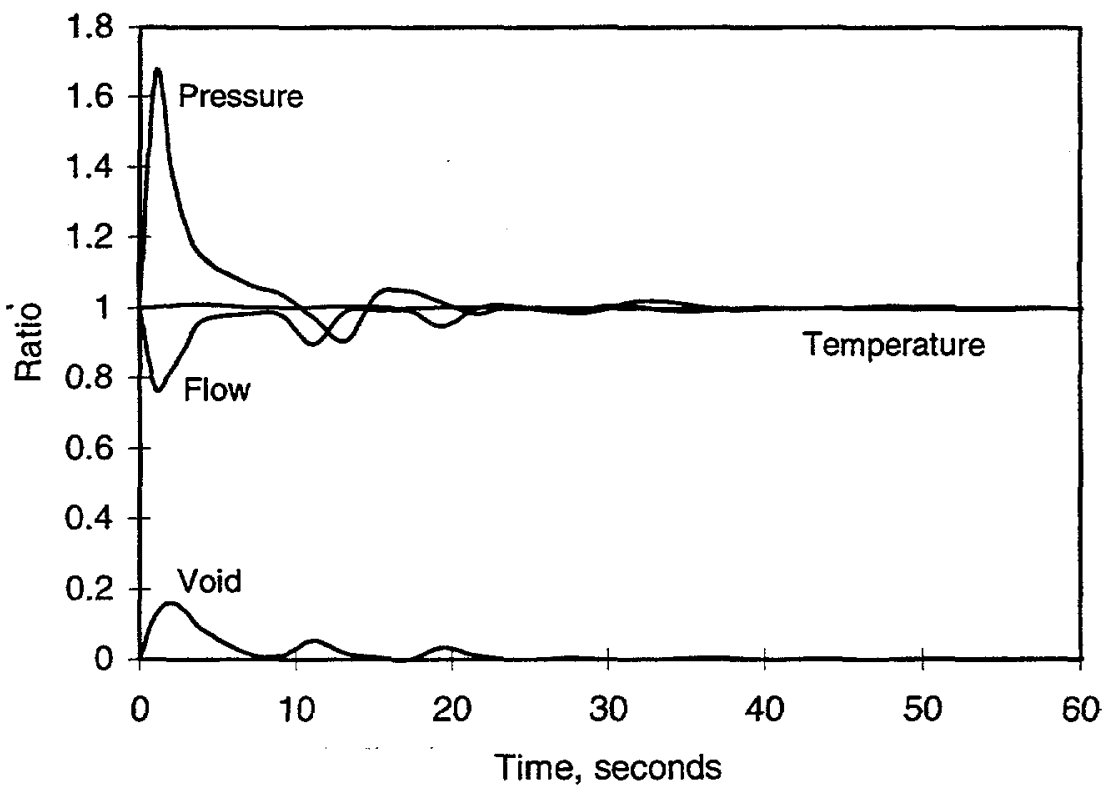

Figure 4.3-2 Inlet boundary conditions for helium tube rupture at TRAC component 350 out to 60 seconds. 


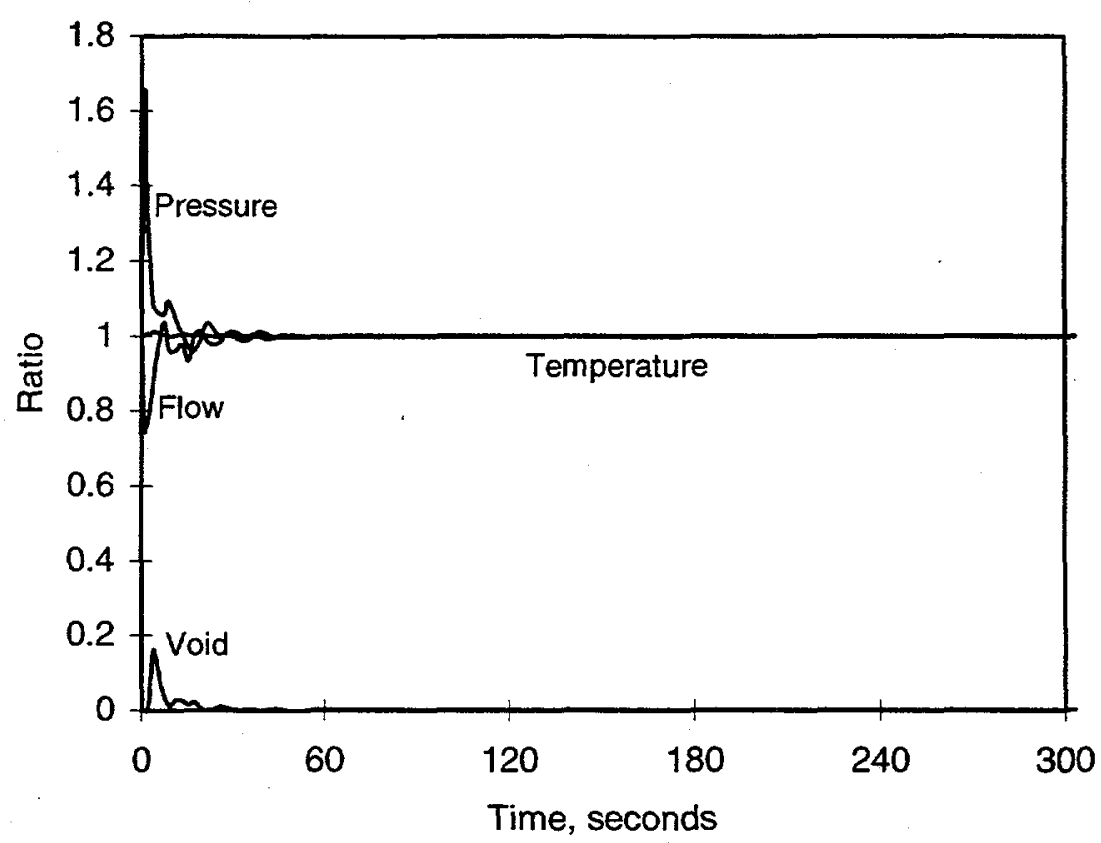

Figure 4.3-3 Inlet boundary conditions for helium tube rupture-at TRAC component 370 out to 300 seconds.

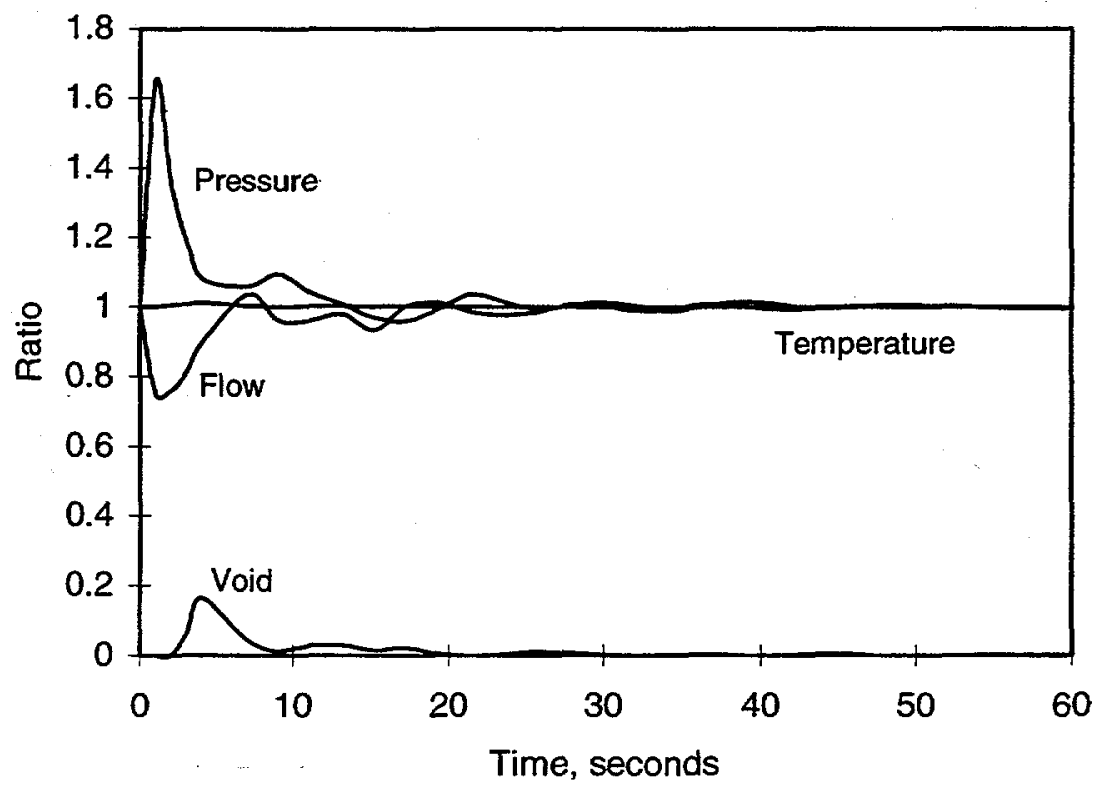

Figure 4.3-4 Inlet boundary conditions for helium tube rupture at TRAC component 370 out to 60 seconds. 


\section{FLOWTRAN-TF Detailed Bin Model Results}

Results from the two sets of FLOWTRAN-TF calculations at the different break locations are presented in the following sections of this report. In both of these sets of calculations, it is assumed that the accelerator beam is not tripped during the accident and that the total deposited power in the plate remains constant at the nominal preincident value of $61.5 \mathrm{~kW}$. At steady-state pre-incident conditions, the initial maximum aluminum temperature is about $100.0 \mathrm{C}$ while the initial maximum lead temperature is about $113 \mathrm{C}$. Maximum metal temperatures occur in the region of the plate nearest the decoupler side and closest to the point of beam impact. The highest metal temperatures were found at axial level 12 in the model. A partial listing of the input deck used for the FLOWTRAN-TF calculations of the plate response to a helium tube rupture at TRAC component 350 is given in Appendix $F$.

\subsection{FLOWTRAN-TF Results for Component 350 Rupture}

Maximum metal temperatures from the FLOWTRAN-TF calculations for a rupture in the helium tube at TRAC component 350 are shown in Fig. 5.1-1. The maximum aluminum temperature found anywhere in the plate cladding and the maximum lead temperature anywhere in the plate are plotted as functions of time into the accident transient. The two figures are plotted on the same scale for easier comparison. The decrease in coolant flow in the early part of the transient leads to a temperature increase in the metal of only about $2.5 \mathrm{C}$. As expected, the metal temperature returns to its initial value as the flow disturbance passes. It is clear from the plotted metal temperatures that surface temperatures in the flow channels following beam trip are not close to local boiling conditions. At typical module pressures, the saturation temperature is around $165 \mathrm{C}$. At no point in the transient does the flow channel become completely voided.

To further illustrate safety margins in the plate type design, operating surface heat fluxes $\left(q_{o h f}\right)$ were compared to the heat fluxes predicted for onset of nucleate boiling ( $\left.q_{\text {onb }}\right)$, onset of significant void formation $\left(q_{\text {osv }}\right)$ and the critical heat flux $\left(q_{\text {chf }}\right)$. Since each of the 12 flow channels has 20 axial cells and at each axial level the surface is composed of from 8 to 17 surface nodes there are many (2760) surface heat fluxes at any time in the calculation. To reduce the number of data points, we have taken the approach of reporting the maximum heat flux at each axial position that is within the channel irrespective of the particular surface node where the maximum occurs. Further we can restrict the examination to the location of the peak axial heat fluxes. The highest operating surface heat fluxes were observed to occur in axial cells 10 through 15 of the 20 cells that were used to discretize the axial dimension for the safety calculations.

To show some representative results, the operating surface heat flux and the calculated limits for the helium tube rupture in component 350 are shown in Figs. 5.1-2 through 5.1-4 for all of the flow channels at axial level 10. Channels 1 and 5 are the rectangular flow channels nearest the decoupler side of the plate while channel 8 is the first annular flow channel on that side (see Fig. 4.1). The decoupler side of the plate absorbs the largest fraction of the deposited power and has a larger fraction of the coolant flow. Channels 4 and 7 are the rectangular flow channels nearest the cavity side of the plate where the deposited power and coolant flow are lowest. 
Results from other axial locations on the plate surface where there is significant deposited power are similar demonstrating a substantial margin between the surface heat flux and the potentially limiting values. The closest approach of the operating heat flux to the limiting values typically occurs in flow channels 1 or 8 . Near the top of the plate, the surface heat flux can reverse as energy flows from the heated water back into the cooler metal. This condition was observed in the flow channels in regions of the plate where the deposited power was low.
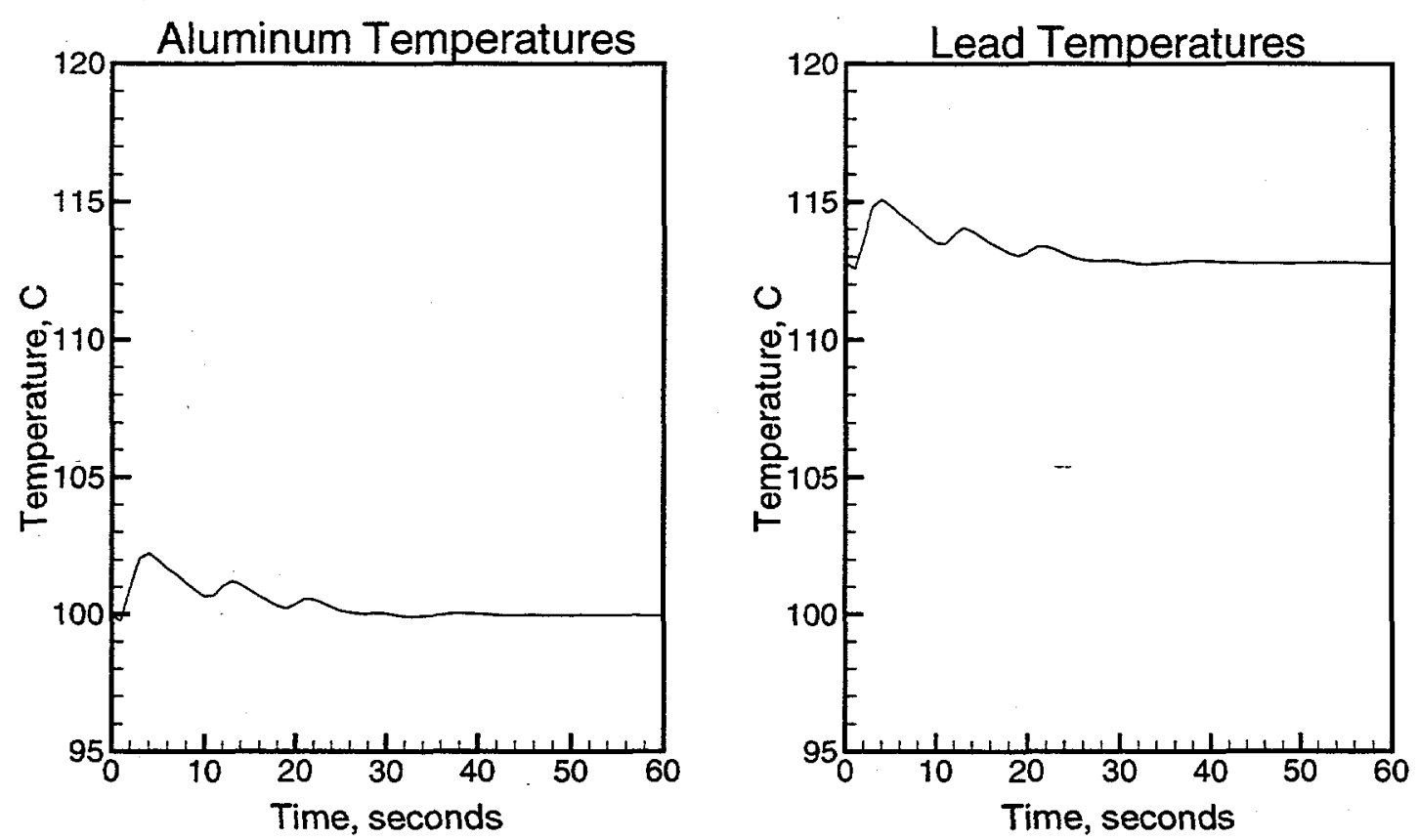

Figure 5.1-1 Maximum metal temperatures for helium tube rupture at TRAC component 350 out to 60 seconds. 
Channel 1

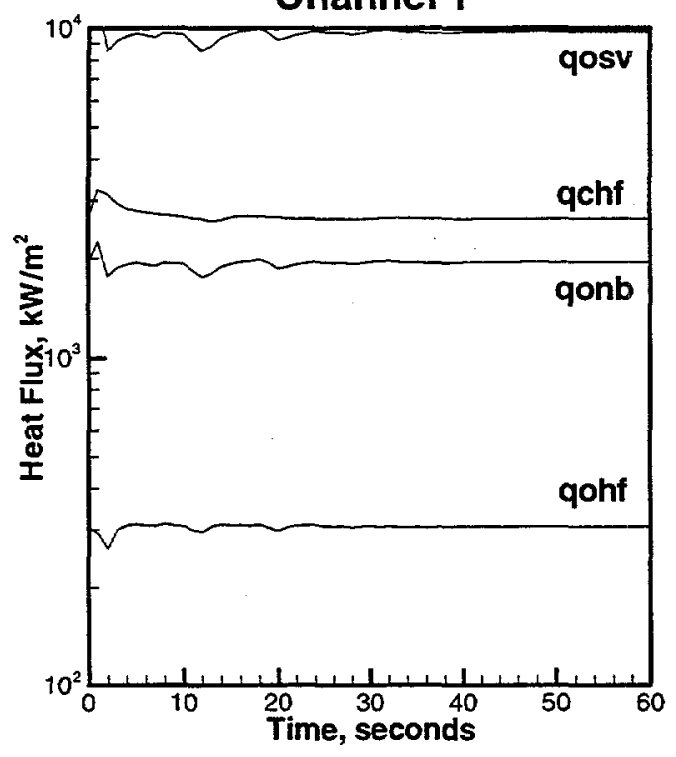

Channel 2

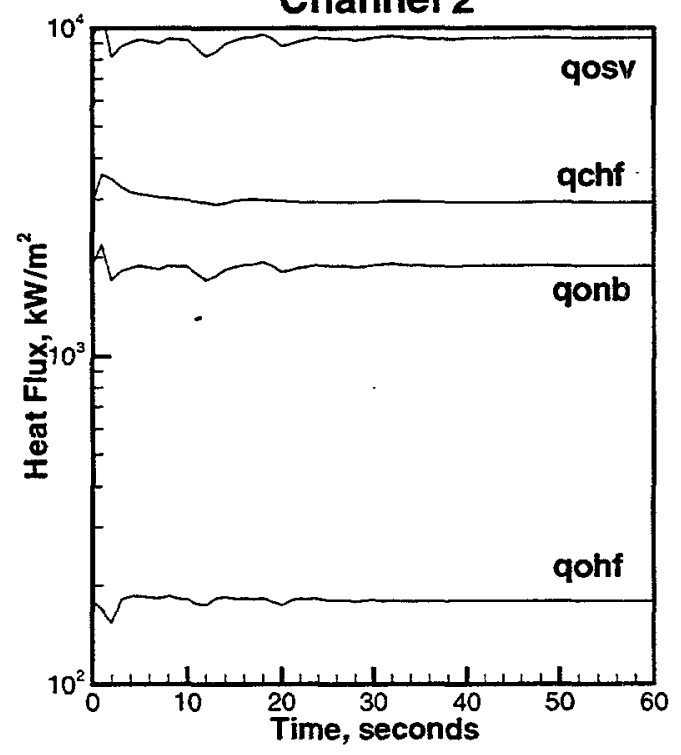

Channel 3

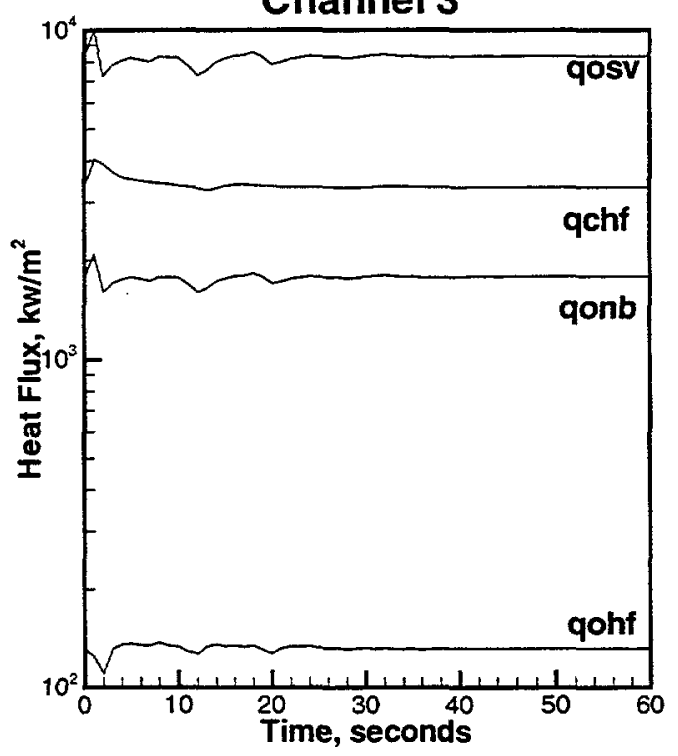

Channel 4

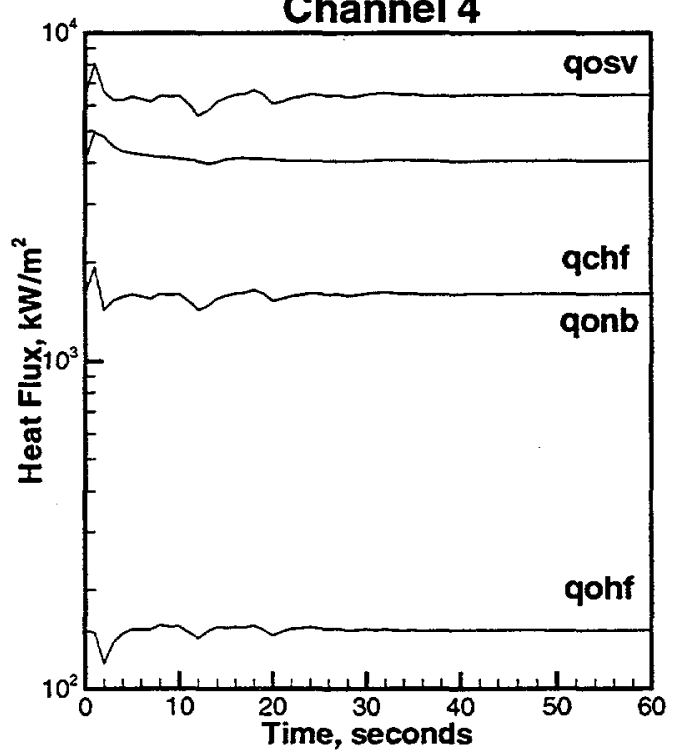

Figure 5.1-2 Surface heat fluxes for helium tube rupture at TRAC component 350 out to 60 seconds, axial level 10, channels 1-4. 

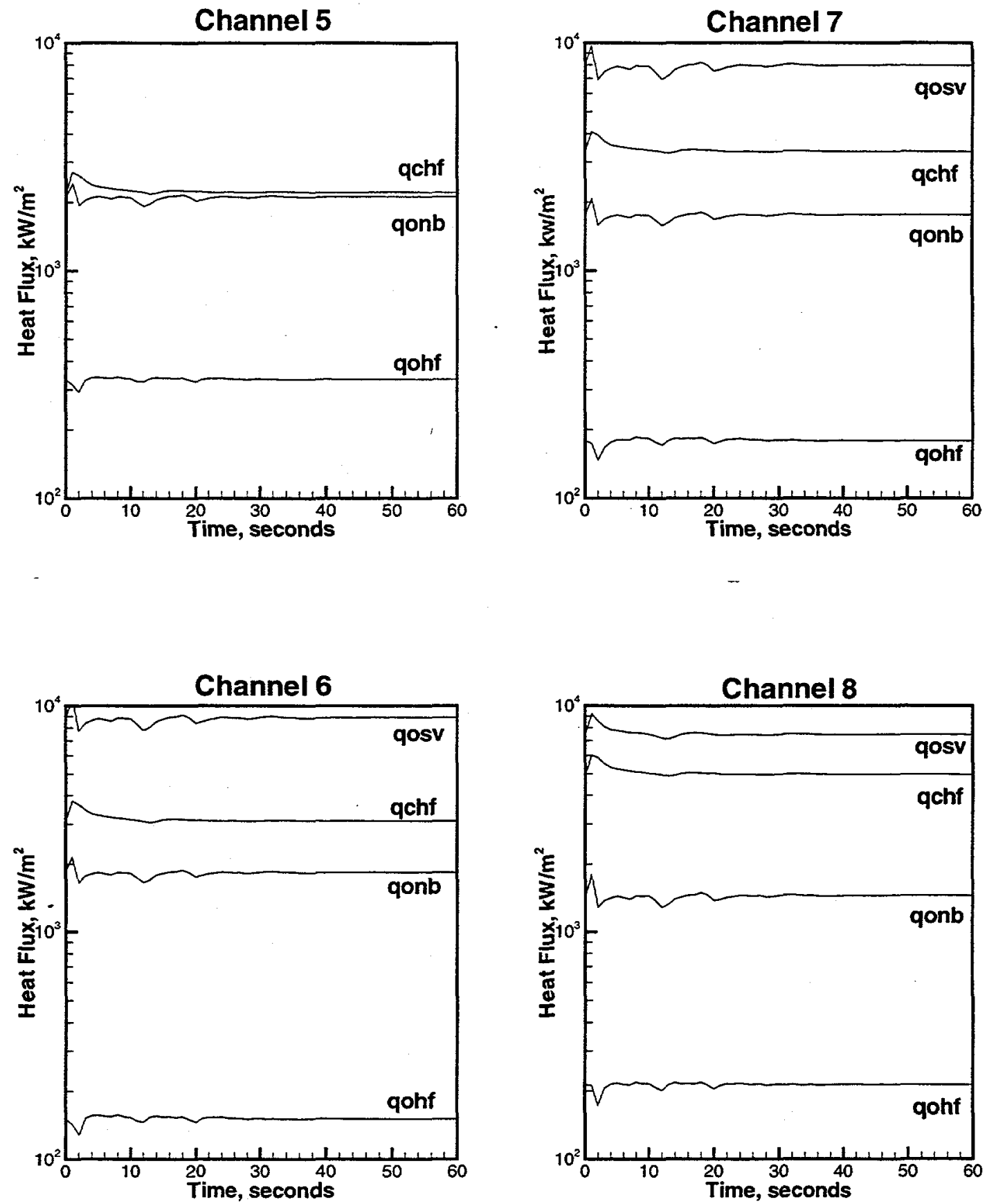

Figure 5.1-3 Surface heat fluxes for helium tube rupture at TRAC component 350 out to 60 seconds, axial level 10, channels 5-8. 

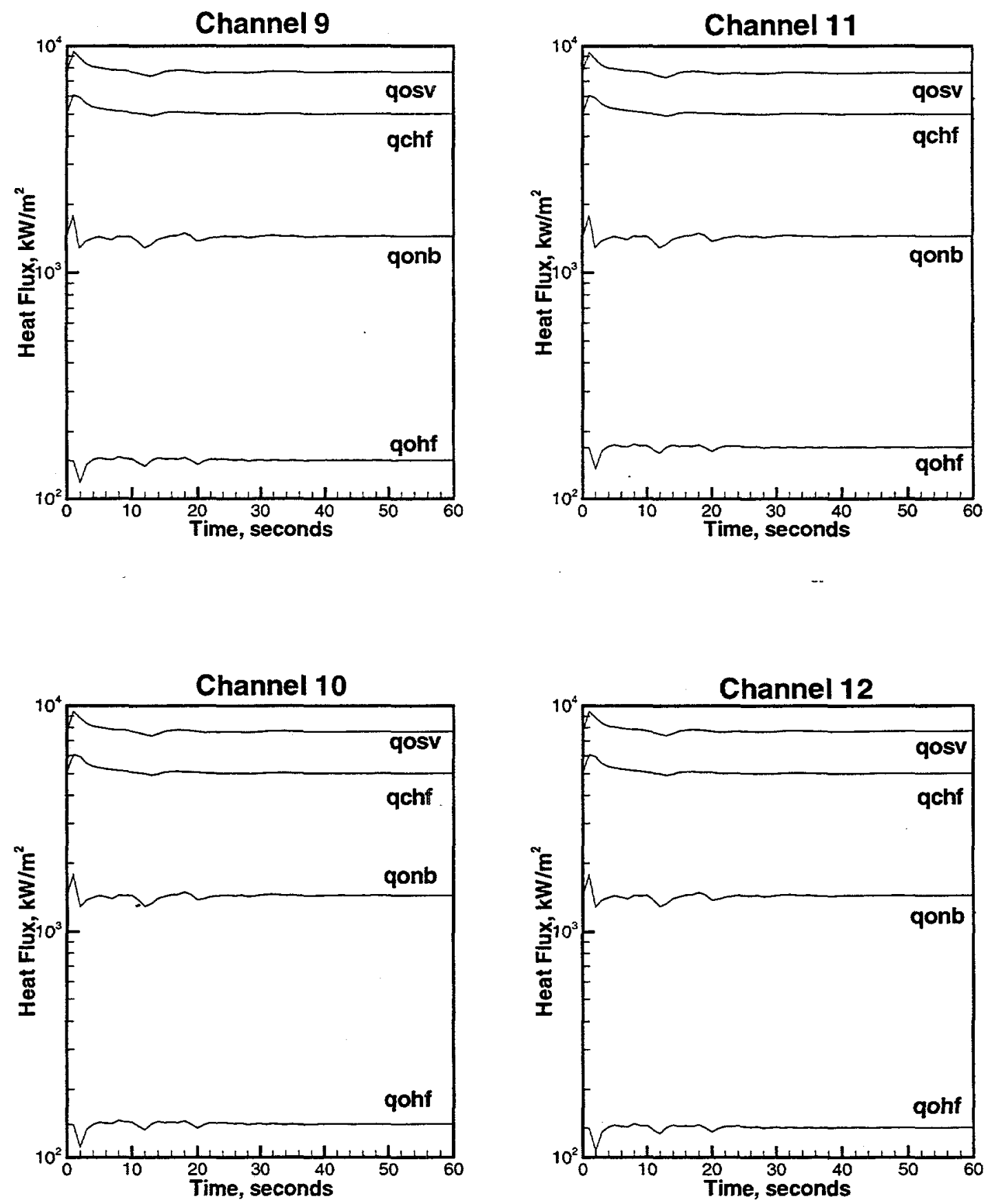

Figure 5.1-4 Surface heat fluxes for helium tube rupture at TRAC component 350 out to 60 seconds, axial level 10, channels 9-12. 


\subsection{FLOWTRAN-TF Results for Component 370 Rupture}

Maximum metal temperatures from the FLOWTRAN-TF calculations for a rupture in the helium tube at TRAC component 370 are shown in Fig. 5.2-1. The maximum aluminum temperature found anywhere in the plate cladding and the maximum lead temperature anywhere in the plate are plotted as functions of time into the accident transient. As with the other helium tube rupture accident, the decrease in coolant flow in the early part of the transient leads to a temperature increase in the metal of only about $2.5 \mathrm{C}$. As in the previous case, the metal temperature returns to its initial value within 60 seconds of the start of the accident as the flow disturbance passes. It is clear from the metal temperatures that surface temperatures in the flow channels following beam trip are not close to local boiling conditions.

Representative results for the operating surface heat flux and the calculated heat flux limits for the helium tube rupture in component 370 are shown in Figs. 5.2-2 through 5.2-4 for all of the flow channels at axial level 10. Channels 1 and 5 are the rectangular flow channels nearest the decoupler side of the plate while channel 8 is the first annular flow channel on that side. Channels 4 and 7 are the rectangular flow channels nearest the cavity side of the plate where deposited power and coolant flow are lowest.

Results from other axial locations on the plate surface are again similar demonstrating a substantial margin between the surface heat flux and the potentially limiting values. As before, the closest approach of the operating heat flux to the limiting values typically occurs in flow channels 1 or 8 . Near the top of the plate, the surface heat flux can reverse as energy flows from the heated water back into the cooler metal. This condition was observed in the flow channels in regions of the plate where the deposited power was lowest.

The plate response to a helium tube rupture at component 370 is very similar to the response for a rupture at component 350 . The most significant disturbance to the coolant flow passes through the channels within the first 10 seconds of the transient and has no major impact on the plate performance. 

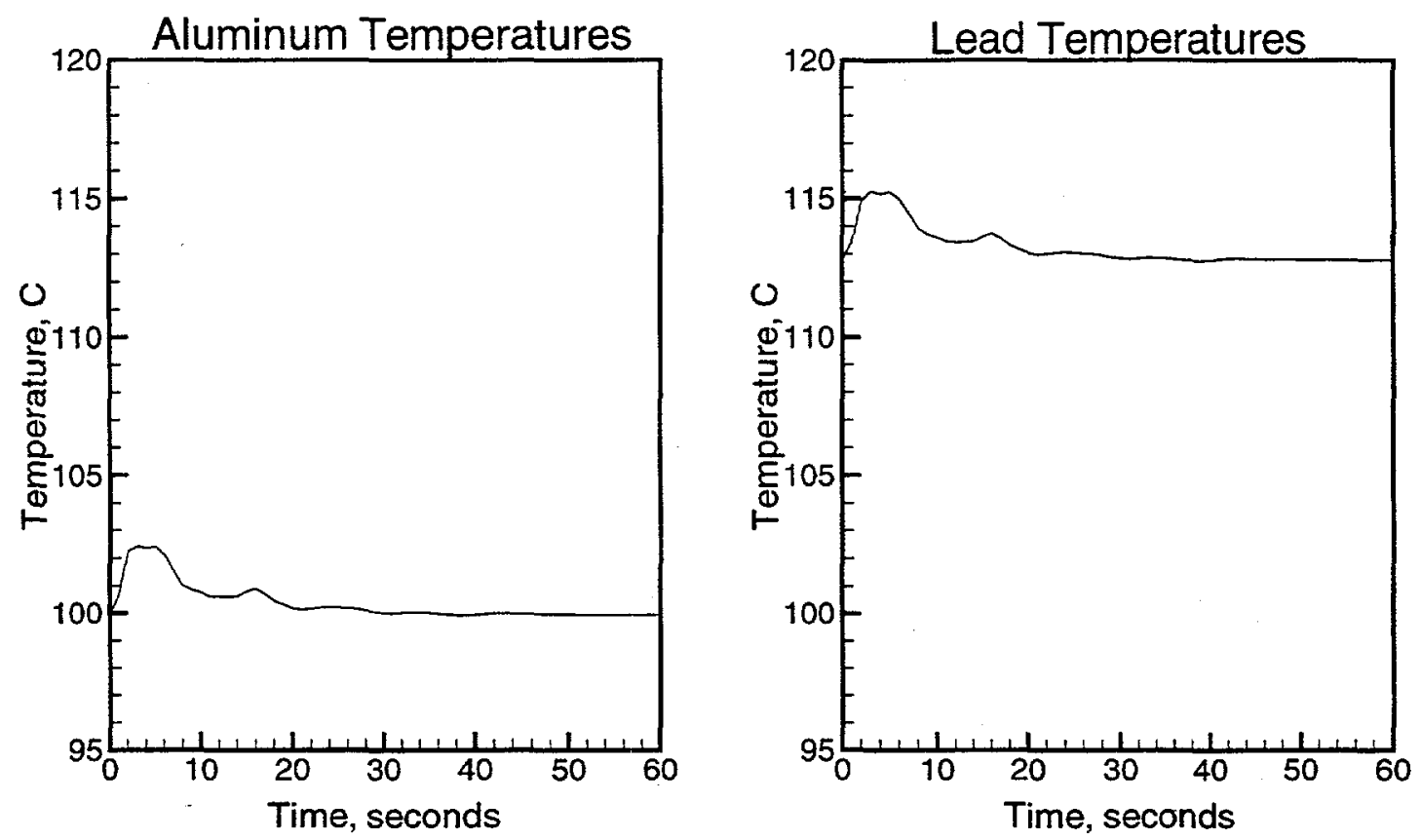

Figure 5.2-1 Maximum metal temperatures for helium tube rupture at TRAC component 370 out to 60 seconds. 

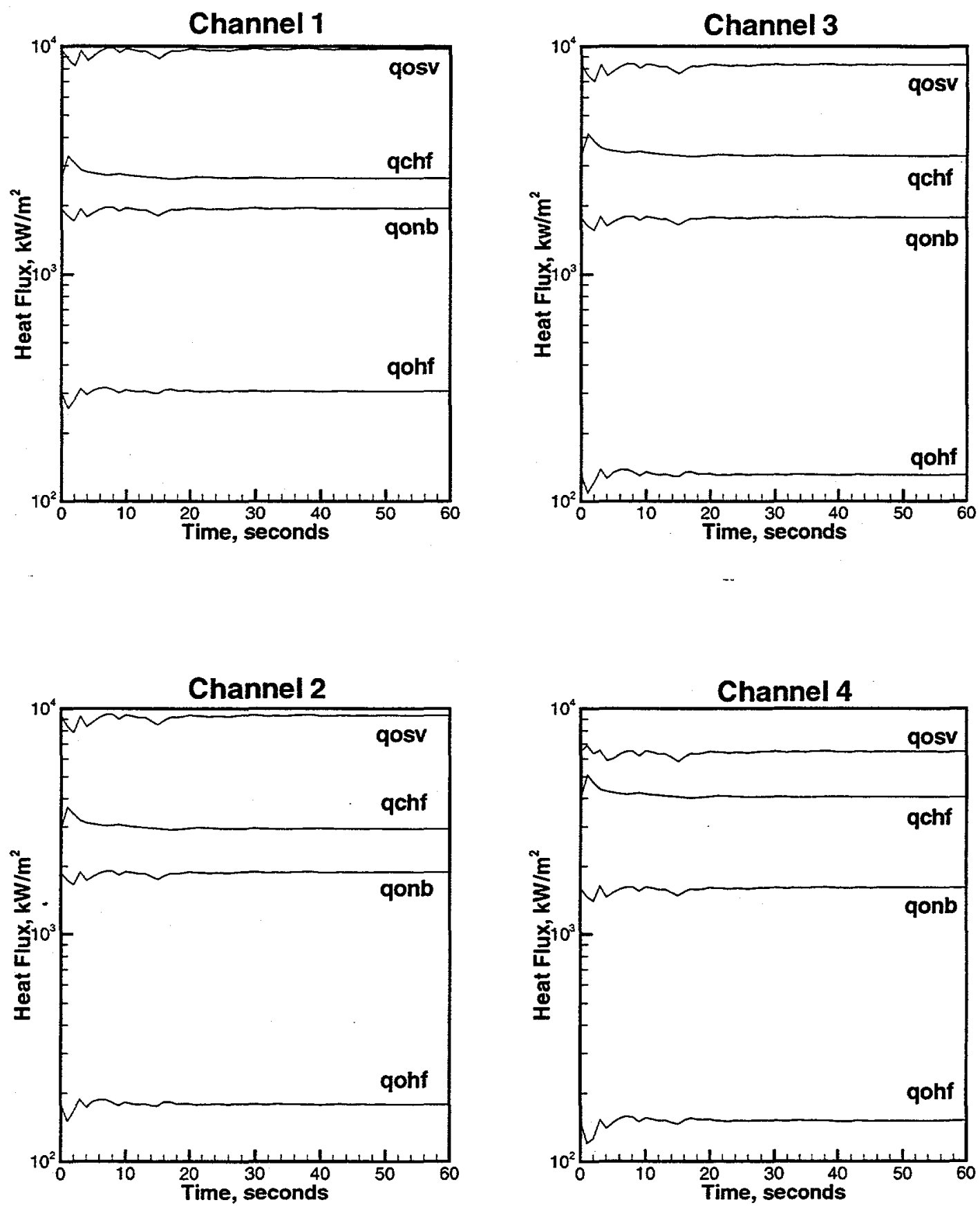

Figure 5.2-2 Surface heat fluxes for helium tube rupture at TRAC component 370 out to 60 seconds, axial level 10, channels $1-4$. 

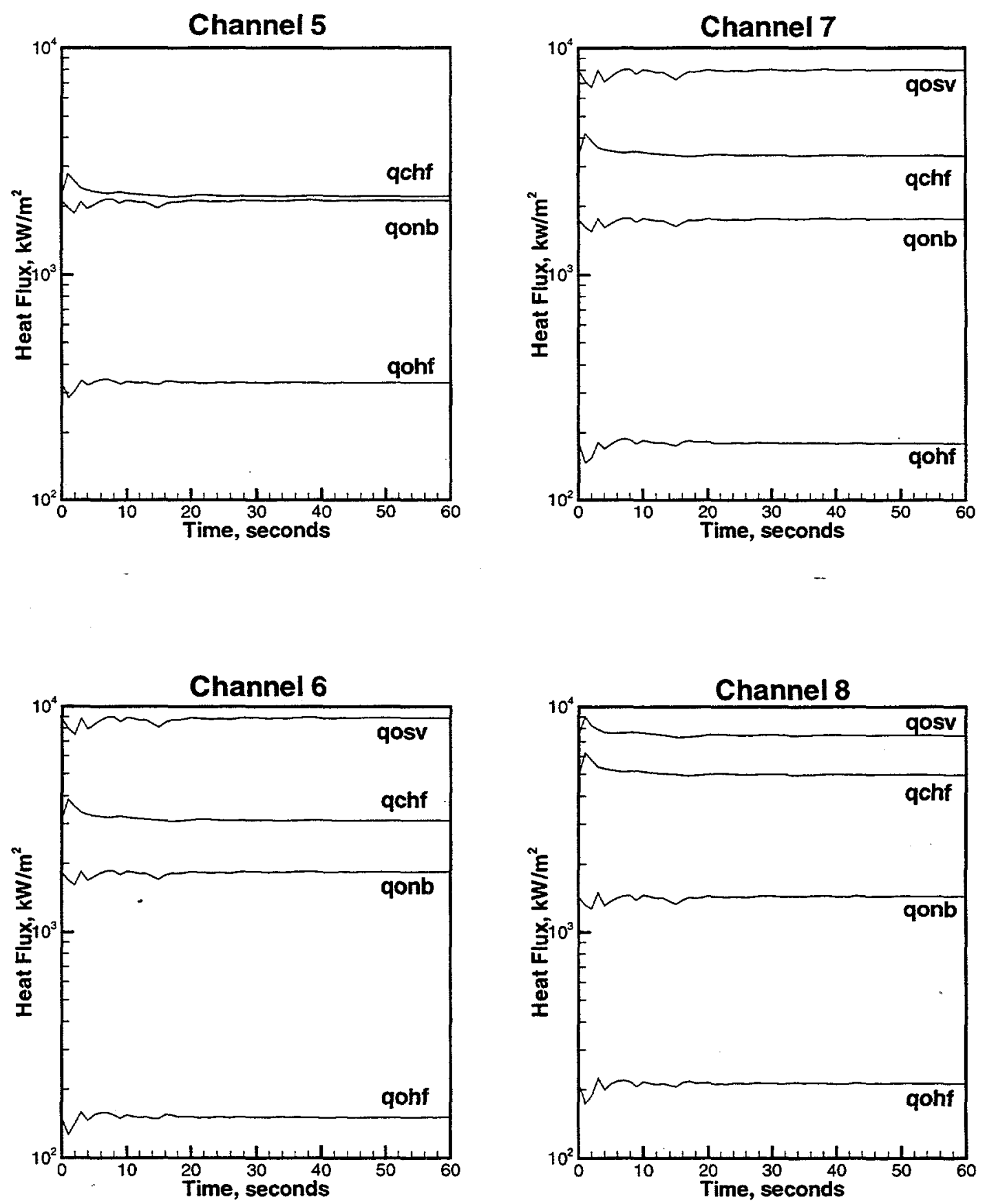

Figure 5.2-3 Surface heat fluxes for helium tube rupture at TRAC component 370 out to 60 seconds, axial level 10 , channels 5-8. 

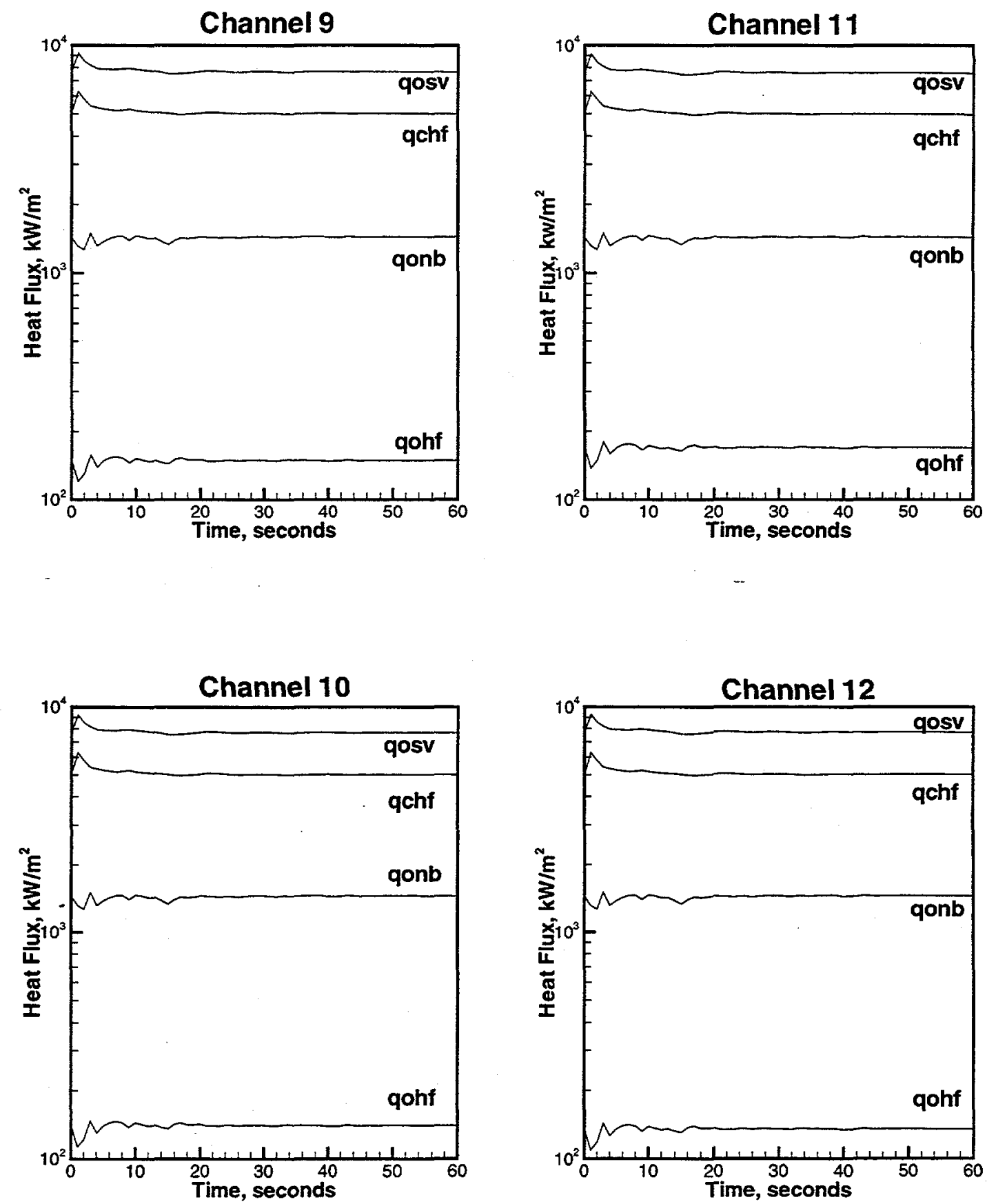

Figure 5.2-4 Surface heat fluxes for helium tube rupture at TRAC component 370 out to 60 seconds, axial level 10, channels 9-12. 


\section{Conclusions}

\subsection{Design Criteria}

Simulations performed using the TRAC system model and the FLOWTRAN-TF detailed bin model show that the APT blanket modules maintain a coolable geometry during the helium tube rupture scenarios. This conclusion is based on the analysis of two Large Break LOHGA accidents internal to the blanket module where the pressurizer relief valve remains closed. The thermal/hydraulic $(T / H)$ design criteria, along with the basis for their development, is discussed in Refs. $[1,8]$. For LOHGA the T/H onset criteria are based on meeting very strict phenomenological limits with a high degree of confidence, as follows:

- for local heated surfaces within the module components, the onset-of-significantvoids (OSV) at a three sigma confidence level; and

- for the remaining unheated piping sections of the blanket system, the onset-of-bulkboiling (OBB) at a three sigma confidence level.

Additional (steady state derived) material design criteria are imposed on the maximum lead and aluminum (Series 6061 - Type T6) metal temperatures acceptable for the module components. The limiting values for these parameters are $327.5 \mathrm{C}$ and $115 \mathrm{C}$ for lead and aluminum, respectively. These material design criteria ensure that a coolable geometry can be maintained throughout the expected lifetime of each module unit.

On a module-by-module basis, the above steady-state material and thermal onset criteria for LOHGA are compared to the FLOWTRAN-TF detailed bin model results. The bin model results for the reference 1 plate-type module are tabulated in Table 6.1-1 (note that only module 1 results are currently available since the design specifications for modules 2 through 6 do not presently exist). However, module 1 should be close to the most limiting module. Additional thermal onset criteria, which are typically considered, are also provided in Table 6.1-1. Note that these are generally more stringent than the imposed design criteria chosen.

The definitions of the criteria ratios in Table 6.1-1 are listed below, as follows:

$$
\begin{gathered}
R_{S U B}=\max \left(\frac{T_{f}-T_{i n}}{T_{s a t}-T_{i n}}\right), \text { liquid subcooling } \\
R_{S U P}=\max \left(\frac{T_{w}-T_{i n}}{T_{s a t}-T_{i n}}\right), \text { wall superheating } \\
R_{O N B}=\max \left(\frac{q_{O H F}^{\prime \prime}}{q_{O N B}^{\prime \prime}}\right), \text { ONB heat flux }
\end{gathered}
$$




$$
\begin{aligned}
& R_{O S V}=\max \left(\frac{q_{O H F}^{\prime \prime}}{q_{O S V}^{\prime \prime}}\right), \text { OSV heat flux } \\
& R_{C H F}=\max \left(\frac{q_{O H F}^{\prime \prime}}{q_{C H F}^{\prime \prime}}\right), \text { CHF heat flux }
\end{aligned}
$$

where:

$$
\begin{aligned}
& q_{O H F}^{\prime \prime} \text { …........... operating heat flux }
\end{aligned}
$$

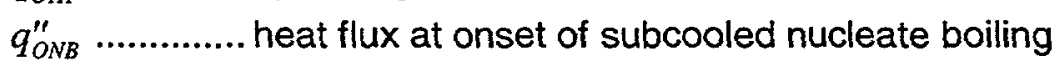

$$
\begin{aligned}
& q_{o S V}^{\prime \prime} \text {.............. heat flux at onset of significant void formation }
\end{aligned}
$$

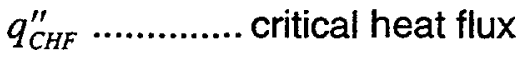

$$
\begin{aligned}
& T_{f} \text {.................. fluid temperature } \\
& T_{\text {in }} \text {.................. fluid temperature at inlet to flow channels, }(53.05 \mathrm{C}) \\
& T_{\text {sat }} \ldots \ldots . . . . . . . . . . \text { local fluid saturation temperature } \\
& T_{w} \text {.................. wall temperature }
\end{aligned}
$$

To evaluate these criteria, the maximum value corresponds to its limit spatially, as well as over the time period of the event sequence. The ratio of OHF-to-CHF is sometimes referred to as the departure from nucleate boiling ratio (DNBR). Predicted thermal onset ratios should not exceed unity.

Confidence bounds are required to establish the acceptable level of probability of exceeding these criteria. The results presented in Table 6.1-1 represent primarily best estimate values (however, some parameters were set to their estimated upper bounds, such as power density). Quantification of overall uncertainties and then their corresponding confidence levels (i.e., operating and modeling uncertainties) have not yet been performed. Future efforts to perform a response surface analysis are planned. At that time quantification of safety margins will be determined.

The. peak blanket metal temperature that occurs in module \#1 during this accident scenario is $115.1 \mathrm{C}$, as predicted by the FLOWTRAN-TF model. This occurs in the lead plate, and is well below the lead melting point, $327.5 \mathrm{C}$. The peak aluminum temperature that occurs in module \#1 is $102.2 \mathrm{C}$, which is well below the long-term temperature limit of $115 \mathrm{C}$. Maximum temperatures are reached about 10 seconds after the start of the accident as the released gas voids the flow channels and decreases coolant flow. However, the transient gas flow passes through the system very quickly and operating parameters return to what are essentially normal operating conditions approximately 60 seconds after the initiation of the accident event. The thermal inertia of the solid is such that the blanket system response to this short transient is very small with little impact on the operating conditions. 
Table 6.1-1 FLOWTRAN-TF model results under helium tube rupture conditions.

\begin{tabular}{|c|c|c|c|c|c|c|c|}
\hline $\begin{array}{l}\text { Wodule } \\
\text { Number }\end{array}$ & $\begin{array}{l}\text { Max } \\
\text { Pb } \\
\text { Temp } \\
\text { (c) }\end{array}$ & $\begin{array}{l}\text { Max } \\
\text { Temp } \\
(C)\end{array}$ & $\begin{array}{c}\text { Max } \\
\text { Subcooling } \\
\text { Ratio }\end{array}$ & $\begin{array}{l}\text { Max } \\
\text { Superheat } \\
\text { Ratio }\end{array}$ & $\begin{array}{l}\text { Max } \\
\text { ONB } \\
\text { Ratio }\end{array}$ & $\begin{array}{l}\text { Max } \\
\text { Osv } \\
\text { Ratio }\end{array}$ & $\begin{array}{l}\text { Max } \\
\text { CHR } \\
\text { Ratio }\end{array}$ \\
\hline 1 & 115.1 & 102.2 & 0.165 & 0.326 & 0.178 & 0.036 & 0.157 \\
\hline 2 & TBD & TBD & TBD & TBD & TBD & TBD & TBD \\
\hline 3 & TBD & TBD & TBD & TBD & TBD & TBD & TBD \\
\hline 4 & TBD & TBD & TBD & TBD & TBD & TBD & TBD \\
\hline 5 & TBD & TBD & TBD & TBD & TBD & TBD & TBD \\
\hline 6 & TBD & TBD & TBD & TBD & TBD & TBD & TBD \\
\hline
\end{tabular}

\subsection{Design Issues}

This analysis indicates that a rupture of a helium tube releasing gas into the blanket cooling system results in no impact on the plant operations. None of the safety systems need to be activated to mitigate the consequences of this accident. The current system design can sustain the sudden release of $1 \mathrm{~m}^{3}$ of helium with no adverse impacts.

\subsection{Predicted Impact}

Blanket conditions during large break helium tube rupture accidents internal to the blanket system fall within all specified thermal/hydraulic design criteria. No off-site impact to people or the environment would occur as a result of a rupture in the helium tubes that suddenly releases $1 \mathrm{~m}^{3}$ of gas into the blanket cooling system assuming that the pressurizer relief valve remains closed. The only on-site consequence would result from the contamination of the cooling water with tritium. 


\section{References}

1. "APT Conceptual Design Report," Los Alamos National Laboratory report, LA-UR97-1329 (April 1997).

2. Personal communication with Jack N. Edwards at LANL, April 22, 1998.

3. L. L. Hamm, S. Y. Lee, M. A. Shadday, and F. G. Smith, III, "APT Blanket System Model Based on Initial Conceptual Design - Integrated 1-D TRAC System Model," Westinghouse Savannah River Company, WSRC-TR-98-0053 (July 1998).

4. L. L. Hamm, S. Y. Lee, M. A. Shadday, and F. G. Smith, III, "Normal operation (NO) of APT Blanket System and Its Components Based on Initial Conceptual Design", Westinghouse Savannah River Company, WSRC-TR-98-0057 (July 1998).

5. R. Kapernick, "Blanket Reference 1 Plate-Type Design for Lateral Row 1 Module", e-mail memo from Los Alamos National Laboratory, Oct. 11, 1997.

6. L. L. Hamm, S. Y. Lee, M. A. Shadday, and F. G. Smith, III, "APT Blanket Detailed Bin Model Based on Initial Plate-Type Design - 3D FLOWTRAN-TF Model", Westinghouse Savannah River Company, WSRC-TR-98-0055 (July 1998).

7. L. L. Hamm, S. Y. Lee, M. A. Shadday, and F. G. Smith, III, "FLOWTRAN-TF Code Modifications made for APT Blanket Safety Analyses", Westinghouse Savannah River Company, WSRC-TR-98-0056 (July 1998).

8. - L. L. Hamm, S. Y. Lee, M. A. Shadday, and F. G. Smith, III, "APT Blanket System Safety Analysis Methodology," Westinghouse Savannah River Company, Westinghouse Savannah River Company, WSRC-TR-98-0052 (May 1998).

9. L. L. Hamm, S. Y. Lee, M. A. Shadday, and F. G. Smith, III, "TRAC Code Modifications made for APT Blanket Safety Analysis," Westinghouse Savannah River Company, WSRC-TR-98-0054 (July 1998).

10. Safety Code Development Group, "TRAC-PF1/MOD2: An Advanced Best Estimate Computer Program for Pressurized Water Reactor Thermal-Hydraulic Analysis," Los Alamos National Laboratory report LA-12031-M, Vol. 1 (NUREG/CR-5673), (July 21, 1993).

11. L. L. Hamm, S. Y. Lee, M. A. Shadday, and F. G. Smith, III, "APT Blanket System - Loss-of-Flow Accident (LOFA) Analyses Based on Initial Conceptual Design Case 1: with Beam Shutdown and Active RHR," Westinghouse Savannah River Company, WSRC-TR-98-0058 (July 1998). 
Date:

$07 / 20 / 98$

Page:

28 of 28

(This Page Intentionally Left Blank) 


\section{Appendix A: TRAC Model Component Nomenclature}

Table A-1 Blanket System Component Descriptions in TRAC Model.

\begin{tabular}{|c|c|c|c|c|}
\hline System & Component Type & $\underset{\#}{C o m p}$ & No of & Descriptions \\
\hline \multirow[t]{25}{*}{$\mathrm{HR}$} & Fixed Header (FH) & 380 & 1 & coolant Supply FH \\
\hline & & 340 & 1 & coolant Return FH \\
\hline & Pressurizer (Pzr) & 760 & 1 & Pzr surge line 1 connected to Supply FH 380 \\
\hline & & 761 & 2 & Pzr surge line 2 \\
\hline & & 762 & 1 & Pzr surge line 3 \\
\hline & & 763 & 1 & Pzr surge line 4 \\
\hline & & 764 & 1 & Pzr surge line 5 \\
\hline & & 765 & 13 & Pzr surge line 6 \\
\hline & & 766 & 9 & primary Pzr \\
\hline & Hot Leg Loop & 20 & 1 & pipe connected to Return $\mathrm{FH} 340$ \\
\hline & & 21 & 1 & plenum for potential break loc. \\
\hline & & 22 & 7 & pipe connection to external loop \\
\hline & & 23 & 1 & pipe connect. for potential break \\
\hline & & 24 & 13 & connection pipe \\
\hline & & 25 & 1 & connection pipe \\
\hline & & 26 & 2 & pipe connected to two pumps \\
\hline & & 27 & 1 & plenum for two pump connection \\
\hline & & 28 & 2 & pump\#1 suction pipe \\
\hline & & 29 & 7 & pump\#2 suction pipe \\
\hline & & 30 & 2 & pump located at cell face 2 \\
\hline & & 31 & 2 & pump located at cell face 2 \\
\hline & & 32 & 3 & check valve located at pump\#1 discharge \\
\hline & & 33 & 3 & check valve located at pump\#2 discharge \\
\hline & & 34 & 1 & pump outlet plenum \\
\hline & & 36 & 1 & connect. pipe between pump and pipe \\
\hline$=$ & & 37 & 1 & HX connect. pipe for potential break \\
\hline & & 38 & 1 & pipe connect. to two HX's inlet plenum \\
\hline & & 40 & 1 & plenum \\
\hline & $\mathrm{HX}$ & 48 & 3 & HX\#1 inlet pipe \\
\hline & & 50 & 4 & 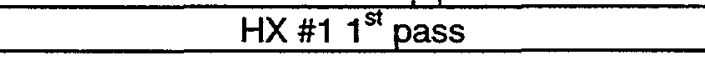 \\
\hline & & 52 & 3 & HX \#1 middle header \\
\hline & & 54 & 4 & $\mathrm{HX} \# 12^{\text {nd }}$ pass \\
\hline & & 49 & 3 & $\mathrm{HX \# 2}$ inlet pipe \\
\hline & & 51 & 4 & 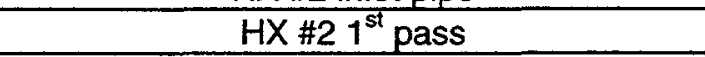 \\
\hline & & 53 & 3 & HX \#2 middle header \\
\hline & & 55 & 4 & $H X \# 22^{\text {nd }}$ pass \\
\hline & & 710 & 1 & HX \#1 secondary side fill \\
\hline & & 711 & 4 & $\mathrm{HX} \# 12^{\text {nd }}$ pass secondary side \\
\hline & & 712 & 3 & $\mathrm{HX} \# 1$ middle header secondary side \\
\hline & & 713 & 4 & $H X \# 11^{\text {st }}$ pass secondary side \\
\hline & & 714 & 1 & $H X \# 1$ secondary side break $B C$ \\
\hline & & 730 & 1 & $H X \# 2$ secondary side fill $B C$ \\
\hline
\end{tabular}


Table A-1 Blanket System Component Descriptions in TRAC Model (continued).

\begin{tabular}{|c|c|c|c|c|}
\hline System & Component Type & Comp & $\begin{array}{l}\text { No } \\
\text { of } \\
\text { Cells }\end{array}$ & Descriptions \\
\hline & & 731 & 4 & $\mathrm{HX} \# 22^{\text {nd }}$ pass secondary side \\
\hline & & 732 & 3 & $\mathrm{HX} \# 2$ middle header secondary side \\
\hline & & 733 & 4 & $\mathrm{HX} \# 21^{\text {st }}$ pass secondary side \\
\hline & & 734 & 1 & $\mathrm{HX} \# 2$ secondary side break $\mathrm{BC}$ \\
\hline \multirow[t]{12}{*}{$\mathrm{HR}$} & Cold Leg Loop & 56 & 3 & $\mathrm{HX} \# 1$ outlet pipe \\
\hline & & 57 & 6 & $\mathrm{HX} \# 2$ outlet pipe \\
\hline & & 60 & 3 & HX outlet plenum merged after two HX's \\
\hline & & 62 & 1 & cold leg pipe \\
\hline & & 63 & 1 & cold leg pipe \\
\hline & & 64 & 13 & cold leg pipe located outside cavity wall \\
\hline & & 65 & 1 & pipe for cold leg pipe break \\
\hline & & 66 & 1 & horizontal cold leg pipe penetration \\
\hline & & 67 & 1 & plenum for internal break on HR loop \\
\hline & & 854 & 2 & HR isolation valve for internal break \\
\hline & & 69 & 1 & plenum for internal LOCA simulation \\
\hline & & 68 & 5 & pipe connect. to FH 340 inside cavity \\
\hline \multirow[t]{9}{*}{$\begin{array}{l}\text { Cavity } \\
\text { Vessel } \\
\end{array}$} & Cold Leg Loop & 850 & 2 & valve located bet. cavity vessel and $H R$ \\
\hline & & 852 & 2 & valve located bet. cavity vessel and HR \\
\hline & & & 1 & plenum for cavity vessel connection \\
\hline & & 828 & 3 & cavity vent valve \\
\hline & & 802 & 1 & break component for cavity vent pressure $B C$ \\
\hline & & 823 & 11 & pipe for cavity lower section simulation \\
\hline & & 824 & 1 & plenum for cavity connection \\
\hline & & 840 & 2 & valve to connect cavity line to Module 1 \\
\hline & & 825 & 4 & pipe for cavity middle section simulation \\
\hline \multirow[t]{8}{*}{$\begin{array}{c}\text { Cavity } \\
\text { Pool }\end{array}$} & Cavity Flood Line & 820 & 13 & $\begin{array}{c}\text { pipe for cavity pool connection to cavity } \\
\text { vessel }\end{array}$ \\
\hline & & 821 & 2 & cavity flood line valve \\
\hline & & 822 & 1 & flood line pipe inside cavity vessel \\
\hline & Cavity Flood Pool & 801 & 1 & break component for cavity pool BC \\
\hline & & 810 & 10 & pipe for top cavity pool section \\
\hline & & 811 & 1 & plenum for middle cavity pool section \\
\hline & & 812 & 7 & pipe for lower cavity pool section \\
\hline & & 813 & 1 & plenum for cavity pool bottom \\
\hline \multirow[t]{10}{*}{ RHR } & RHR Loop & 621 & 1 & pipe located to return $\mathrm{FH}$ \\
\hline & + & 623 & 10 & pipe located inside the cavity vessel \\
\hline & & 624 & 1 & pipe located outside the cavity vessel \\
\hline & & 625 & 18 & pipe bet. RHR pump and pipe comp. \#624 \\
\hline & & 630 & 2 & RHR pump located at face 2 \\
\hline & & 640 & 3 & check valve located at pump discharge \\
\hline & & 652 & 4 & HX tubes \\
\hline & & 660 & 16 & pipe at the cold leg side \\
\hline & & 661 & 1 & pipe located before cavity vessel \\
\hline & & 662 & 8 & cold leg pipe inside cavity vessel \\
\hline
\end{tabular}


WESTINGHOUSE SAVANNAH RIVER COMPANY

APT BLANKET SYSTEM FOR LOHGA

(HELIUM SUPPLY RUPTURE INTO BLANKET MODULE)
Report:

Section:

Date:

Page:
WSRC-TR-98-00177

Appendix A

07/16/98

3 of 12

Table A-1 Blanket System Component Descriptions in TRAC Model (continued).

\begin{tabular}{|c|c|c|c|c|}
\hline System & Component Type & Comp & $\begin{array}{l}\text { No } \\
\text { of } \\
\text { Cells }\end{array}$ & Deseriptions \\
\hline & & 663 & 1 & cold leg pipe connected to supply FH \\
\hline & & 672 & 1 & fill for $H X$ secondary side $B C$ \\
\hline & & 671 & 4 & HX secondary shell side \\
\hline & & 673 & 1 & break comp. for $\mathrm{HX}$ secondary side $\mathrm{BC}$ \\
\hline \multirow[t]{9}{*}{ Module } & Module 1 Flow & 454 & 7 & pipe connected to supply FH \\
\hline & & 80 & 1 & $\begin{array}{l}\text { plenum for potential internal break simulation } \\
\text { at Module } 1\end{array}$ \\
\hline & & 375 & 5 & $\begin{array}{l}\text { pipe connection bet. Suuply FH and Module } \\
1 \text { upper plenum }\end{array}$ \\
\hline & & 370 & 1 & upper plenum for Module 1 downcomer \\
\hline & & 360 & 5 & Module 1 downflow region \\
\hline & & 350 & 1 & $\begin{array}{c}\text { middle plenum bet. Module } 1 \text { downflow and } \\
\text { upflow regions }\end{array}$ \\
\hline & & 300 & 5 & Module 1 upflow region \\
\hline & & 330 & 1 & upper plenum for module 1 upflow region \\
\hline & & 335 & 5 & $\begin{array}{c}\text { connection pipe after Module } 1 \text { upper } \\
\text { plenum }\end{array}$ \\
\hline \multirow[t]{9}{*}{-} & & 429 & 4 & pipe connected to return $\mathrm{FH}$ \\
\hline & Module 2 Flow & 173 & 7 & pipe coonected to supply FH \\
\hline & & 81 & 1 & $\begin{array}{l}\text { plenum for potential internal break simulation } \\
\text { at Module } 2\end{array}$ \\
\hline & & 82 & 3 & pipe connection \\
\hline & & 172 & 1 & upper plenum for Module 2 downcomer \\
\hline & & 158 & 6 & Module 2 downflow region \\
\hline & & 147 & $\overline{1}$ & $\begin{array}{c}\text { middle plenum bet. Module } 2 \text { downflow and } \\
\text { upflow regions }\end{array}$ \\
\hline & & 102 & 6 & Module 2 upflow region \\
\hline & & 133 & 1 & upper plenum for module 2 upflow region \\
\hline \multirow[t]{14}{*}{ 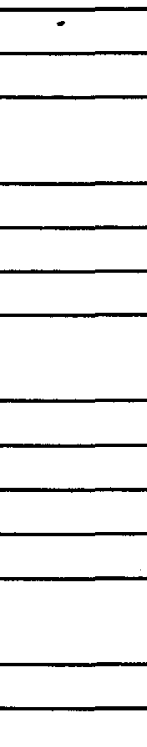 } & & 136 & 7 & pipe connected to return FH \\
\hline & Module 3 Flow & 415 & 7 & pipe connected to supply FH \\
\hline & & 85 & 1 & $\begin{array}{l}\text { plenum for potential internal break simulation } \\
\text { at Module } 3\end{array}$ \\
\hline & & 86 & 3 & pipe connection \\
\hline & & 479 & 1 & upper plenum for Module 3 downcomer \\
\hline & & 478 & 5 & Module 3 downflow region \\
\hline & & 418 & 1 & $\begin{array}{c}\text { middle plenum bet. Module } 3 \text { downflow and } \\
\text { upflow regions }\end{array}$ \\
\hline & & 409 & 5 & Module 3 upflow region \\
\hline & & 423 & 1 & upper plenum for module 3 upflow region \\
\hline & & 417 & 7 & pipe connected to return FH \\
\hline & Module 4 Flow & 485 & 7 & pipe connected to supply FH \\
\hline & & 87 & 1 & $\begin{array}{l}\text { plenum for potential internal break simulation } \\
\text { at Module } 4\end{array}$ \\
\hline & & 88 & 3 & pipe connection \\
\hline & & 489 & 1 & upper plenum for Module 4 downcomer \\
\hline
\end{tabular}


Table A-1 Blanket System Component Descriptions in TRAC Model (continued).

\begin{tabular}{|c|c|c|c|c|}
\hline System & Component Type & Comp & $\begin{array}{l}\text { No of } \\
\text { Cells }\end{array}$ & Descriptions \\
\hline & & 480 & 6 & Module 4 downflow region \\
\hline & & 419 & 1 & $\begin{array}{c}\text { middle plenum bet. Module } 4 \text { downflow and } \\
\text { upflow regions }\end{array}$ \\
\hline & & 412 & 6 & Module 4 upflow region \\
\hline & & 483 & 1 & upper plenum for module 4 upflow region \\
\hline & & 484 & 7 & pipe connected to return $\mathrm{FH}$ \\
\hline \multirow[t]{31}{*}{ Module } & Module 5 Flow & 513 & 7 & pipe connected to supply FH \\
\hline & & 89 & 1 & $\begin{array}{l}\text { plenum for potential internal break } \\
\text { simulation at Module } 5\end{array}$ \\
\hline & & 90 & 3 & pipe connection \\
\hline & & 510 & 1 & upper plenum for Module 5 downcomer \\
\hline & & 507 & 6 & Module 5 downflow region \\
\hline & & 503 & 1 & $\begin{array}{l}\text { middle plenum bet. Module } 4 \text { downflow and } \\
\text { upflow regions }\end{array}$ \\
\hline & & 500 & 6 & Module 5 upflow region \\
\hline & & 508 & 1 & upper plenum for Module 5 upflow region \\
\hline & & 511 & 7 & pipe connected to return $\mathrm{FH}$ \\
\hline & Module 6 Flow & 541 & 7 & pipe connected to supply FH \\
\hline & & 83 & 1 & $\begin{array}{c}\text { plenum for potential internal break } \\
\text { simulation at Module } 6\end{array}$ \\
\hline & & 84 & 1 & pipe connection \\
\hline & & 538 & 1 & upper plenum for Module 6 decoupler \\
\hline & & 535 & 5 & Module 6 downcomer region \\
\hline & & 531 & 1 & $\begin{array}{l}\text { middle plenum bet. Module } 6 \text { decoupler and } \\
\text { main heated regions }\end{array}$ \\
\hline & & 528 & 5 & Module 6 main heated region \\
\hline & & 536 & 1 & $\begin{array}{c}\text { upper plenum for module } 6 \text { main heated } \\
\text { region }\end{array}$ \\
\hline & & 539 & 12 & pipe connected to return $\mathrm{FH}$ \\
\hline & $\begin{array}{c}\text { Module } 1 \text { Heater } \\
\text { Structure }\end{array}$ & 901 & 5 & Al tube structure in Row 1 \\
\hline & & 951 & 5 & Lead zone with Al cladding in Row 1 \\
\hline & & 984 & 5 & Al tube structure in decoupler \\
\hline & $\begin{array}{l}\text { Module } 2 \text { Heater } \\
\text { Structure } \\
\end{array}$ & 905 & 6 & Al tube structure in Row 2 \\
\hline & & 955 & 6 & Lead zone with Al cladding in Row 2 \\
\hline & & 916 & 6 & Al tube structure in Row 3 \\
\hline & & 966 & 6 & Lead zone with Al cladding in Row 3 \\
\hline & $\begin{array}{l}\text { Module } 3 \text { Heater } \\
\text { Structure }\end{array}$ & 911 & 5 & Al tube structure in Row 1 \\
\hline & & 961 & 5 & Lead zone with Al cladding in Row 1 \\
\hline & & 988 & 5 & Al tube structure in decoupler \\
\hline & $\begin{array}{l}\text { Module } 4 \text { Heater } \\
\text { Structure }\end{array}$ & 912 & 6 & Al tube structure in Row 1 \\
\hline & & 962 & 6 & Lead zone with Al cladding in Row 1 \\
\hline & & 931 & 6 & Al tube structure in Row 2 \\
\hline
\end{tabular}


Table A-1 Blanket System Component Descriptions in TRAC Model (continued).

\begin{tabular}{|c|c|c|c|c|}
\hline System & Component Type & Comp & $\begin{array}{l}\text { No } \\
\text { of } \\
\text { Cells }\end{array}$ & Descriptions \\
\hline & & 978 & 6 & Lead zone with Al cladding in Row 2 \\
\hline & $\begin{array}{l}\text { Module } 5 \text { Heater } \\
\text { Structure }\end{array}$ & 913 & 6 & Al tube structure in Row 2 \\
\hline & & 963 & 6 & Lead zone with Al cladding in Row 2 \\
\hline & & 932 & 6 & Al tube structure in Row 2 \\
\hline & & 979 & 6 & Lead zone with Al cladding in Row 2 \\
\hline & $\begin{array}{c}\text { Module } 6 \text { Heater } \\
\text { Structure }\end{array}$ & 915 & 5 & Al tube structure in Row 2 \\
\hline & & 965 & 5 & Lead zone with Al cladding in Row 2 \\
\hline
\end{tabular}

Table A-2 Steady State Conditions.

\begin{tabular}{|c|c|c|}
\hline Parameter & Units & $\begin{array}{c}\text { Calculated } \\
\text { Values }\end{array}$ \\
\hline Total power deposited in blanket modules & $\mathrm{MW}$ & 56.5 \\
\hline Total flow rate & $\mathrm{kg} / \mathrm{sec}$ & 1569 \\
& $\mathrm{gpm}$ & 25252 \\
\hline Pressure in cold-leg fixed header & $\mathrm{MPa}$ & 0.7325 \\
& $\mathrm{psia}$ & 106.24 \\
\hline Pressure in hot-leg fixed header & $\mathrm{MPa}$ & 0.4563 \\
& $\mathrm{psia}$ & 66.180 \\
\hline Pressurizer (cell \#1) pressure & $\mathrm{MPa}$ & 0.7311 \\
& $\mathrm{psia}$ & 106.03 \\
\hline Pump \#1 suction pressure & $\mathrm{MPa}$ & 0.2751 \\
& $\mathrm{psia}$ & 39.90 \\
\hline Pump \#1 discharge pressure & $\mathrm{MPa}$ & 1.0356 \\
& $\mathrm{psia}$ & 150.20 \\
\hline Pump \#2 suction pressure & $\mathrm{MPa}$ & 0.2958 \\
& $\mathrm{psia}$ & 42.91 \\
\hline Pump \#2 discharge pressure & $\mathrm{MPa}$ & 1.0409 \\
& $\mathrm{psia}$ & 150.97 \\
\hline Temperature in cold-leg fixed header & $\mathrm{C}$ & 49.43 \\
& $\mathrm{~F}$ & 121.0 \\
\hline Temperature in hot-leg fixed header & $\mathrm{C}$ & 58.03 \\
& $\mathrm{~F}$ & 136.5 \\
\hline Max. fluid temperature of the hottest & $\mathrm{C}$ & 71.95 \\
module & $\mathrm{F}$ & 161.5 \\
\hline
\end{tabular}




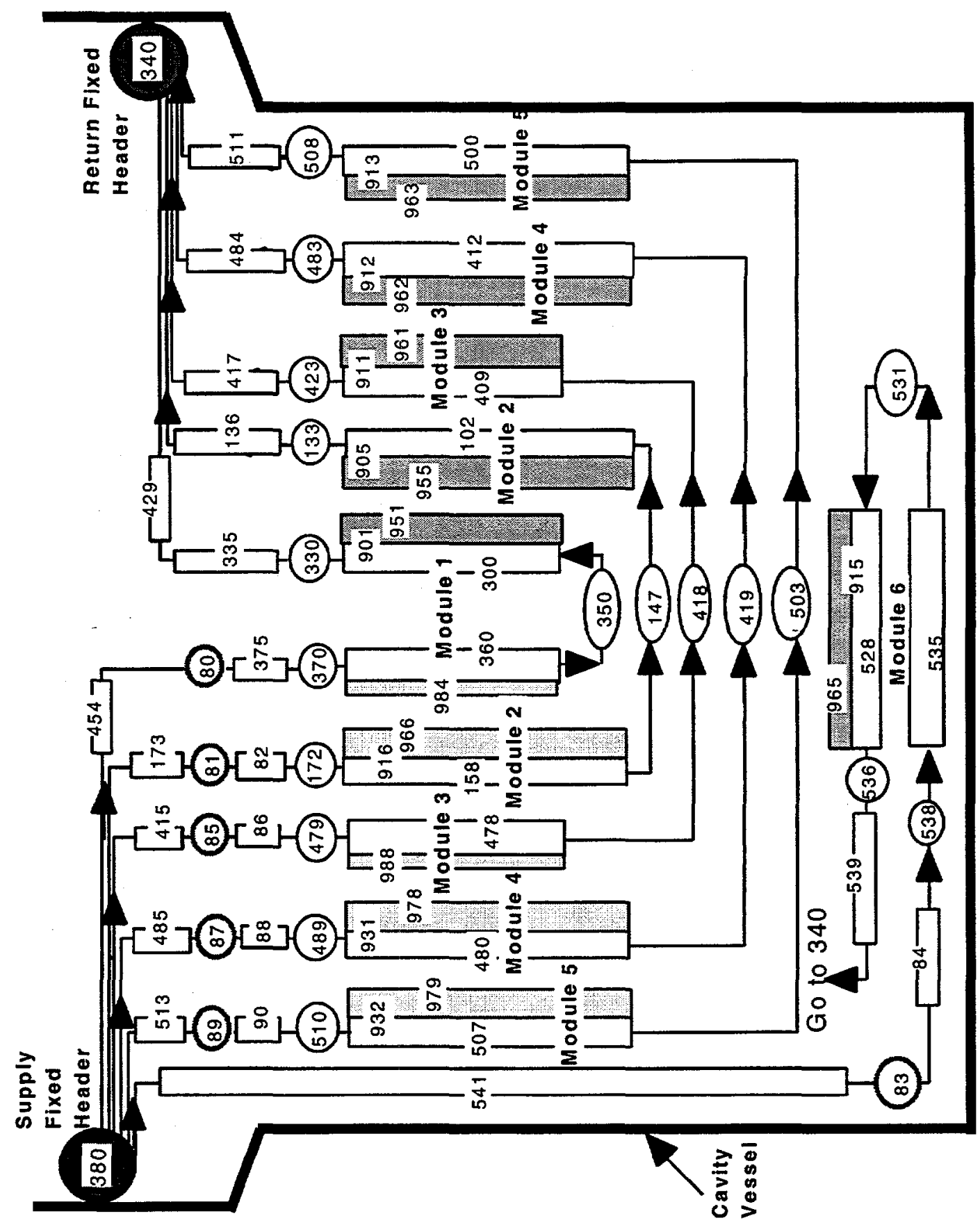

Figure A-1 6 blanket module layout for safety analysis. 


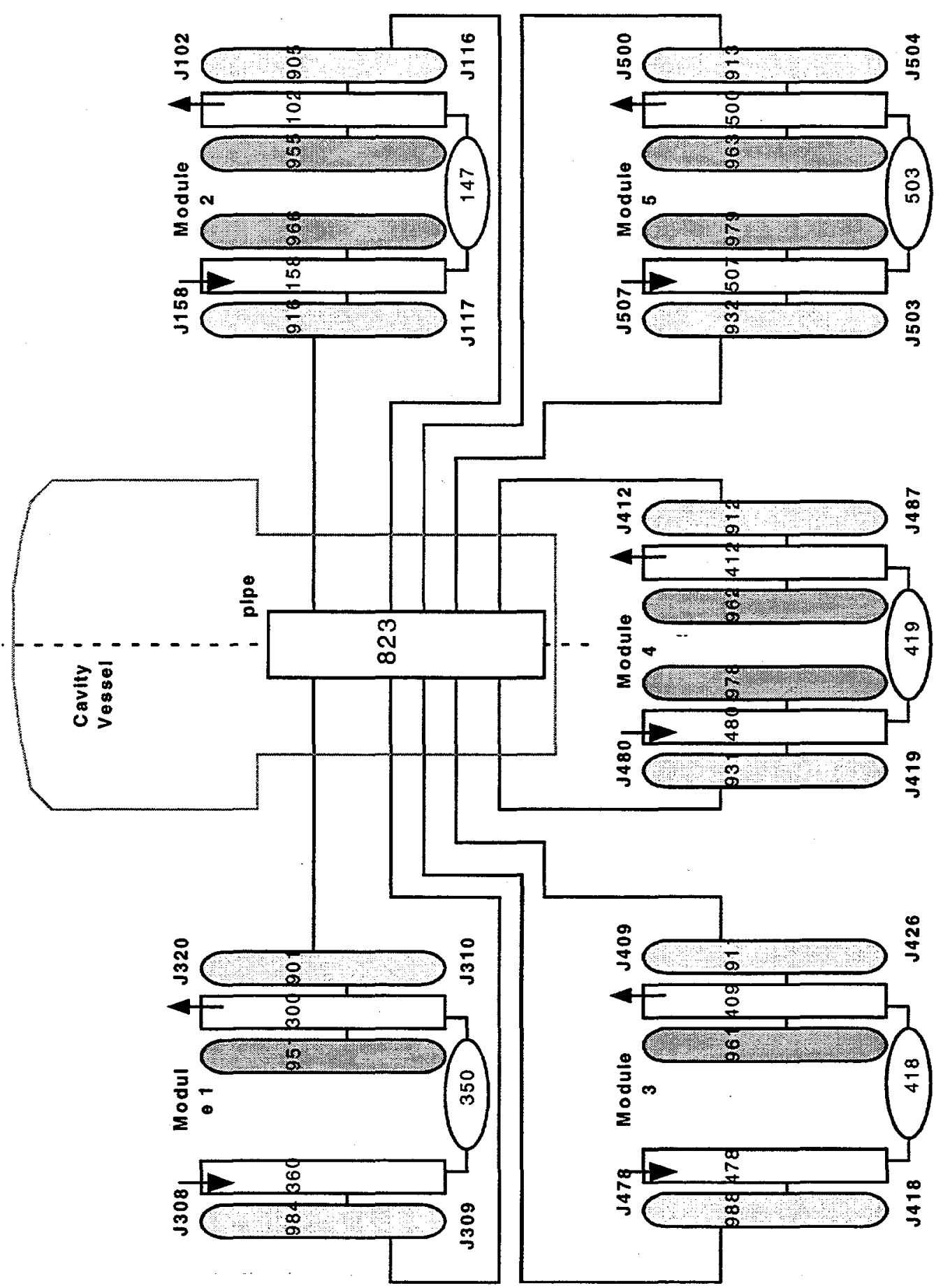

Figure A-2 TRAC component layout for the cavity vessel and blanket module heat structures. 


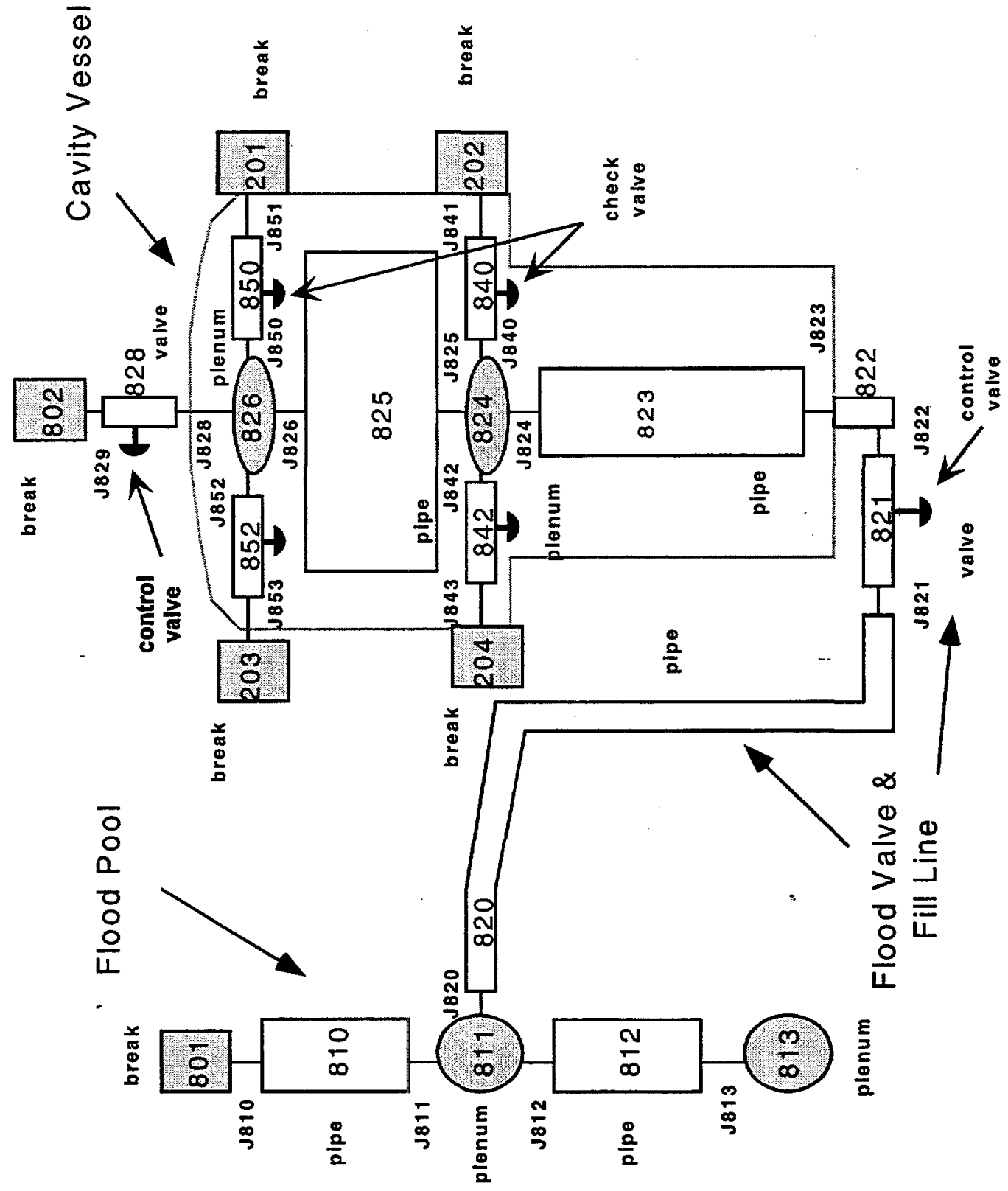

Figure A-3 TRAC component layout for the cavity vessel and cavity flood system. 


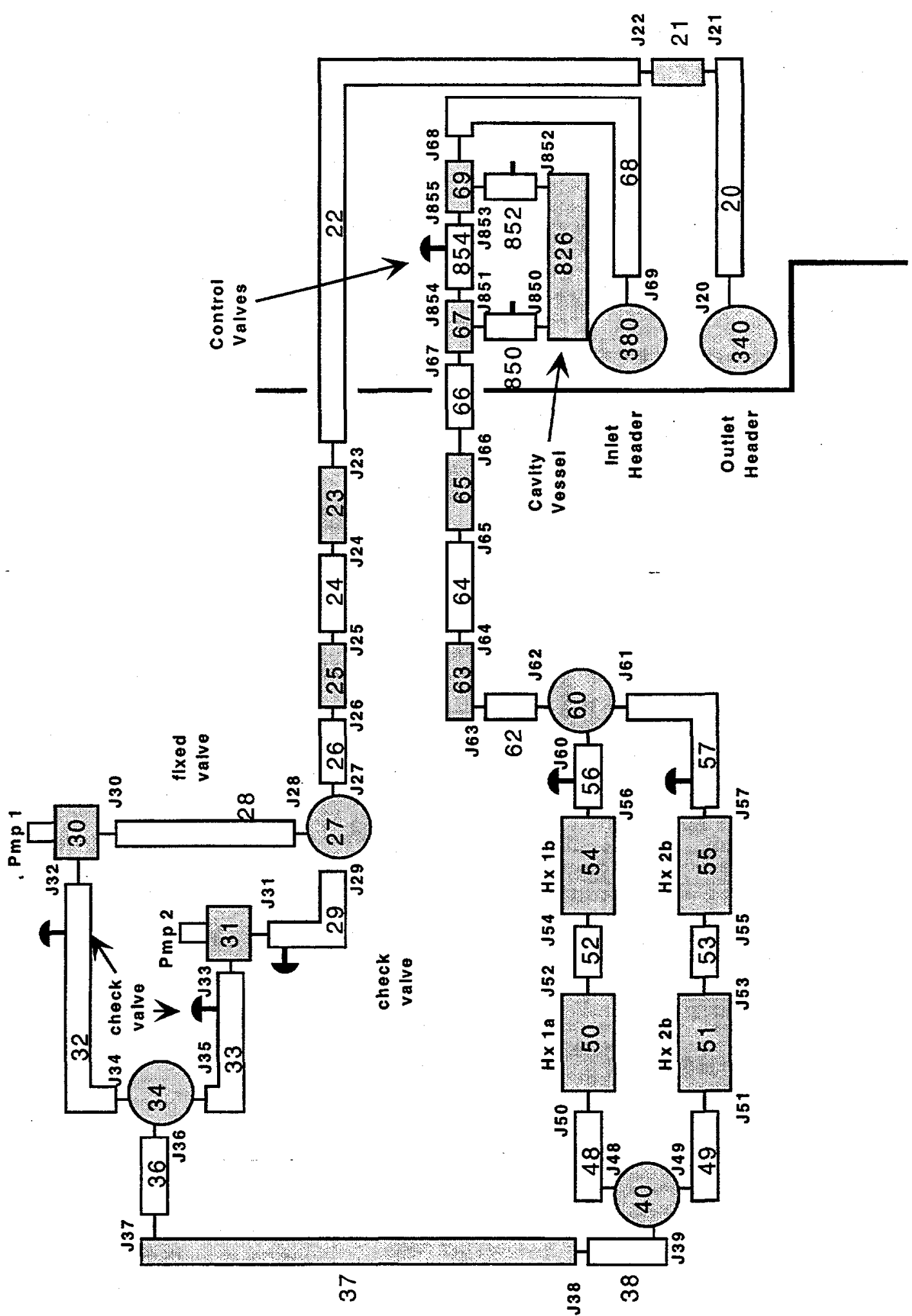

Figure A-4 TRAC component layout for blanket primary HR coolant loop. 


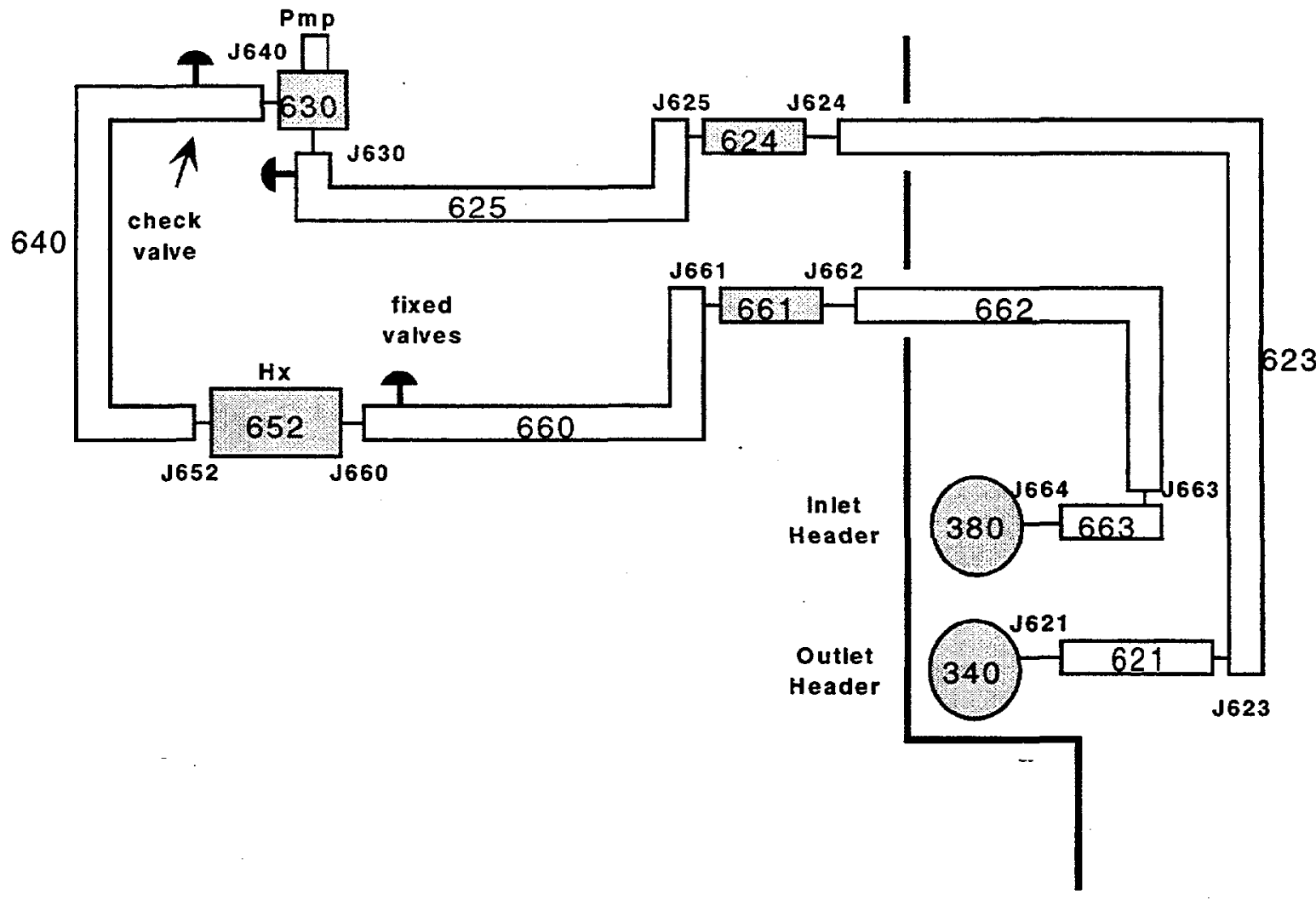

Figure A-5 TRAC component layout for blanket primary RHR coolant loop. 


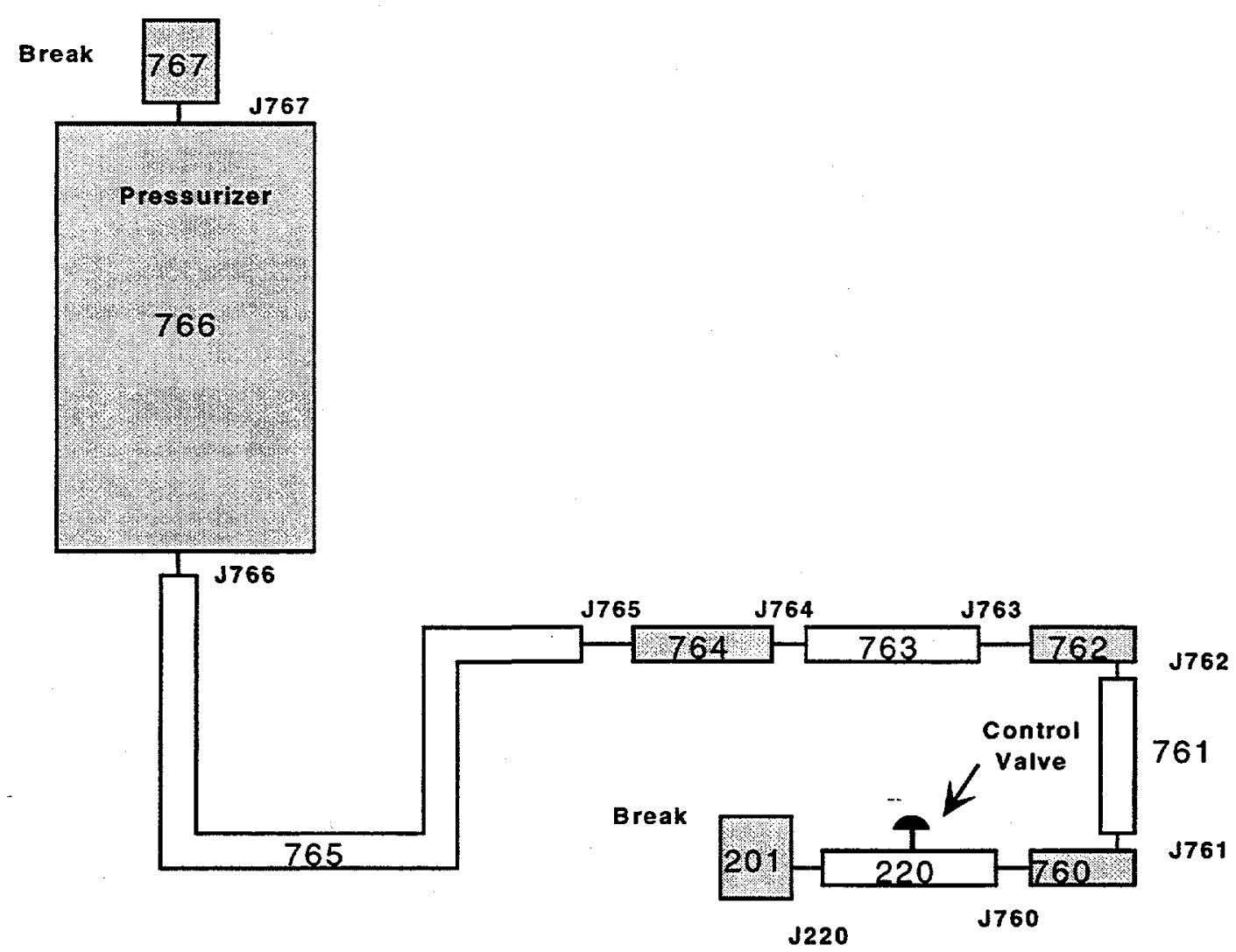

Figure A-6 TRAC component layout for blanket primary pressurizer and surge line. 
WESTINGHOUSE SAVANNAH RIVER COMPANY

Report: WSRC-TR-98-00177

Section:

APT BLANKET SYSTEM FOR LOHGA

Date:

Appendix A

(HELIUM SUPPLY RUPTURE INTO BLANKET MODULE)

Page:

$07 / 16 / 98$

12 of 12

(This Page Intentionally Left Blank) 


\section{Appendix B: LOHGA (Case 1) TRAC Results}

\section{Appendix B1 LOHGA (Case 1) TRAC Plenum Component Figures}

The following figures are from a TRAC simulation for Case 1 of a LOHGA (Helium supply plenum break near decoupler inlet):

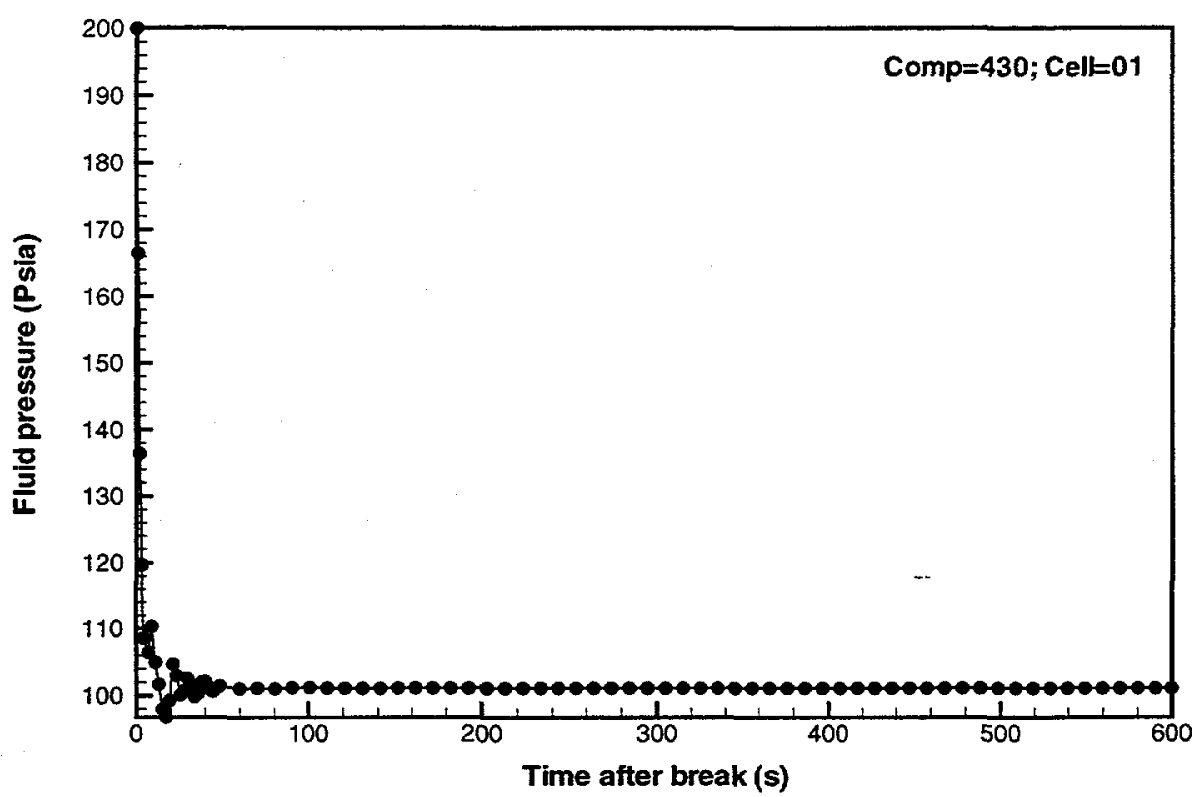

Figure B-1a Helium gas supply fluid pressures for a LOHGA (Case 1: helium supply plenum break near decoupler inlet). 


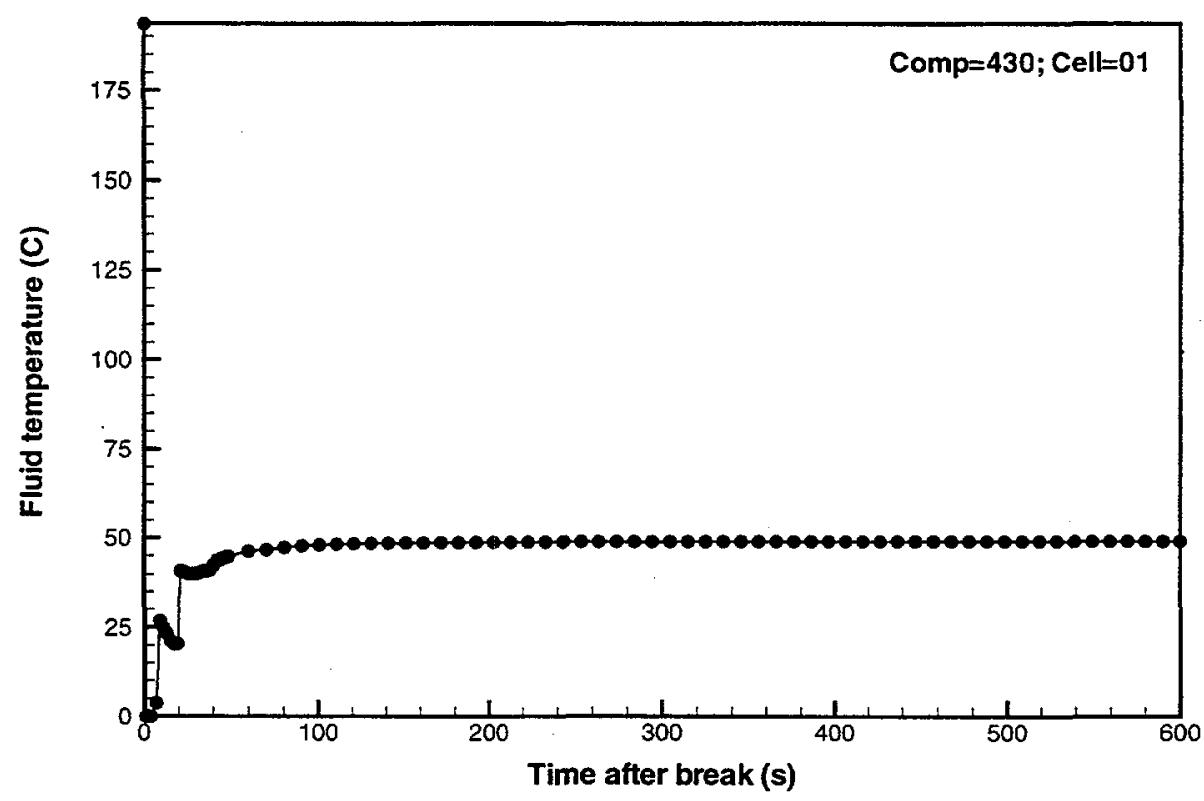

Figure B-1b Helium gas supply fluid temperatures for a LOHGA (Case 1: helium supply plenum break near decoupler inlet).

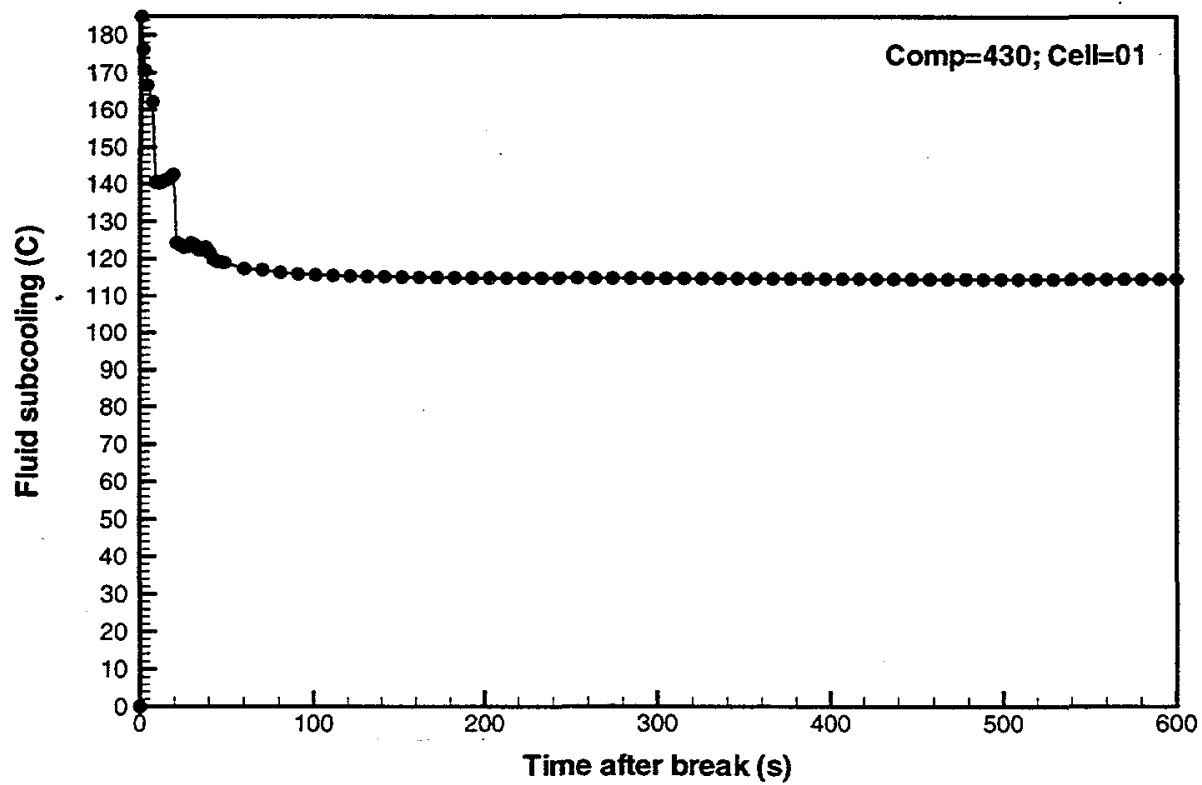

Figure B-1c Helium gas supply fluid subcoolings for a LOHGA (Case 1: helium supply plenum break near decoupler inlet). 


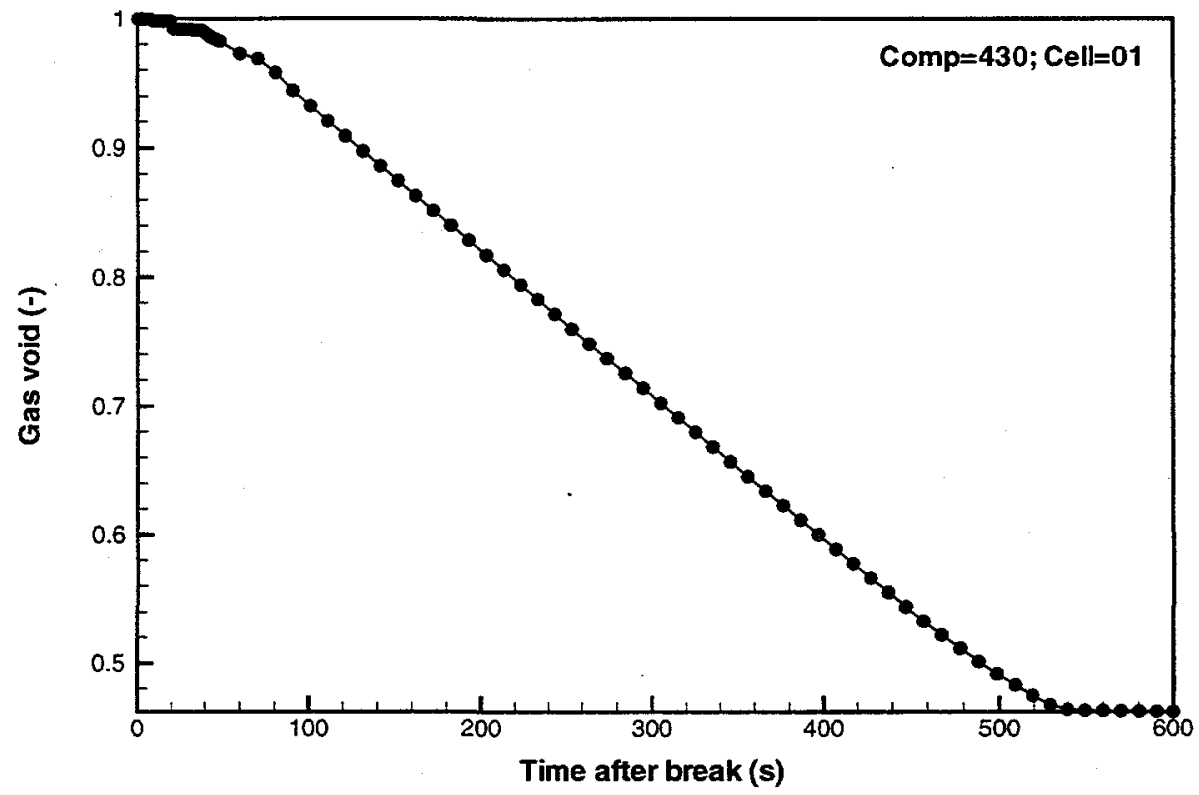

Figure B-1d Helium gas supply void fractions for a LOHGA-(Case 1: helium supply plenum break near decoupler inlet).

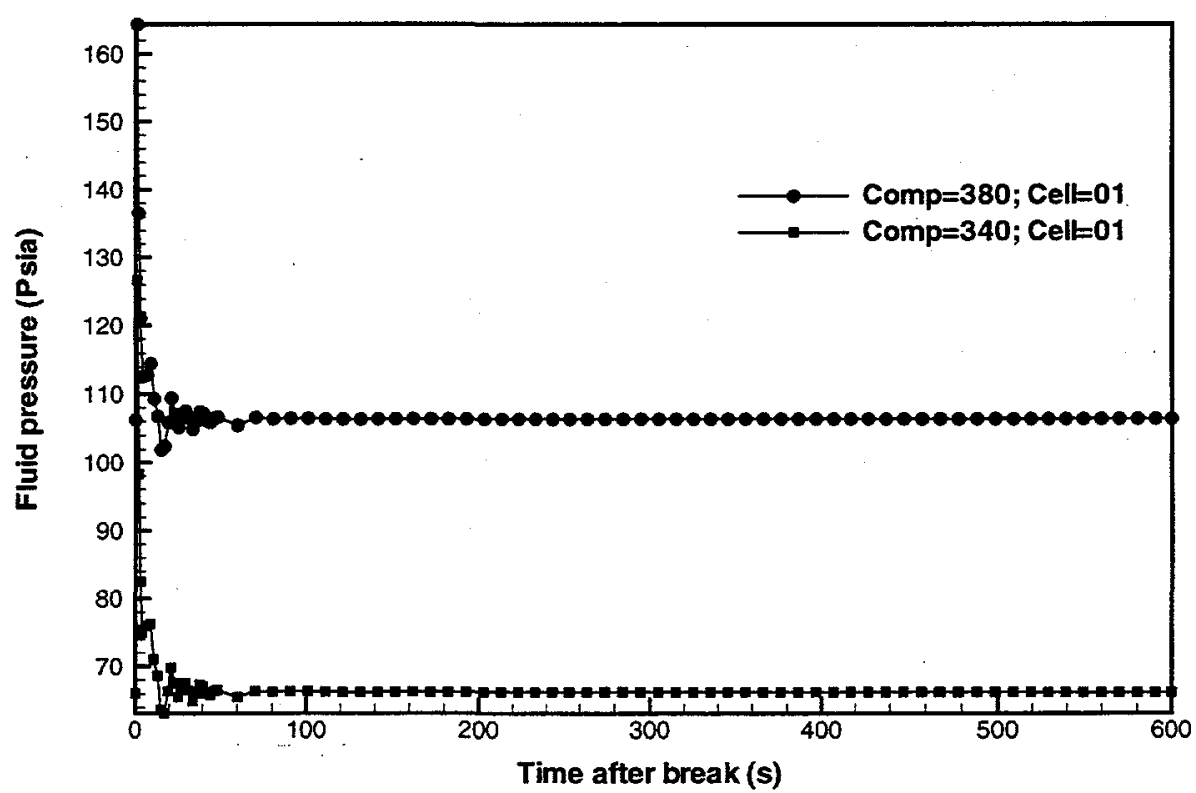

Figure B-2a Fixed header fluid pressures for a LOHGA (Case 1: helium supply plenum break near decoupler inlet). 


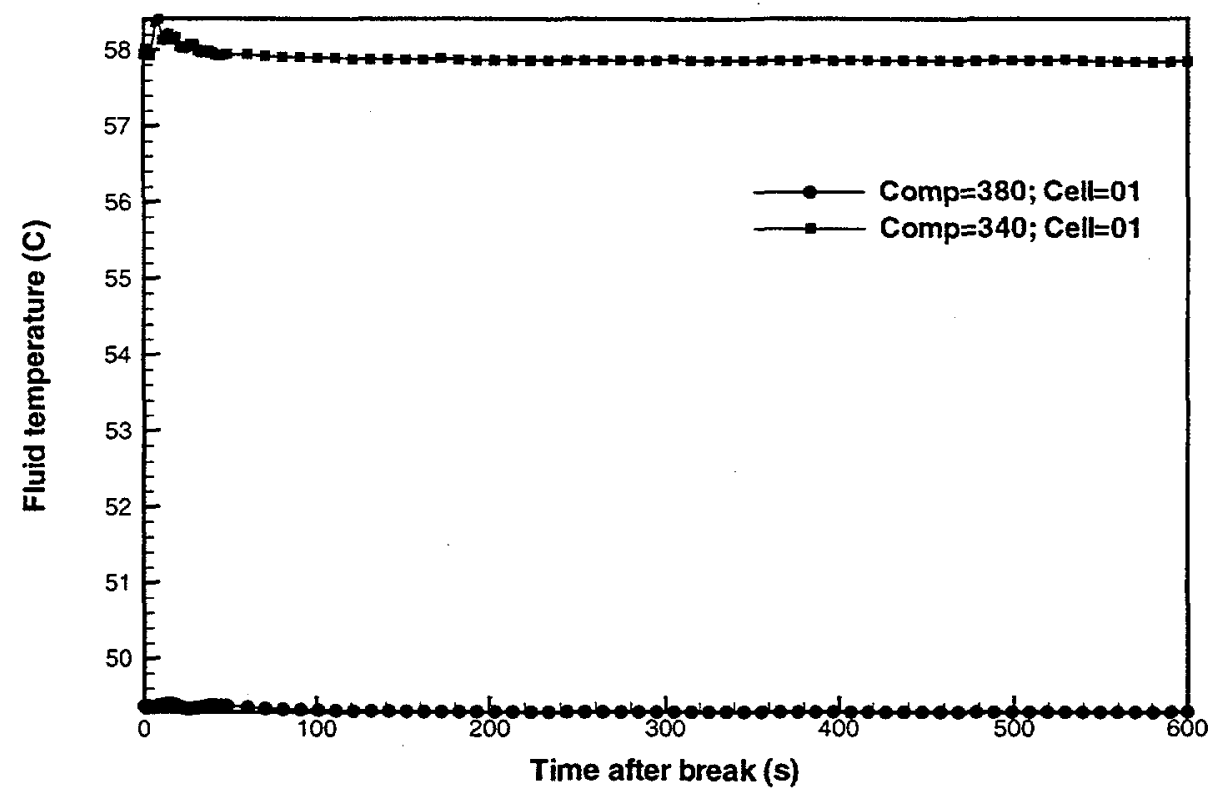

Figure B-2b Fixed header fluid temperatures for a LOHGA (Case t: helium supply plenum break near decoupler inlet).

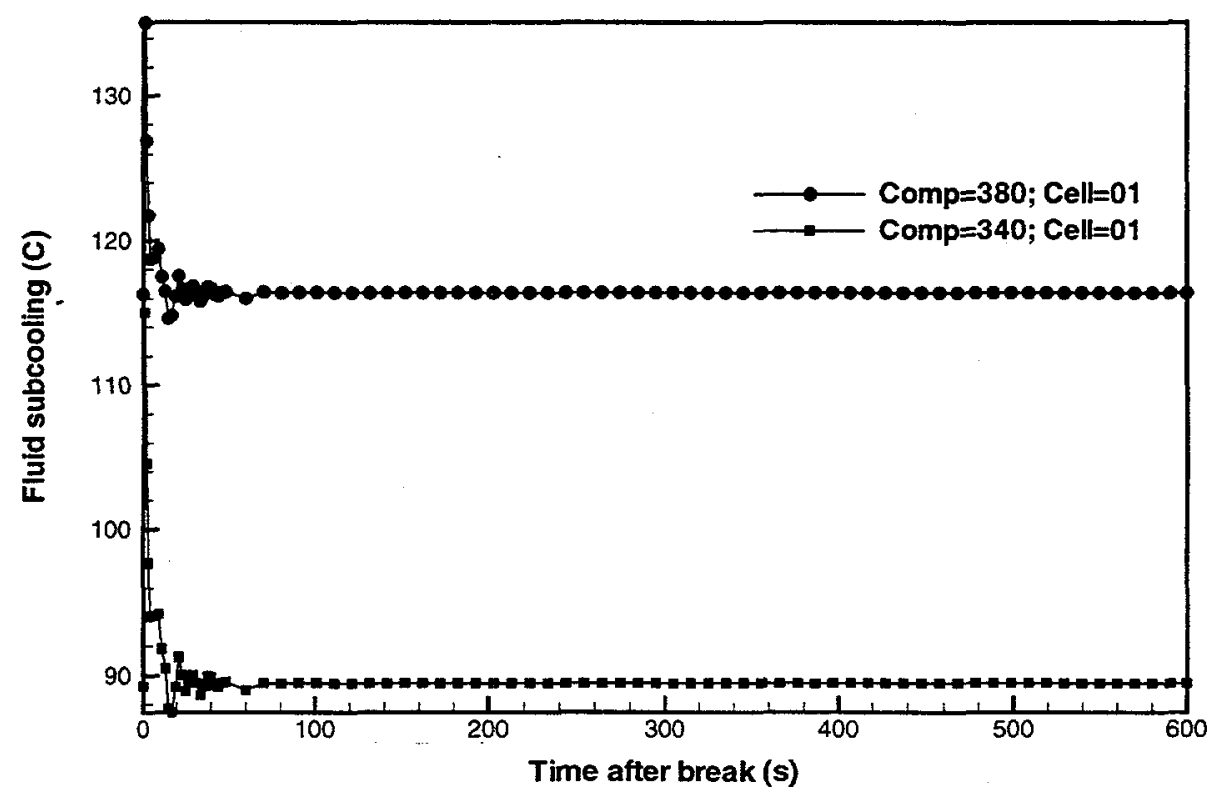

Figure B-2c Fixed header fluid subcoolings for a LOHGA (Case 1: helium supply plenum break near decoupler inlet). 


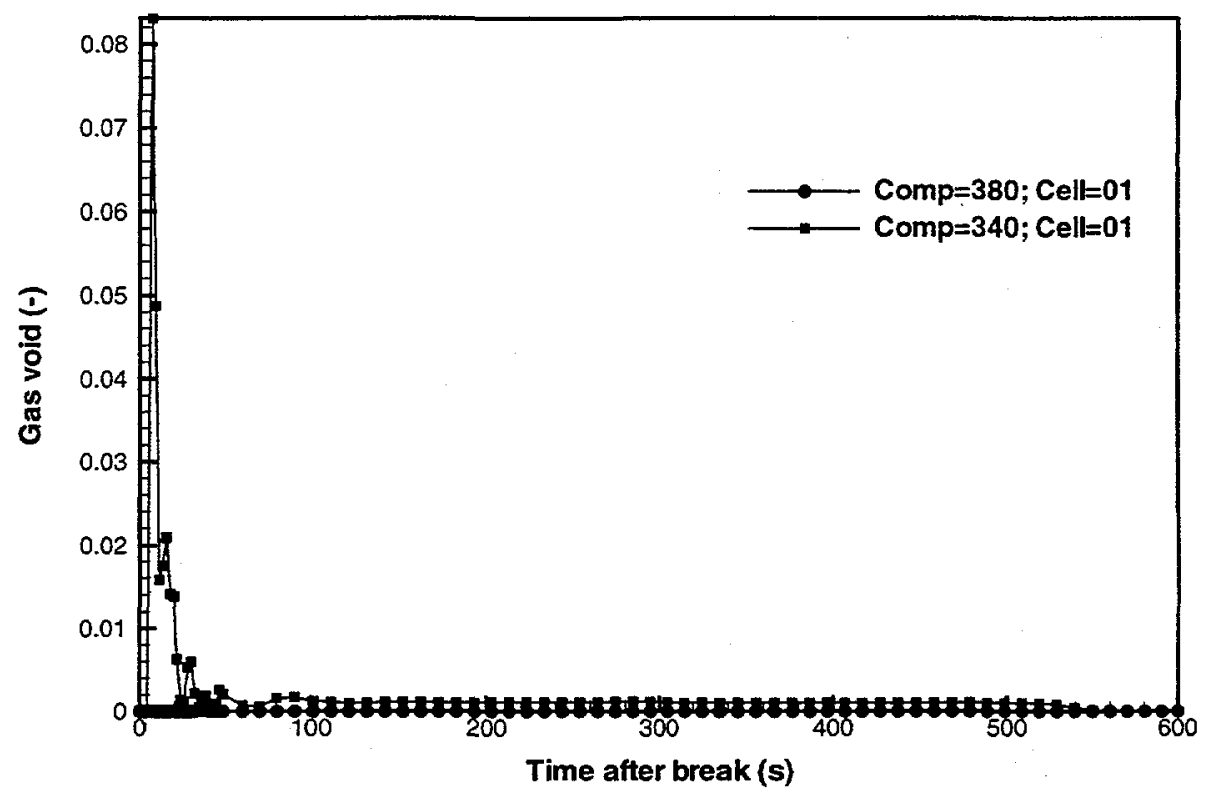

Figure B-2d Fixed header void fractions for a LOHGA (Case-1: helium supply plenum break near decoupler inlet).

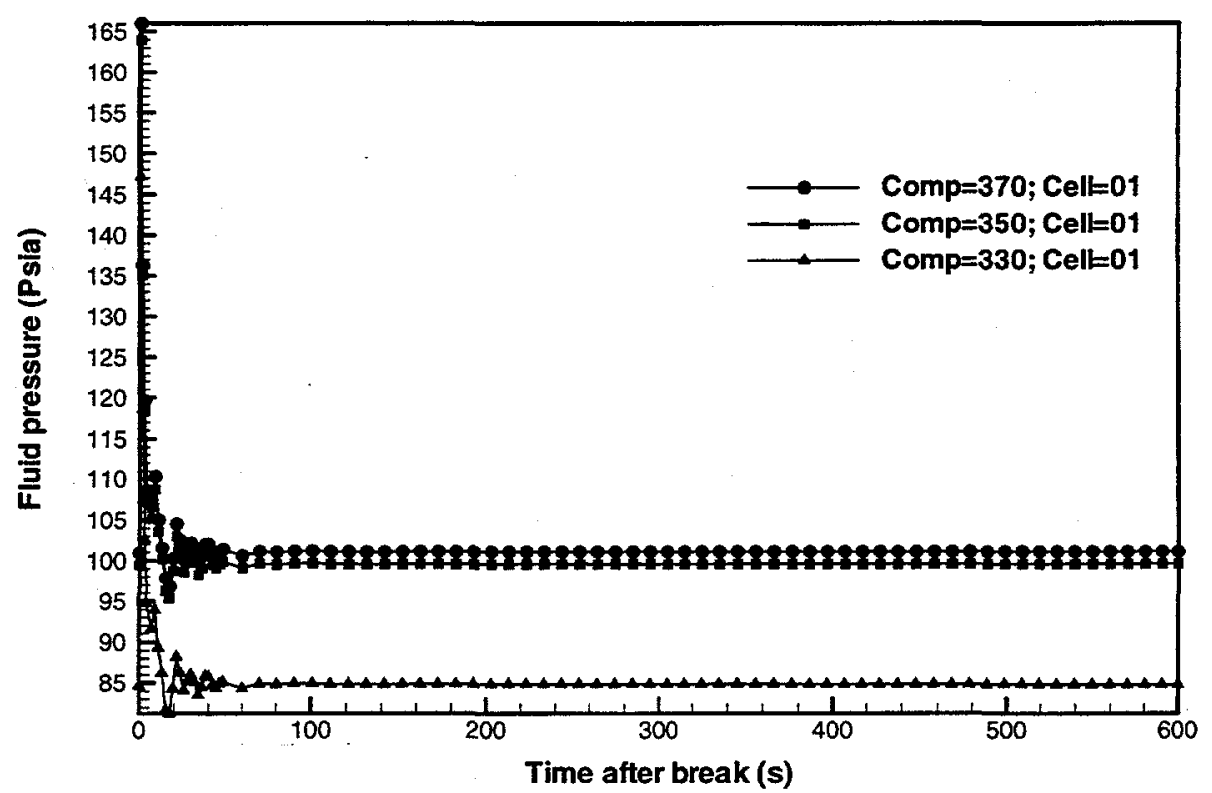

Figure B-3a Module 1 plenum fluid pressures for a LOHGA (Case 1: helium supply plenum break near decoupler inlet). 


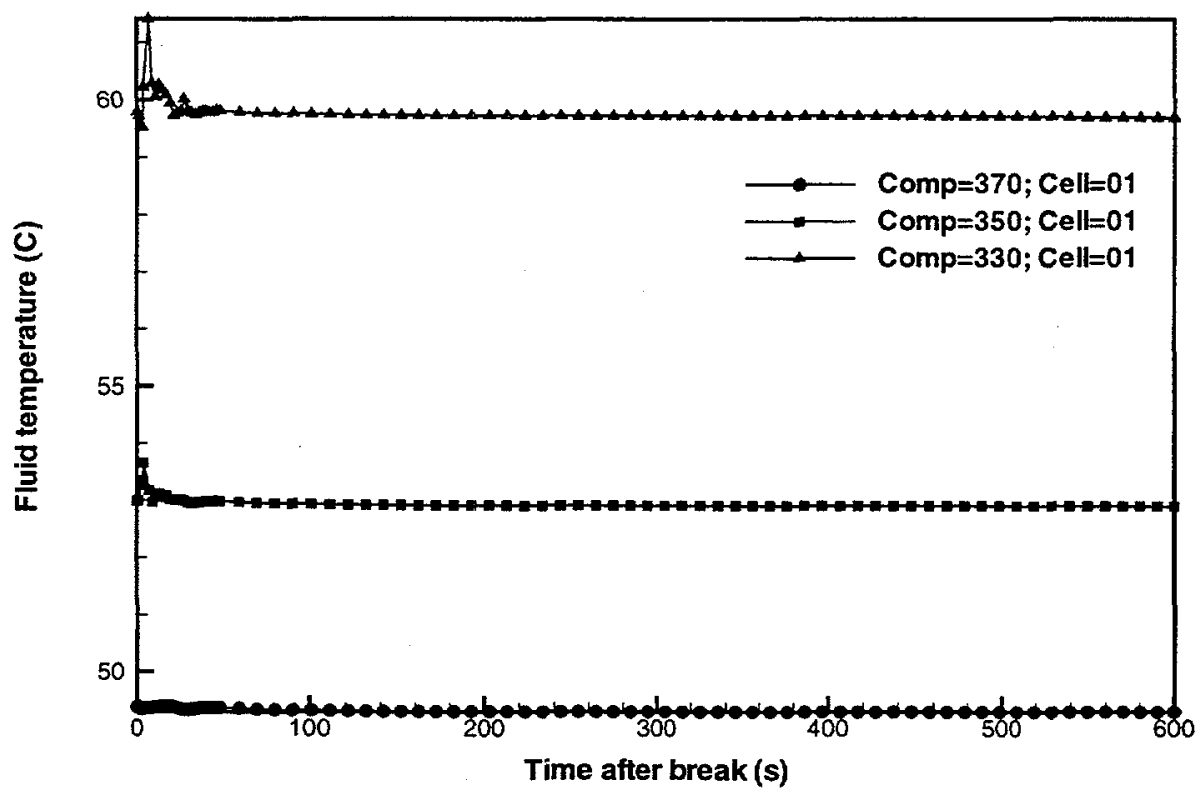

Figure B-3b Module 1 plenum fluid temperatures for a LOHGA (Case-1: helium supply plenum break near decoupler inlet).

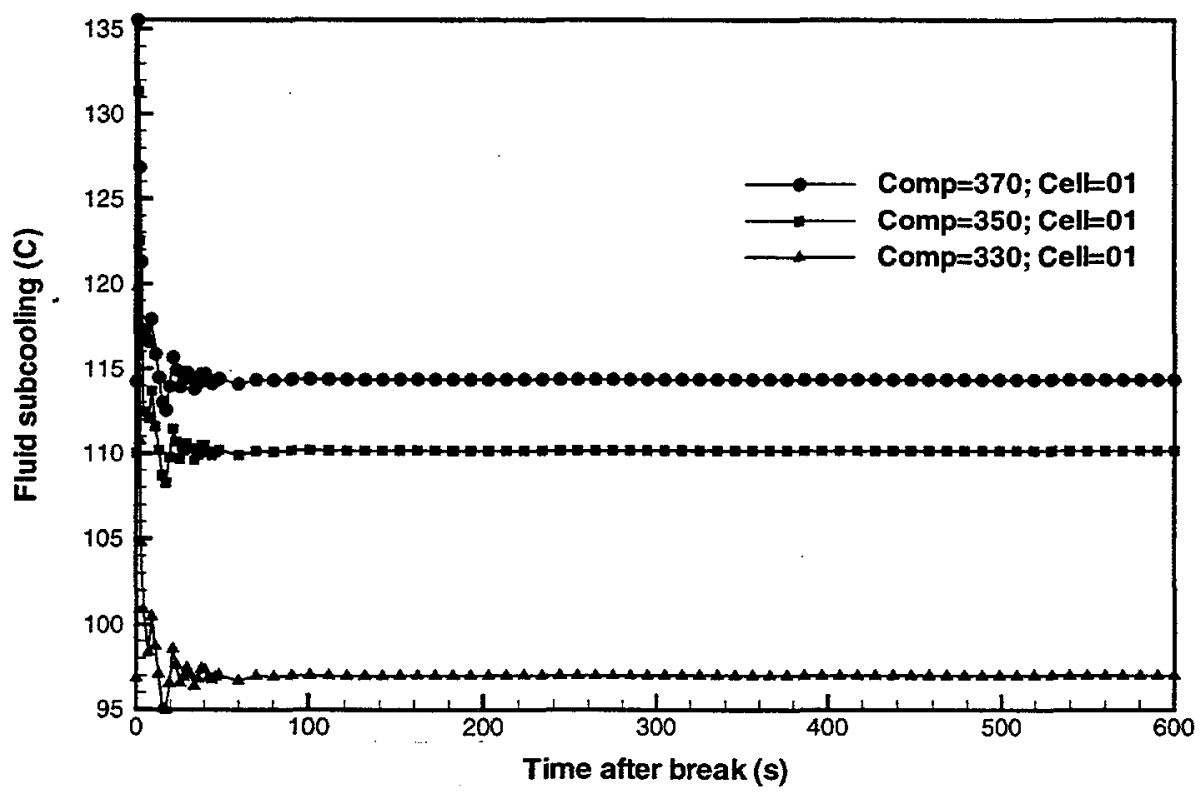

Figure B-3c Module 1 plenum fluid subcoolings for a LOHGA (Case 1: helium supply plenum break near decoupler inlet). 


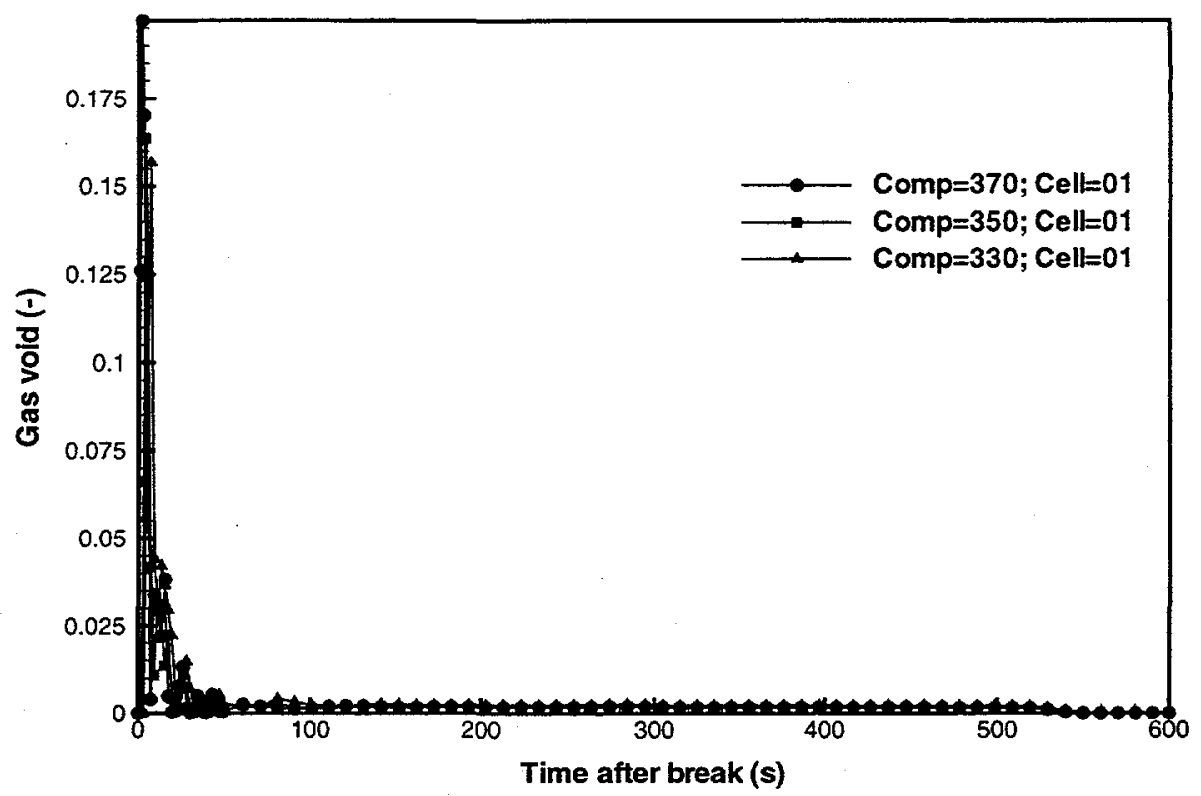

Figure B-3d Module 1 plenum void fractions for a LOHGA (Case 1: helium supply plenum break near decoupler inlet).

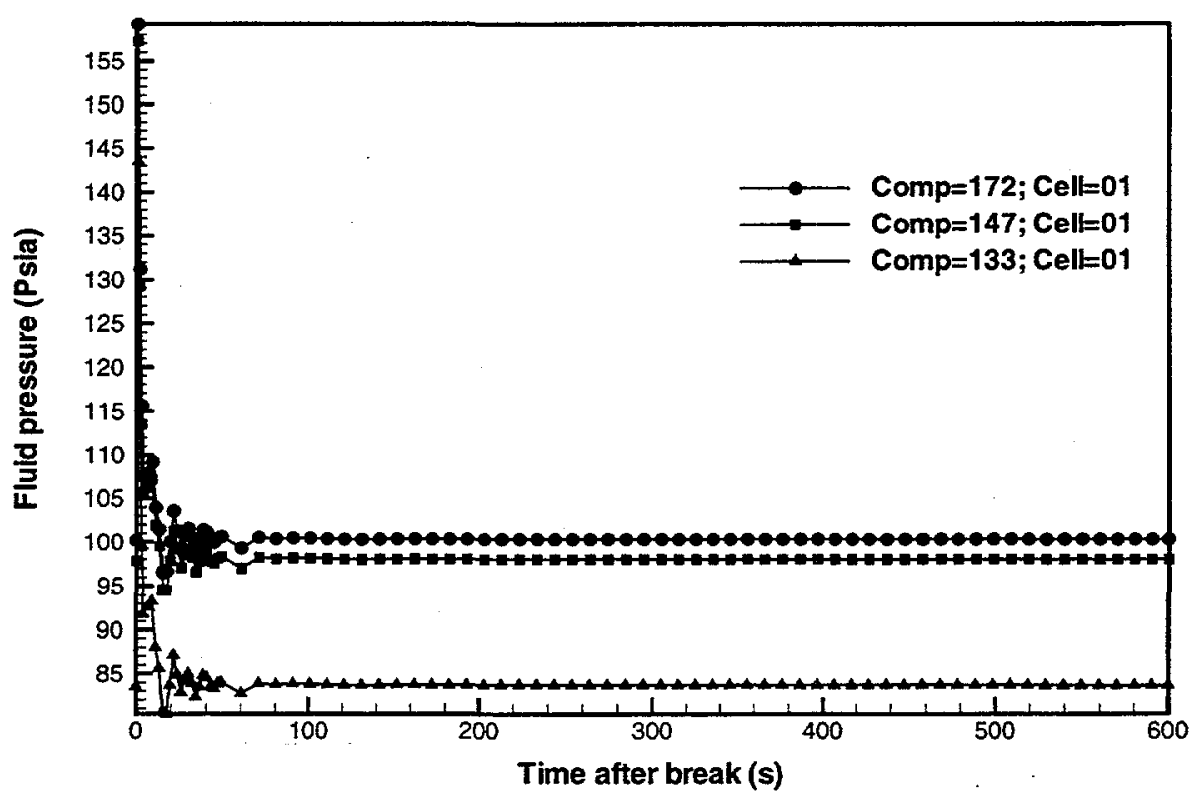

Figure B-4a Module 2 plenum fluid pressures for a LOHGA (Case 1: helium supply plenum break near decoupler inlet). 


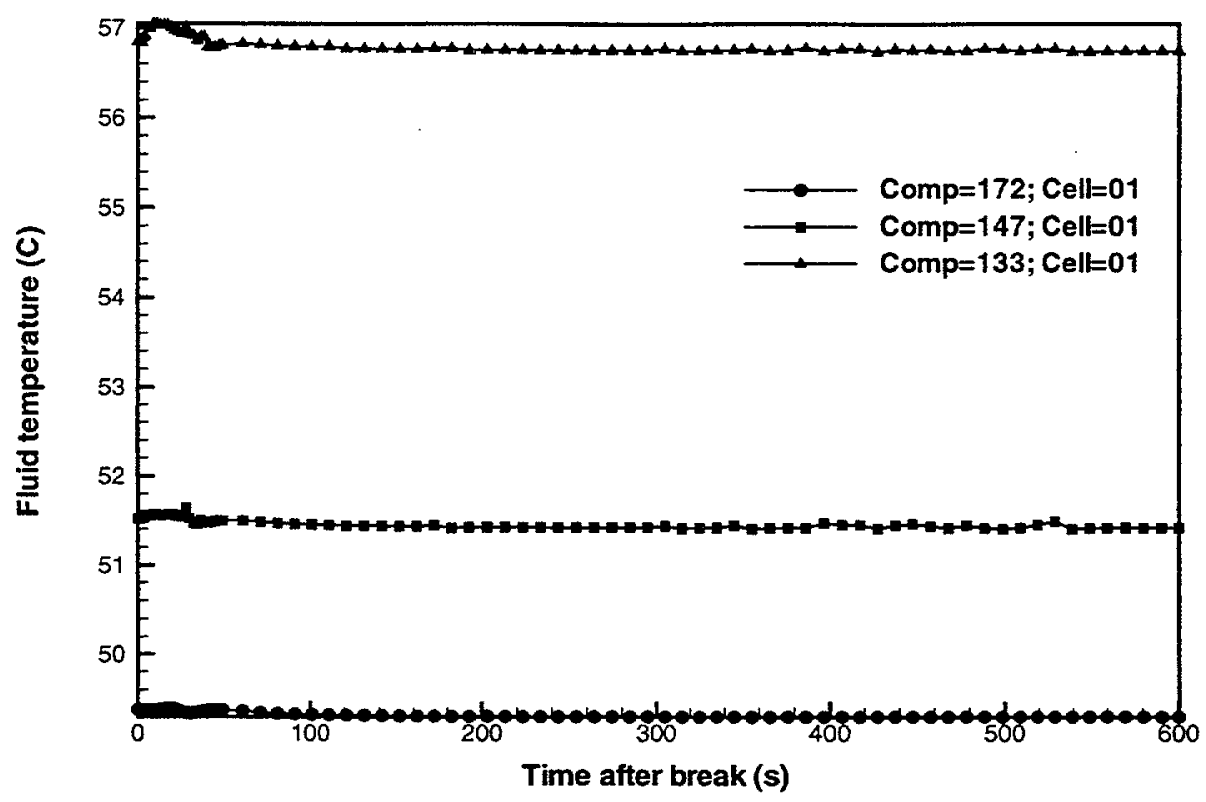

Figure B-4b Module 2 plenum fluid temperatures for a LOHGA (Case-1: helium supply plenum break near decoupler inlet).

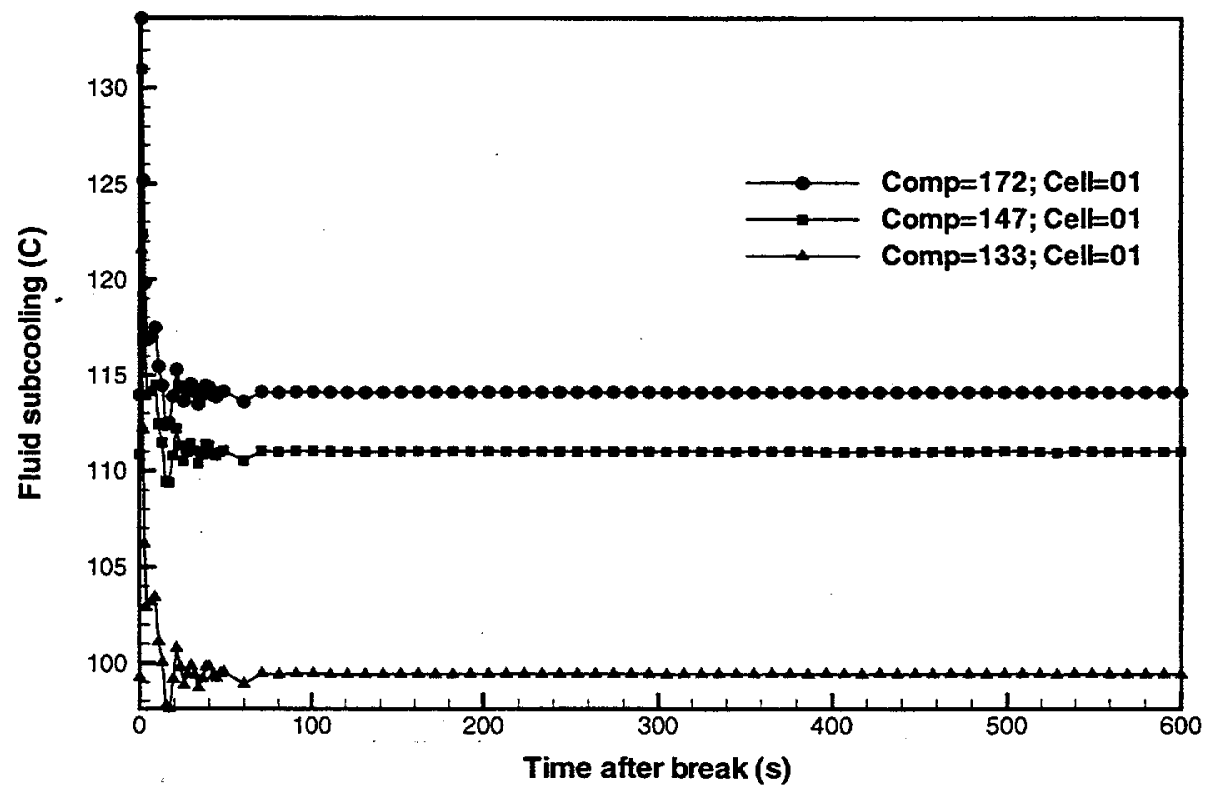

Figure B-4c Module 2 plenum fluid subcoolings for a LOHGA (Case 1: helium supply plenum break near decoupler inlet). 


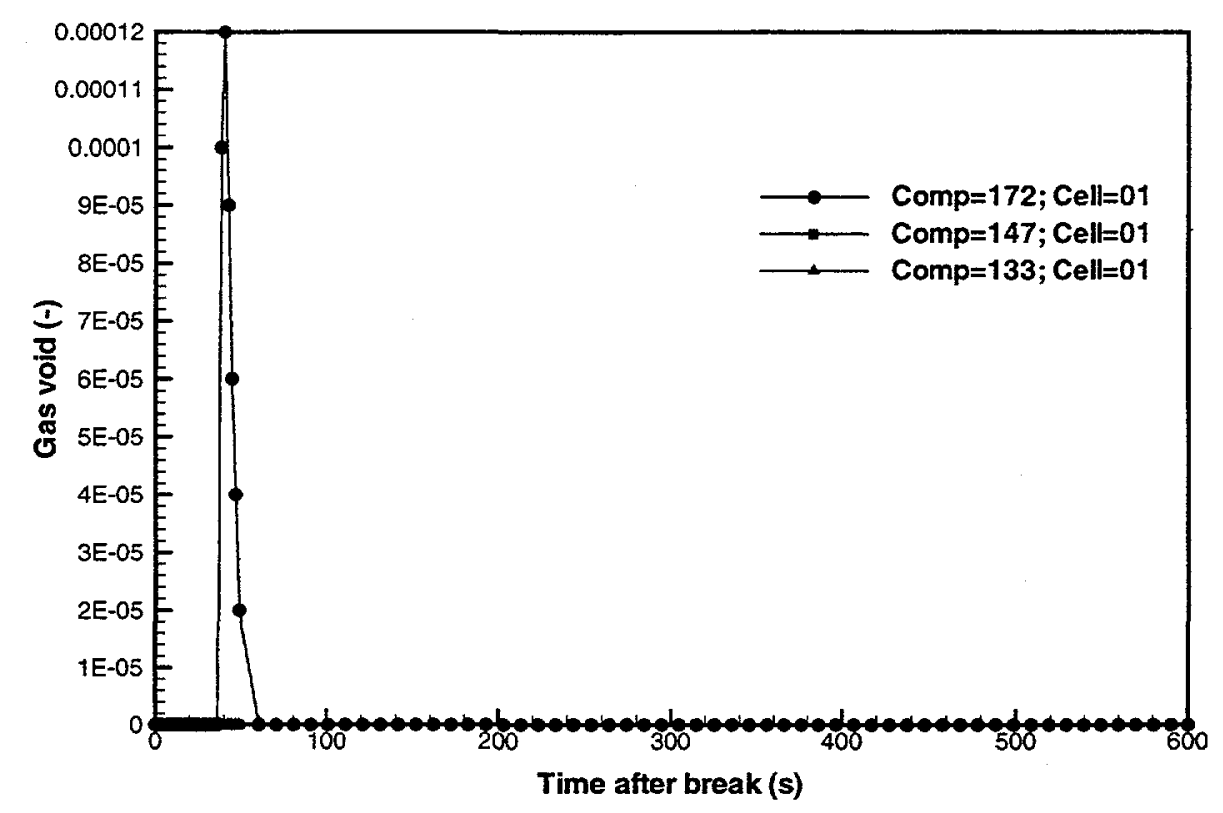

Figure B-4d Module 2 plenum void fractions for a LOHGA (Case 1: helium supply plenum break near decoupler inlet).

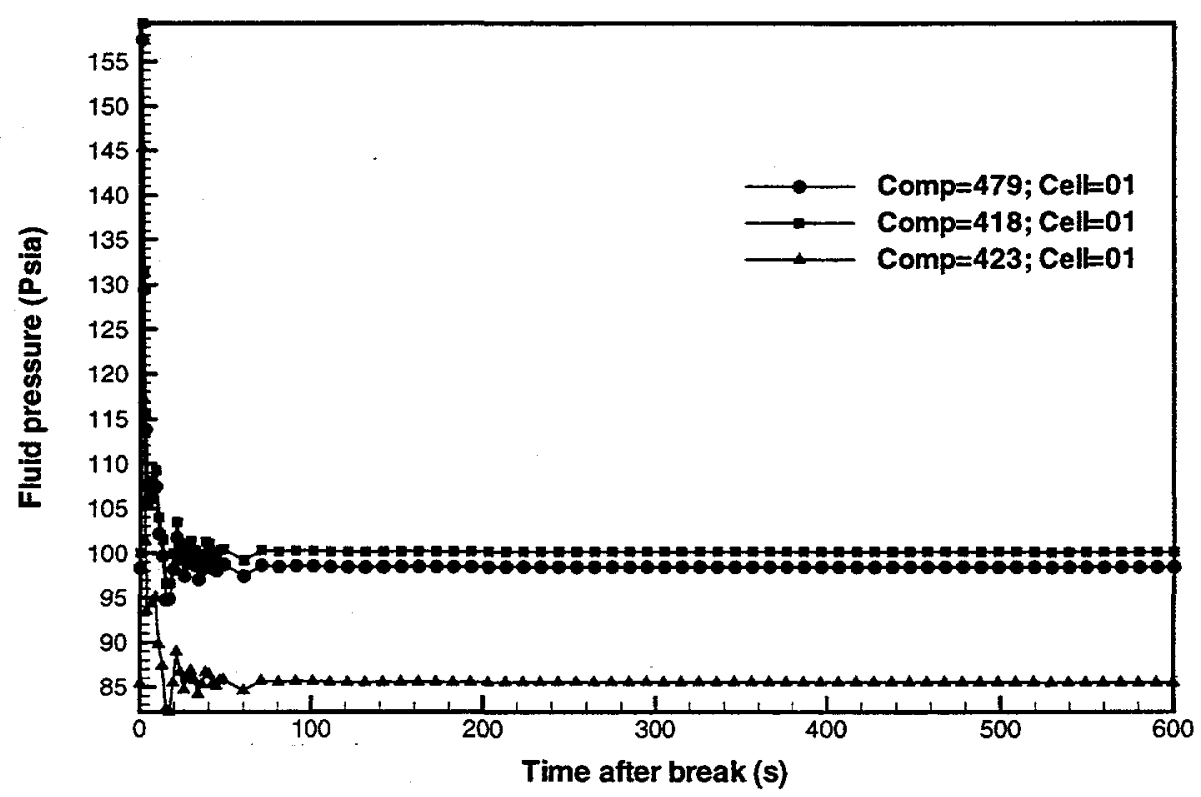

Figure B-5a Module 3 plenum fluid pressures for a LOHGA (Case 1: helium supply plenum break near decoupler inlet). 


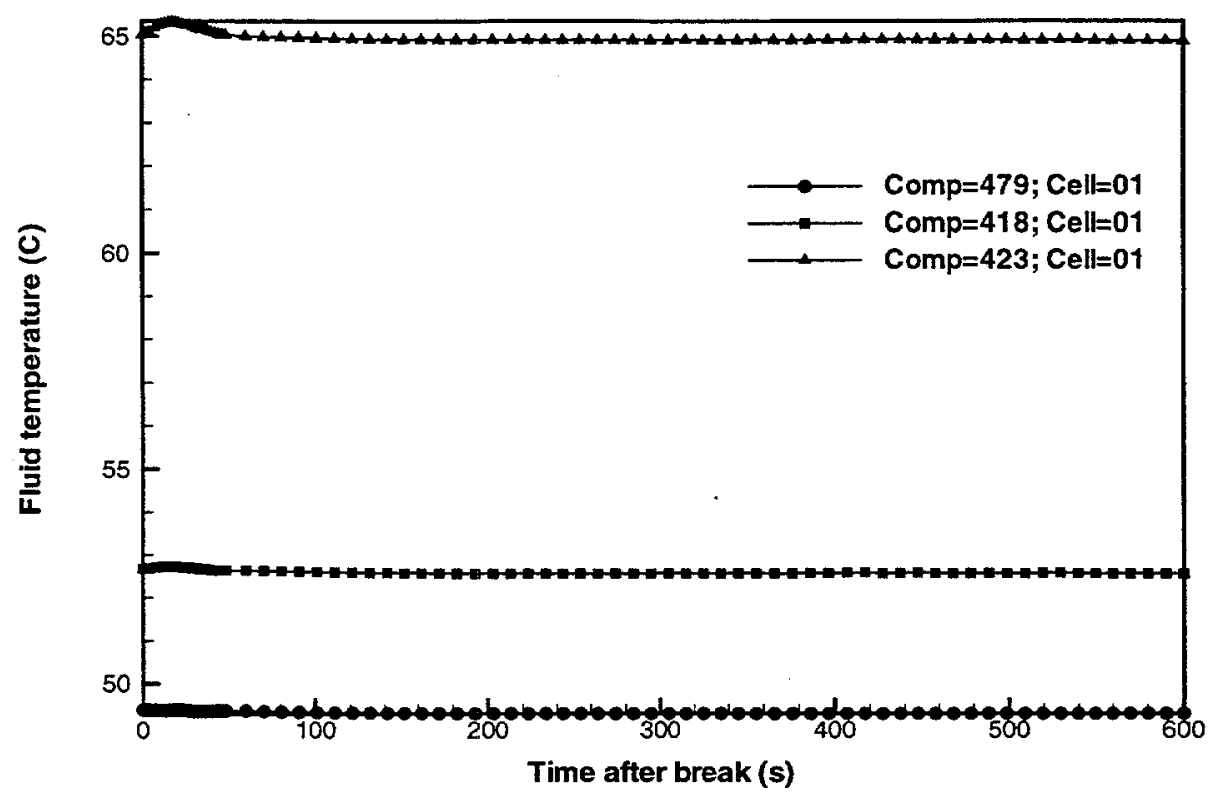

Figure B-5b Module 3 plenum fluid temperatures for a LOHGA (Case-1: helium supply plenum break near decoupler inlet).

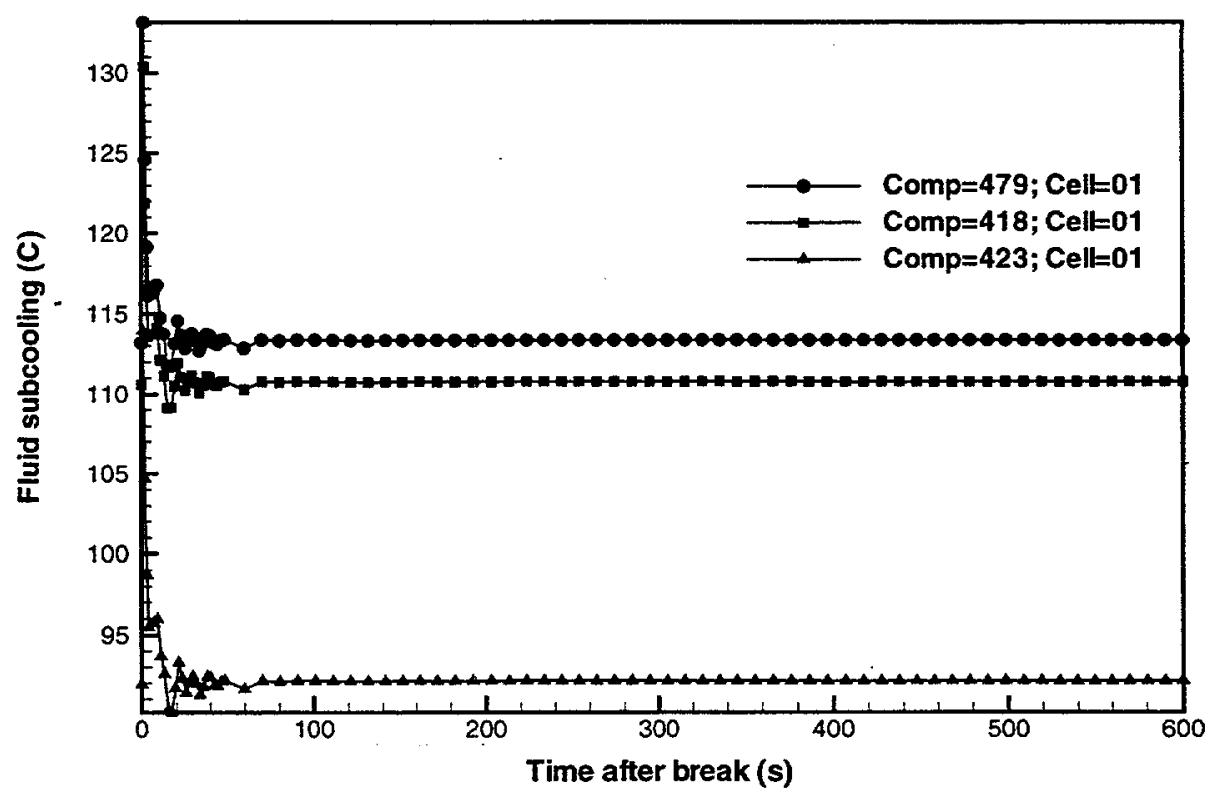

Figure B-5c Module 3 plenum fluid subcoolings for a LOHGA (Case 1: helium supply plenum break near decoupler inlet). 


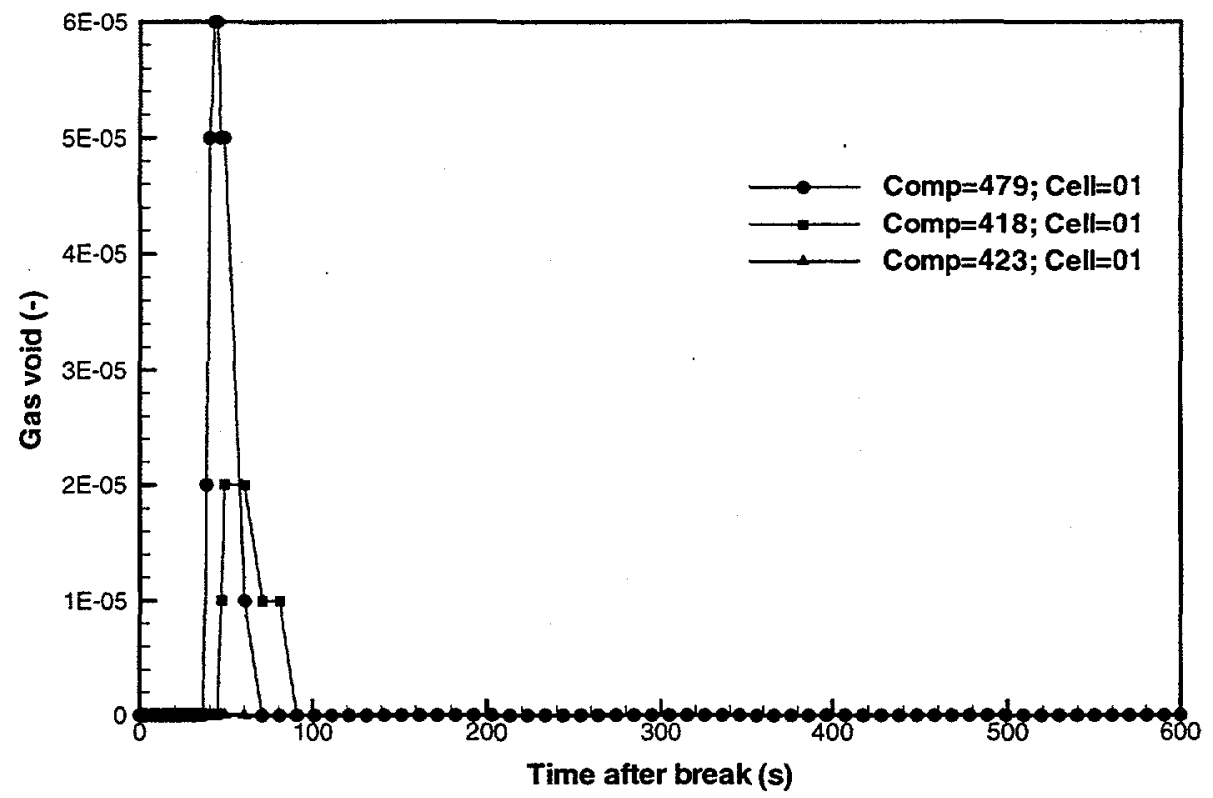

Figure B-5d Module 3 plenum void fractions for a LOHGA (Case 1: helium supply plenum break near decoupler inlet).

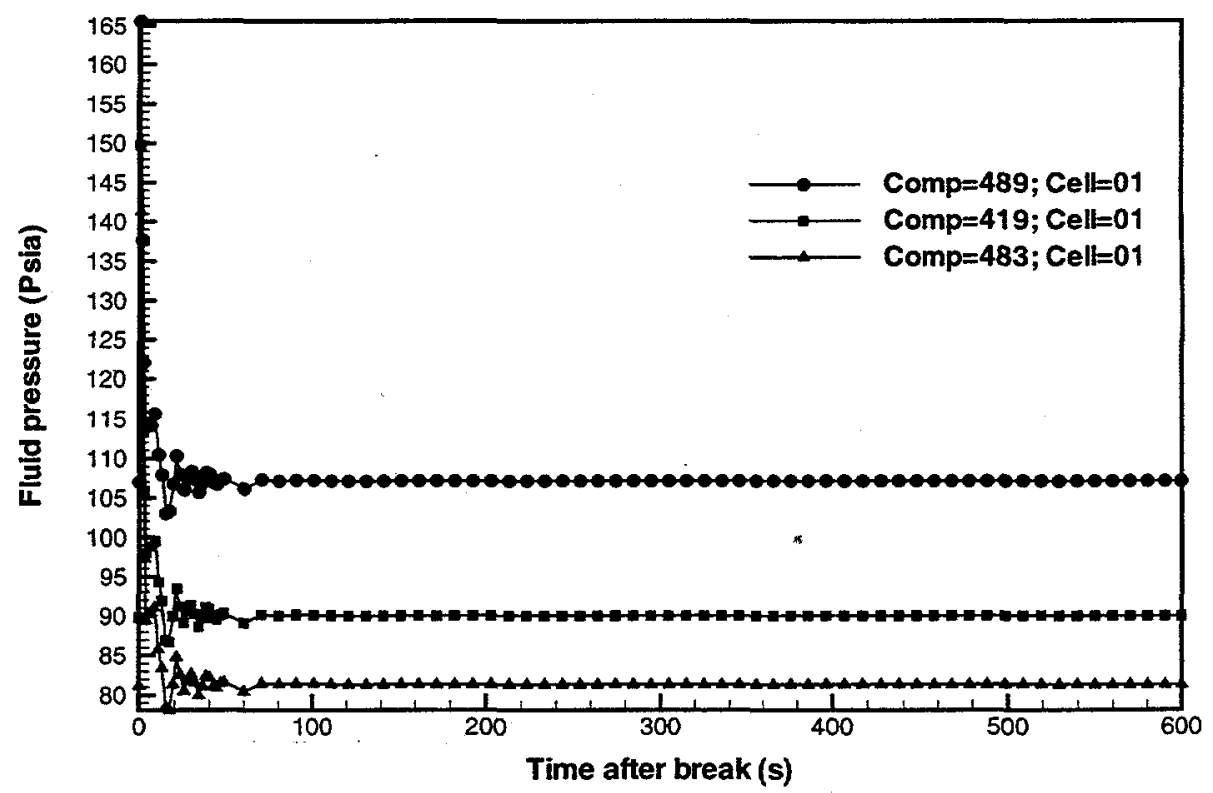

Figure B-6a Module 4 plenum fluid pressures for a LOHGA (Case 1: helium supply plenum break near decoupler inlet). 


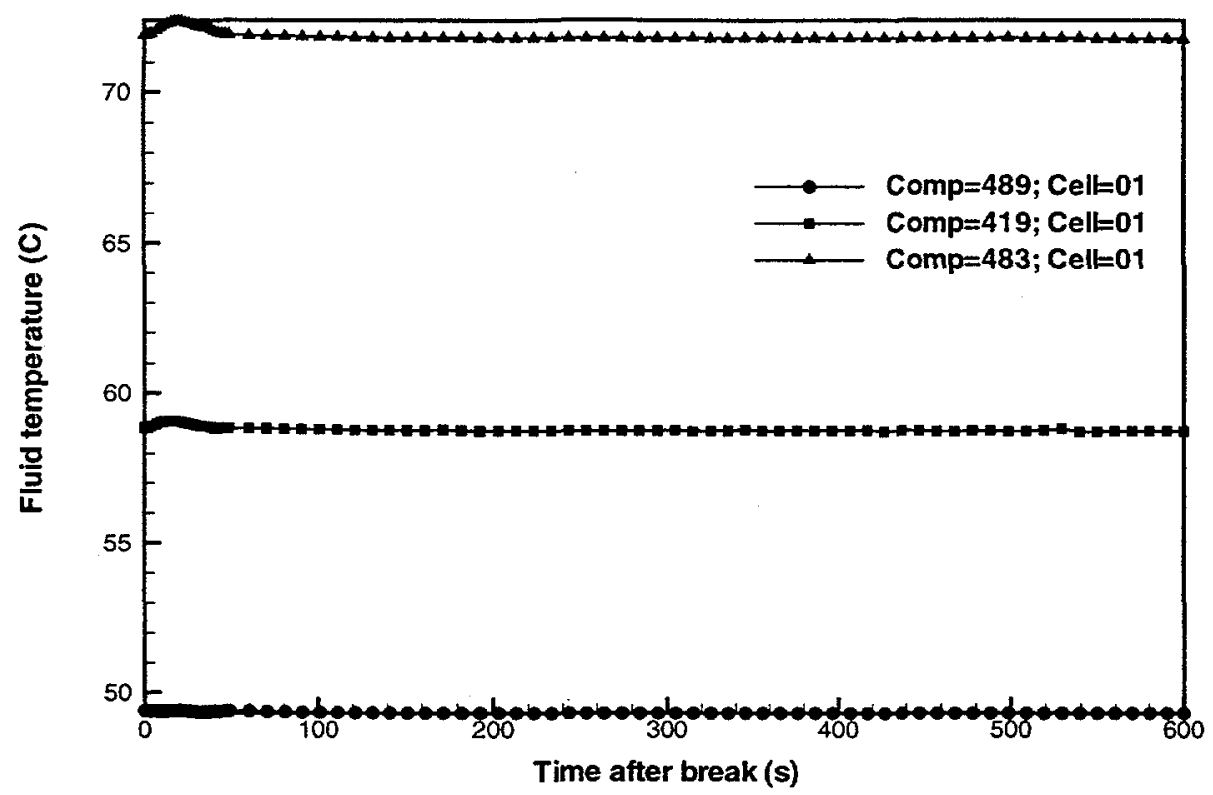

Figure B-6b Module 4 plenum fluid temperatures for a LOHGA (Case-1: helium supply plenum break near decoupler inlet).

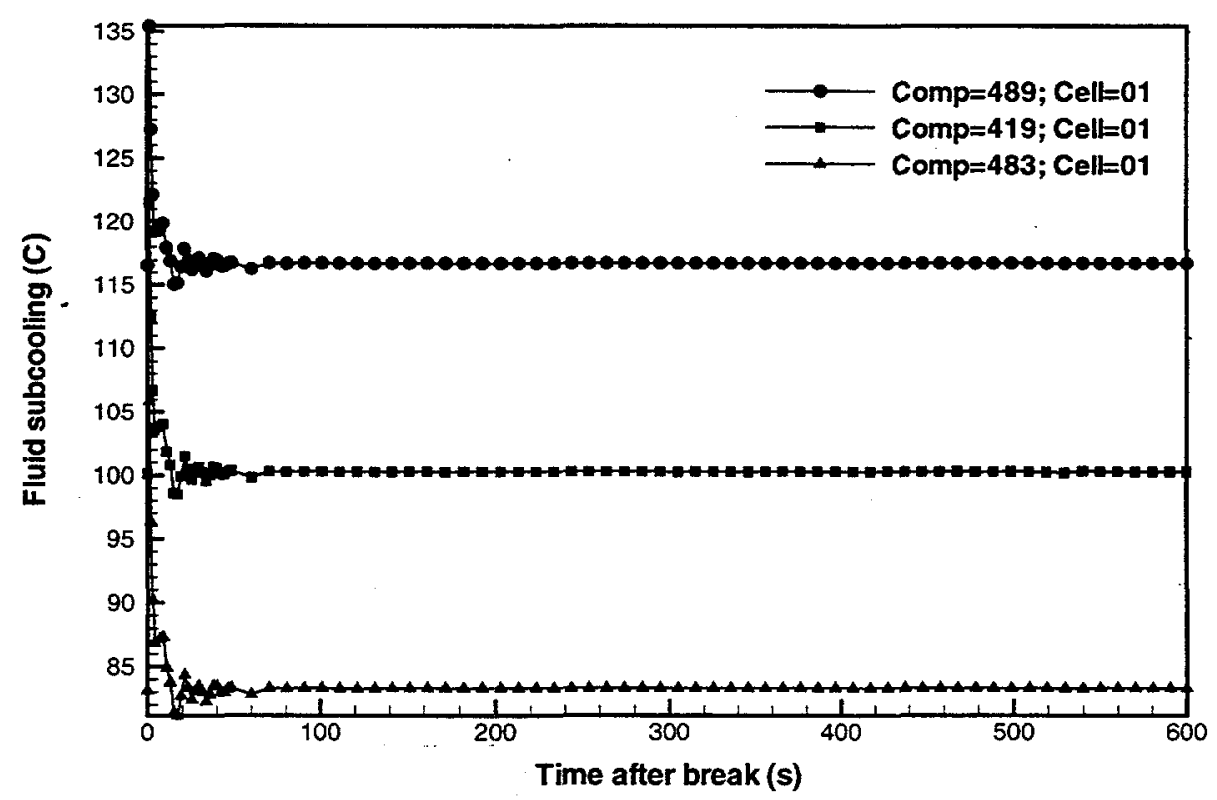

Figure B-6c Module 4 plenum fluid subcoolings for a LOHGA (Case 1: helium supply plenum break near decoupler inlet). 


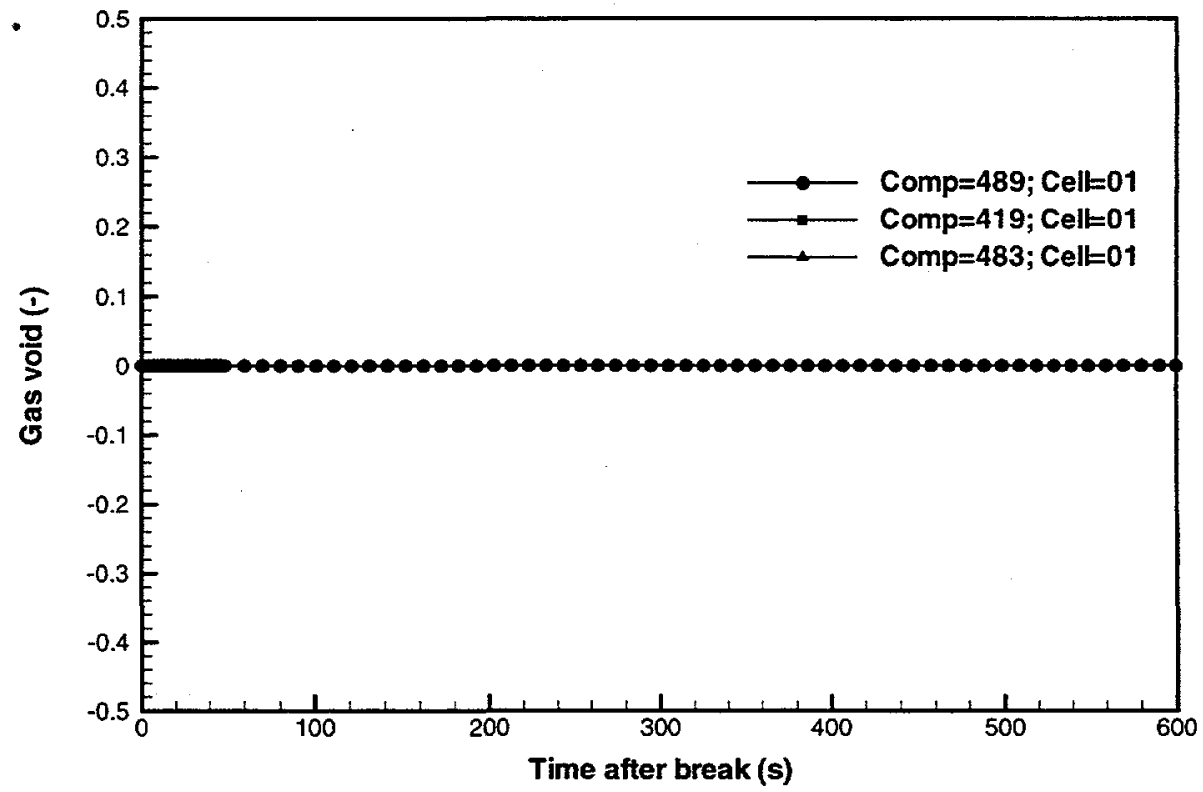

. Figure B-6d Module 4 plenum void fractions for a LOHGA (Case 1: helium supply plenum break near decoupler inlet).

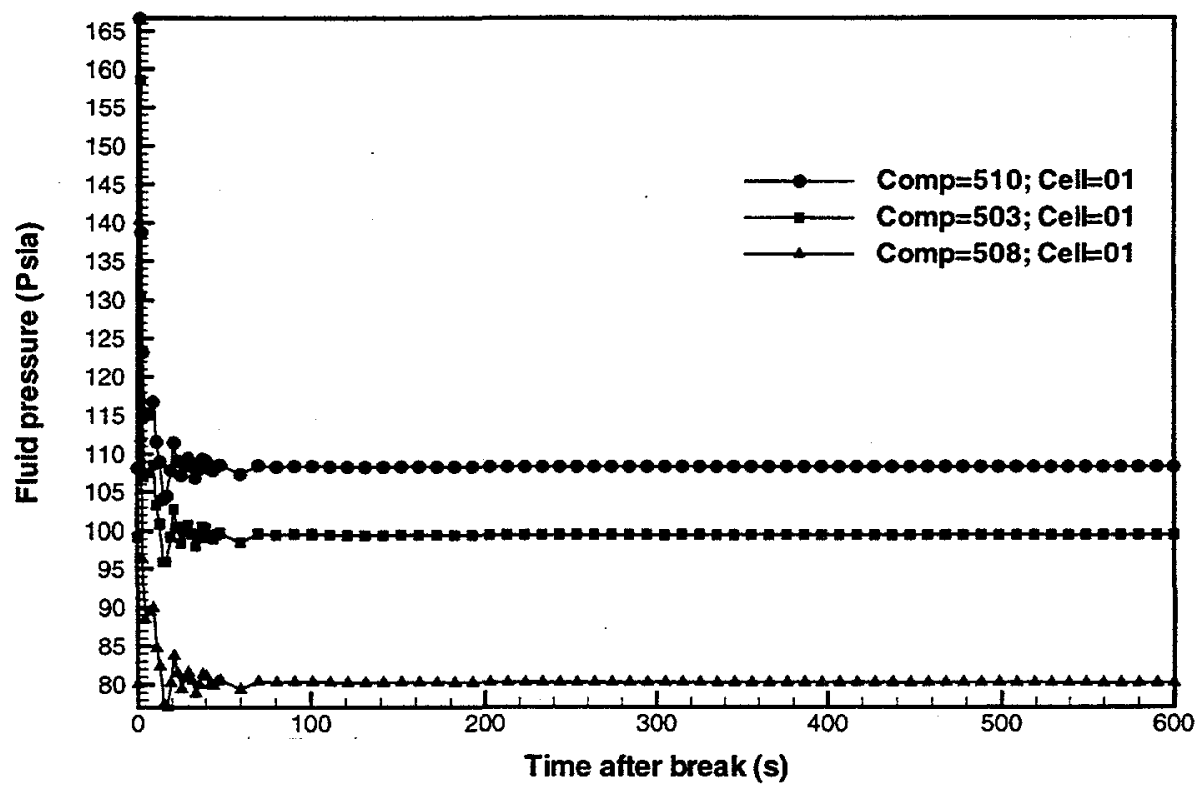

Figure B-7a Module 5 plenum fluid pressures for a LOHGA (Case 1: helium supply plenum break near decoupler inlet). 


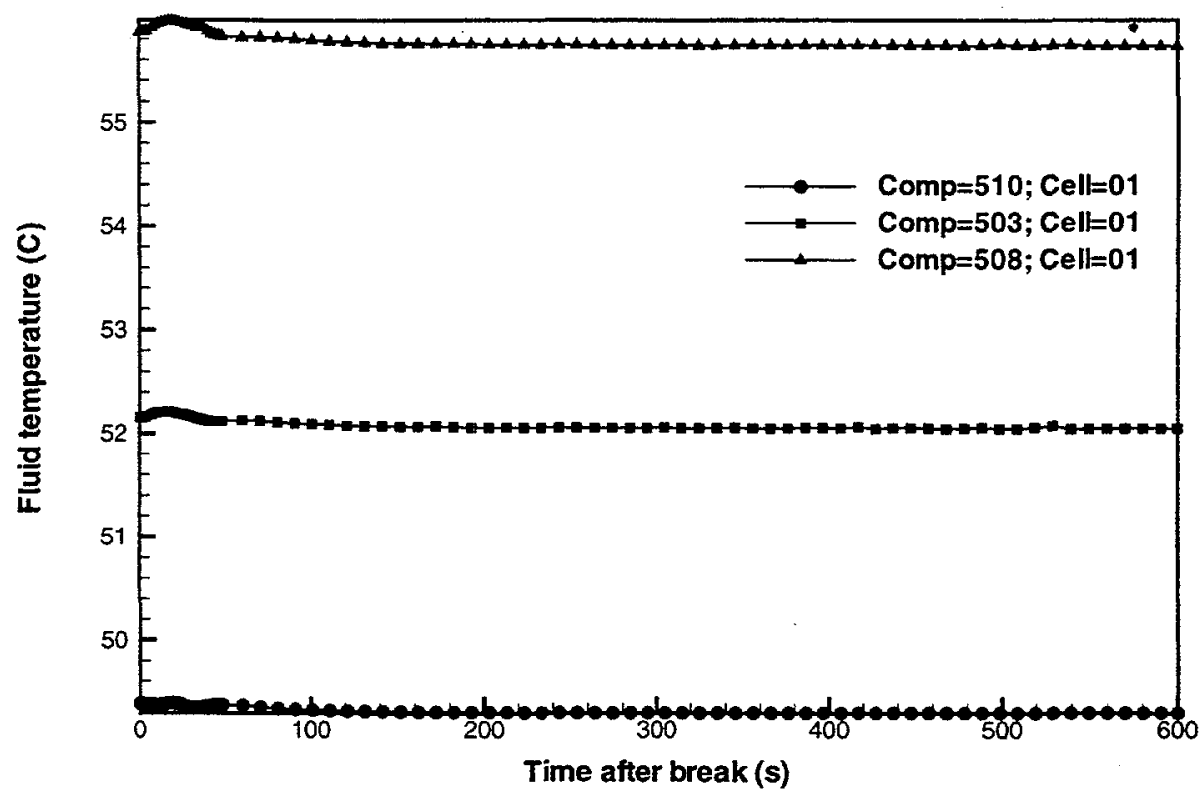

Figure B-7b Module 5 plenum fluid temperatures for a LOHGA (Case 1: helium supply plenum break near decoupler inlet).

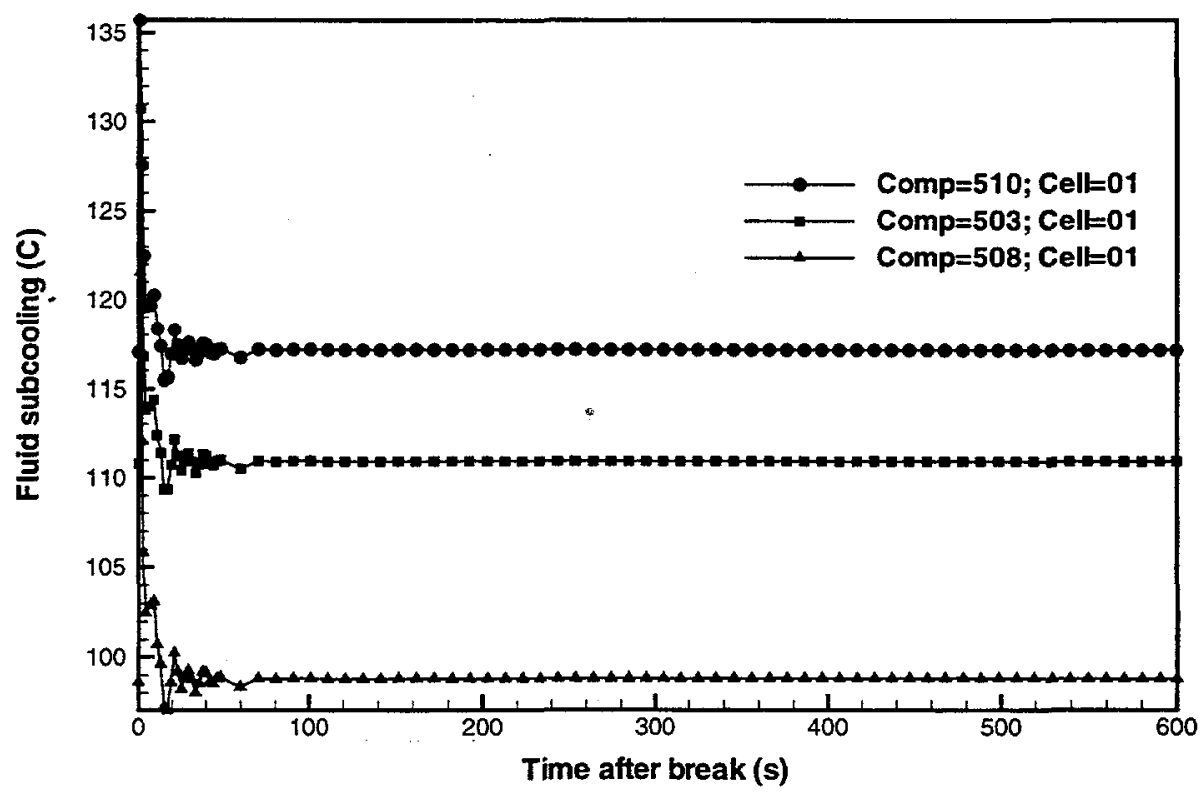

Figure B-7c Module 5 plenum fluid subcoolings for a LOHGA (Case 1: helium supply plenum break near decoupler inlet). 


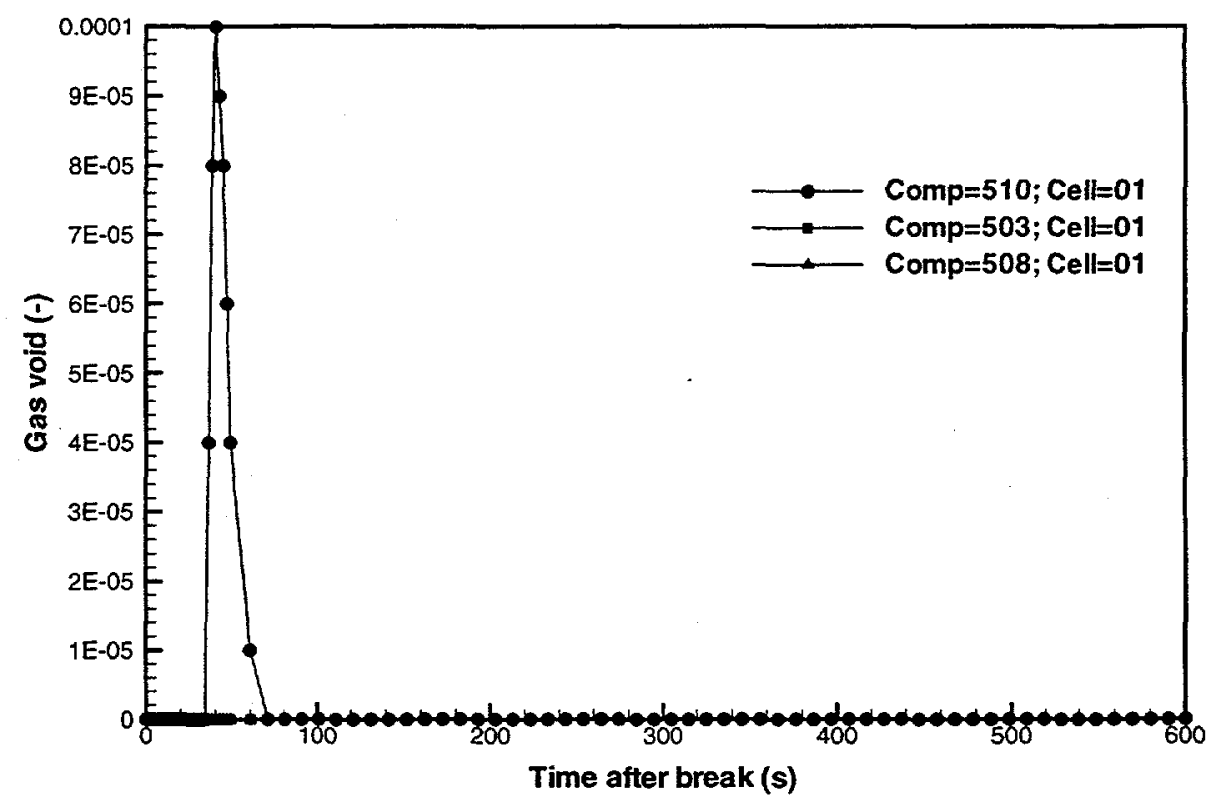

Figure B-7d Module 5 plenum void fractions for a LOHGA (Case 1: helium supply plenum break near decoupler inlet).

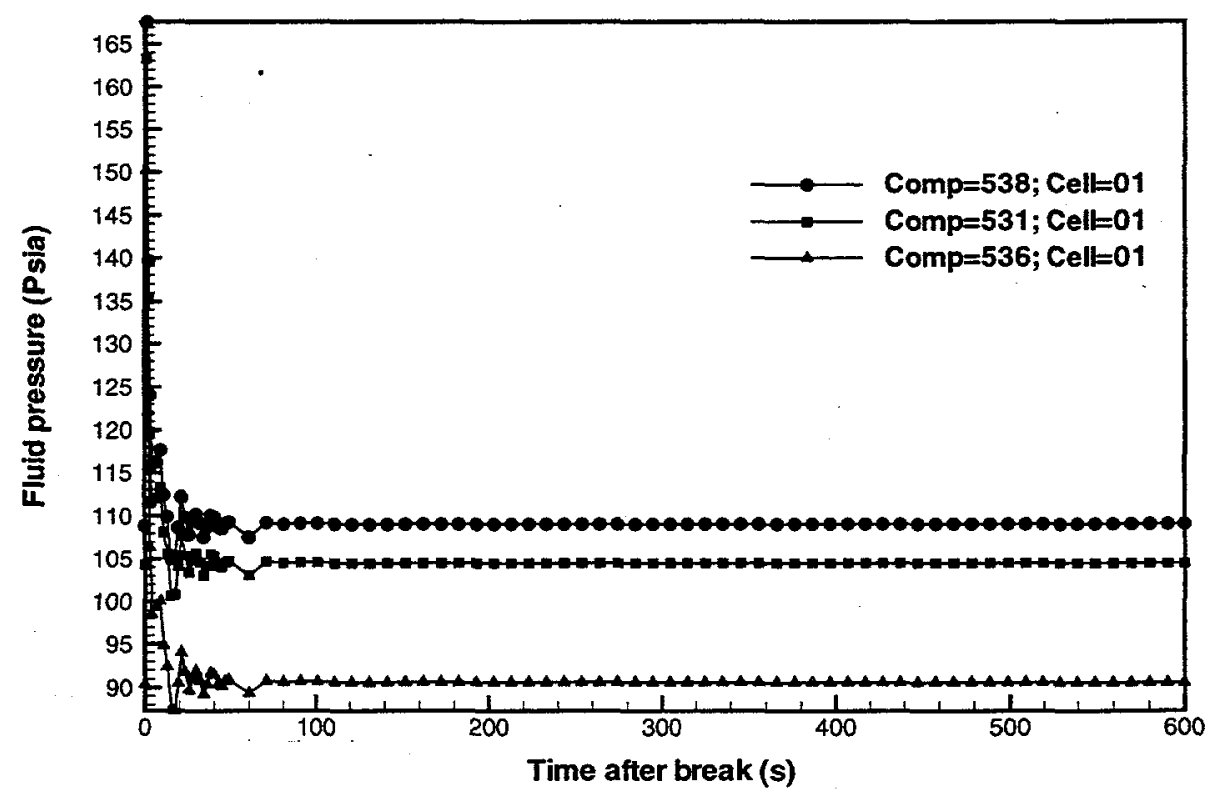

Figure B-8a Module 6 plenum fluid pressures for a LOHGA (Case 1: helium supply plenum break near decoupler inlet). 


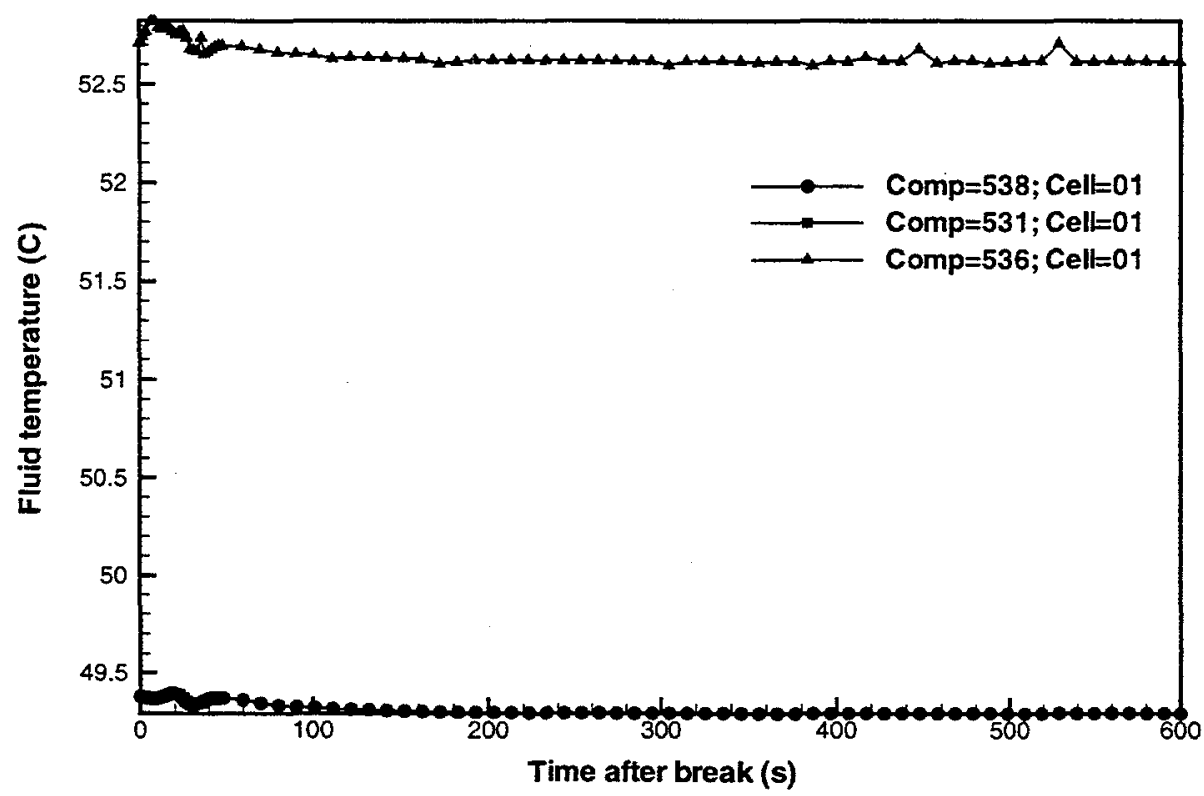

Figure B-8b Module 6 plenum fluid temperatures for a LOHGA (Case-1: helium supply plenum break near decoupler inlet).

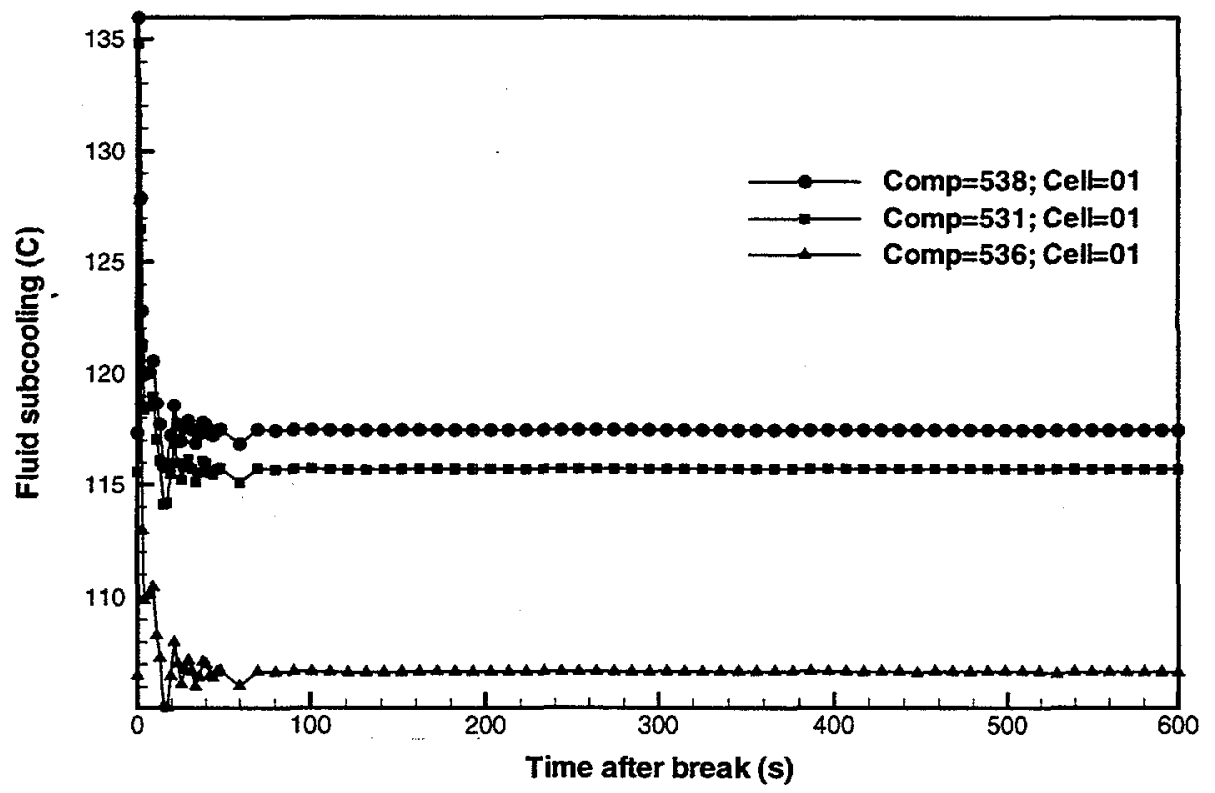

Figure B-8c Module 6 plenum fluid subcoolings for a LOHGA (Case 1: helium supply plenum break near decoupler inlet). 


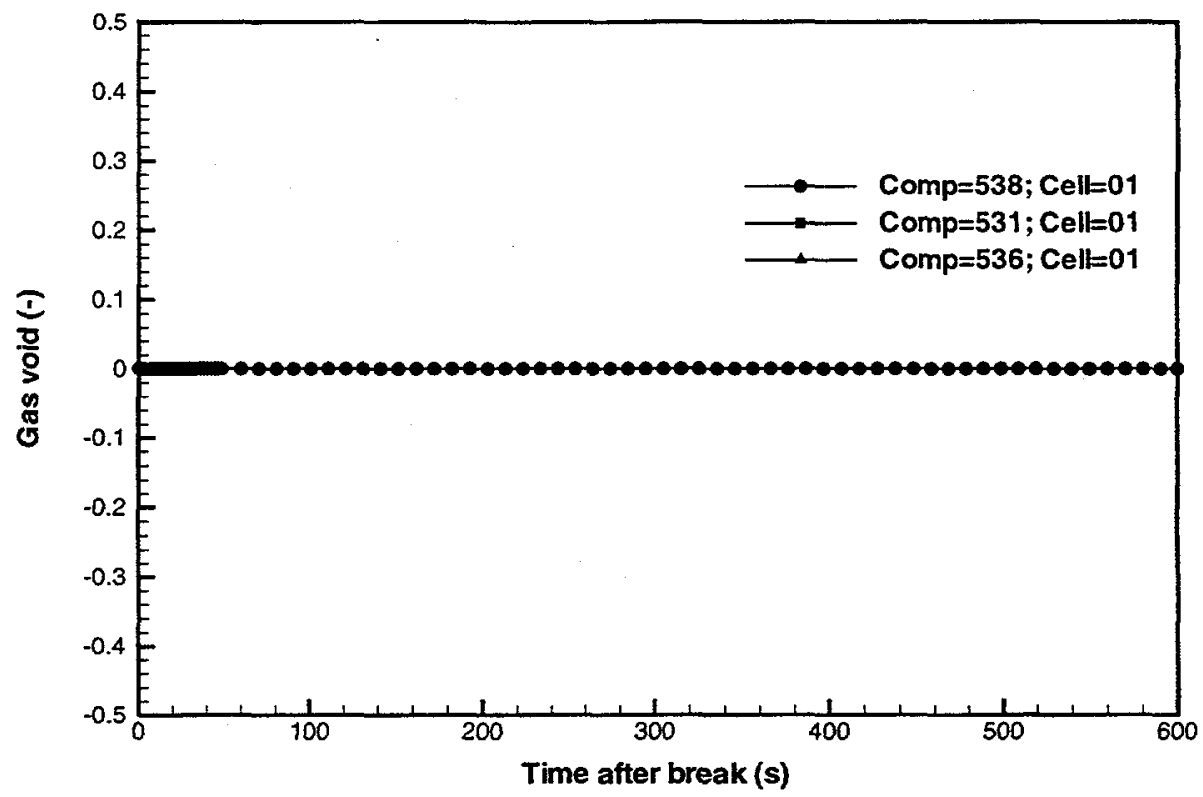

Figure B-8d Module 6 plenum void fractions for a LOHGA (Case 1: helium supply plenum break near decoupler inlet).

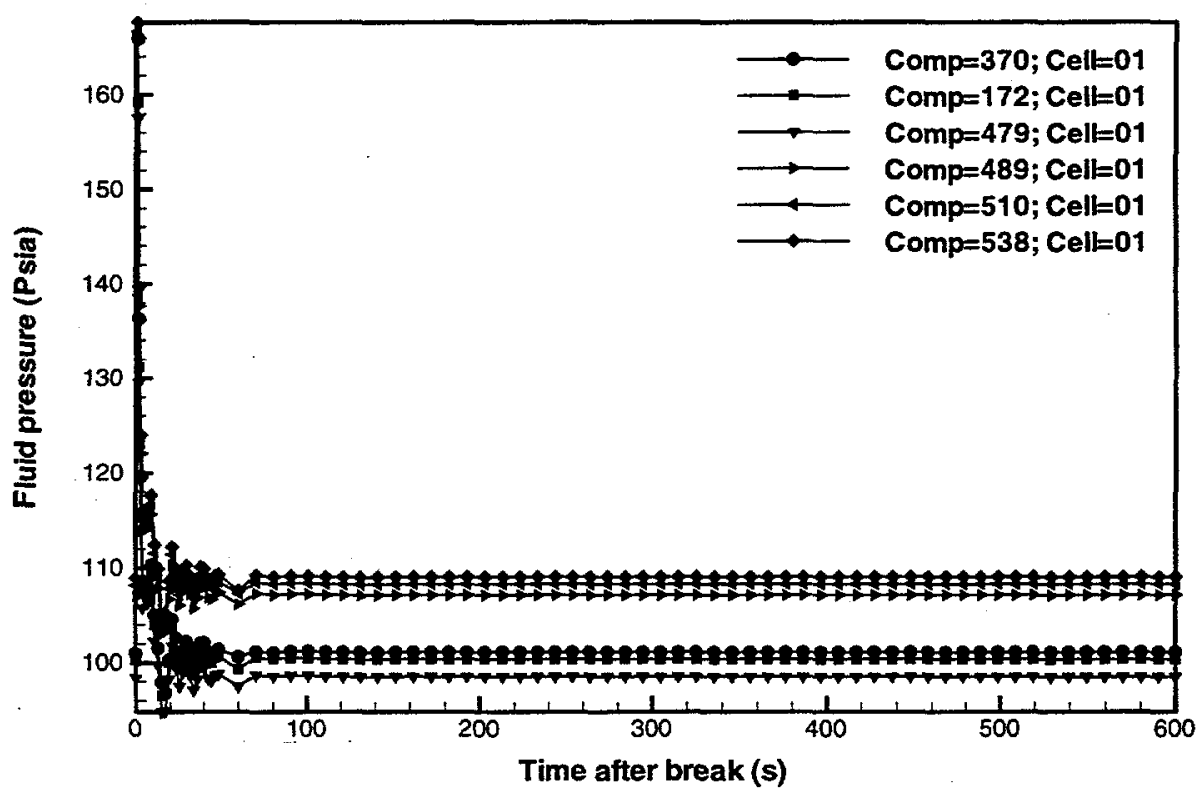

Figure B-9a Module inlet plenum fluid pressures for a LOHGA (Case 1: helium supply plenum break near decoupler inlet). 


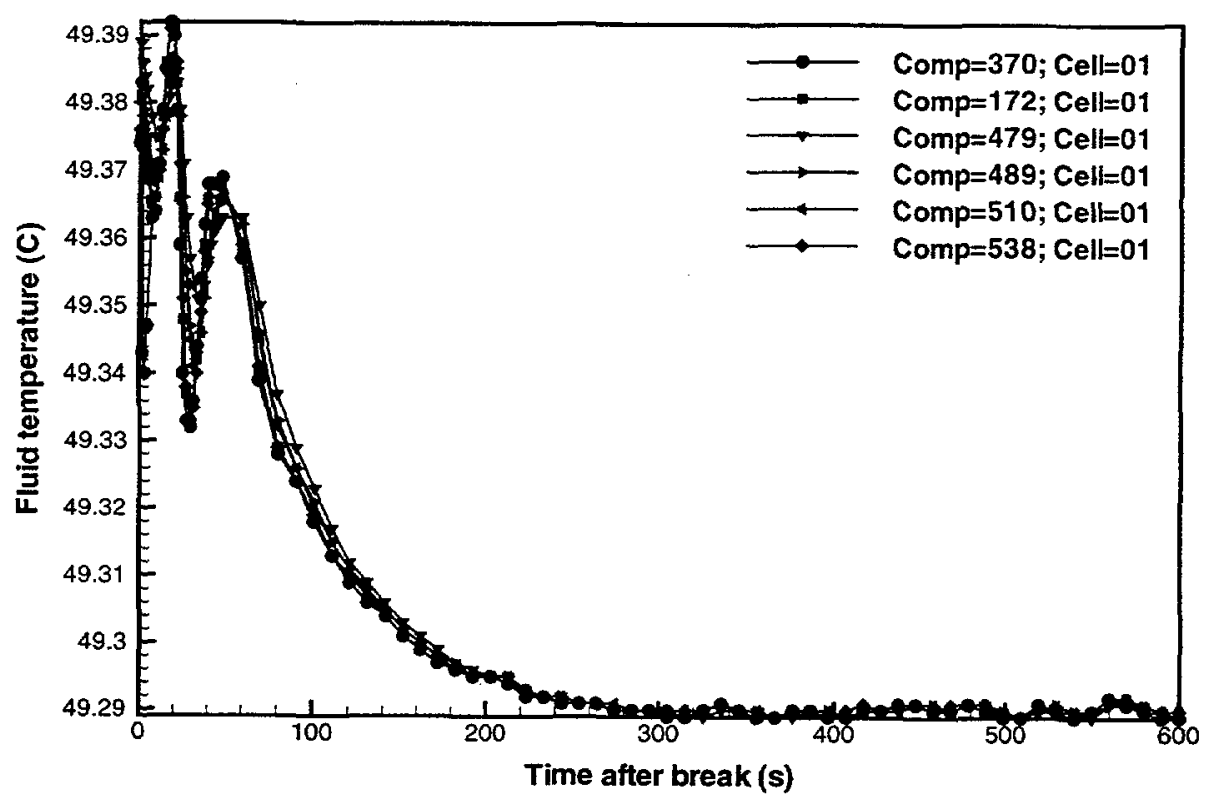

Figure B-9b Module inlet plenum fluid temperatures for a LOHGA (Case 1: helium supply plenum break near decoupler inlet).

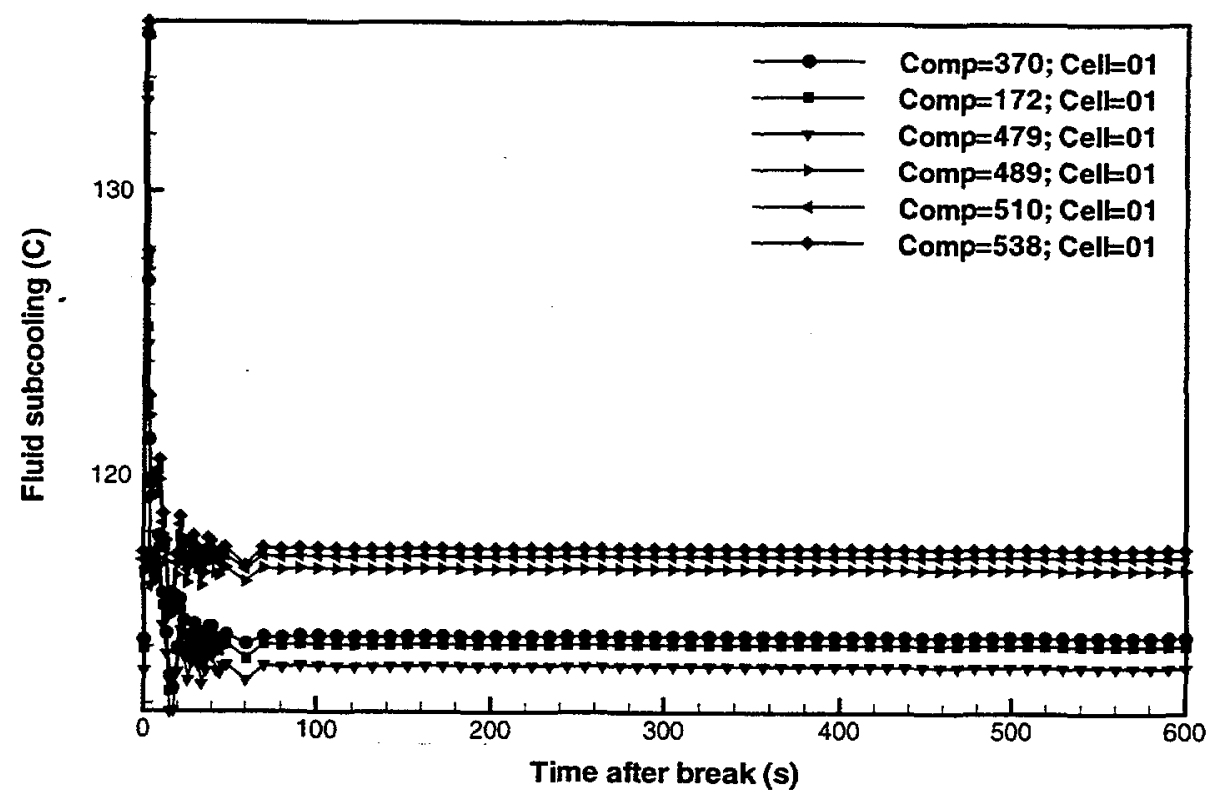

Figure B-9c Module inlet plenum fluid subcoolings for a LOHGA (Case 1: helium supply plenum break near decoupler inlet). 


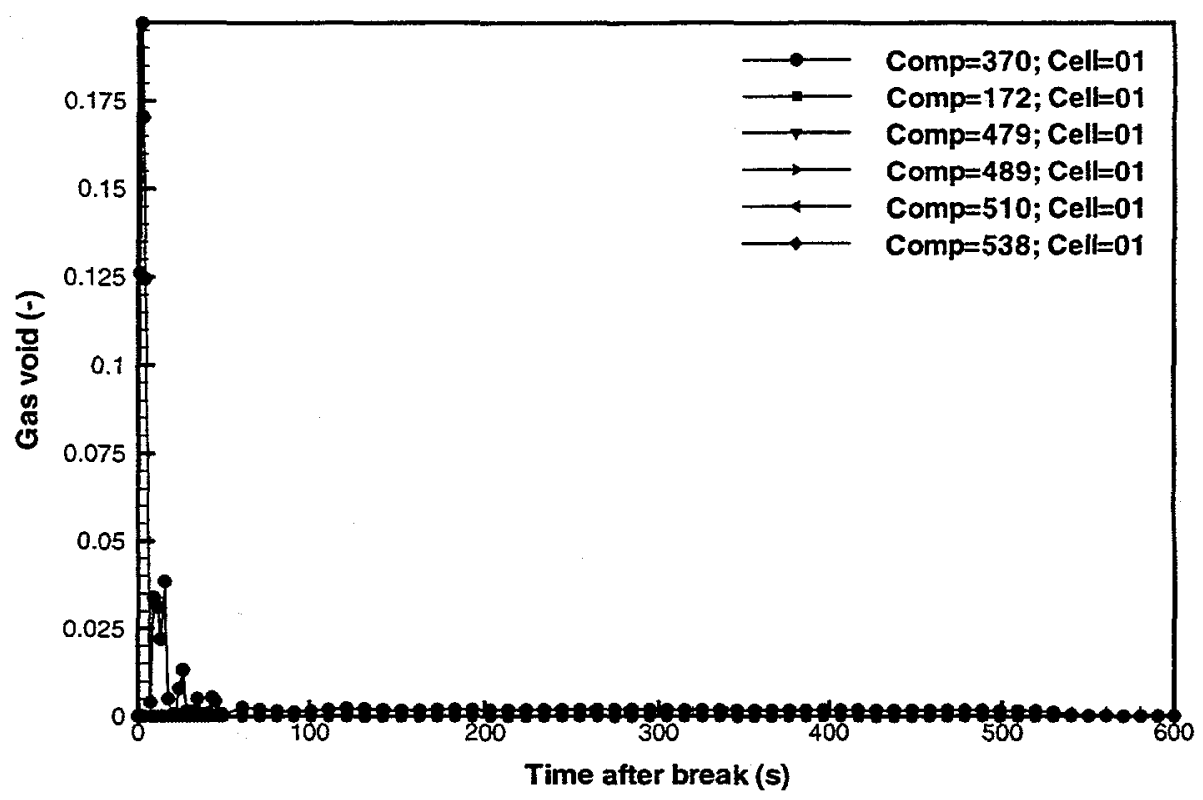

Figure B-9d Module inlet plenum void fractions for a LOHGA (Case 1: helium supply plenum break near decoupler inlet).

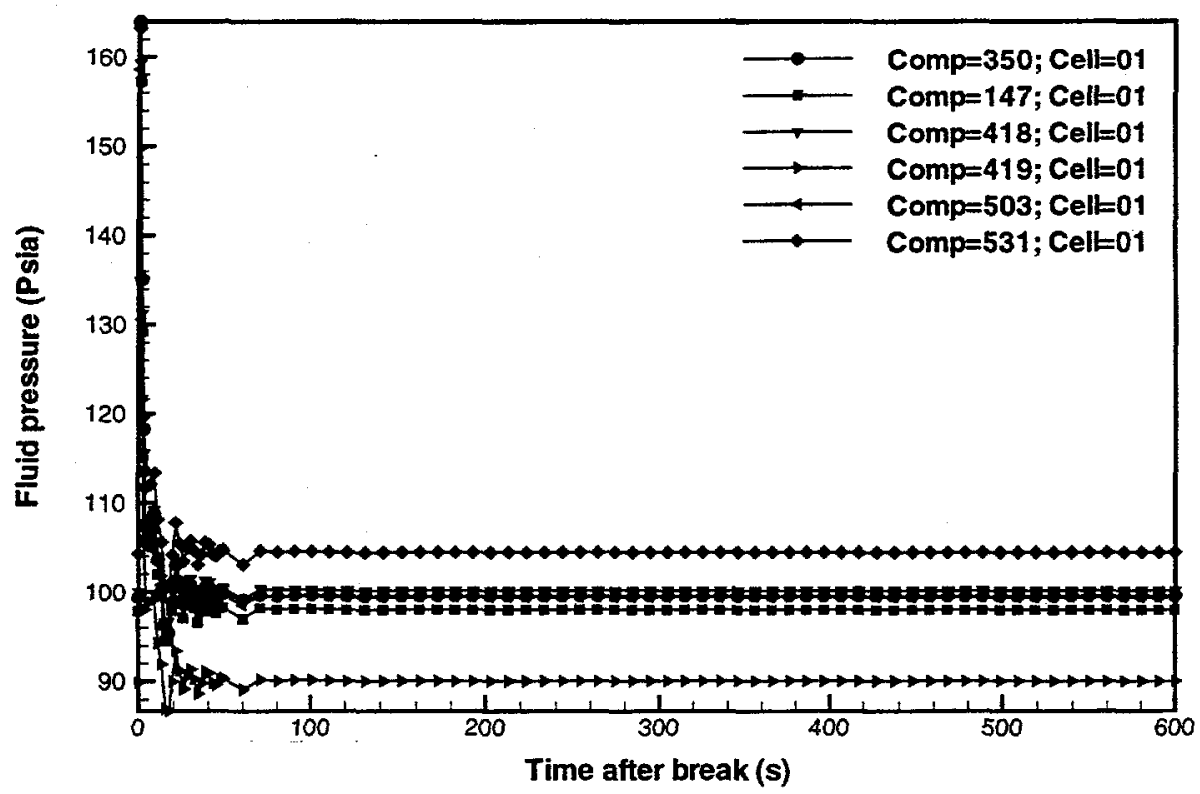

Figure B-10a Module middle plenum fluid pressures for a LOHGA (Case 1: helium supply plenum break near decoupler inlet). 


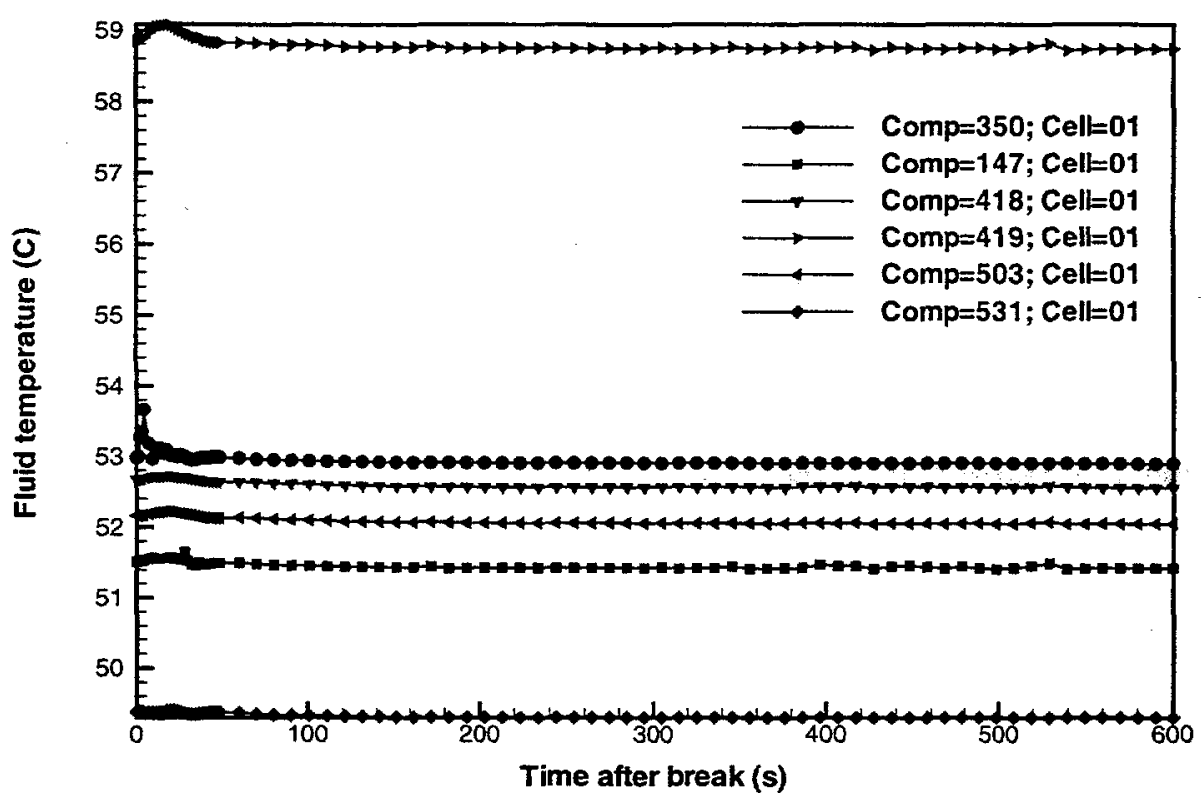

Figure B-10b Module middle plenum fluid temperatures for a LOHGA (Case 1: helium supply plenum break near decoupler inlet).

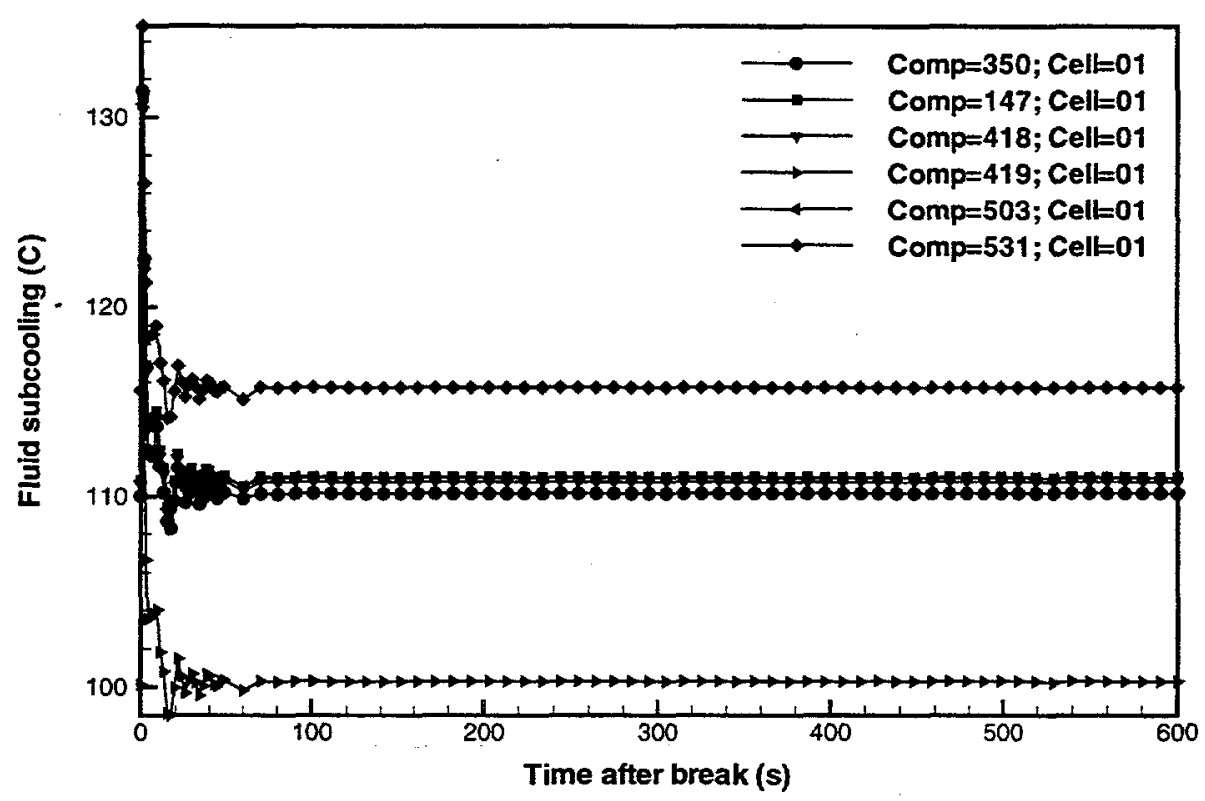

Figure B-10c Module middle plenum fluid subcoolings for a LOHGA (Case 1: helium supply plenum break near decoupler inlet). 


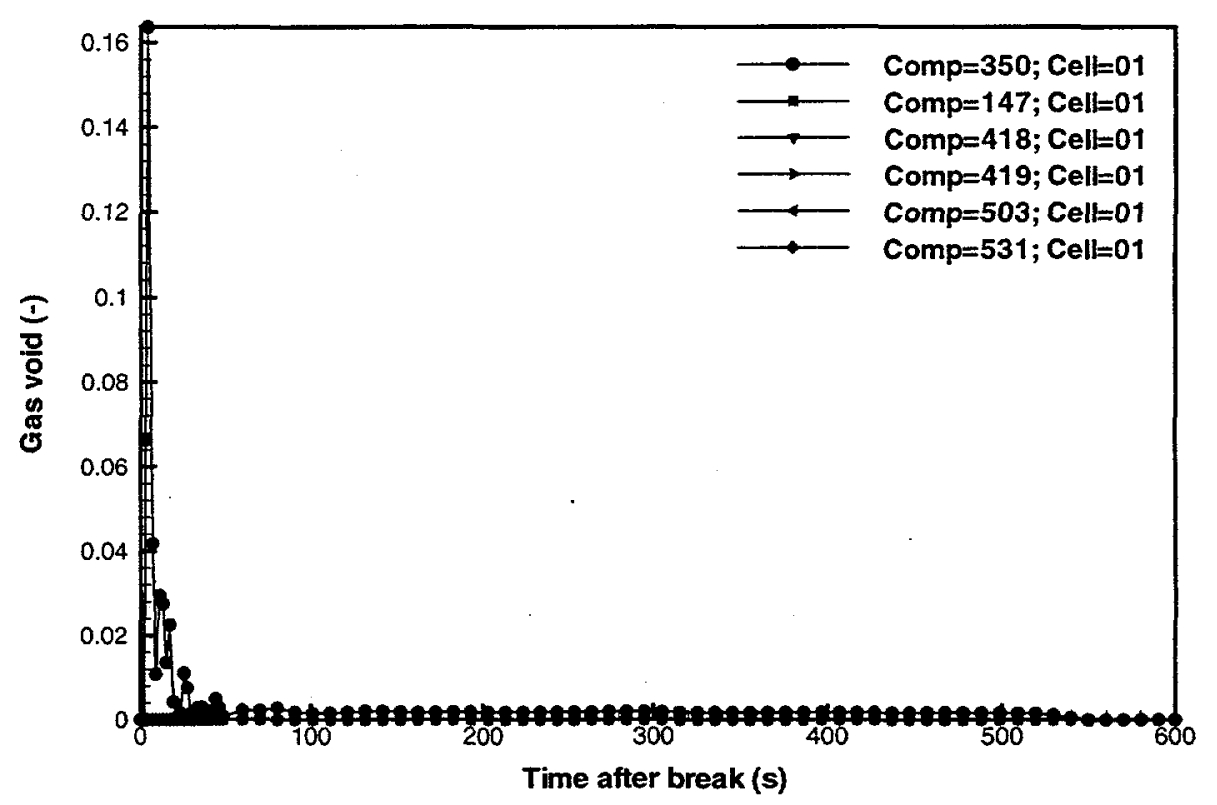

Figure B-10d Module middle plenum void fractions for a LOHGA (Case 1: helium supply plenum break near decoupler inlet).

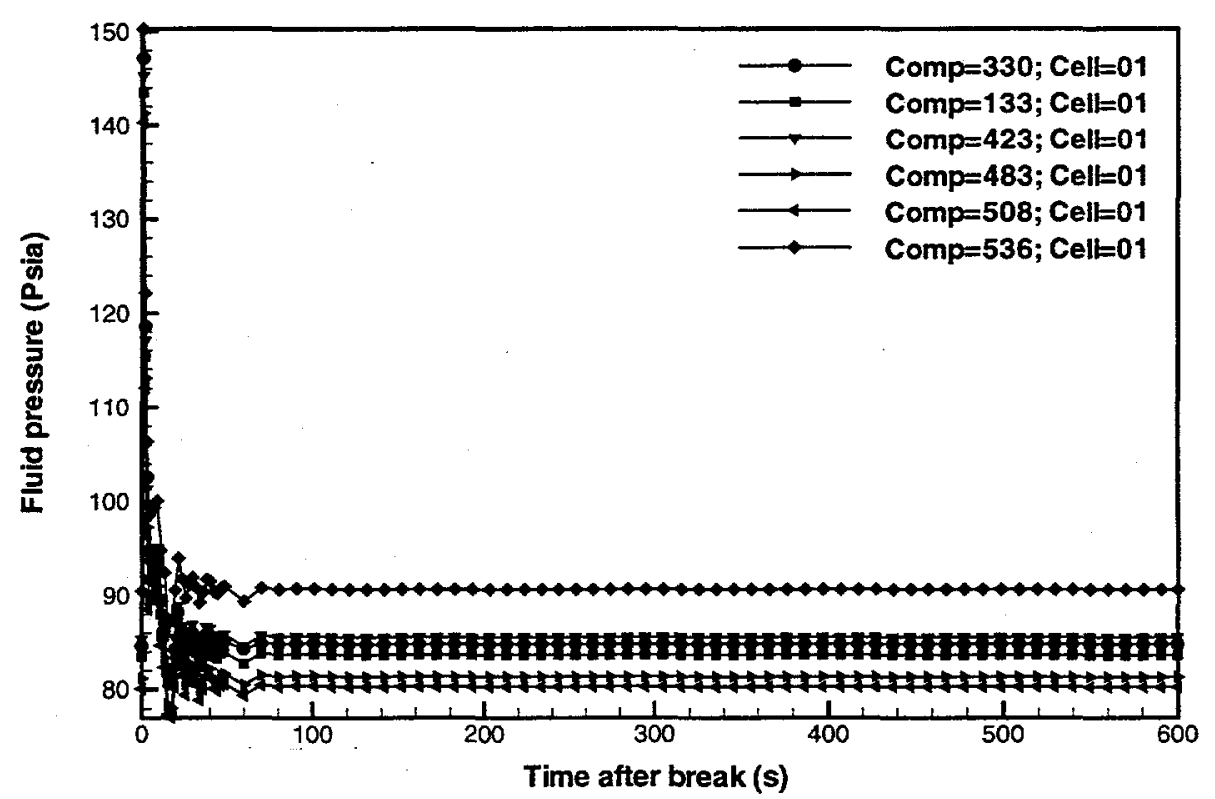

Figure B-11a Module outlet plenum fluid pressures for a LOHGA (Case 1: helium supply plenum break near decoupler inlet). 


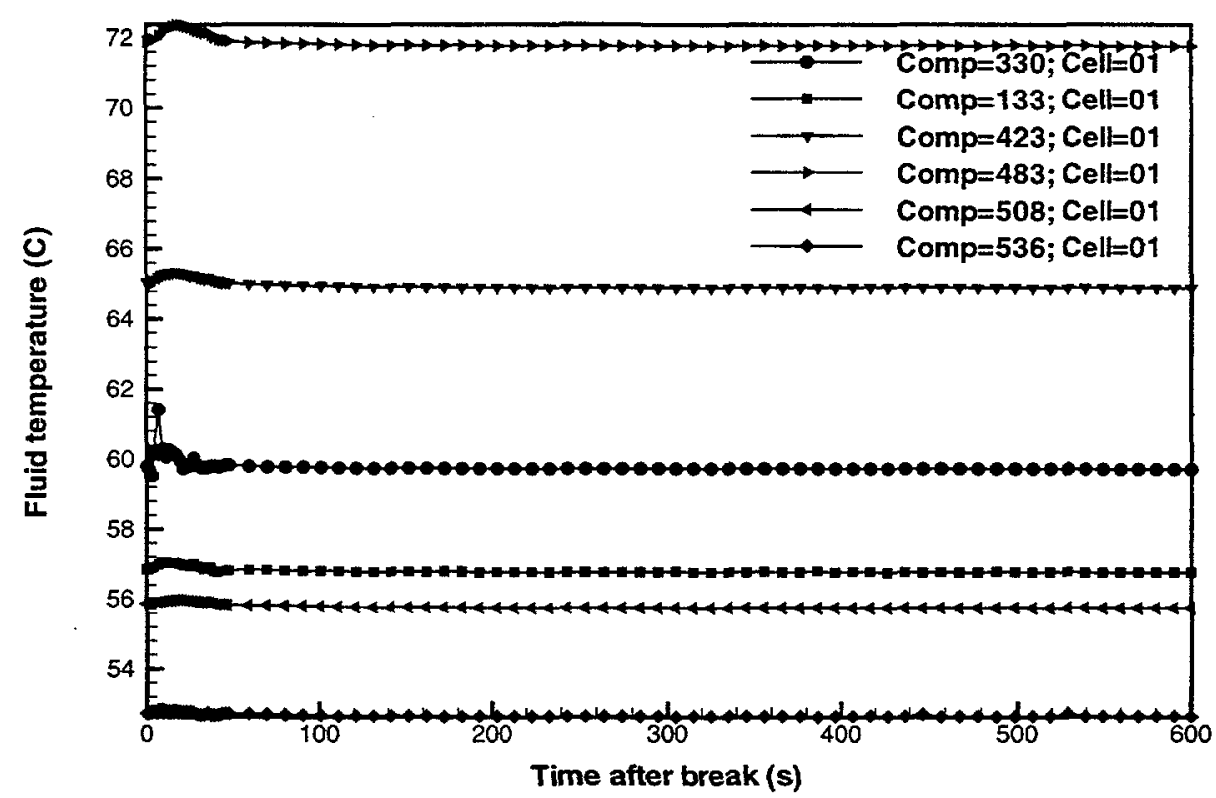

Figure B-11b Module outlet plenum fluid temperatures for a LOHGA (Case 1: helium supply plenum break near decoupler inlet).

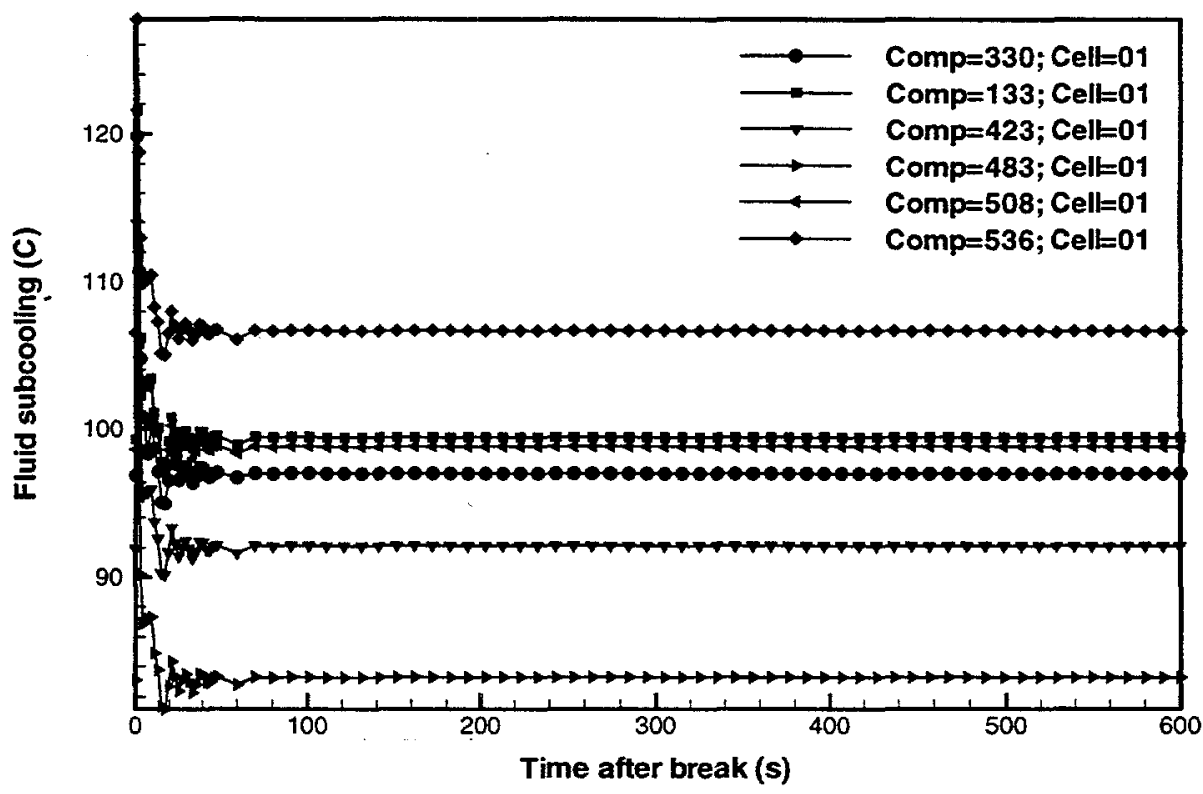

Figure B-11c Module outlet plenum fluid subcoolings for a LOHGA (Case 1: helium supply plenum break near decoupler inlet). 


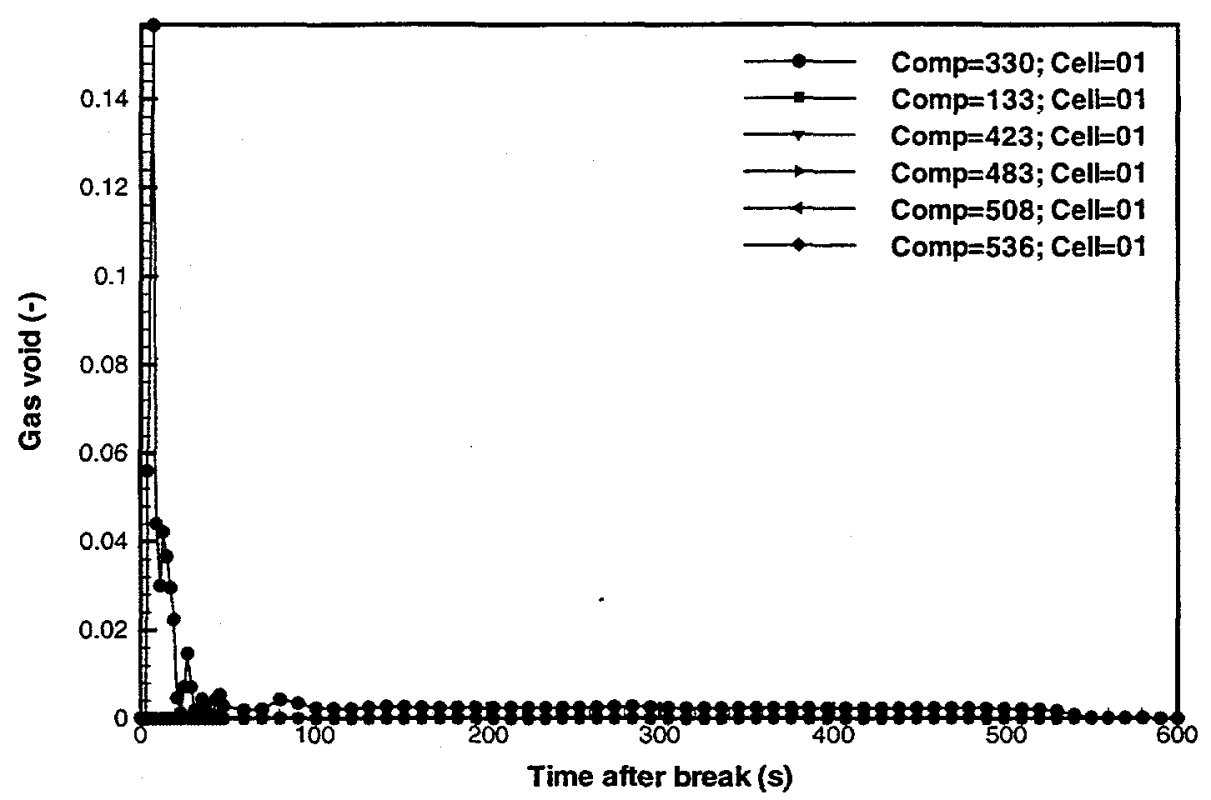

Figure B-11d Module outlet plenum void fractions for a LOHGA (Case 1: helium supply plenum break near decoupler inlet). 


\section{Appendix B2 LOHGA (Case 1) TRAC Pipe, Pump, and Valve Component} Figures

The following figures are from a TRAC simulation for Case 1 of a LOHGA (Helium supply plenum break near decoupler inlet):

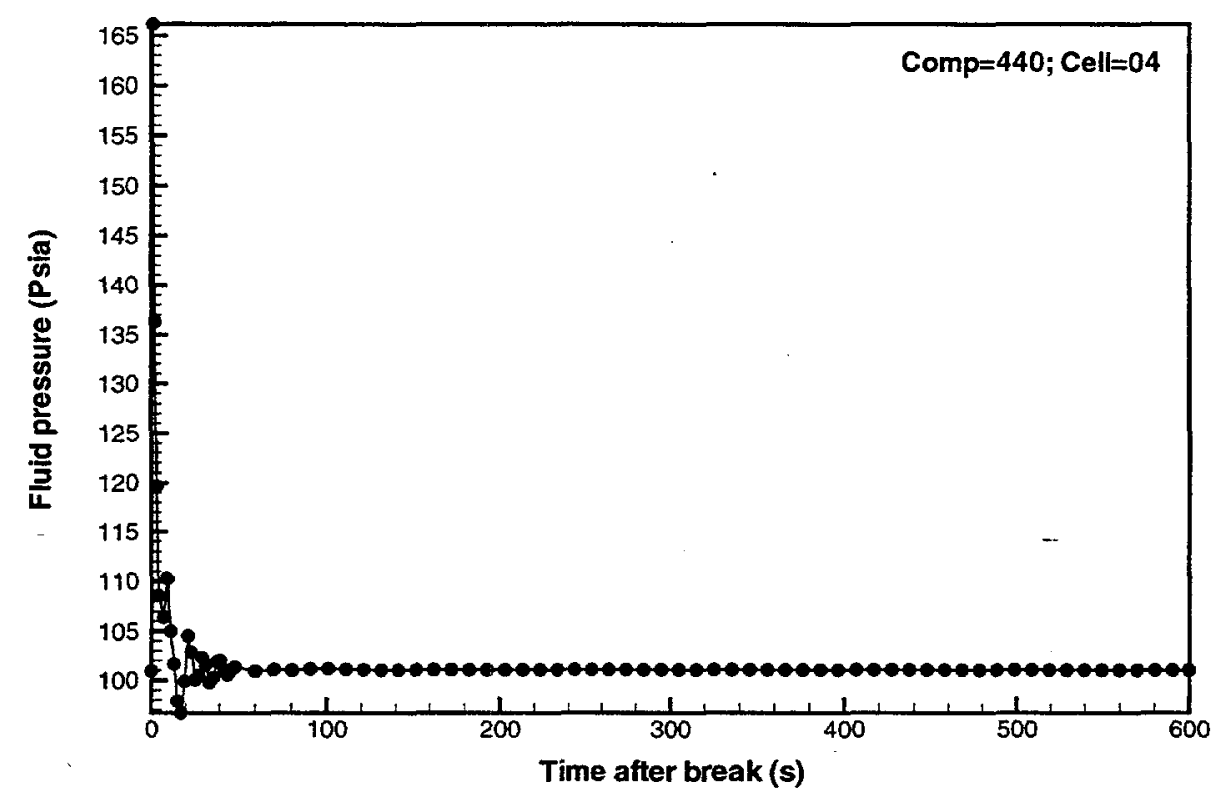

Figure B-12a Helium gas line fluid pressures for a LOHGA (Case 1: helium supply plenum break near decoupler inlet). 


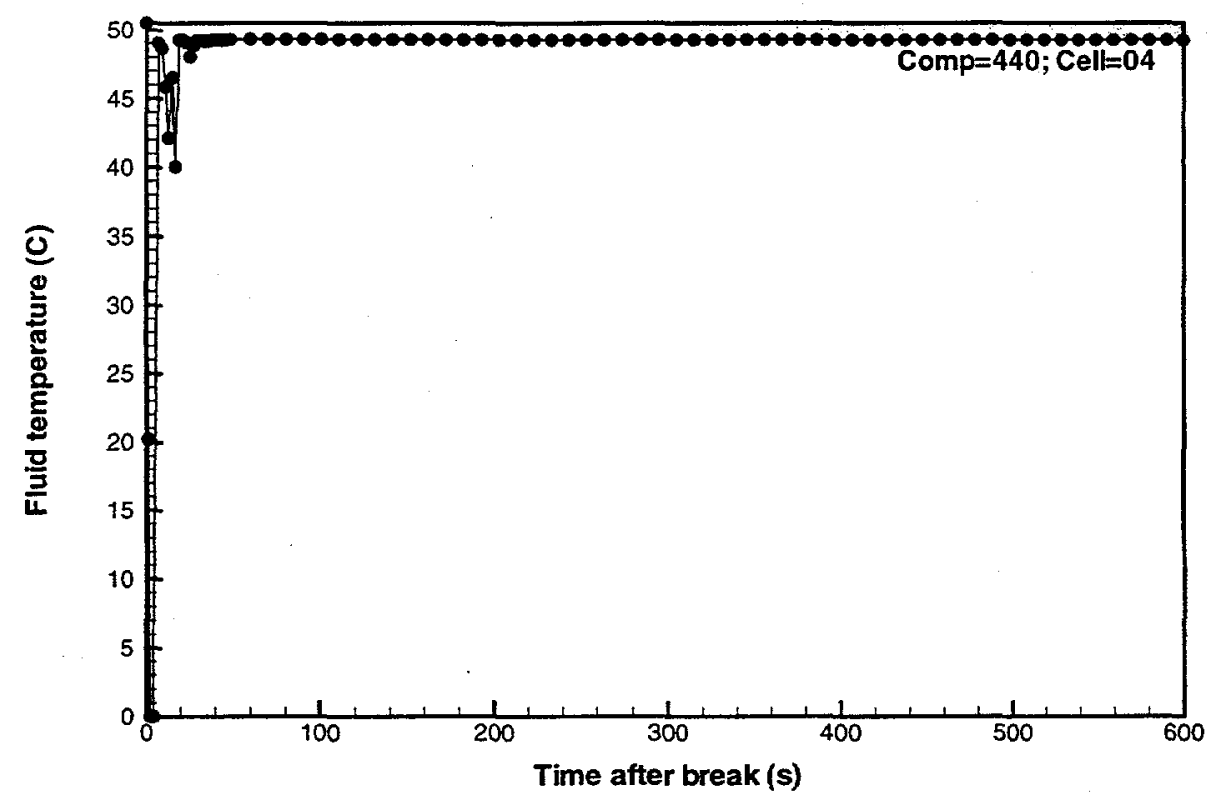

Figure B-12b Helium gas line fluid temperatures for a LOHGA (Case 1: helium supply plenum break near decoupler inlet).

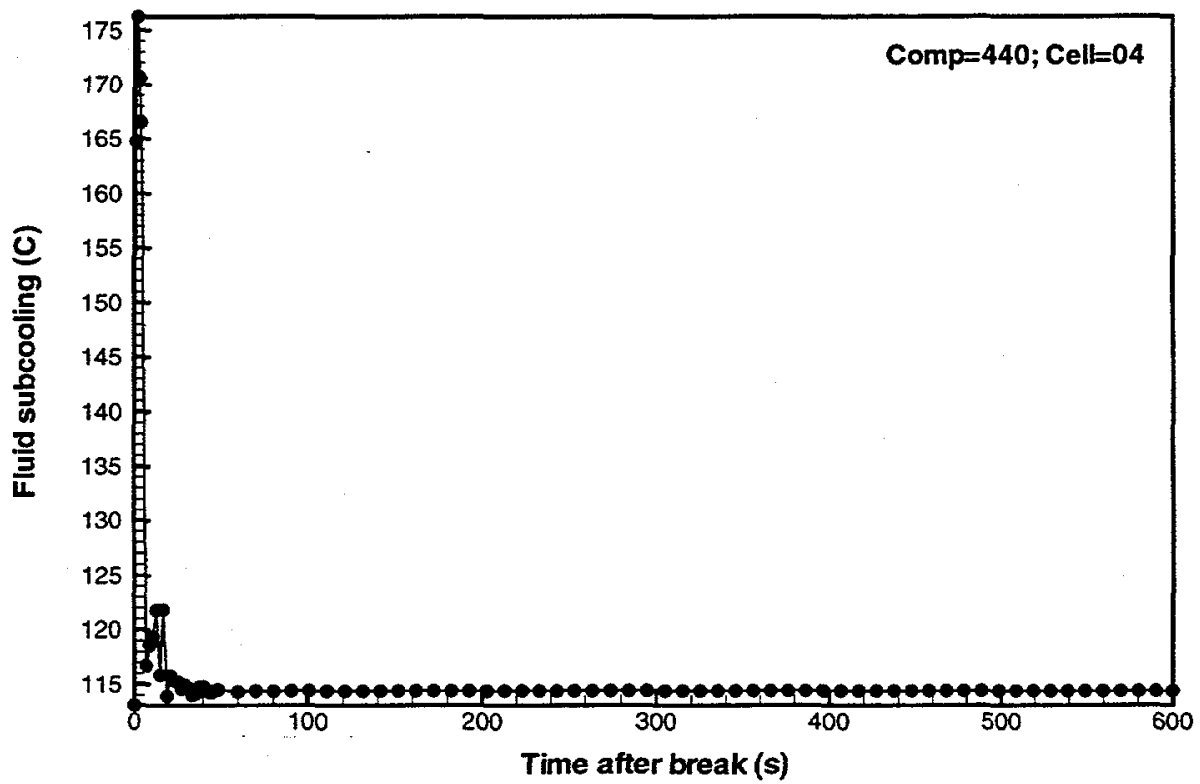

Figure B-12c Helium gas line fluid subcoolings for a LOHGA (Case 1: helium supply plenum break near decoupler inlet). 


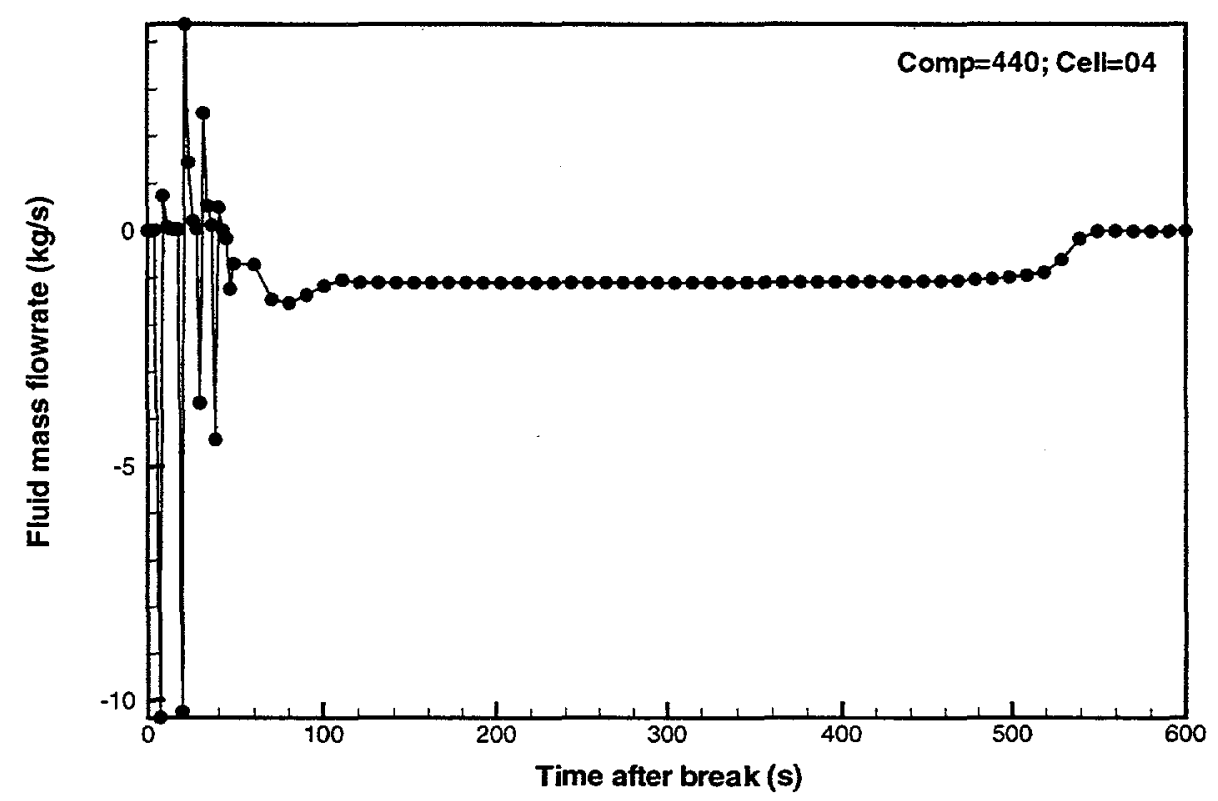

Figure B-12d Helium gas line liquid mass flowrates for a LOHGA (Case 1: helium supply plenum break near decoupler inlet).

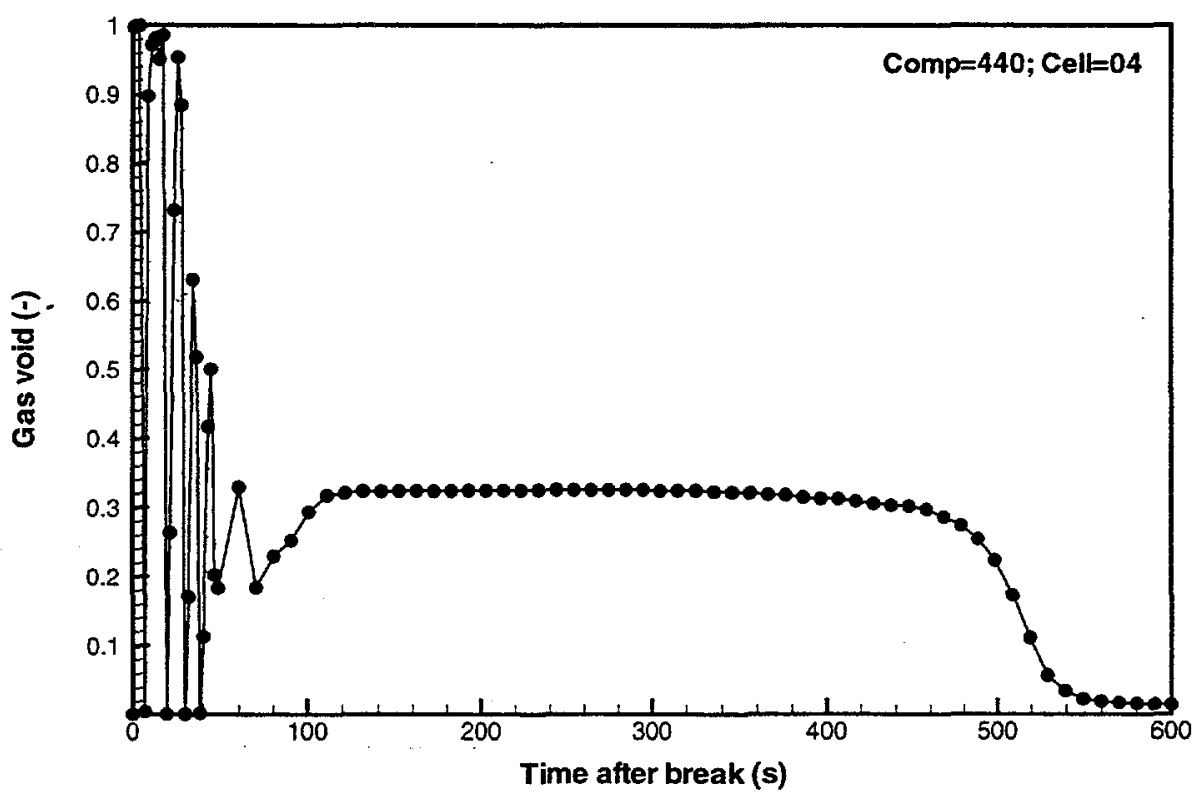

Figure B-12e Helium gas line void fractions for a LOHGA (Case 1: helium supply plenum break near decoupler inlet). 


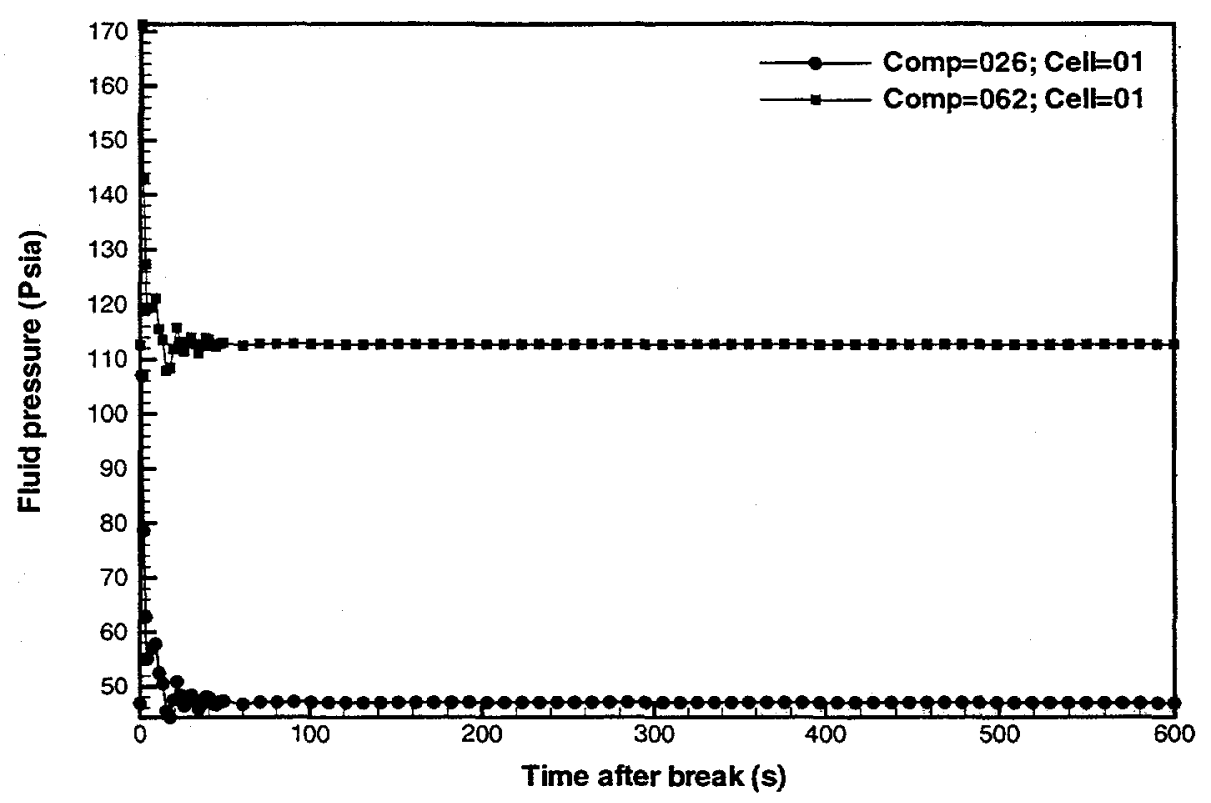

Figure B-13a Primary HR hot and cold leg piping fluid pressures for a LOHGA (Case 1: helium supply plenum break near decoupler inlet).

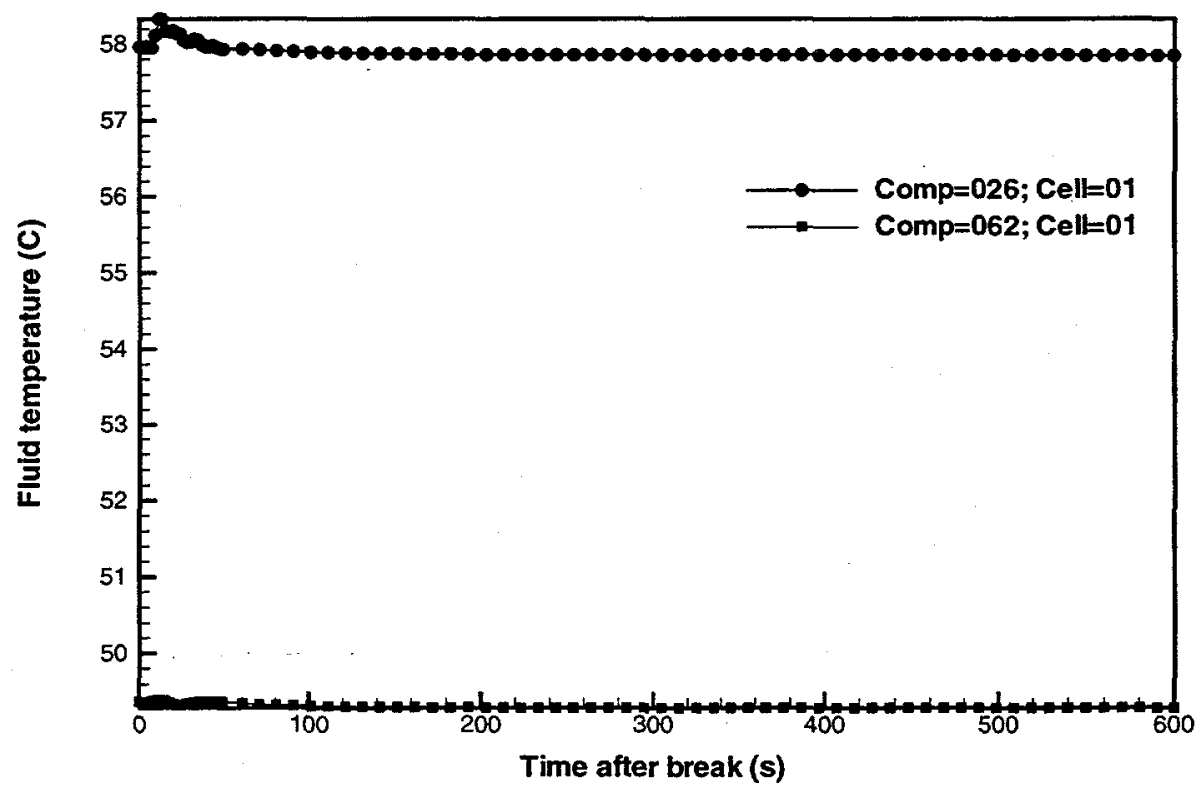

Figure B-13b Primary HR hot and cold leg piping fluid temperatures for a LOHGA (Case 1: helium supply plenum break near decoupler inlet). 


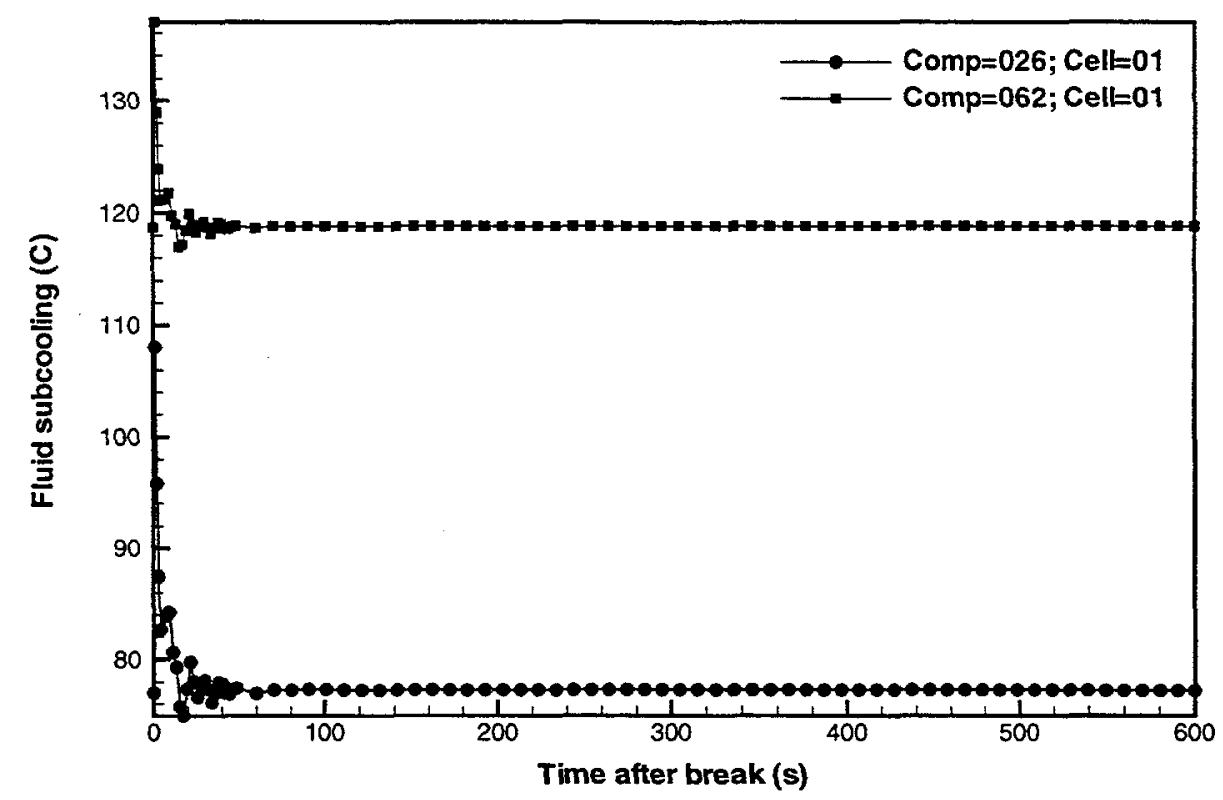

Figure B-13c Primary HR hot and cold leg piping fluid subcoolings for a LOHGA (Case 1: helium supply plenum break near decoupler inlet).

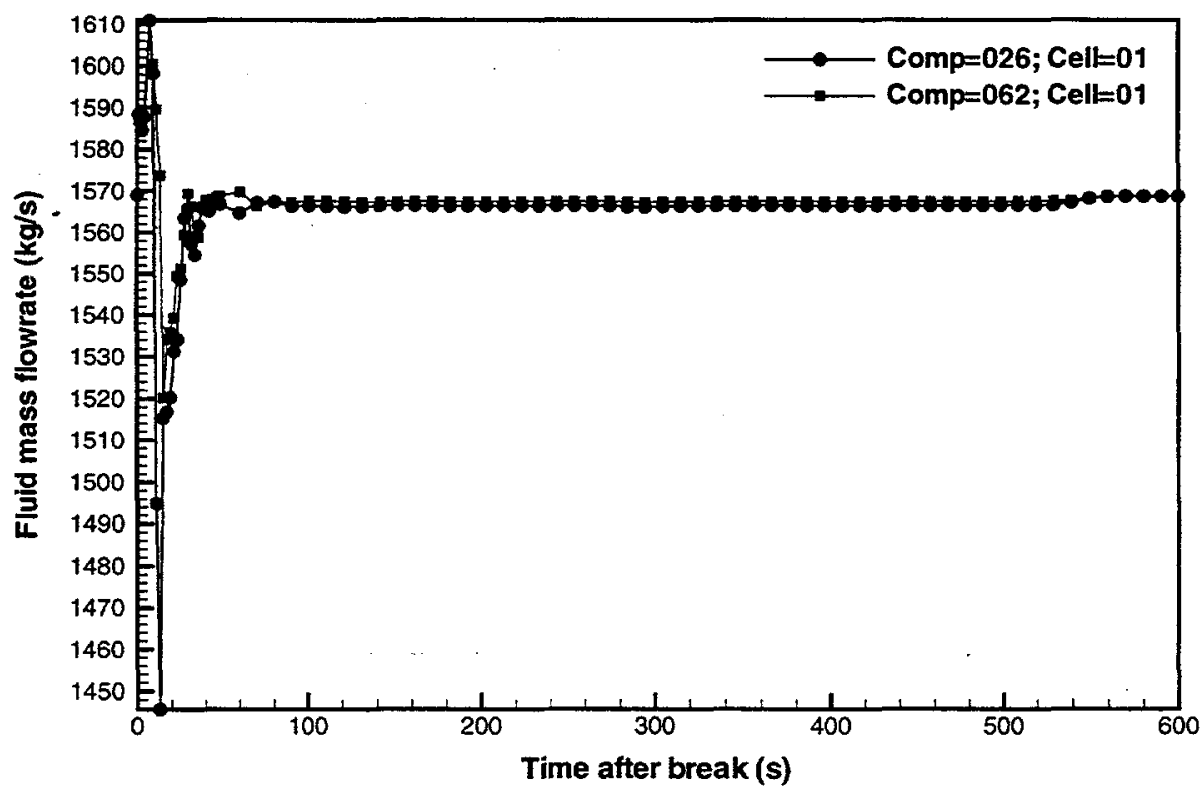

Figure B-13d Primary HR hot and cold leg piping liquid mass flowrates for a LOHGA (Case 1: helium supply plenum break near decoupler inlet). 


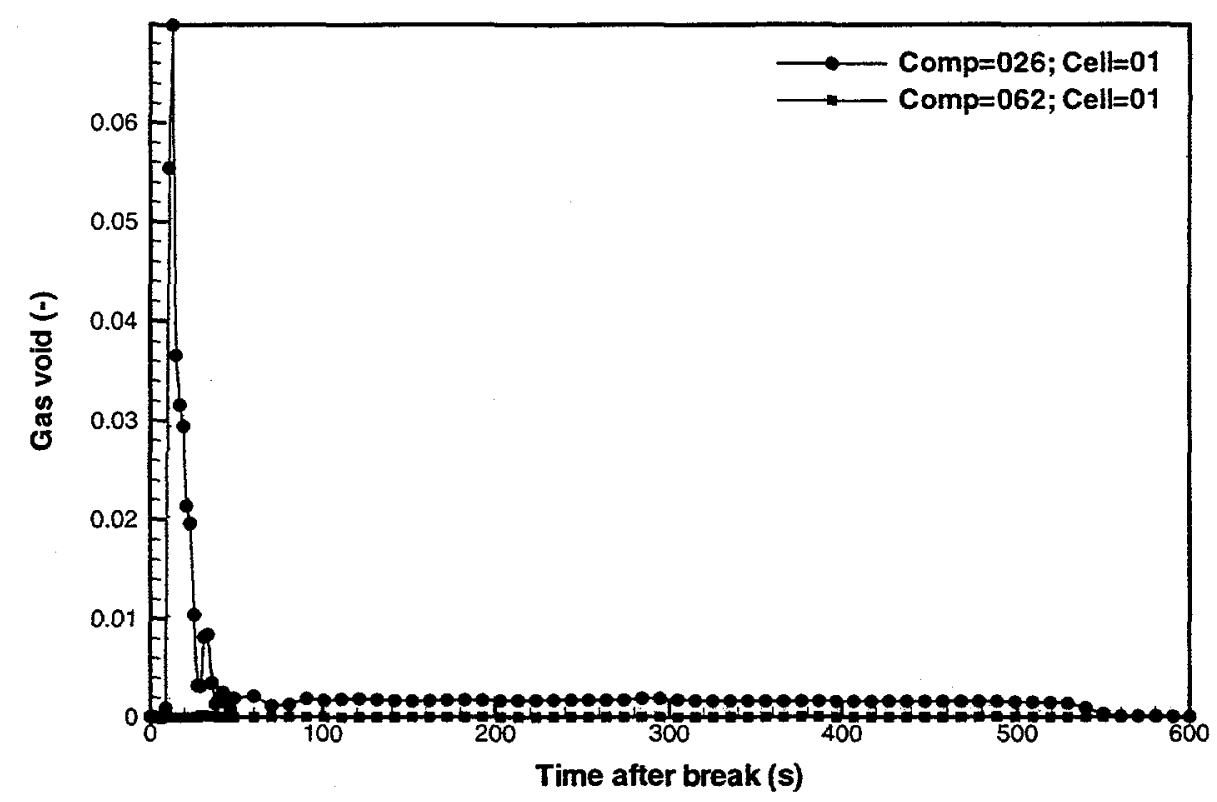

Figure B-13e Primary HR hot and cold leg piping void fractions for a LOHGA (Case 1: helium supply plenum break near decoupler inlet).

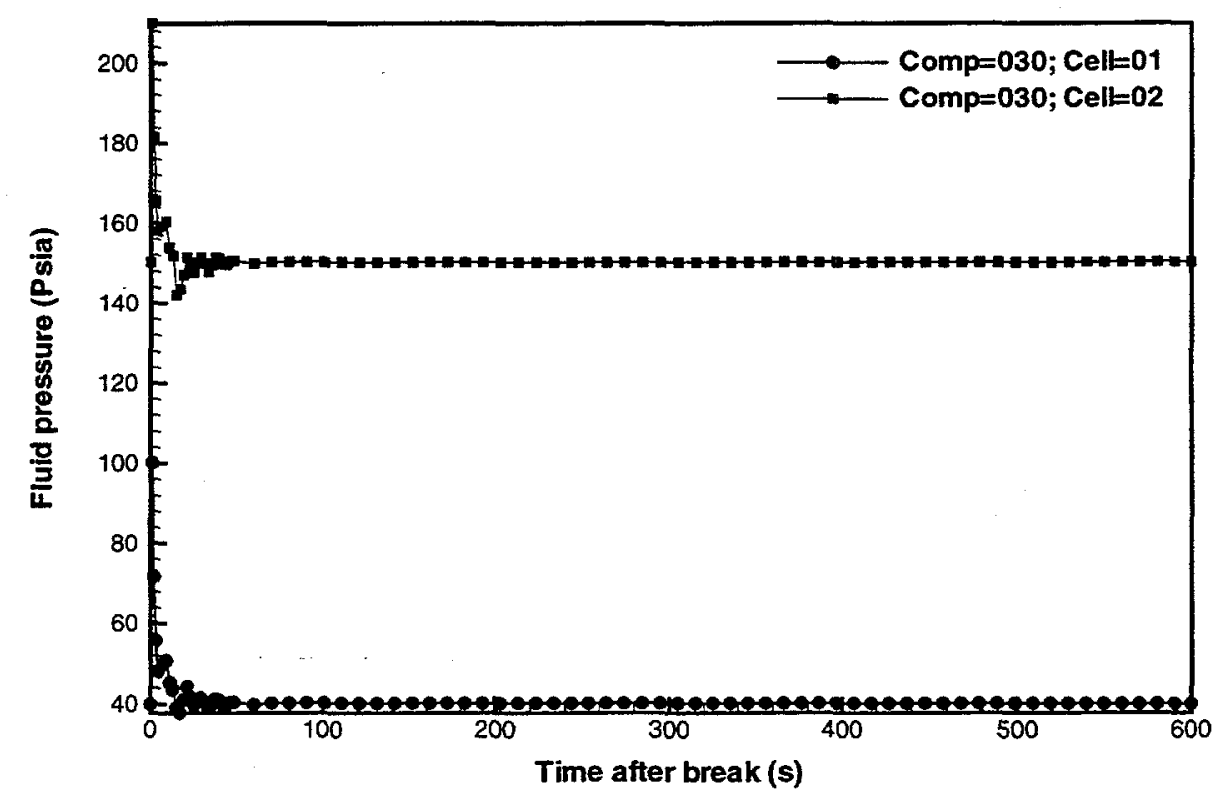

Figure B-14a Primary HR pump 1 fluid pressures for a LOHGA (Case 1: helium supply plenum break near decoupler inlet). 


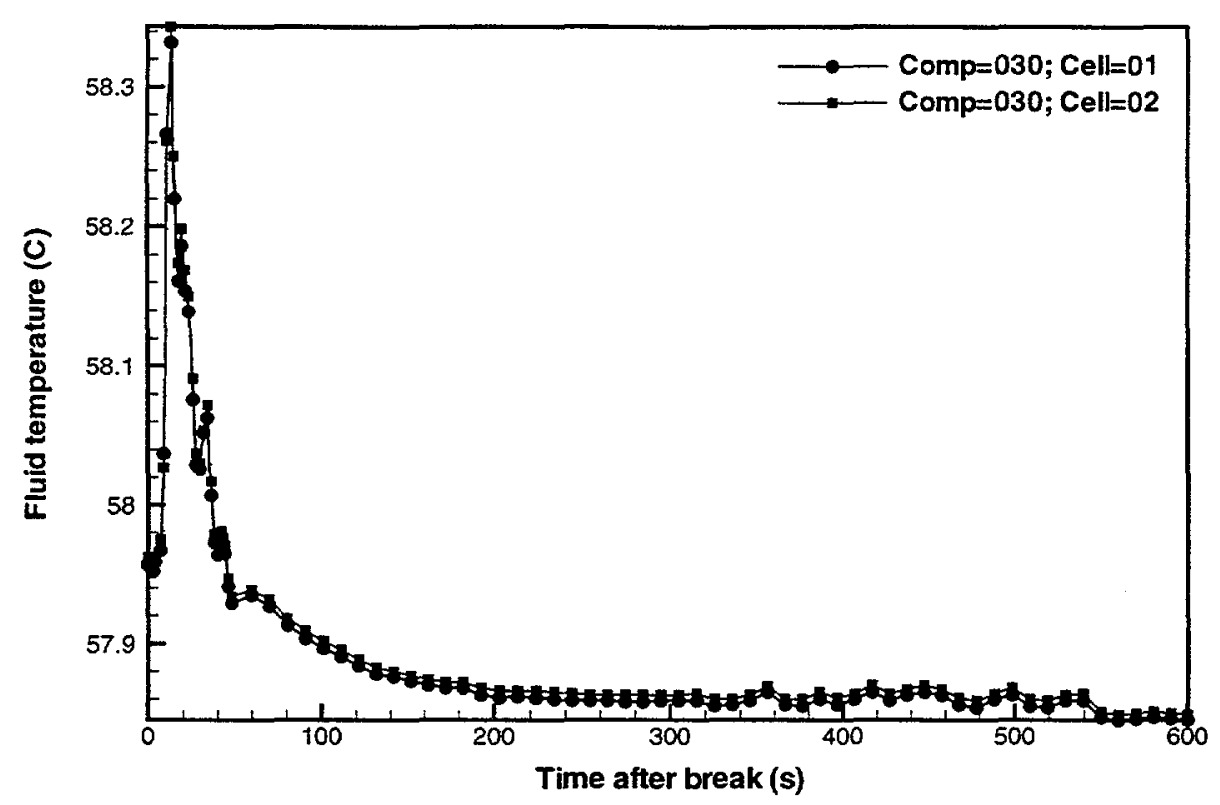

Figure B-14b Primary HR pump 1 fluid temperatures for a LOHGA tCase 1: helium supply plenum break near decoupler inlet).

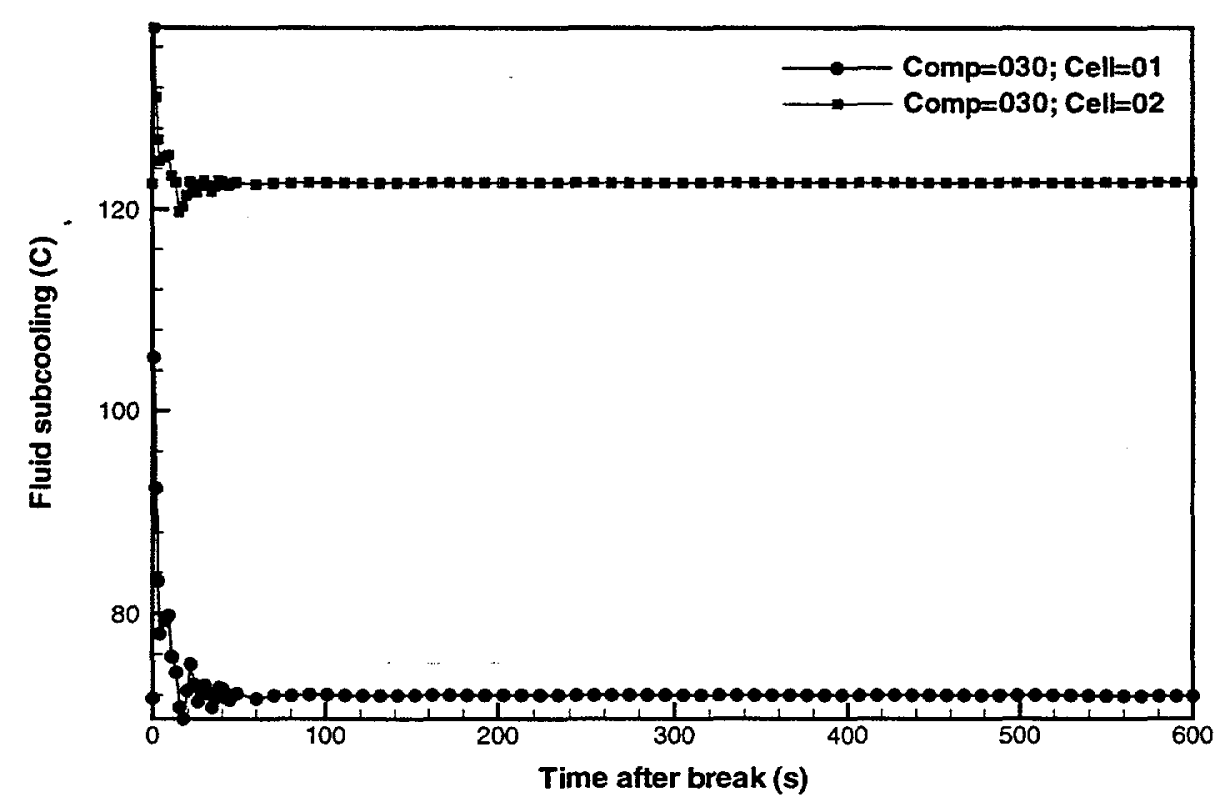

Figure B-14c Primary HR pump 1 fluid subcoolings for a LOHGA (Case 1: helium supply plenum break near decoupler inlet). 


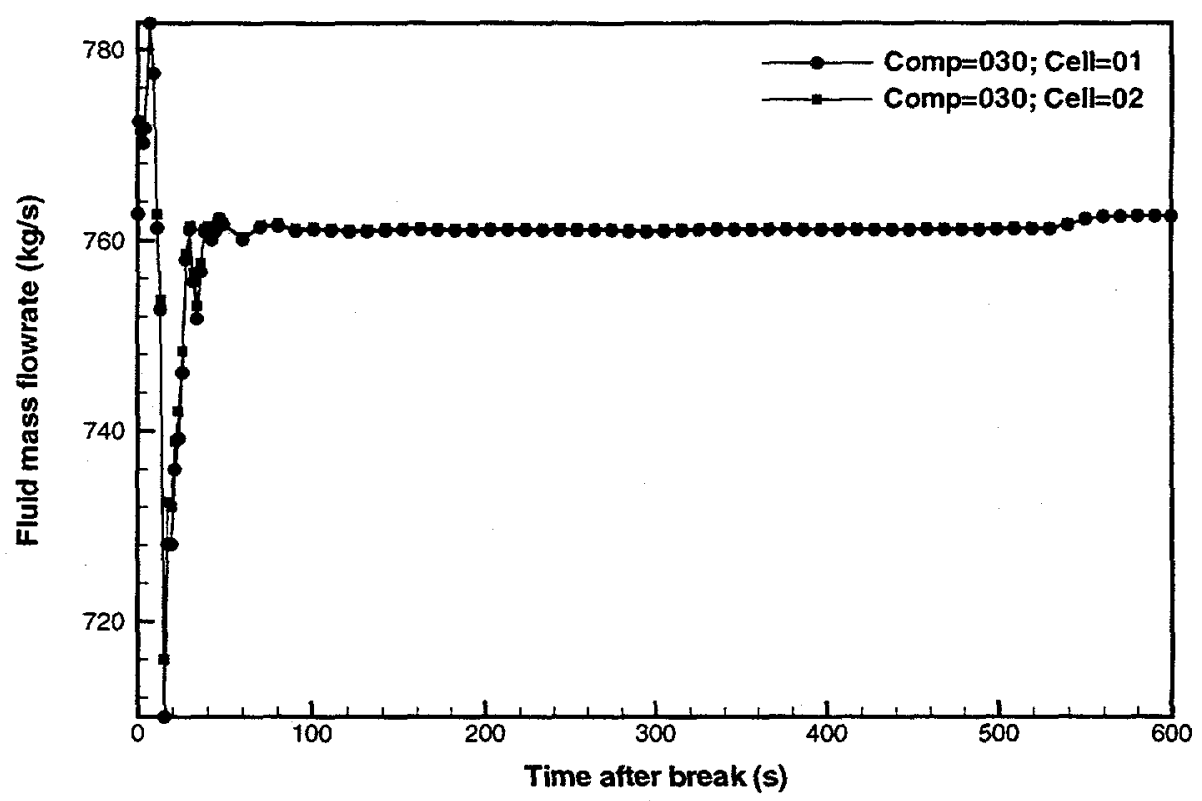

Figure B-14d Primary HR pump 1 liquid mass flowrates for a LOHGA (Case 1: helium supply plenum break near decoupler inlet).

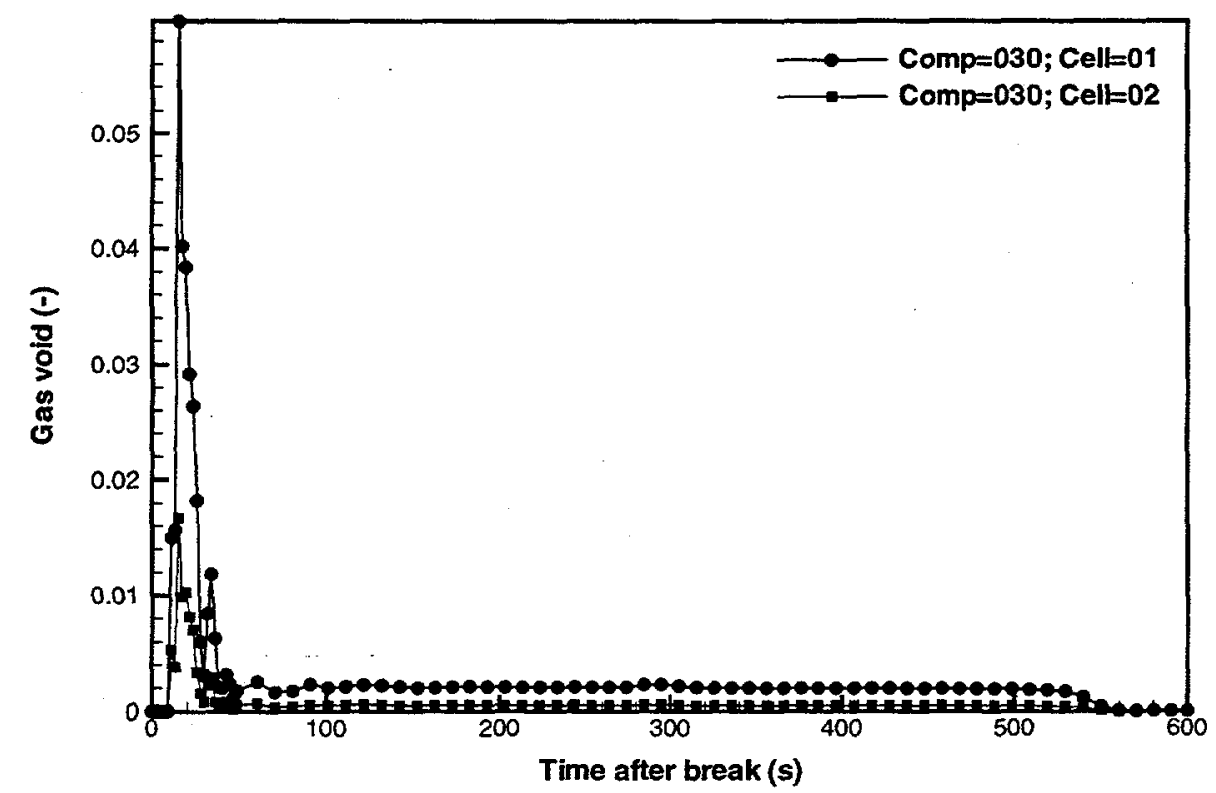

Figure B-14e Primary HR pump 1 void fractions for a LOHGA (Case 1: helium supply plenum break near decoupler inlet). 


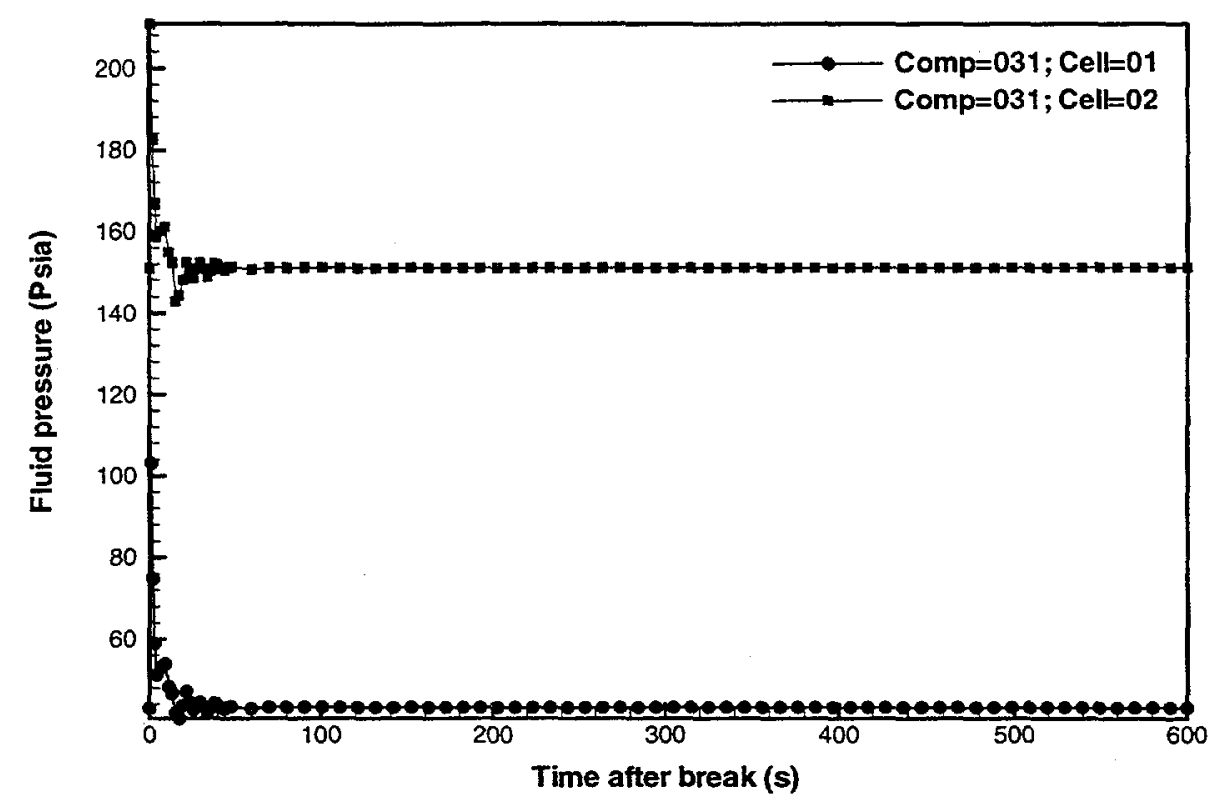

Figure B-15a Primary HR pump 2 fluid pressures for a LOHGA (Case 1: 'helium supply plenum break near decoupler inlet).

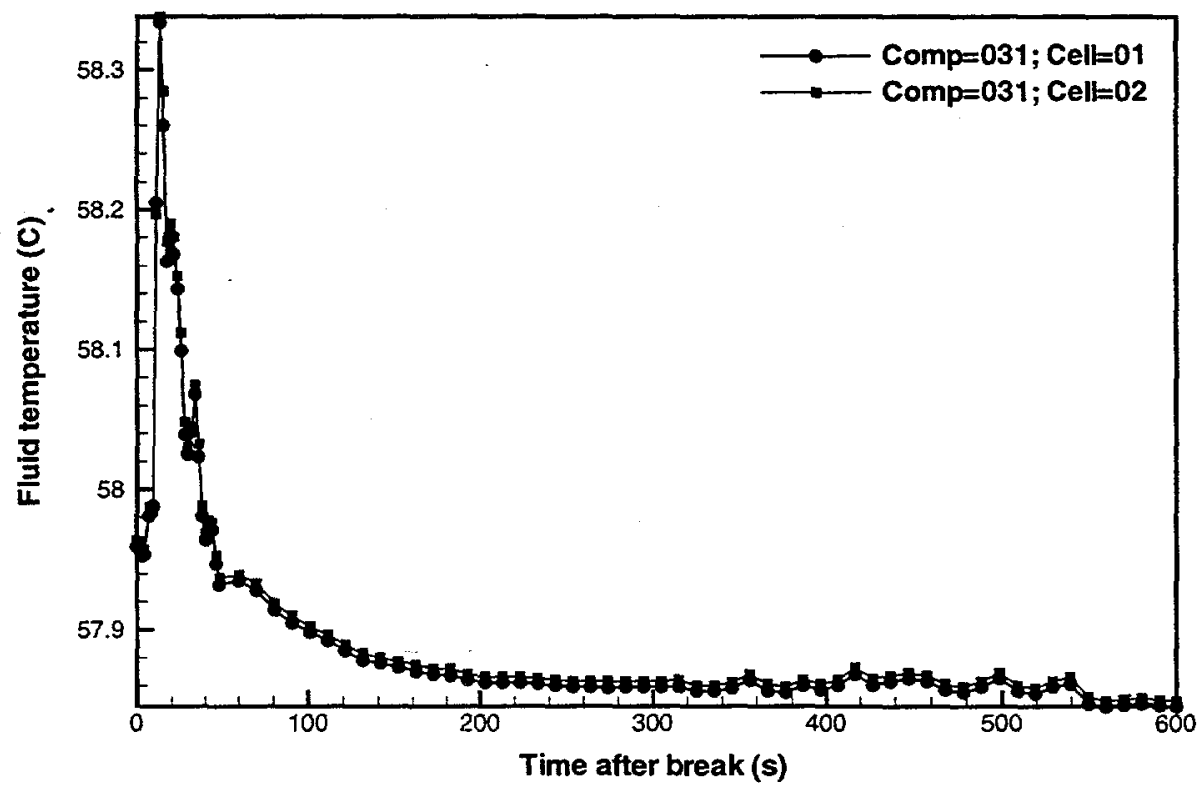

Figure B-15b Primary HR pump 2 fluid temperatures for a LOHGA (Case 1: helium supply plenum break near decoupler inlet). 


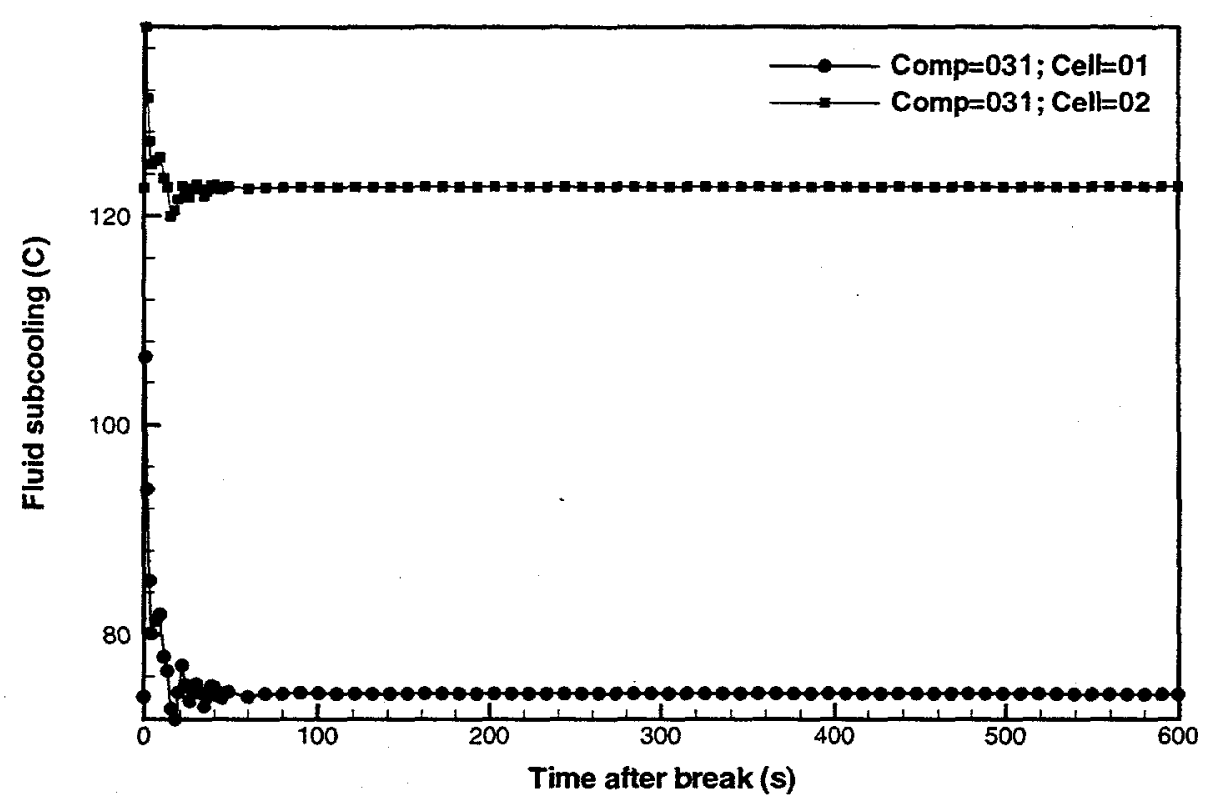

Figure B-15c Primary HR pump 2 fluid subcoolings for a LOHGA (Case 1: helium supply plenum break near decoupler inlet).

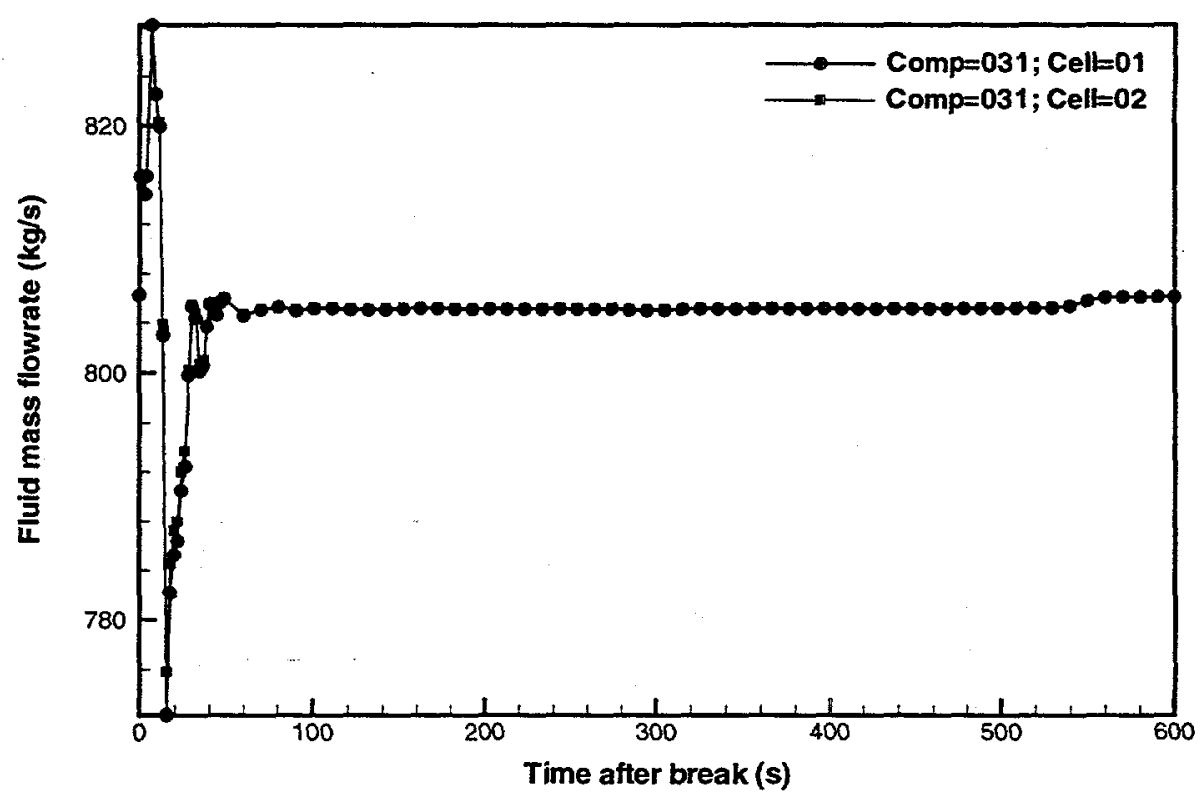

Figure B-15d Primary HR pump 2 liquid mass flowrates for a LOHGA (Case 1: helium supply plenum break near decoupler inlet). 


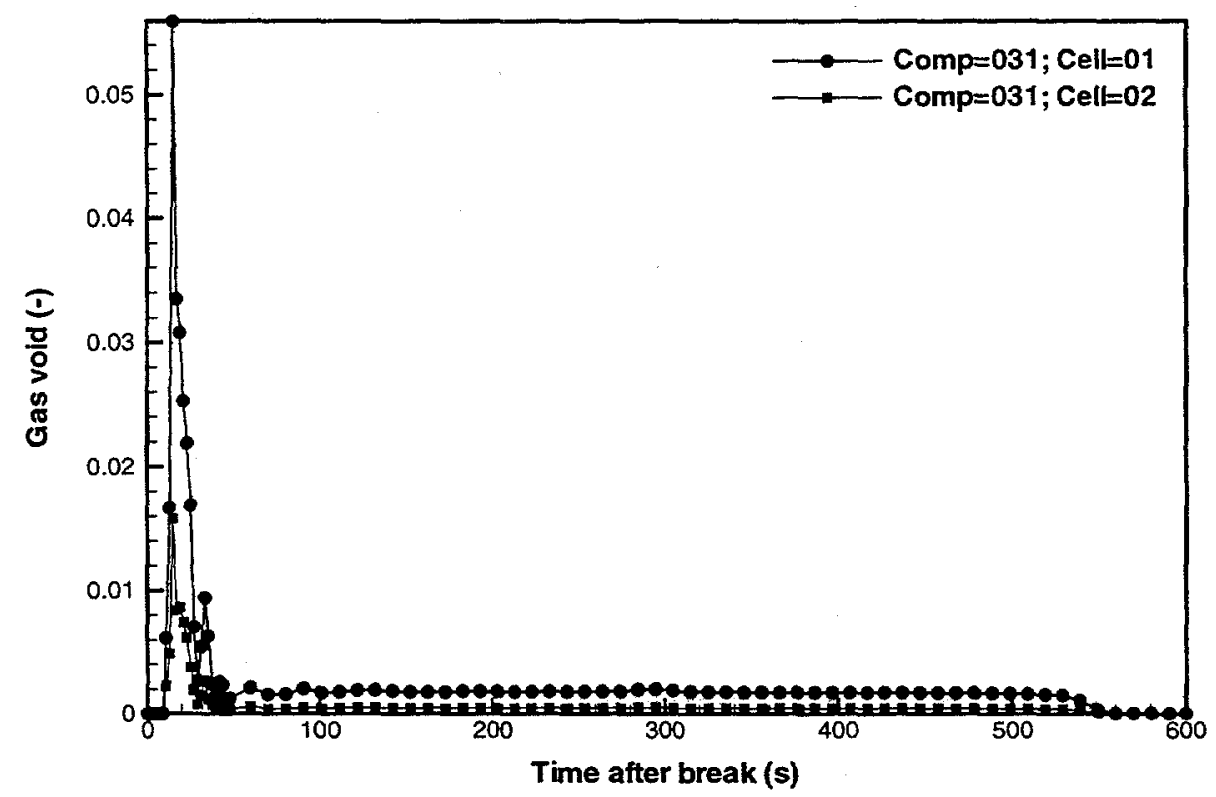

Figure B-15e Primary HR pump 2 void fractions for a LOHGA (Case-1: helium supply plenum break near decoupler inlet).

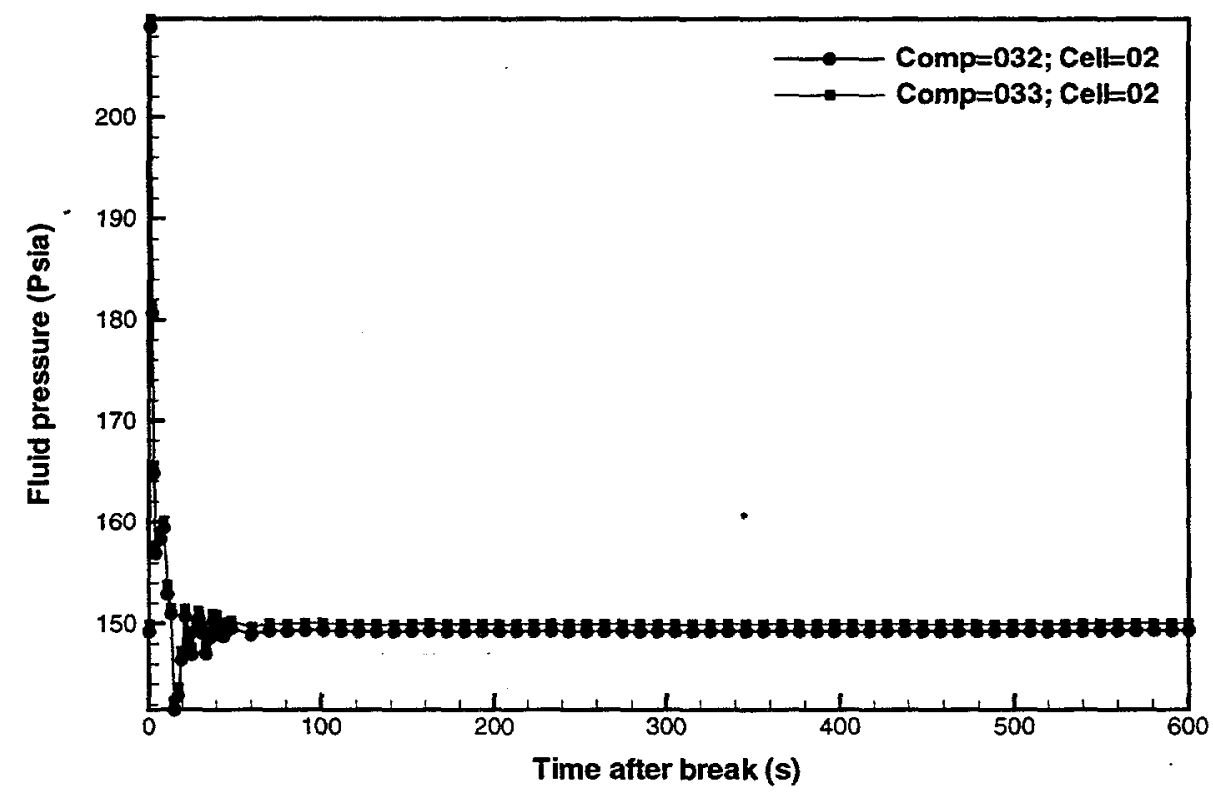

Figure B-16a Primary HR pump discharge piping fluid pressures for a LOHGA (Case 1: helium supply plenum break near decoupler inlet). 


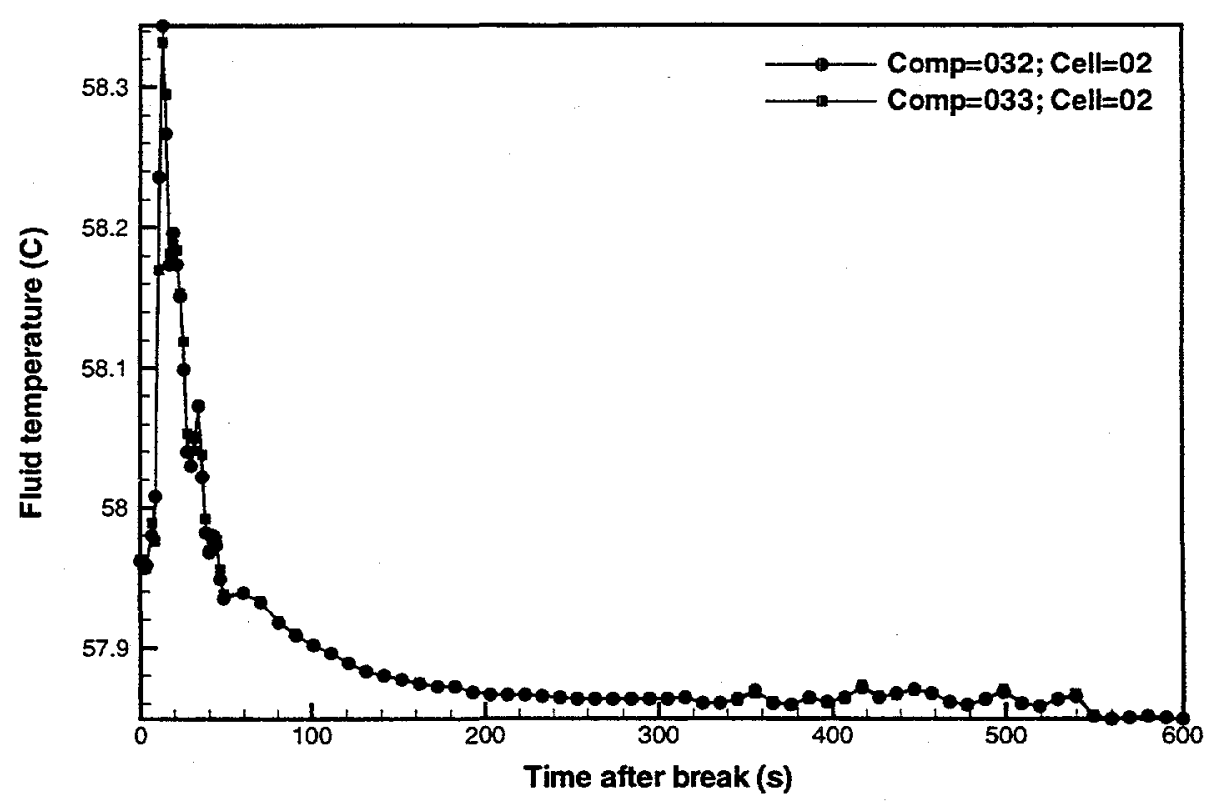

Figure B-16b Primary HR pump discharge piping fluid temperatures for a LOHGA (Case 1: helium supply plenum break near decoupler inlet).

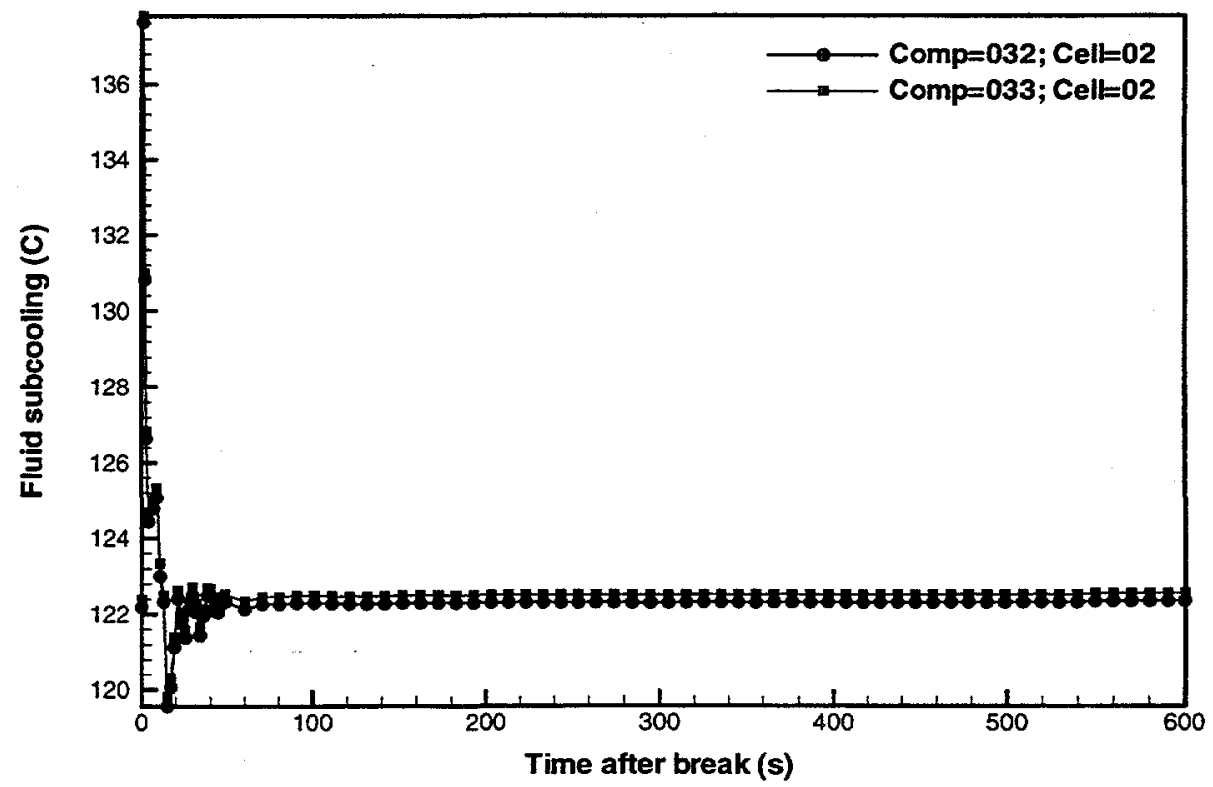

Figure B-16c Primary HR pump discharge piping fluid subcoolings for a LOHGA (Case 1: helium supply plenum break near decoupler inlet). 


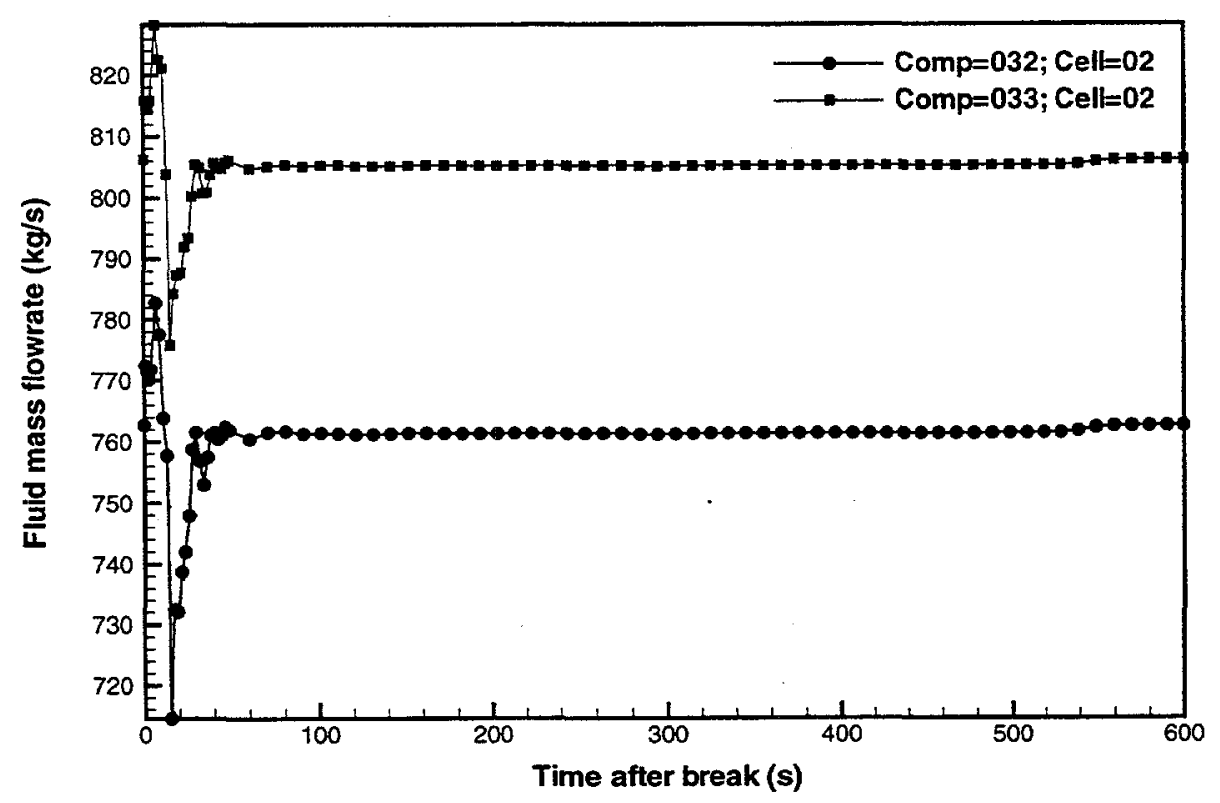

Figure B-16d Primary HR pump discharge piping liquid mass flowrates for a LOHGA (Case 1: helium supply plenum break near decoupler inlet).

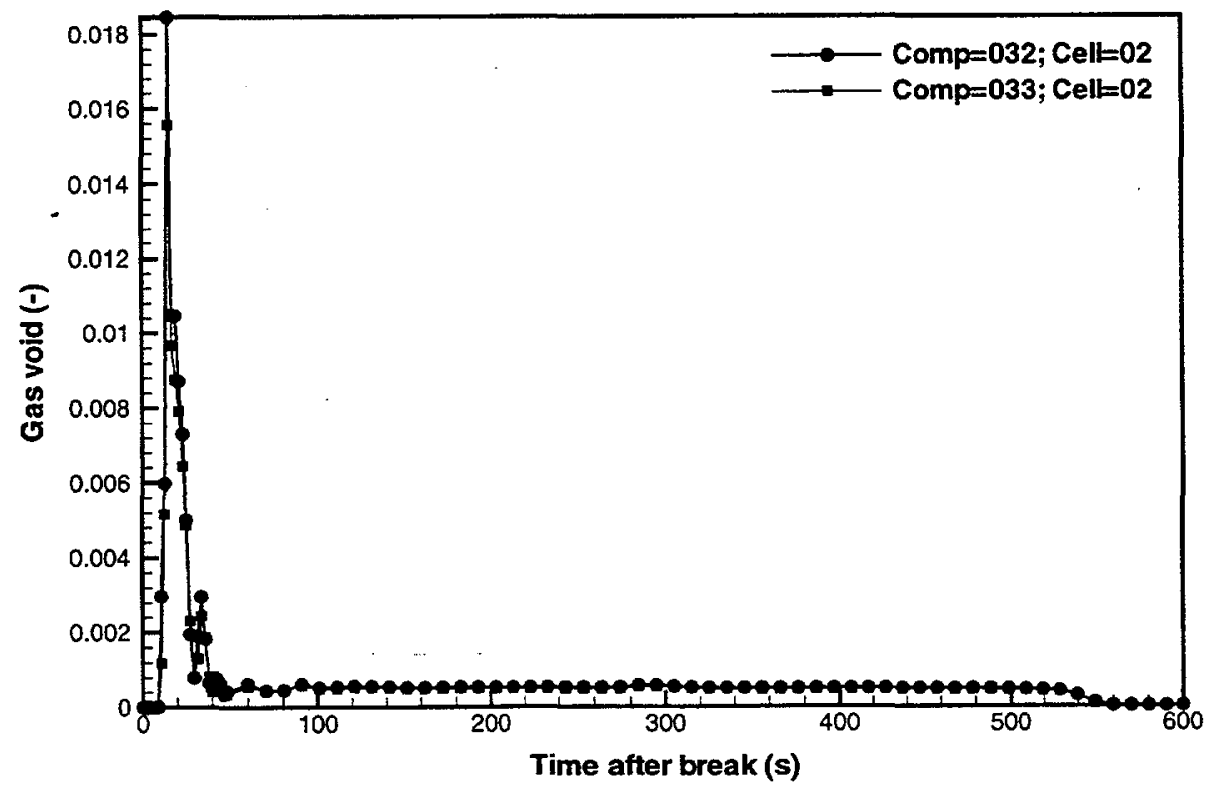

Figure B-16e Primary HR pump discharge piping void fractions for a LOHGA (Case 1: helium supply plenum break near decoupler inlet). 


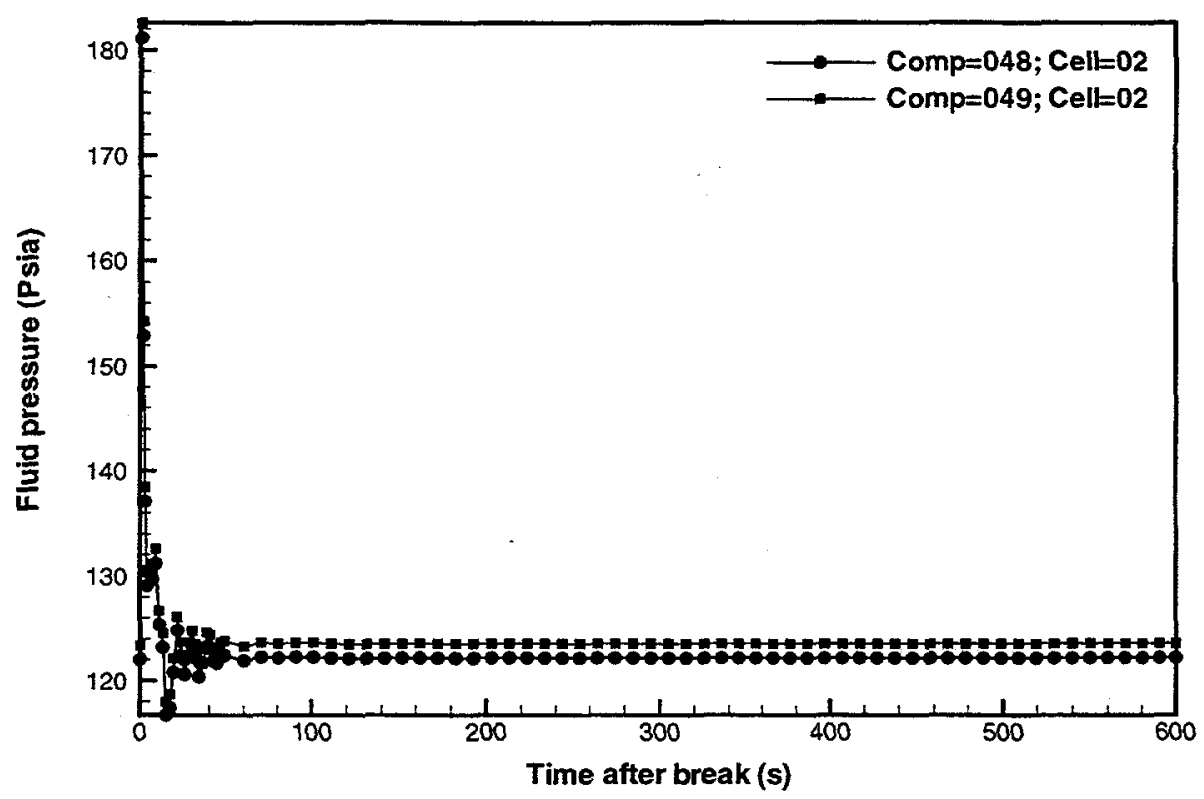

Figure B-17a Primary HR heat exchanger inlet piping fluid pressures for a LOHGA (Case 1: helium supply plenum break near decoupler inlet).

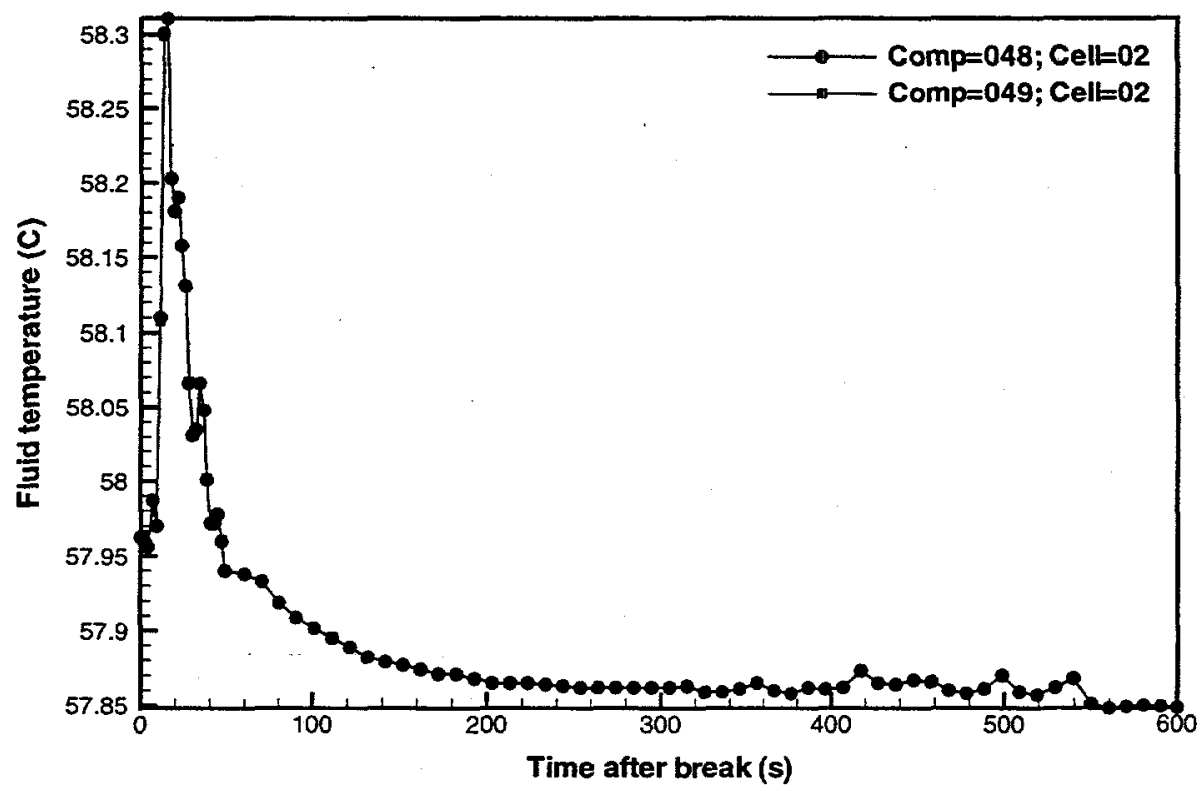

Figure B-17b Primary HR heat exchanger inlet piping fluid temperatures for a LOHGA (Case 1: helium supply plenum break near decoupler inlet). 


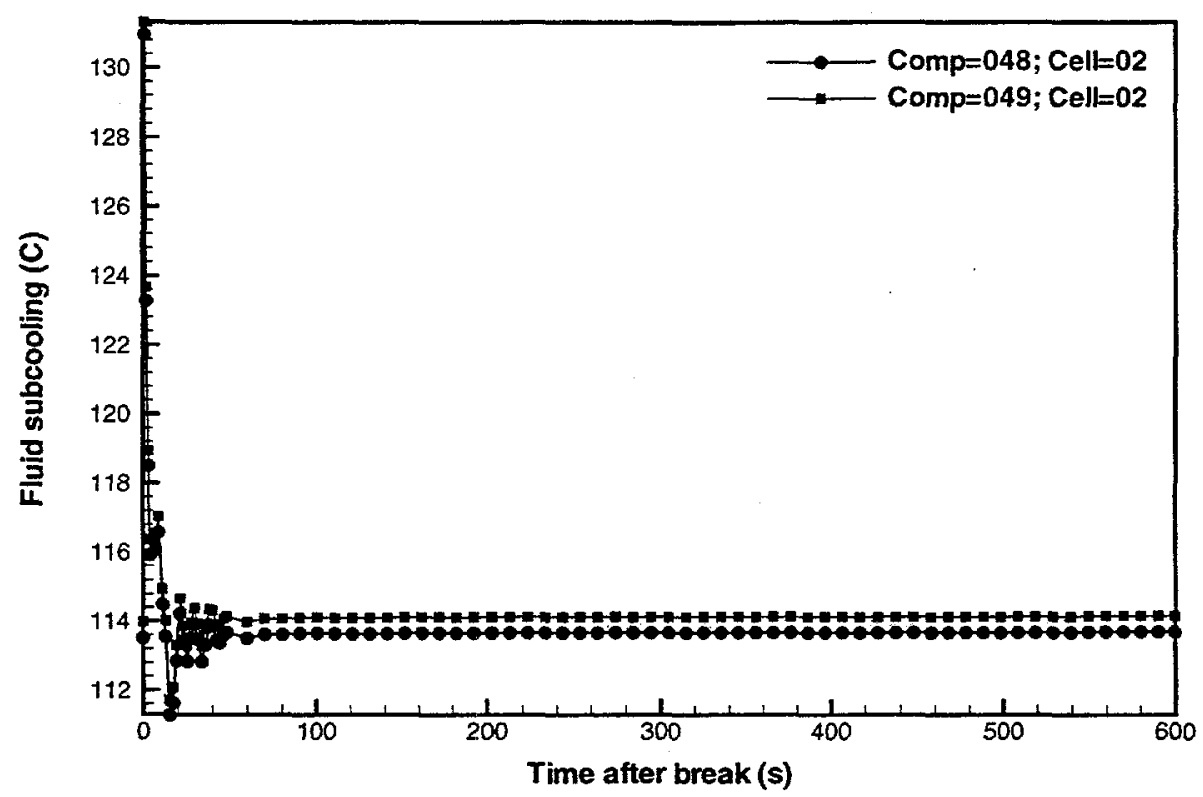

Figure B-17c Primary HR heat exchanger inlet piping fluid subcoolings for a LOHGA (Case 1: helium supply plenum break near decoupler inlet).

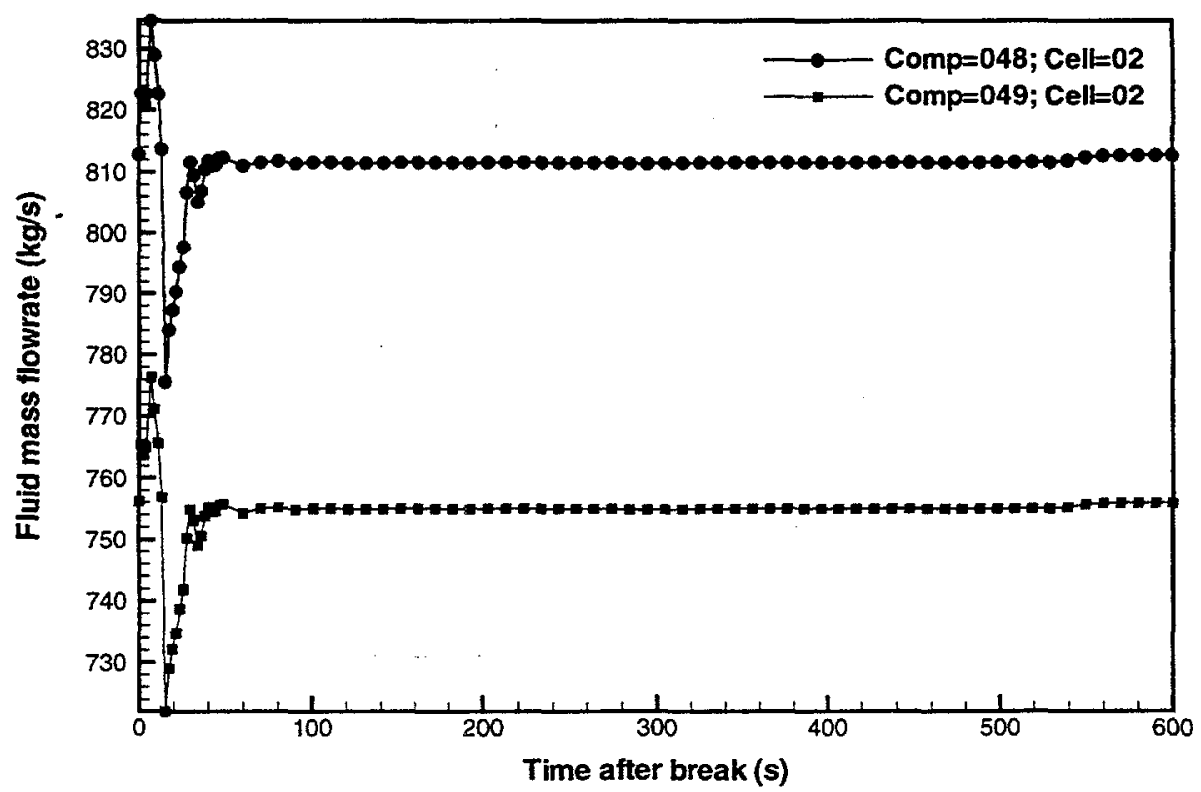

Figure B-17d Primary HR heat exchanger inlet piping liquid mass flowrates for a LOHGA (Case 1: helium supply plenum break near decoupler inlet). 


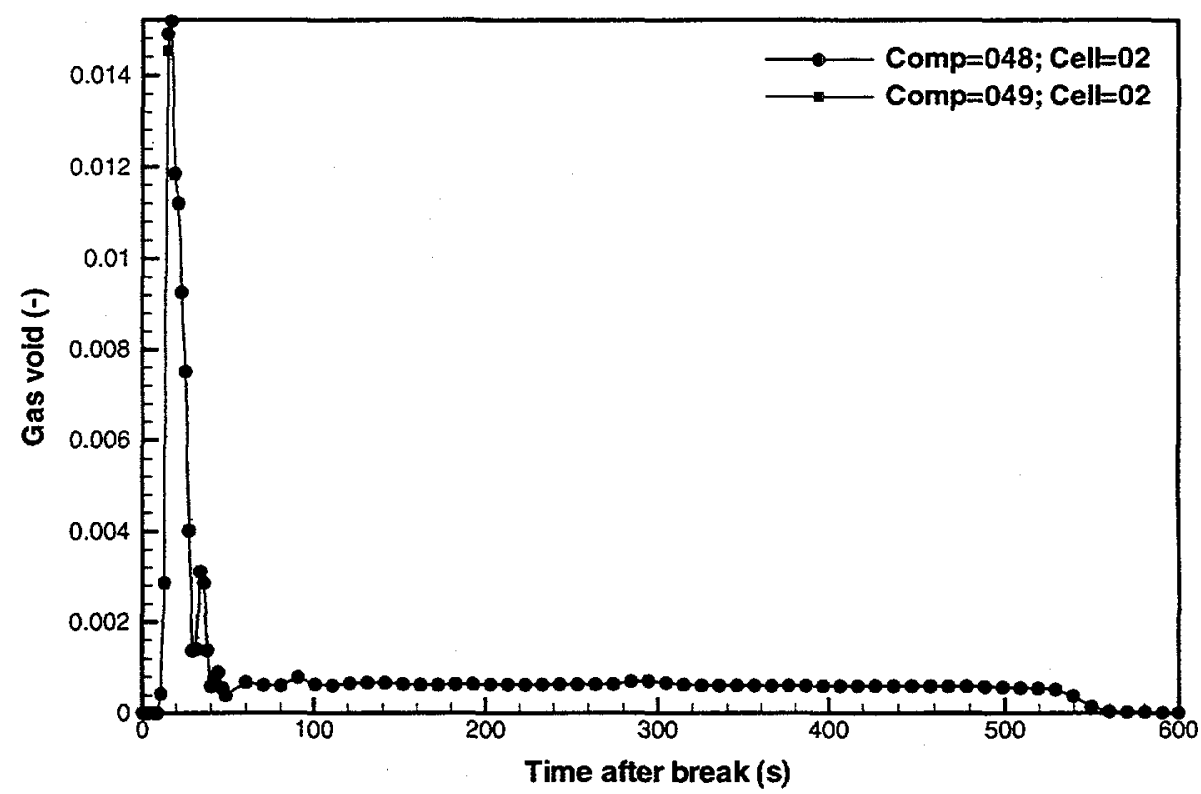

Figure B-17e Primary HR heat exchanger inlet piping void fractions for a LOHGA (Case 1: helium supply plenum break near decoupler inlet).

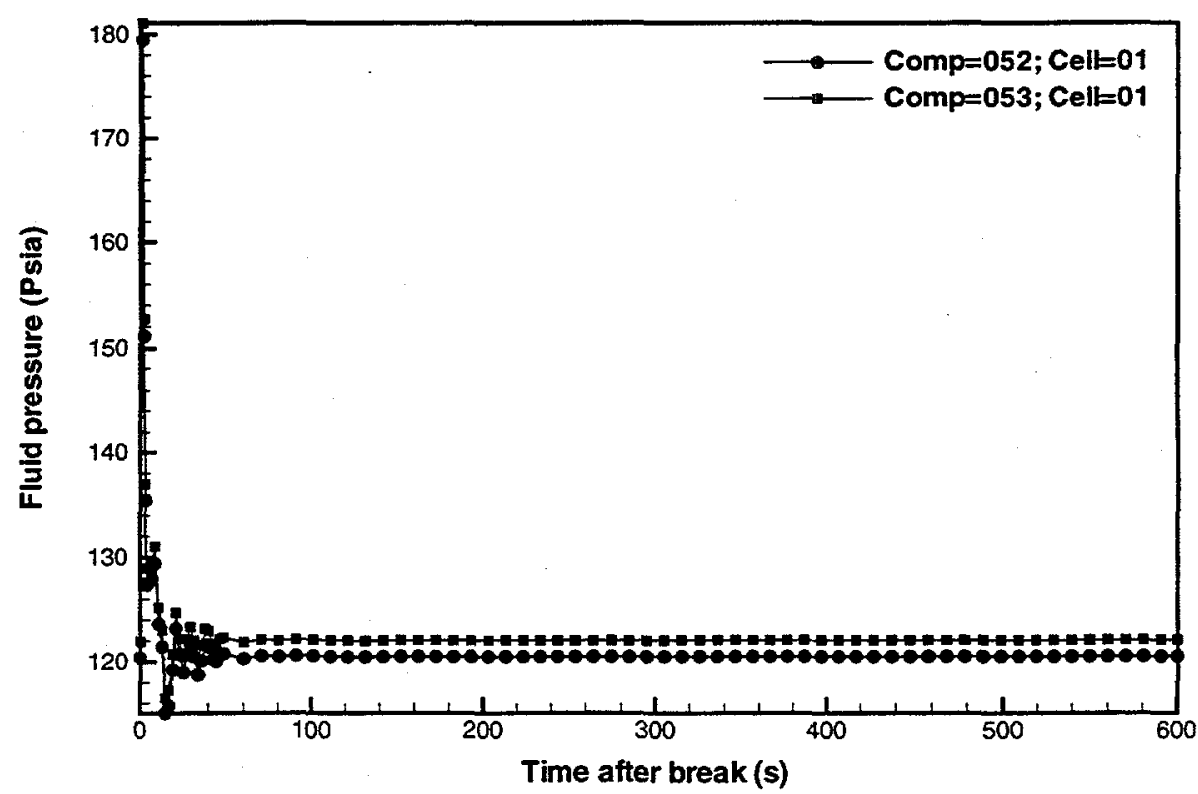

Figure B-18a Primary HR heat exchanger first pass outlet piping fluid pressures for a LOHGA (Case 1: helium supply plenum break near decoupler inlet). 


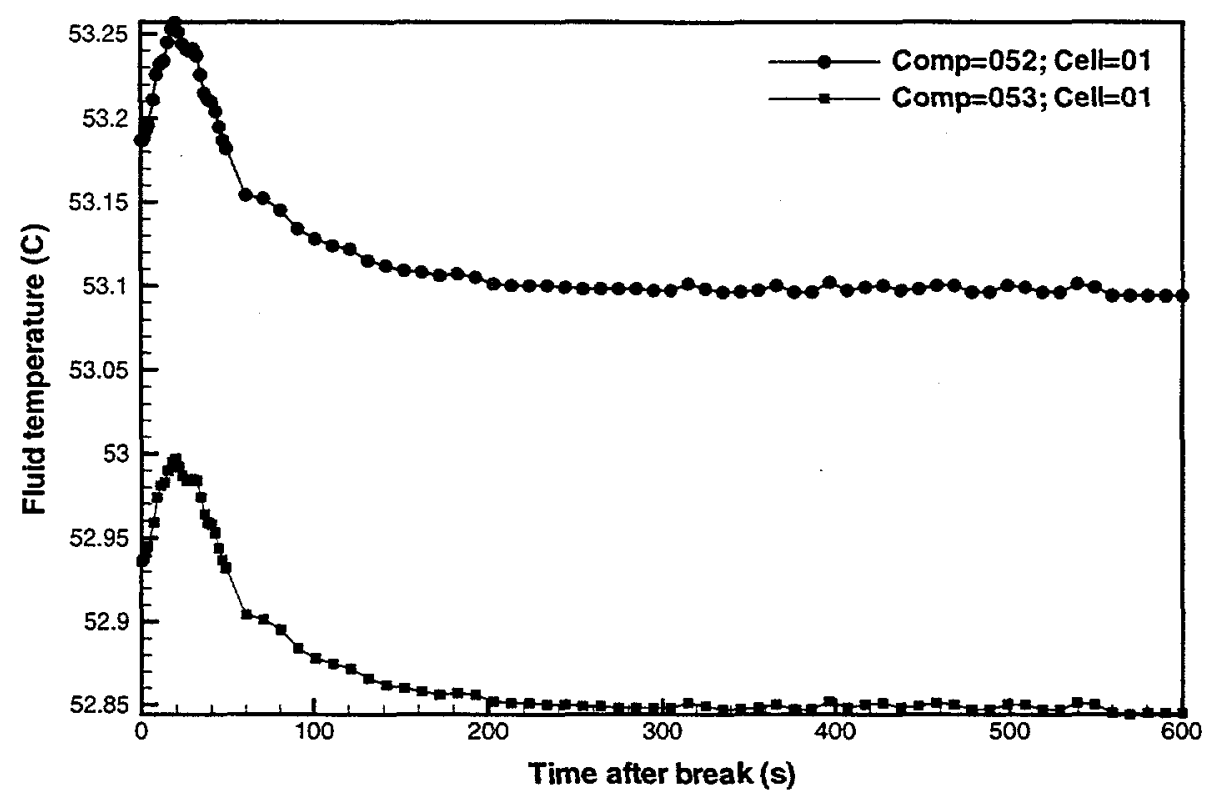

Figure B-18b Primary HR heat exchanger first pass outlet piping fluid temperatures for a LOHGA (Case 1: helium supply plenum break near decoupler inlet).

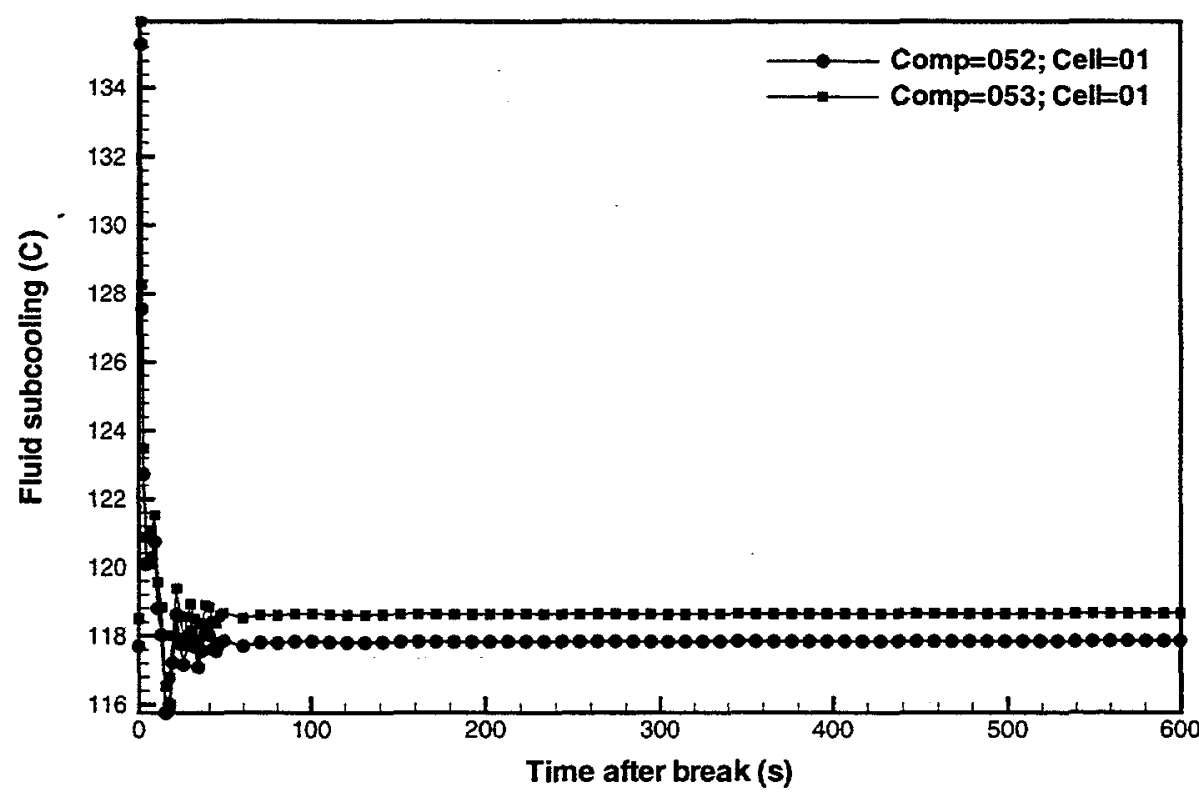

Figure B-18c Primary HR heat exchanger first pass outlet piping fluid subcoolings for a LOHGA (Case 1: helium supply plenum break near decoupler inlet). 


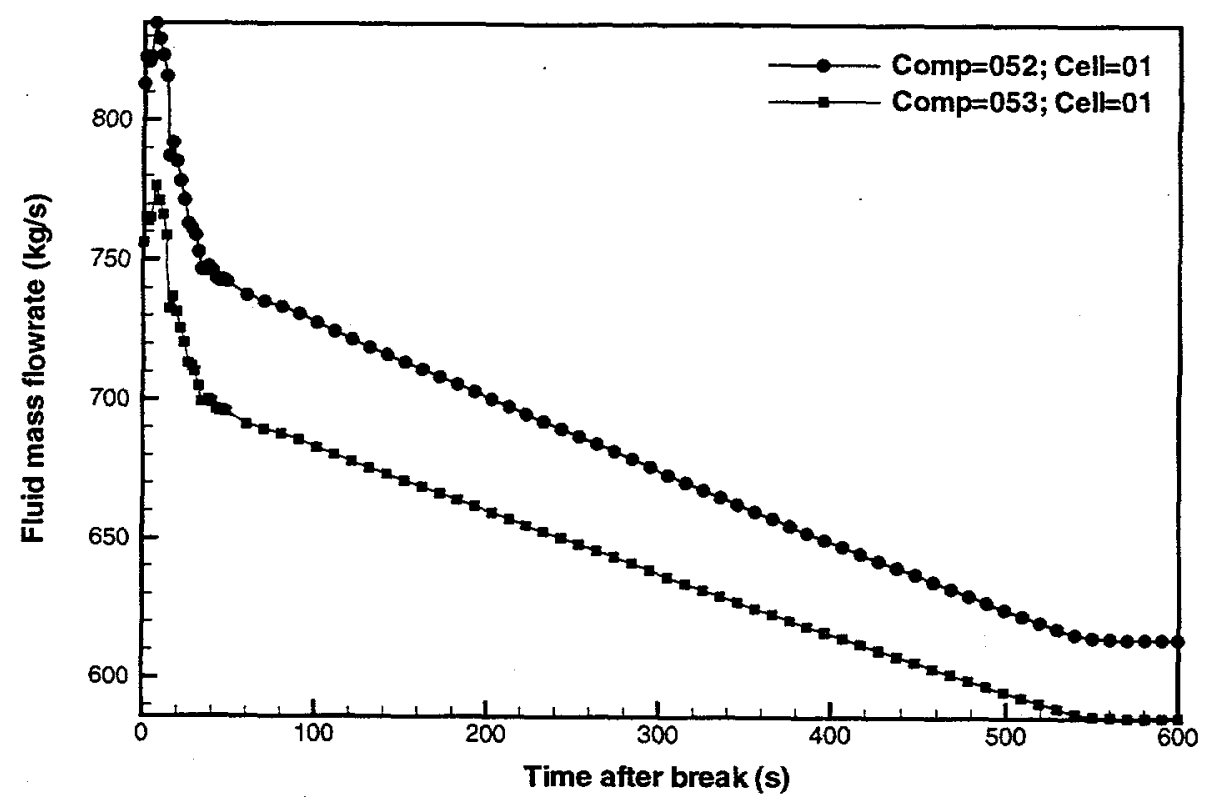

Figure B-18d Primary HR heat exchanger first pass outlet piping liquid mass flowrates for a LOHGA (Case 1: helium supply plenum break near decoupler inlet).

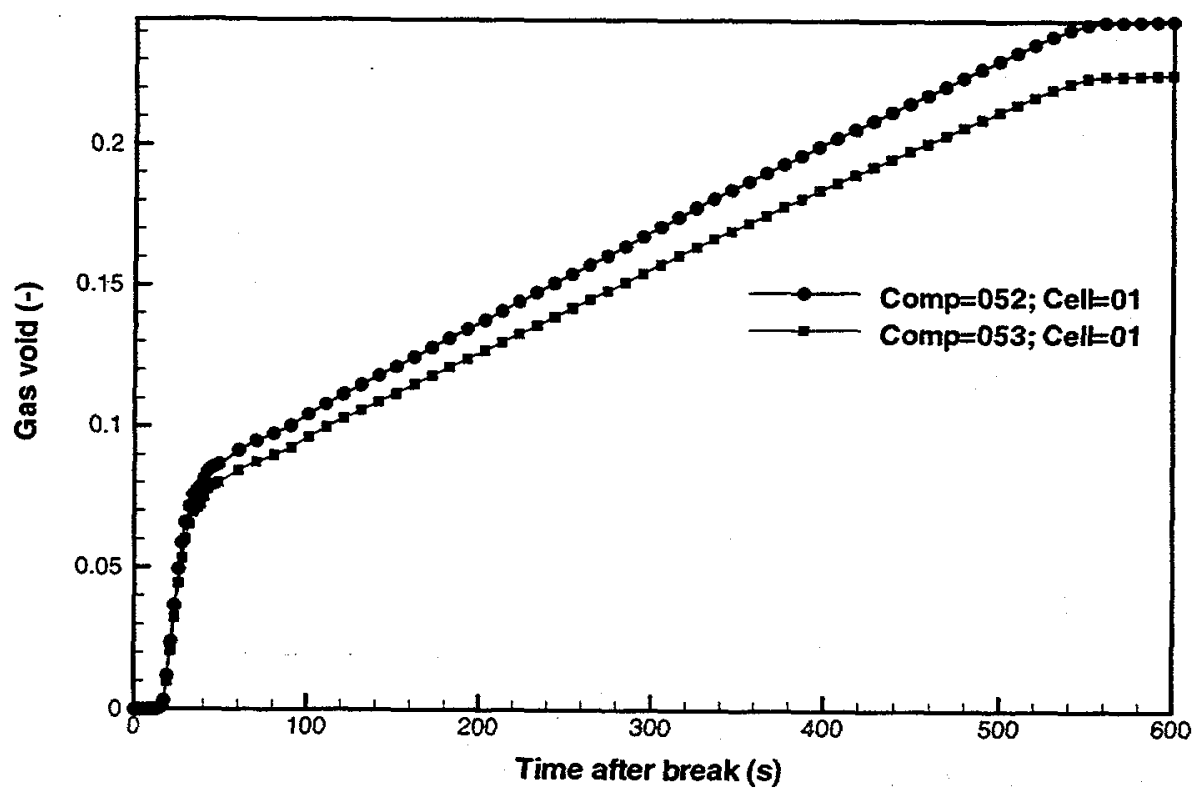

Figure B-18e Primary HR heat exchanger first pass outlet piping void fractions for a LOHGA (Case 1: helium supply plenum break near decoupler inlet). 


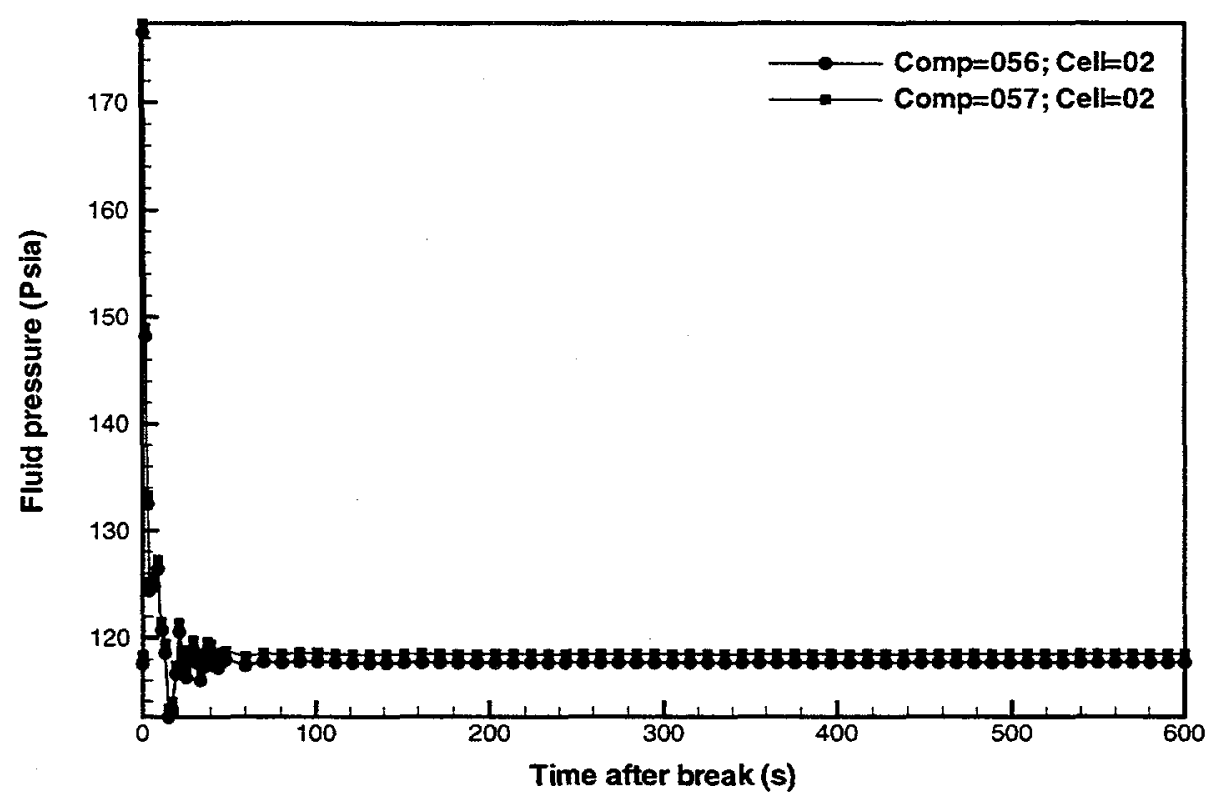

Figure B-19a Primary HR heat exchanger outlet piping fluid pressures for a LOHGA (Case 1: helium supply plenum break near decoupler inlet).

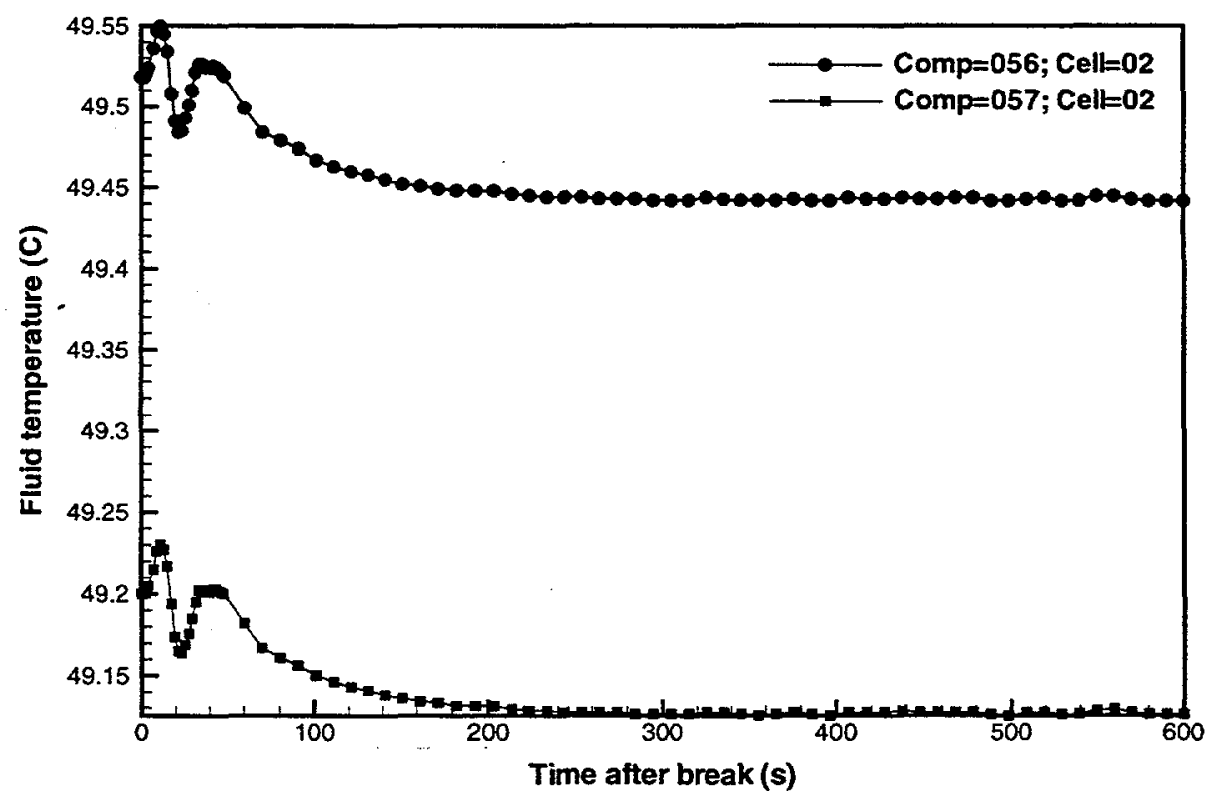

Figure B-19b Primary HR heat exchanger outlet piping fluid temperatures for a LOHGA (Case 1: helium supply plenum break near decoupler inlet). 


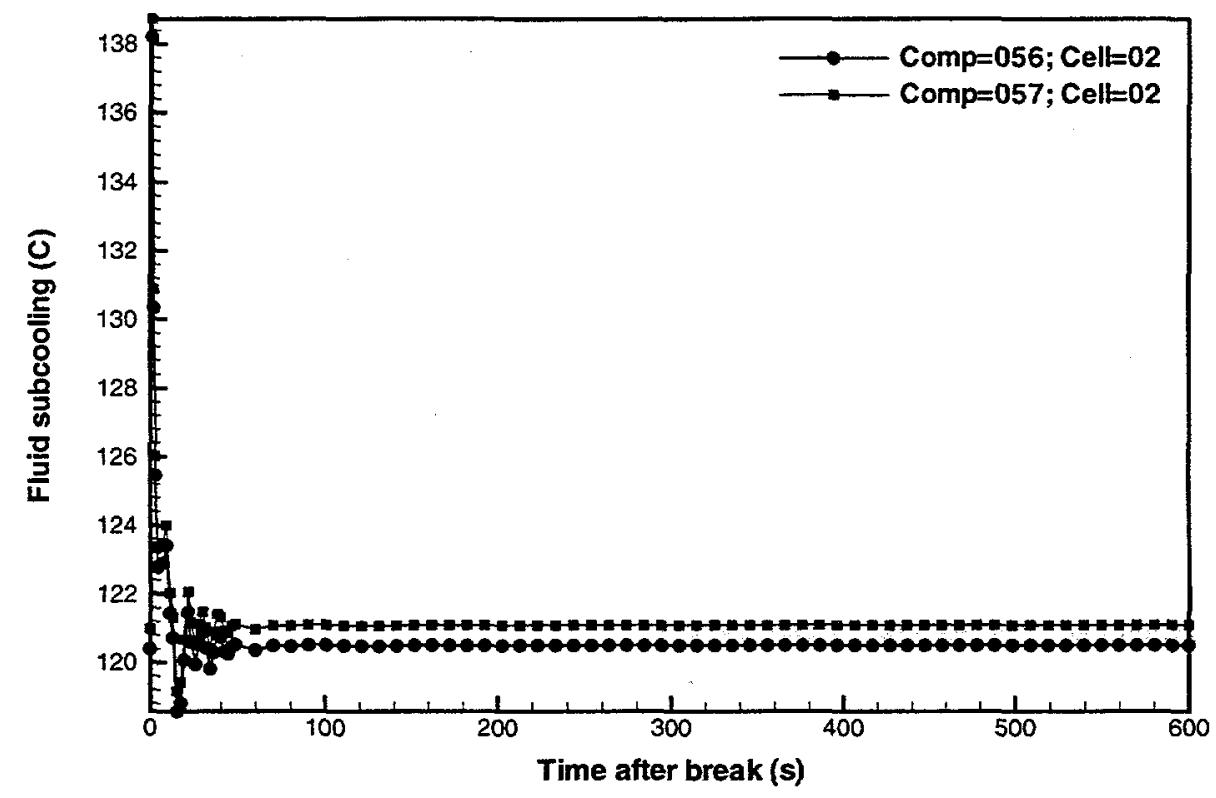

Figure B-19c Primary HR heat exchanger outlet piping fluid subcoolings for a LOHGA (Case 1: helium supply plenum break near decoupler inlet).

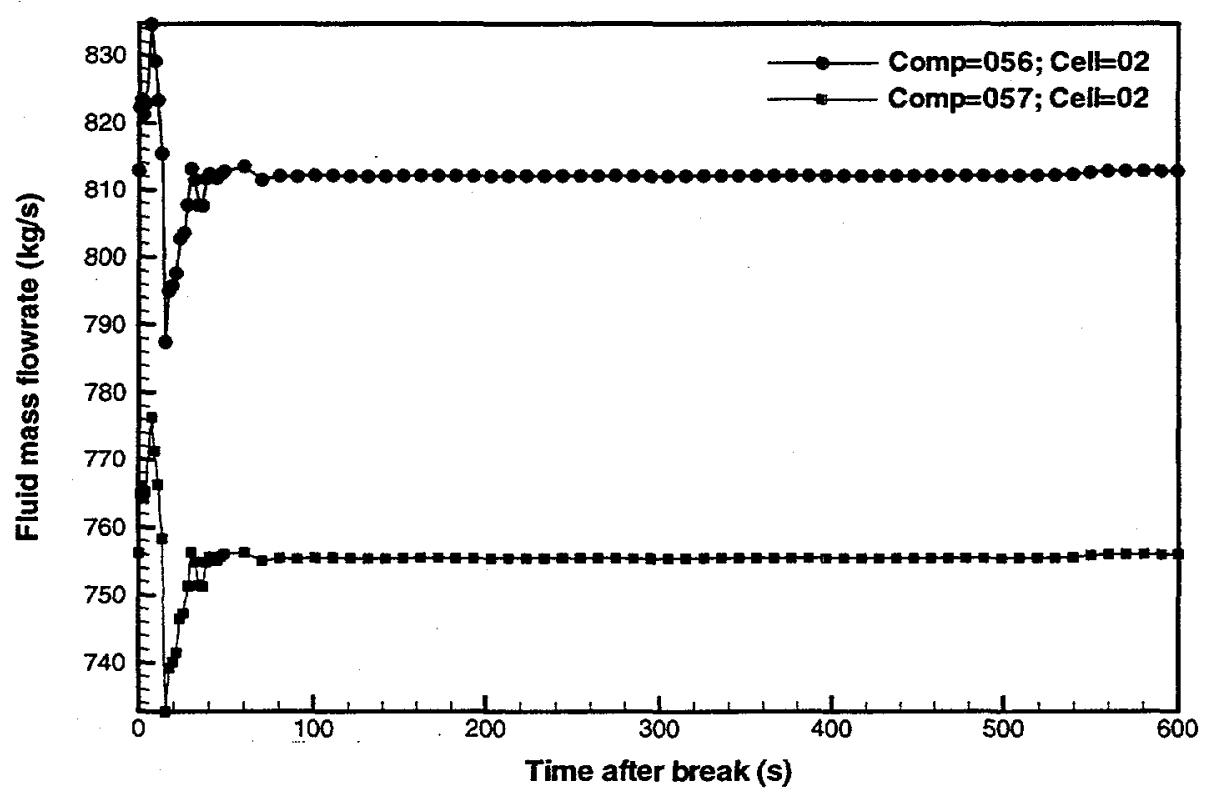

Figure B-19d Primary HR heat exchanger outlet piping liquid mass flowrates for a LOHGA (Case 1: helium supply plenum break near decoupler inlet). 


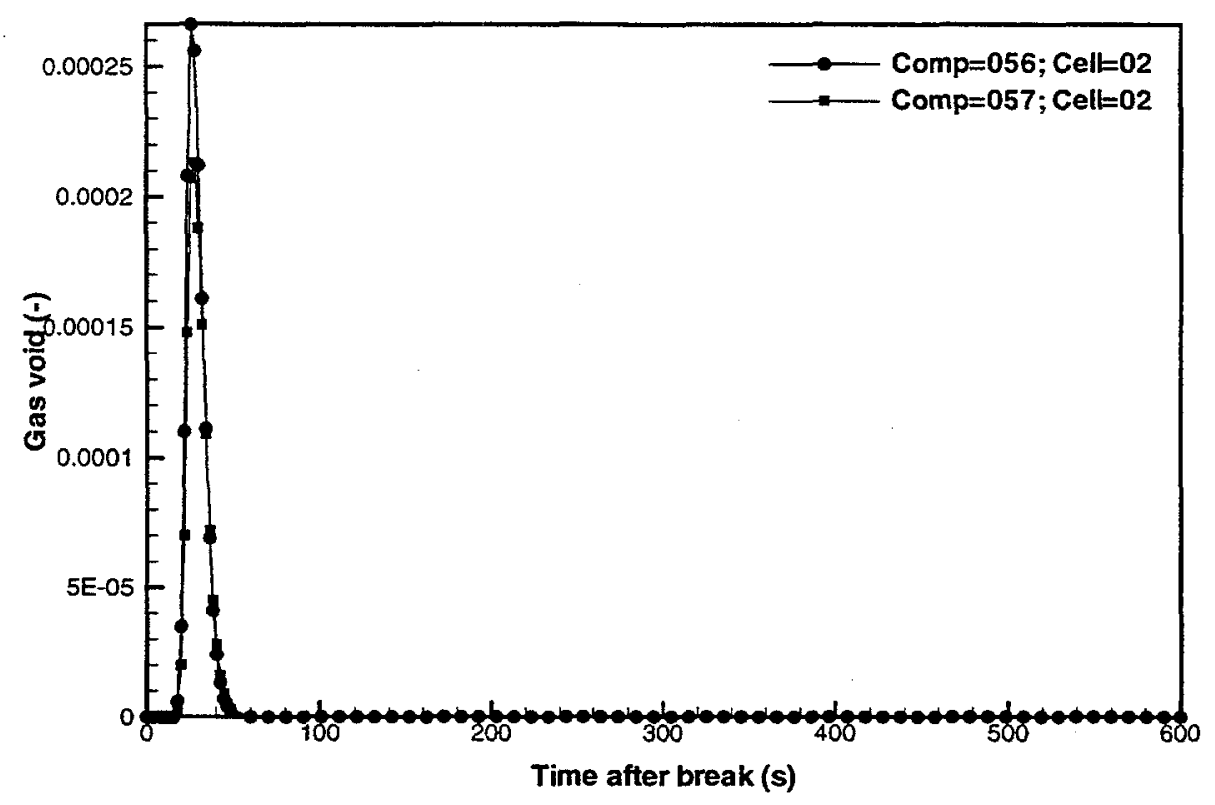

Figure B-19e Primary HR heat exchanger outlet piping void fractions for a LOHGA (Case 1: helium supply plenum break near decoupler inlet).

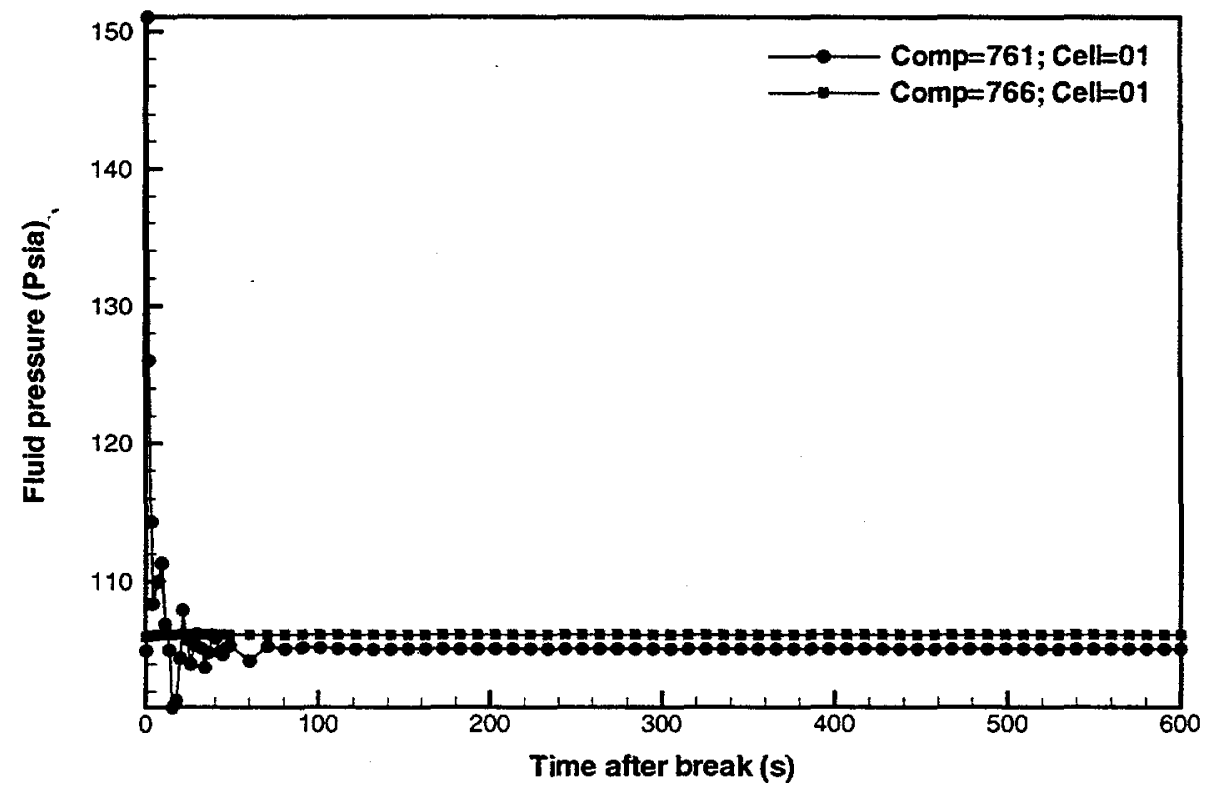

Figure B-20a Primary HR pressurizer and surge line fluid pressures for a LOHGA (Case 1: helium supply plenum break near decoupler inlet). 


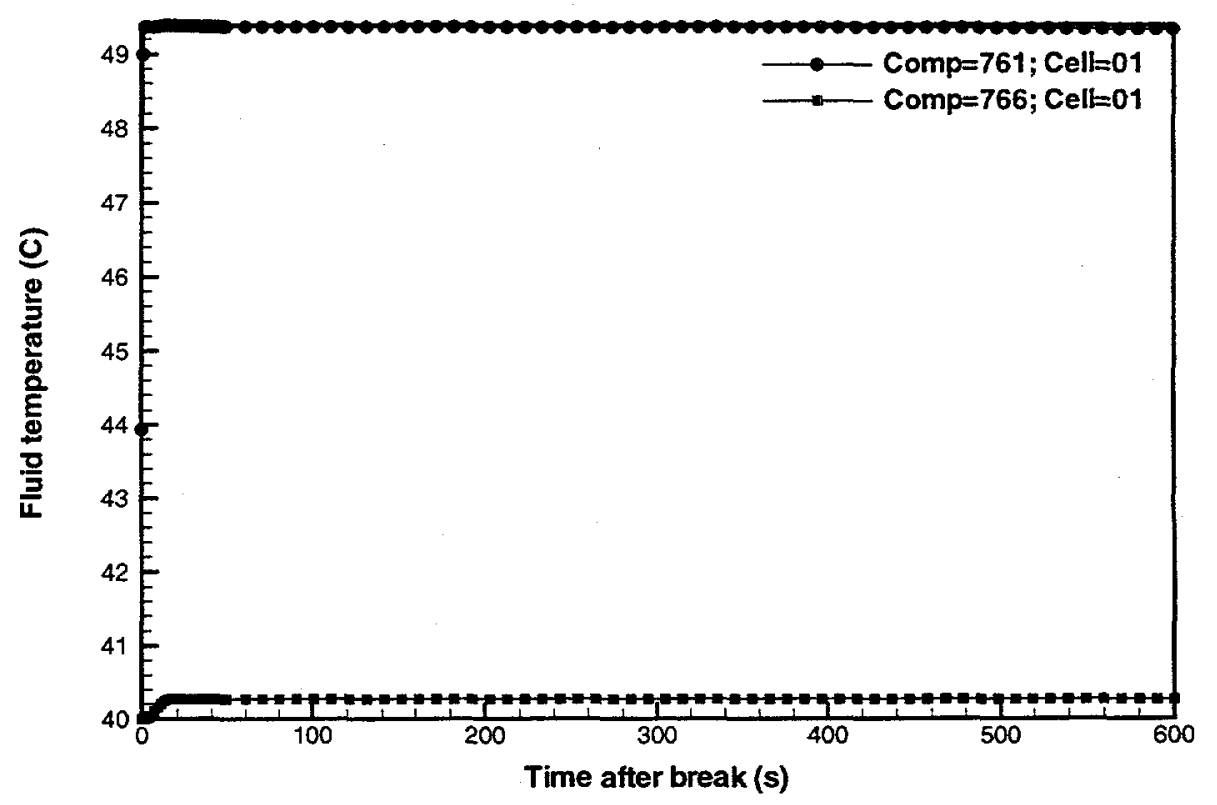

Figure B-20b Primary HR pressurizer and surge line fluid temperatures for a LOHGA (Case 1: helium supply plenum break near decoupler inlet).

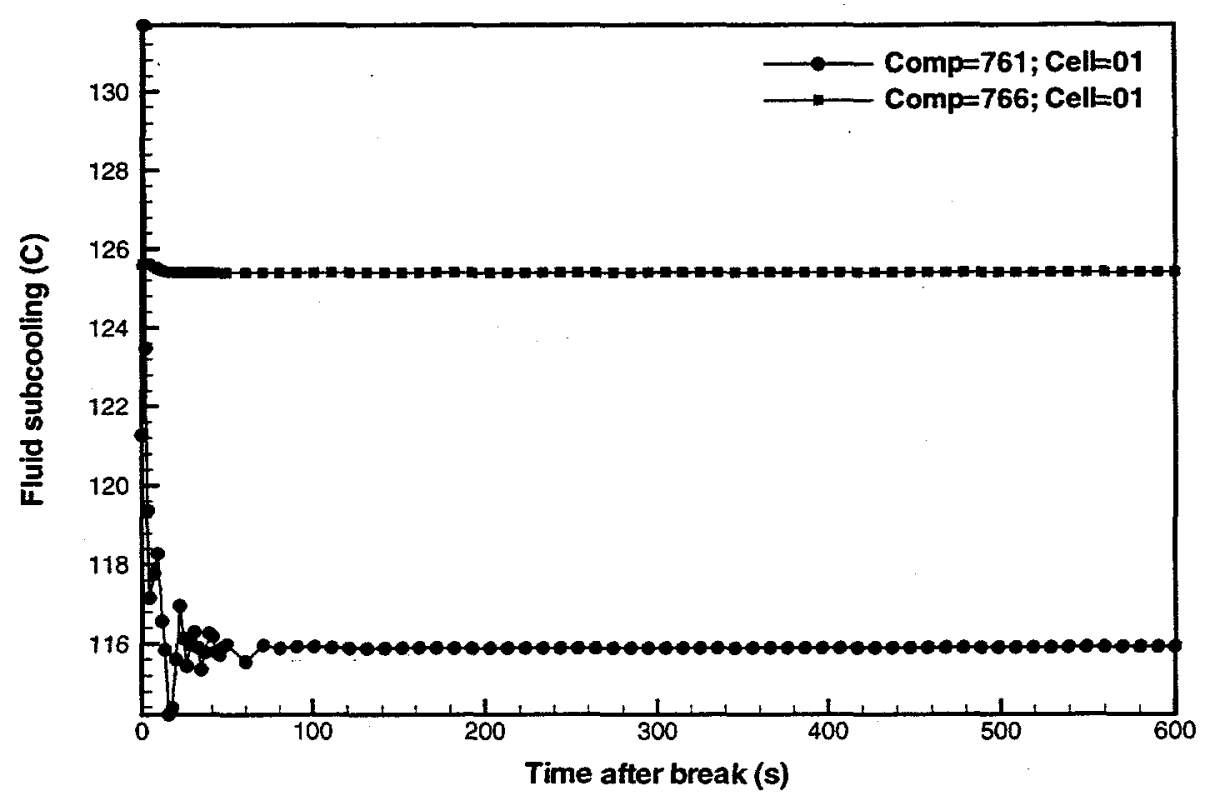

Figure B-20c Primary HR pressurizer and surge line fluid subcoolings for a LOHGA (Case 1: helium supply plenum break near decoupler inlet). 


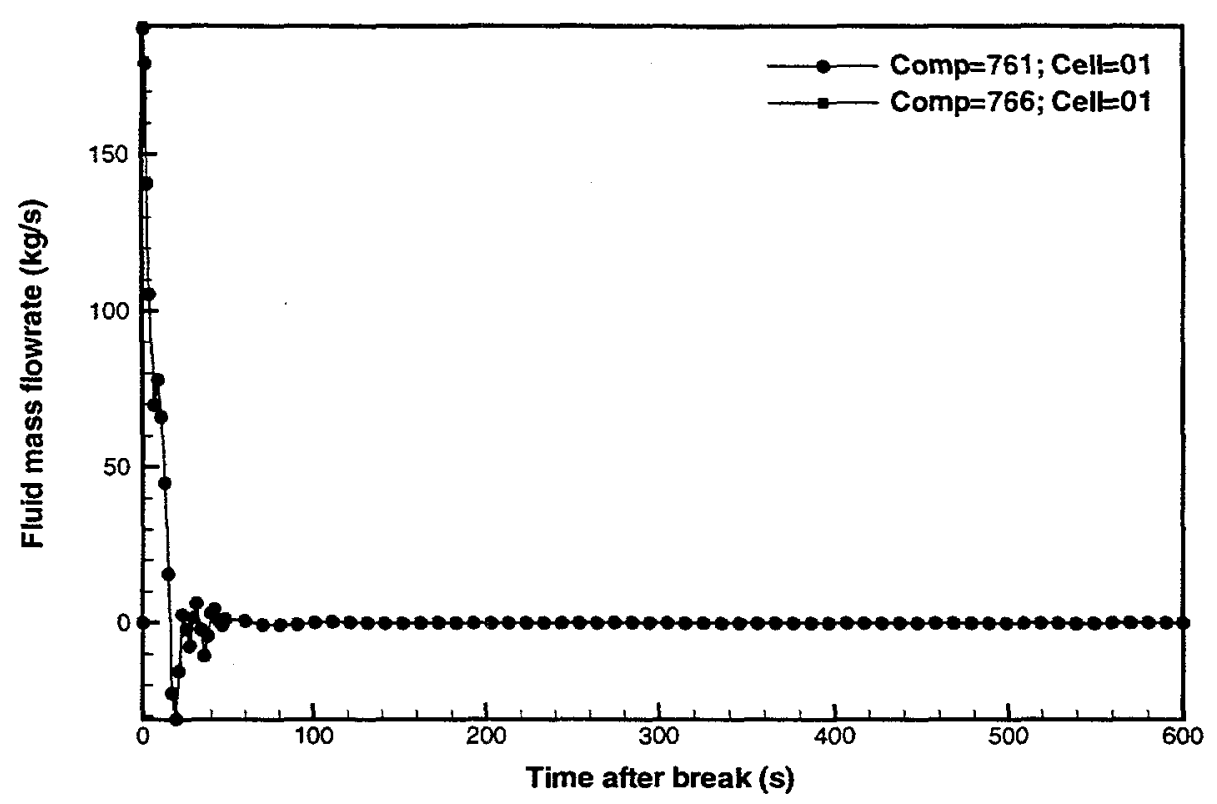

Figure B-20d Primary HR pressurizer and surge line liquid mass flowrates for a LOHGA (Case 1: helium supply plenum break near decoupler inlet).

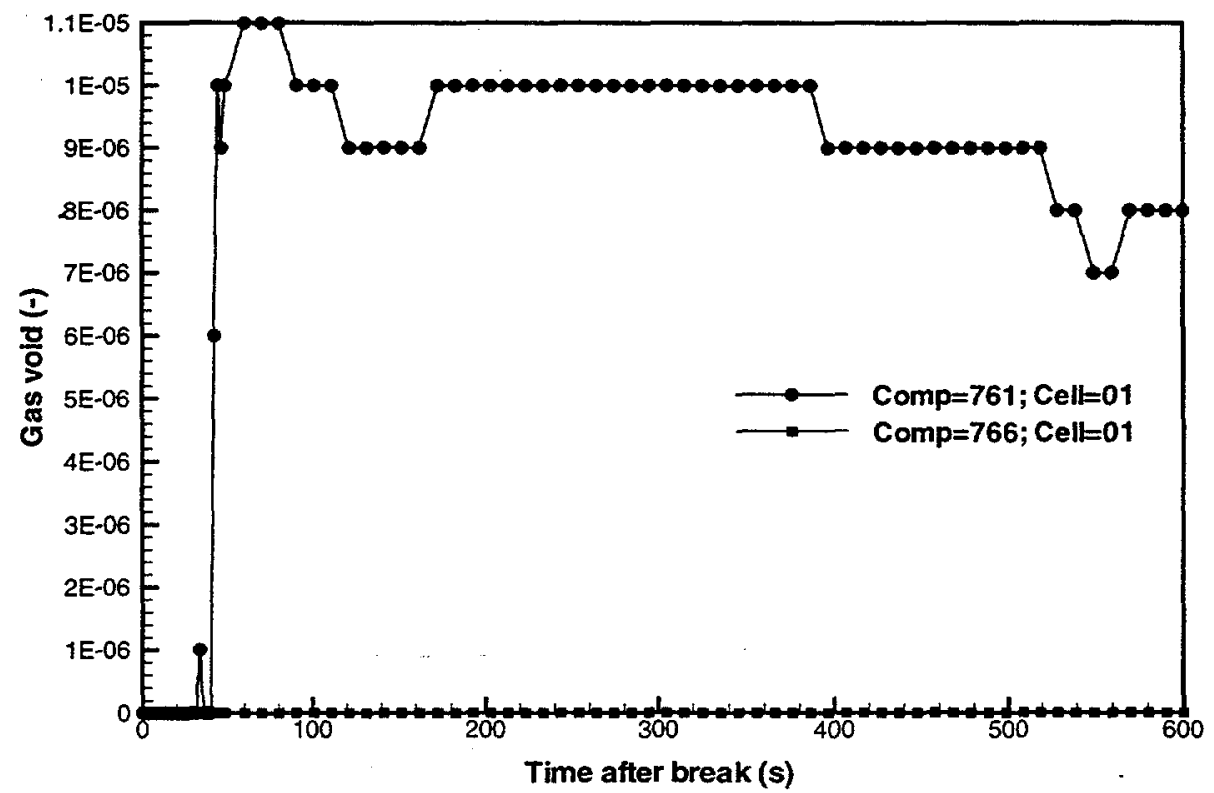

Figure B-20e Primary HR pressurizer and surge line void fractions for a LOHGA (Case 1: helium supply plenum break near decoupler inlet). 


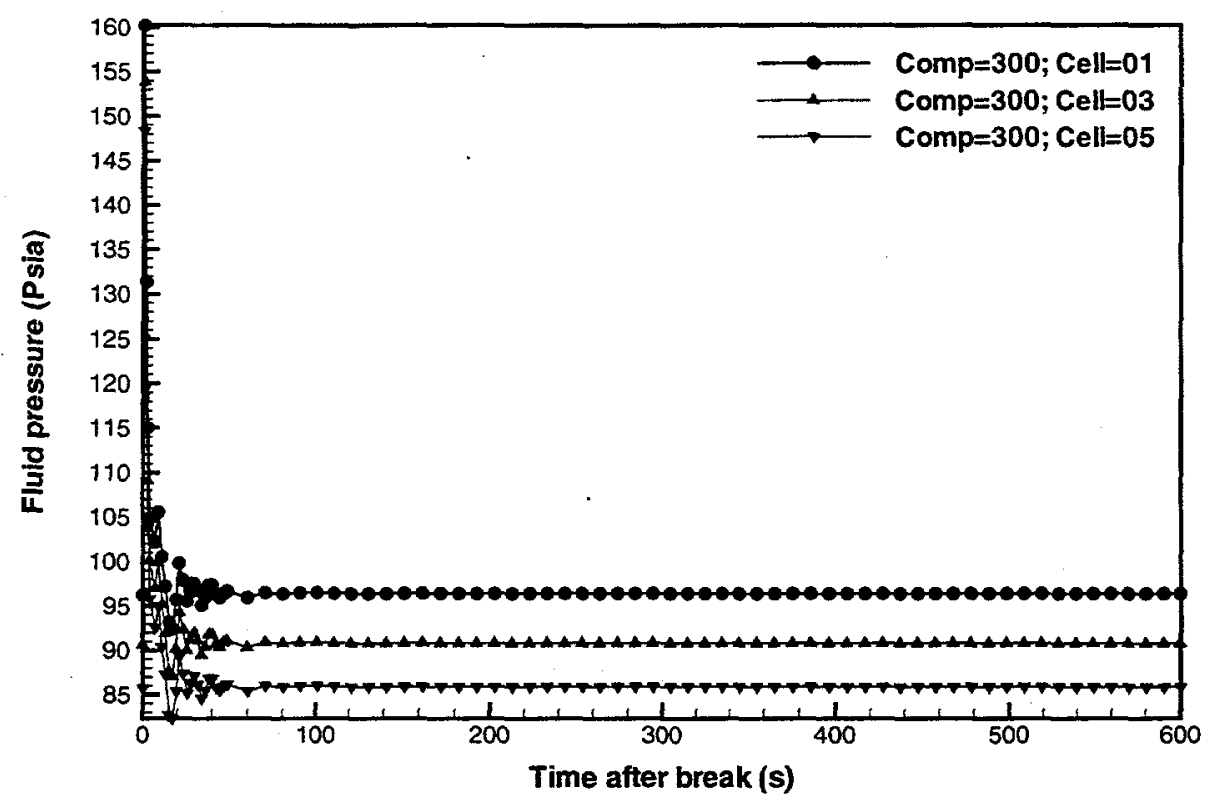

Figure B-21a Module 1 row 1 channel fluid pressures for a LOHGA (Case 1: helium supply plenum break near decoupler inlet).

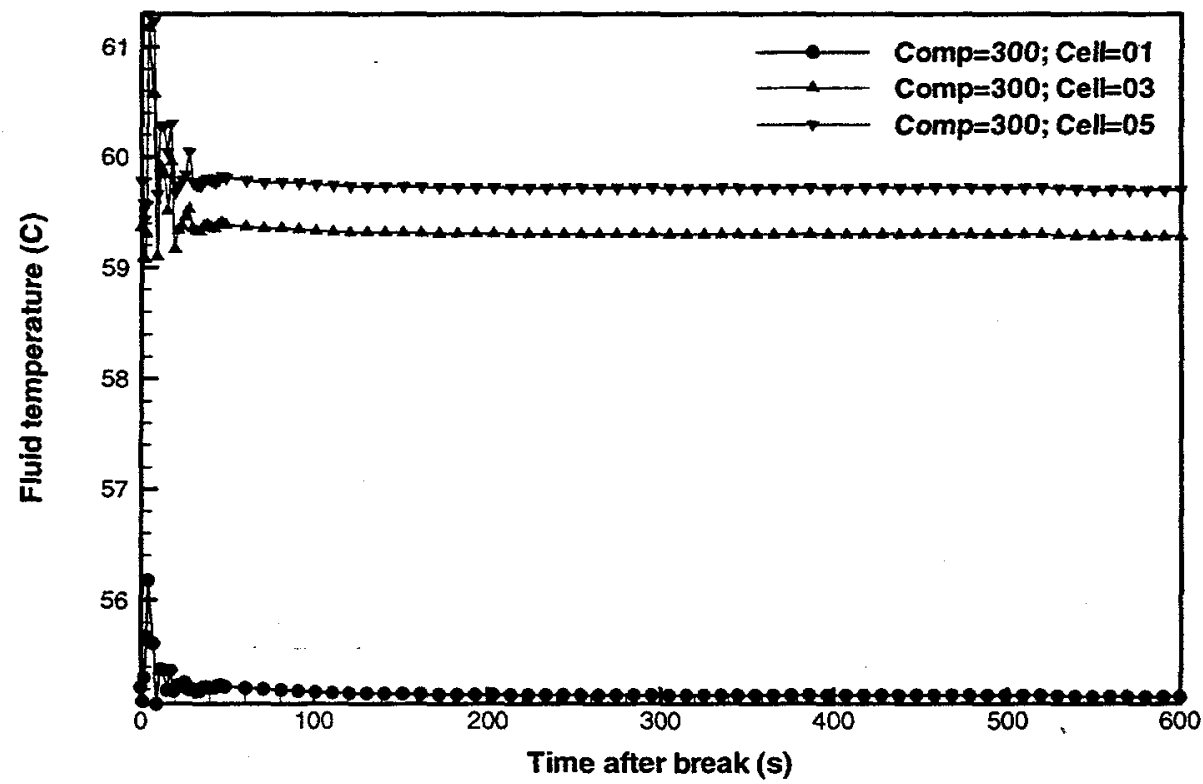

Figure B-21b Module 1 row 1 channel fluid temperatures for a LOHGA (Case 1: helium supply plenum break near decoupler inlet). 


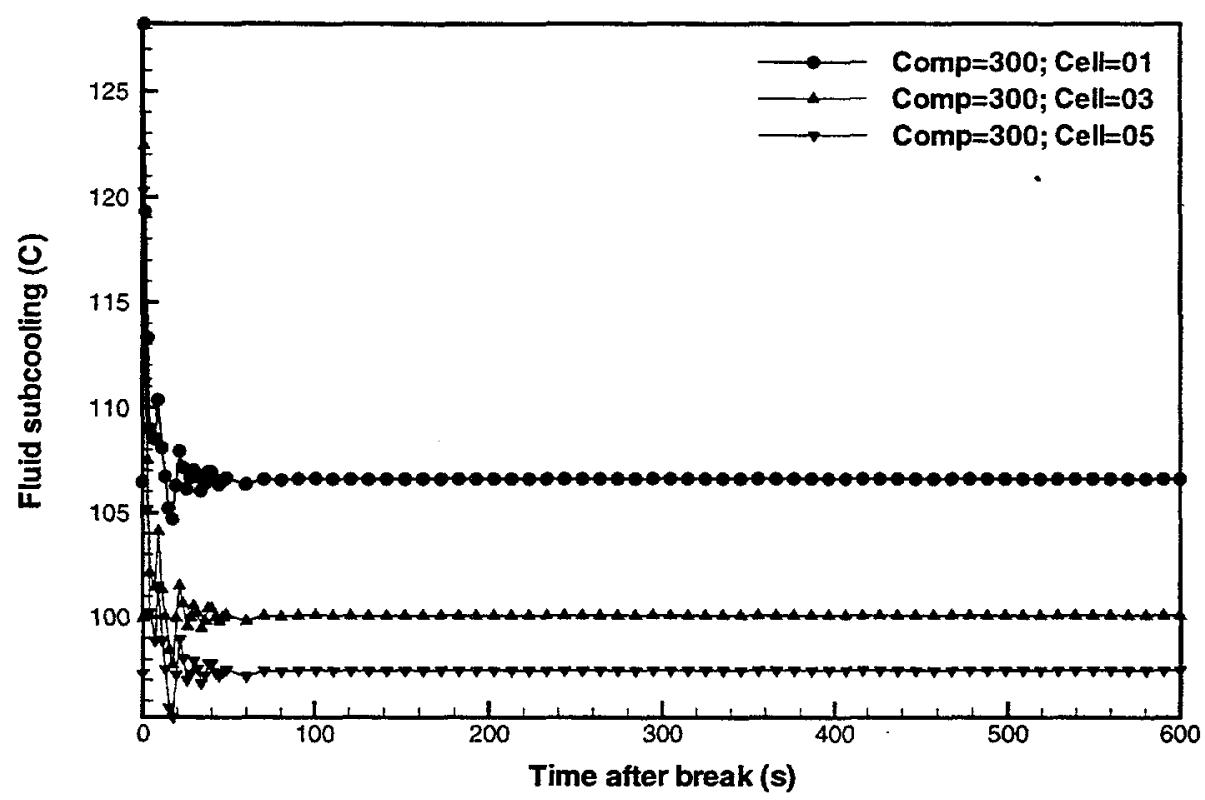

Figure B-21c Module 1 row 1 channel fluid subcoolings for a LOHGA-(Case 1: helium supply plenum break near decoupler inlet).

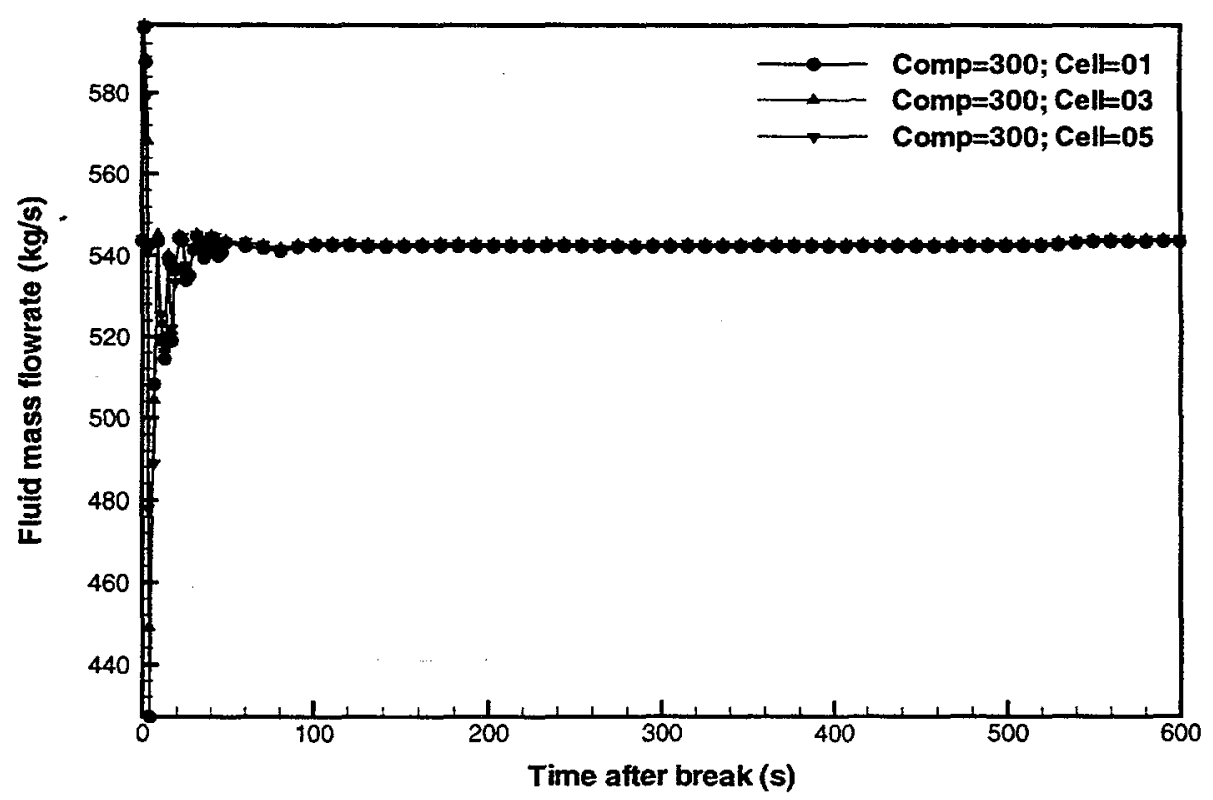

Figure B-21d Module 1 row 1 channel liquid mass flowrates for a LOHGA (Case 1: helium supply plenum break near decoupler inlet). 


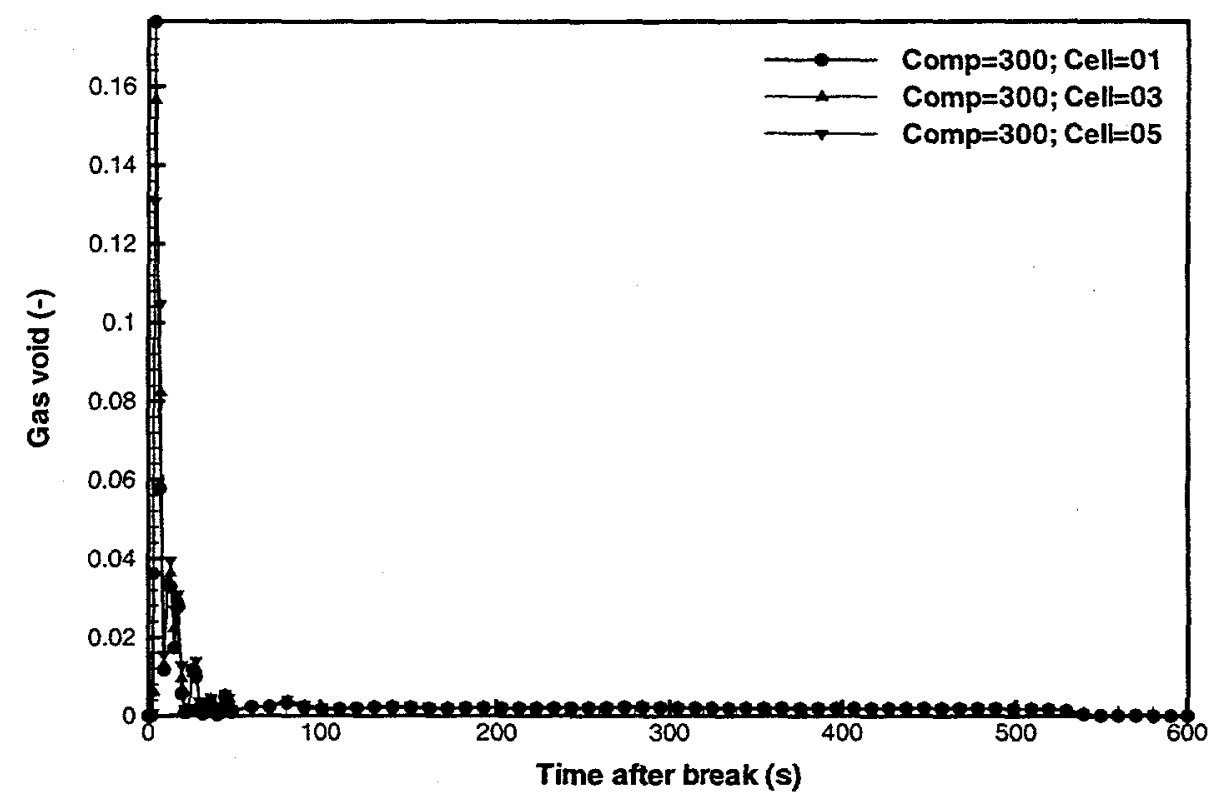

Figure B-21e Module 1 row 1 channel void fractions for a LOHGA (Case 1: helium supply plenum break near decoupler inlet).

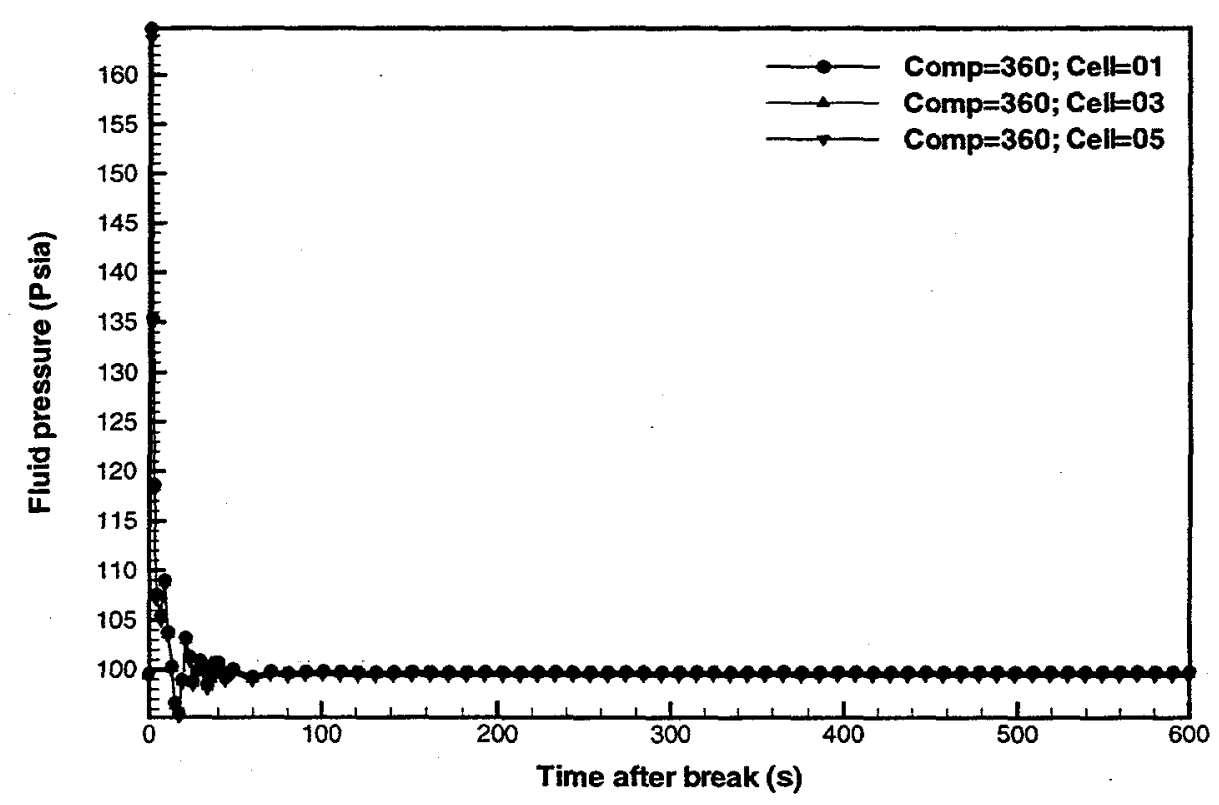

Figure B-22a Module 1 decoupler channel fluid pressures for a LOHGA (Case 1: helium supply plenum break near decoupler inlet). 


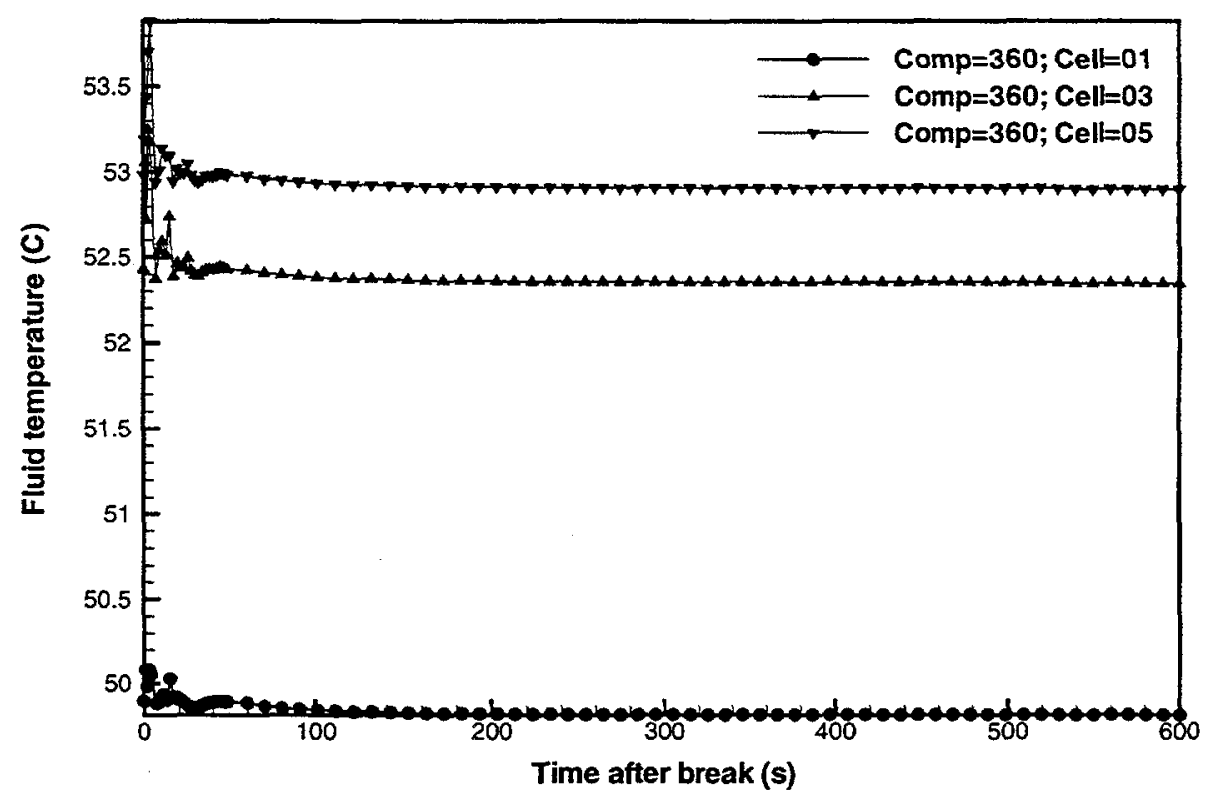

Figure B-22b Module 1 decoupler channel fluid temperatures for a tOHGA (Case 1: helium supply plenum break near decoupler inlet).

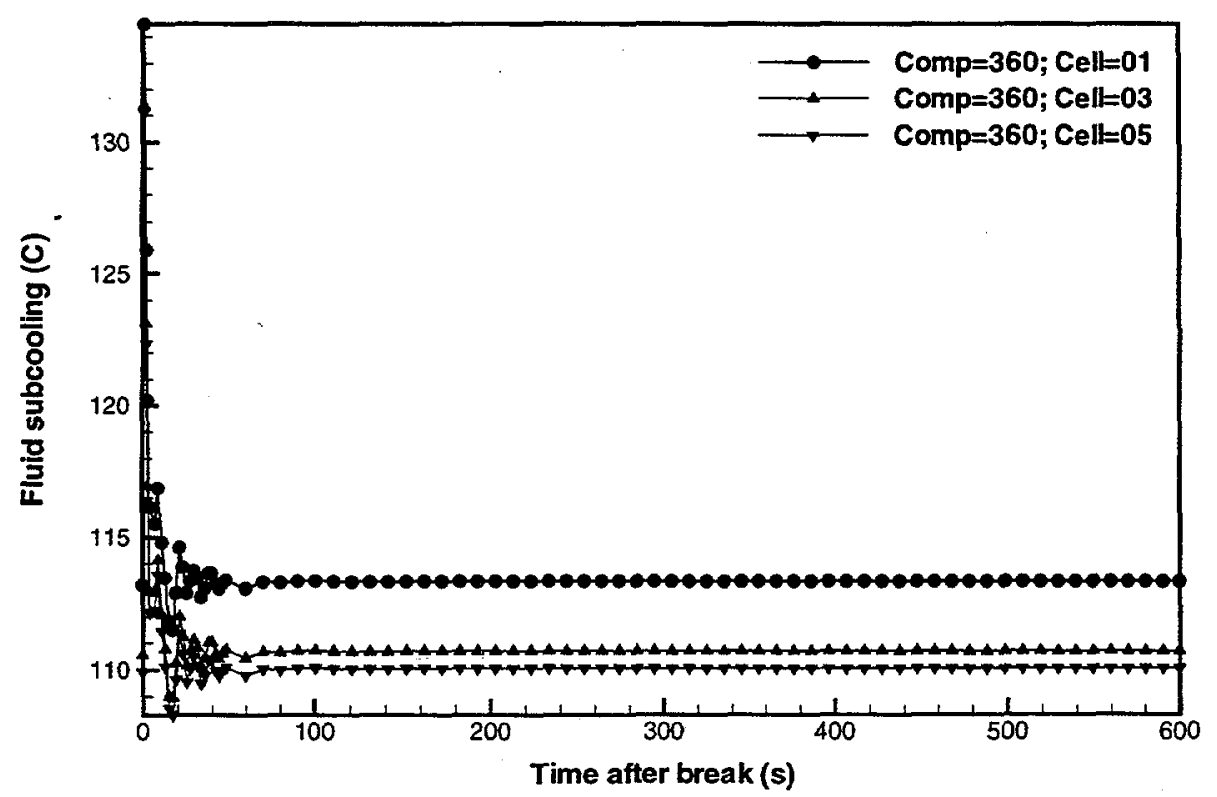

Figure B-22c Module 1 decoupler channel fluid subcoolings for a LOHGA (Case 1: helium supply plenum break near decoupler inlet). 


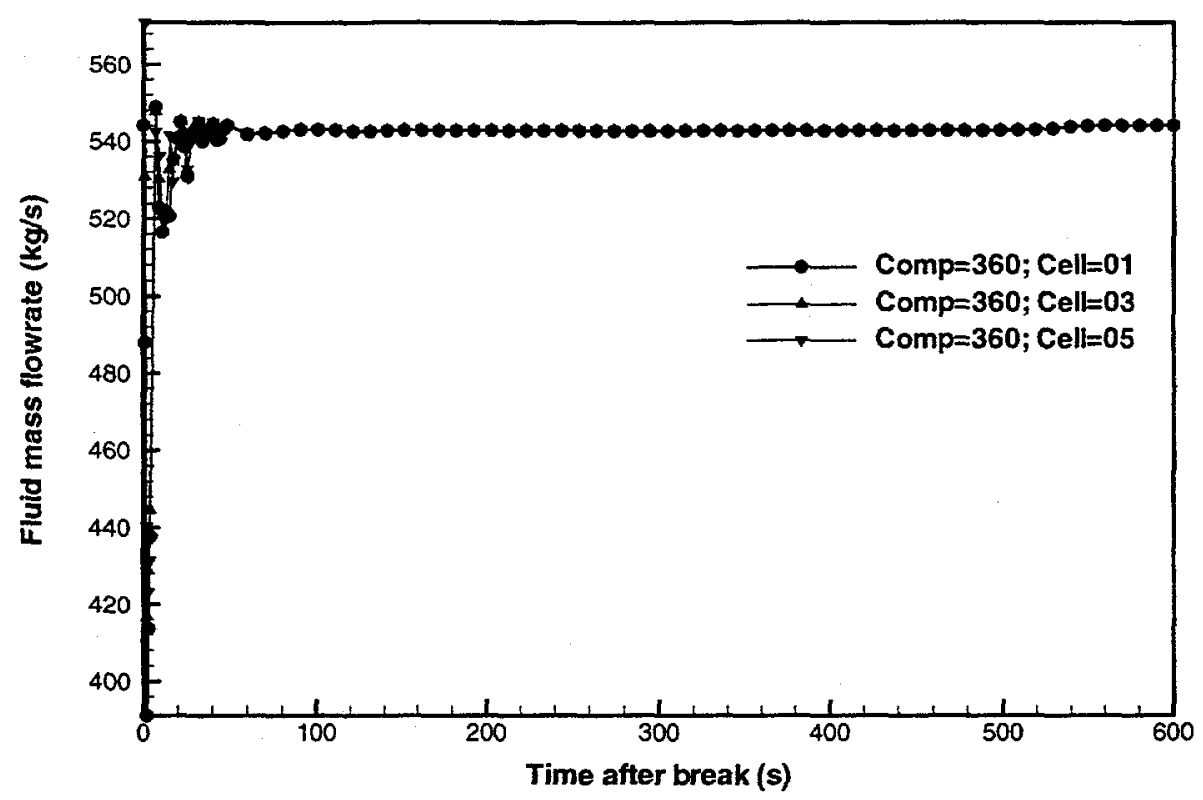

Figure B-22d Module 1 decoupler channel liquid mass flowrates for a LOHGA (Case 1: helium supply plenum break near decoupler inlet).

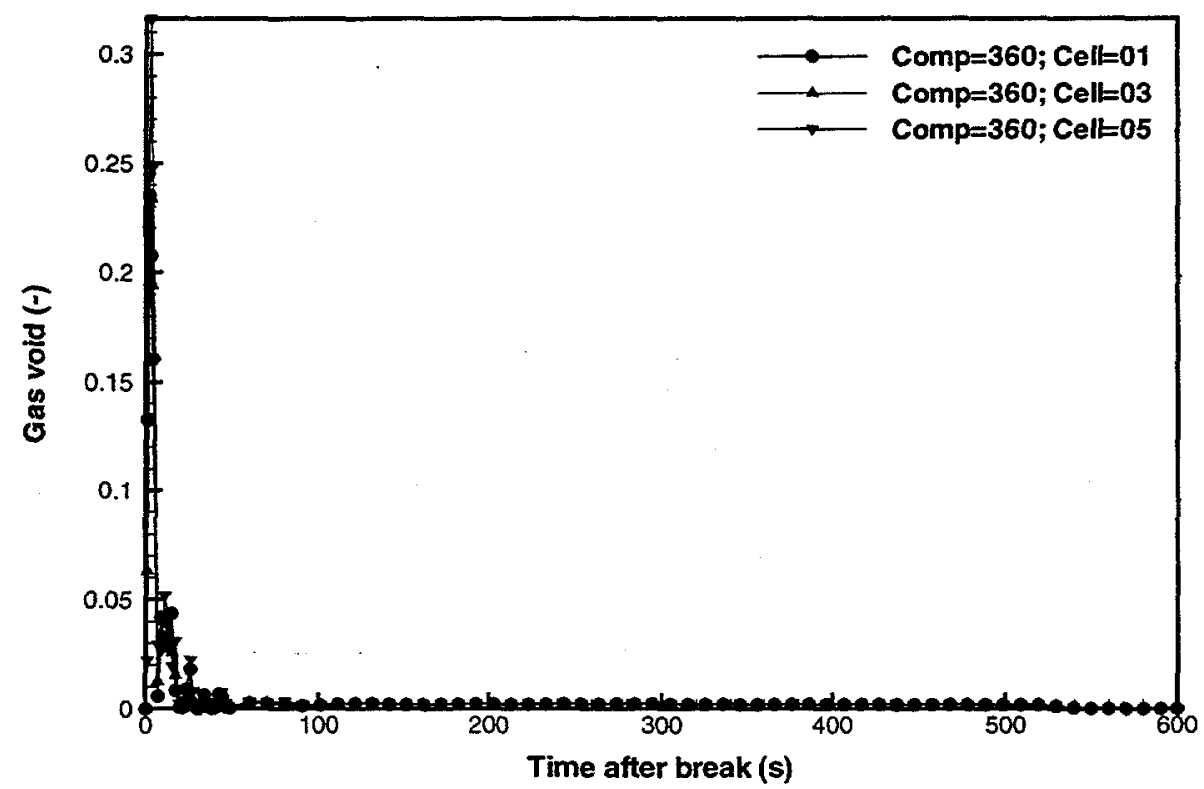

Figure B-22e Module 1 decoupler channel Module 2 channel void fractions for a LOHGA (Case 1: helium supply plenum break near decoupler inlet). 


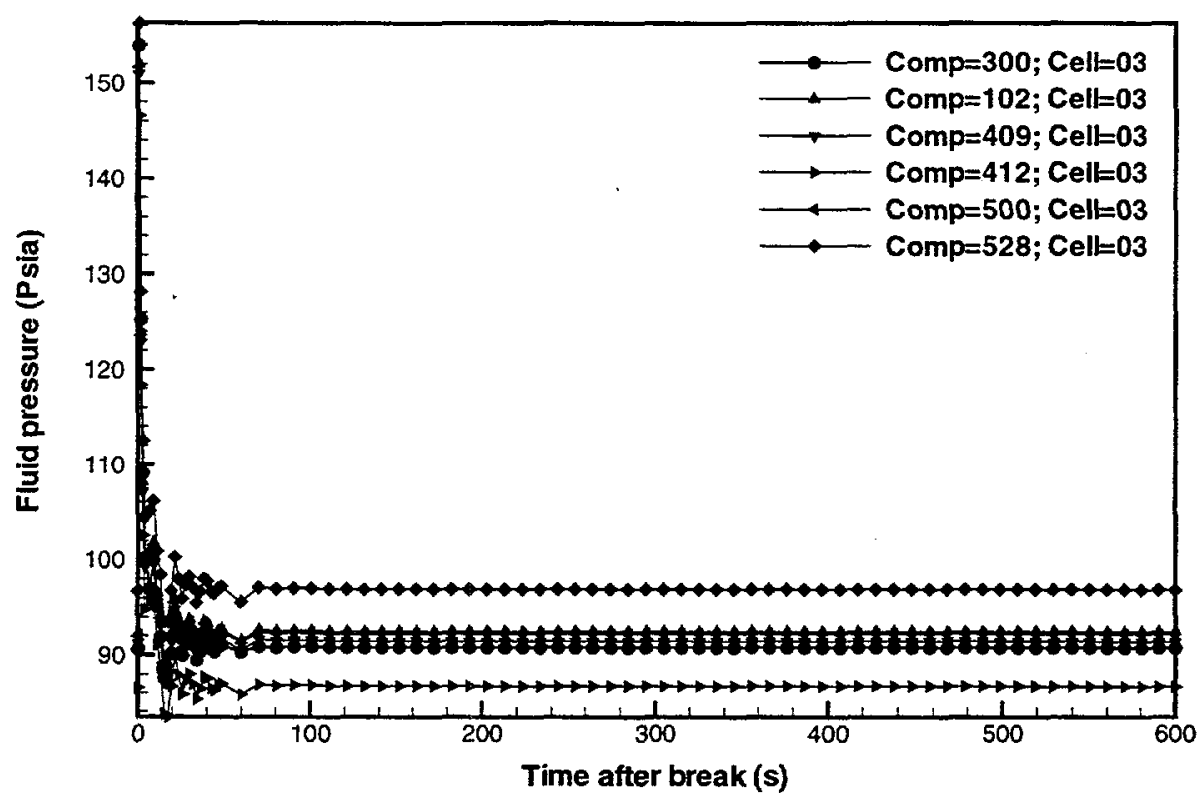

Figure B-23a Mid-plane module fluid pressures for a LOHGA (Case 1: helium supply plenum break near decoupler inlet).

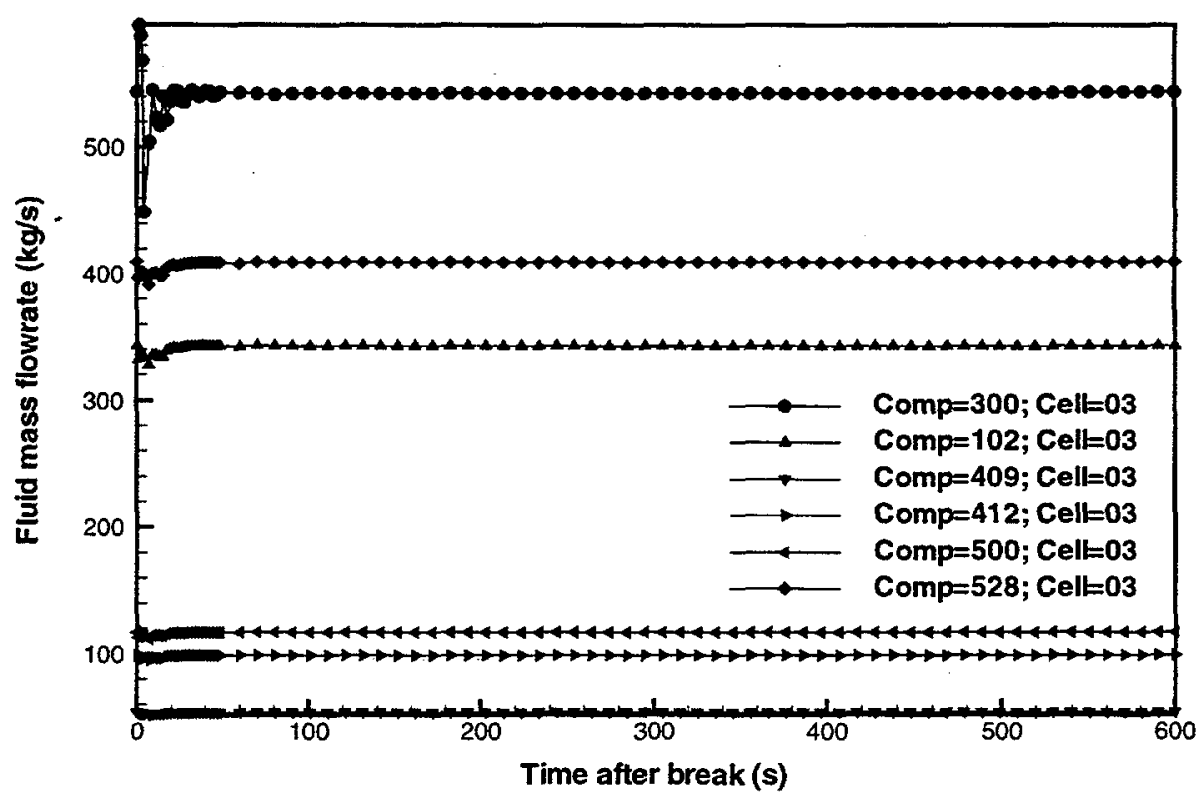

Figure B-23b Mid-plane module fluid temperatures for a LOHGA (Case 1: helium supply plenum break near decoupler inlet). 


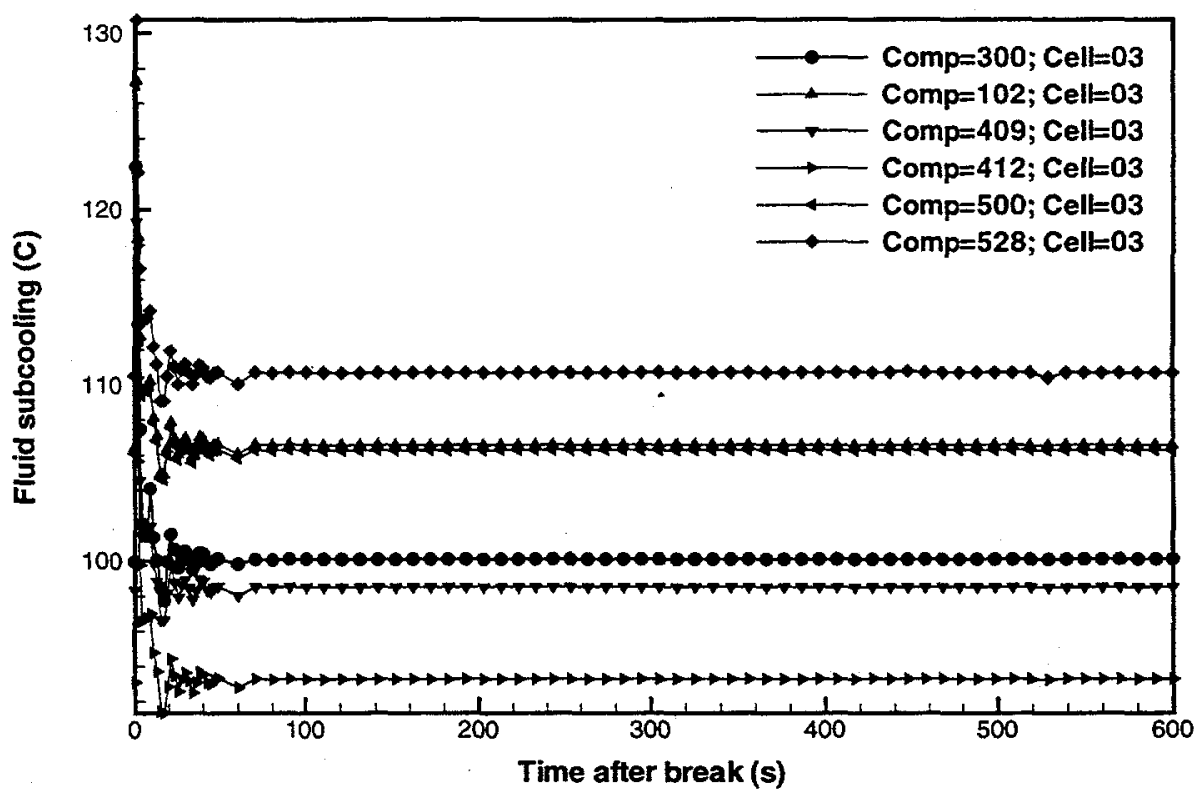

Figure B-23c Mid-plane module fluid subcoolings for a LOHGA (Case 1: helium supply plenum break near decoupler inlet).

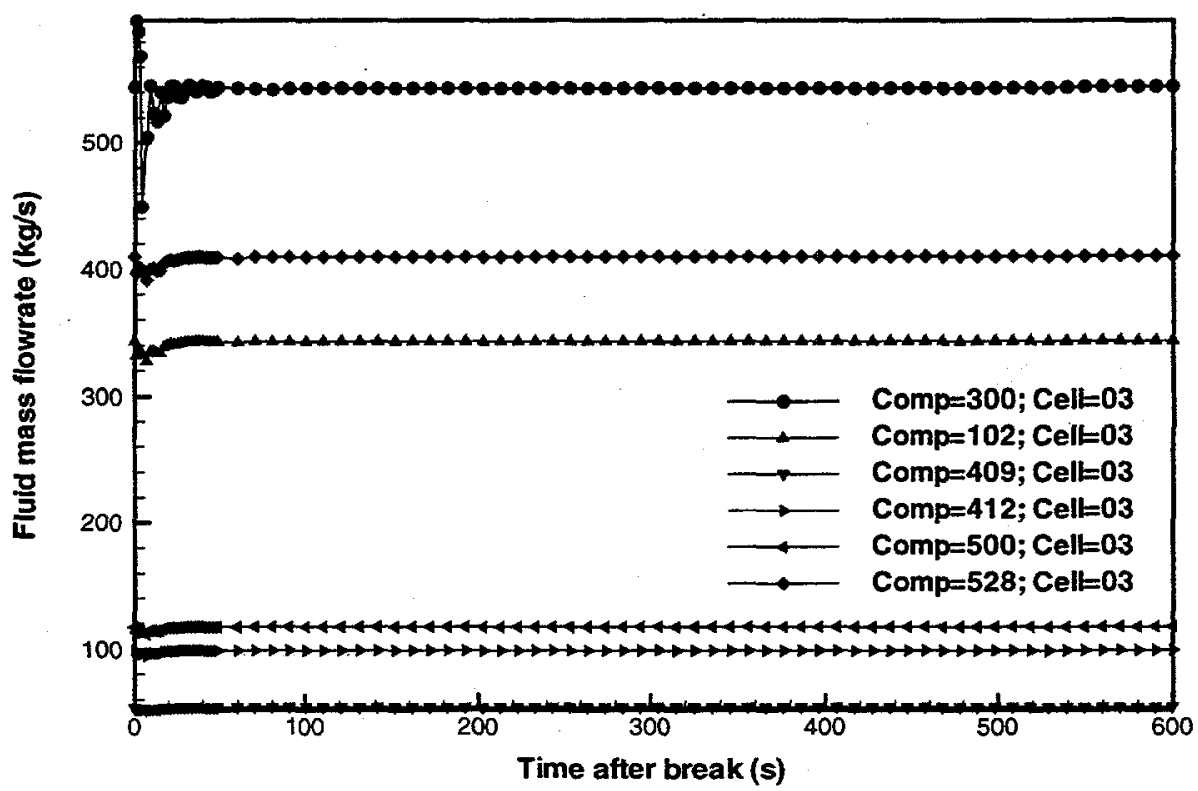

Figure B-23d Mid-plane module liquid mass flowrates for a LOHGA (Case 1: helium supply plenum break near decoupler inlet). 


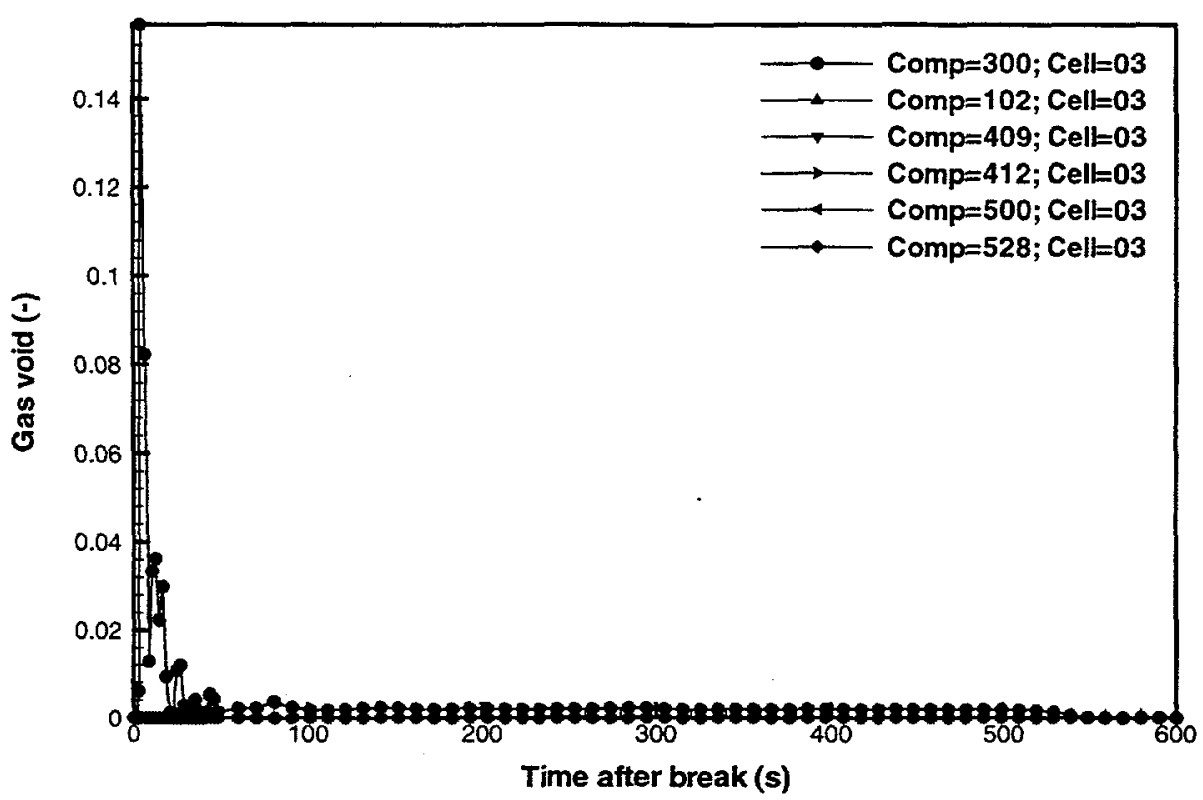

Figure B-23e Mid-plane module void fractions for a LOHGA (Case t: helium supply plenum break near decoupler inlet).

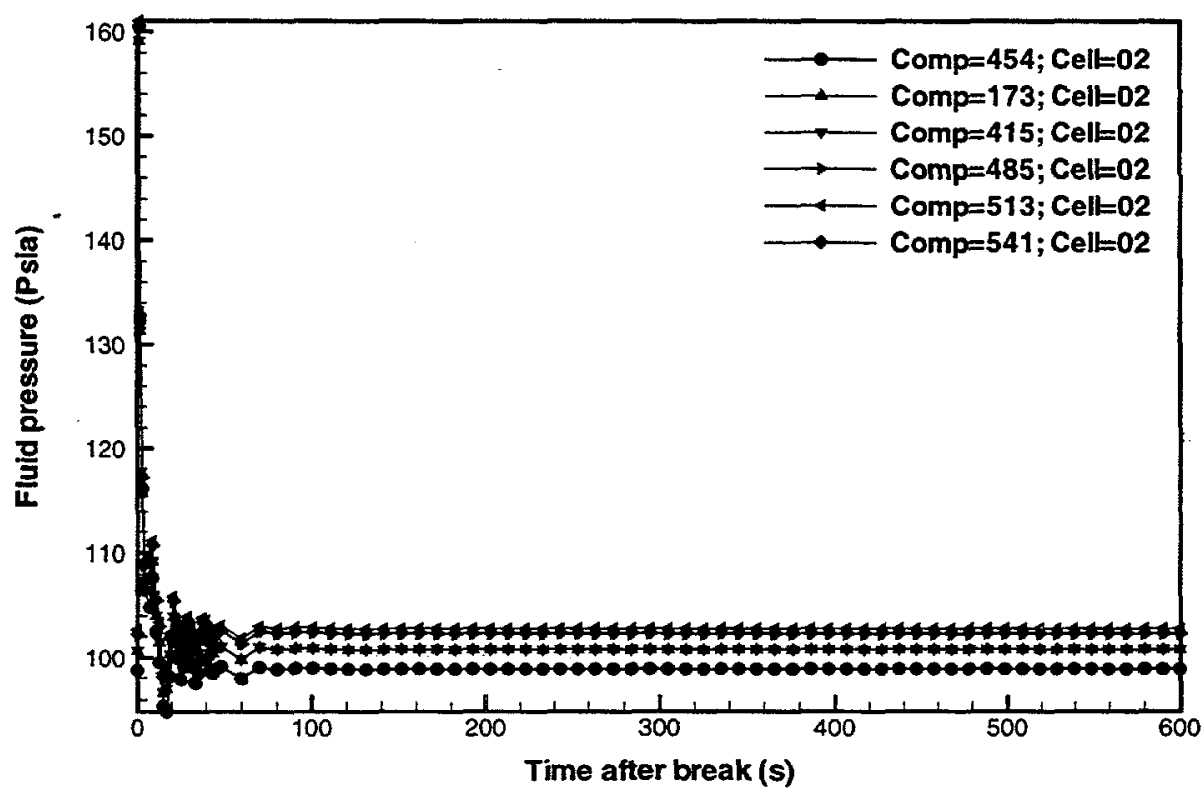

Figure B-24a Module inlet fluid pressures for a LOHGA (Case 1: helium supply plenum break near decoupler inlet). 


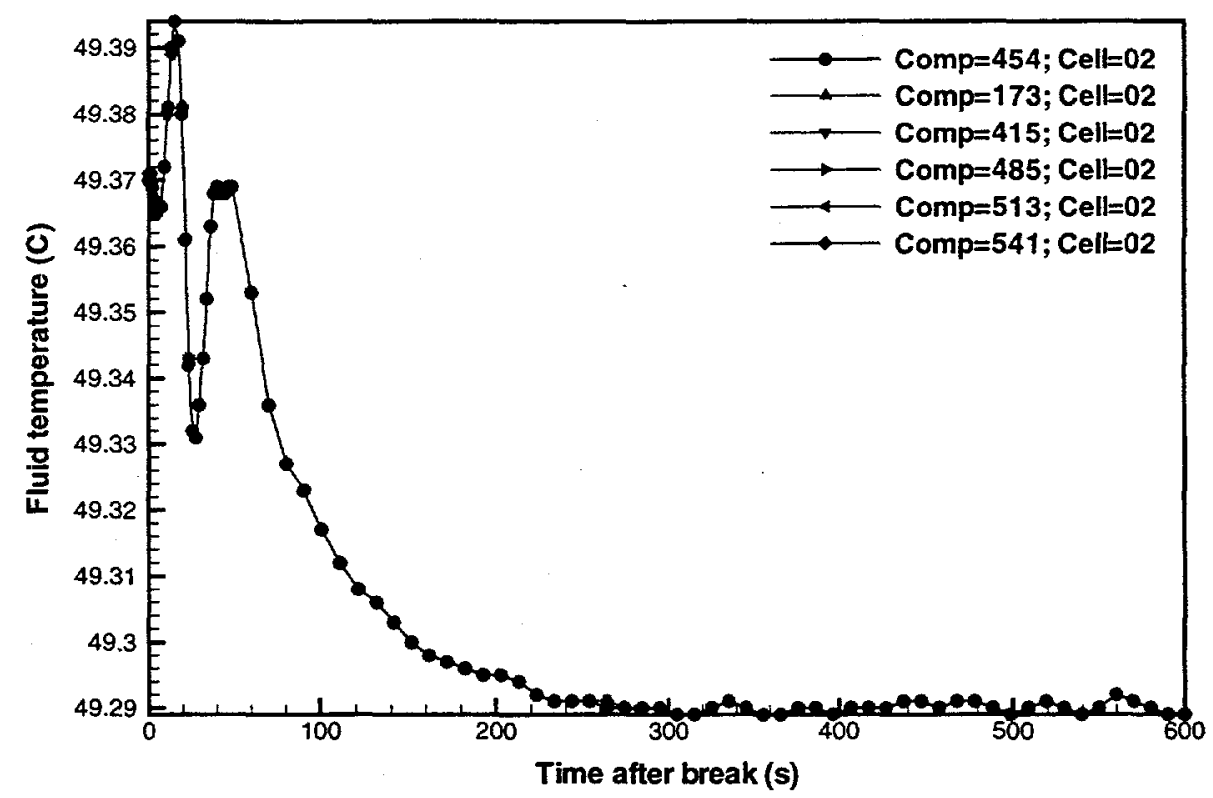

Figure B-24b Module inlet fluid temperatures for a LOHGA (Case 1: helium supply plenum break near decoupler inlet).

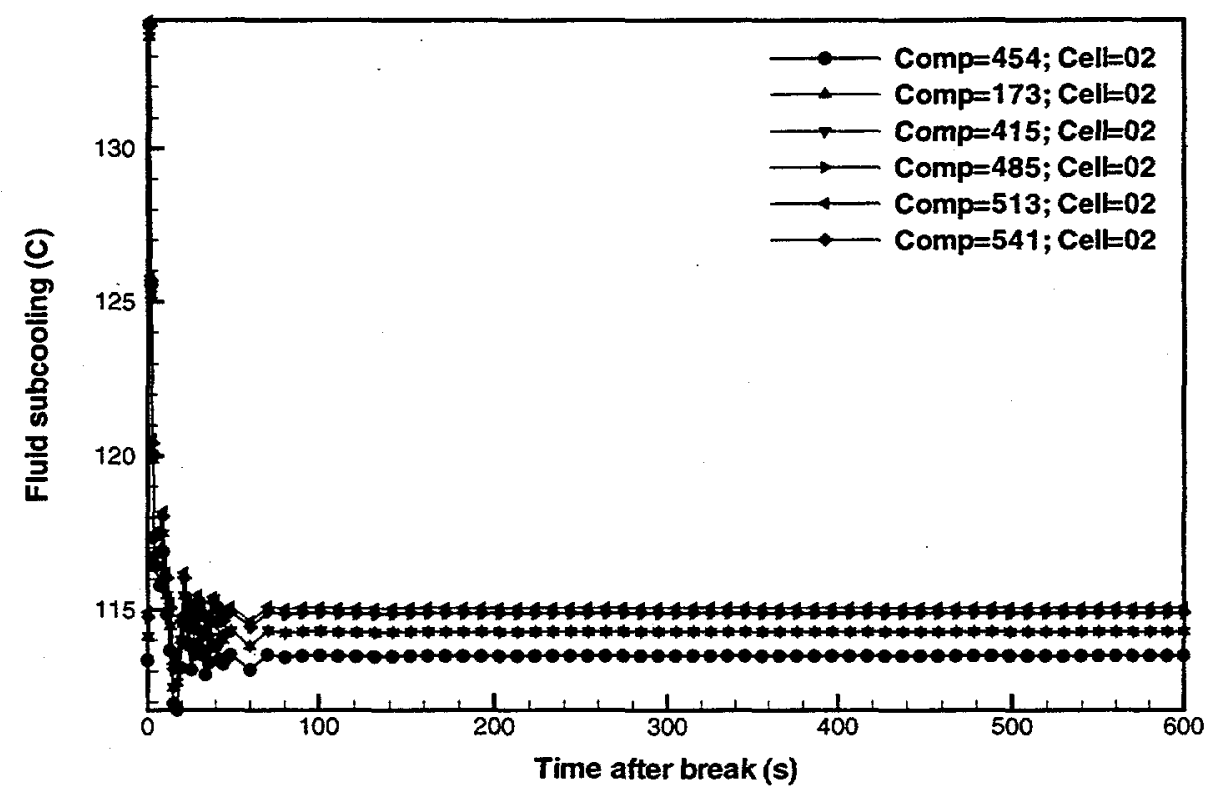

Figure B-24c Module inlet fluid subcoolings for a LOHGA (Case 1: helium supply plenum break near decoupler inlet). 


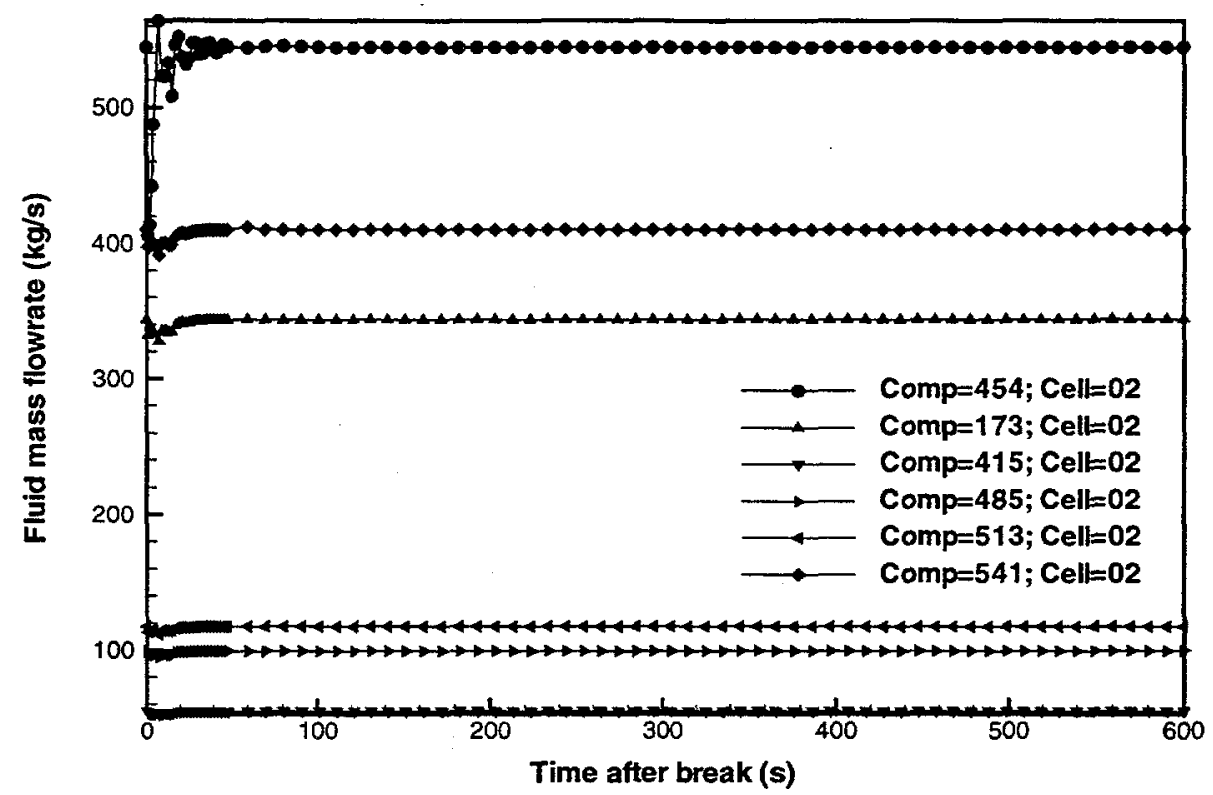

Figure B-24d Module inlet liquid mass flowrates for a LOHGA (Case-1: helium supply plenum break near decoupler inlet).

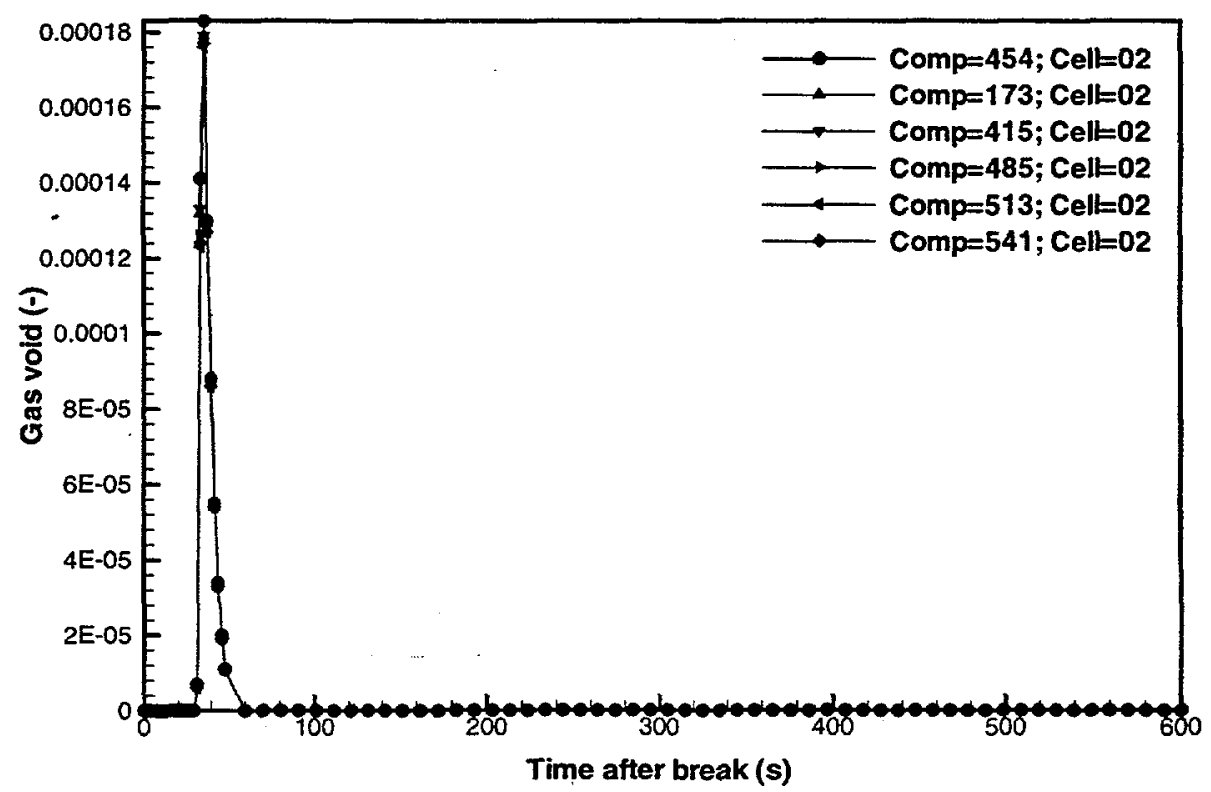

Figure B-24e Module inlet void fractions for a LOHGA (Case 1: helium supply plenum break near decoupler inlet). 


\section{Appendix C: LOHGA (Case 2) TRAC Results}

\section{Appendix C1 LOHGA (Case 2) TRAC Plenum Component Figures}

The following figures are from a TRAC simulation for Case 2 of a LOHGA (Helium supply plenum break near decoupler outlet):

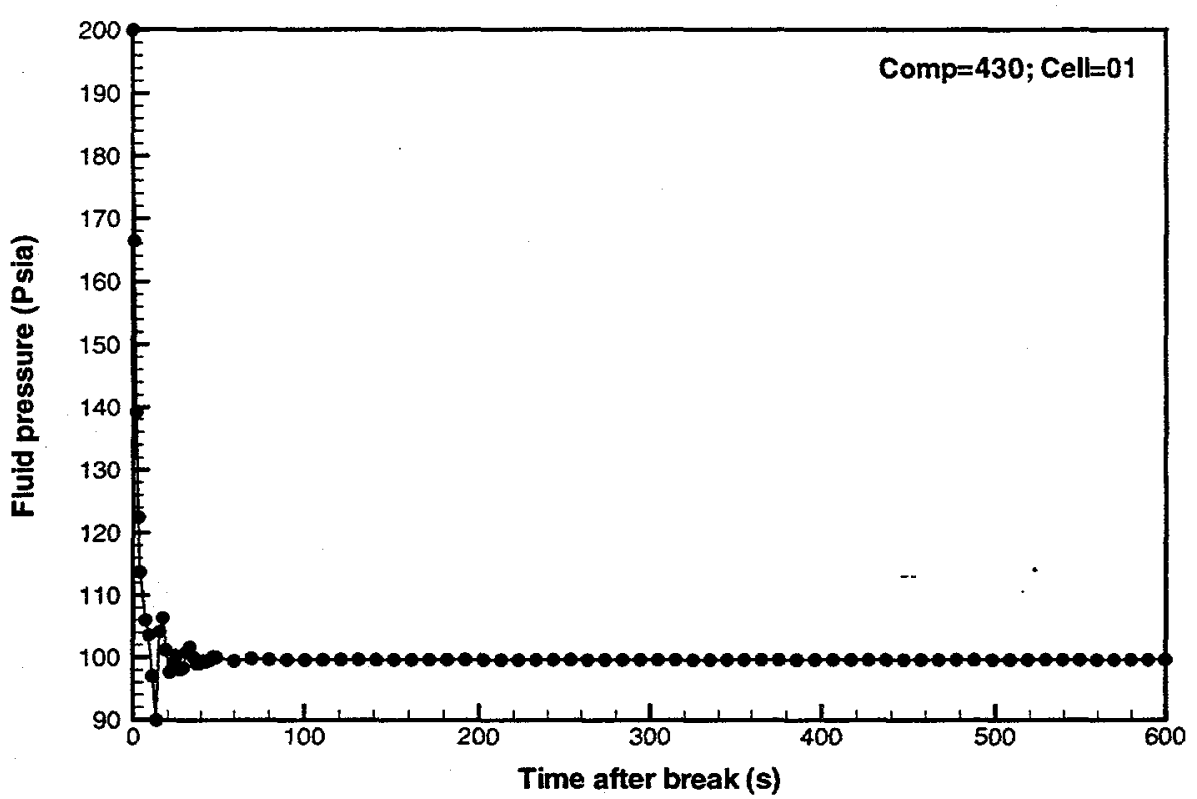

Figure C-1a Helium gas supply fluid pressures for a LOHGA (Case 2: helium supply plenum break near decoupler outlet). 


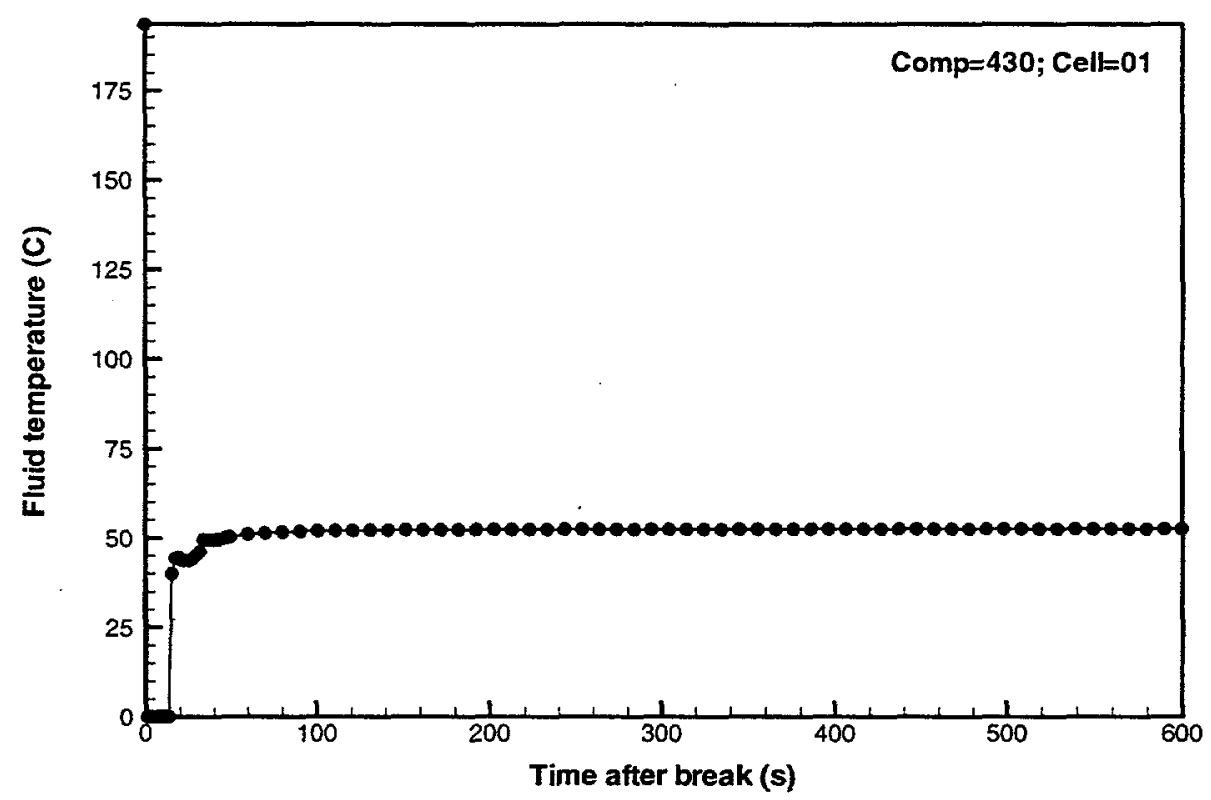

Figure C-1b Helium gas supply fluid temperatures for a LOHGA (Case 2: helium supply plenum break near decoupler outlet).

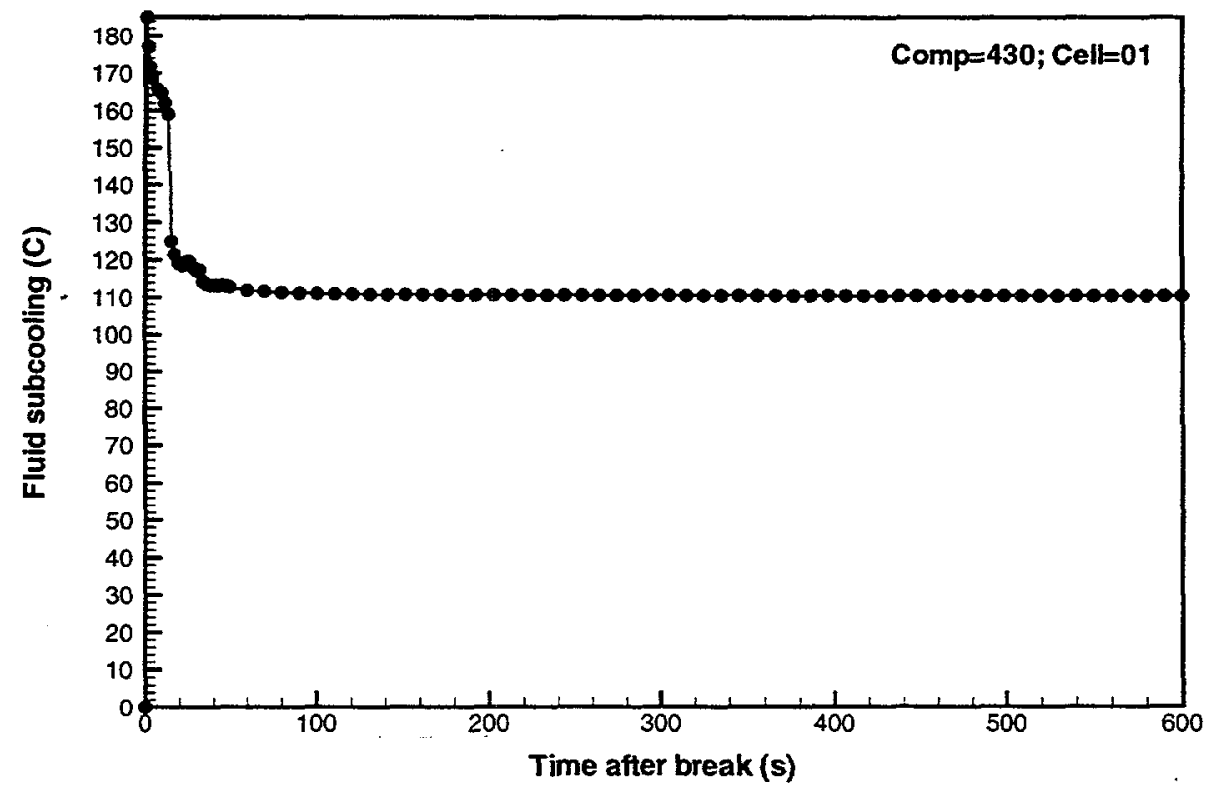

Figure C-1c Helium gas supply fluid subcoolings for a LOHGA (Case 2: helium supply plenum break near decoupler outlet). 


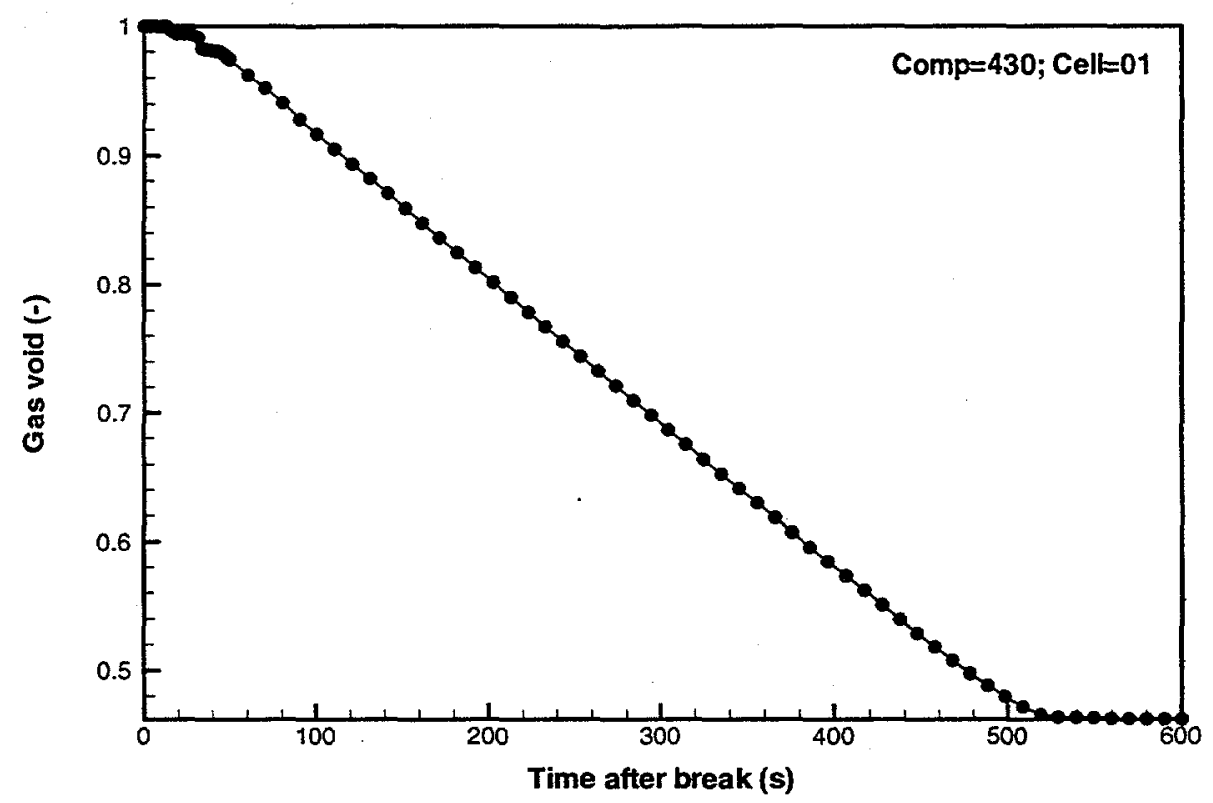

Figure C-1d Helium gas supply void fractions for a LOHGA (Case 2: helium supply plenum break near decoupler outlet).

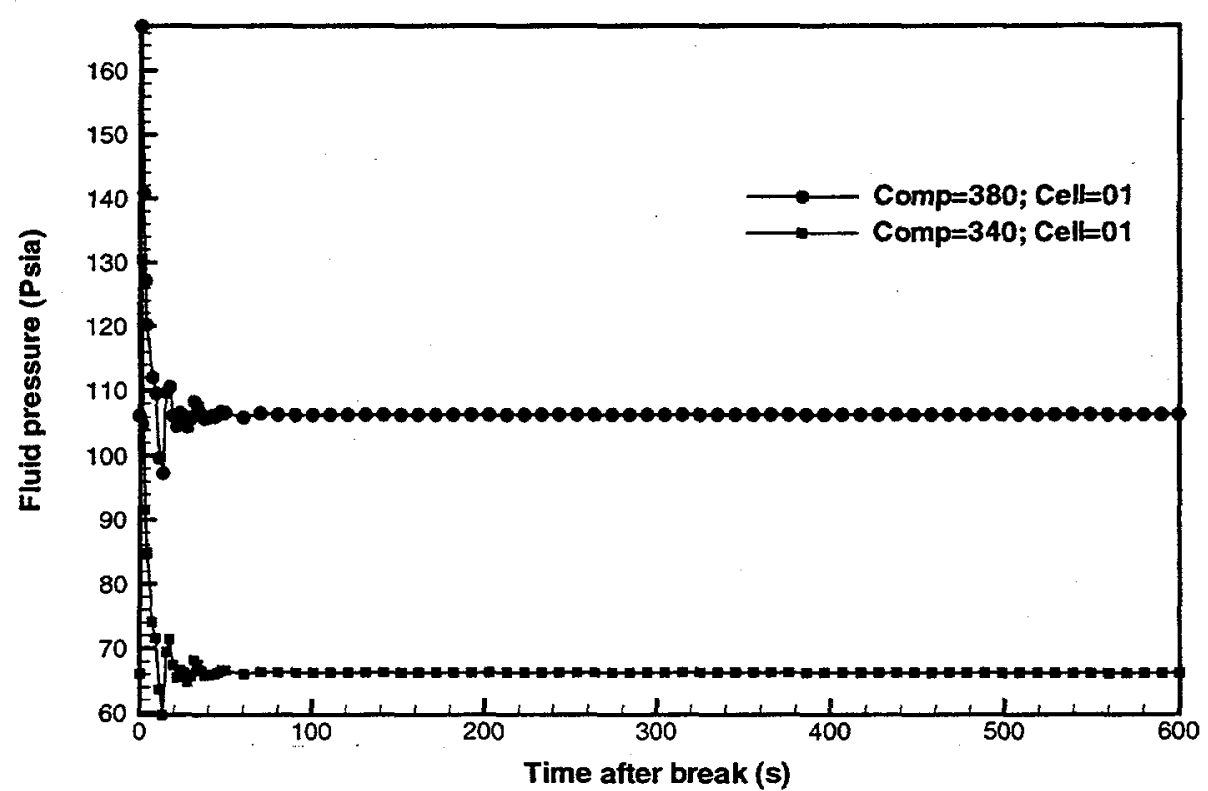

Figure C-2a Fixed header fluid pressures for a LOHGA (Case 2: helium supply plenum break near decoupler outlet). 


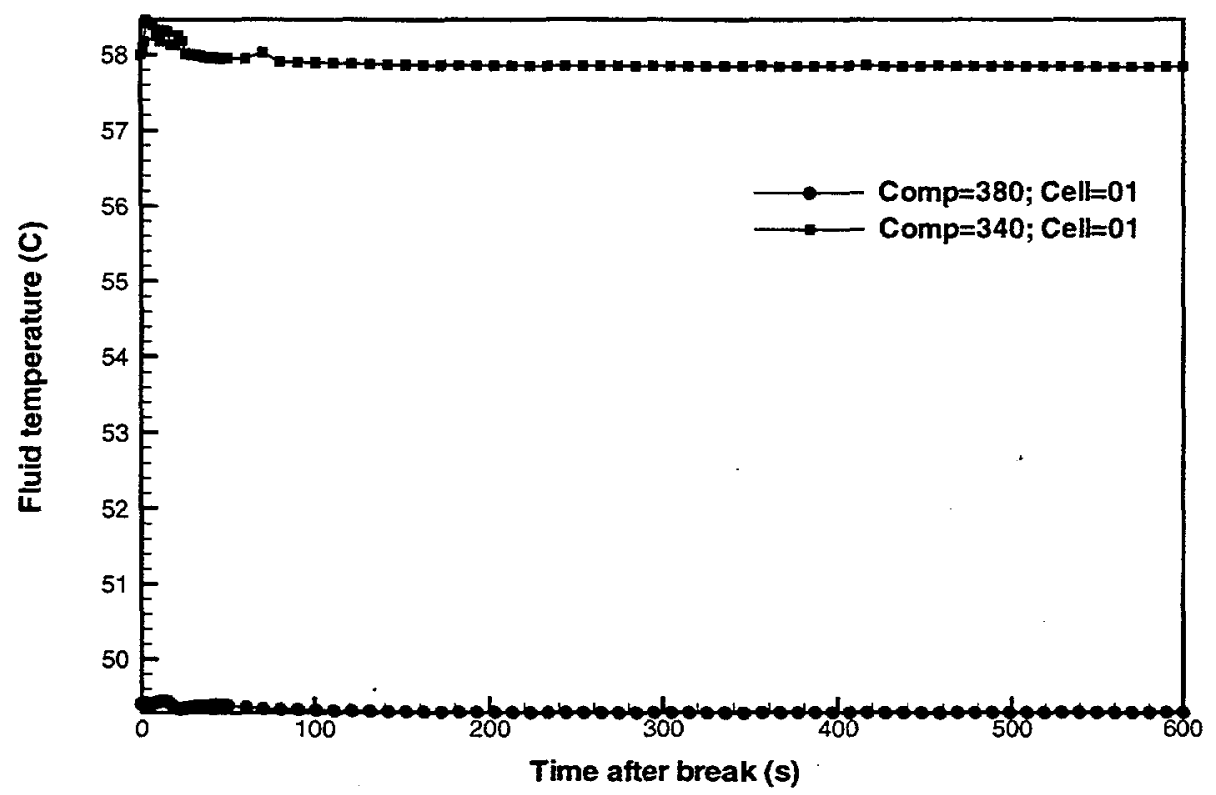

Figure C-2b Fixed header fluid temperatures for a LOHGA (Case 2: helium supply plenum break near decoupler outlet).

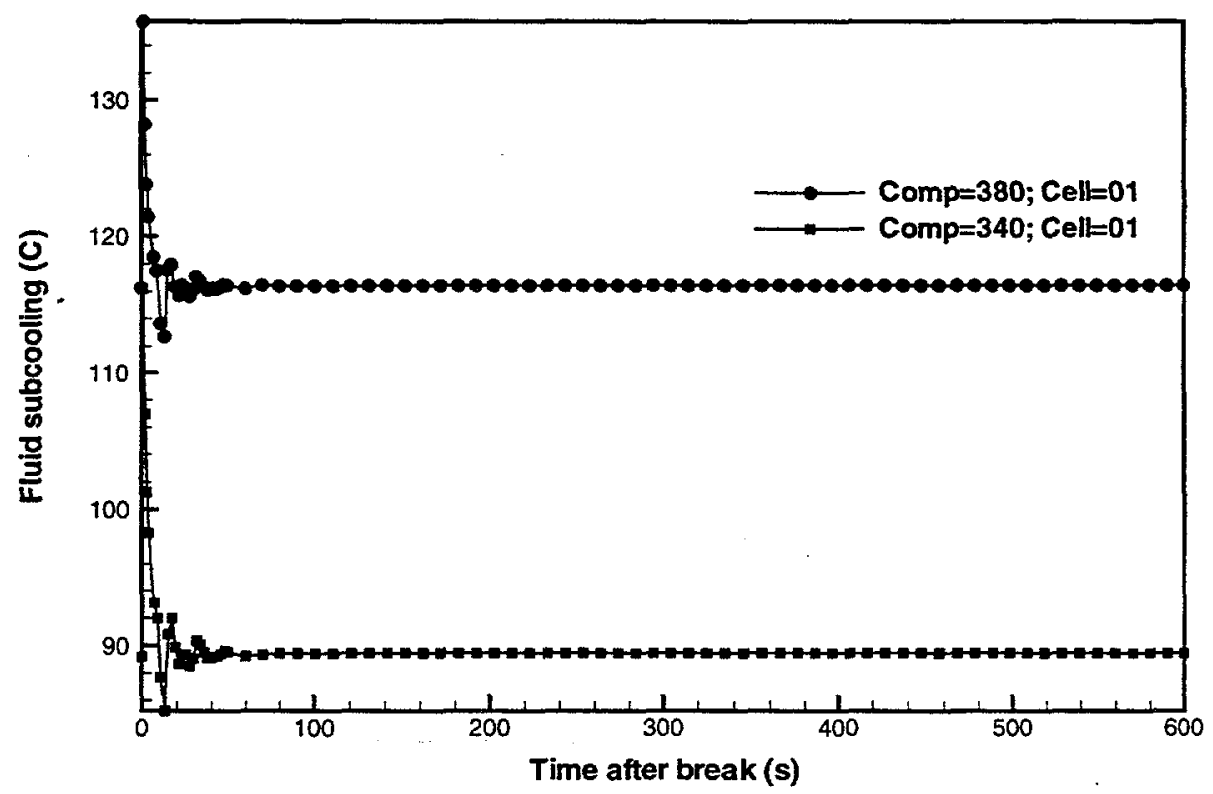

Figure C-2c Fixed header fluid subcoolings for a LOHGA (Case 2: helium supply plenum break near decoupler outlet). 


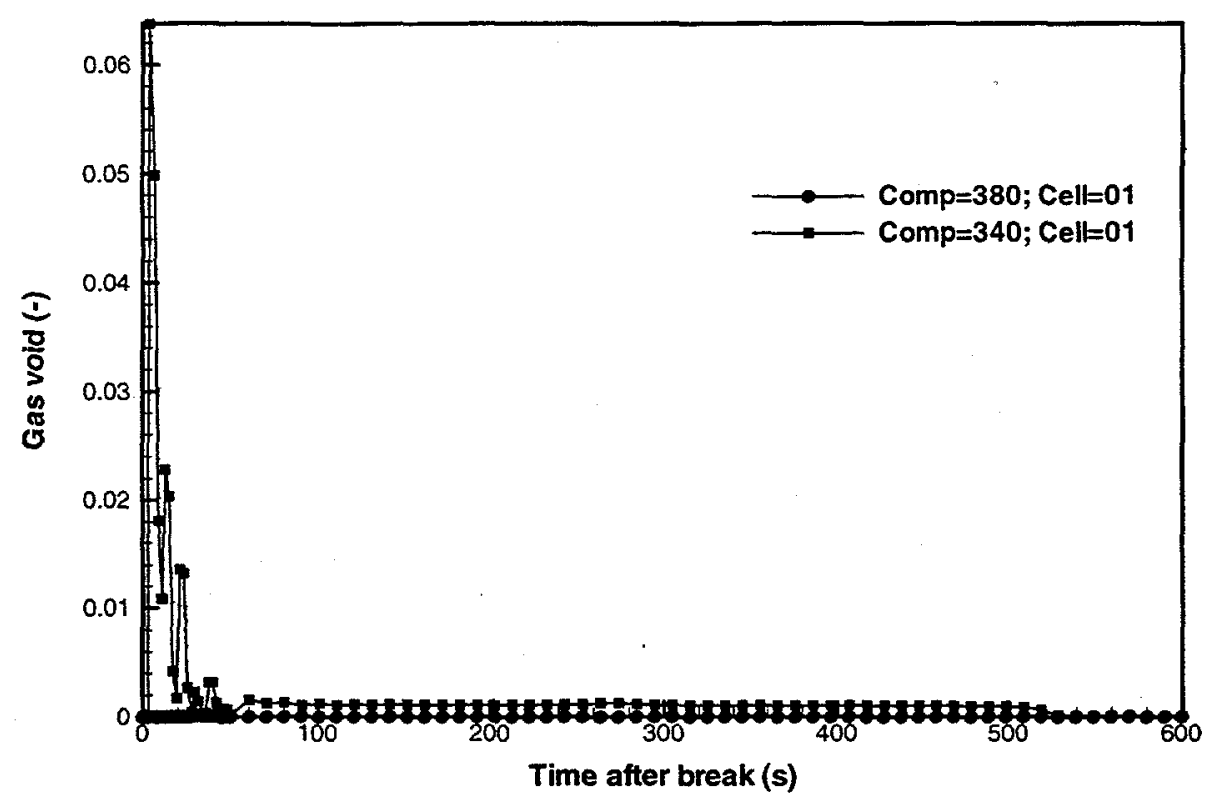

Figure C-2d Fixed header void fractions for a LOHGA (Case 2: helium supply plenum break near decoupler outlet).

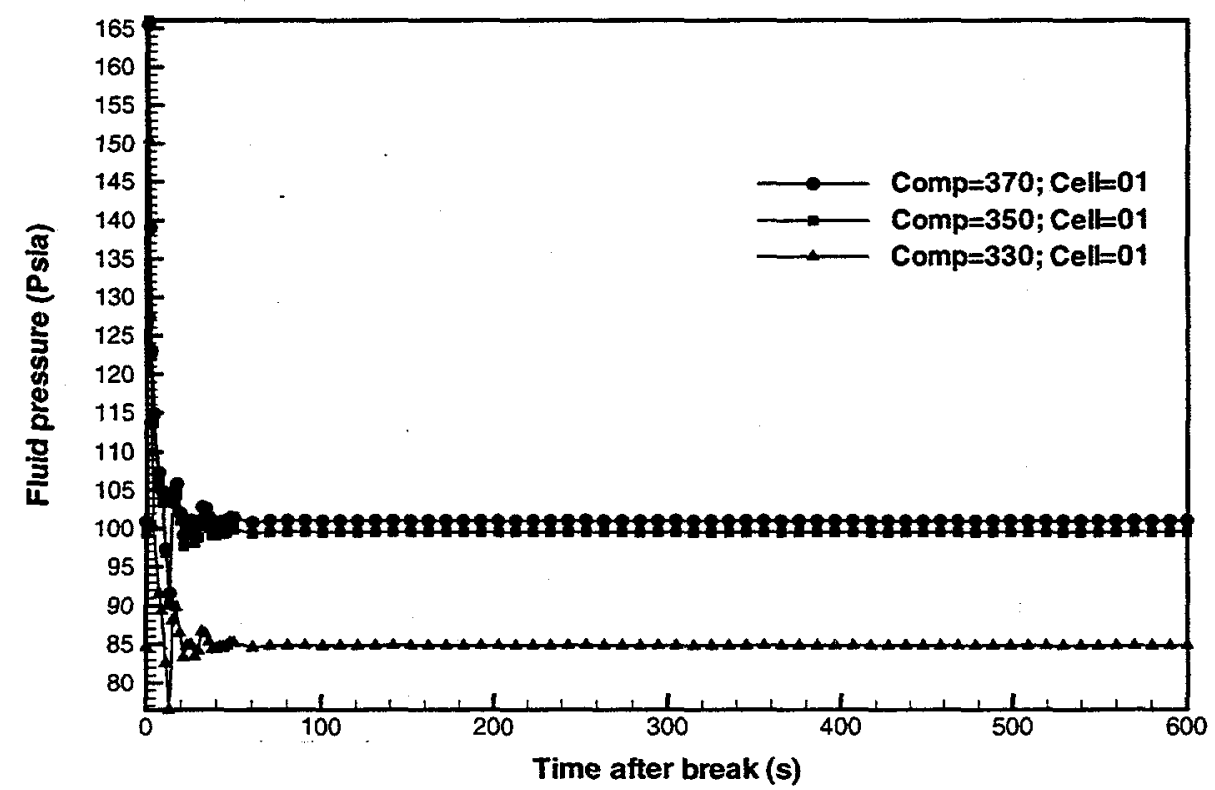

Figure C-3a Module 1 plenum fluid pressures for a LOHGA (Case 2: helium supply plenum break near decoupler outlet). 


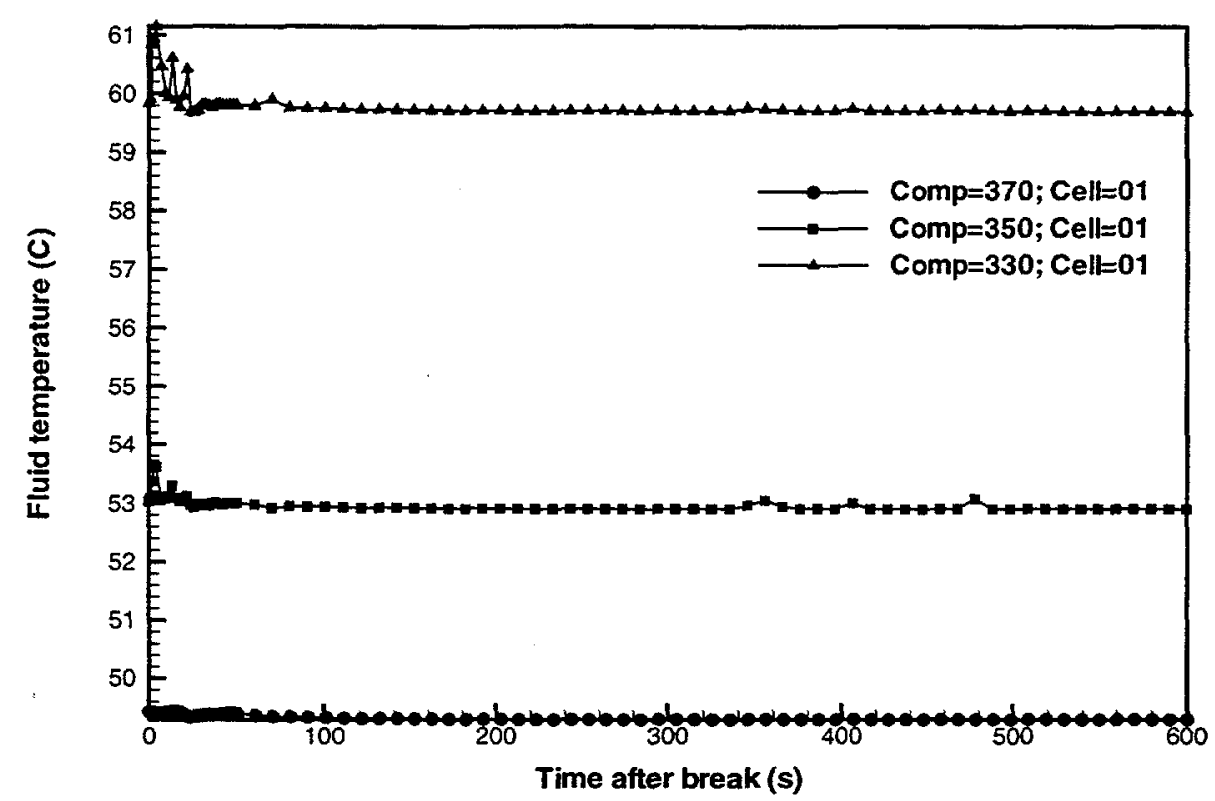

Figure C-3b Module 1 plenum fluid temperatures for a LOHGA (Case 2 : helium supply plenum break near decoupler outlet).

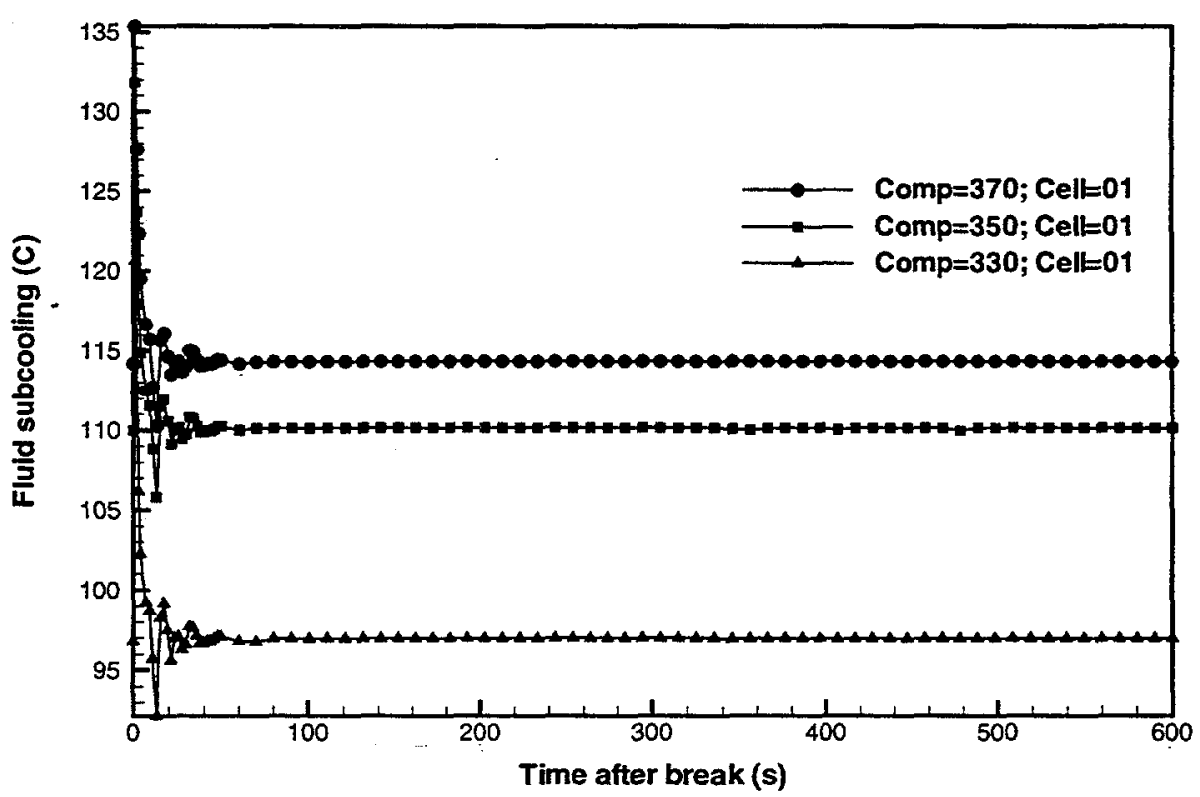

Figure C-3c Module 1 plenum fluid subcoolings for a LOHGA (Case 2: helium supply plenum break near decoupler outlet). 


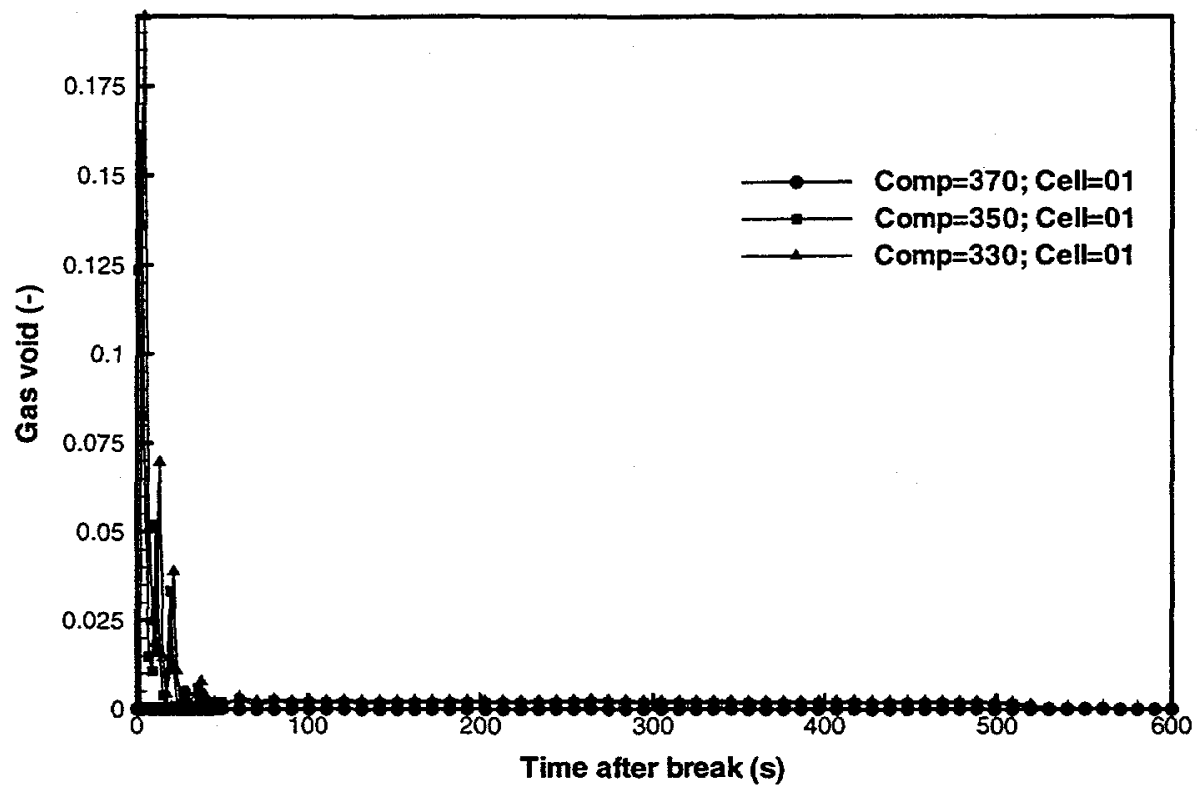

Figure C-3d Module 1 plenum void fractions for a LOHGA (Case 2: helium supply plenum break near decoupler outlet).

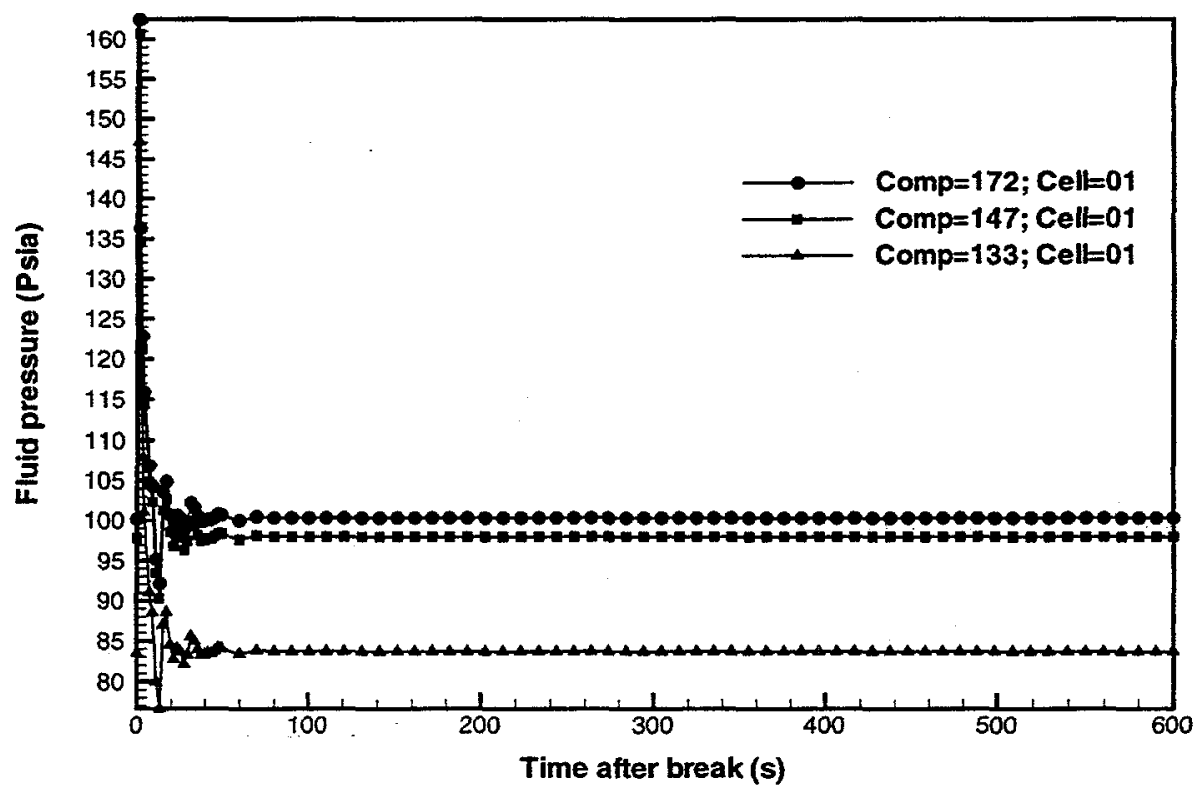

Figure C-4a Module 2 plenum fluid pressures for a LOHGA (Case 2: helium supply plenum break near decoupler outlet). 


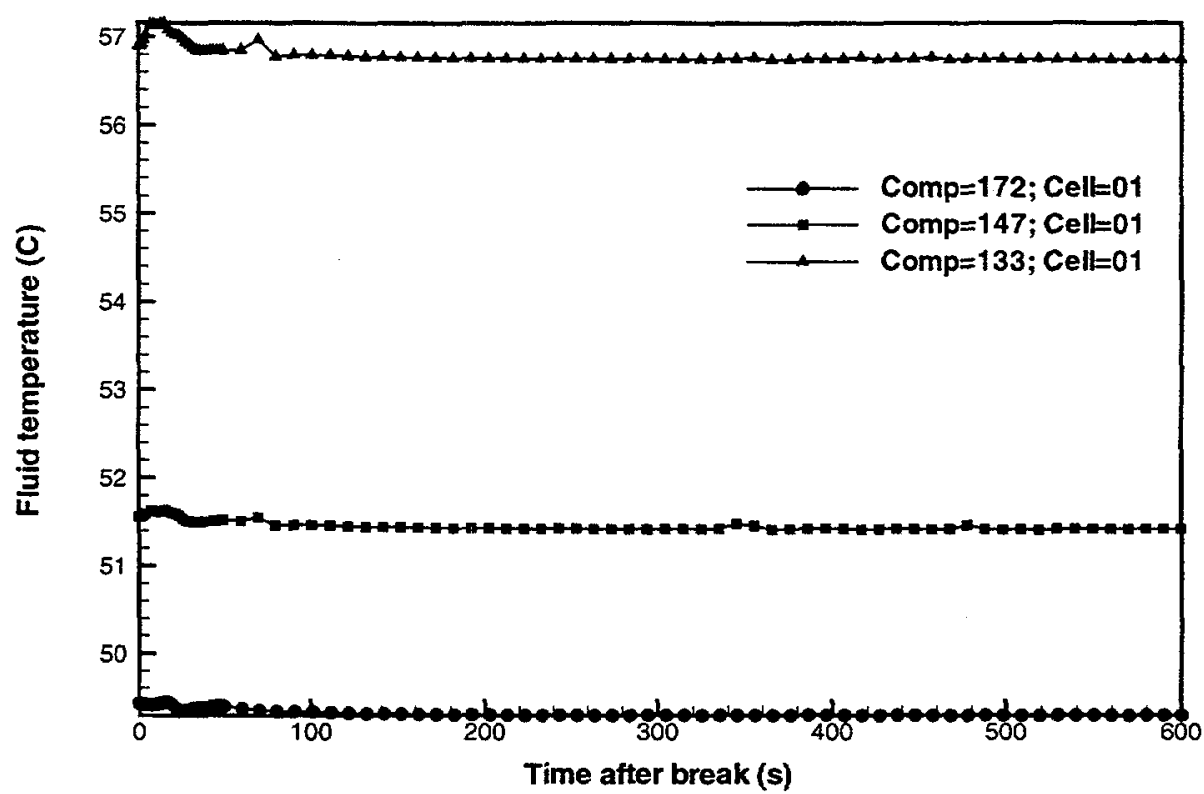

Figure C-4b Module 2 plenum fluid temperatures for a LOHGA (Case-2: helium supply plenum break near decoupler outlet).

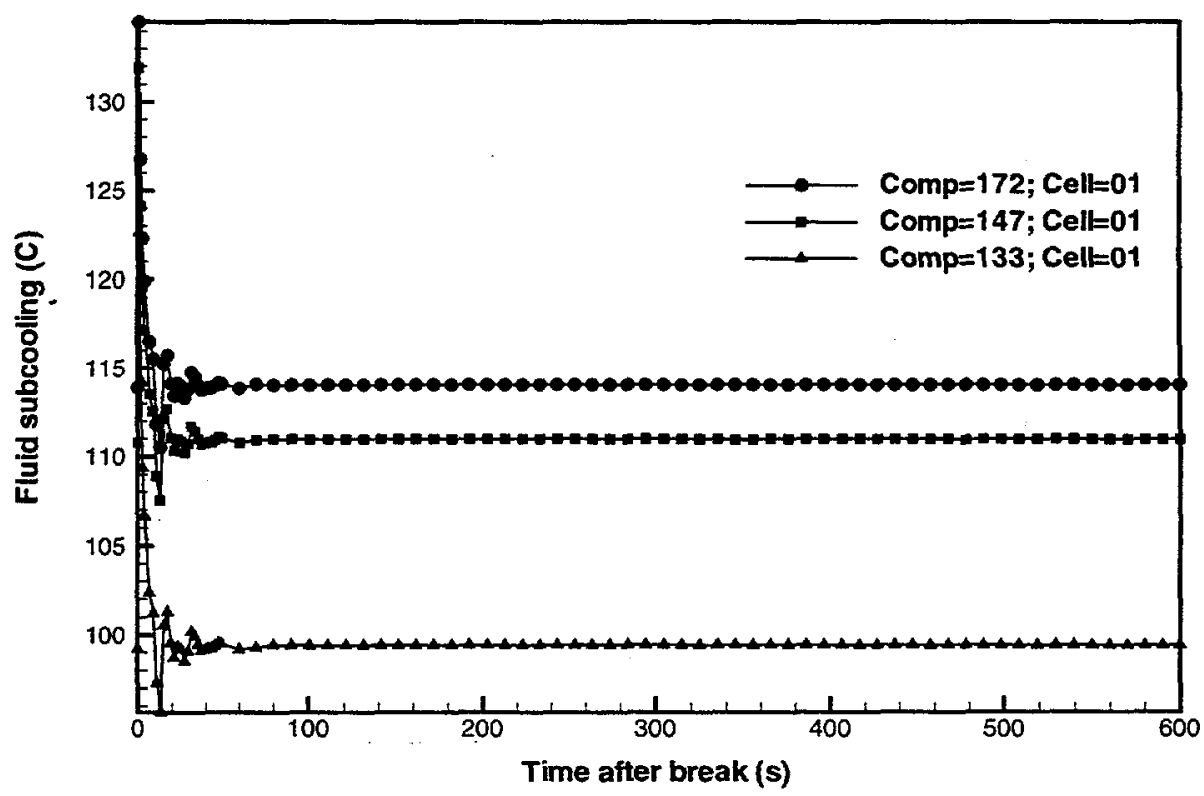

Figure C-4c Module 2 plenum fluid subcoolings for a LOHGA (Case 2: helium supply plenum break near decoupler outlet). 


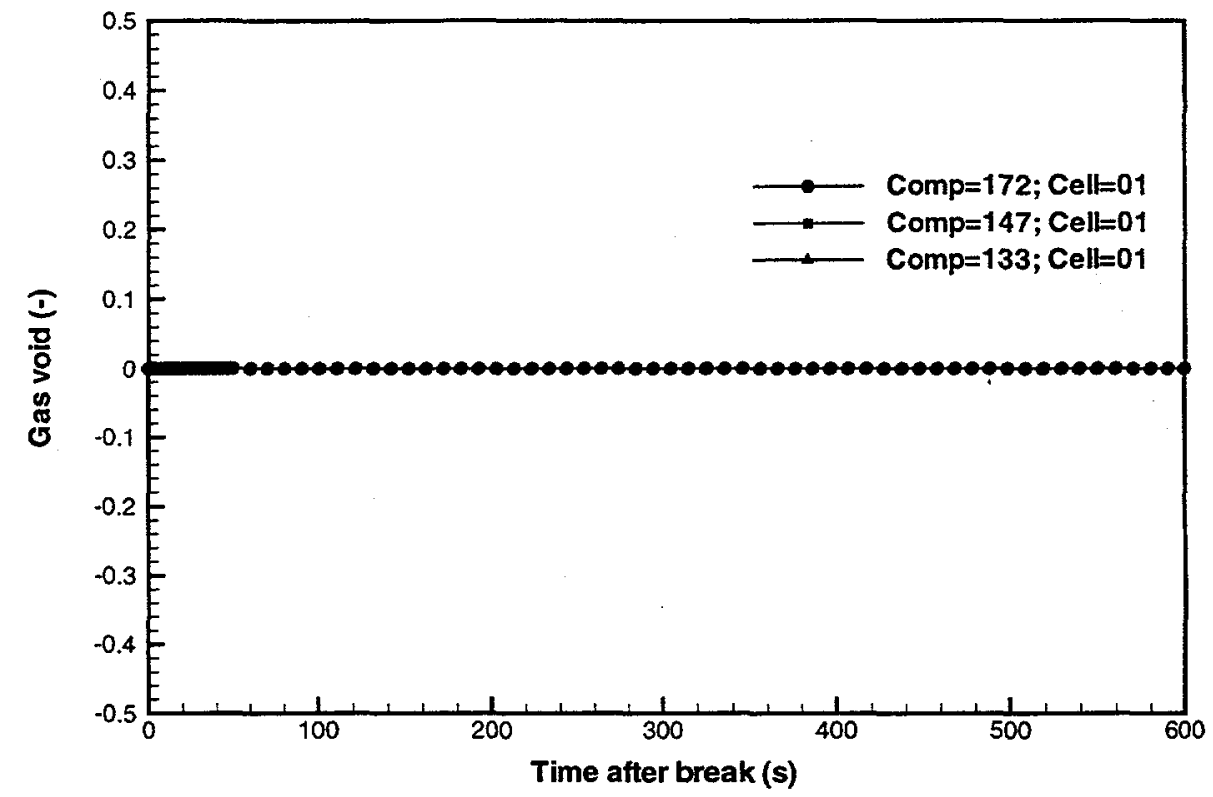

Figure C-4d Module 2 plenum void fractions for a LOHGA (Case 2: helium supply plenum break near decoupler outlet).

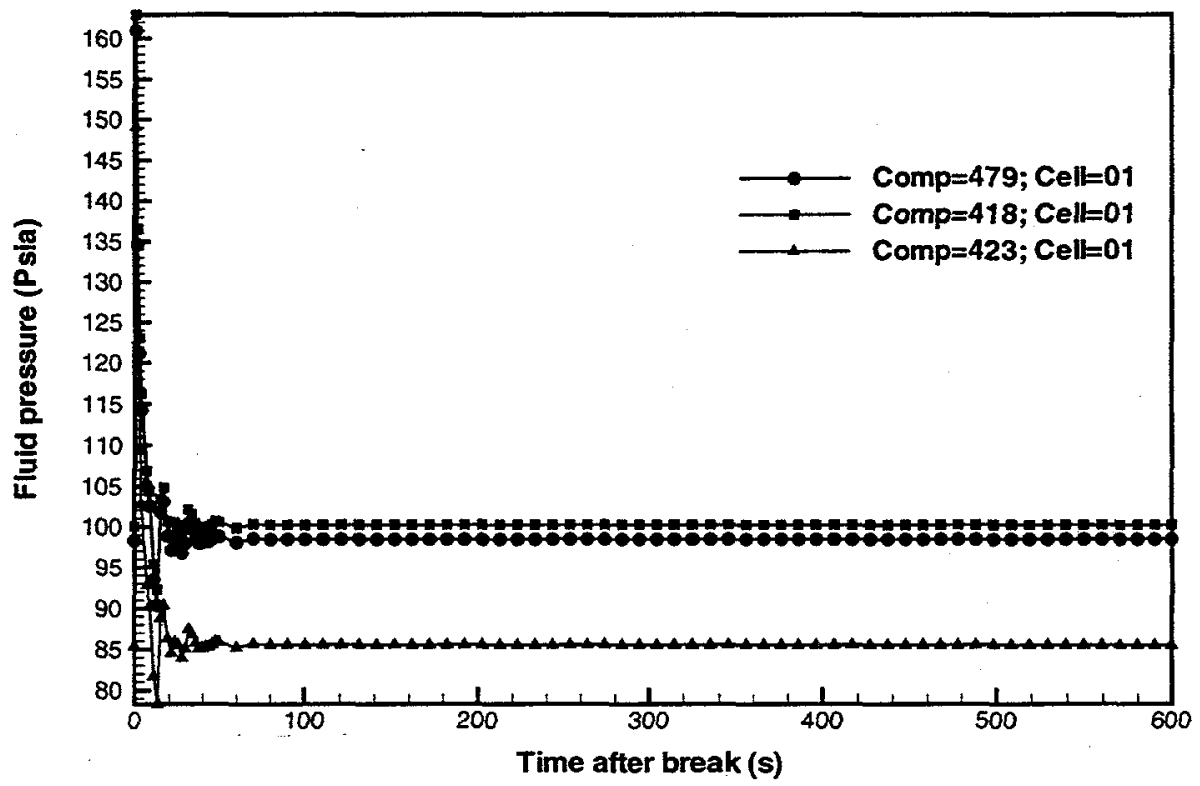

Figure C-5a Module 3 plenum fluid pressures for a LOHGA (Case 2: helium supply plenum break near decoupler outlet). 


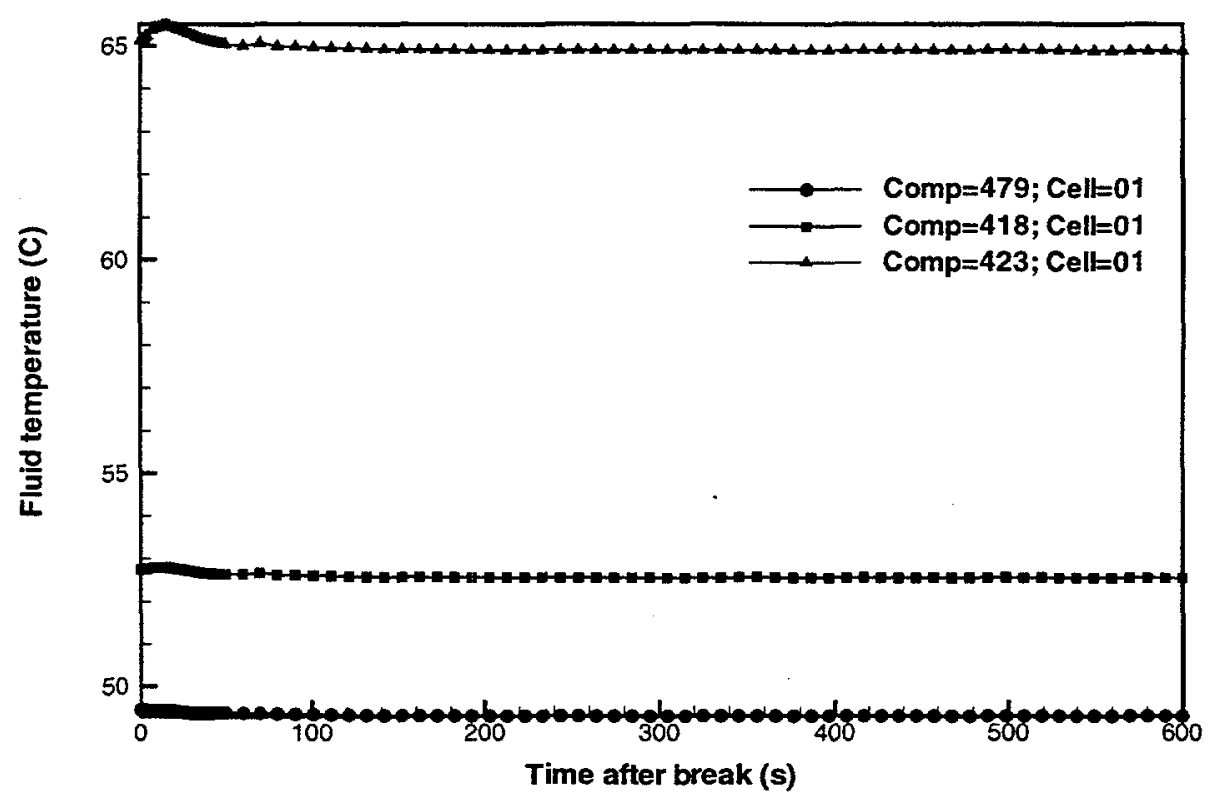

Figure C-5b Module 3 plenum fluid temperatures for a LOHGA (Case 2: helium supply plenum break near decoupler outlet).

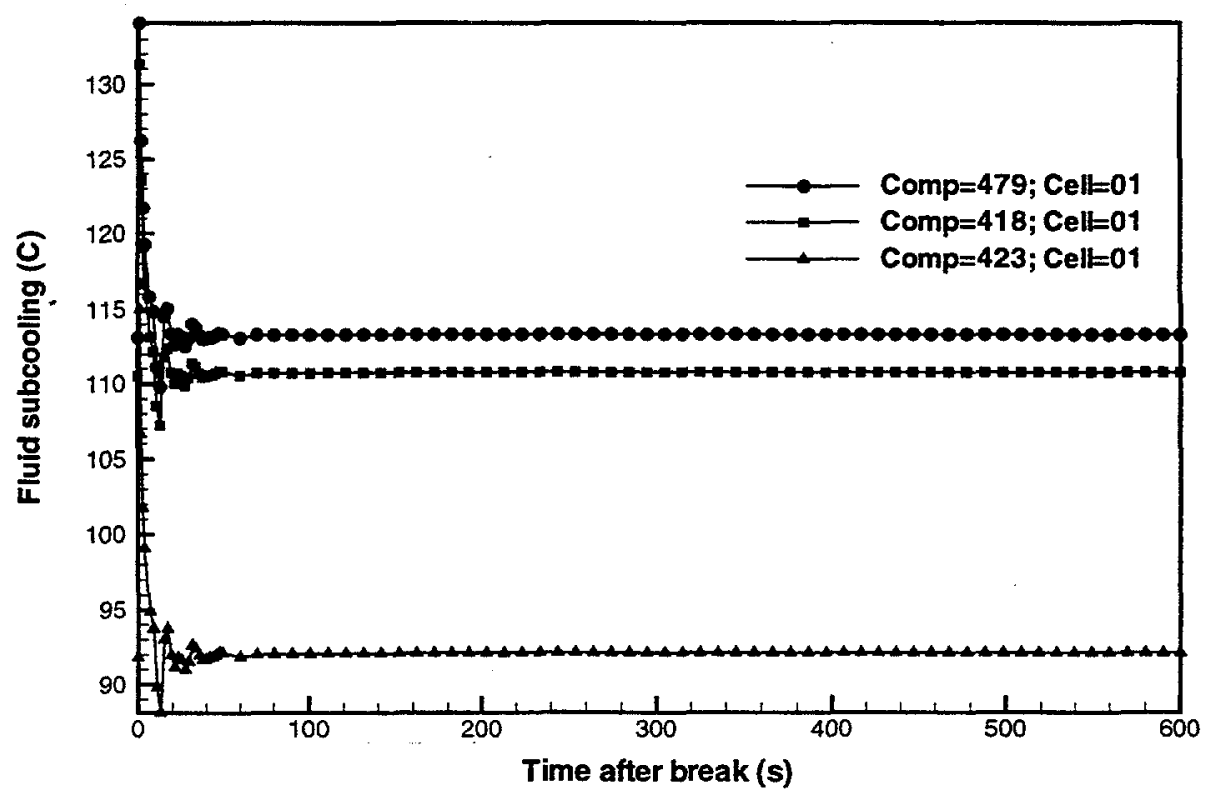

Figure C-5c Module 3 plenum fluid subcoolings for a LOHGA (Case 2: helium supply plenum break near decoupler outlet). 


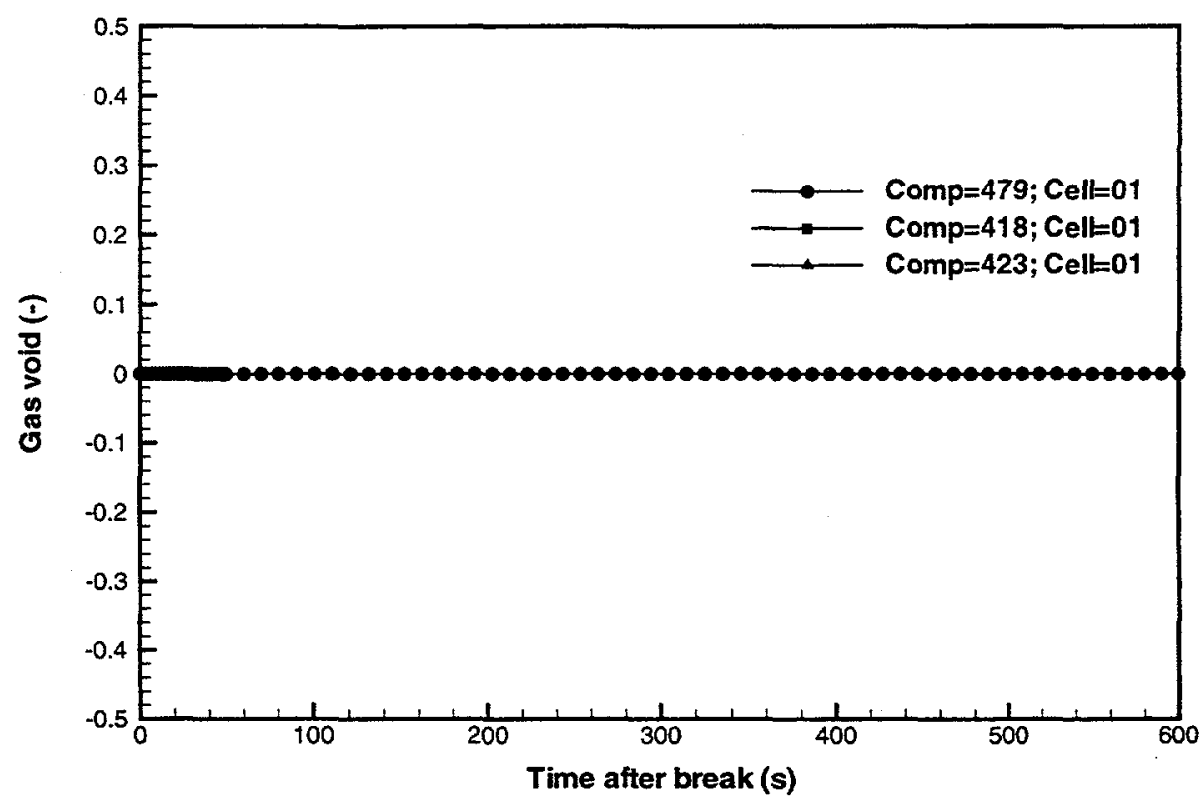

Figure C-5d Module 3 plenum void fractions for a LOHGA (Case 2: helium supply plenum break near decoupler outlet).



Figure C-6a Module 4 plenum fluid pressures for a LOHGA (Case 2: helium supply plenum break near decoupler outlet). 


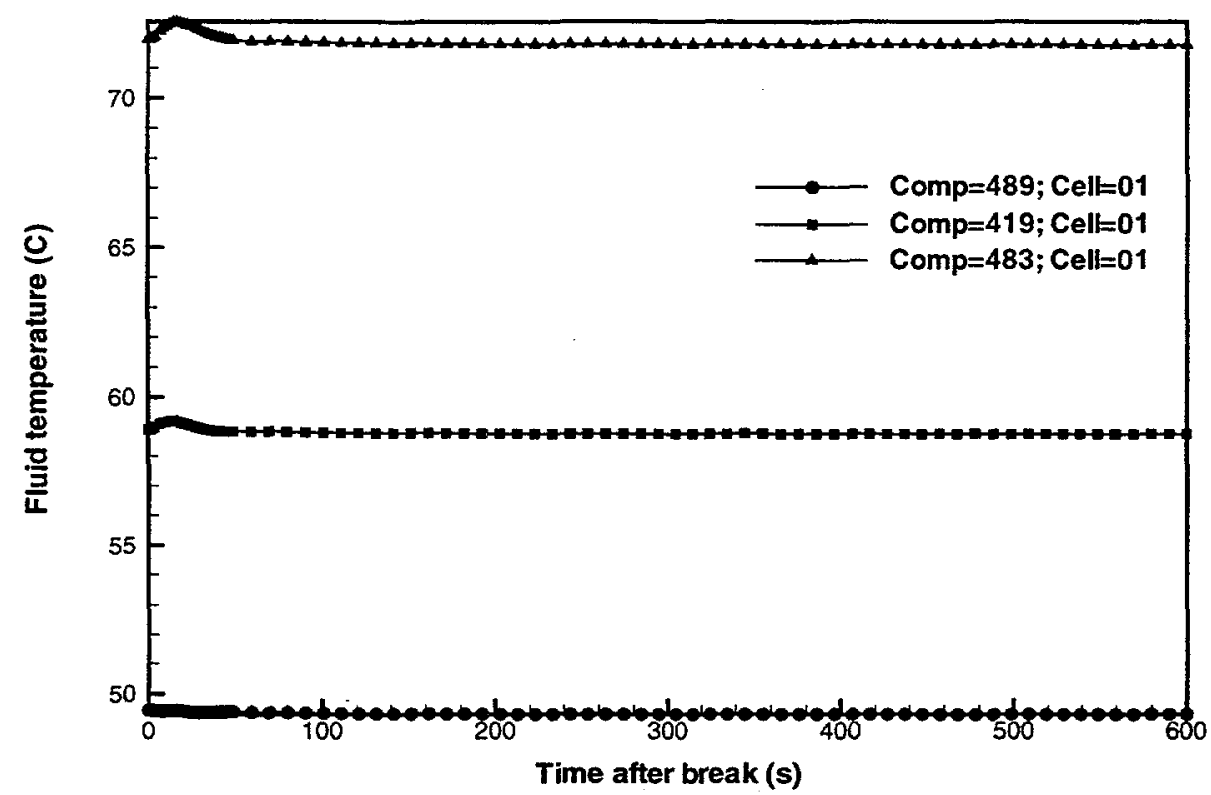

Figure C-6b Module 4 plenum fluid temperatures for a LOHGA (Case 2: helium supply plenum break near decoupler outlet).

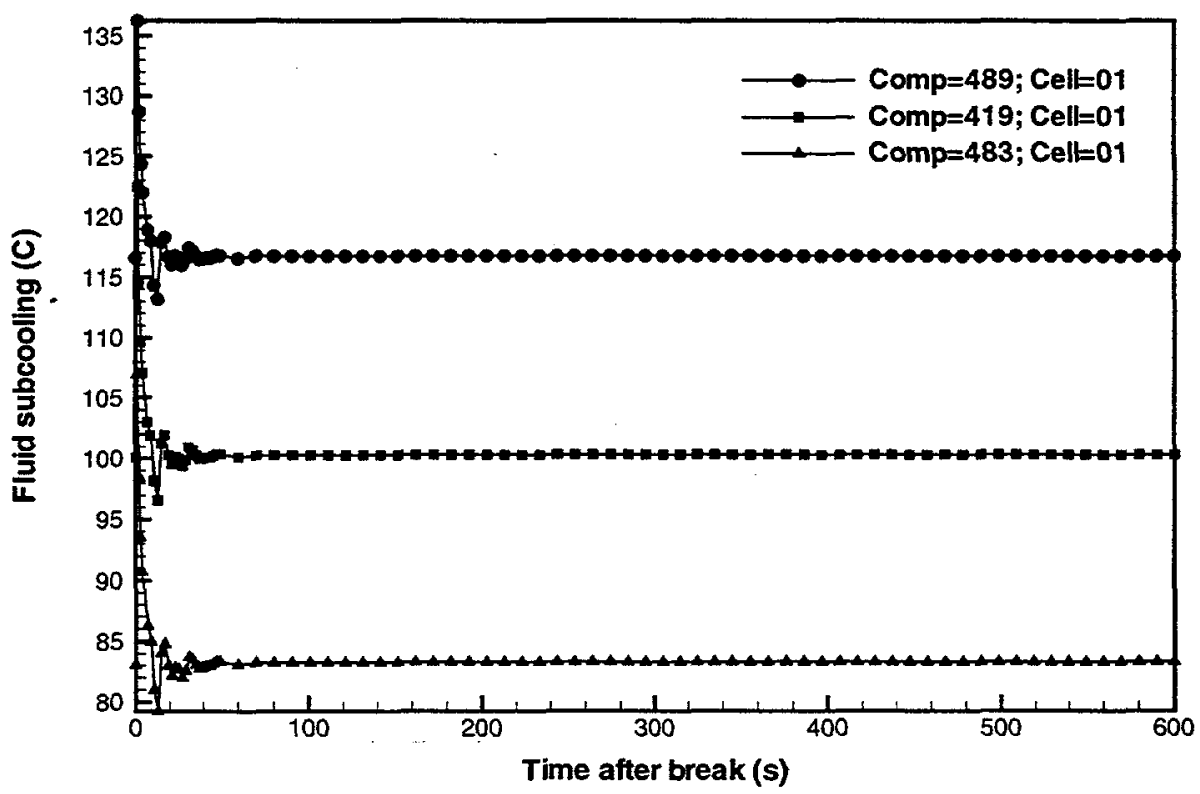

Figure C-6c Module 4 plenum fluid subcoolings for a LOHGA (Case 2: helium supply plenum break near decoupler outlet). 


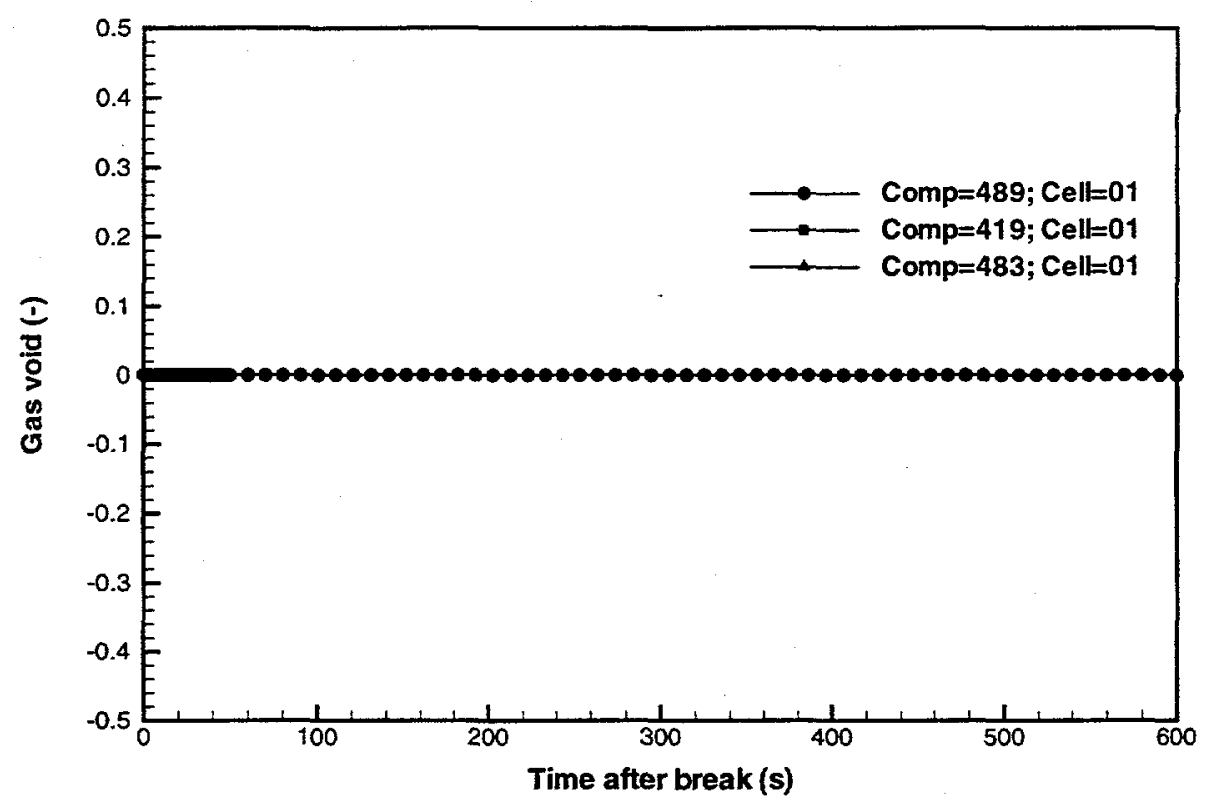

Figure C-6d Module 4 plenum void fractions for a LOHGA (Case 2: helium supply plenum break near decoupler outlet).

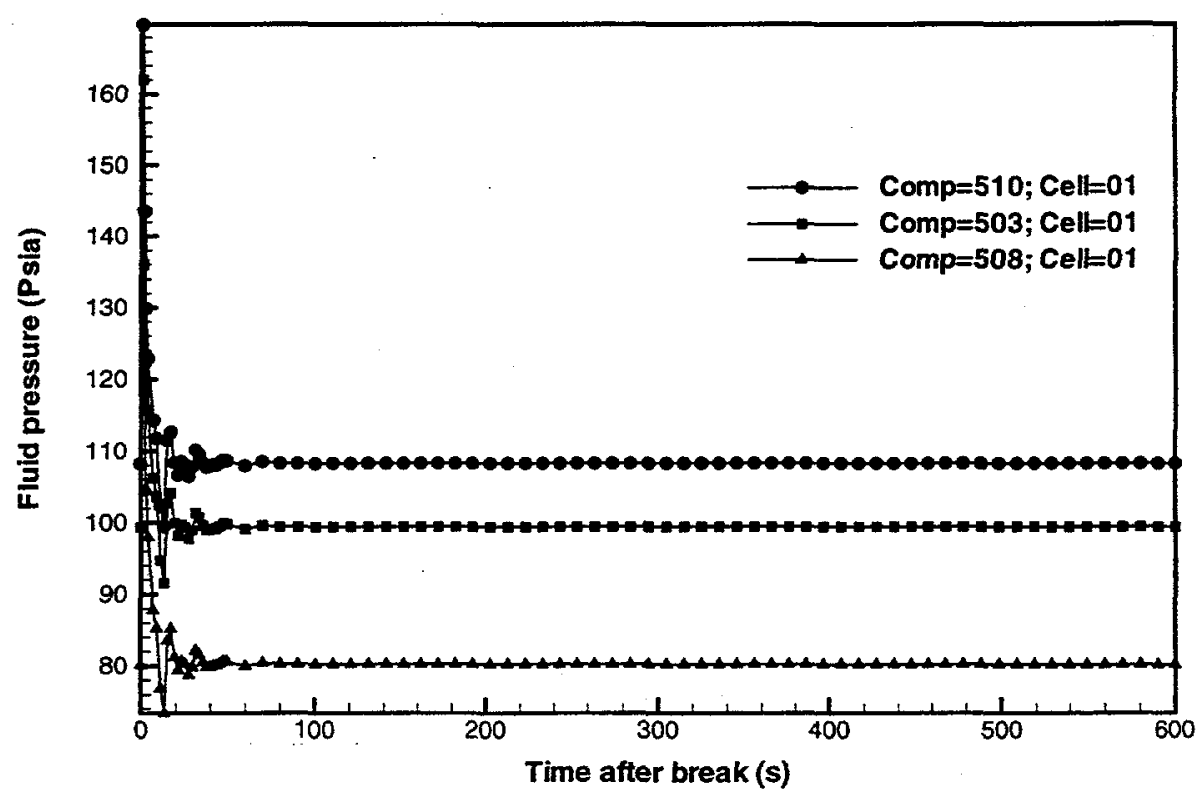

Figure C-7a Module 5 plenum fluid pressures for a LOHGA (Case 2: helium supply plenum break near decoupler outlet). 


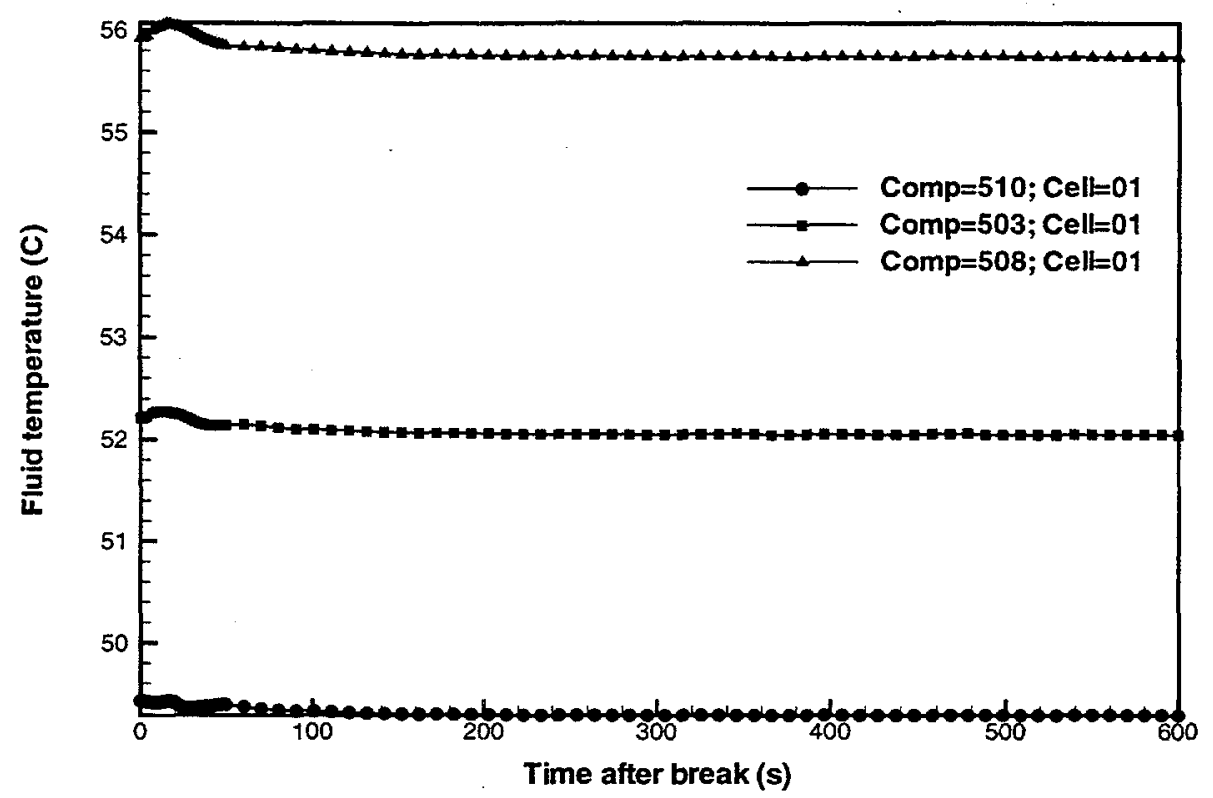

Figure C-7b Module 5 plenum fluid temperatures for a LOHGA (Case 2: helium supply plenum break near decoupler outlet).

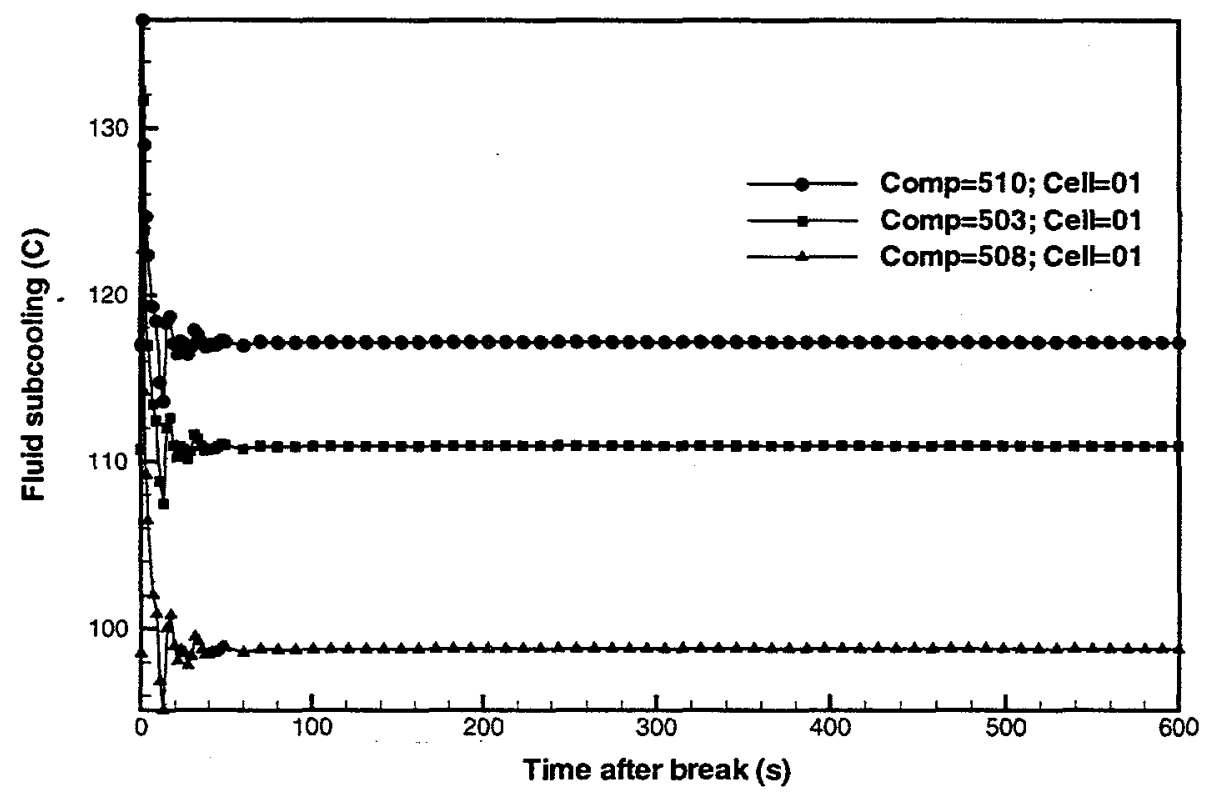

Figure C-7c Module 5 plenum fluid subcoolings for a LOHGA (Case 2: helium supply plenum break near decoupler outlet). 


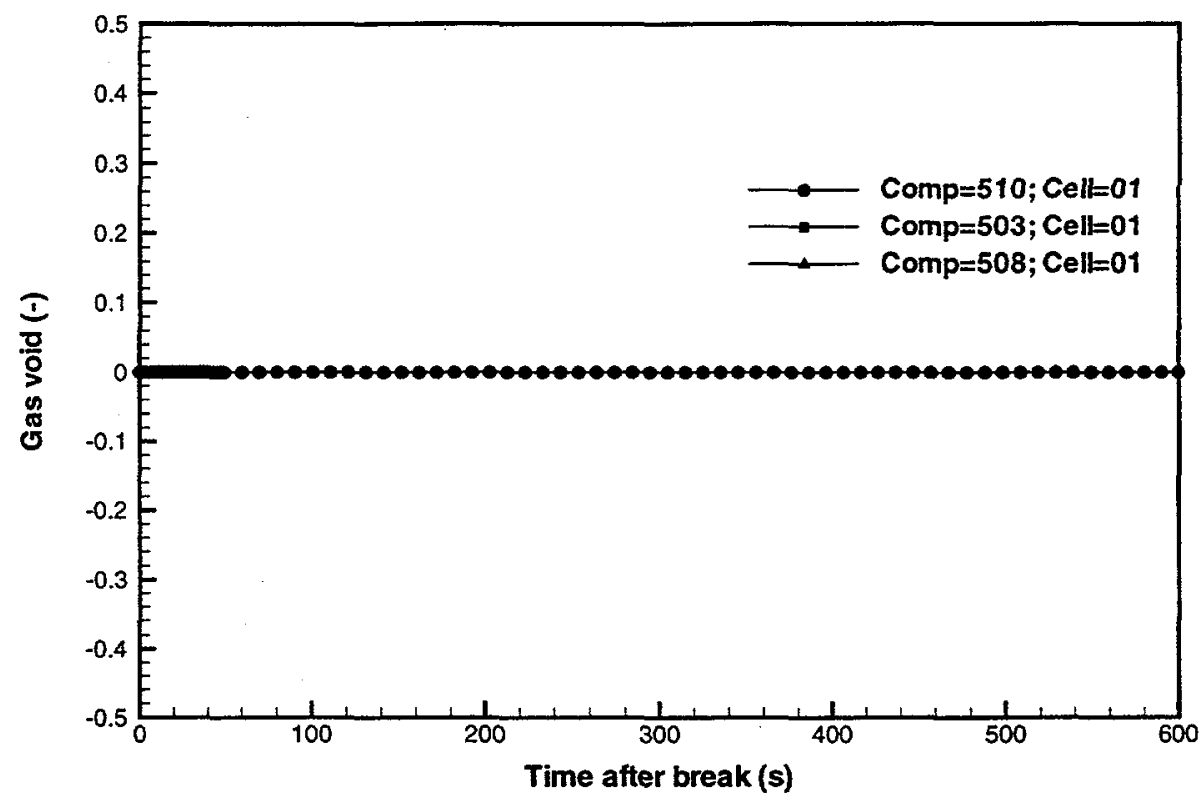

Figure C-7d Module 5 plenum void fractions for a LOHGA (Case 2: helium supply plenum break near decoupler outlet).

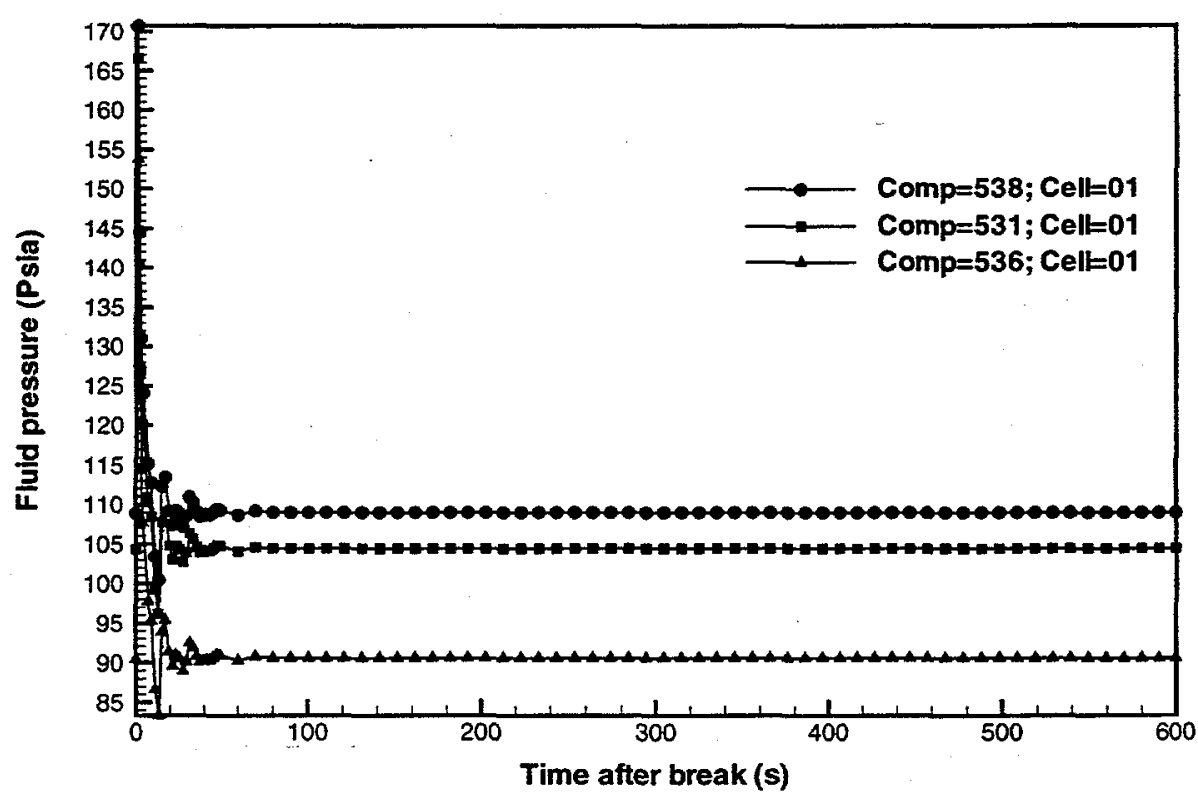

Figure C-8a Module 6 plenum fluid pressures for a LOHGA (Case 2: helium supply plenum break near decoupler outlet). 


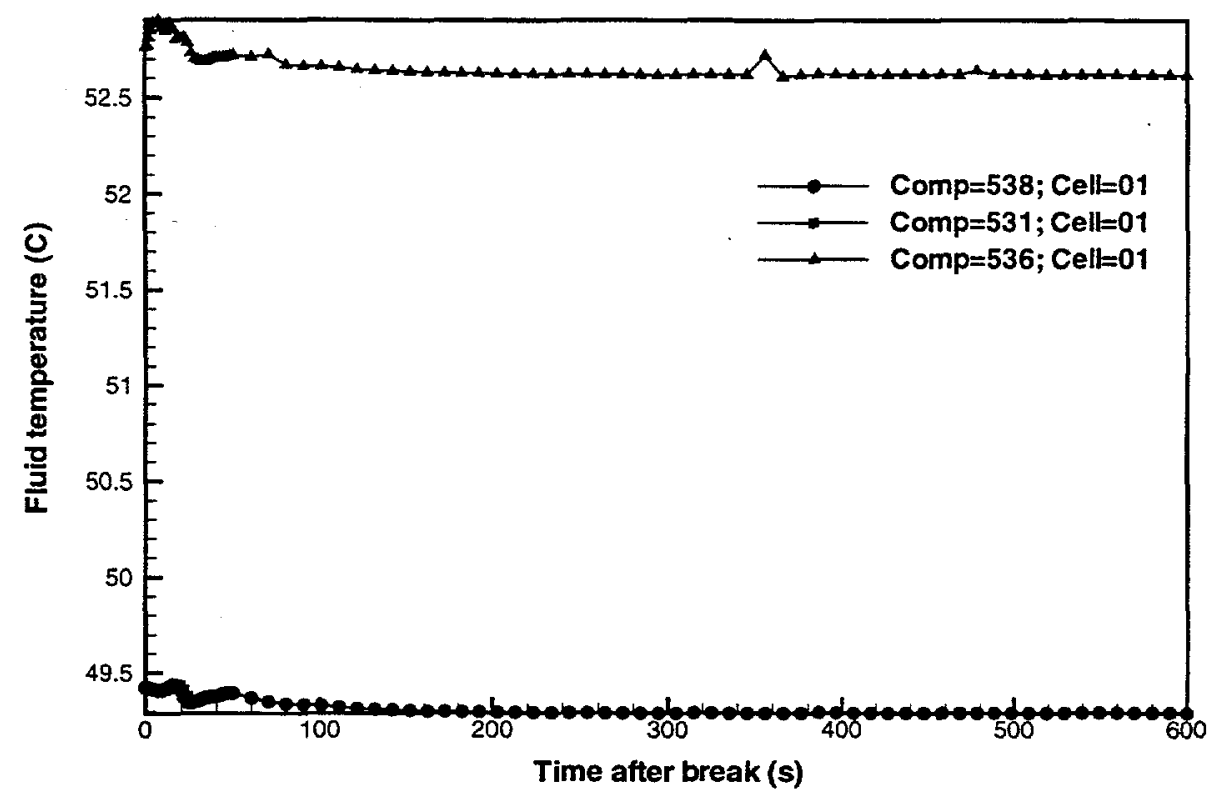

Figure $\mathrm{C}-8 \mathrm{~b}$ Module 6 plenum fluid temperatures for a LOHGA (Case-2: helium supply plenum break near decoupler outlet).

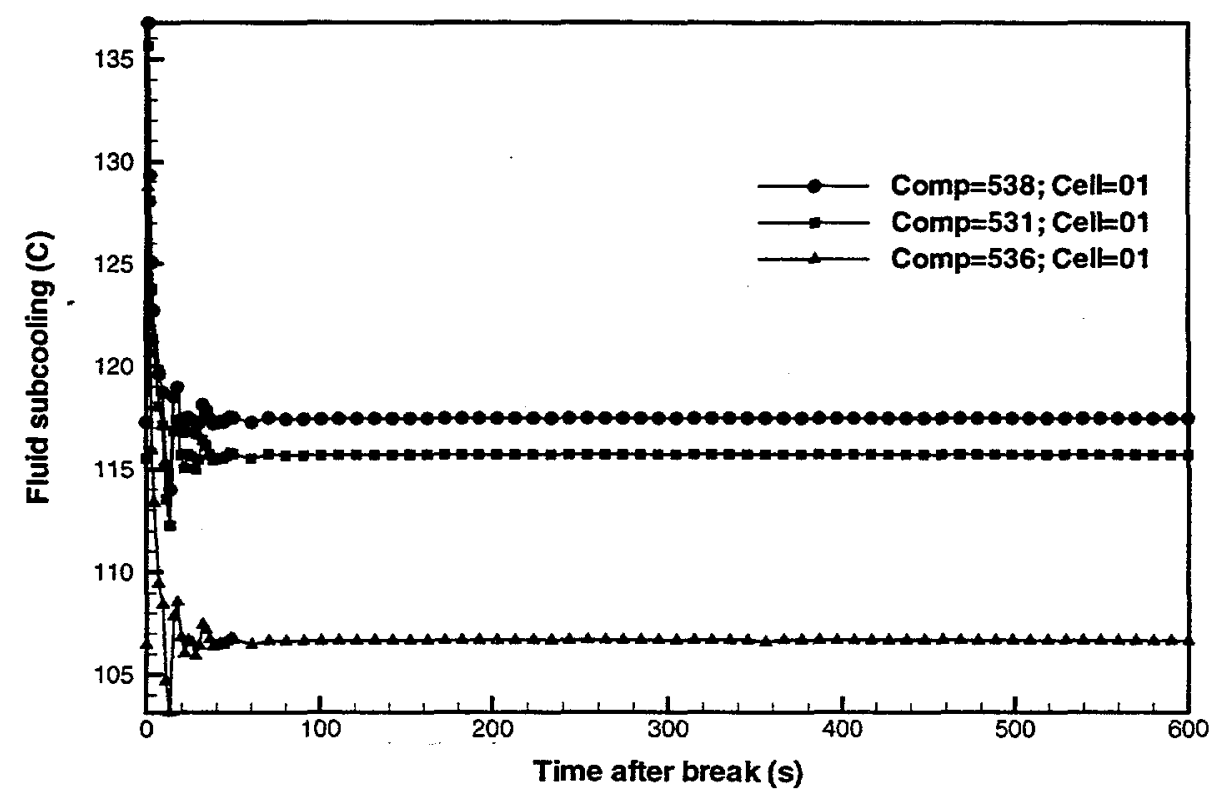

Figure $\mathrm{C}-8 \mathrm{c}$ Module 6 plenum fluid subcoolings for a LOHGA (Case 2: helium supply plenum break near decoupler outlet). 


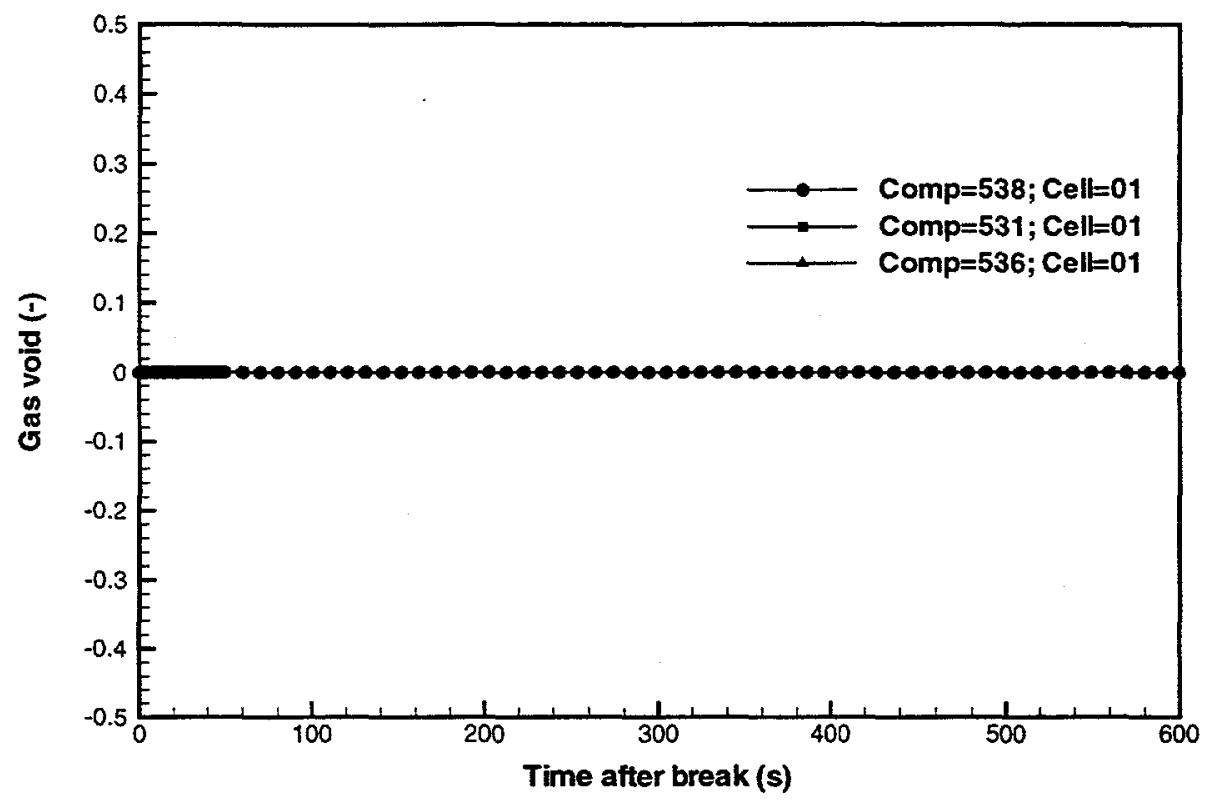

Figure C-8d Module 6 plenum void fractions for a LOHGA (Case 2: helium supply plenum break near decoupler outlet).

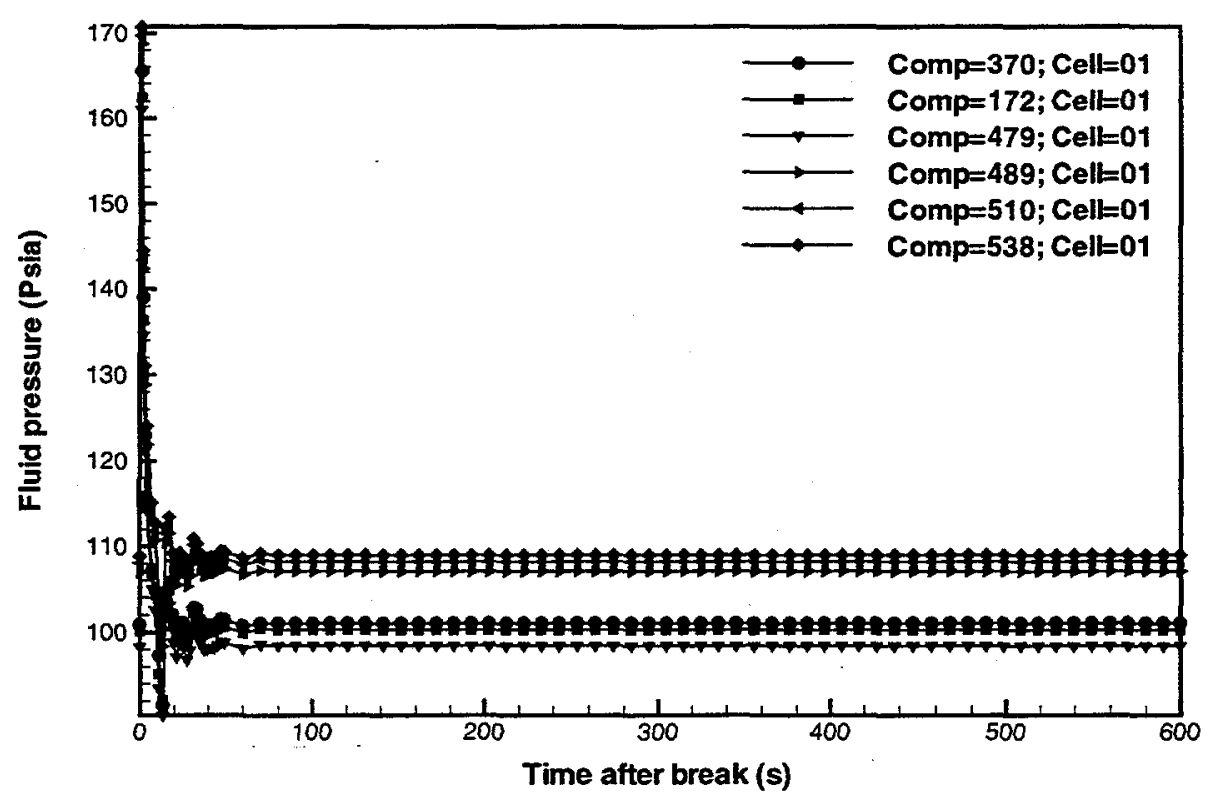

Figure C-9a Module outlet plenum fluid pressures for a LOHGA (Case 2: helium supply plenum break near decoupler outlet). 


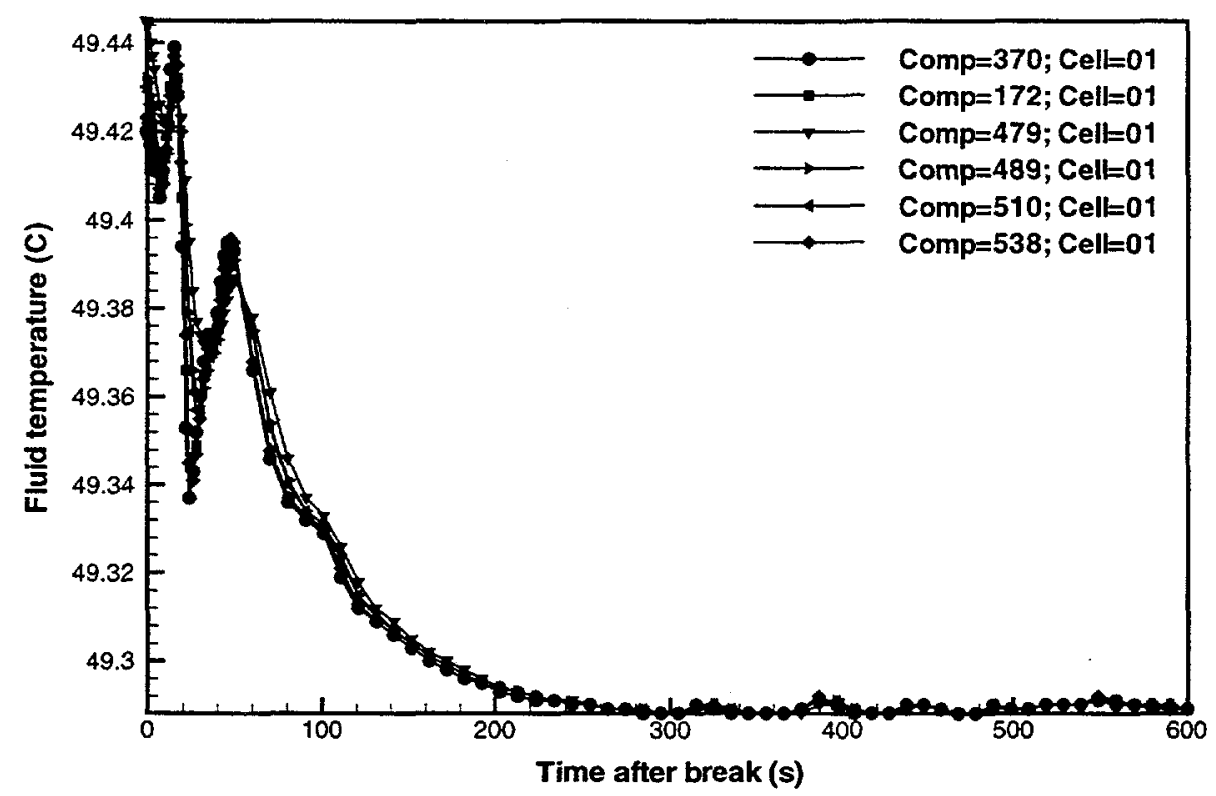

Figure C-9b Module outlet plenum fluid temperatures for a LOHGA (Case 2: helium supply plenum break near decoupler outlet).

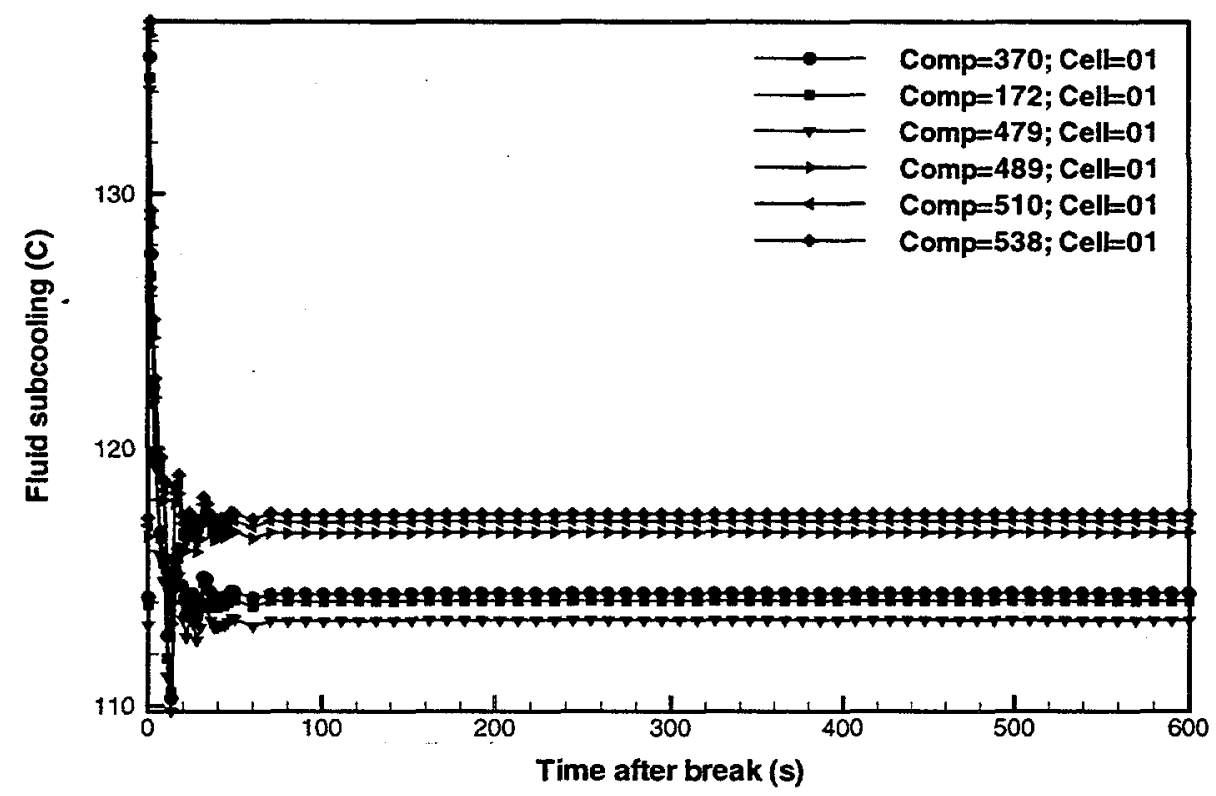

Figure C-9c Module outlet plenum fluid subcoolings for a LOHGA (Case 2: helium supply plenum break near decoupler outlet). 


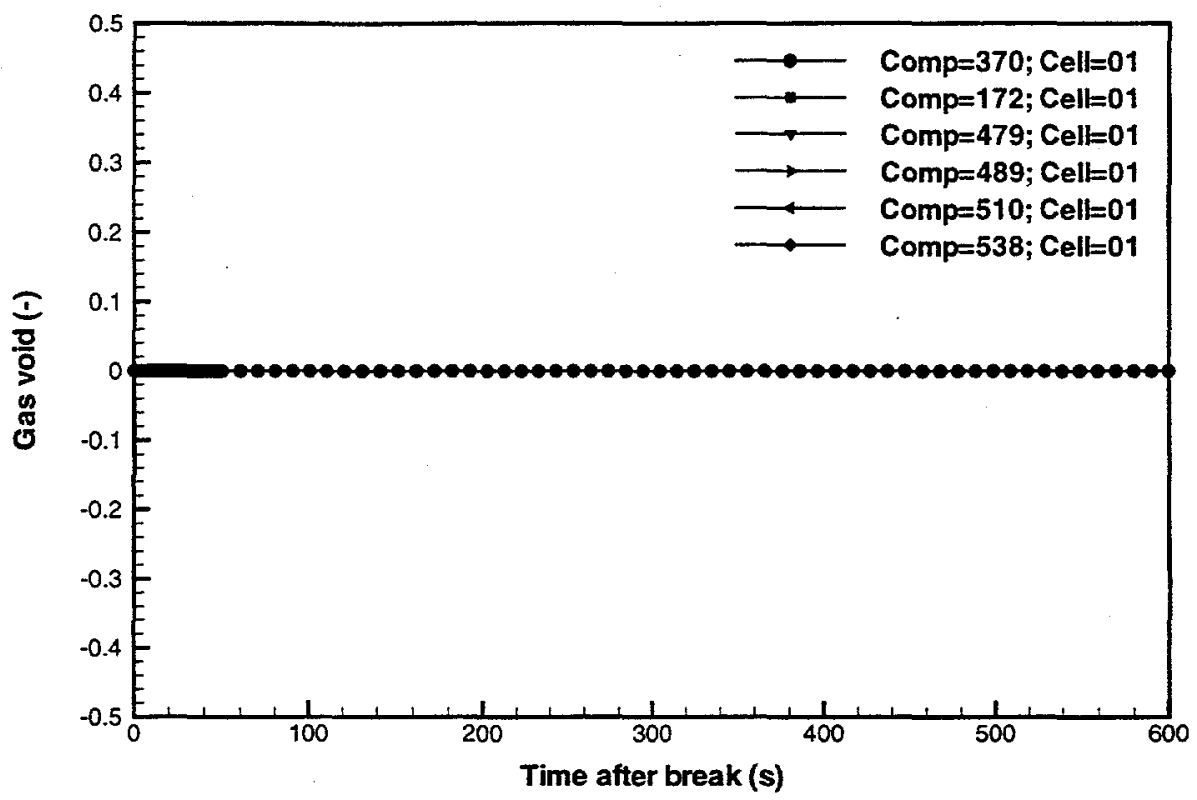

Figure C-9d Module outlet plenum void fractions for a LOHGA (Case 2: helium supply plenum break near decoupler outlet).

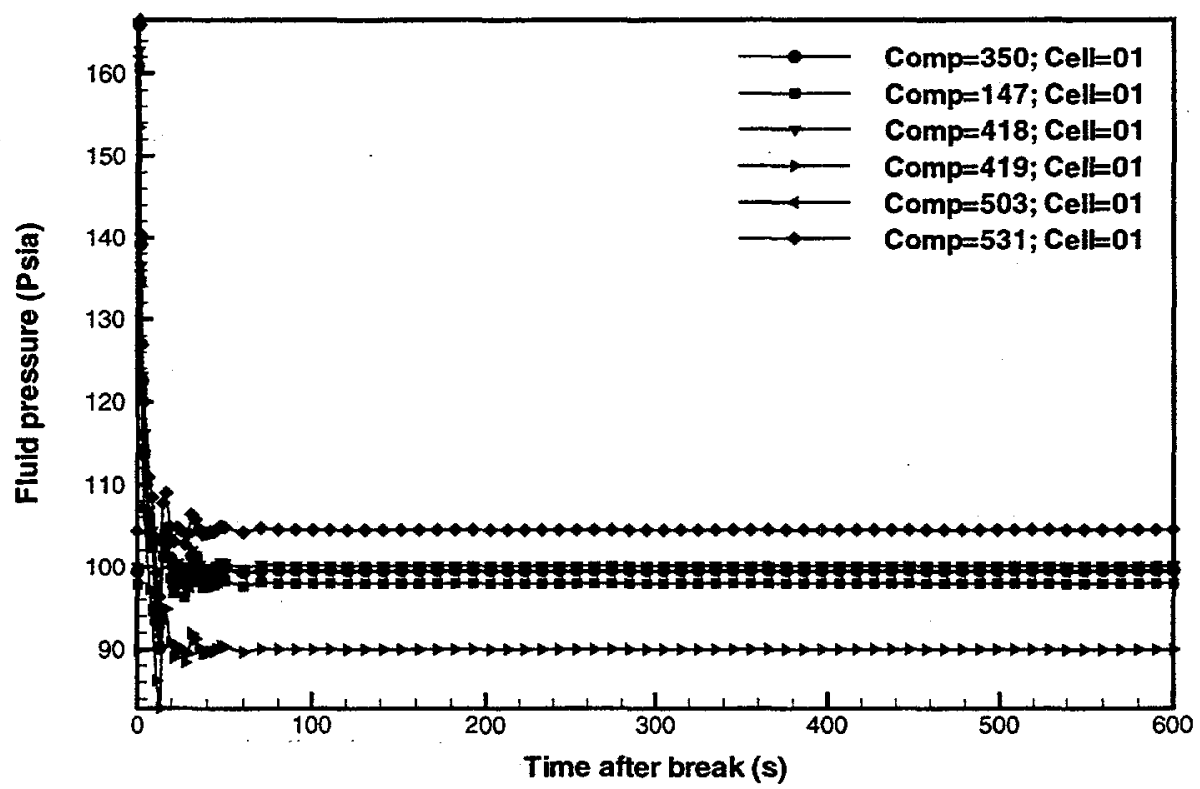

Figure $\mathrm{C}-10 \mathrm{a}$ Module middle plenum fluid pressures for a LOHGA (Case 2: helium supply plenum break near decoupler outlet). 


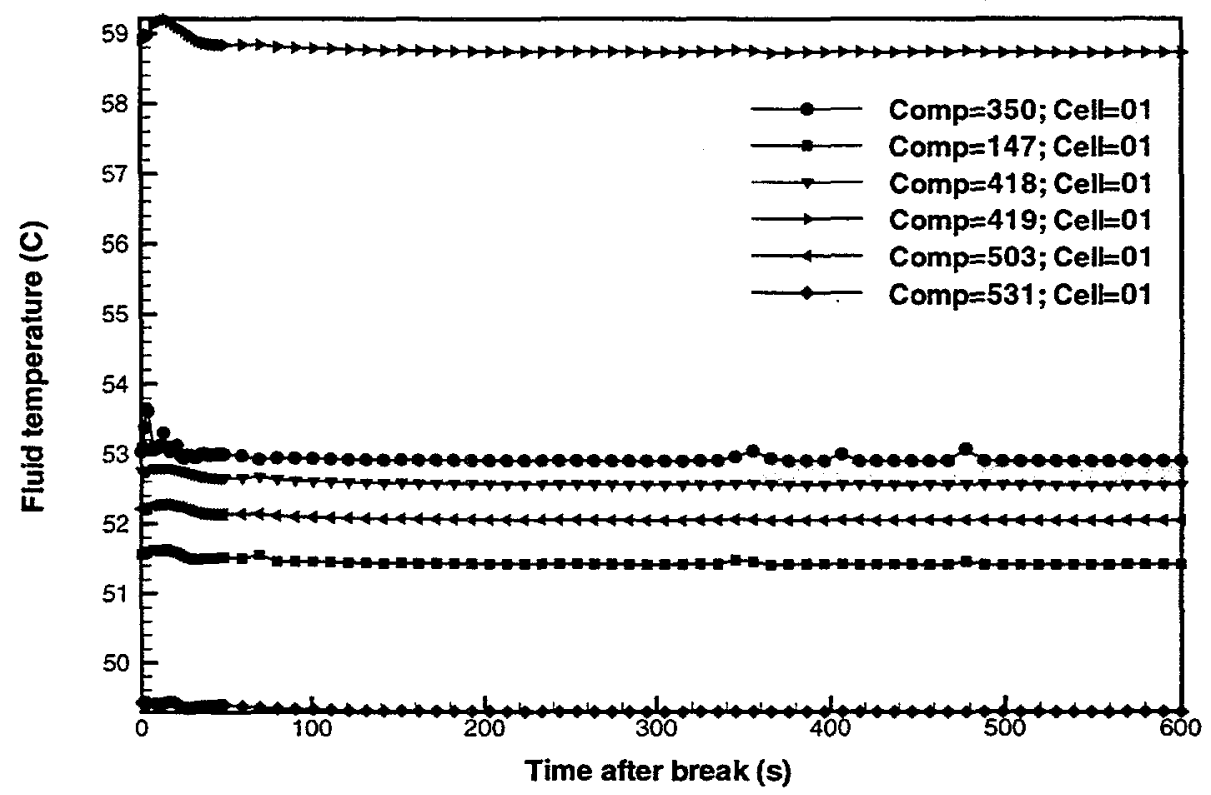

Figure C-10b Module middle plenum fluid temperatures for a LOHGA (Case 2: helium supply plenum break near decoupler outlet).

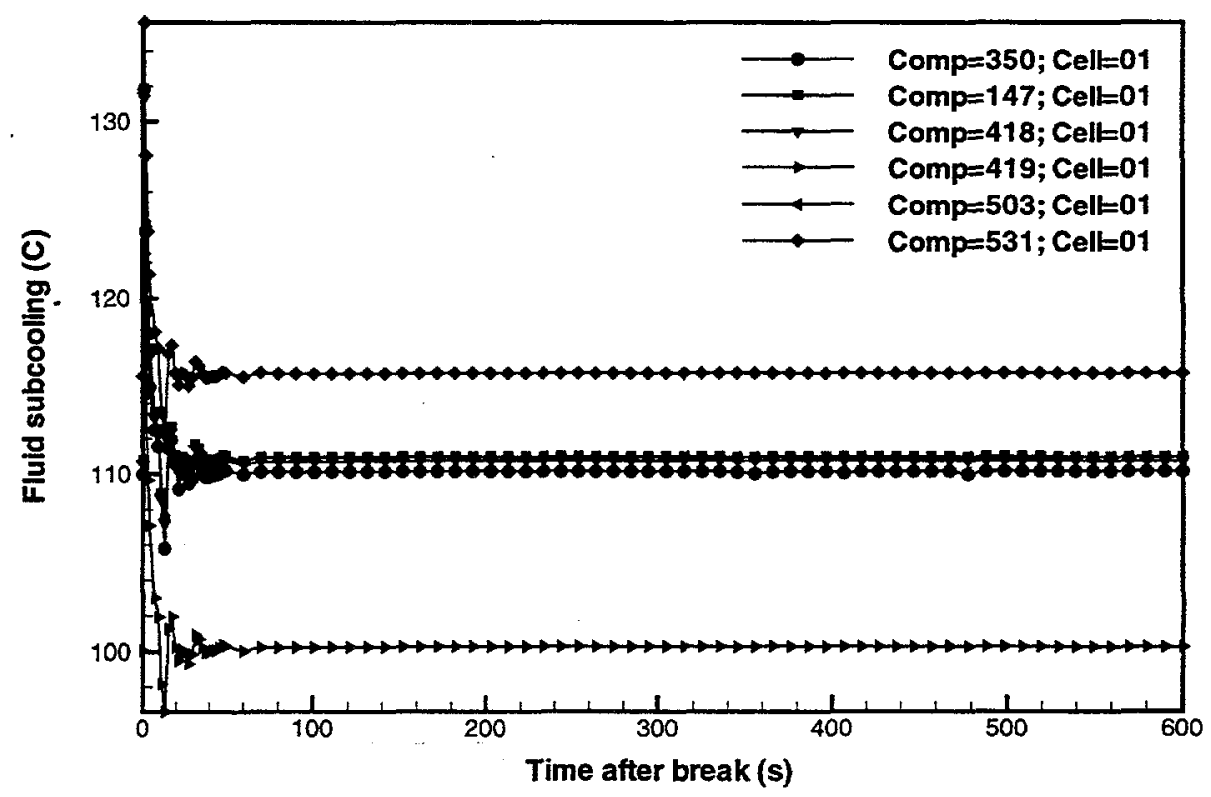

Figure C-10c Module middle plenum fluid subcoolings for a LOHGA (Case 2: helium supply plenum break near decoupler outlet). 


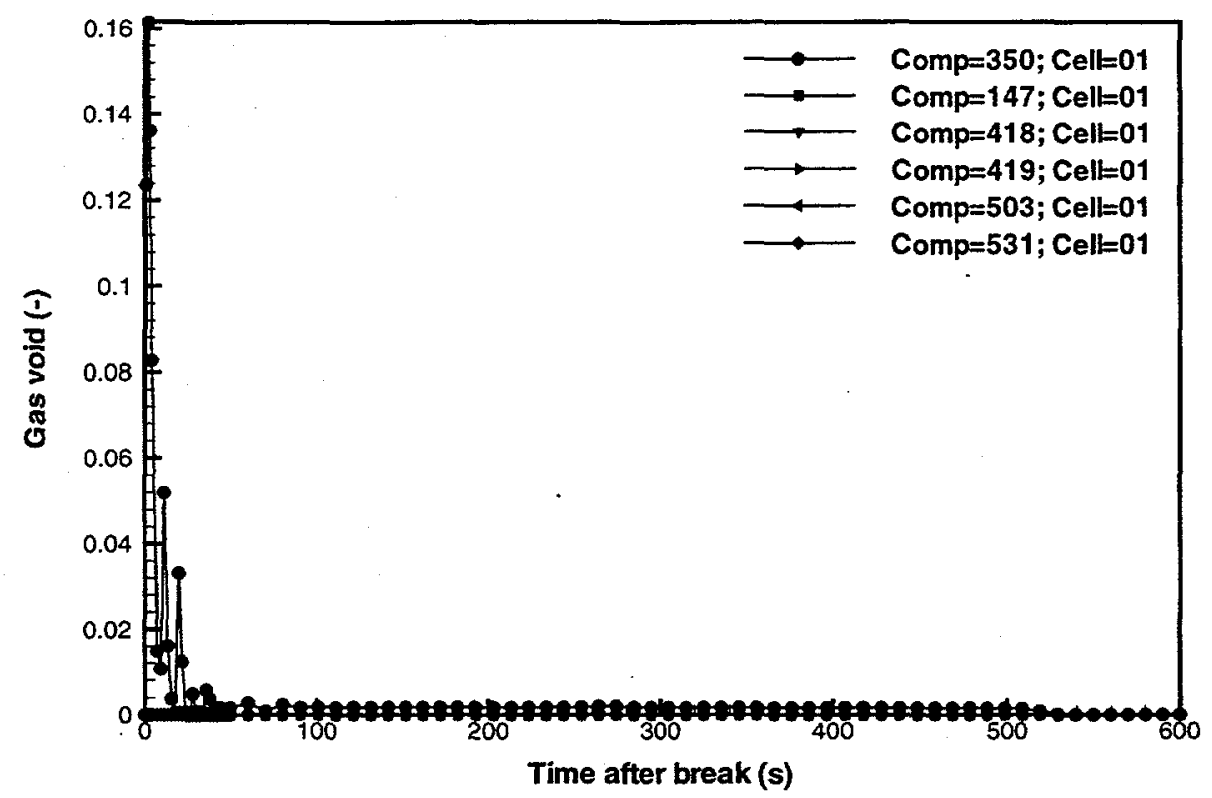

Figure C-10d Module middle plenum void fractions for a LOHGA (Case 2: helium supply plenum break near decoupler outlet).

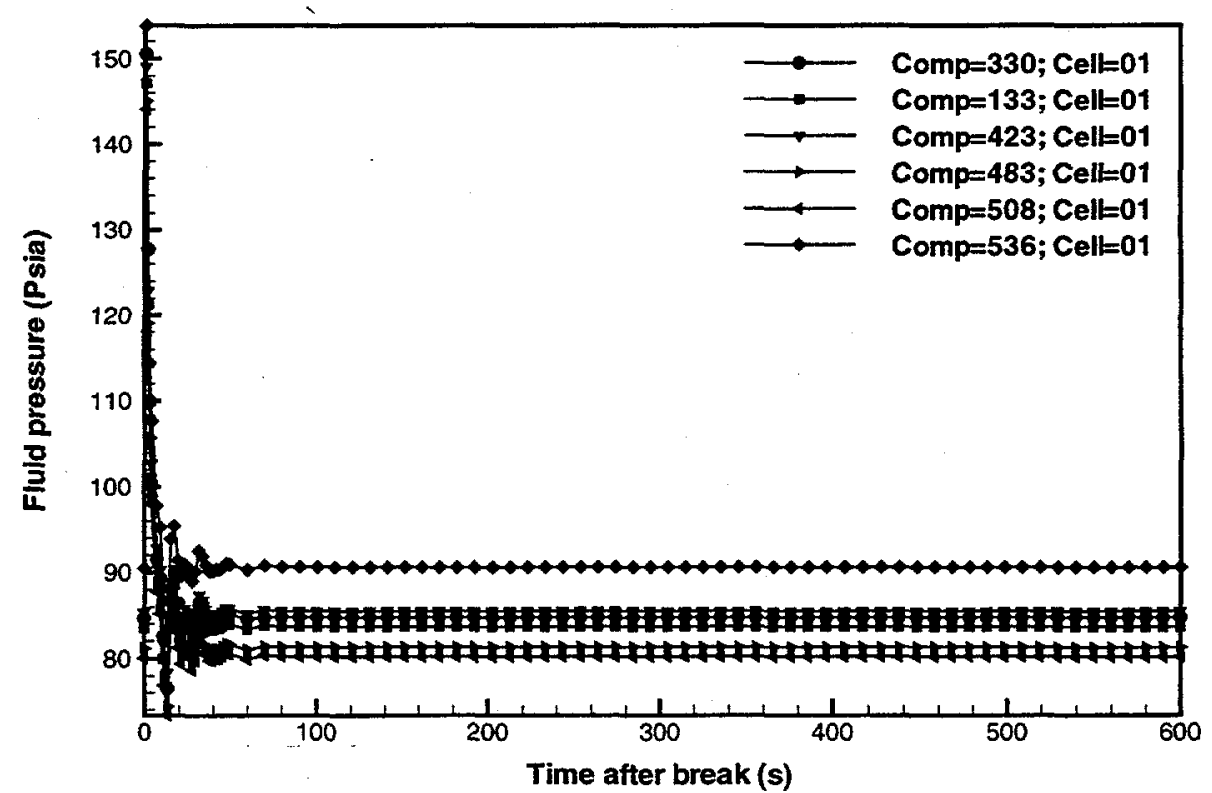

Figure C-11a Module outlet plenum fluid pressures for a LOHGA (Case 2: helium supply plenum break near decoupler outlet). 


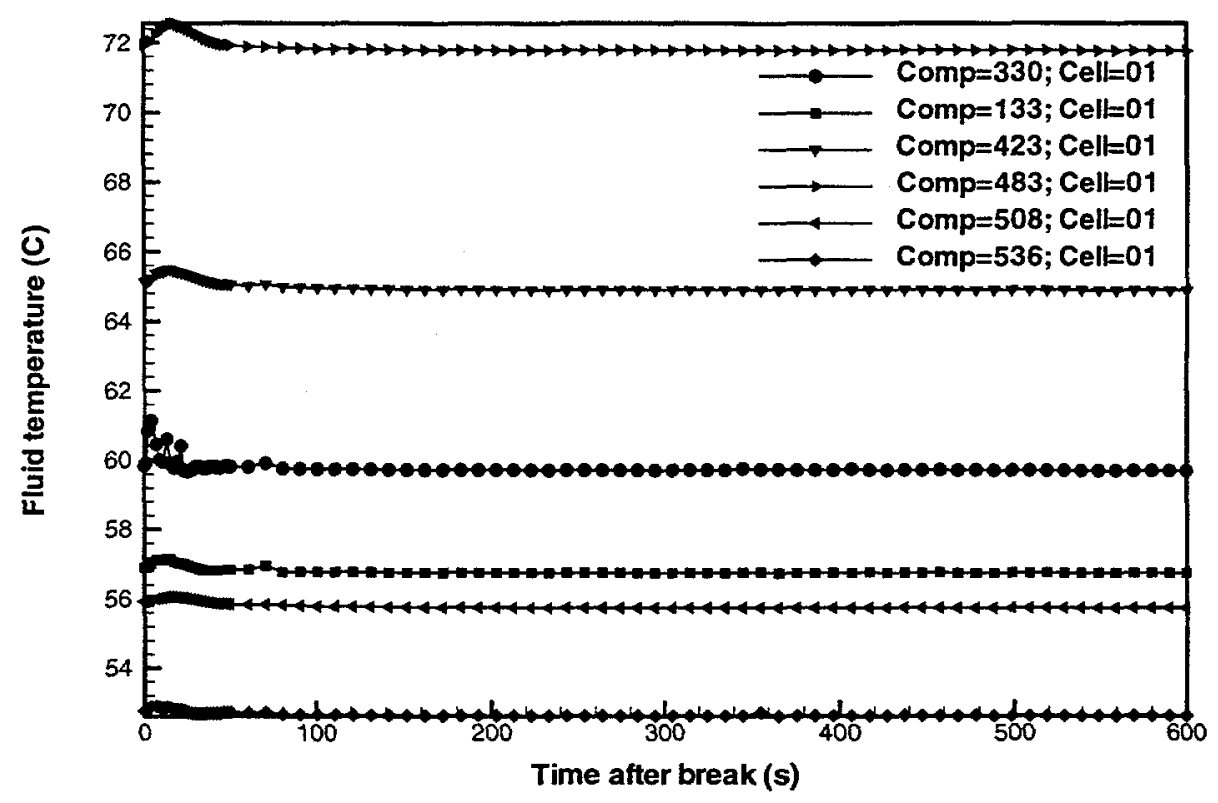

Figure C-11b Module outlet plenum fluid temperatures for a LOHGA-(Case 2: helium supply plenum break near decoupler outlet).

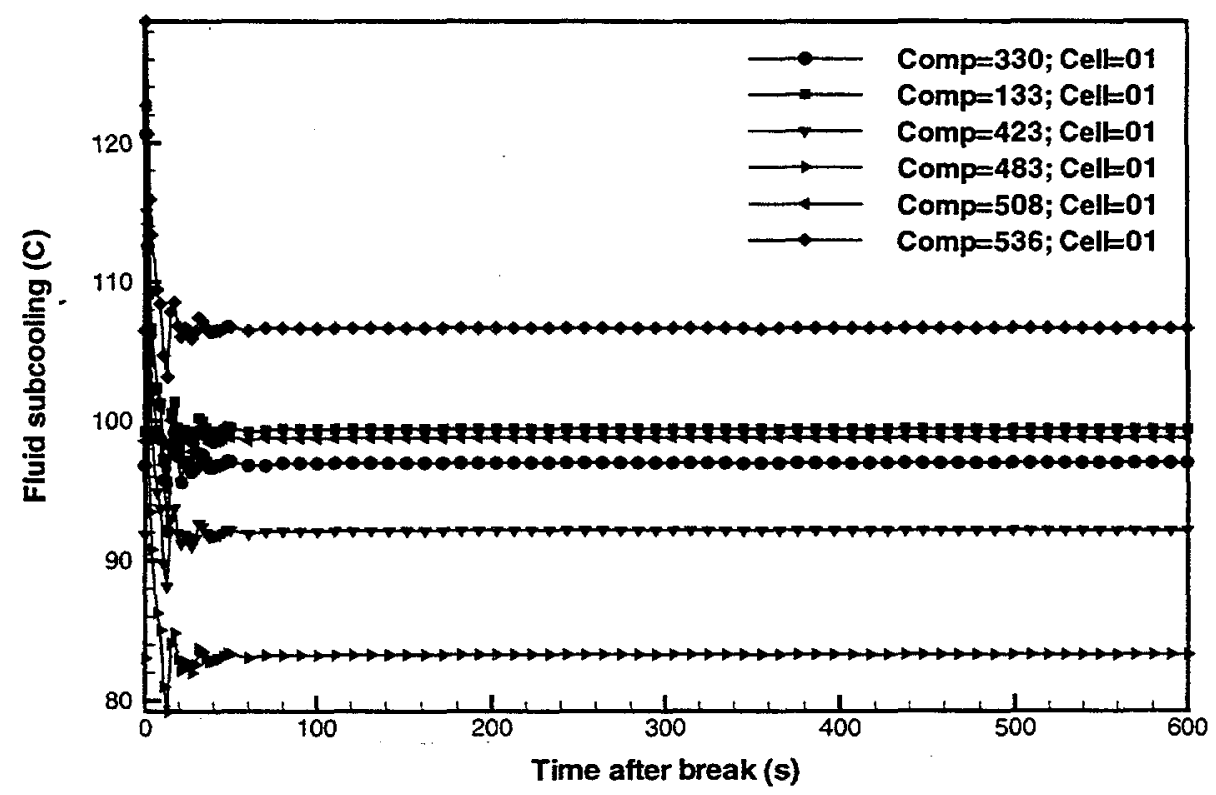

Figure C-11c Module outlet plenum fluid subcoolings for a LOHGA (Case 2: helium supply plenum break near decoupler outlet). 


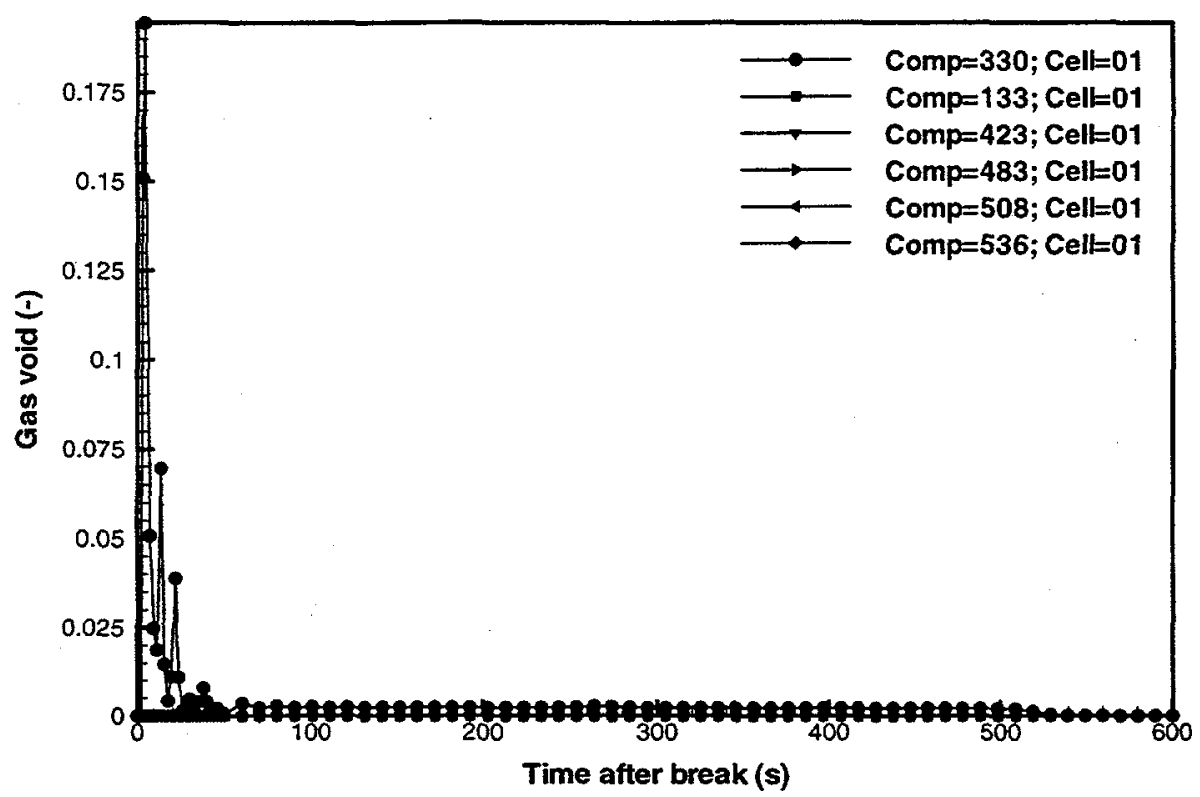

Figure C-11d Module outlet plenum void fractions for a LOHGA (Case 2: helium supply plenum break near decoupler outlet). 


\section{Appendix C2 LOHGA (Case 2) TRAC Pipe, Pump, and Valve Component Figures}

The following figures are from a TRAC simulation for Case 2 of a LOHGA (Helium supply plenum break near decoupler outlet):

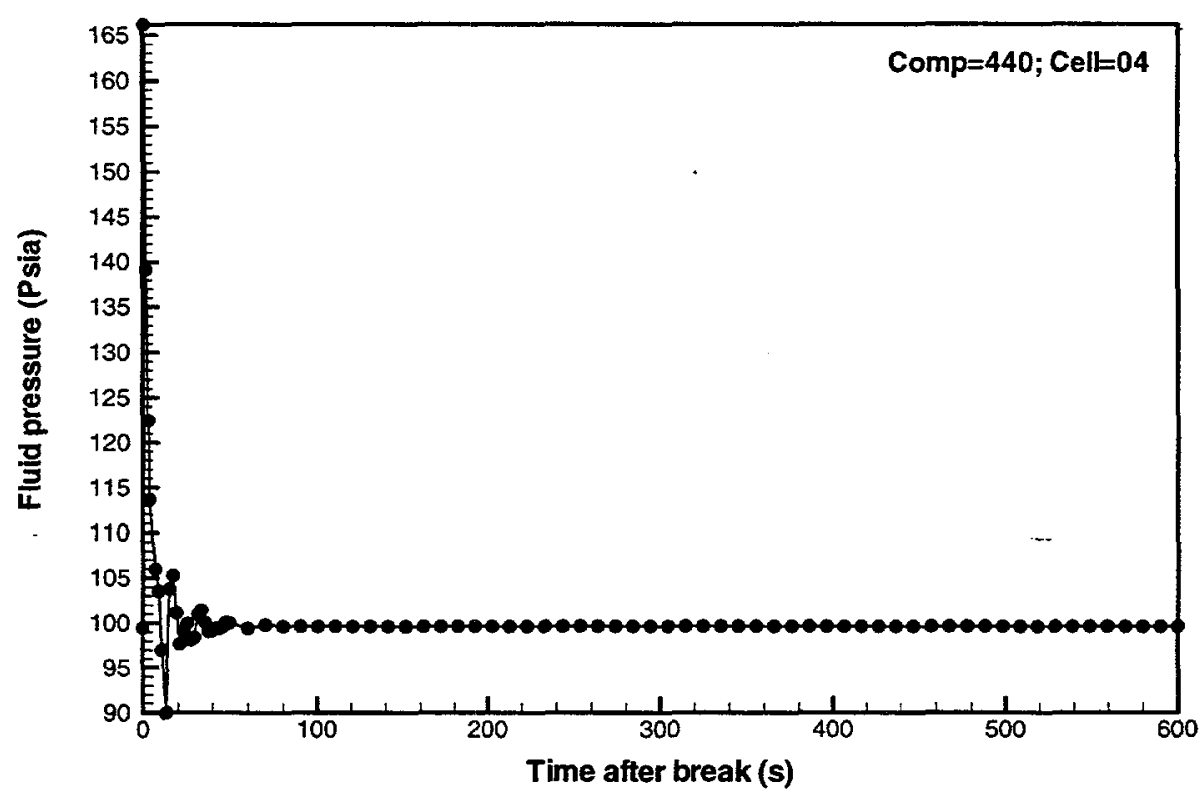

Figure C-12a Helium gas line fluid pressures for a LOHGA (Case 2: helium supply plenum break near decoupler outlet). 


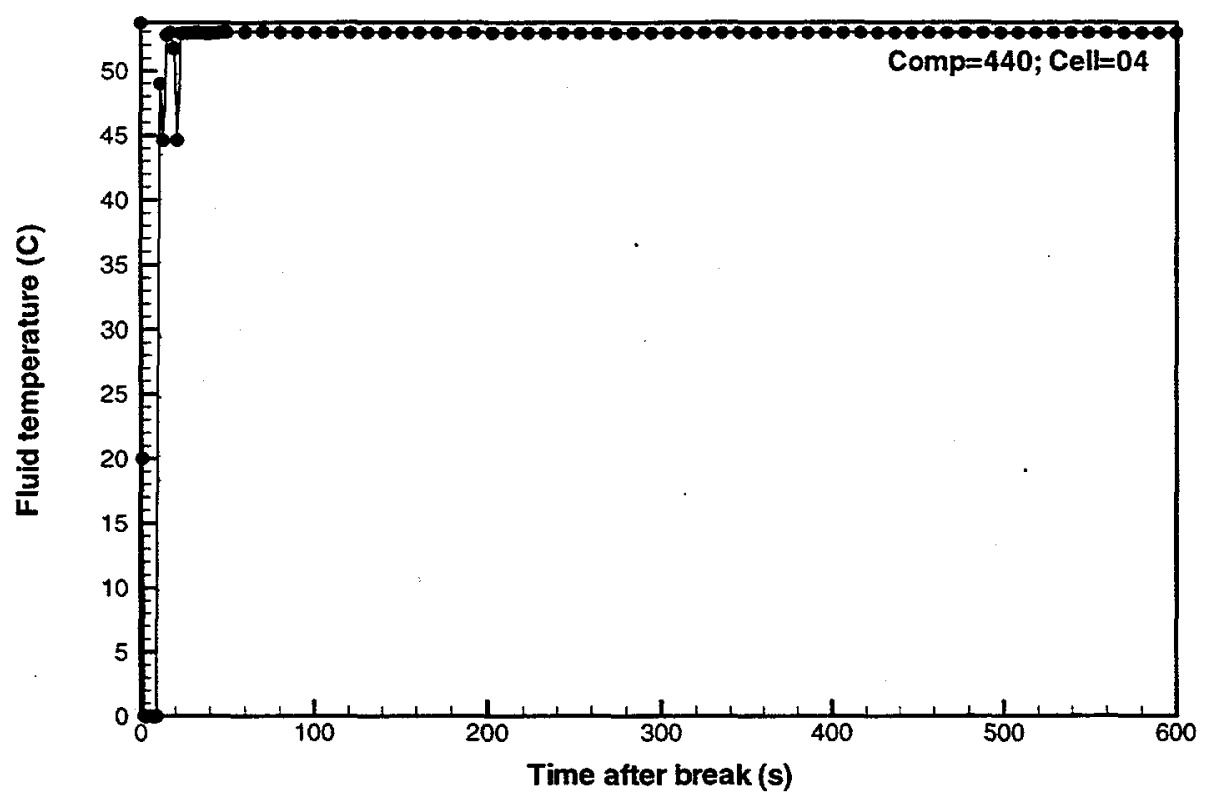

Figure $\mathrm{C}-12 \mathrm{~b}$ Helium gas line fluid temperatures for a LOHGA (Case 2: helium supply plenum break near decoupler outlet).

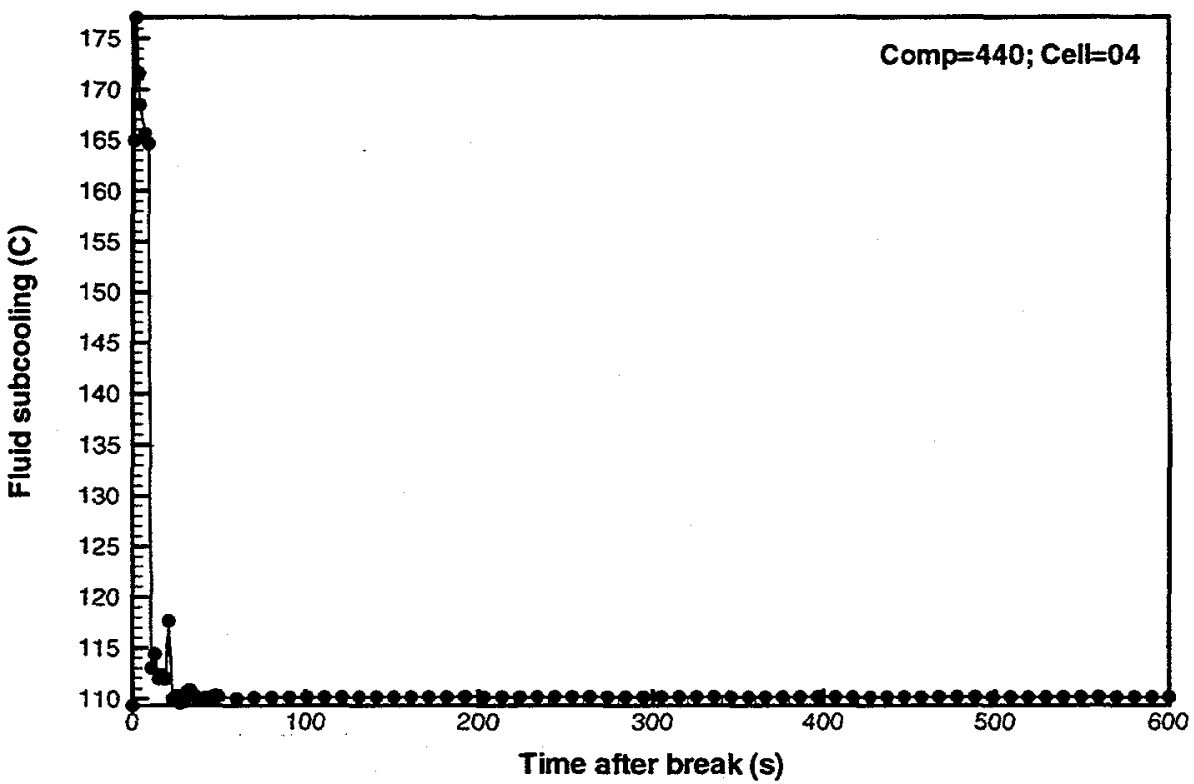

Figure C-12c Helium gas line fluid subcoolings for a LOHGA (Case 2: helium supply plenum break near decoupler outlet). 


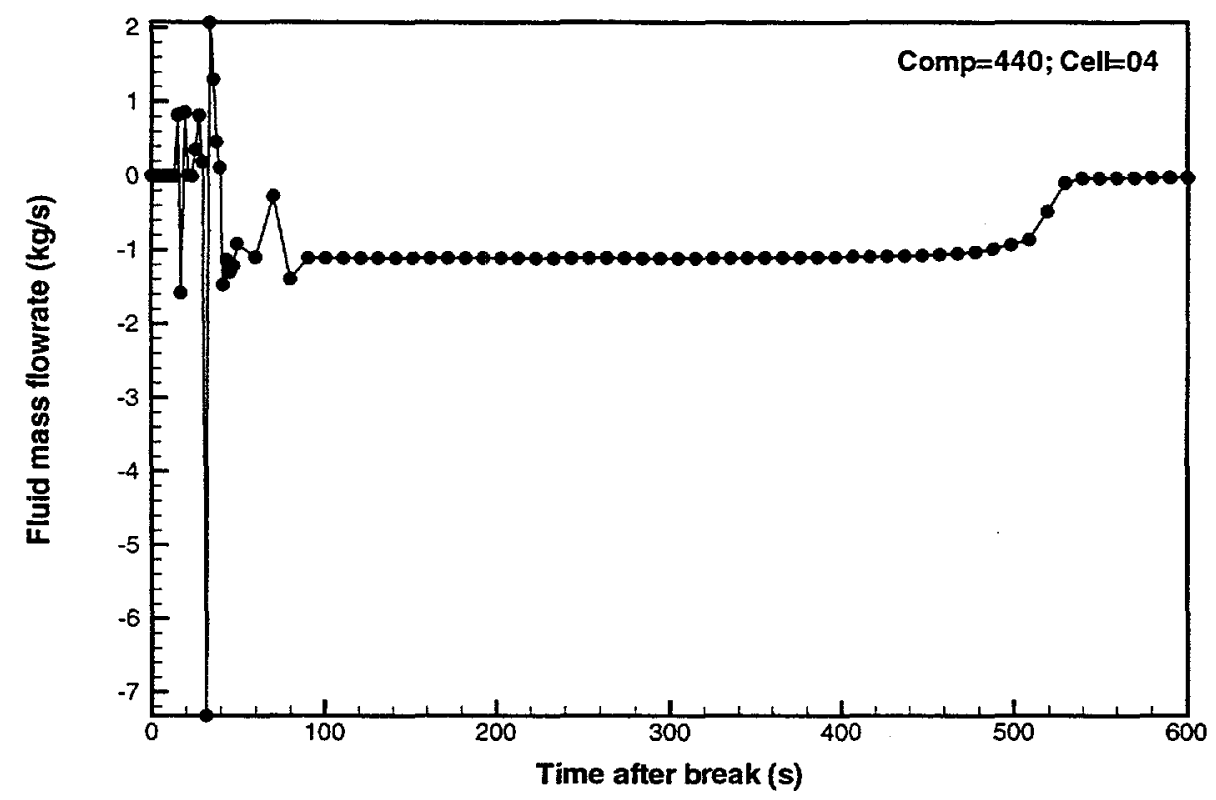

Figure C-12d Helium gas line liquid mass flowrates for a LOHGA (Case 2: helium supply plenum break near decoupler outlet).

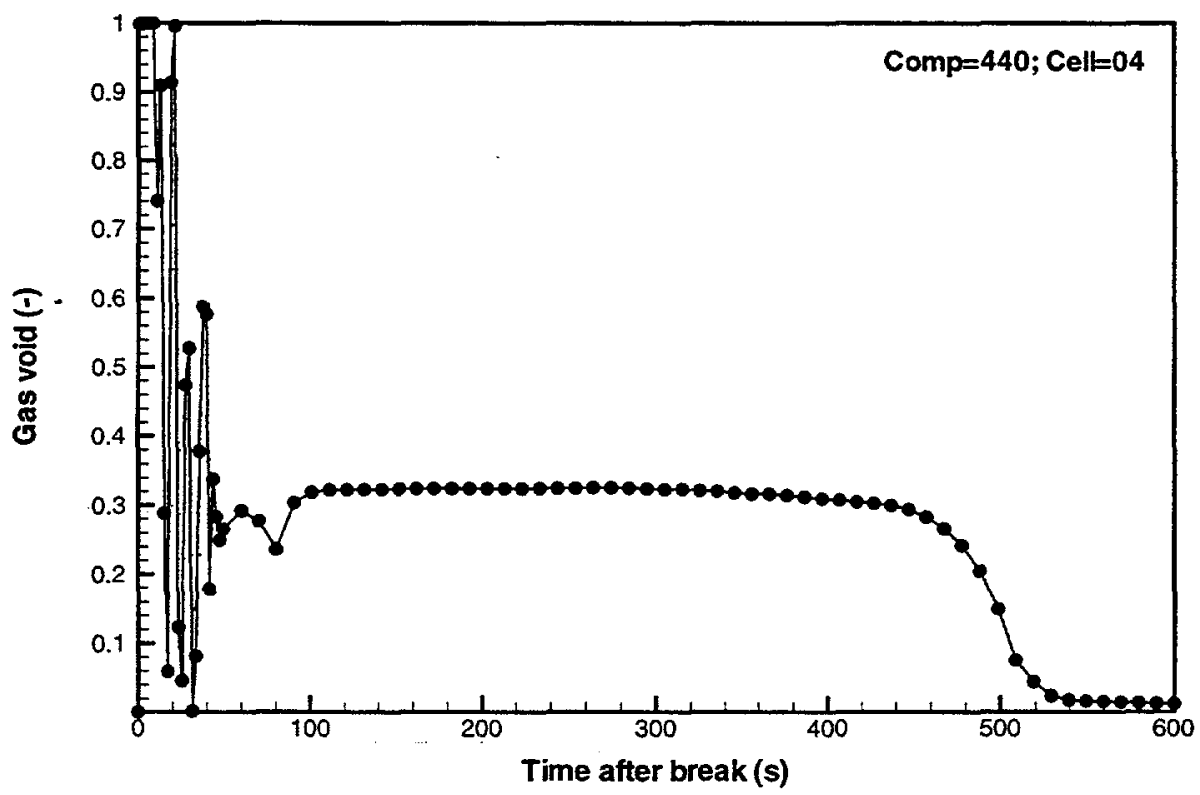

Figure $\mathrm{C}-12 \mathrm{e}$ Helium gas line void fractions for a LOHGA (Case 2: helium supply plenum break near decoupler outlet). 


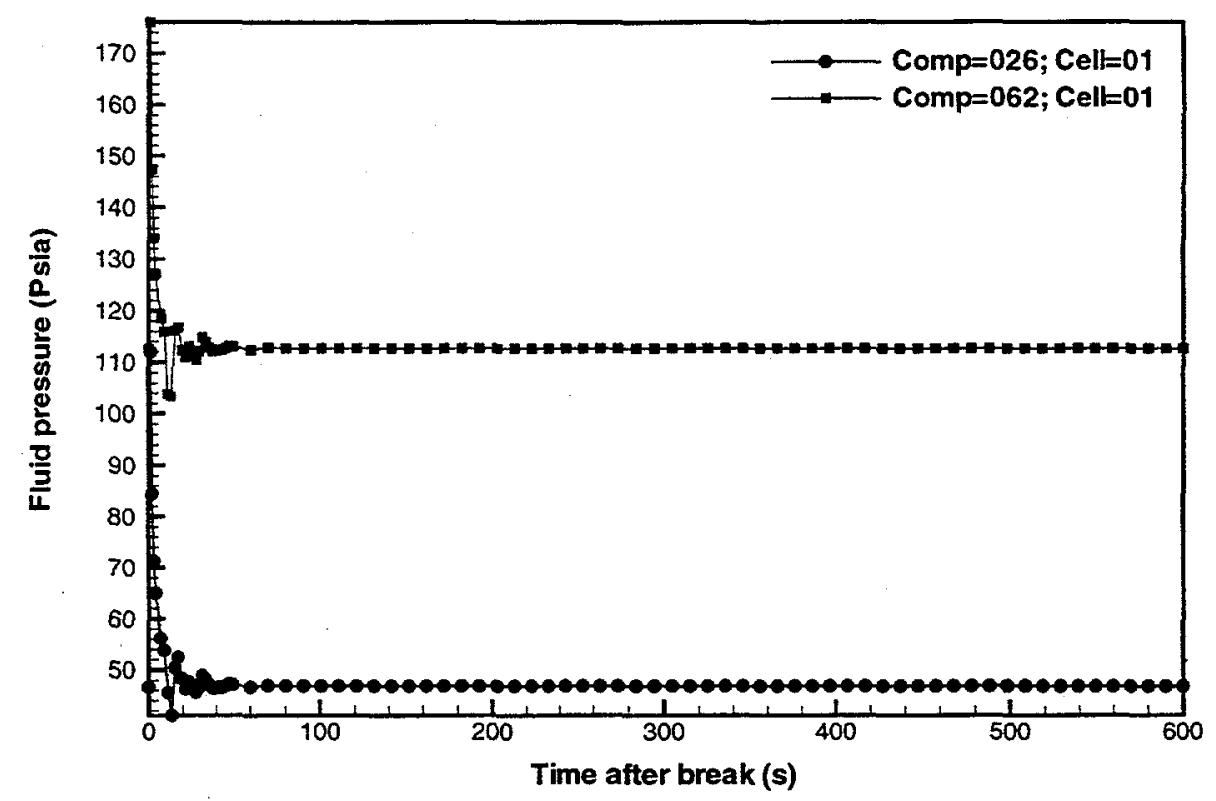

Figure C-13a Primary HR hot and cold leg piping fluid pressures for a LOHGA (Case 2: helium supply plenum break near decoupler outlet).

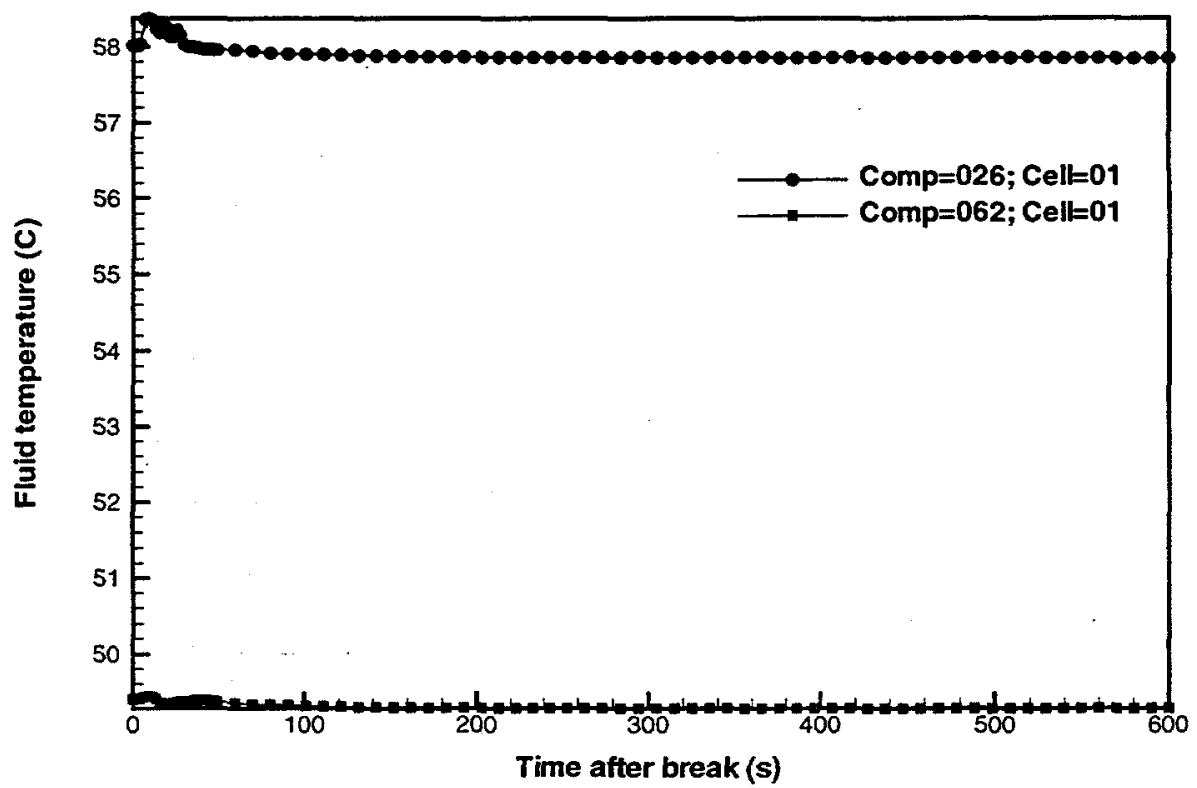

Figure C-13b Primary HR hot and cold leg piping fluid temperatures for a LOHGA (Case 2: helium supply plenum break near decoupler outlet). 


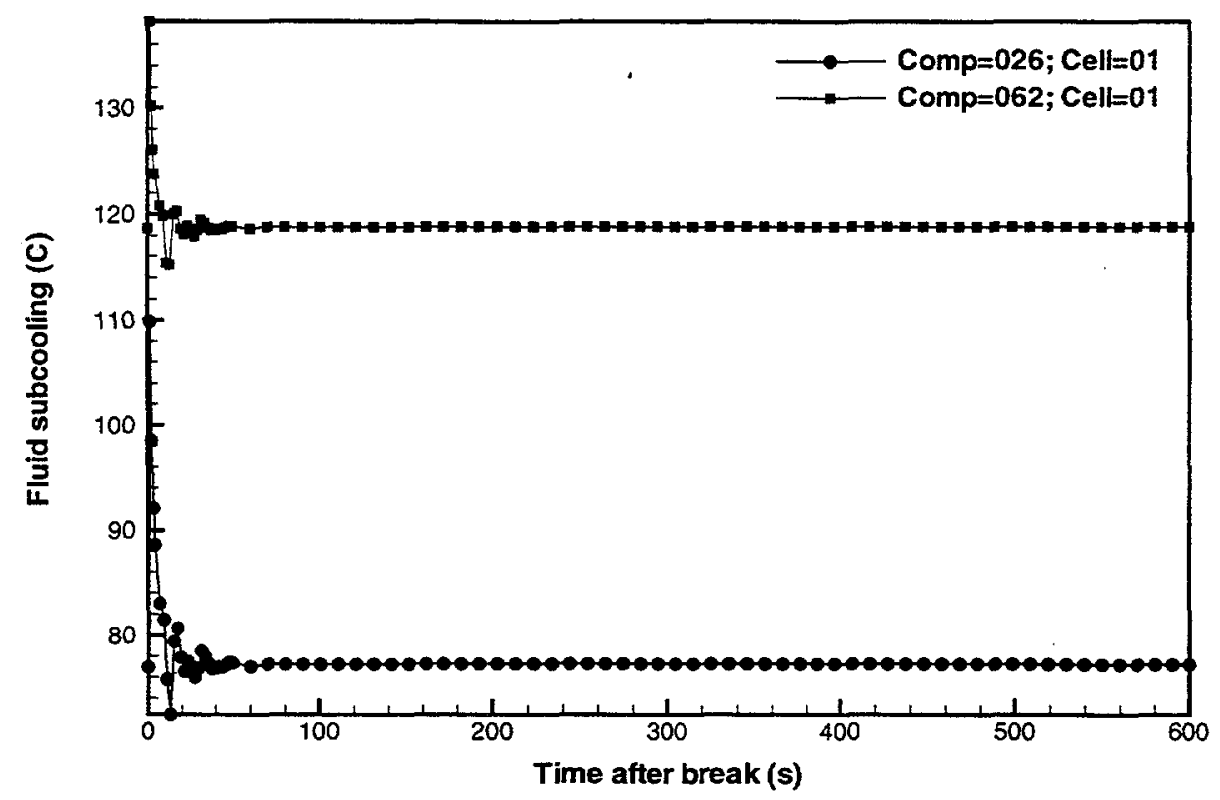

Figure C-13c Primary HR hot and cold leg piping fluid subcoolings for a LOHGA (Case 2: helium supply plenum break near decoupler outlet).

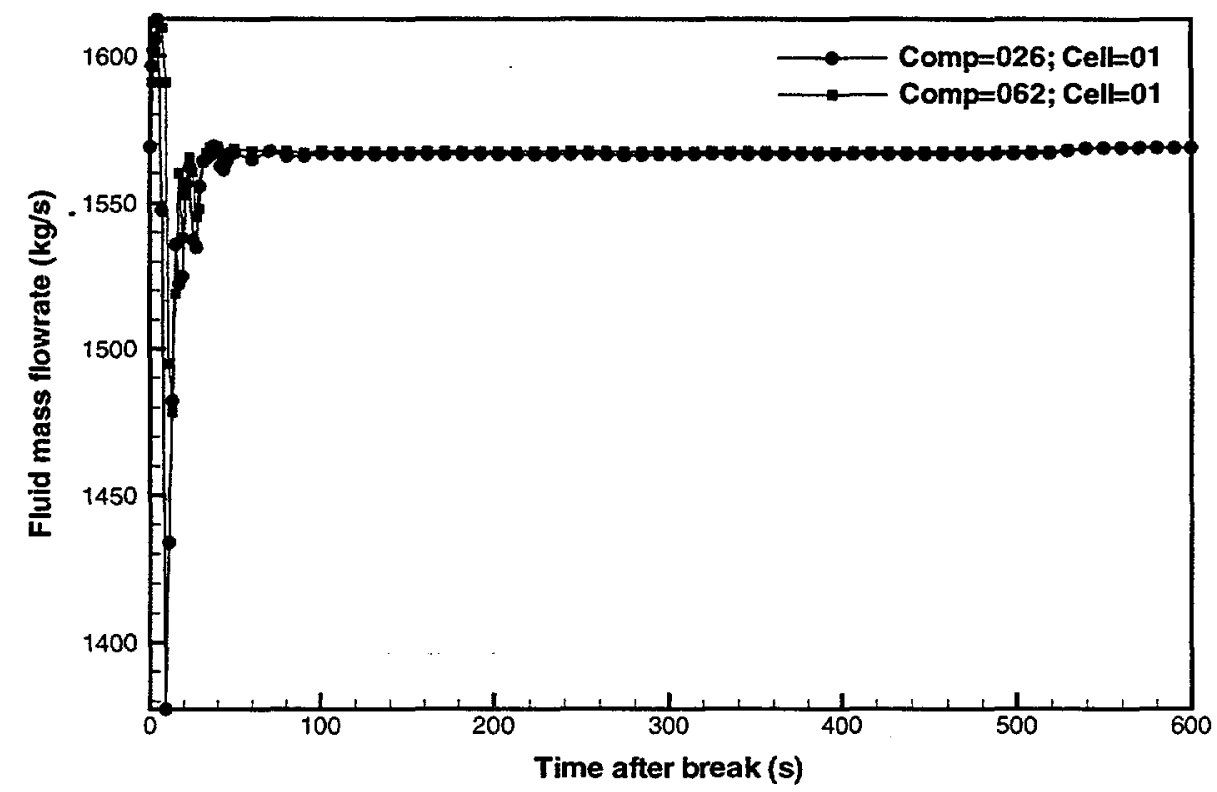

Figure C-13d Primary HR hot and cold leg piping liquid mass flowrates for a LOHGA (Case 2: helium supply plenum break near decoupler outlet). 


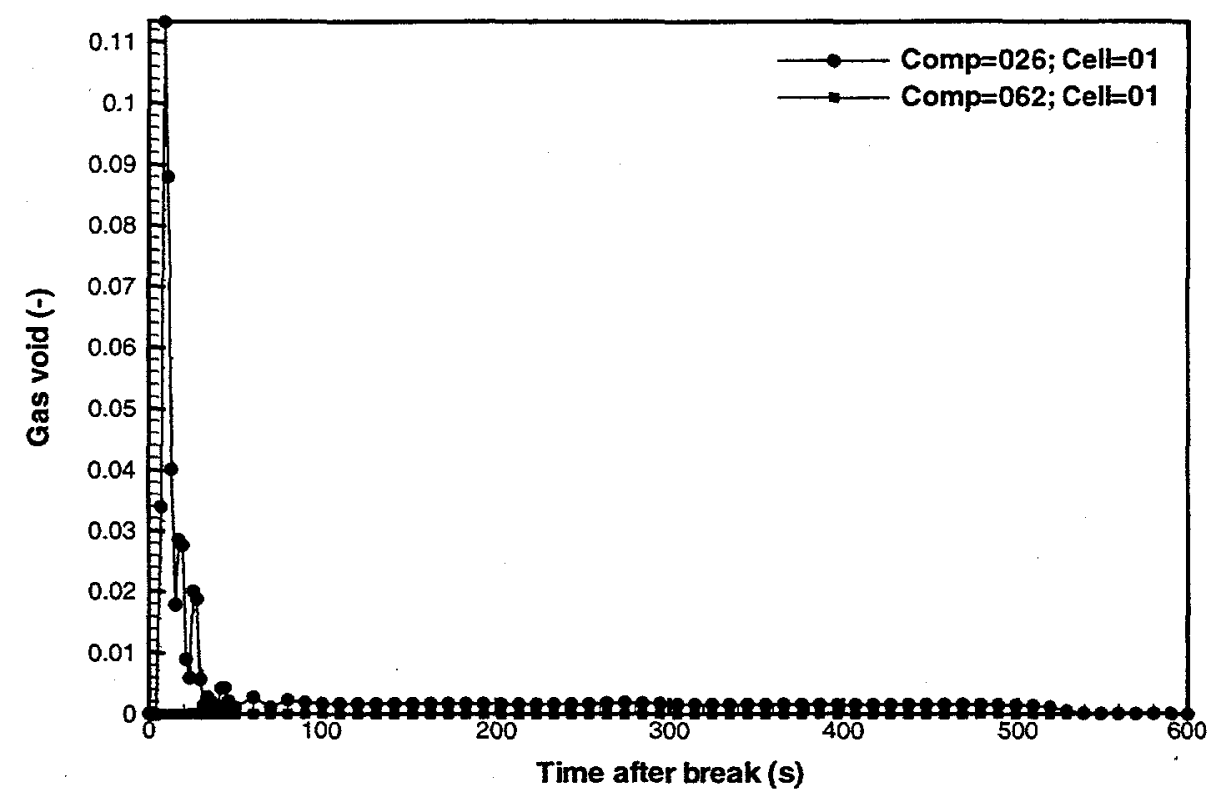

Figure C-13e Primary HR hot and cold leg piping void fractions for a LOHGA (Case 2: helium supply plenum break near decoupler outlet).

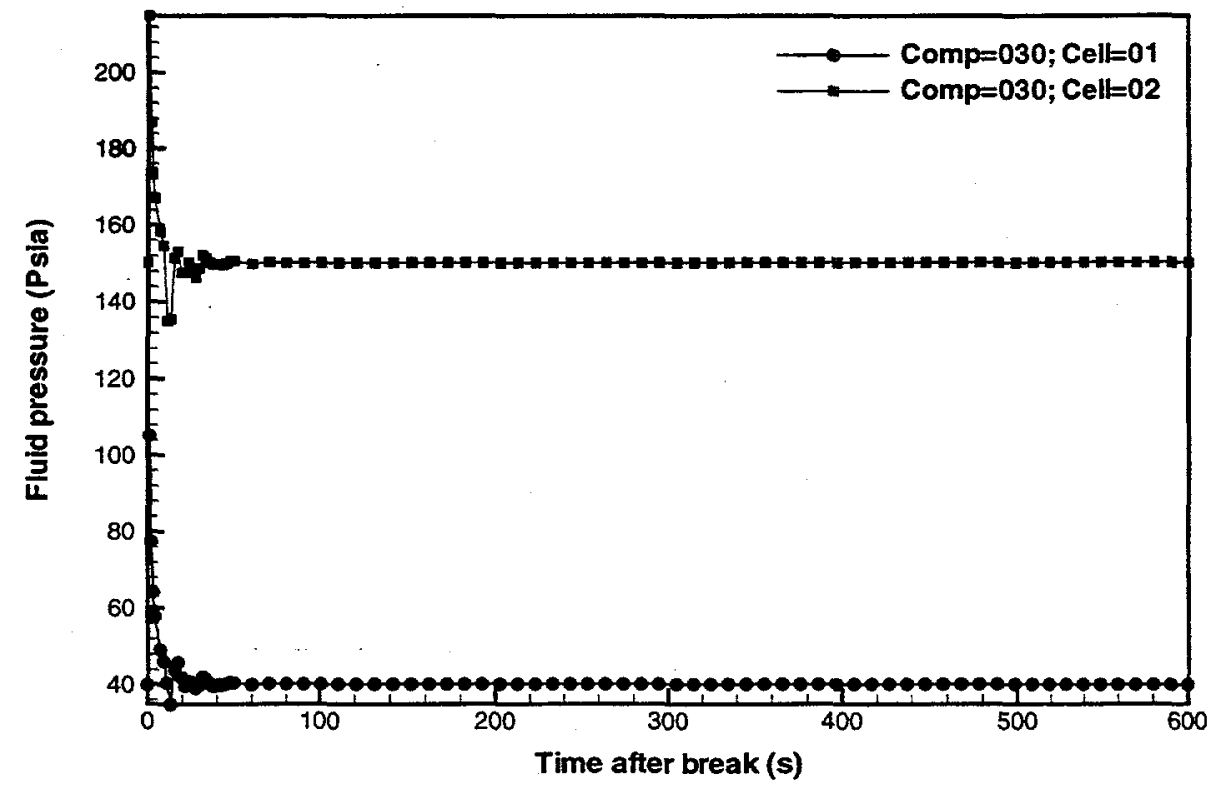

Figure C-14a Primary HR pump 1 fluid pressures for a LOHGA (Case 2: helium supply plenum break near decoupler outlet). 


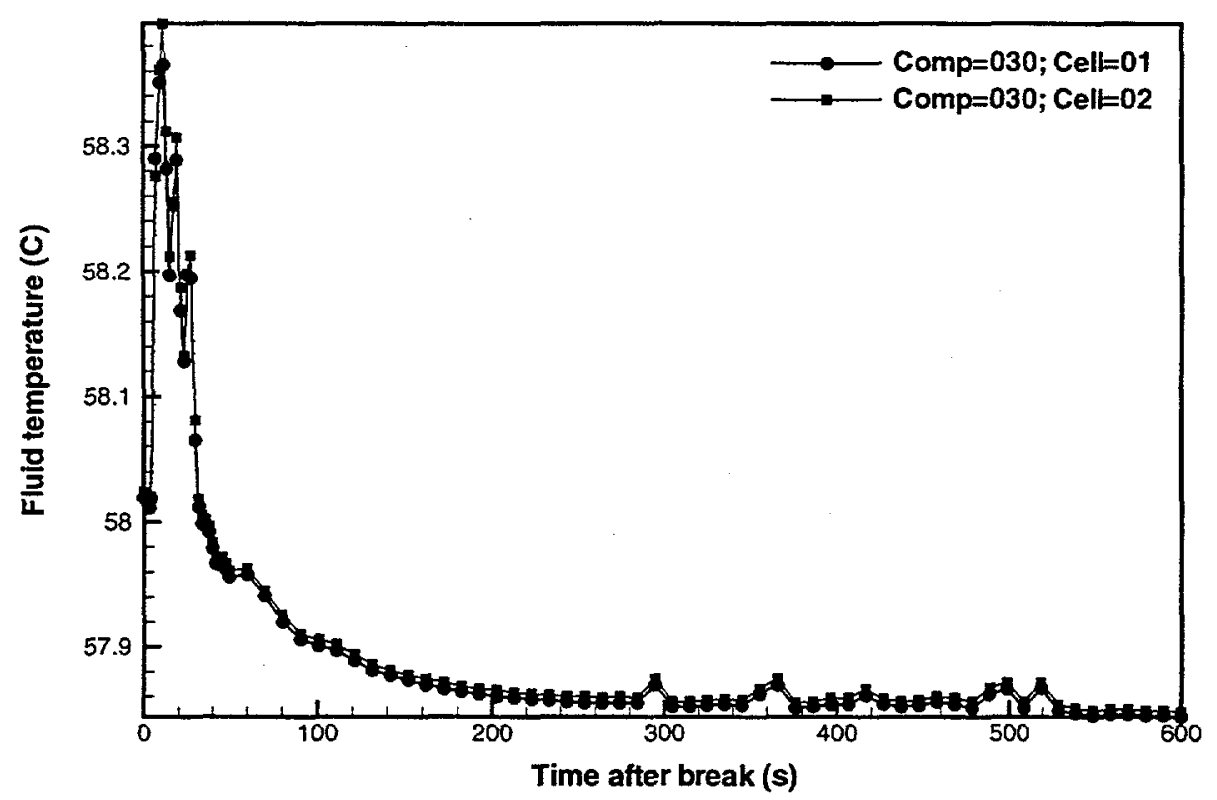

Figure C-14b Primary HR pump 1 fluid temperatures for a LOHGA (Case 2: helium supply plenum break near decoupler outlet).

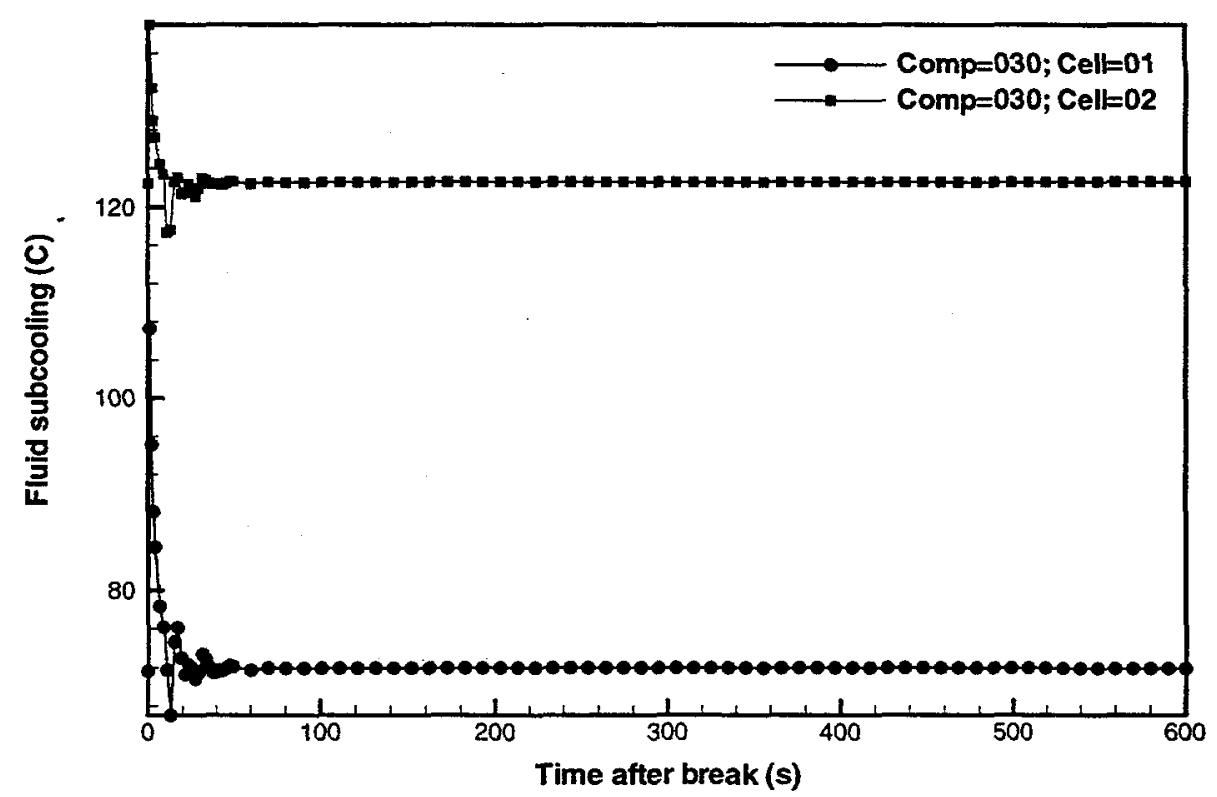

Figure C-14c Primary HR pump 1 fluid subcoolings for a LOHGA (Case 2: helium supply plenum break near decoupler outlet). 


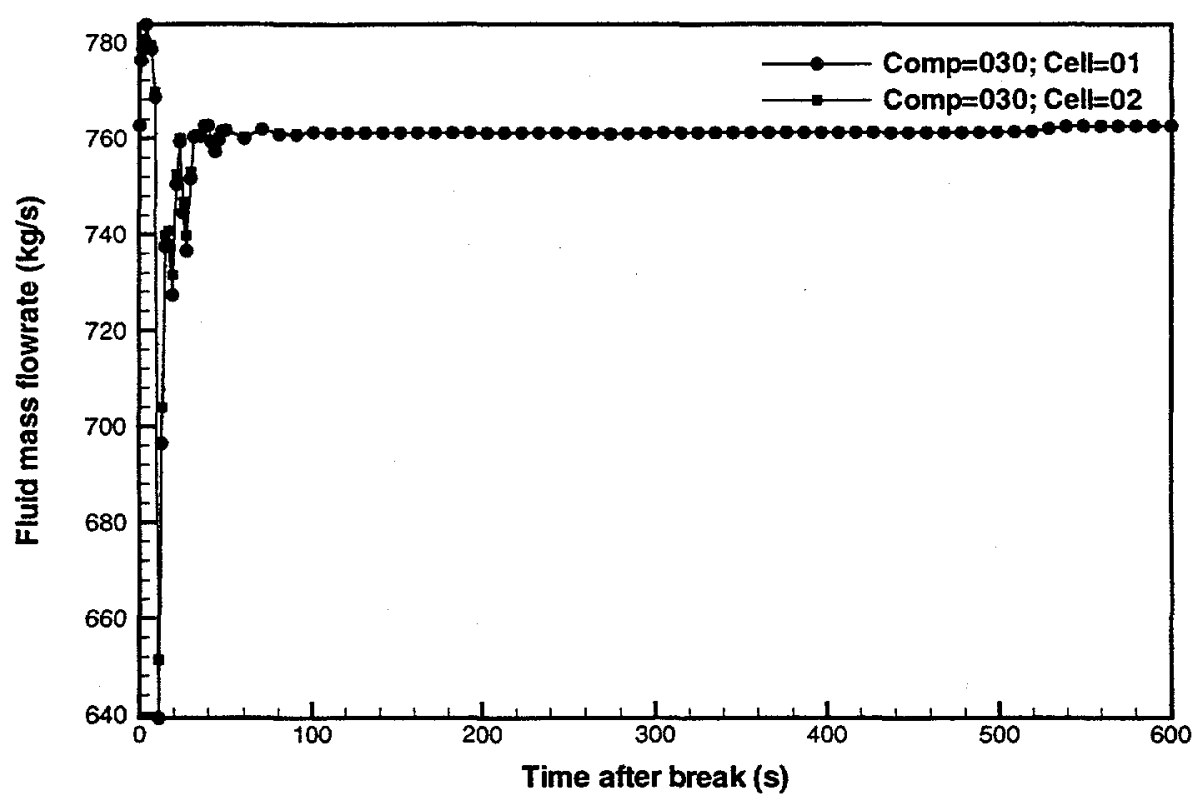

Figure C-14d Primary HR pump 1 liquid mass flowrates for a-LOHGA (Case 2: helium supply plenum break near decoupler outlet).

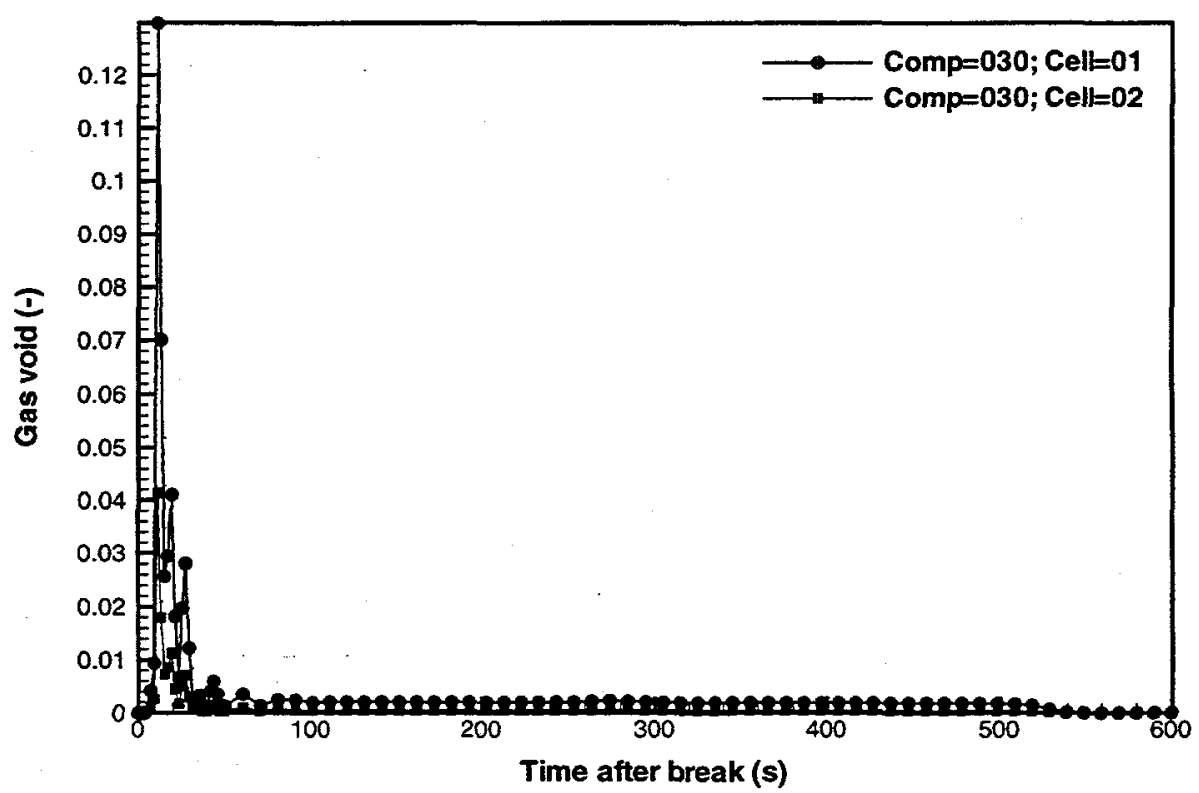

Figure C-14e Primary HR pump 1 void fractions for a LOHGA (Case 2: helium supply plenum break near decoupler outlet). 


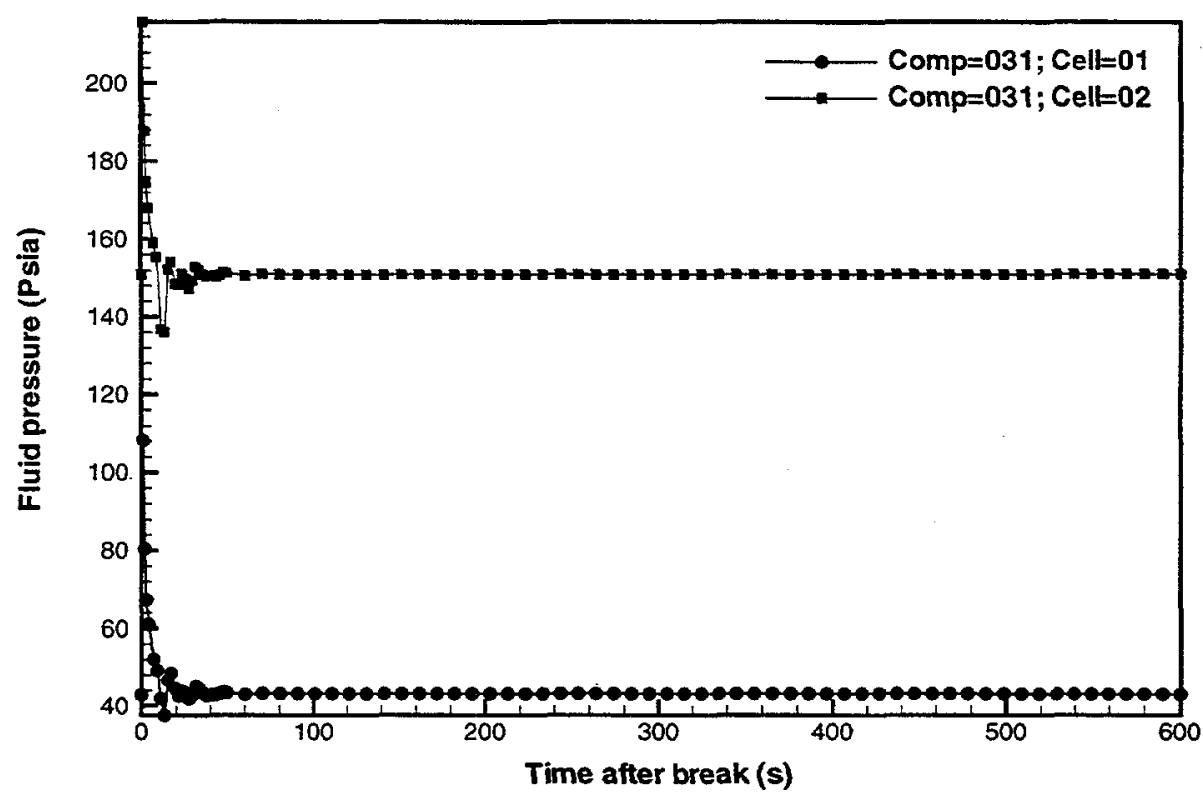

Figure C-15a Primary HR pump 2 fluid pressures for a LOHGA (Case 2: helium supply plenum break near decoupler outlet).

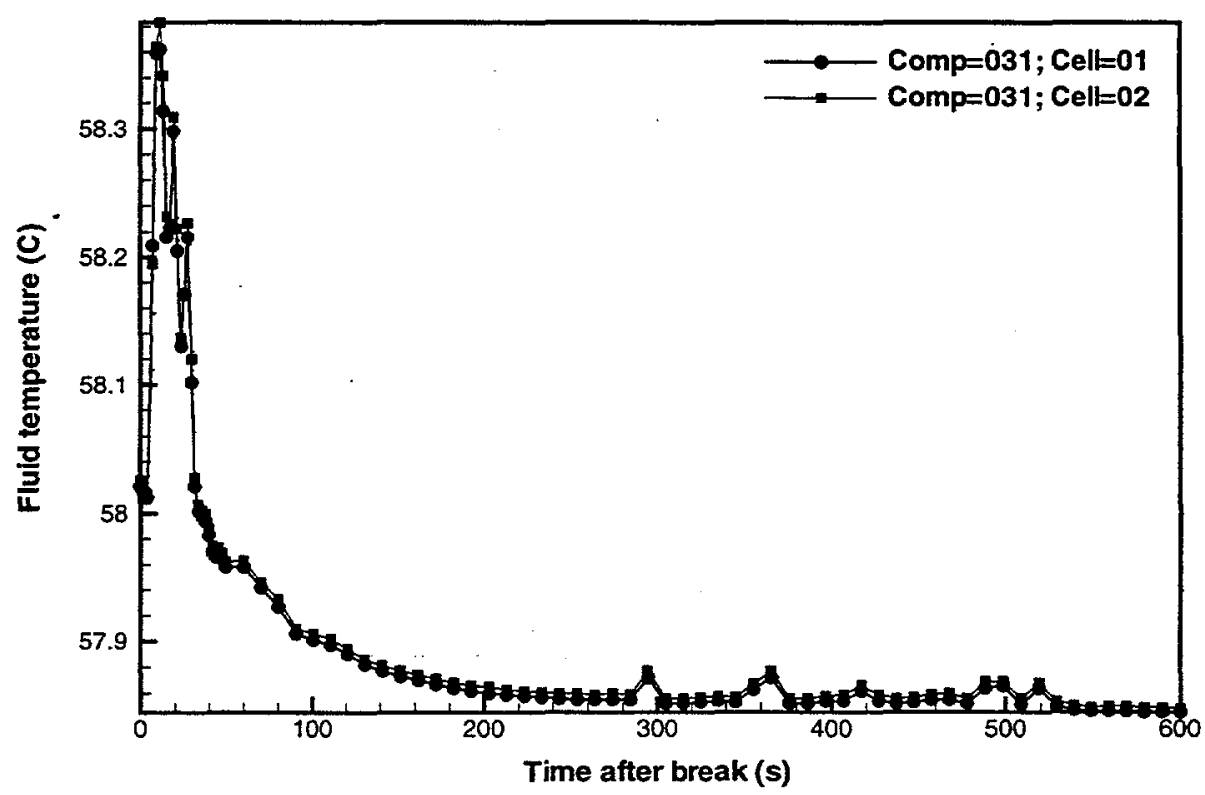

Figure C-15b Primary HR pump 2 fluid temperatures for a LOHGA (Case 2: helium supply plenum break near decoupler outlet). 


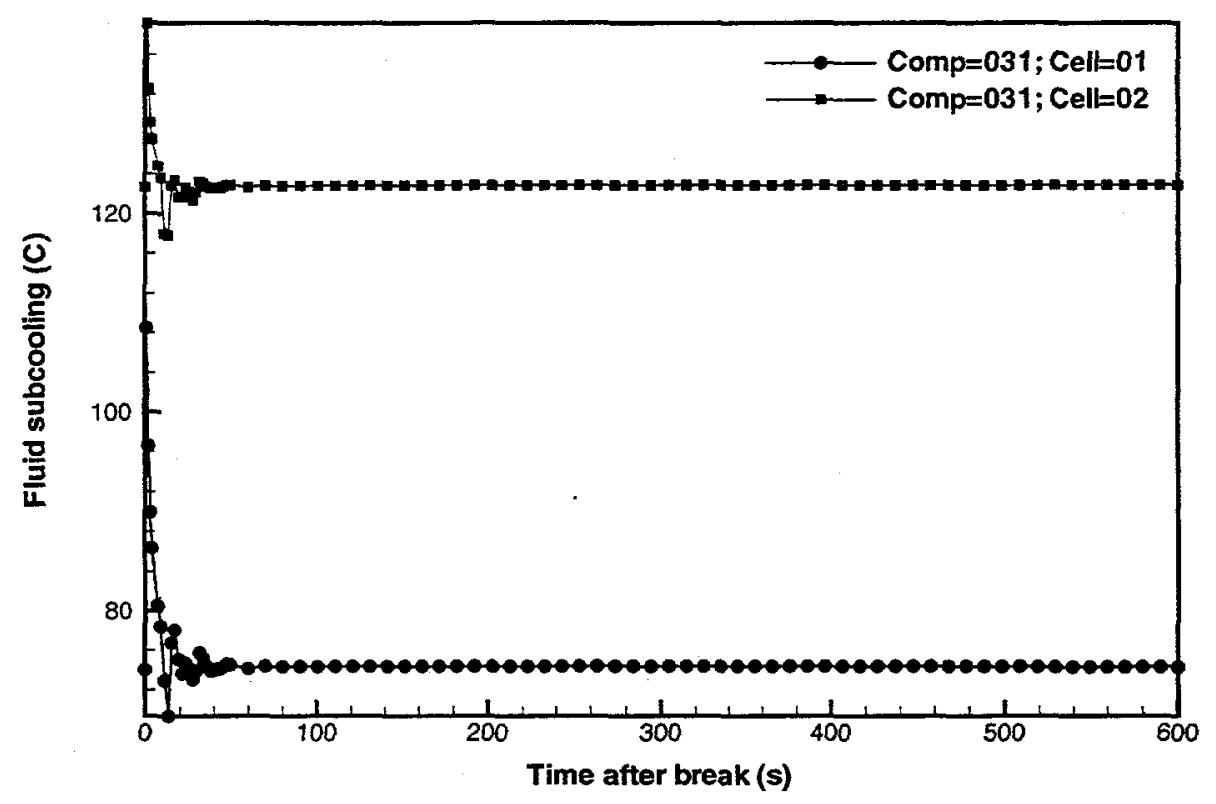

Figure C-15c Primary HR pump 2 fluid subcoolings for a LOHGA (Case 2: helium supply plenum break near decoupler outlet).

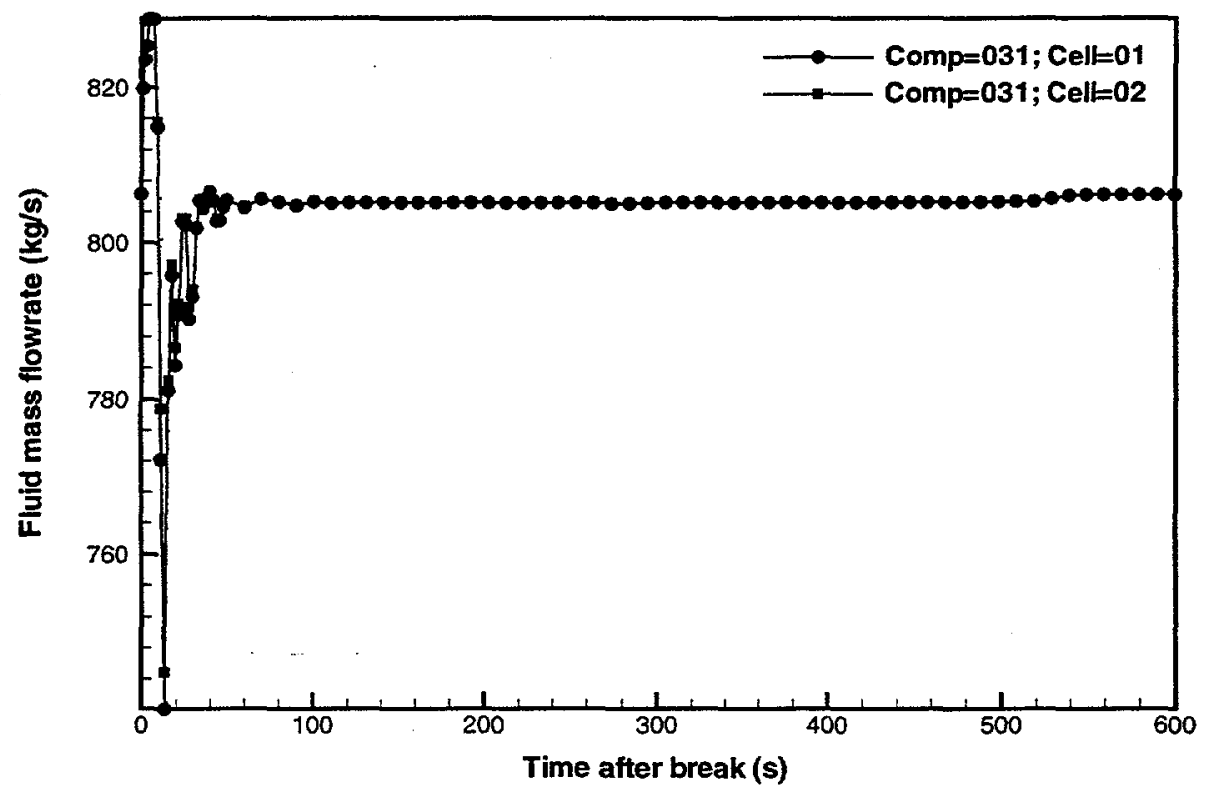

Figure C-15d Primary HR pump 2 liquid mass flowrates for a LOHGA (Case 2: helium supply plenum break near decoupler outlet). 


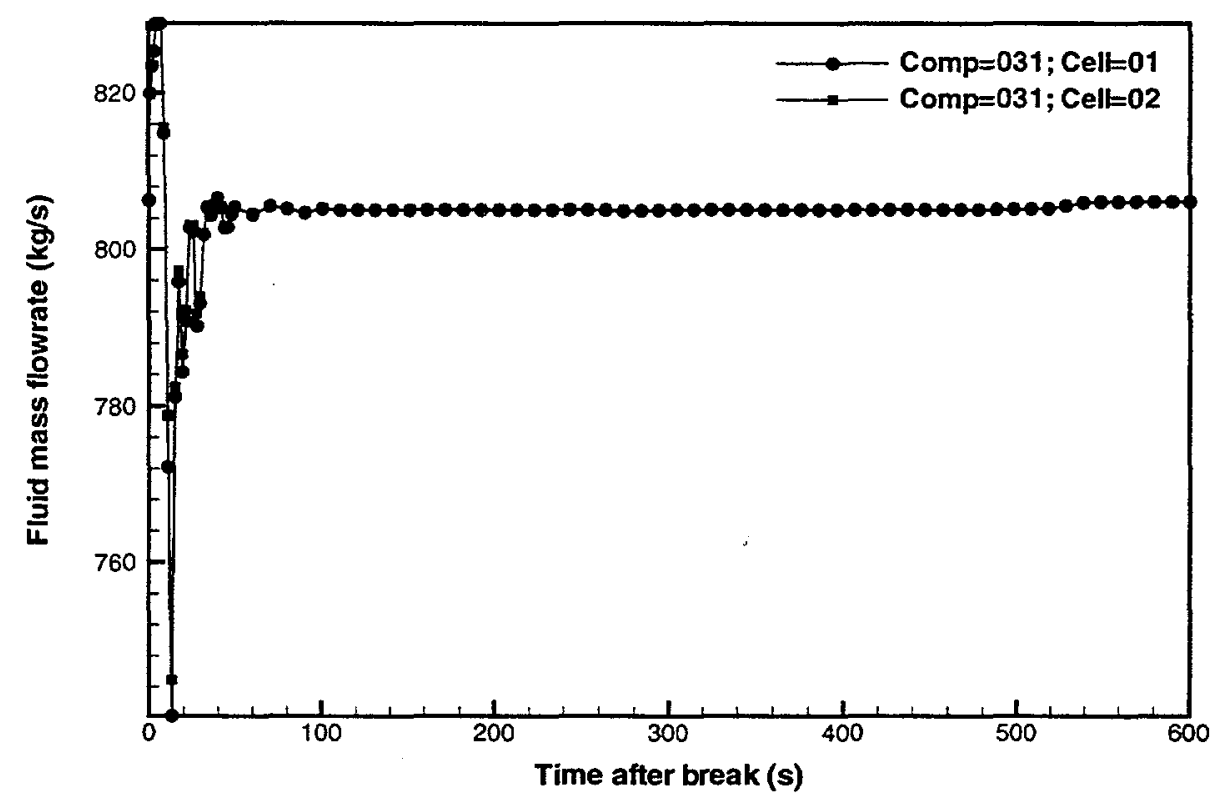

Figure C-15e Primary HR pump 2 void fractions for a LOHGA (Case-2: helium supply plenum break near decoupler outlet).

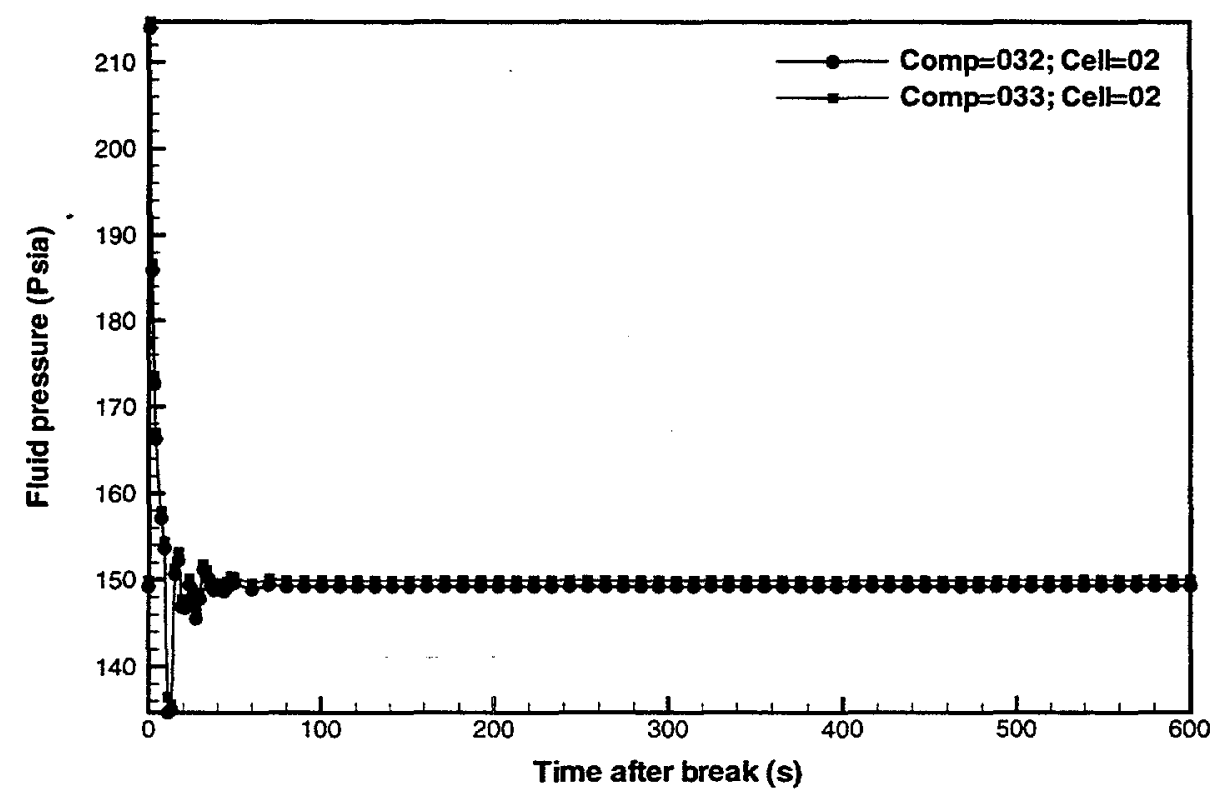

Figure $\mathrm{C}: 16$ a Primary HR pump discharge piping fluid pressures for a LOHGA (Case 2: helium supply plenum break near decoupler outlet). 


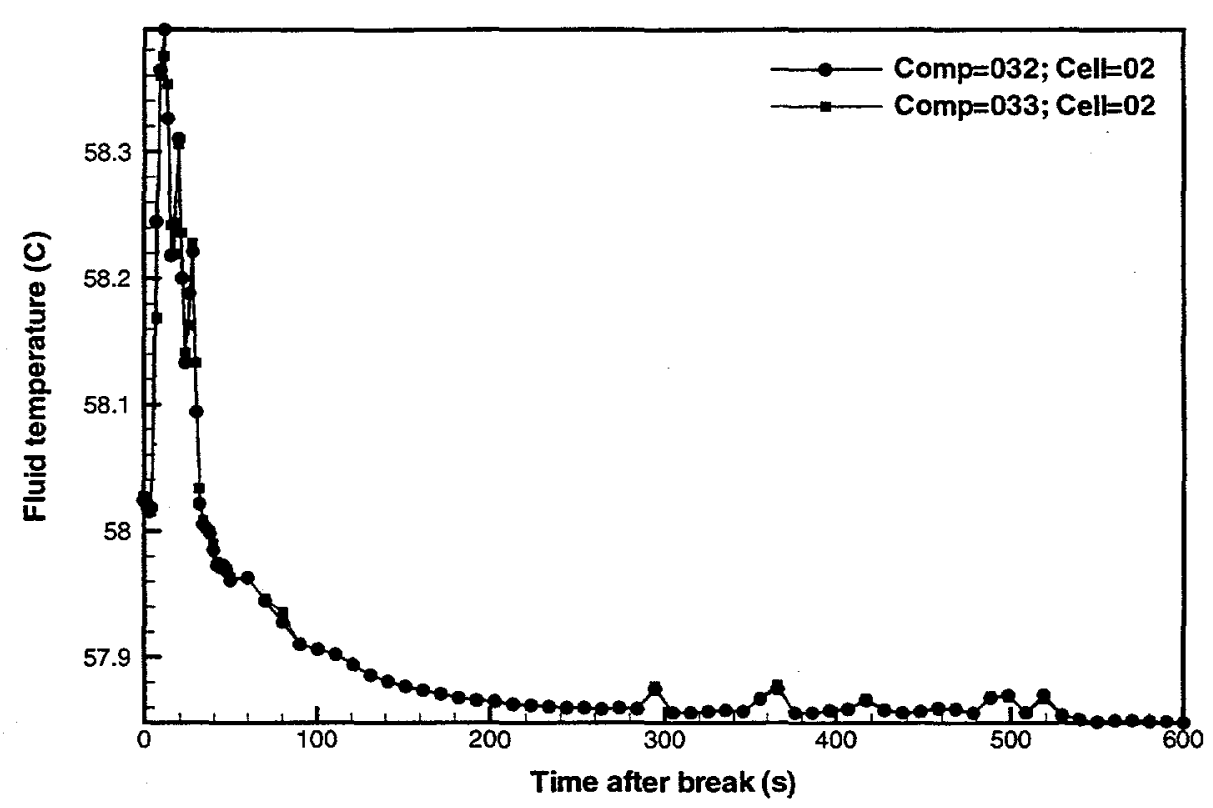

Figure C-16b Primary HR pump discharge piping fluid temperatures for a LOHGA (Case 2: helium supply plenum break near decoupler outlet).

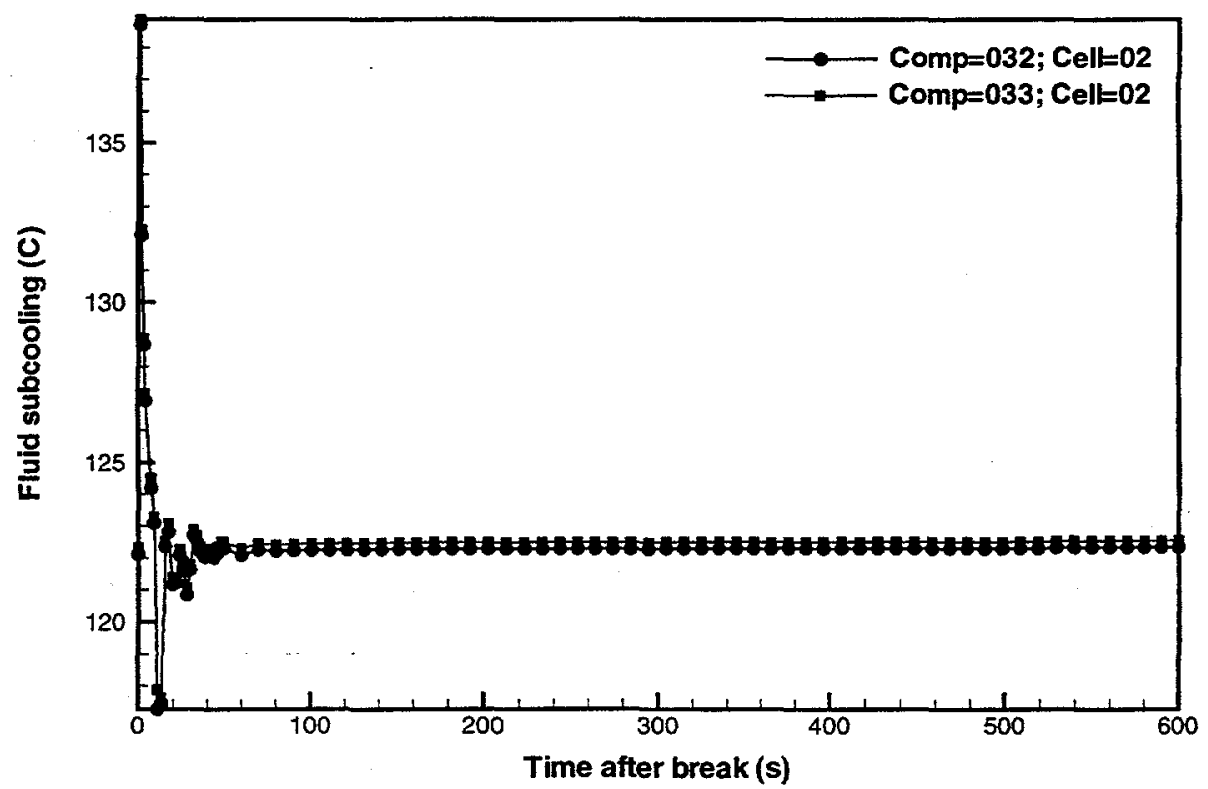

Figure C-16c Primary HR pump discharge piping fluid subcoolings for a LOHGA (Case 2: helium supply plenum break near decoupler outlet). 


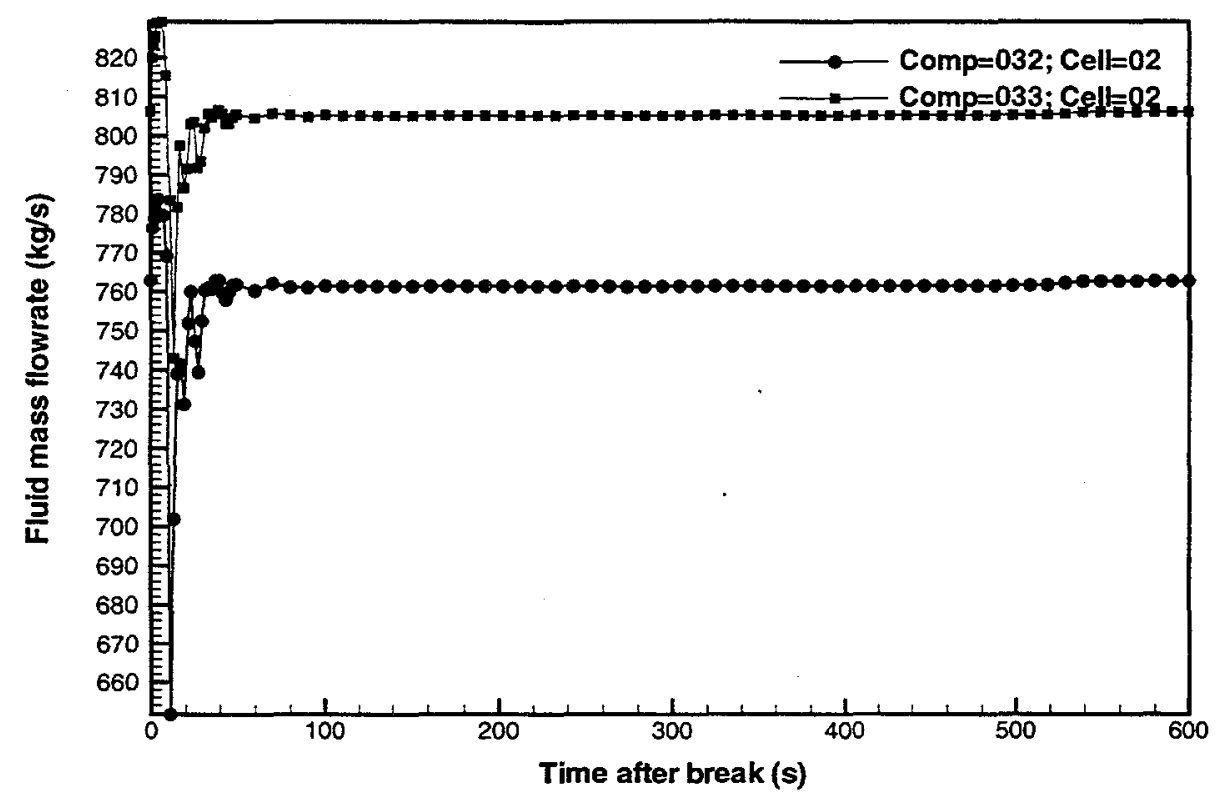

Figure C-16d Primary HR pump discharge piping liquid mass flowrates for a LOHGA (Case 2: helium supply plenum break near decoupler outlet).

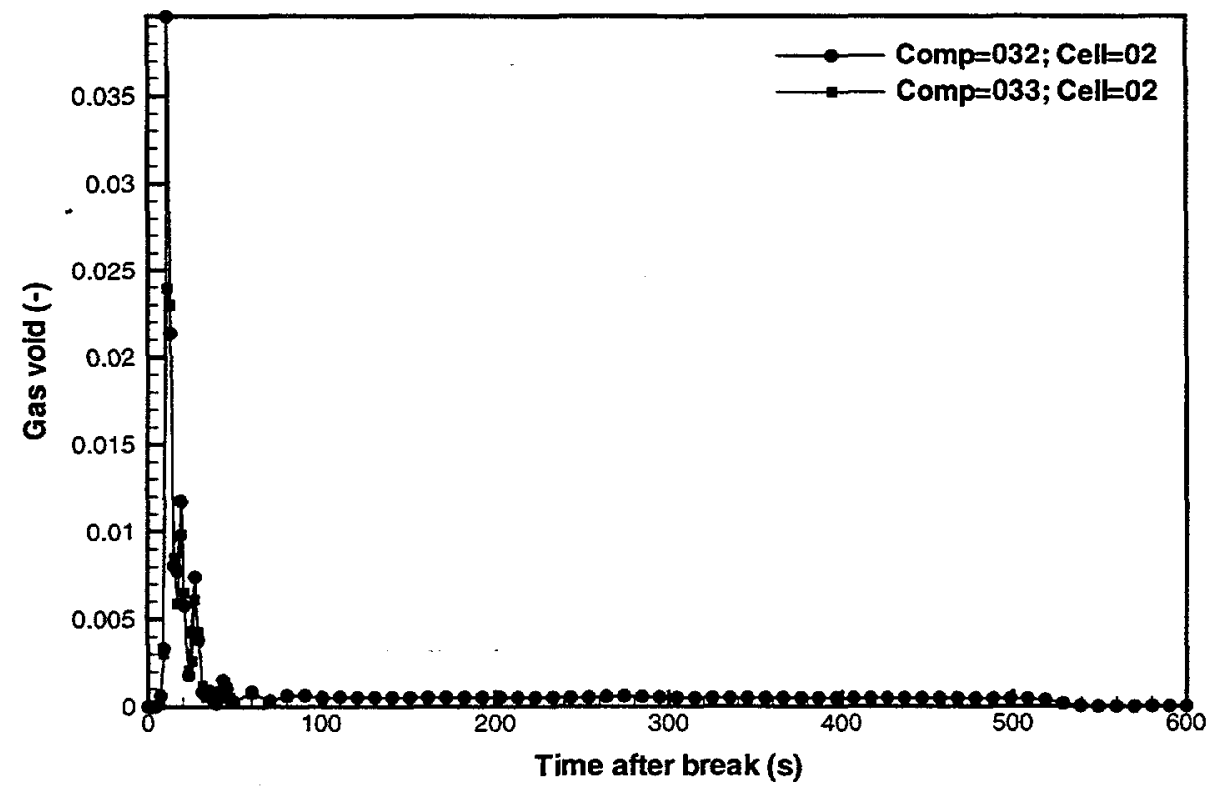

Figure C-16e Primary HR pump discharge piping void fractions for a LOHGA (Case 2: helium supply plenum break near decoupler outlet). 


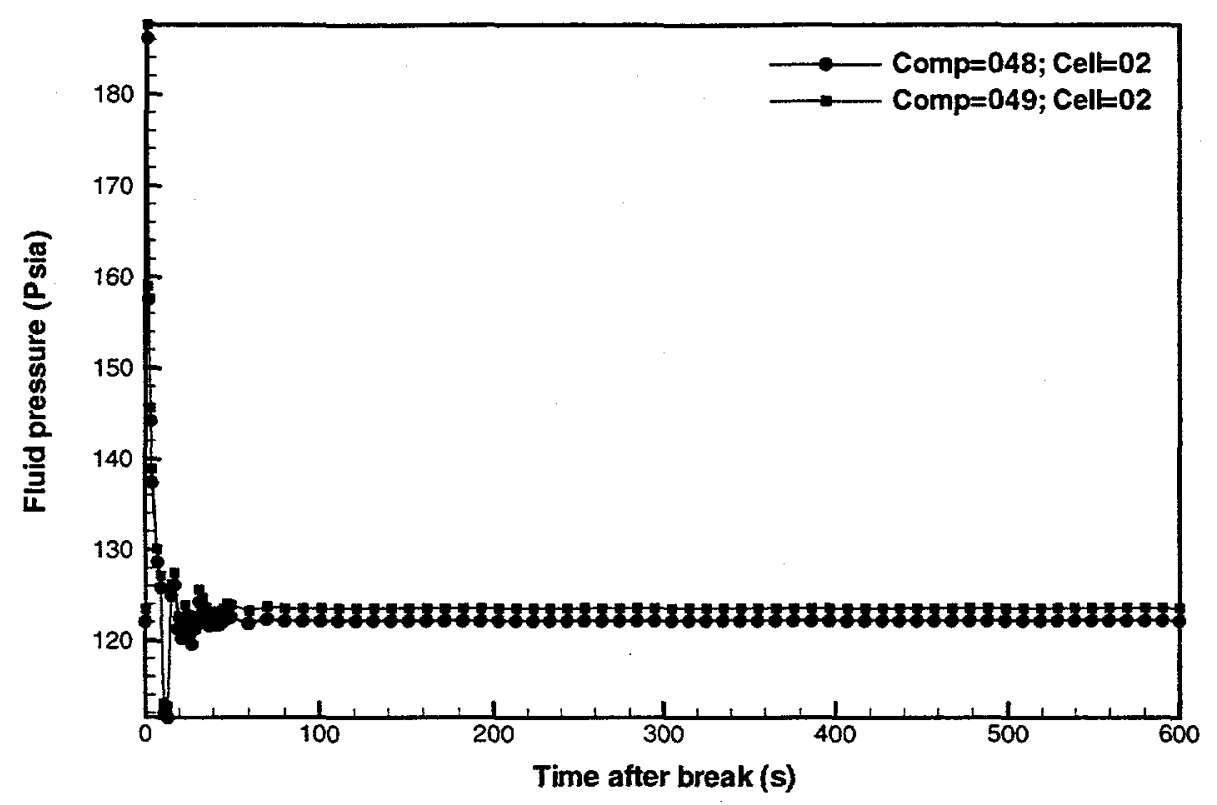

Figure $\mathrm{C}-17$ a Primary HR heat exchanger outlet piping fluid pressures for a LOHGA (Case 2: helium supply plenum break near decoupler outlet).

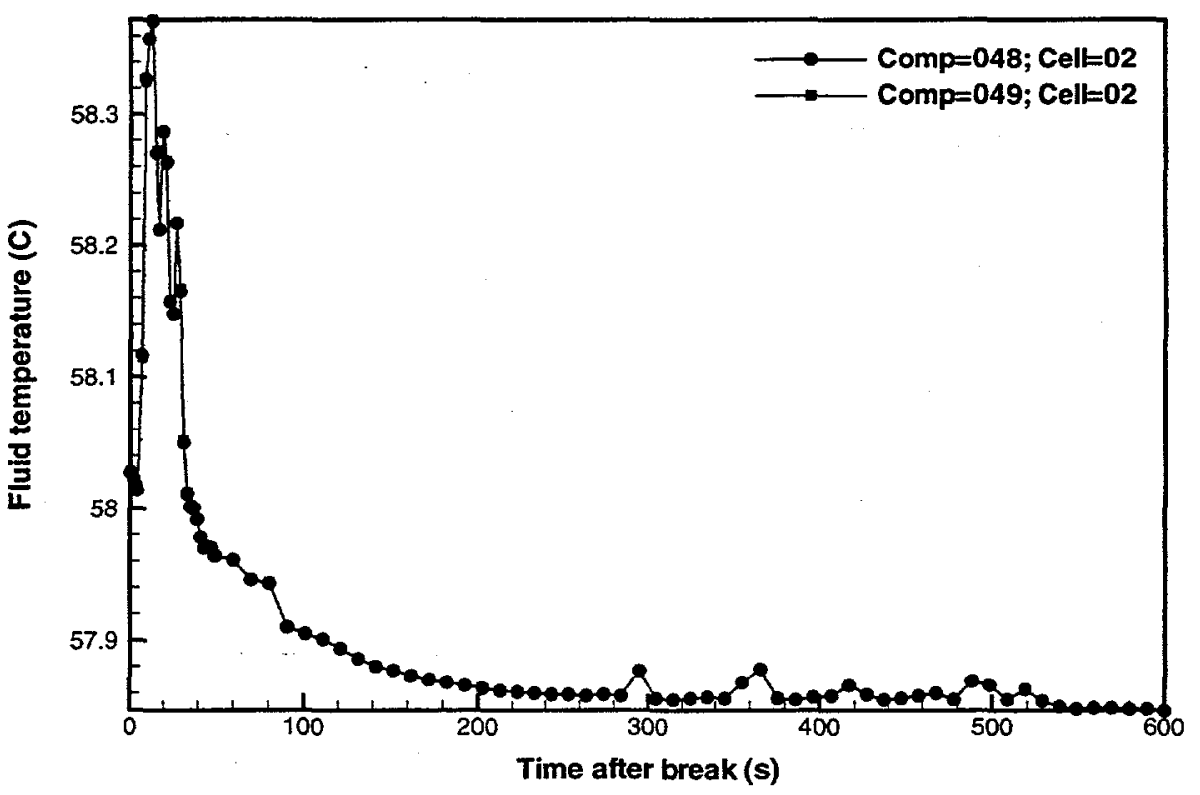

Figure C-17b Primary HR heat exchanger outlet piping fluid temperatures for a LOHGA (Case 2: helium supply plenum break near decoupler outlet). 


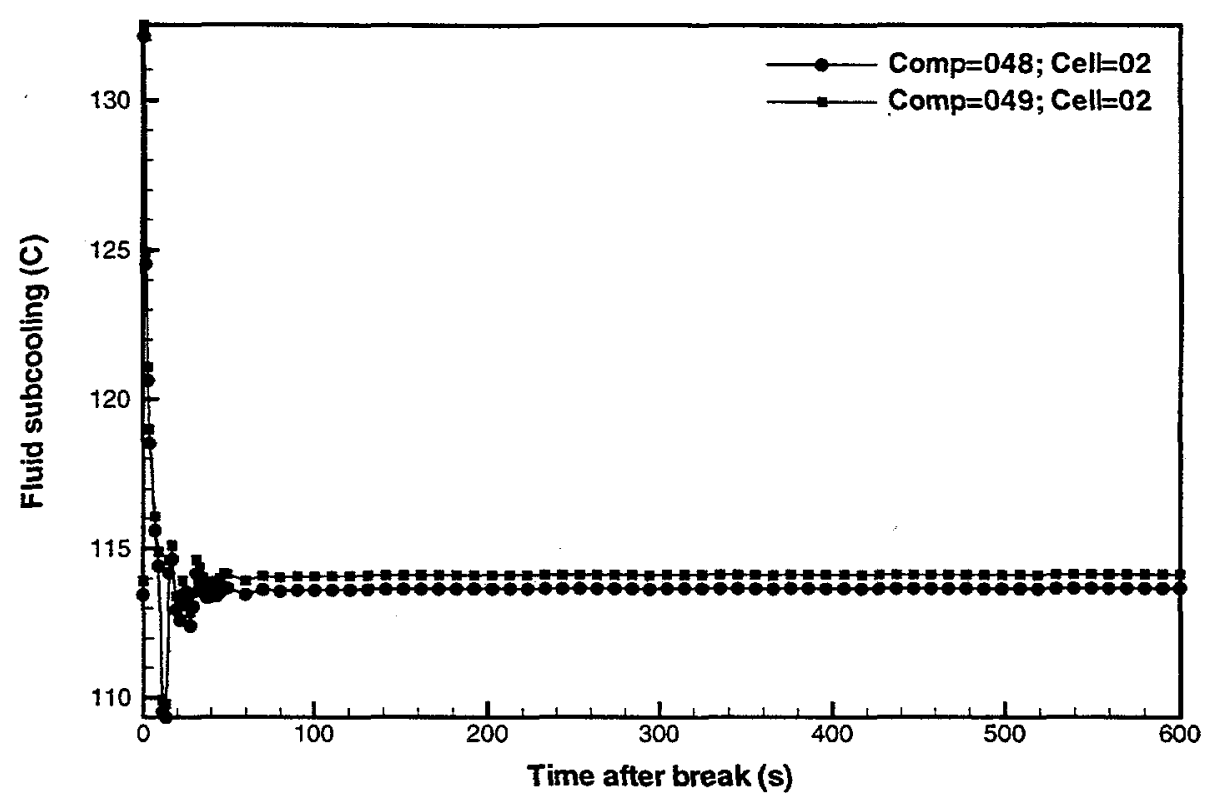

Figure C-17c Primary HR heat exchanger outlet piping fluid subcoolings for a LOHGA (Case 2: helium supply plenum break near decoupler outlet).

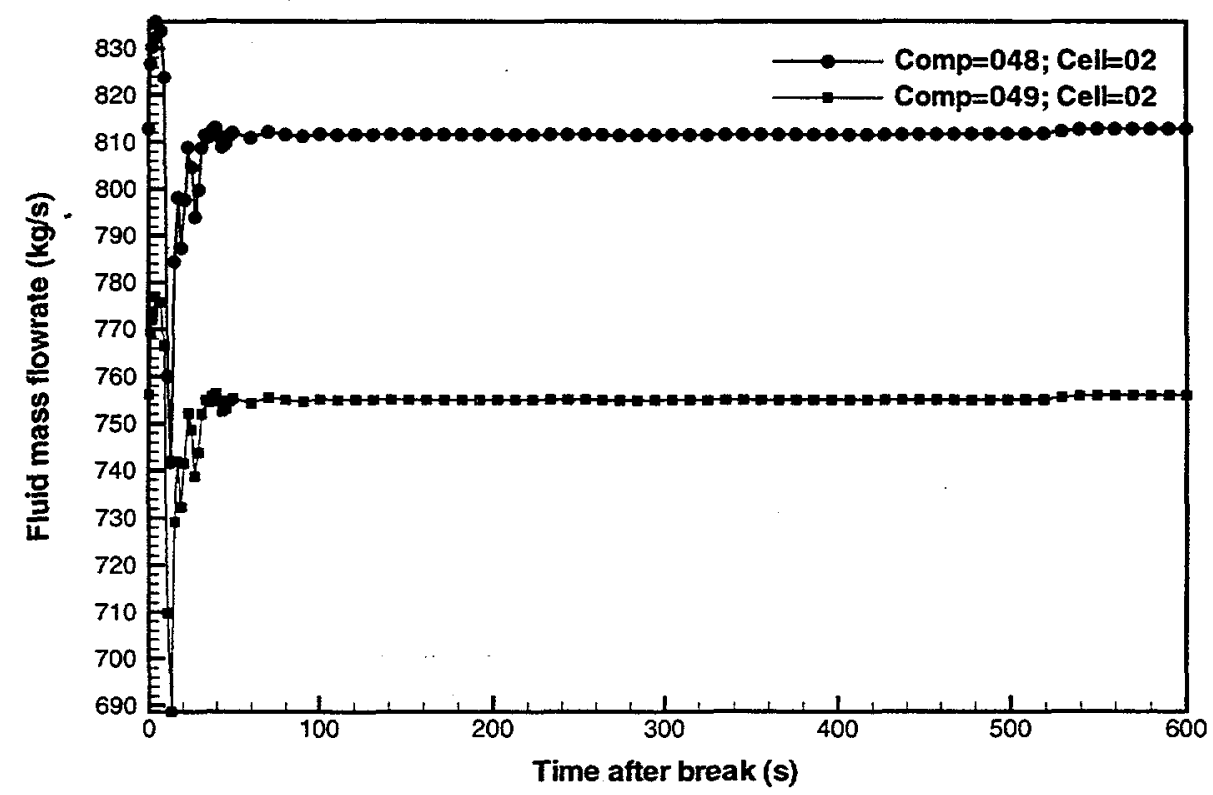

Figure C-17d Primary HR heat exchanger outlet piping liquid mass flowrates for a LOHGA (Case 2: helium supply plenum break near decoupler outlet). 


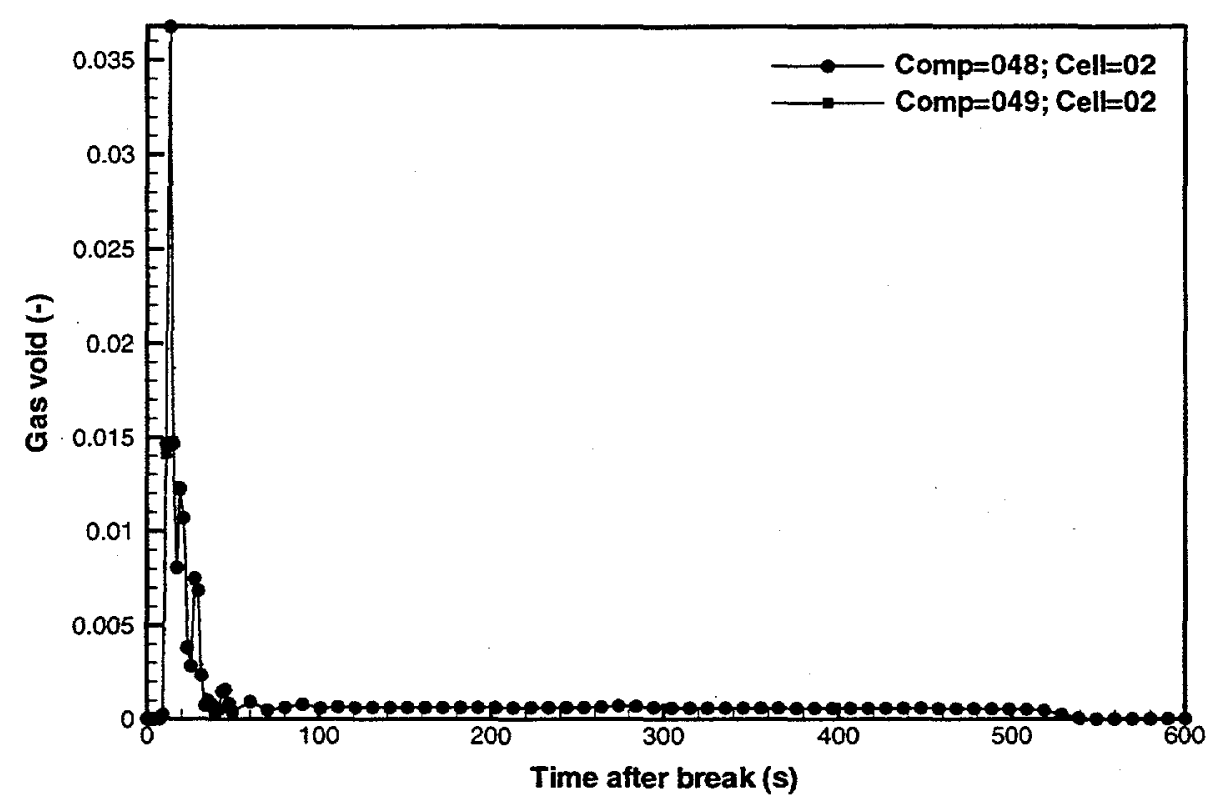

Figure C-17e Primary HR heat exchanger outlet piping void fractions for a LOHGA (Case 2: helium supply plenum break near decoupler outlet).

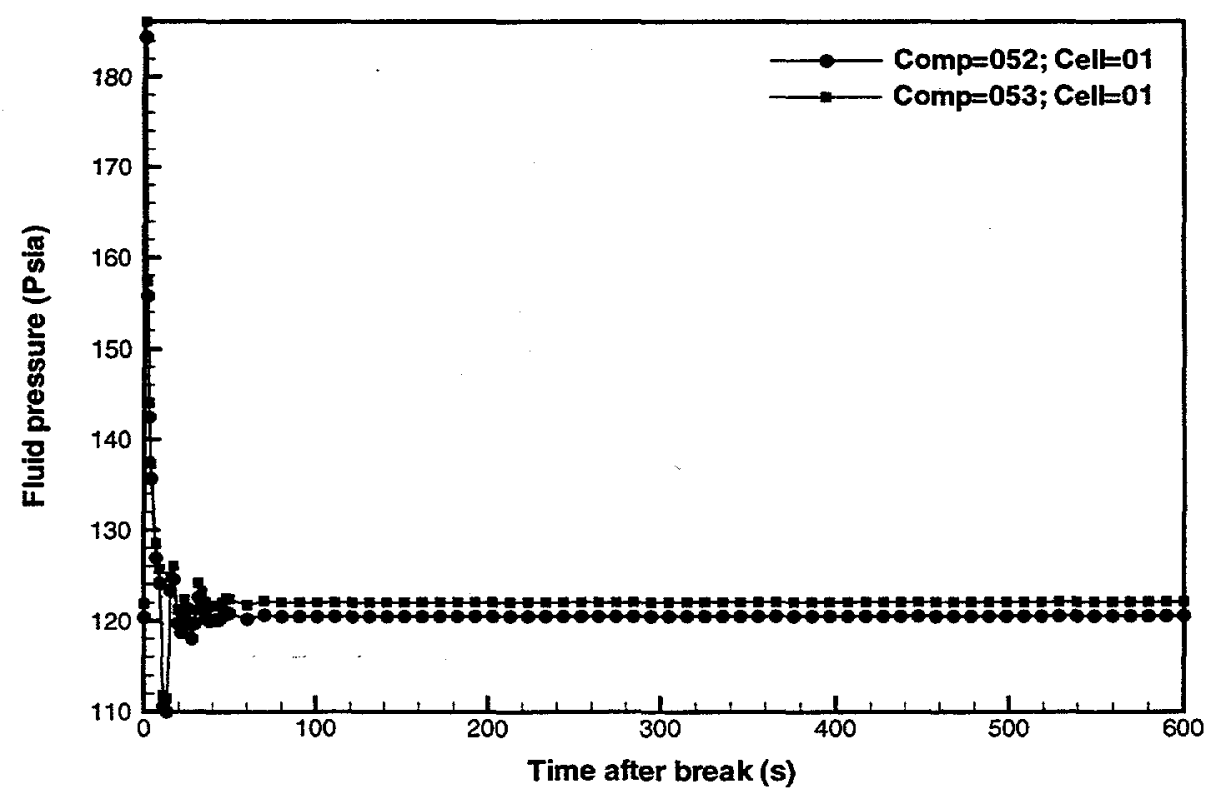

Figure C-18a Primary HR heat exchanger first pass outlet piping fluid pressures for a LOHGA (Case 2: helium supply plenum break near decoupler outlet). 


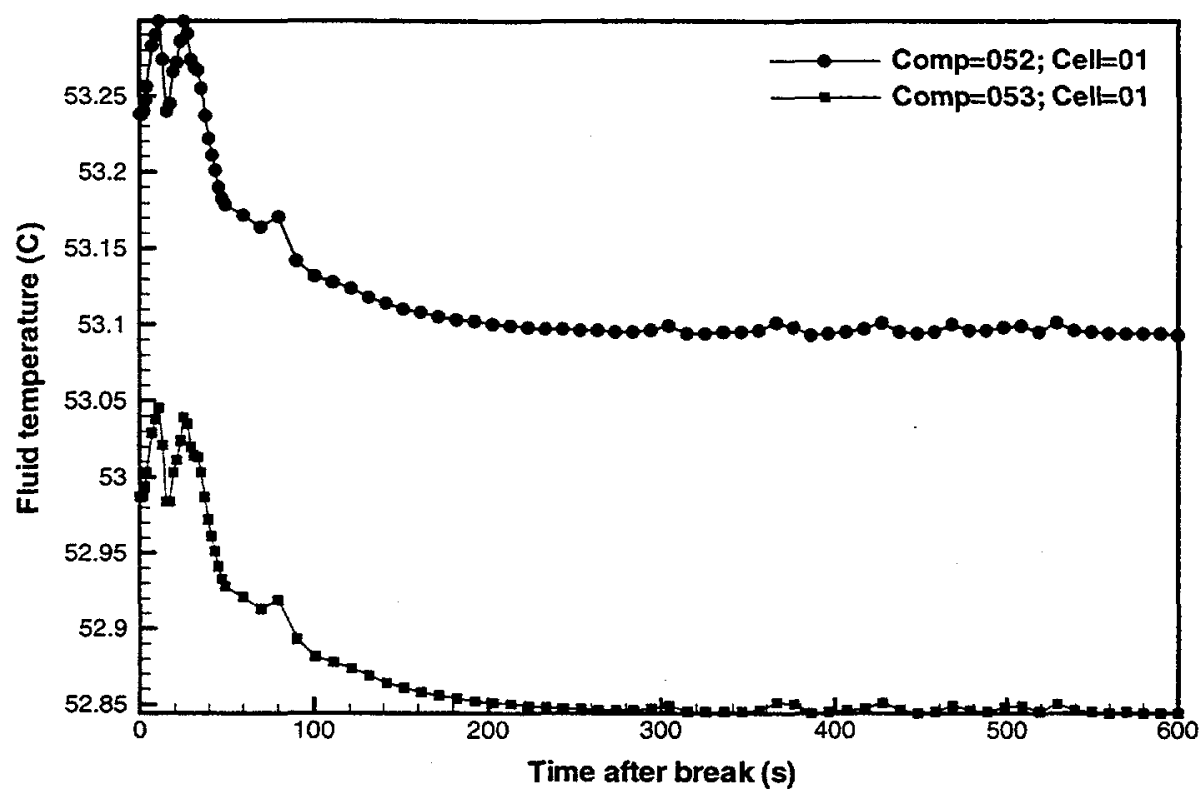

Figure C-18b Primary HR heat exchanger first pass outlet piping fluid temperatures for a LOHGA (Case 2: helium supply plenum break near decoupler outlet).

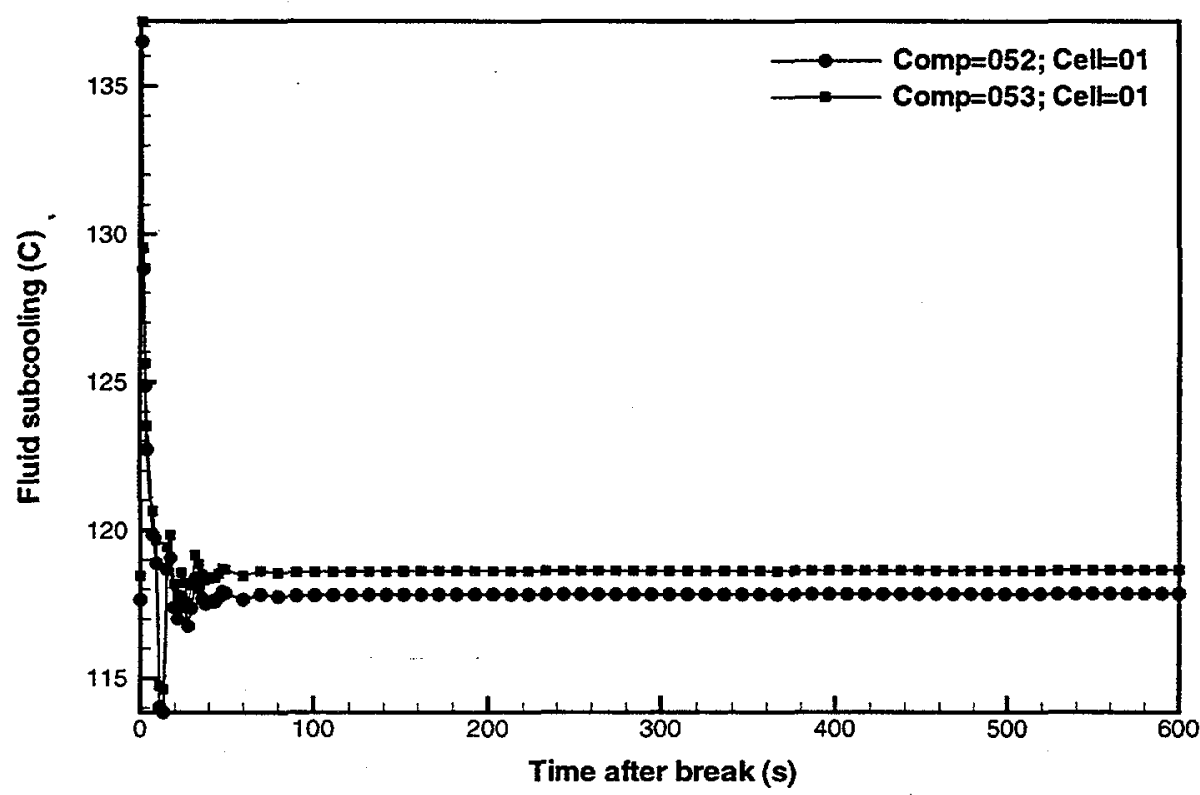

Figure C-18c Primary HR heat exchanger first pass outlet piping fluid subcoolings for a LOHGA (Case 2: helium supply plenum break near decoupler outlet). 


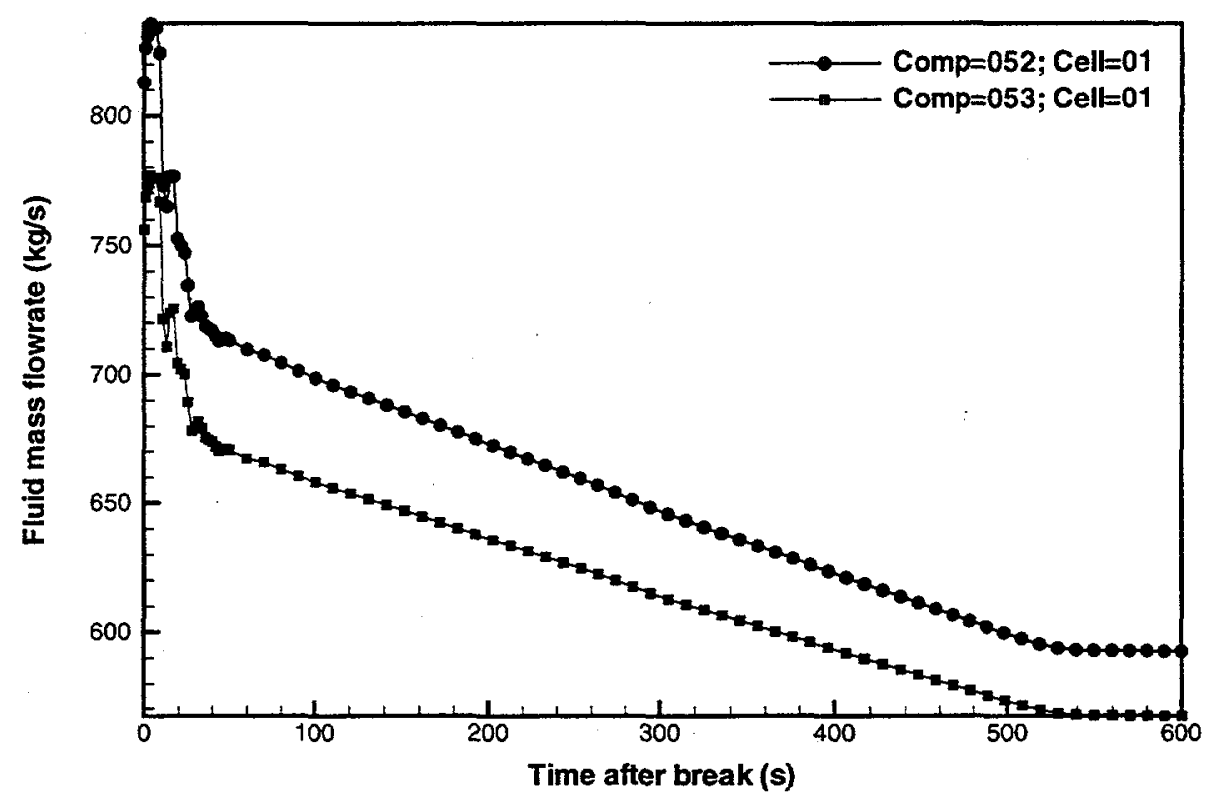

Figure C-18d Primary HR heat exchanger first pass outlet piping liquid mass flowrates for a LOHGA (Case 2: helium supply plenum break near decoupler outlet).

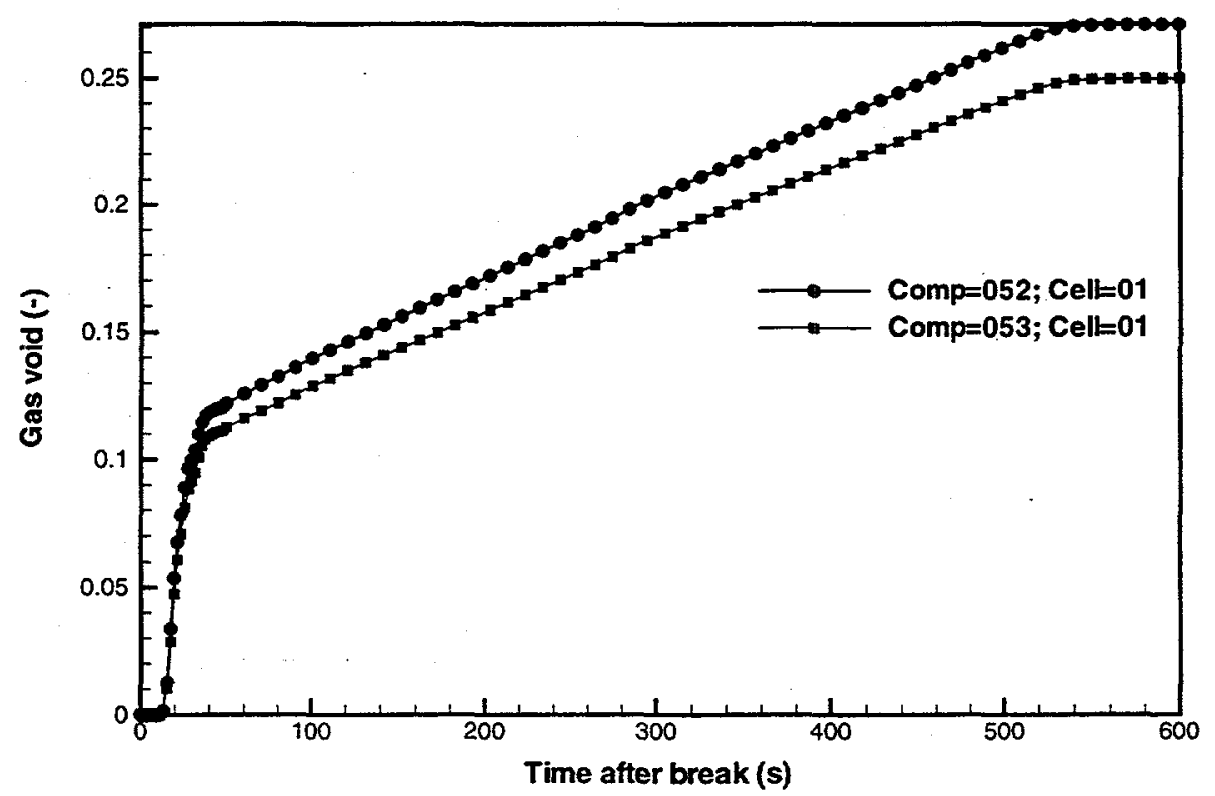

Figure C-18e Primary HR heat exchanger first pass outlet piping void fractions for a LOHGA (Case 2: helium supply plenum break near decoupler outlet). 


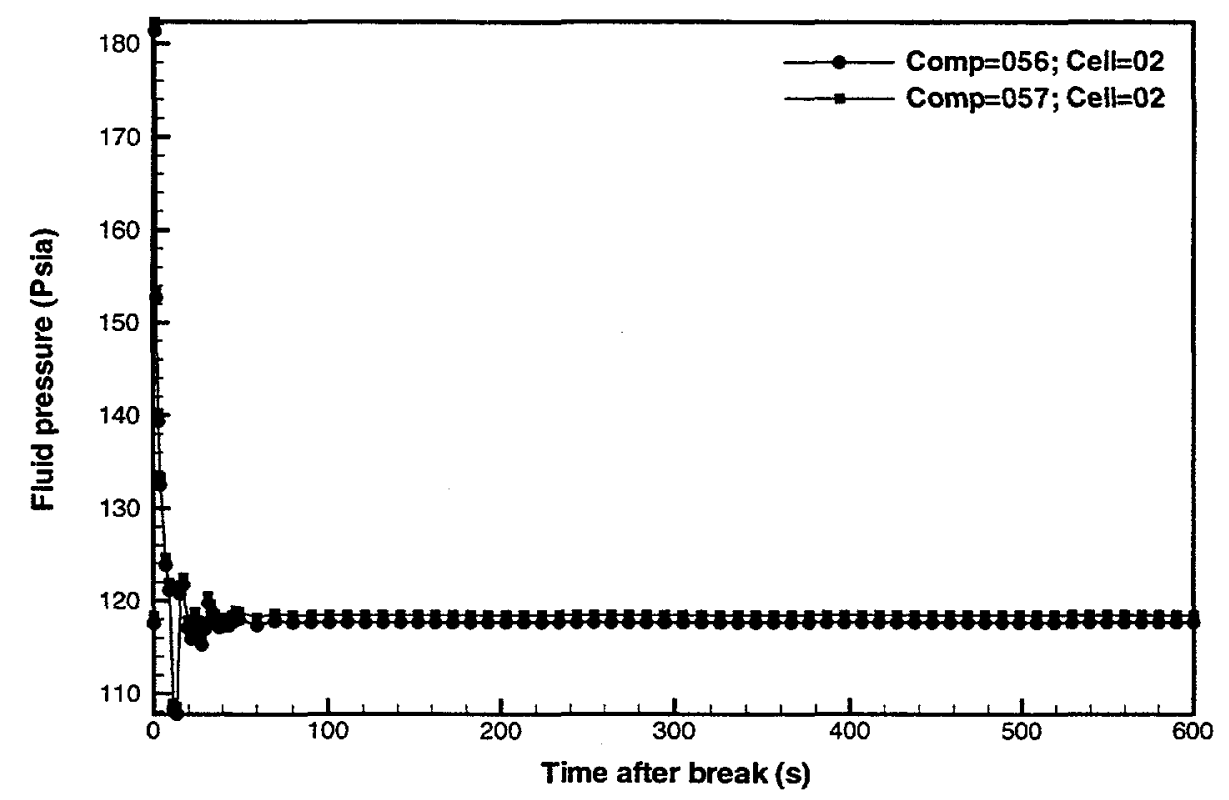

Figure C-19a Primary HR heat exchanger outlet piping fluid pressures for a LOHGA (Case 2: helium supply plenum break near decoupler outlet).

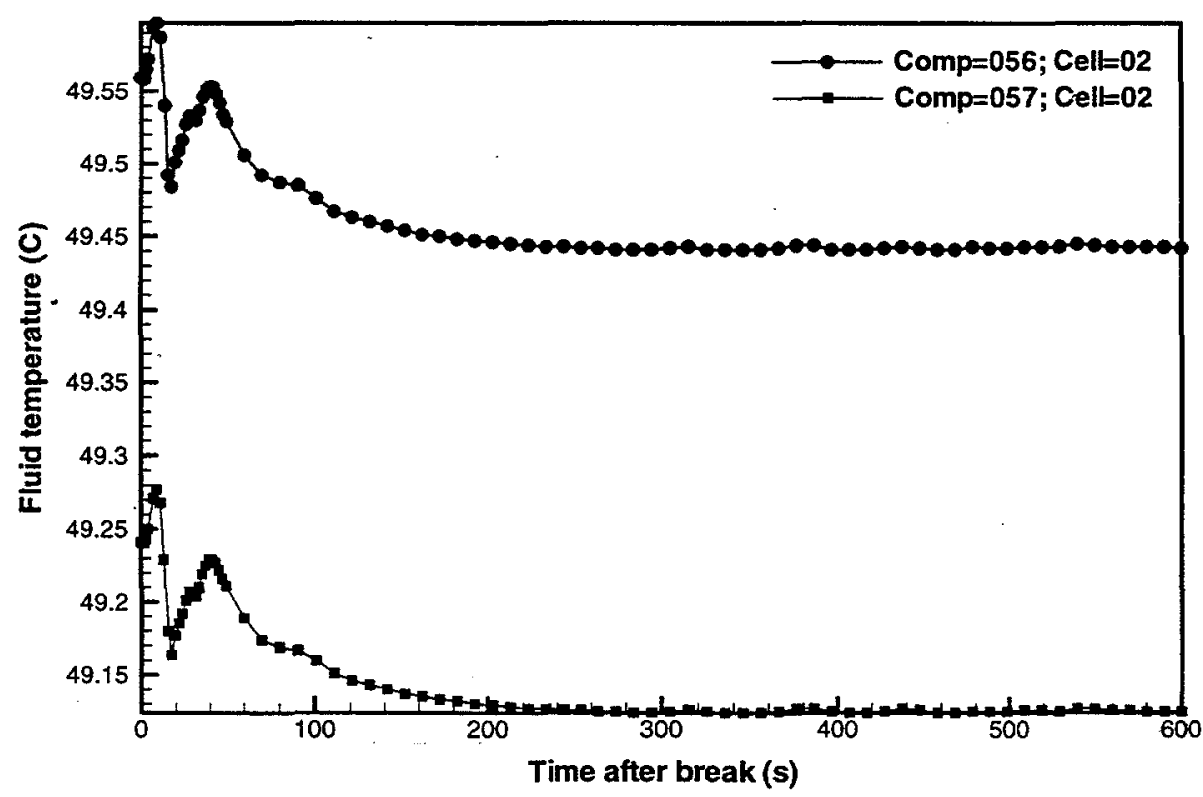

Figure C-19b Primary HR heat exchanger outlet piping fluid temperatures for a LOHGA (Case 2: helium supply plenum break near decoupler outlet). 


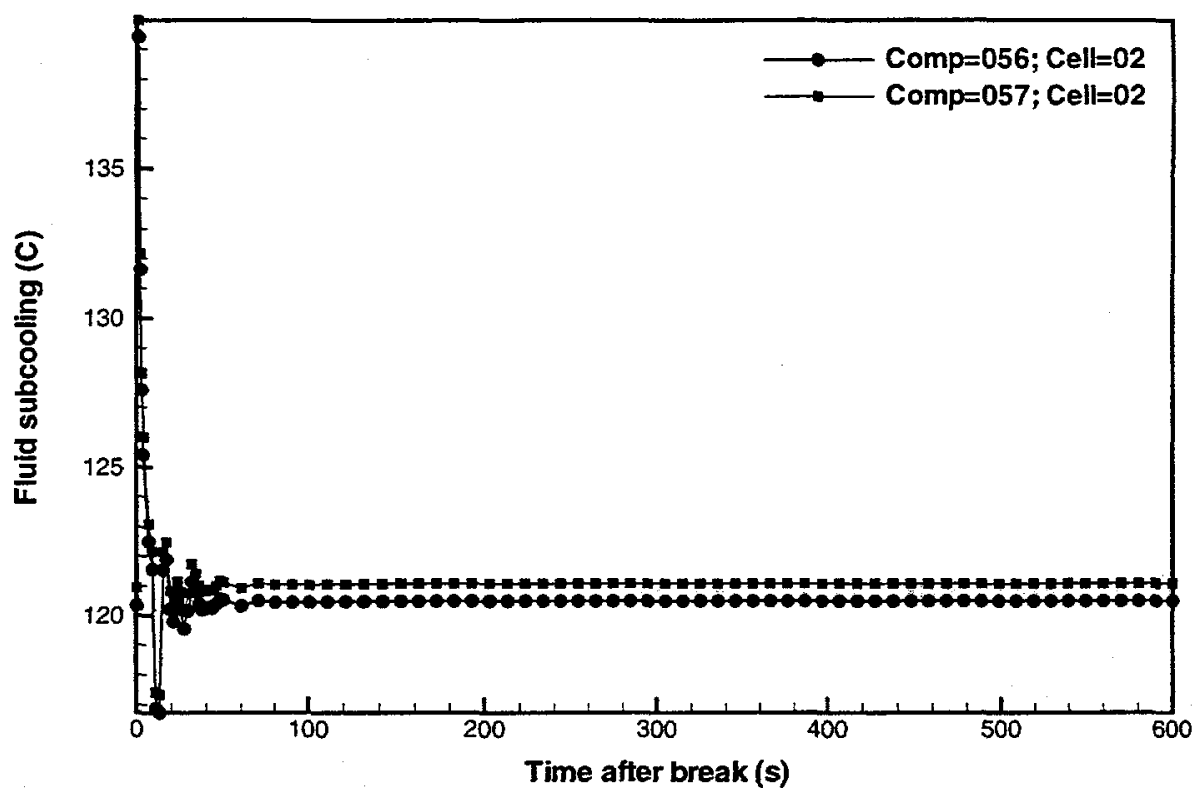

Figure C-19c Primary HR heat exchanger outlet piping fluid subcoolings for a LOHGA (Case 2: helium supply plenum break near decoupler outlet).

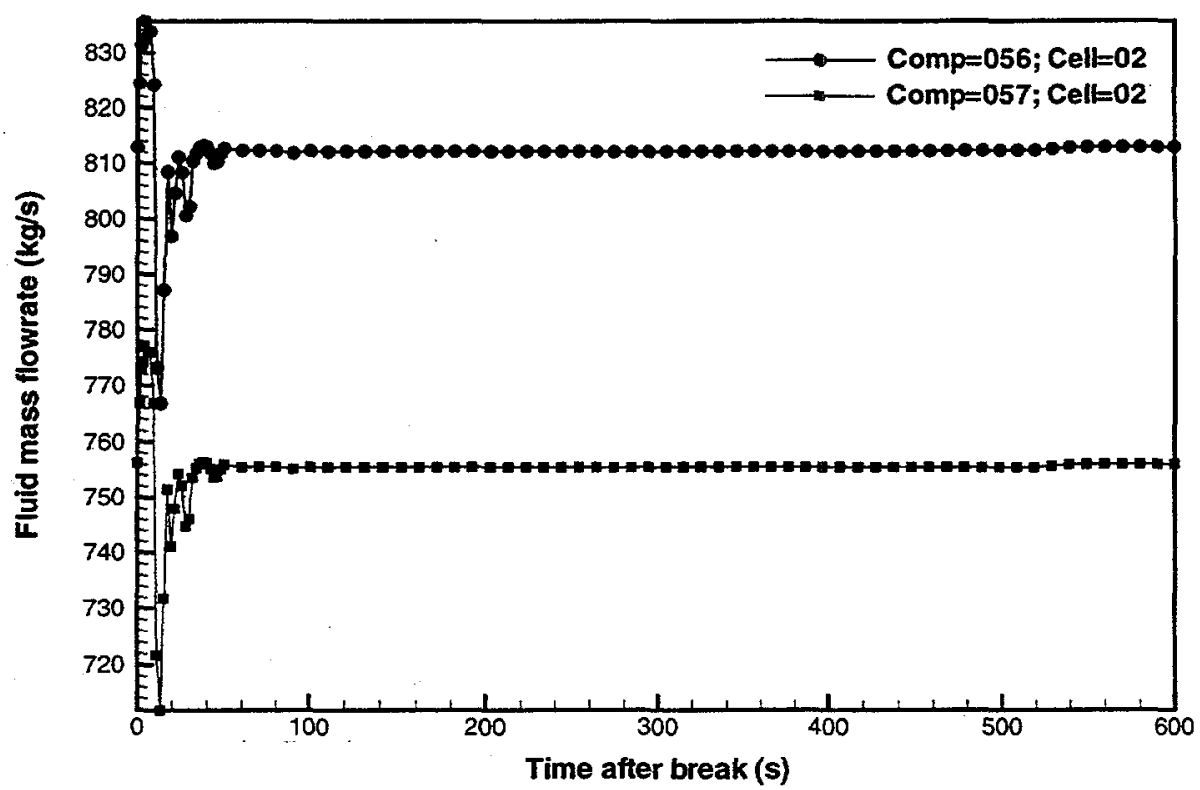

Figure C-19d Primary HR heat exchanger outlet piping liquid mass flowrates for a LOHGA (Case 2: helium supply plenum break near decoupler outlet). 


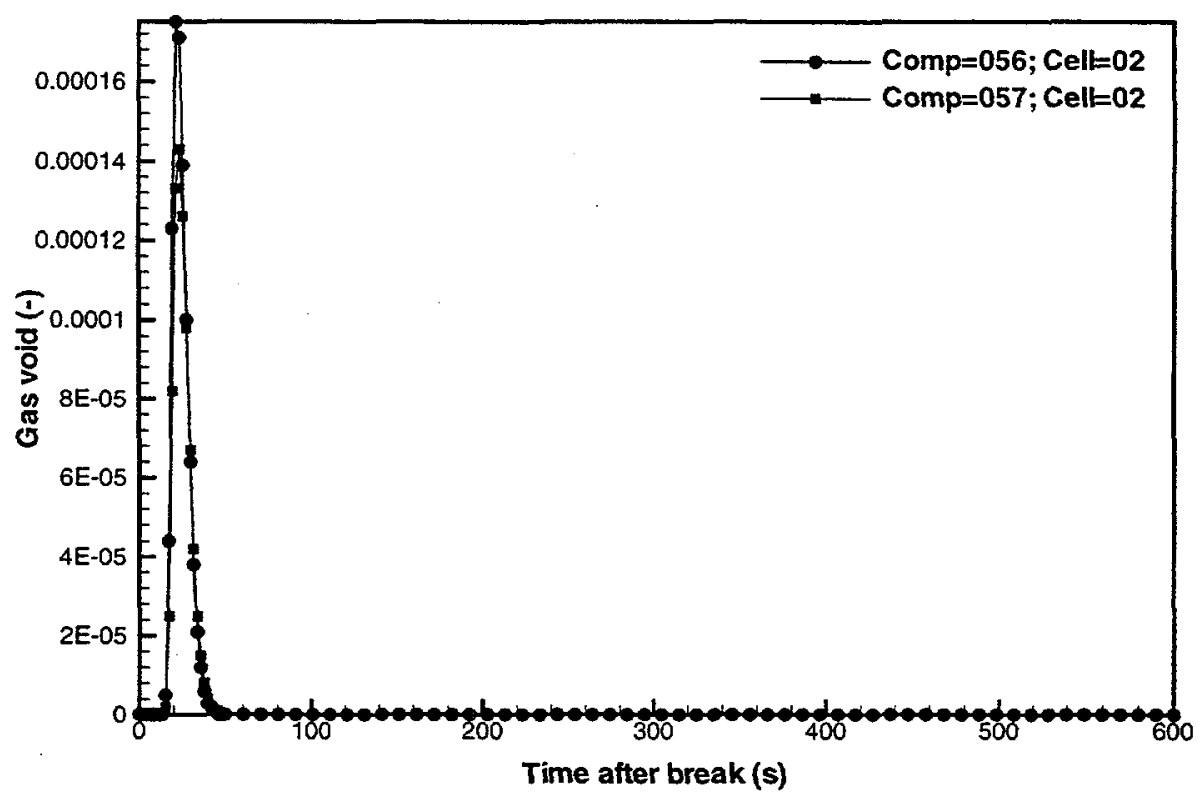

Figure C-19e Primary HR heat exchanger outlet piping void fractions for a LOHGA (Case 2: helium supply plenum break near decoupler outlet).

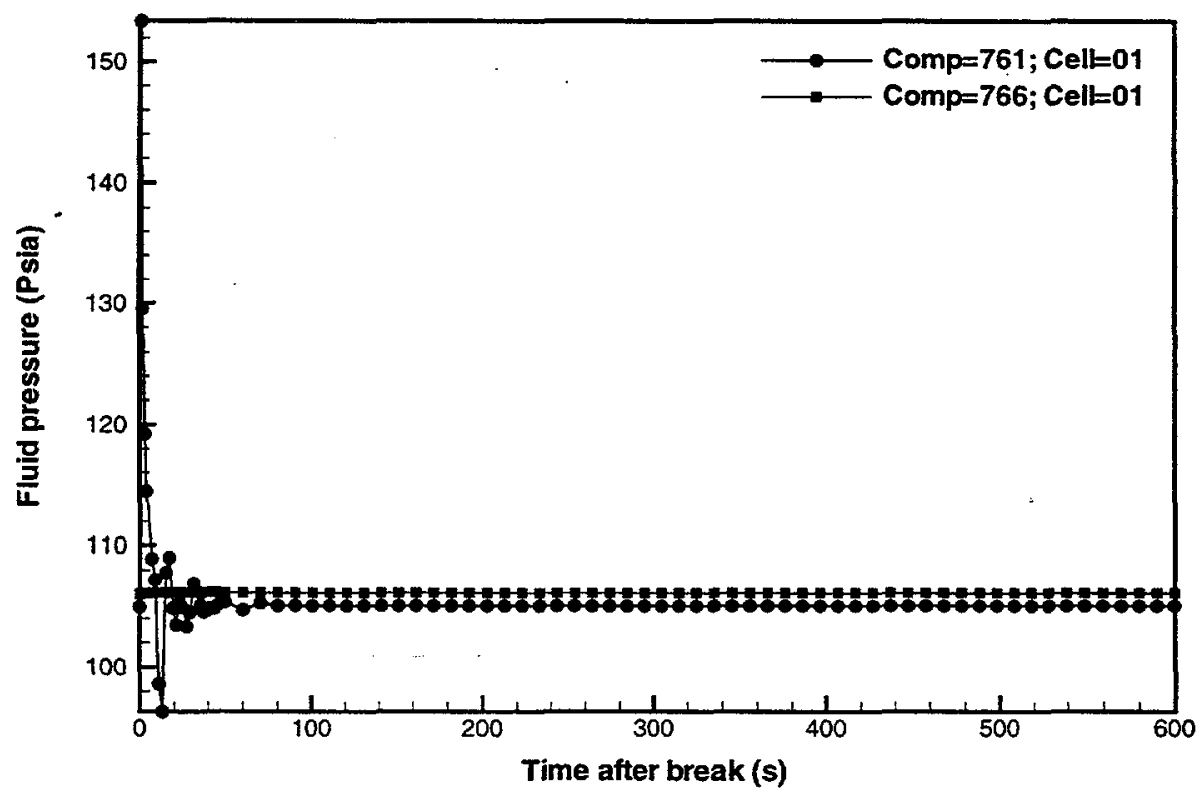

Figure C-20a Primary HR pressurizer and surge line fluid pressures for a LOHGA (Case 2: helium supply plenum break near decoupler outlet). 


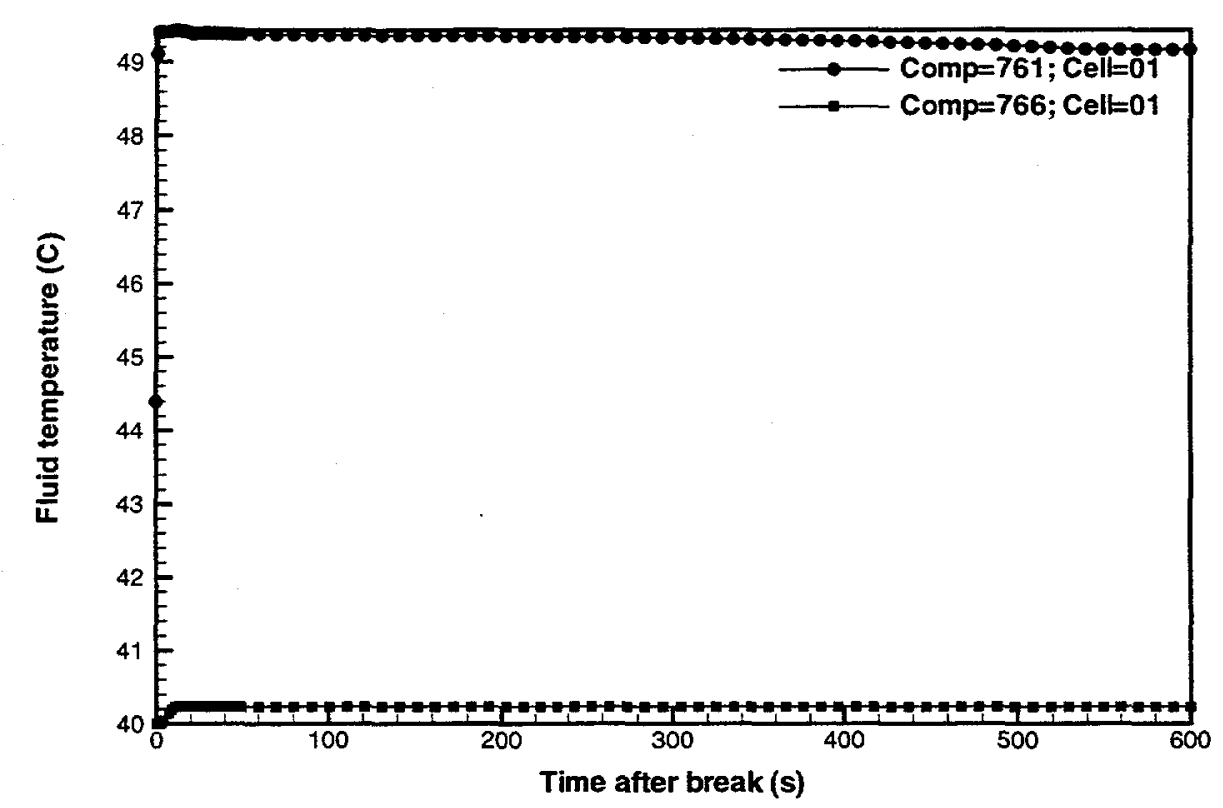

Figure C-20b Primary HR pressurizer and surge line fluid temperatures for a LOHGA (Case 2: helium supply plenum break near decoupler outlet).

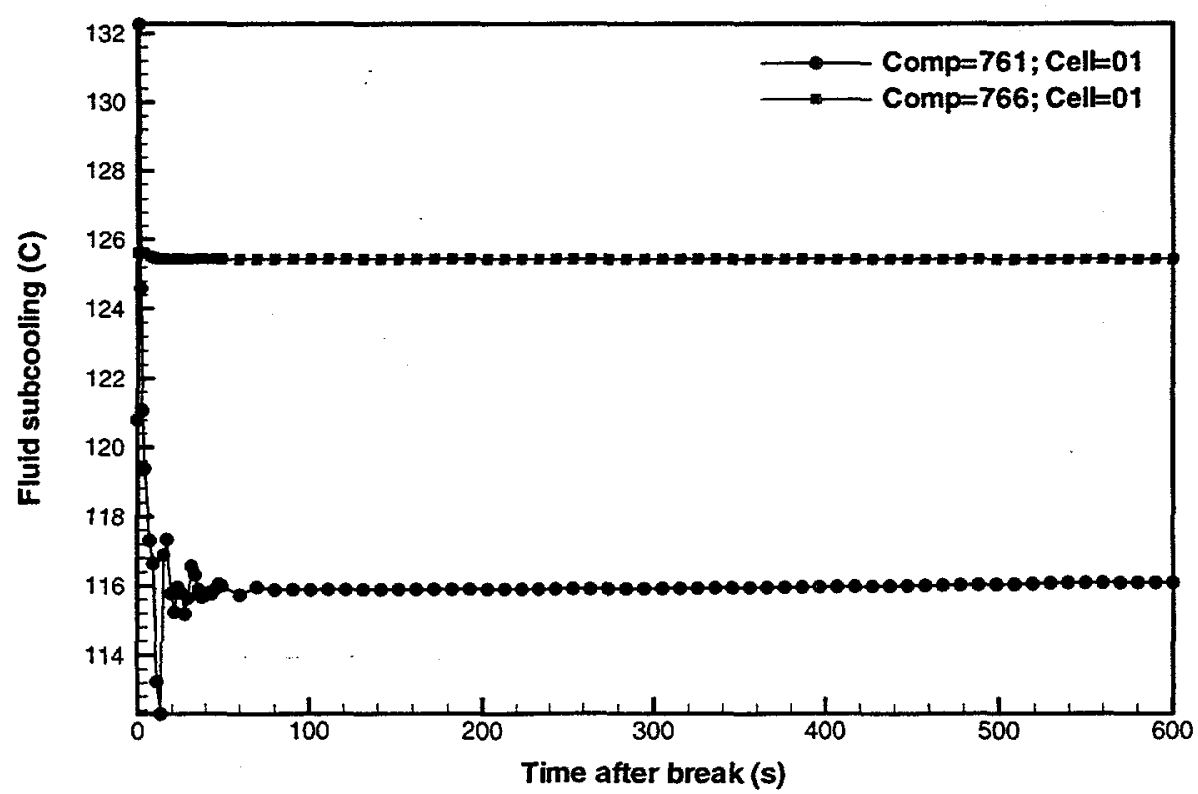

Figure C-20c Primary HR pressurizer and surge line fluid subcoolings for a LOHGA (Case 2: helium supply plenum break near decoupler outlet). 


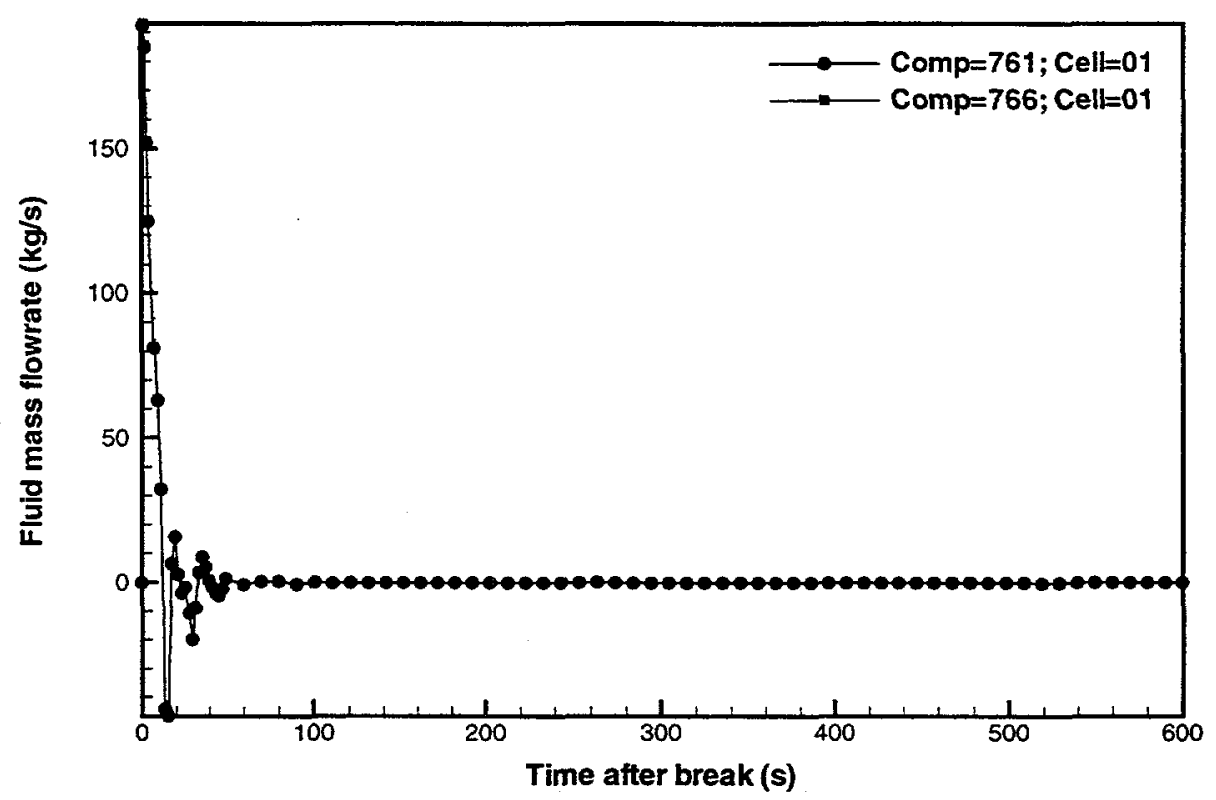

Figure C-20d Primary HR pressurizer and surge line liquid mass flowrates for a LOHGA (Case 2: helium supply plenum break near decoupler outlet).

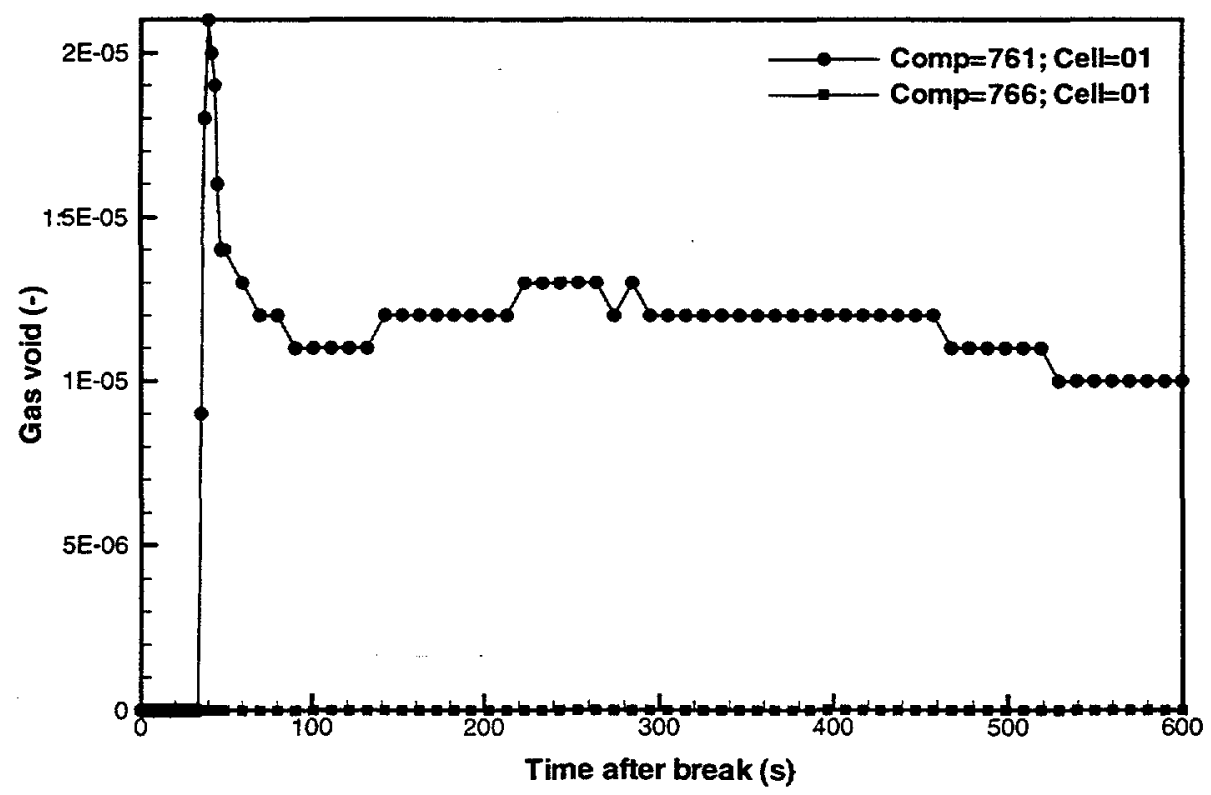

Figure C-20e Primary HR pressurizer and surge line void fractions for a LOHGA (Case 2: helium supply plenum break near decoupler outlet). 


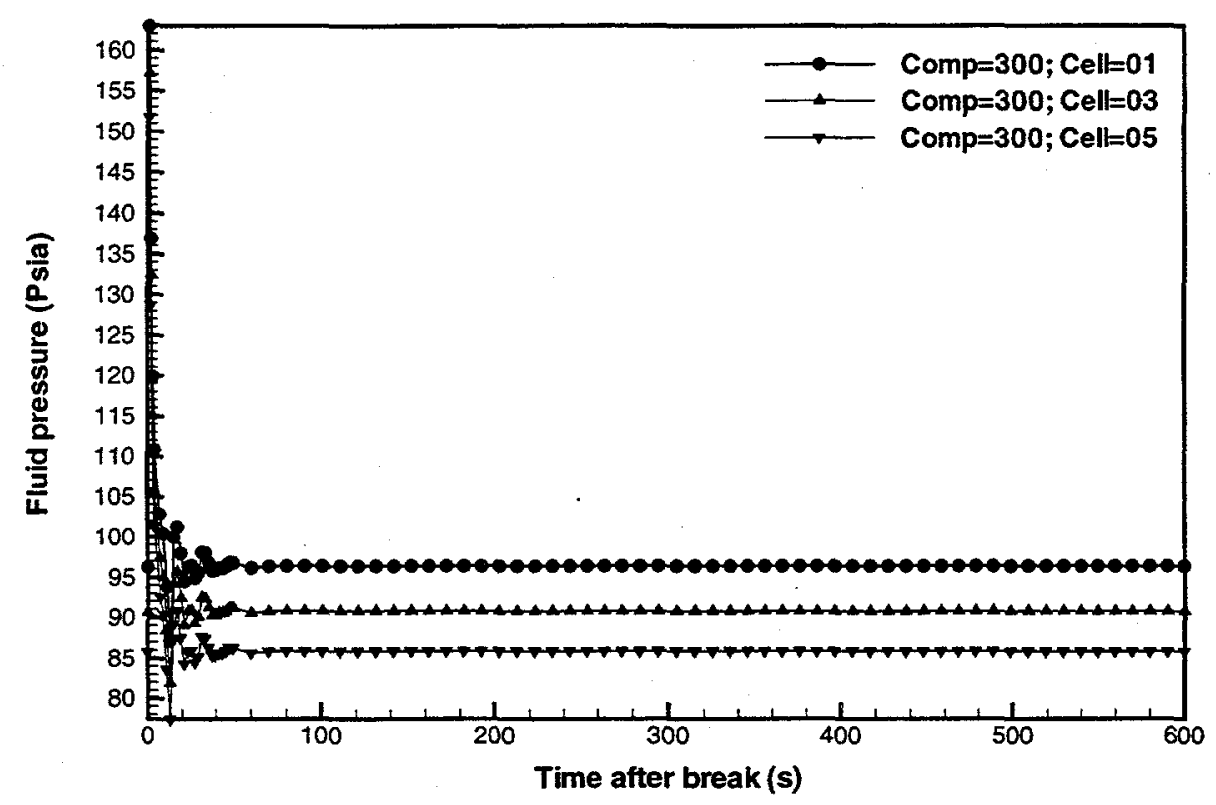

Figure C-21a Module 1 row 1 channel fluid pressures for a LOHGA (Case 2: helium supply plenum break near decoupler outlet).

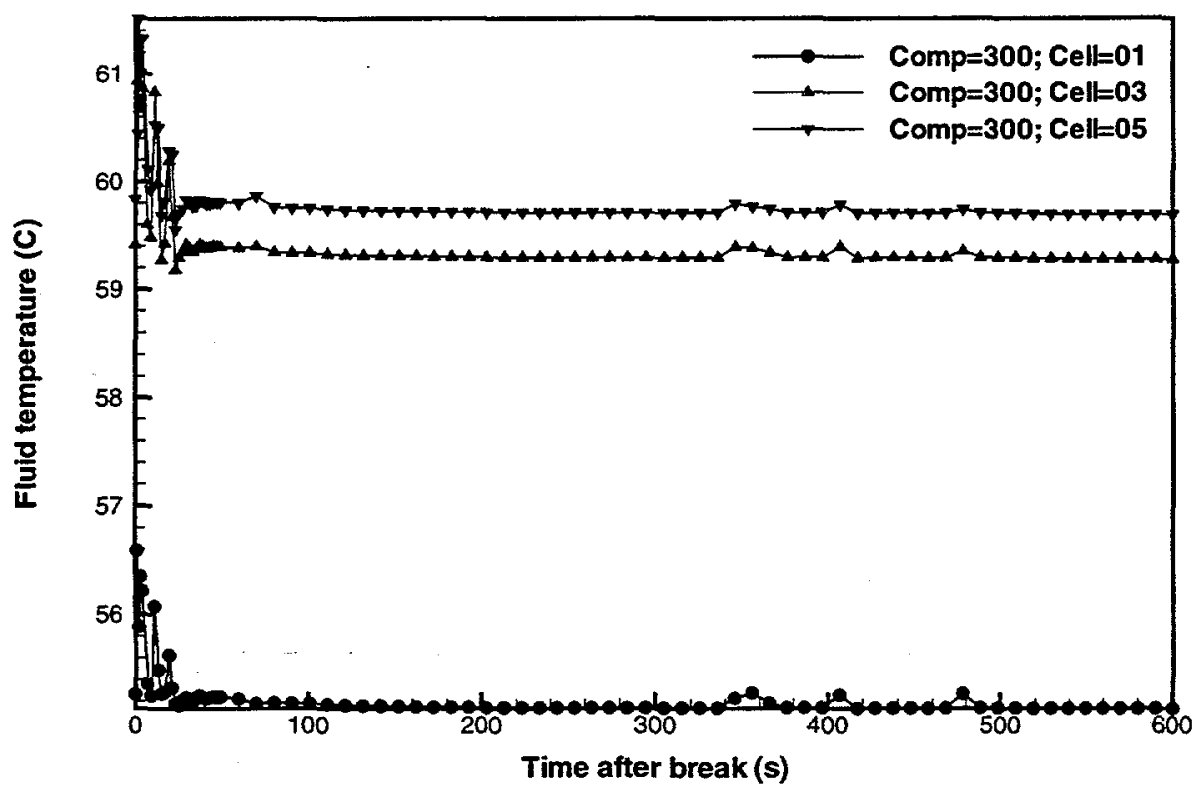

Figure C-21b Module 1 row 1 channel fluid temperatures for a LOHGA (Case 2: helium supply plenum break near decoupler outlet). 


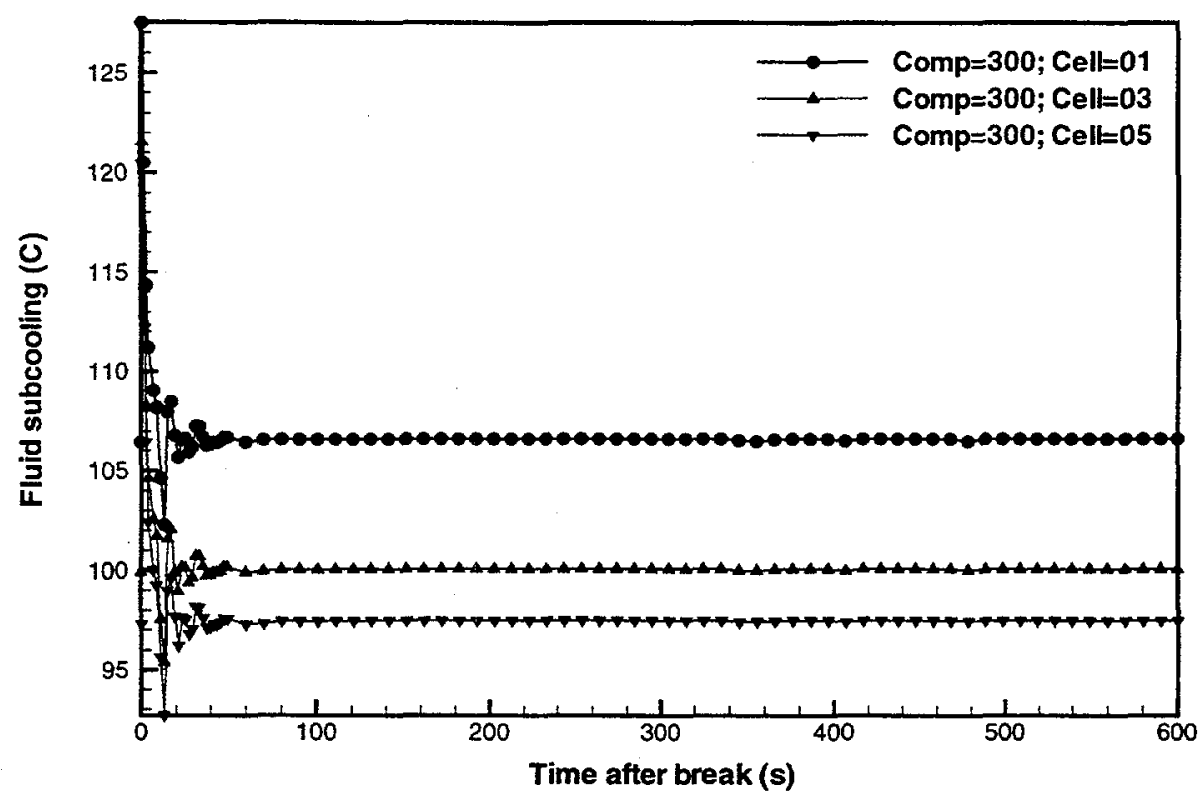

Figure C-21c Module 1 row 1 channel fluid subcoolings for a LOHGA (Case 2: helium supply plenum break near decoupler outlet).

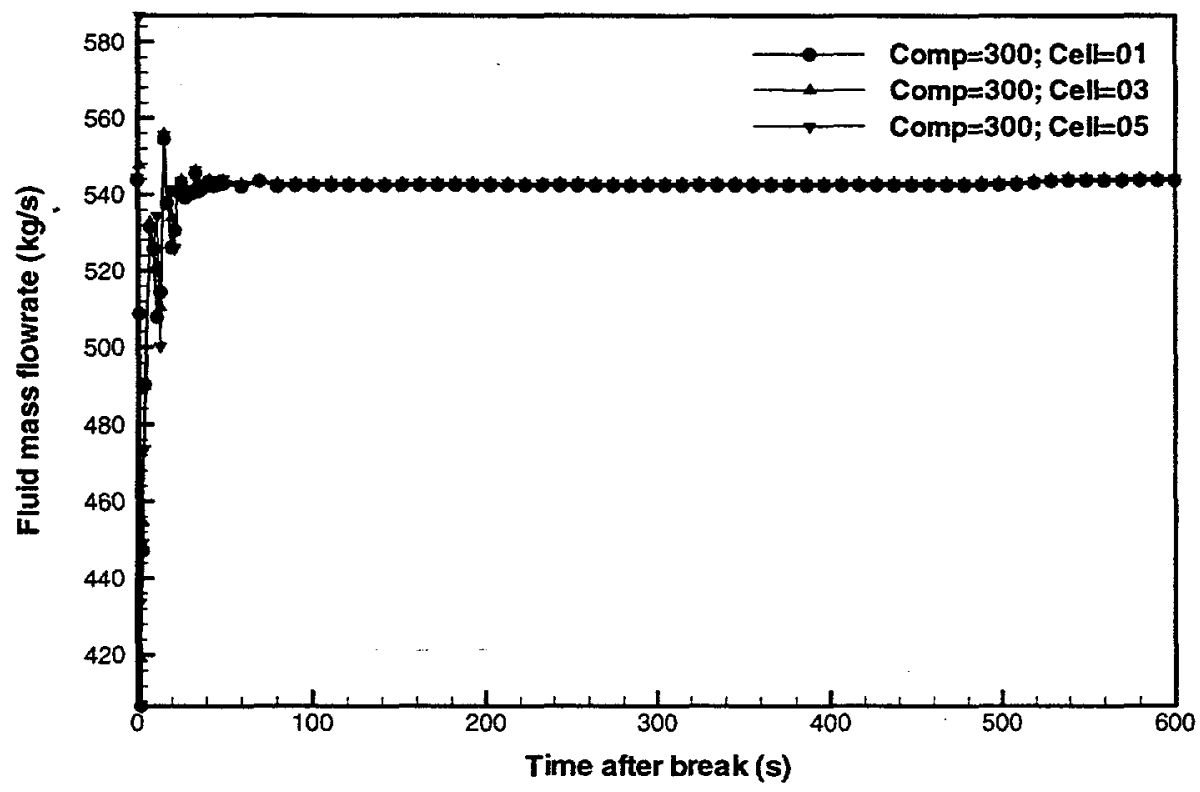

Figure C-21d Module 1 row 1 channel liquid mass flowrates for a LOHGA (Case 2: helium supply plenum break near decoupler outlet). 


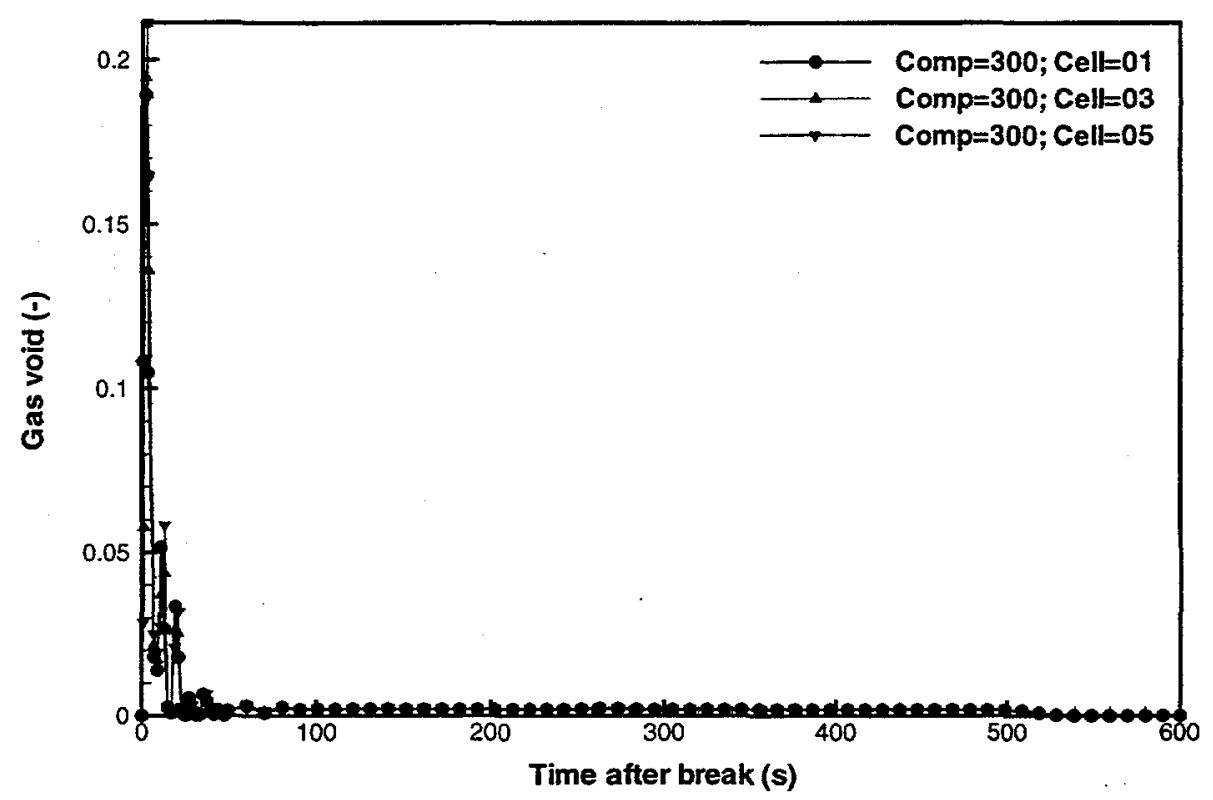

Figure C-21e Module 1 row 1 channel void fractions for a LOHGA (Case 2: helium supply plenum break near decoupler outlet).

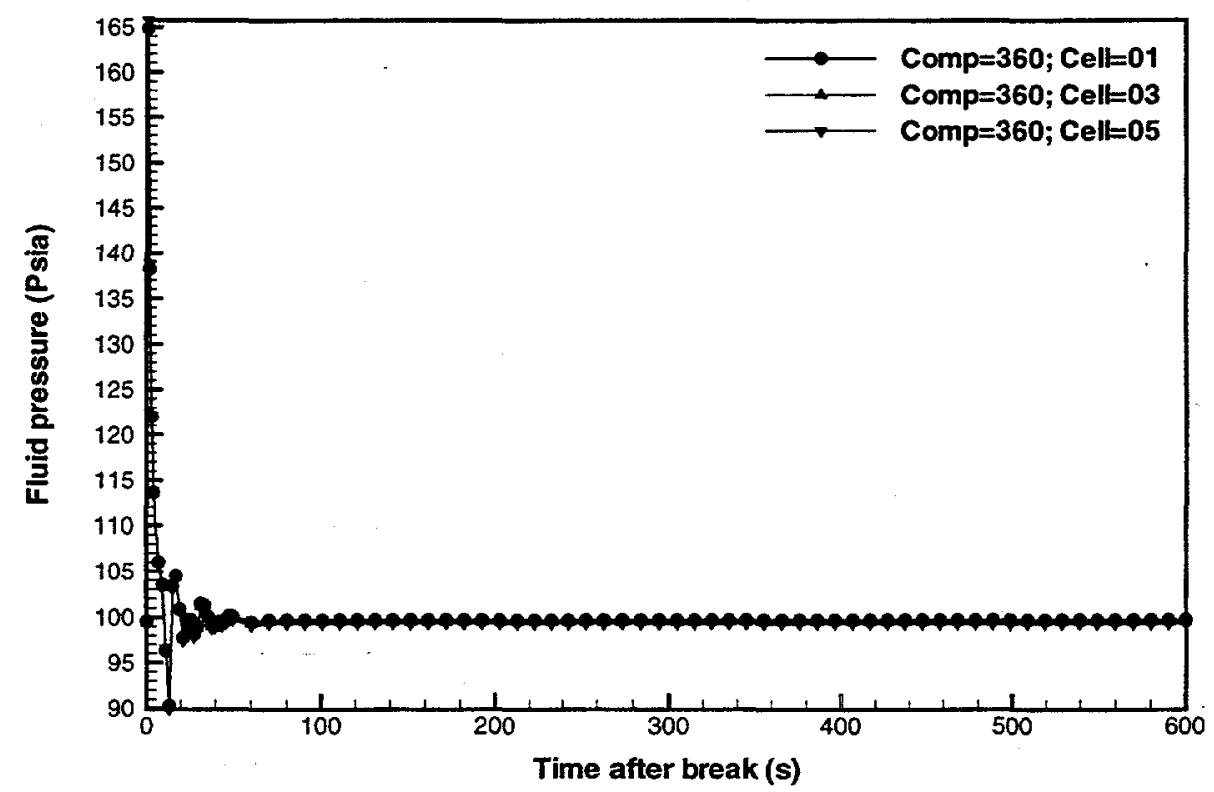

Figure C-22a Module 1 decoupler channel fluid pressures for a LOHGA (Case 2: helium supply plenum break near decoupler outlet). 


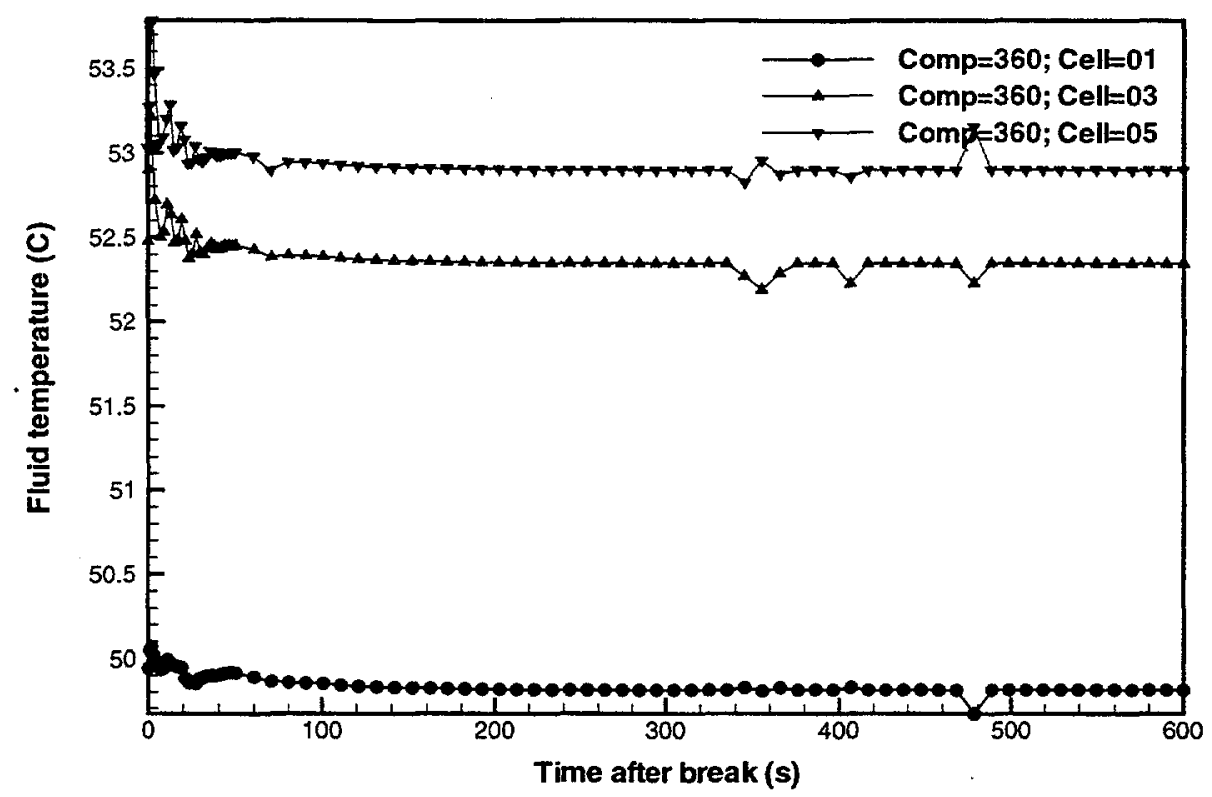

Figure C-22b Module 1 decoupler channel fluid temperatures for a LOHGA (Case 2: helium supply plenum break near decoupler outlet).

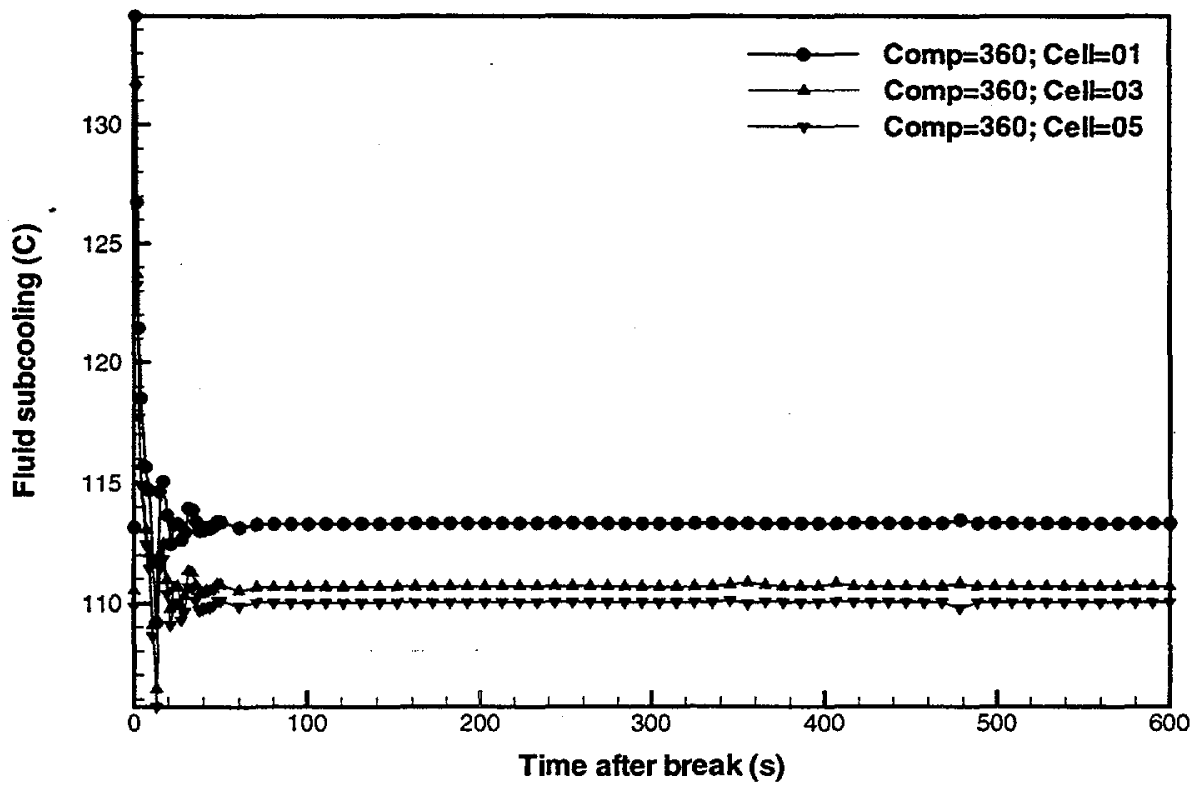

Figure C-22c Module 1 decoupler channel fluid subcoolings for a LOHGA (Case 2: helium supply plenum break near decoupler outlet). 


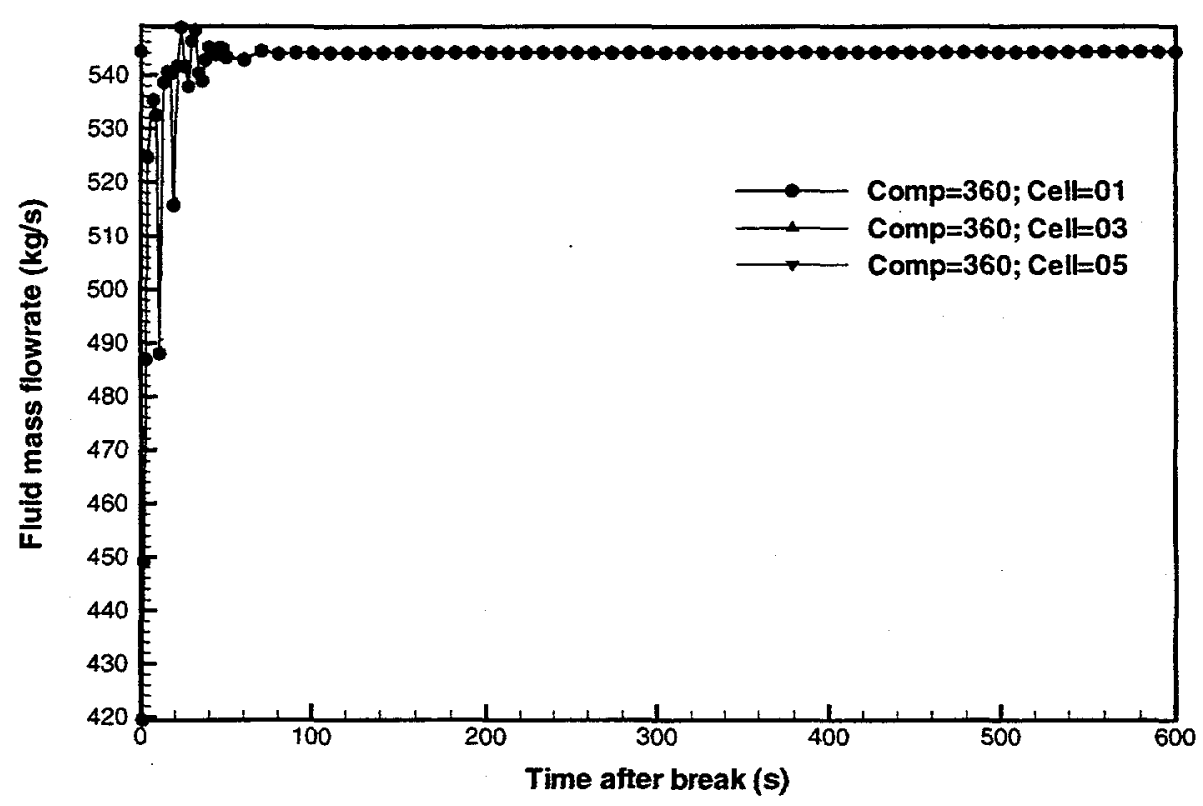

Figure C-22d Module 1 decoupler channel liquid mass flowratës for a LOHGA (Case 2: helium supply plenum break near decoupler outlet).

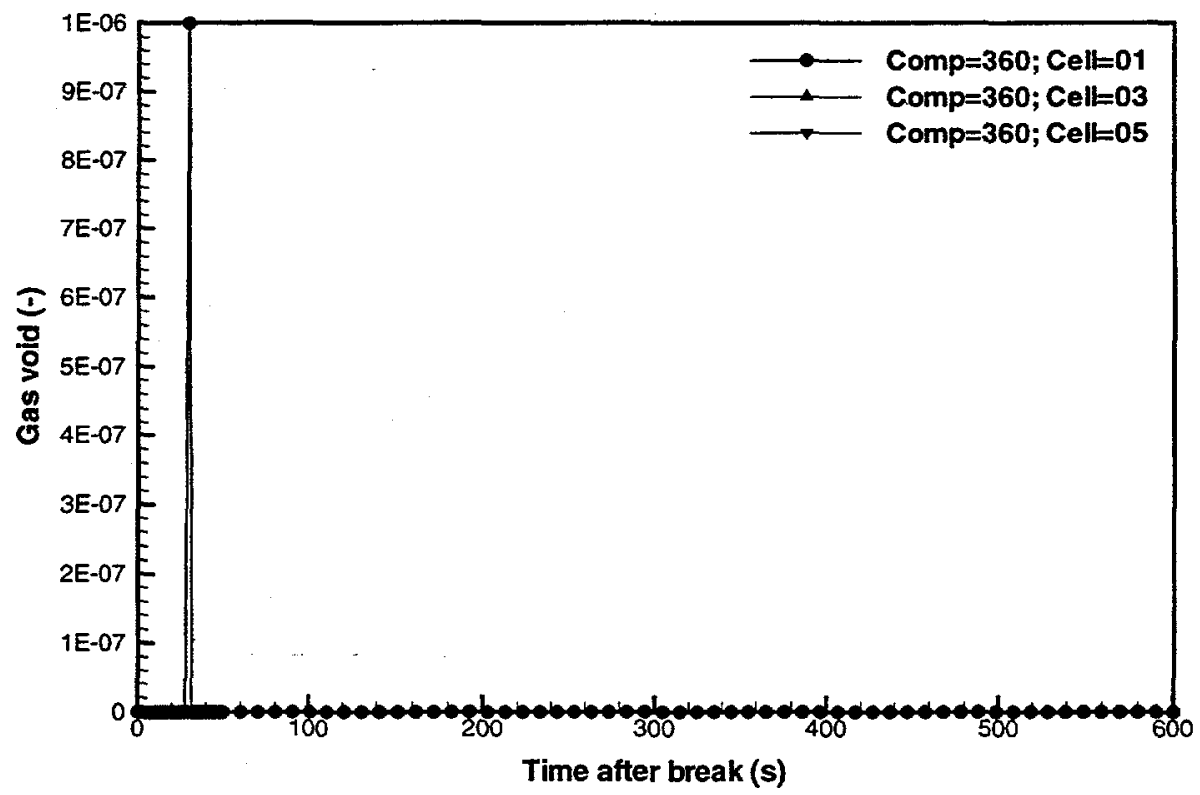

Figure C-22e Module 1 decoupler channel Module 2 channel void fractions for a LOHGA (Case 2: helium supply plenum break near decoupler outlet). 


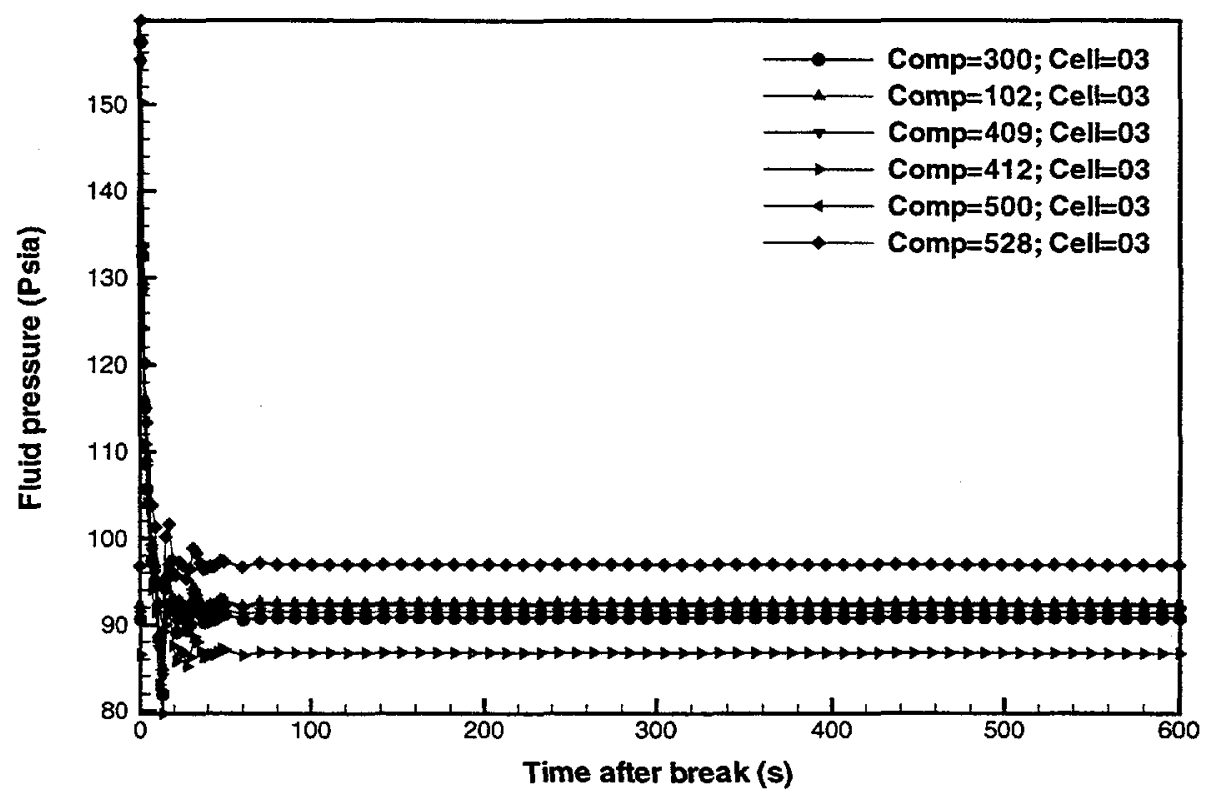

Figure C-23a Mid-plane module fluid pressures for a LOHGA (Case 2: helium supply plenum break near decoupler outlet).

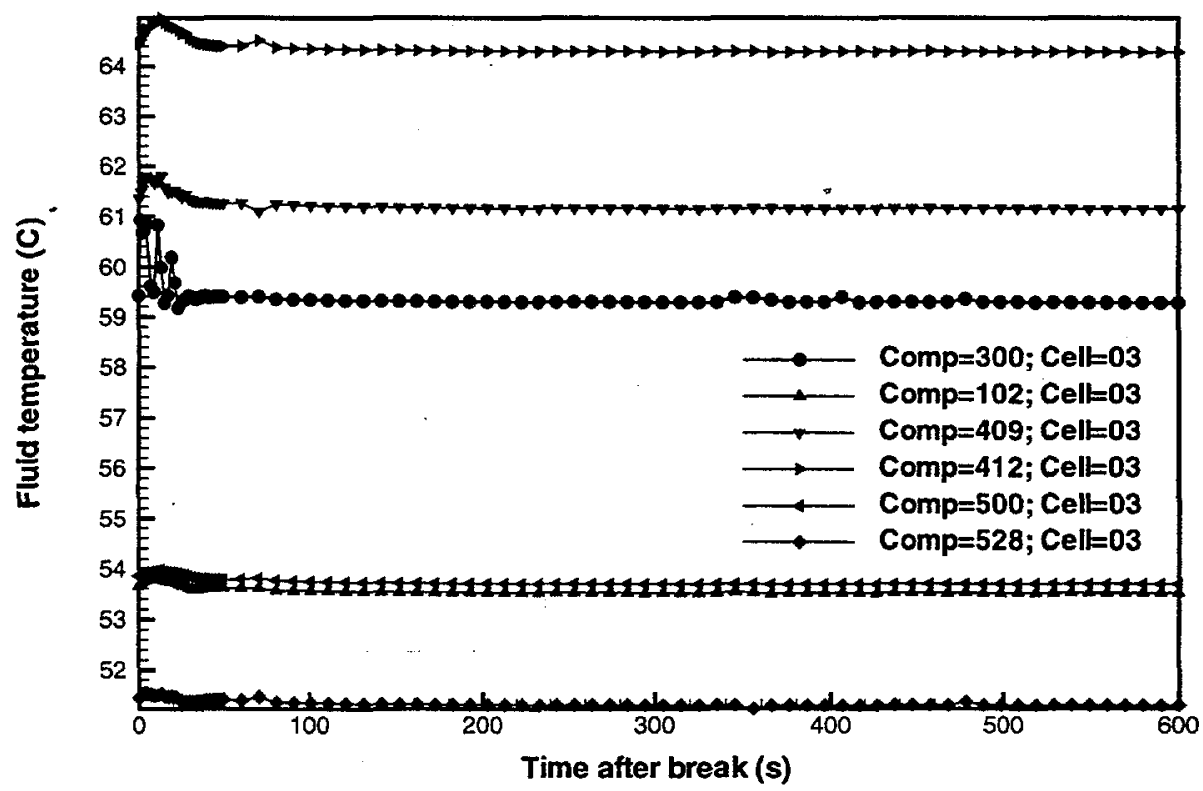

Figure C-23b Mid-plane module fluid temperatures for a LOHGA (Case 2: helium supply plenum break near decoupler outlet). 


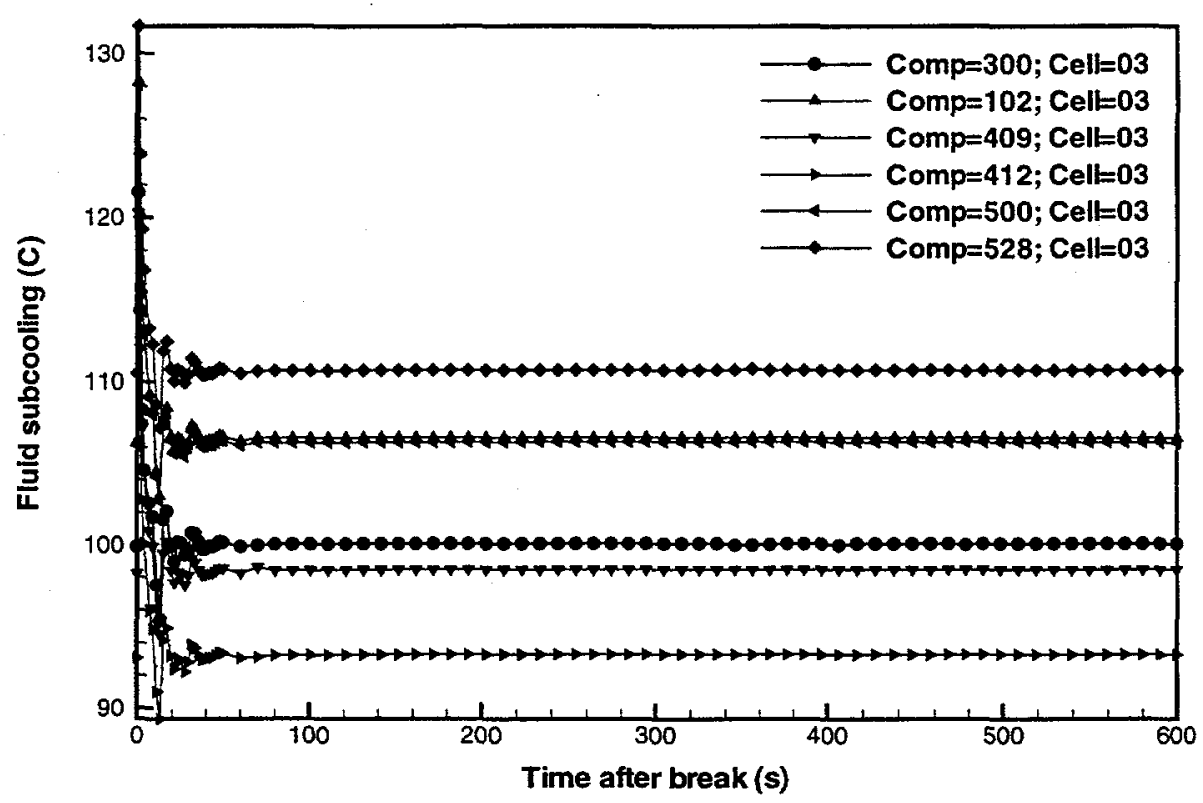

Figure C-23c Mid-plane module fluid subcoolings for a LOHGA (Case 2: helium supply plenum break near decoupler outlet).

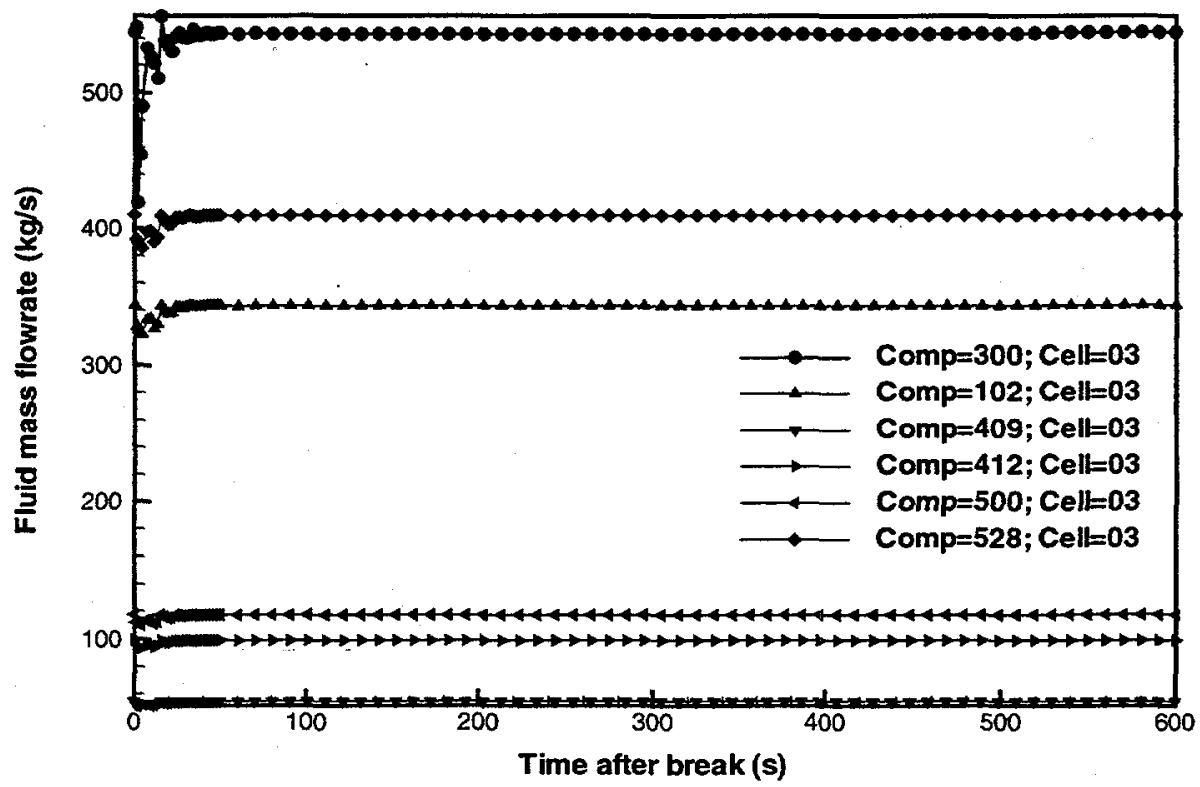

Figure C-23d Mid-plane module liquid mass flowrates for a LOHGA (Case 2: helium supply plenum break near decoupler outlet). 


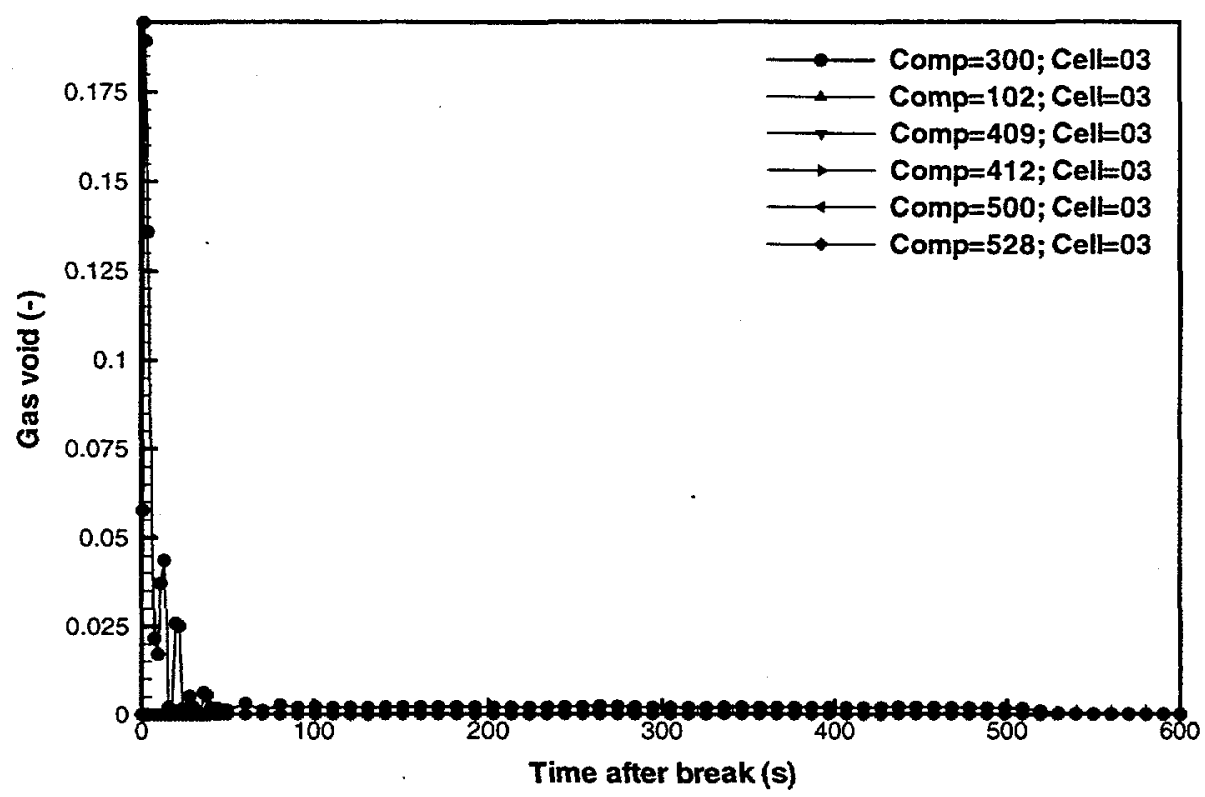

Figure C-23e Mid-plane module void fractions for a LOHGA (Case 2: helium supply plenum break near decoupler outlet).

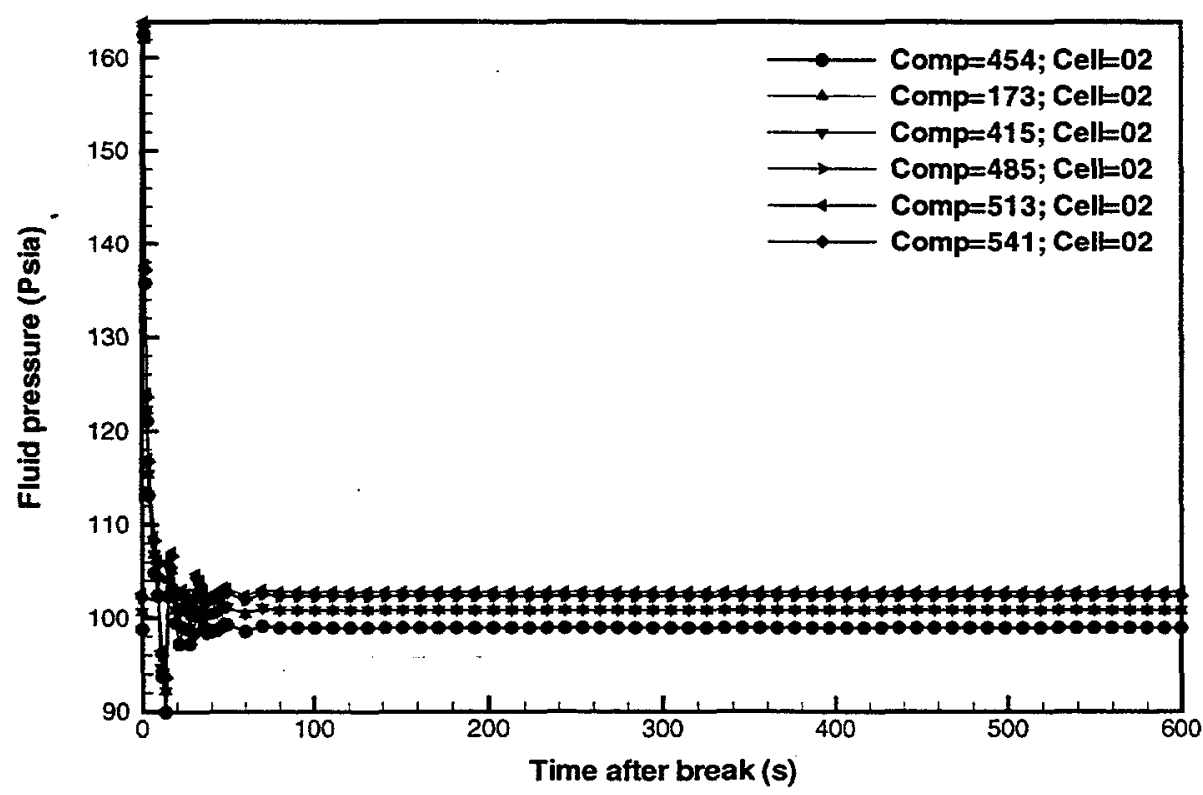

Figure C-24a Module inlet fluid pressures for a LOHGA (Case 2: helium supply plenum break near decoupler outlet). 


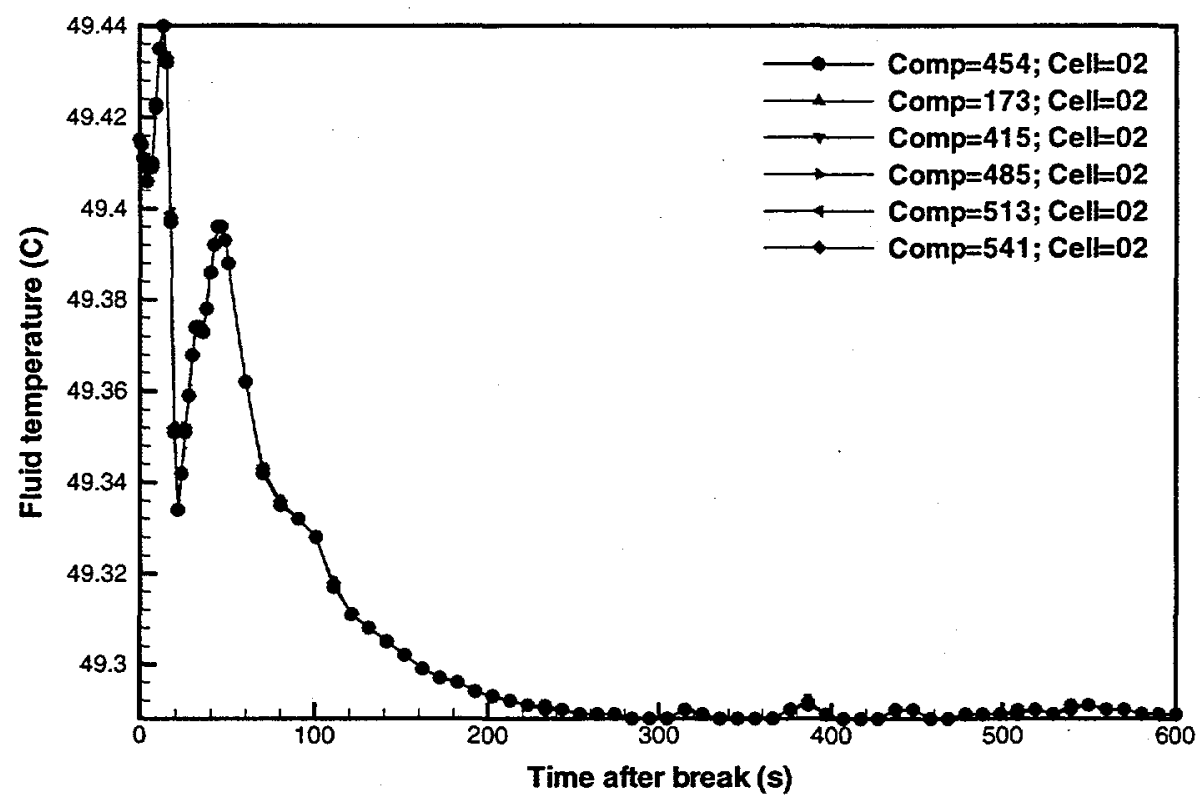

Figure C-24b Module inlet fluid temperatures for a LOHGA tCase 2: helium supply plenum break near decoupler outlet).

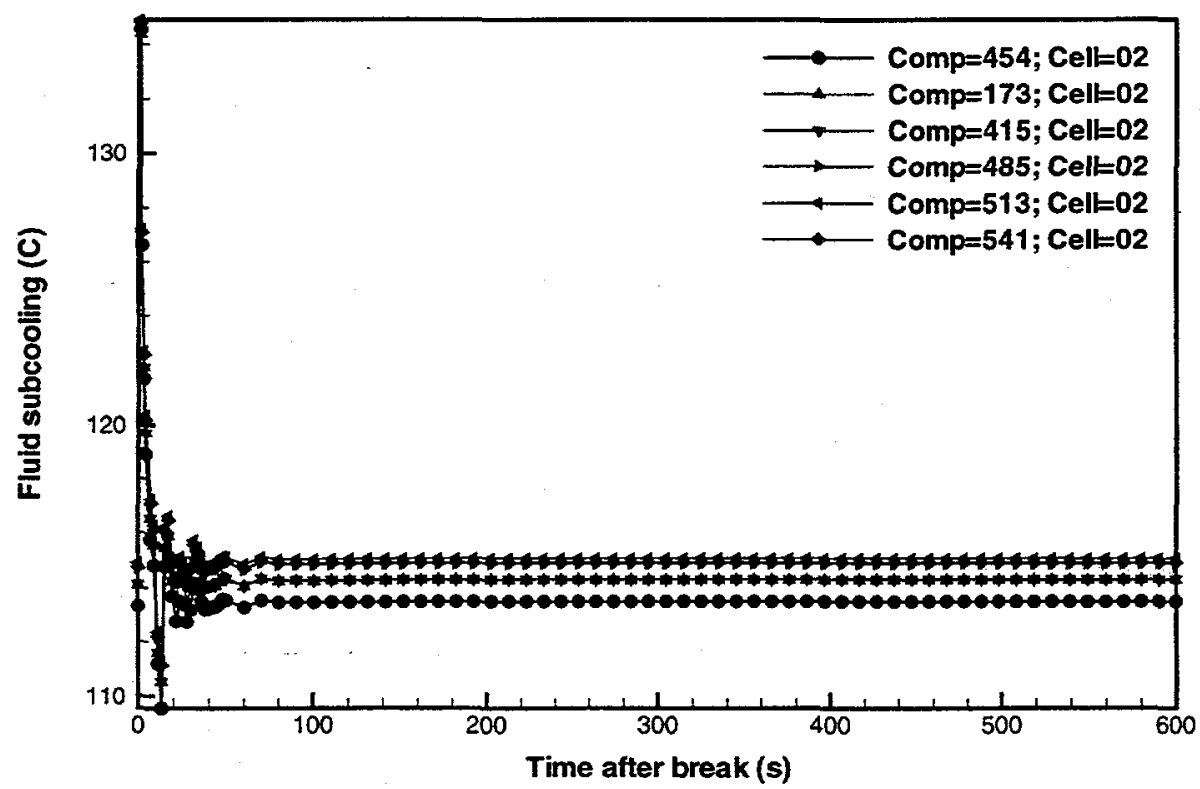

Figure C-24c Module inlet fluid subcoolings for a LOHGA (Case 2: helium supply plenum break near decoupler outlet). 


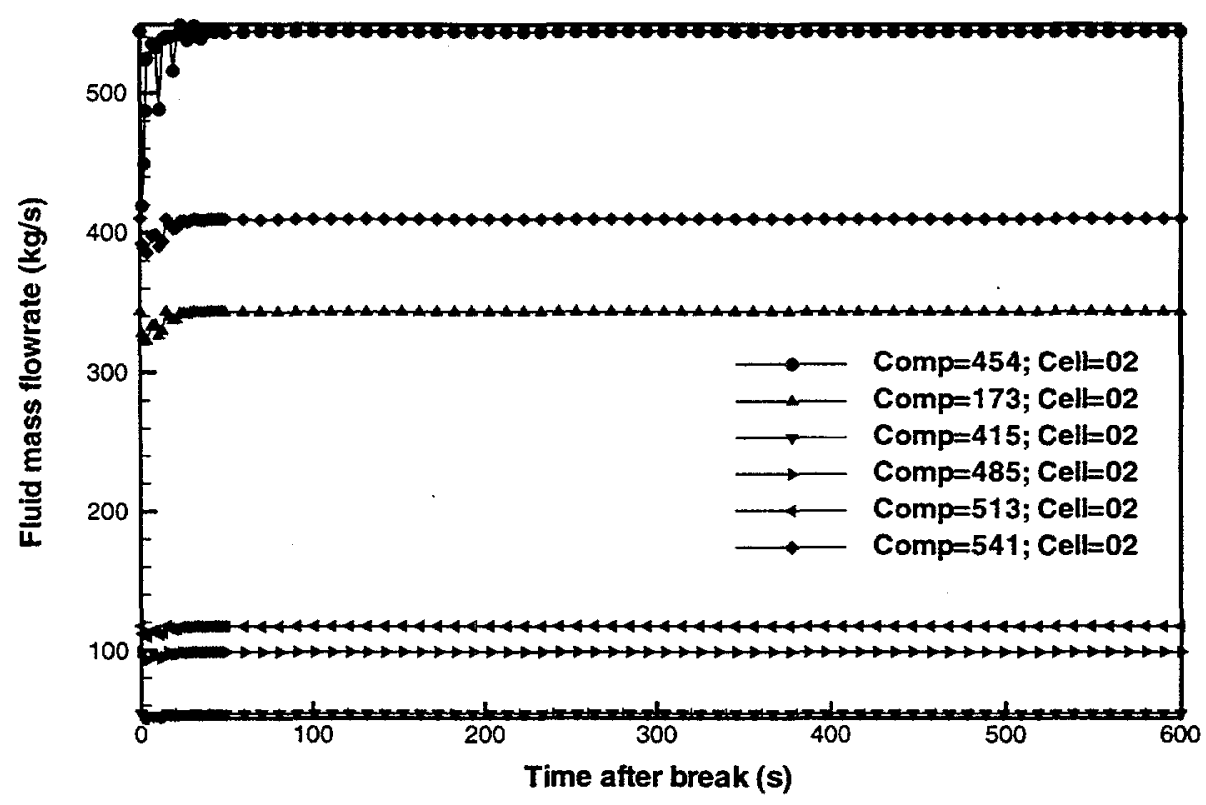

Figure C-24d Module inlet liquid mass flowrates for a LOHGA (Case-2: helium supply plenum break near decoupler outlet).

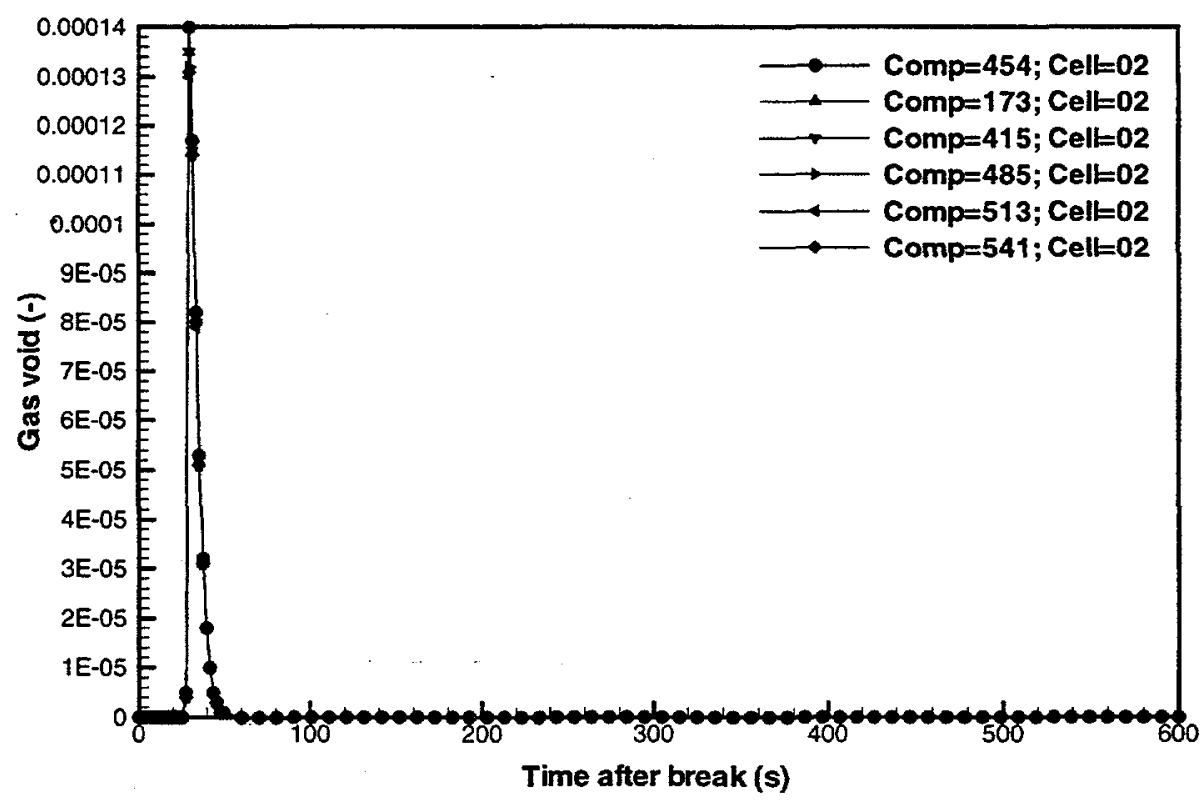

Figure C-24e Module inlet void fractions for a LOHGA (Case 2: helium supply plenum break near decoupler outlet). 


\section{Appendix D: TRAC Standard Input File for LOHGA}

The file listed below represents the TRAC code "tracin" file that corresponds to the LOHGA for the blanket system. This input deck assumes that a TRAC restart file ("trcrst") exists based on normal operation (NO).

\section{Input file tracin:}

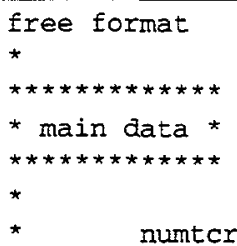

41 ieos

0 inopt

nimat 2 id2o

0

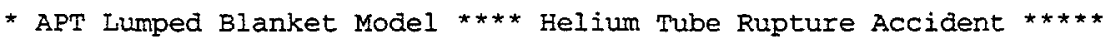
He-reservoir was added $-(4 / 22 / 1998)$

SRS Pressurizer was updated - elev. level down (1/22/1998)

SRS Primary Coolant Loop and RHR Loop were added (12/28/1997)

SRS Pump homologous Curve for Primary/RHR were added (12/28/1997)

6 Module Lumped Model with Primary Coolant Loop and RHR Loop

6 Modules - Lateral (R1/Dec) Module, R2/R3 Module,

3 Backstop Modules, Low Power Module (12/18/1997)

Hydraulic RHR Loop added as of $12 / 22 / 1997$

- This is based on check valve with flow reversal control logic.

Number of material $=2$ ( $\mathrm{Al}$ and $\mathrm{Pb}$ )

- This is single combined nod model without He comp (12/12/1997)

- Aluminum, lead material table got from Ref. (3/5, 1997)

- This is I module loop model without prmary coolant and RHR loops

- adding two upper modules (L14B-back / L14F-front) as of 7/18/1997.

- Row2/3 power updated (4/23/1997).

- R2/R3 axial power distribution has been updated as of $4 / 25 / 1997$.

- Al and lead material properties updated already.

- Unit cell cal. should be checked.

- K-loss values for each comp and elevation levels need be checked.

- Control signal variable was added (4/25/1997).

- Module 5678 connection to fixed header was updated (5/28/97).

- Blanket primary loop pipe size (14 inch) was updated (5/28/1997).

- Lower modules (module $1516 \quad 17$ 18) were added/updated (5/29/1997).

- Backstop 1st module was updated (5/29/1997).

- Backstop 2nd and 3rd modules were updated (5/30/1997).

- R2/R3 lateral modules were updated from 9 to 11 bins (6/3/1997).

- Lateral module 1 to 4 decay powers were updated (6/23/1997).

- Power for each module was updated from the 6/9/97 e-mail except for snout and top modules $(6 / 26 / 97)$.

- Decay power fraction for each module was updated from the 6/9/97 e-mail except for snout and top modules $(6 / 26 / 97)$.

- Power for each module was updated from the $6 / 9 / 97$ e-mail for snout module $(7 / 8 / 97)$.

- Decay power fraction for each module was updated from the 6/9/97 e-mail for snout module $(7 / 8 / 97)$.

- Single loop to connect two front lateral modules was updated (7/16/97).

- Single loop to connect two back lateral modules was updated $(7 / 16 / 97)$. 


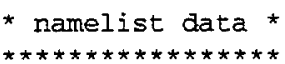

1

* HR hot leg

\begin{tabular}{|c|c|}
\hline s & * pipe \\
\hline s & * plenum \\
\hline.$s$ & * pipe \\
\hline s & * pipe \\
\hline$s$ & * pipe \\
\hline s & * pipe \\
\hline $\mathbf{s}$ & * pipe \\
\hline
\end{tabular}

* HR pumps

\begin{tabular}{|c|c|c|c|}
\hline 27 & $\mathbf{s}$ & $\star$ & plenum \\
\hline 28 & $s$ & $\star$ & pipe \\
\hline 30 & $s$ & $\star$ & pump \\
\hline 32 & $\mathbf{s}$ & $\star$ & valve \\
\hline 29 & $\mathbf{s}$ & $\star$ & pipe \\
\hline 31 & $\mathbf{s}$ & $\star$ & pump \\
\hline 33 & 5 & * & valve \\
\hline 34 & $s$ & * & plenum \\
\hline
\end{tabular}

* HR pump-to-hx piping

$$
\begin{array}{ll}
36 & s \text { * pipe } \\
37 & s \text { * pipe } \\
38 & s \text { * pipe }
\end{array}
$$

* HR hx's

$$
\begin{aligned}
& 40 \quad \mathrm{~s} * \text { plenum } \\
& 48 \text { s } \text { * pipe } \\
& 50 \text { s * pipe }
\end{aligned}
$$

HR pump suction pipe HR pump suction pipe (bk) HR pump suction pipe HR pump suction pipe (bk) HR pump suction pipe IR pump suction pipe (bk) HR punp suction pipe

HR pump suction plenum IR pump \#1 inlet pipe HR pump \#1 HR pump \#1 outlet pipe HR pump \#2 inlet pipe HR pump \#2 HR pump \#2 outlet pipe HR pump discharge plenum

HR pump dicharge pipe HR pump dicharge pipe (bk) HR pump dicharge pipe

$\mathrm{HR} h \mathrm{~h}$ inlet plenum IR $h \times 1$ inlet pipe HR hx 1 tubes 1 st pass 


\begin{tabular}{|c|c|c|}
\hline 52 & $c$ & * nin \\
\hline 54 & $\mathrm{~s}$ & * pipe \\
\hline 56 & $\mathrm{~s}$ & * pipe \\
\hline 49 & $s$ & * pipe \\
\hline 51 & $s$ & * pipe \\
\hline 53 & $s$ & * pipe \\
\hline 55 & $s$ & * pipe \\
\hline 57 & $s$ & * pipe \\
\hline 60 & $s$ & * plenum \\
\hline \multicolumn{3}{|c|}{ * HR cold leg } \\
\hline 62 & $s$ & * pipe \\
\hline 63 & $s$ & * pipe \\
\hline 64 & $\mathrm{~s}$ & * pipe \\
\hline 65 & $s$ & * pipe \\
\hline 66 & $\mathrm{~s}$ & * pipe \\
\hline 67 & $s$ & * plenum \\
\hline 68 & $\mathbf{s}$ & * pipe \\
\hline
\end{tabular}

* HR hx Secondary Side

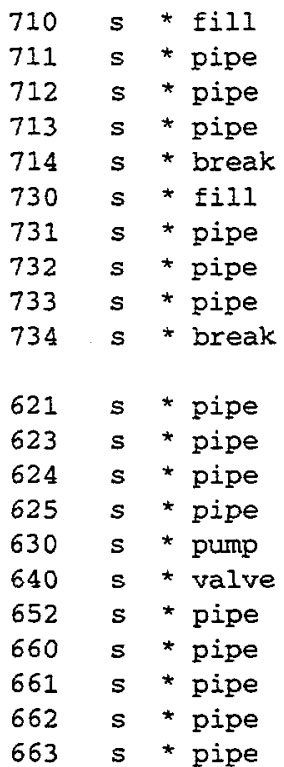

* RHB hx Secondary Side

\begin{tabular}{|c|c|c|}
\hline 672 & $s$ & * fill \\
\hline 671 & $\mathbf{s}$ & * pipe \\
\hline 673 & $s$ & * break \\
\hline 300 & $s$ & * pipe \\
\hline 330 & $s$ & * plenum \\
\hline 335 & $s$ & * pipe \\
\hline 340 & $s$ & * plenum \\
\hline 350 & $s$ & * plenum \\
\hline 360 & $s$ & * pipe \\
\hline 370 & $s$ & * plemum \\
\hline 375 & $\mathrm{~s}$ & * pipe \\
\hline 380 & $s$ & * plenum \\
\hline 429 & $\mathrm{~s}$ & * pipe \\
\hline 454 & $s$ & * pipe \\
\hline 173 & $s$ & * pipe \\
\hline 172 & $s$ & * plenum \\
\hline 158 & $s$ & * pipe \\
\hline 147 & s & * plenum \\
\hline
\end{tabular}

HR hx 1 mid-header

HR hx 1 tubes 2nd pass

HR hx 1 outlet pipe

HR hx 2 inlet pipe

HR hx 2 tubes 1 st pass

$\mathrm{HR}$ hx 2 mid-header

HR hx 2 tubes 2 nd pass

$\mathrm{HR} h \mathrm{hx} 2$ outlet pipe

HR hx outlet plenum

HR hx discharge pipe

$H R$ hx discharge pipe (bk)

$H R$ hx discharge pipe

HR $\mathrm{hx}$ discharge pipe (bk)

$H R$ hx discharge pipe

HR $h x$ discharge pipe (bk)

$H R$ hx discharge pipe

HR hx Secondary Side-1

HR hx Secondary Side-1

HR hx Secondary Side-1

HR hx Secondary Side-1

HR hx Secondary Side-1

HR hx Secondary Side-2

HR hx Secondary Side-2

HR hx Secondary Side-2

HR hx Secondary Side-2

HR hx Secondary Side-2

RHR hot leg sect 1 (bk)

RHR hot leg sect 2

RHR hot leg sect 3 (bk)

RHR hot leg sect 4

RHR primary pump

RHR pump discharge valve

RHR primary heat exchanger tubes

RHR cold leg sect 1

RHR cold leg sect 2 (bk)

RHR cold leg sect 3

RHR cold leg sect 4 (bk)

RHR Ix Secondary Side

RHR hx Secondary side

RHR $h x$ Secondary side
L1 Blanket Row1
L1 Blanket Row1 Plenum
L1 pipe conn. $330-340$
I1 outlet header
L1 lower plenum
L1 decoupler
L1 decoupler upper plenum
L1 pipe conn. $370-380$
L1 inlet header
L1 connect hot header-tee
L1 connect cold header-tee
LI Blanket Row1
L1 Blanket Row1 Plenum
L1 pipe conn. $330-340$
I.1 outlet header
L1 lower plenum 


\begin{tabular}{|c|c|c|}
\hline 133 & $s$ & * plenum \\
\hline 136 & $s$ & * pipe \\
\hline 541 & $s$ & * pipe \\
\hline 538 & $\mathrm{~s}$ & * plenum \\
\hline 535 & $s$ & * pipe \\
\hline 531 & $s$ & * plenum \\
\hline 528 & s & * pipe \\
\hline 536 & $s$ & * plenum \\
\hline 539 & $s$ & * pipe \\
\hline 415 & $s$ & * pipe \\
\hline 479 & $s$ & * plenum \\
\hline 478 & $\mathbf{s}$ & * pipe \\
\hline 418 & $s$ & * plenum \\
\hline 409 & $s$ & * pipe \\
\hline 423 & $s$ & * plenum \\
\hline 417 & $s$ & * pipe \\
\hline 485 & $s$ & * pipe \\
\hline 489 & $\mathbf{s}$ & * plenum \\
\hline 480 & $s$ & * pipe \\
\hline 419 & $s$ & * plenum \\
\hline 412 & $s$ & * pipe \\
\hline 483 & $s$ & * plenum \\
\hline 484 & $s$ & * pipe \\
\hline
\end{tabular}

513 s * pipe

$510 s *$ plenum

$507 \mathrm{~s}$ * pipe

$503 \mathrm{~s} *$ plenum

$500 \mathrm{~s} *$ pipe

508 s * plenum

511 s * pipe

$\begin{array}{lll}901 & \mathrm{~s} & * \operatorname{rod} \\ 951 & \mathrm{~s} & * \operatorname{rod} \\ 984 & \mathrm{~s} & * \operatorname{rod} \\ 905 & \mathrm{~s} & * \operatorname{rod} \\ 955 & \mathrm{~s} & * \operatorname{rod} \\ 916 & \mathrm{~s} & * \operatorname{rod} \\ 966 & \mathrm{~s} & * \operatorname{rod} \\ 915 & \mathrm{~s} & * \operatorname{rod} \\ 965 & \mathrm{~s} & * \operatorname{rod} \\ 911 & \mathrm{~s} & * \operatorname{rod} \\ 961 & \mathrm{~s} & * \operatorname{rod} \\ 988 & \mathrm{~s} & * \operatorname{rod} \\ 912 & \mathrm{~s} & * \operatorname{rod} \\ 962 & \mathrm{~s} & * \operatorname{rod} \\ 931 & \mathrm{~s} & * \operatorname{rod} \\ 978 & \mathrm{~s} & * \operatorname{rod} \\ 913 & \mathrm{~s} & * \operatorname{rod} \\ 963 & \mathrm{~s} & * \operatorname{rod} \\ 932 & \mathrm{~s} & * \operatorname{rod} \\ 979 & \mathrm{~s} & * \operatorname{rod} \\ 919 & \mathrm{~s} & * \operatorname{rod}\end{array}$

Ll decouplex

Il decouplex upper plenum

II Blanket Row1

II Blanket Rowl PIenum

Il pipe conn. $330-340$

II outlet header

I1 lower plenum

II decoupler

II decoupler upper plenum

II Blanket Row I

II Blanket Row Plenum

I1 pipe conn. $330-340$

I 1 outlet header

II lower plenum

Il decoupler

I1 decoupler upper plenum

2nd DNS Blanket Row 1

2nd DNS Blanket Row Plenum

2nd DNS pipe conn. $330-340$

2nd DNS outlet header

2nd DNS lower plenum

2nd DNS decoupler

2nd DNS dec upper plenum

Third DNS Blanket Row1

Third DNS Row1 Plenum

Third DNS pipe conn. 330-340

Third DNS outlet header

Third DNS lower plenum

Third DNS decoupler

Third DNS dec upper plenum

annular aluminum rod
cylindrical lead rod
cylindrical lead rod

annular aluminum rod

cylindrical lead rod

cylindrical lead rod

cylindrical lead rod

annular aluminum rod

cylindrical lead rod

annular aluminum rod cylindrical lead rod cylindrical lead rod

annular aluminum rod cylindrical lead rod cylindrical lead rod cylindrical lead rod

annular aluminum rod cylindrical lead rod cylindrical lead rod cylindrical lead rod

annular ss rod 
WESTINGHOUSE SAVANNAH RIVER COMPANY

APT BLANKET SYSTEM FOR LOHGA

(HELIUM SUPPLY RUPTURE INTO BLANKET MODULE)
Report:

Section:

Date:

Page:
WSRC-TR-98-00177

Appendix D

$07 / 16 / 98$

annular ss rod

annular ss rod

annular ss rod

annular ss rod

$52 \mathrm{e}$

$6 e$

$\operatorname{prptb}(3, i)$

$1.2970 \mathrm{e}+02$

$8.9538 \mathrm{e}+02$ $\operatorname{prptb}(4, i)$

3. $4592 \mathrm{e}+01$

$3.3382 e+01$ prptb $(5, i)$

$2.8000 e-01$

$2.8000 e-01$

e

* aluminum material

\begin{abstract}
prptb $(1, i)$
$2.7300 e+02$

$3.0000 e+02$

$3.7300 e+02$

$4.7300 e+02$

$6.7300 e+02$

1. $0000 e+10$
\end{abstract}

e

$\star$

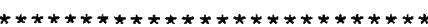

* css data

*

*

$* * * * * * * * * * * * * * * * * * * * * * * * * *$

* control-parameter data *

$\star * \star * * \star * \star * \star * \star * * * * * * * * \star * * * * * *$

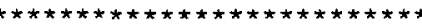

* signal variables

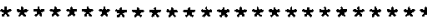

$\star$

* time

$$
\text { * }
$$

idsv

1

isvm

ilcn

0

$i \operatorname{col} 1$

0

$\operatorname{icn} 2$

* pressure difference across RHR check valve idsv

isvm

il cn

icn 1

0

$i \cos 2$

0

* Elapse time since RHR pump activated iasv isvn

ilcn

3

0

$i \operatorname{con} 1$

0

icn2

* Elapse time since HR pumps activated iasv isvm ilcn
5.0000 e- 02

.0000e-02

5.0000e-02

5. 0000 e- 02

$5.0000 e-02$ $\operatorname{prptb}(5, i)$

5 of 8 
WESTINGHOUSE SAVANNAH RIVER COMPANY

APT BLANKET SYSTEM FOR LOHGA

(HELIUM SUPPLY RUPTURE INTO BLANKET MODULE)
Report:

Section:

Date:

Page:
WSRC-TR-98-00177

Appendix D

$07 / 16 / 98$

* pressure difference across HR check valve

* iasv isvn ilcn

5

0

32

icn 1

$\operatorname{ien} 2$

* pressure difference across HR check valve 
* trips from off to on at setp(2), starts RHR pump.

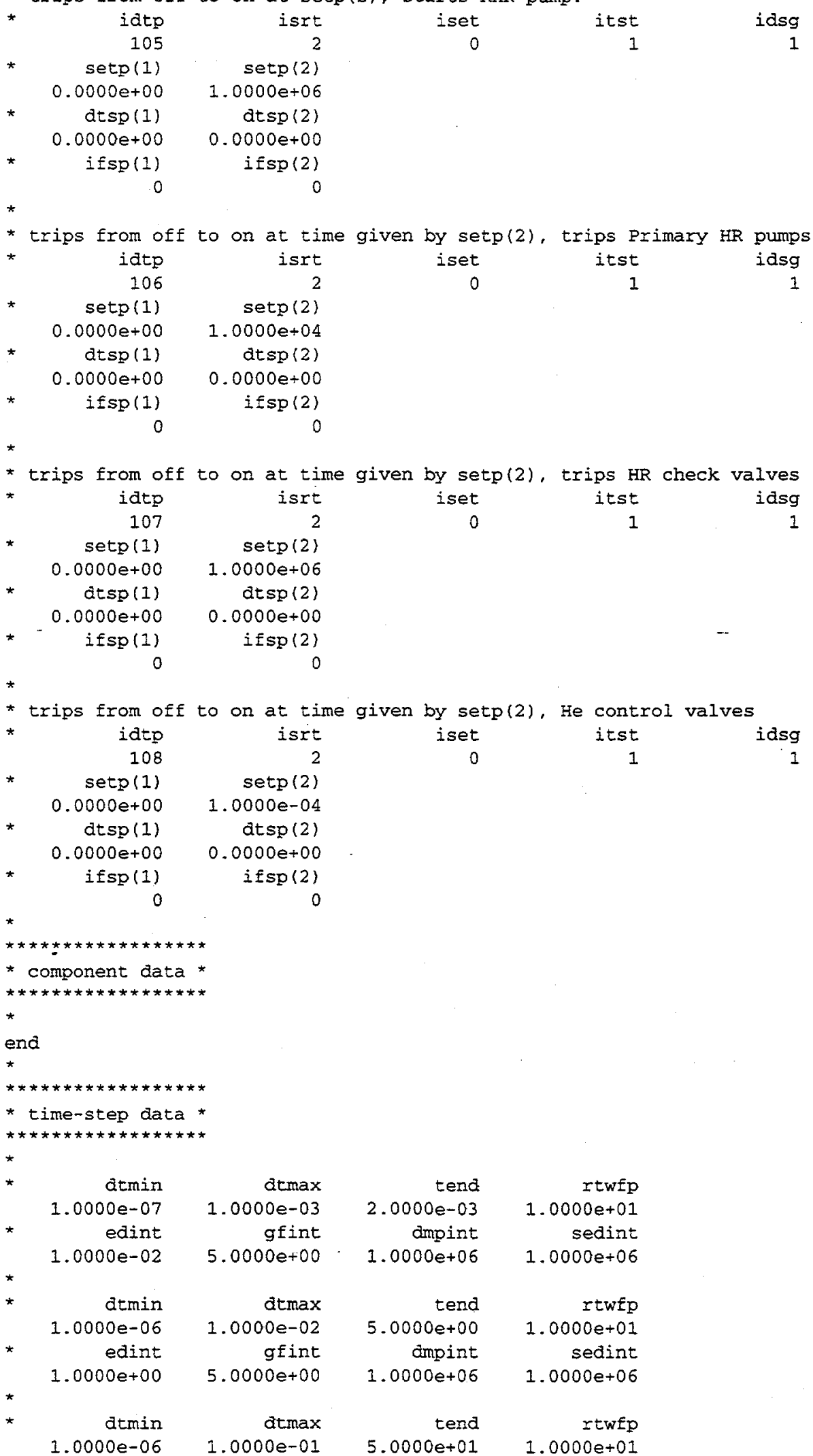


WESTINGHOUSE SAVANNAH RIVER COMPANY

APT BLANKET SYSTEM FOR LOHGA

(HELIUM SUPPLY RUPTURE INTO BLANKET MODULE)
Report:

Section:

Date:

Page:
WSRC-TR-98-00177

Appendix D

$07 / 16 / 98$

8 of 8 .
* edint

$2.0000 e+00$

*

* demir

$1.0000 e-06$

edint

$1.0000 e+01$

*

* endflag

$-1.0000 e+00$ gfint
$5.0000 e+00$

dmpint

$1.0000 e+06$

dtmax

$3.0000 e-01$

gfint

$5.0000 e+00$

$6.0000 e+02$

ampint

$1.0000 e+06$
$1.0000 e+01$

sedint

$1.0000 e+06$

sedint

$1.0000 e+06$

rtwfp

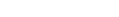




\section{Appendix E: TRAC Graphics Input File for LOHGA}

The file listed below represents the TRAC code "graphin" file that corresponds to the LOHGA for the blanket system. This input deck contains the various graphics points selected for output to the "tecsum.grf" file.

\section{Input file graphin:}

\begin{tabular}{|c|c|c|c|c|c|}
\hline \multicolumn{6}{|l|}{$\begin{array}{c}\text { mpoints } / \\
97\end{array}$} \\
\hline / component & $\operatorname{cel} 1$ & ictype & itee/ & & \\
\hline 340 & 1 & 1 & 1 & Fixed outlet he & eader \\
\hline 380 & 1 & 1 & 1 & Fixed inlet hea & ader \\
\hline 454 & 2 & 0 & 1 & Module 1 pipe & \\
\hline 173 & 2 & 0 & 1 & Module 2 pipe & \\
\hline 415 & 2 & 0 & 1 & Module 3 pipe & \\
\hline 485 & 2 & 0 & 1 & Module 4 pipe & \\
\hline 513 & 2 & 0 & 1 & Module 5 pipe & \\
\hline 541 & 2 & 0 & 1 & Module 6 pipe & \\
\hline 360 & 1 & 0 & 1 & Module 1 Dec & \\
\hline 300 & 1 & 0 & 1 & Module 1 Row 1 & \\
\hline 102 & 1 & 0 & 1 & Module 2 Row2 & \\
\hline-409 & 1 & 0 & 1 & Module 3 Row 1 & $\ldots$ \\
\hline 412 & 1 & 0 & 1 & Module 4 Row & \\
\hline 500 & 1 & 0 & 1 & Module 5 Row2 & \\
\hline 528 & 1 & 0 & 1 & Module 6 Low Po & ower \\
\hline 360 & 3 & 0 & 1 & Module 1 Dec & \\
\hline 300 & 3 & 0 & 1 & Module 1 Row 1 & \\
\hline 102 & 3 & 0 & 1 & Module 2 Row2 & \\
\hline 409 & 3 & 0 & 1 & Module 3 Row 1 & \\
\hline 412 & 3 & 0 & 1 & Mocule 4 Row 1 & \\
\hline 500 & 3 & 0 & 1 & Module 5 Row2 & \\
\hline 528 & 3 & 0 & 1 & Module 6 Low Po & ower \\
\hline 360 & 5 & 0 & 1 & Module 1 Dec & \\
\hline 300 & 5 & 0 & 1 & Module I Row 1 & \\
\hline 102 & 6 & 0 & 1 & Module 2 Row2 & \\
\hline 409 & 5 & 0 & 1 & Module 3 Row 1 & \\
\hline 412 & 6 & 0 & 1 & Module 4 Row 1 & \\
\hline 500 & 6 & 0 & 1 & Module 5 Row2 & \\
\hline 528 & 5 & 0 & 1 & Module 6 Low Po & ower \\
\hline 430 & 1 & 1 & 1 & Helium Reserv P & Plenum \\
\hline 370 & 1 & 1 & 1 & Module 1 Inlet & Plenum \\
\hline 350 & 1 & 1 & 1 & Module 1 Middle & Plenum \\
\hline 330 & 1 & 1 & $I$ & Module 1 outlet & Plenum \\
\hline 172 & 1 & 1 & 1 & Module 2 Inlet & Plenum \\
\hline 147 & 1 & 1 & 1 & Module 2 midale & Plenum \\
\hline 133 & 1 & 1 & 1 & Module 2 outlet & Plenum \\
\hline 479 & 1 & 1 & 1 & Module 3 Inlet & Plenum \\
\hline 418 & 1 & 1 & 1 & Module 3 Middle & Plenum \\
\hline 423 & 1 & 1 & 1 & Module 3 outlet & Elenum \\
\hline 489 & 1 & 1 & 1 & Module 4 Inlet & Plenum \\
\hline
\end{tabular}


419

483

510

503

508

538

531

536

26

440

30

30

31

31

32

33

37

48

50

52

51

53

56

$\begin{array}{lll}1 & 1 & 1 \\ 1 & 1 & 1 \\ 1 & 1 & 1 \\ 1 & 1 & 1 \\ 1 & 1 & 1 \\ 1 & 1 & 1 \\ 1 & 1 & 1 \\ 1 & 1 & 1\end{array}$

1

4

2

1

2

2

2

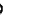

4

4

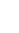

4

2

2

\section{1}

1
3
3

1

1$$
1
$$$$
\begin{aligned}
& 1 \\
& 5 \\
& 1 \\
& 5 \\
& 1 \\
& 6 \\
& 1 \\
& 6 \\
& 1 \\
& 5 \\
& 1 \\
& 5 \\
& 1 \\
& 6 \\
& 1 \\
& 6 \\
& 1 \\
& 6 \\
& 1 \\
& 6
\end{aligned}
$$

1 Module 4 Midale Plenum 1 Module 4 outlet Plenum 1 Module 5 Inlet Plenum 1 Module 5 Midale Plenum 1 Module 5 outlet Plenum 1 Module 6 Inlet Plenum 1 Module 6 Middle Plenum Module 6 outlet Plenum

Hot leg pump suction line

1 He Pipe Inlet valve

PCL Pump 1 suction

PCL Pump 1 Discharge

PCL Pump 2 suction

PCL Pump 2 Discharge

PCL Pump 1 check valve

PCL Pump 2 check valve

PCL Pump to HX

PCL $\mathrm{Hx} 1$ inlet

PCL $\mathrm{HX} I$ iniet

PCL $\mathrm{Hx} 1$ inlet

PCI $\mathrm{Hx} 1$ inlet

PCI HX 1 inlet

PCI $H \times I$ outlet

PCL $\mathrm{Hx} 2$ inlet

PCL HX 2 outlet

Cold leg $\mathrm{Hx}$ discharge line Cold leg $\mathrm{Hx}$ aischarge line

RHR Pump Suction

RHR Pump Discharge

RHR Hx inlet

RHR Hx outlet

Pzr Pressure Signal

Pzr Bottom Pressure

Hot Module (1) upflow inside Hot Module (1) upflow inside Hot Module (I) upflow outside Hot Module (1) upflow outside Hot Module (2) upflow inside Hot Module (2) upflow inside Hot Module (2) upflow outside Hot Module (2) upflow outside Hot Module (3) upflow inside Hot Module (3) upflow inside Hot Module (3) upflow outside Hot Module (3) upflow outside Hot Module (4) upflow inside Hot Module (4) upflow inside Hot Module (4) upflow outside Hot Module (4) upflow outside Hot Module (5) upflow inside Hot Module (5) upflow inside Hot Module (5) upflow outside Hot Module (5) upflow outside 
965

965

965

965

$\begin{array}{ll}1 & 3 \\ 5 & 3 \\ 1 & 3\end{array}$

1

1

2
Hot Module (6) upflow inside Hot Module (6) upflow inside Hot Module (6) upflow outside Hot Module (6) upflow outside

INPUT NOTES:

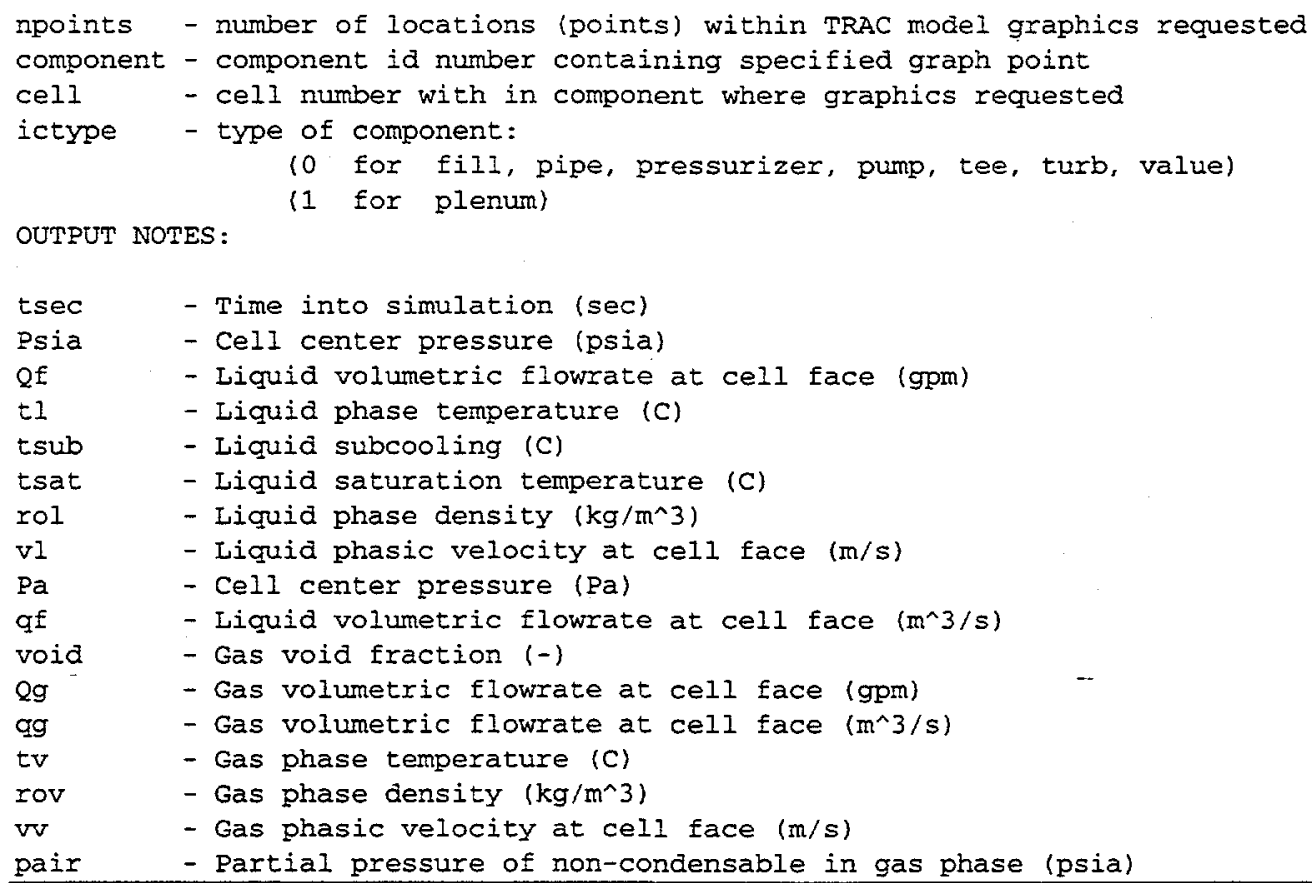




\section{Appendix F: FLOWTRAN-TF Input File for LOHGA}

Below is an abridged listing of the FLOWTRAN-TF input deck for LOHGA. The finite element input of the solid geometric parameters used in the heat conduction calculations and the fluid geometry input are identical to the values given in Ref. [6] and have been edited from this listing to save space.

\section{Input file apt.in:}

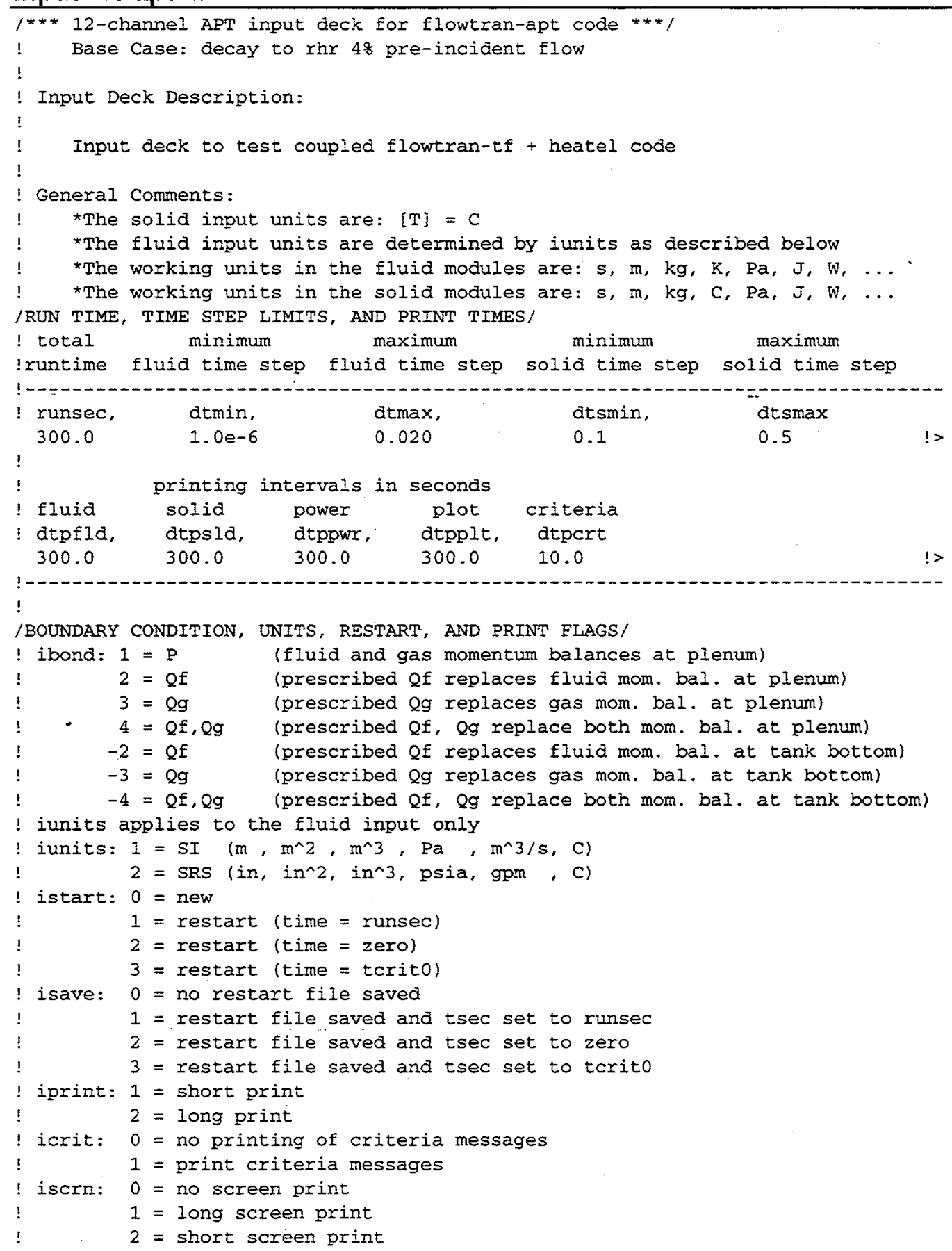


istdy: -1 = grid generation only

0 = transient from tsec to runsec

1 = steady state from tsec

2 = steady state at tsec

3 = steady state at tsec followed by a transient to runsec

igpsk: $0=$ no node renumbering

1 = optimum node renumbering using Gibbs-Poole-stockmeyer-King

2 = optimum node renumbering using Gibbs-poole-stockmeyer-Cuthill-Mckee

inorm: 0 = use unnormalized axial power shapes

1 = normalize the axial power shape

ibond, iunits, istart, isave, iprint

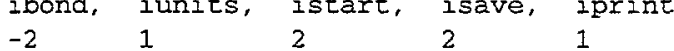

icrit, iscrn, istdy, ippu, ibpu

$\begin{array}{lllll}0 & 2 & 0 & 0 & 0\end{array}$

Page:

2 of 18

! igpsk inorm

10

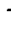

/REFERENCE PRESSURE, COMPRESSIBILITY FACTOR, .../

ilq: Iiquid identification, $1=\mathrm{H} 2 \mathrm{O}, 2=\mathrm{D} 2 \mathrm{O}$

pref: reference pressure used in subroutine inner to compute

relative changes in the dp's

factor: multiplier to drho, fluid/dP to increase fluid compressibility

vminz: minimum absolute velocity for full donoring in $z$ direction

tol: accchk parameter

tolss: steady state tolerance on dhmix/dt, (J/m^3-s)

tolts: relative tolerance on solid temperature

ttol: relative tolerance on solid time step change

dtsup: wall superheat reduction, $C$

htdamp: solid-fluid heat transfer damping factor

cidamp: interfacial drag damping factor

xaO: source/sink air mass fraction

dtf: perturbation to liquid temperature for derivative estimation

atg: perturbation to gas temperature for derivative estimation

nstdy: maximum iterations for steady-state (istay > 0)

nmat: maximum number of solid materials

delox: surface oxide layer thickness $(\mathrm{m})$

tkox: oxide thermal conductivity $(\mathrm{W} / \mathrm{m}-\mathrm{K})$

! ilq, pref, factor, vminz

$5.0 e+5 \quad 1.0 \quad 0.05$

!

! tol, tolss, tolts, ttol

$\begin{array}{llll}1.0 & 10.0 & 0.1 & 0.1\end{array}$

! dtsup, htdamp, cidamp, xao

$\begin{array}{llll}\text { dtsup, htdamp, cidamp, } & \text { xa0 } \\ 0.0 & 1.0 & 0.1 & 0.0\end{array}$

I dtf atg nstdy, nmat

$\begin{array}{lllll}1.0 & 1.0 & 9000 & 9 & \text { !> }\end{array}$

!

! delox, tkox

$5.08 e-5 \quad 2.16$

!

/BOILING CURVE AND INTERPHASE TRANSPORT OPTIONS/

iboil: 0 = use specified heat transfer coefficient

1 = forced convection (SRL)

2 = forced convection (Dittus-Boelter)

3 = forced convection (Sieder-Tate) 


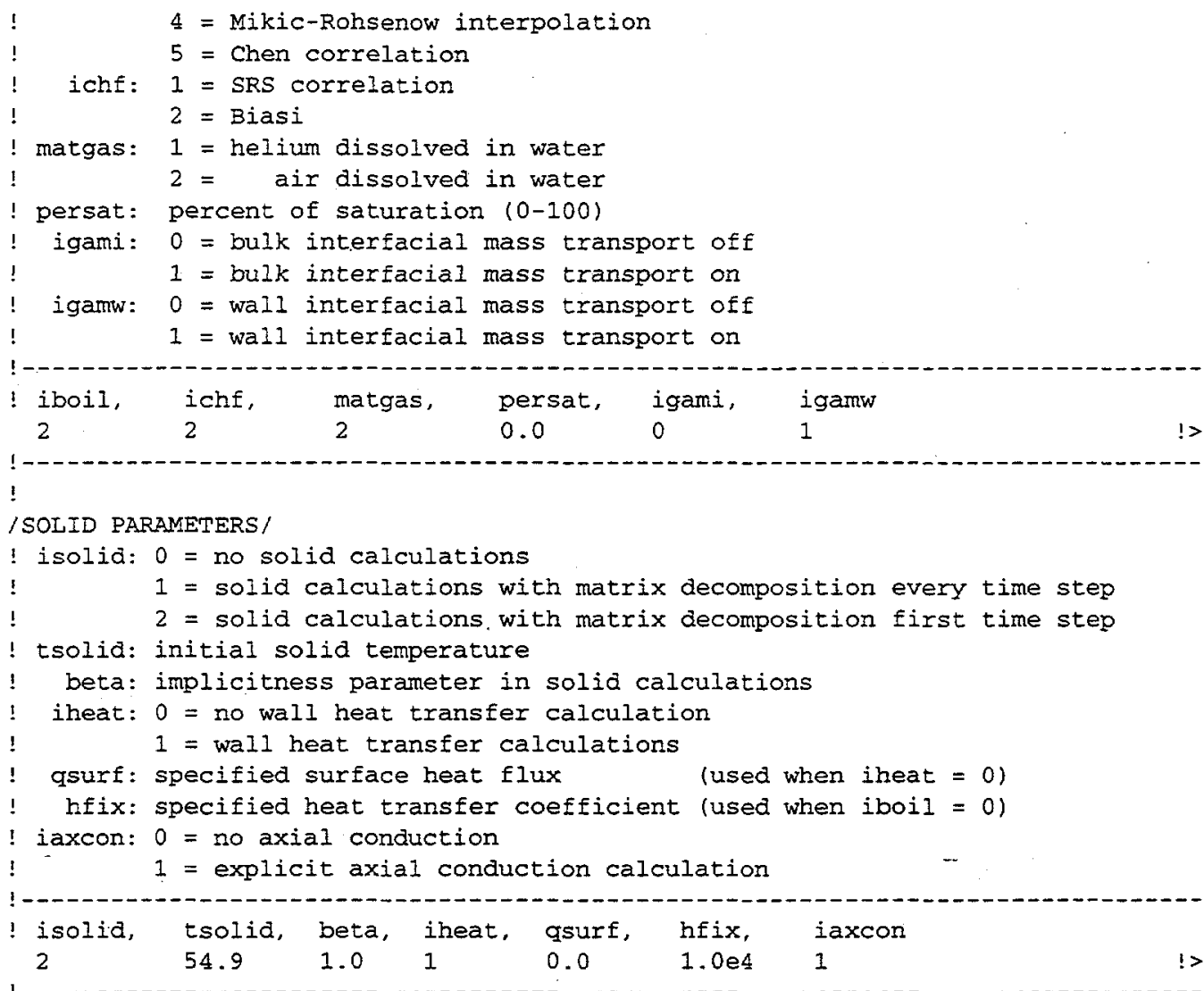

I INNER ITERATION OPTIONS \& NEWTON ITERATION PARAMETERS

irebal: $0=$ no coarse mesh rebalance

1 = coarse mesh rebalance on first pass

2 = coarse mesh rebalance on each pass

ncmr: number of coarse mesh rebalances when irebal $=1$ or 2

epsin: inner iteration convergence criterion for relative dp error

initmx: max. number of inner iterations allowed

epsp: newton iteration convergence criterion for absolute $p$ error in $\mathrm{Pa}$

epsalp: newton iteration convergence criterion for absolute alp error

epstg: newton iteration convergence criterion for absolute tg error in $\mathrm{K}$

epstf: newton iteration convergence criterion for absolute $t f$ error in $k$

epsxa: newton iteration convergence criterion for absolute xa error

| nitmax: |nitmax| = max. number of newton iterations allowed

If nitmax is positive and |nitmax| iterations are reached, then then computations continue using the mth iterate values from the $\mid$ nitmax| iteration.

If nitmax is negative and |nitmax| iterations are reached, then a new time step with a time step reduction is requested.

\begin{tabular}{|c|c|c|c|c|c|c|c|}
\hline irebal, ncms, & epsin, & & & & & initnx & \\
\hline 11 & $1.0 e-5$ & $\cdots$ & & & & 200 & $1>$ \\
\hline$!$ & $\begin{array}{l}\text { epsy, } \\
1.0 \mathrm{e}-5\end{array}$ & $\begin{array}{l}\text { epsf, } \\
0.01\end{array}$ & & & & $\begin{array}{l}\text { nitysi } \\
50\end{array}$ & $>$ \\
\hline 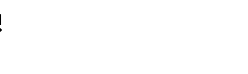 & $\begin{array}{l}\text { epsp. } \\
50.0\end{array}$ & $\begin{array}{l}\text { epsalp, } \\
0.0005\end{array}$ & $\begin{array}{l}\text { epstg, } \\
0.05\end{array}$ & $\begin{array}{l}\text { epstf. } \\
0.05\end{array}$ & $\begin{array}{l}\text { epsxa, } \\
0.005\end{array}$ & $\begin{array}{l}\text { nitmax } \\
-100\end{array}$ & $>$ \\
\hline
\end{tabular}

/NUMBER OF SPLINE PROFILES AND DATA POINTS/

! ndata: number of data groups 
! itime: number of time snapshots for axial power profiles

! ndata, itime

10

Page:

4 of 18

! npdat: number of data points per data set

$!$ nset: number of data sets in data group

!- n-

! npdat nset

$\begin{array}{rlr}3 & 1 & !> \\ 29 & 1 & !> \\ 13 & 1 & !> \\ 81 & 1 & !> \\ 81 & 1 & !> \\ 81 & 1 & !> \\ 81 & 1 & !> \\ 81 & 1 & !> \\ 81 & 1 & !> \\ 81 & 1 & !>\end{array}$

!

/GEOMETRIC DIMENSIONS:/

! nchn: number of flow channels

nzt: number of top section axial cells (>=2)

$\mathrm{nz}$ : number of middle section axial cell layers $(>=3)$

nzb: number of bottom section axial cells (>=2)

I nchn nż nz, nzb
12
2
$20 \quad 2$

!

/POWER ITERATION INPUT/

power: initial power in $\mathrm{kW}$

maxpi: maximum number of power iterations

! tolpow: tolerance on power limit

! ncrit: number of criteria used to check for power limit

! power, maxpi, tolpow, ncrit

$\begin{array}{llllll}61.5 & 1 & 0.005 & 8 & !>\end{array}$

!

SENSITIVITY VARIABLES INPUT SECTION

/SENSITIVITY PARAMETERS/

! cizfac: axial interfacial drag multiplying factor

! xcofh, xreh, xcofl, xrel, xkmet, xcvmet

\begin{tabular}{|c|c|c|c|c|c|}
\hline 1.0 & 1.0 & 1.0 & 1.0 & 1.0 & 1.0 \\
\hline $\operatorname{xhti}$ & xhgi, & xkgi, & $x p h i$ & & \\
\hline 1.0 & 1.0 & 1.0 & 1.0 & & \\
\hline cizfac & xfric, & plnht, & cipIn, & formhs, & alphs \\
\hline 1.0 & 1.0 & 8.75 & 1.0 & $5: 382$ & 0.05 \\
\hline aIb2, & als2. & ala2, & expbs. & expsa & \\
\hline 0.25 & 0.52 & 0.75 & 4.0 & 4.0 & \\
\hline
\end{tabular}


Solid mesh input for finite element regions, nodes and side boundary conditions is identical to that shown in reference [6]

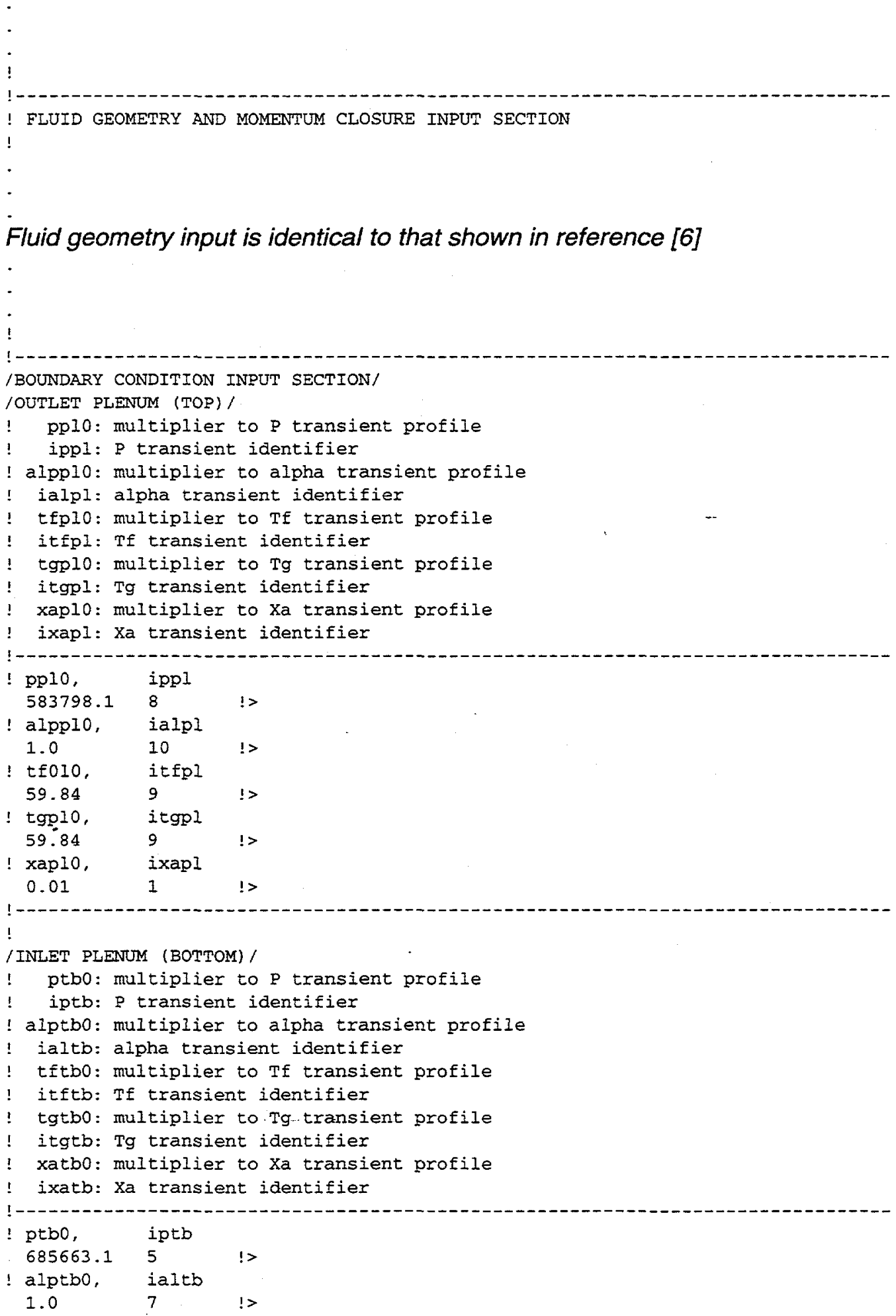


! tetbo,

itftb

53.03

6

! tgtbo,

53.03

itgtb

xatbo,

6

!>

0.99

ixatio

$!$

/INLET FLOW DATA/

! The following inlet flow data is always used to initialize

! axial velocities and will also be used to define the

! appropriate prescribed flowrate for $t>0$ if ibond $=2,3$, or 4 .

! qfino: multiplier to of transient profile

! iqfin: $Q f$ transient identifier

! ggino: multiolier to $\mathrm{Qg}$ transient profile

! iqgin: Qg transient identifier

! qfino = Nominal APT total flow 12 half channels, transient

! -

! qfino, iqfin

$-1.508 e-34 \quad: 1>$

! ggino, iqgin

$0.0 \quad 1 \quad$ ! >$$
!
$$

/INITIAL CONDITION INPUT SECTION/

! If iseto $>0$ then initial conditions are

! input for fluid parameters at each axial level

! iseto

$$
0
$$

!

/CRITERIA CHECKING FLAGS AND PEAKING FACTORS/

! checking flags for criteria \#1 \#2 \#3

!peaking factors for criteria $\begin{array}{rrr}0 & 0 & 0\end{array}$

$\begin{array}{rrrrrr}\# 4 & \# 5 & \# 6 & \# 7 & \# 8 & \\ 0 & 0 & 0 & 0 & 0 & \text { !> } \\ \# 4 & \# 5 & \# 6 & \# 7 & \# 8 & \\ 1.0 & 1.0 & 1.0 & 1.0 & 1.0 & \text { !> }\end{array}$

/CRITERIA CHECKING TIME/

! time to begin criteria checking, sec

terito

terito

0.0 ! !

$!$

! POWER INPUT

!

/POWER PROEILE SPLINE POINTERS/

! DECAY HEAT TRANSIENT

1

!

/AXIAL SPLINE POINTERS AND TIMES/

$\begin{array}{llll}2 & 0.0 & 2 & 600.00\end{array}$

!

/TRANSIENT DATA SET INPUT SECTION/

$!$

/DATA SET NUMBER 1/

! enter data set label below

! $-1-0-10-0$

data set 1 - UNIFORM

! itype: 1 = linear spline

! itype: $\begin{aligned} 1 & =\text { linear spline } \\ 0 & =\text { cubic spline }\end{aligned}$

$x, y$ : data pairs 


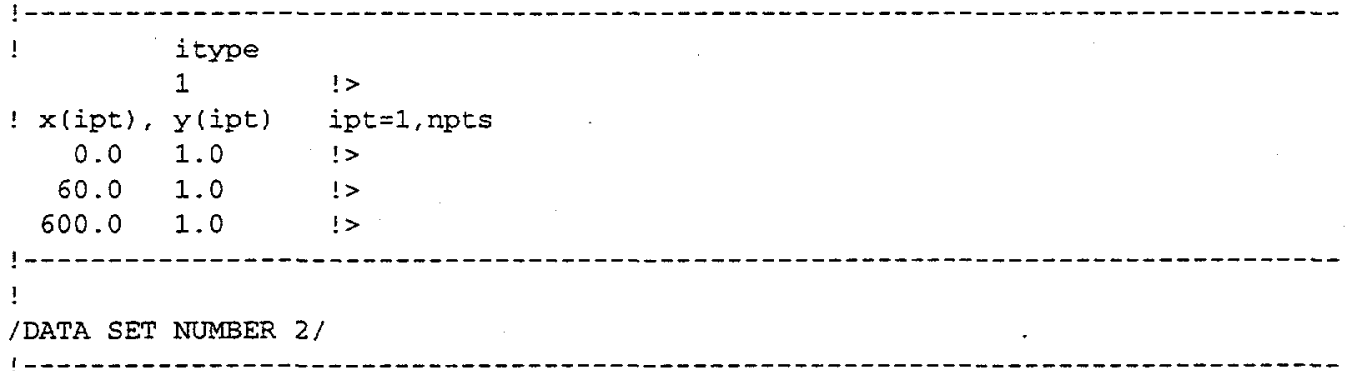

data set 2 - NON-UNIFORM AXIAL POWER PROFILE

! itype

1

! $x$ (ipt), $y$ (ipt)

$0.00 \quad 0.032$

!>

$0.10 \quad 0.043$

$0.20 \quad 0.049$

$0.30 \quad 0.074$

$0.40 \quad 0.093$

$0.50 \quad 0.124$

$0.60 \quad 0.165$

$0.70 \quad 0.217$

$0.80 \quad 0.317$

$0.90 \quad 0.508$

$1.00 \quad 0.943$

$1.10 \quad 1.446$

$1.20 \quad 1.658$

$1.30 \quad 1.754$

$1.40 \quad 1.783$

$1.50 \quad 1.827$

$1.60 \quad 1.870$

$1.70 \quad 1.881$

$1.80 \quad 1.915$

$1.90 \quad 1.915$

$2.00 \quad 1.887$

$2.10 \quad 1.864$

$2.20 \quad 1.790$

$2.30 \quad 1.660$

2.40

1.423

2.50

0.932

2.60

0.506

2.70

0.313

2.80

0.229

ipt $=1, n p t s$

!>

!>

!>

!>

!>

!>

!>

!>

!>

!>

!>

!>

!>

!>

i>

!>

$!>$

!>

!>

!>

!>

!>

!>

!>

!>

$!>$

!>

!>

/DATA SET NUMBER 3/

data set 3 - DECAY POWER CURVE $(+1$ second time delay)

$! \quad$ itype

1 ! $1>$

! $x$ (ipt), $y$ (ipt) ipt $=1$, npts

$0.00 \quad 1.000000000 E+00$

$1.00 \quad 1.000000000 E+00$

$1.01 \quad 1.298500039 \mathrm{E}-02$

$2.00 \quad 9.929999709 \mathrm{E}-03$

$3.00 \quad 8.652999997 \mathrm{E}-03$

$6.007 .736000232 \mathrm{E}-03$

$11.00 \quad 7.573999930 \mathrm{E}-03$

$21.00 \quad 7.437000051 E-03$

$61.00 \quad 7.073000073 \mathrm{E}-03$

$121.00 \quad 6.672000047 \mathrm{E}-03$ 
WESTINGHOUSE SAVANNAH RIVER COMPANY

APT BLANKET SYSTEM FOR LOHGA (HELIUM SUPPLY RUPTURE INTO BLANKET MODULE)
Report: WSRC-TR-98-00177

Section:

Appendix $F$

Date:

$07 / 16 / 98$

Page:

8 of 18 .
301.00
$5.936999805 E-03$
$601.00 \cdot 5.392999854 \mathrm{E}-03$
$1201.00 \quad 4.968000110 \mathrm{E}-03$

!

/DATA SET NUMBER $4 /$

data set 4 - COOLANT FLOW TRANSIENT

! itype

1

! $x$ (ipt),

0.00

$y$ (ipt) $\quad i p t=1$, npts

1.01

$1.00000 \mathrm{E}+00$

2.01

3.01

4.02

7.08

9.08

11.11

13.21

15.23

17.27

19.35

21.44

23.47

25.54

27.59

29.59

31.63

33.65

35.66

37.66

39.66

41.66

43.66

45.66

47.66

49.66

60.11

70.16

80.33

90.53

100.73

110.93

121.13

131.33

141.53

151.73

161.93

172.13

182.33

192.53

202.73

212.93

223.13

233.33

243.53

253.73

263.93

274.13

284.33

294.53

$7.70408 \mathrm{E}-01$

$8.24990 \mathrm{E}-01$

8. $94548 \mathrm{E}-01$

$9.64089 E-01$

$9.83655 \mathrm{E}-01$

9.78418E-01

8.96791E-01

9.89722E-01

9.93217E-01

$9.92801 \mathrm{E}-01$

9. $47602 \mathrm{E}-01$

$9.95208 \mathrm{E}-01$

$1.00834 \mathrm{E}+00$

$9.95067 E-01$

$9.88241 \mathrm{E}-01$

$1.00349 \mathrm{E}+00$

$1.00732 E+00$

$9.92899 \mathrm{E}-01$

$9.90142 \mathrm{E}-01$

$9.97259 \mathrm{E}-01$

1. $00133 \mathrm{E}+00$

$1.00026 E+00$

$9.99043 E-01$

$1.00113 \mathrm{E}+00$

$1.00091 \mathrm{E}+00$

$9.98134 \mathrm{E}-01$

$9.98925 \mathrm{E}-01$

1. $00018 E+00$

9.99198E-01

$9.99566 \mathrm{E}-01$

9.99585E-01

$9.99590 \mathrm{E}-01$

9.99574E-01

9.99591E-01

$9.99590 \mathrm{E}-01$

9.99584E-0I

9. $99592 \mathrm{E}-01$

$9.99588 \mathrm{E}-0 \mathrm{I}$

9.99587E-01

$9.99592 \mathrm{E}-01$

$9.99588 \mathrm{E}-01$

$9.99587 \mathrm{E}-01$

$9.99588 \mathrm{E}-01$

$9.99583 E-01$

$9.99584 \mathrm{E}-01$

9.99512E-0I

$9.99359 E-01$

9.99408E-01

9. $99512 \mathrm{E}-01$

9. $99590 \mathrm{E}-01$ 
304.73

314.93

325.13

335.33

345.53

355.73

365.93

376.13

386.33

396.53

406.73

416.93

427.13

437.33

447.53

457.73

467.93

478.13

488.33

498.53

508.73

518.93

529.13

539.33

549.53

559.73

569.93

580.13

590.33

600.23

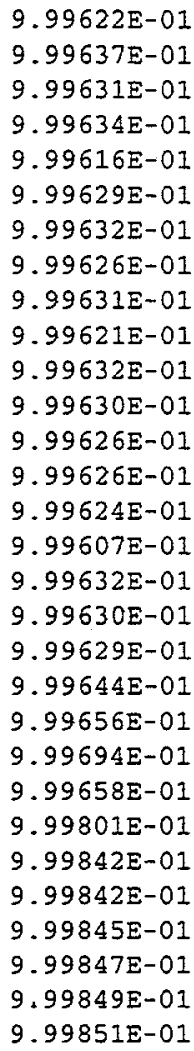


WESTINGHOUSE SAVANNAH RIVER COMPANY

APT BLANKET SYSTEM FOR LOHGA

(HELIUM SUPPLY RUPTURE INTO BLANKET MODULE)
Report:

Section:

Date:

Page:
WSRC-TR-98-00177

Appendix $\mathrm{F}$

$07 / 16 / 98$ 10 of 18
45.66
$1.00110 E+00$
47.66
$1.00556 \mathrm{E}+00$
49.66
$1.00536 \mathrm{E}+00$
60.11
$9.98976 \mathrm{E}-01$
70.16
$1.00096 \mathrm{E}+00$
80.33
$1.00252 E+00$
90.53
$1.00137 \mathrm{E}+00$
100.73
$1.00115 \mathrm{E}+00$
110.93
121.13
$1.00139 \mathrm{E}+00$
1. $00120 \mathrm{E}+00$
$1.00131 \mathrm{E}+00$
131.33
141.53
$1.00123 \mathrm{E}+00$
151.73
1. $00128 \mathrm{E}+00$
161.93
172.13
$1.00125 \mathrm{E}+00$
$1.00126 \mathrm{E}+00$
182.33
$1.00125 E+00$
1. $00125 E+00$
192.53
202.73
$1.00125 \mathrm{E}+00$
$1.00124 E+00$
212.93
223.13
233.33
$1.00124 E+00$
$1.00124 \mathrm{E}+00$
$1.00124 E+00$
243.53
253.73
263.93
$1.00136 \mathrm{E}+00$
$1.00129 E+00$
$1.00127 \mathrm{E}+00$
284.33
$1.00121 \mathrm{E}+00$
$1.00123 \mathrm{E}+00$
294.53
304.73
$1.00119 E+00$
$1.00121 E+00$
$1.00119 \mathrm{E}+00$
325.13
335.33
$1.00120 E+00$
$1.00119 \mathrm{E}+00$
$1.00122 \mathrm{E}+00$
$1.00117 \mathrm{E}+00$
355.73
365.93
376.13
1. $00119 \mathrm{E}+00$
$1.00118 \mathrm{E}+00$
396.53
406.73
$1.00118 \mathrm{E}+00$
1. $00119 \mathrm{E}+00$
$1.00114 \mathrm{E}+00$
$1.00118 E+00$
427.13
437.33 .
447.53
$1.00116 E+00$
457.73
$1.00116 E+00$
$1.00111 \mathrm{E}+00$
$1.00117 \mathrm{E}+00$
$1.00116 E+00$
478.13
488.33
$1.00116 \mathrm{E}+00$
$1.00116 \mathrm{E}+00$
$1.00113 \mathrm{E}+00$
$1.00110 \mathrm{E}+00$
$1.00119 E+00$
$1.00113 E+00$
$1.00106 \mathrm{E}+00$
$1.00106 \mathrm{E}+00$
$1.00106 \mathrm{E}+00$
$1.00106 \mathrm{E}+00$
$1.00106 E+00$
580.13
590.33
$1.00106 \mathrm{E}+00$
600.23

$!$

/DATA SET NUMBER 6/

data set 6 - INLET TEMPERATURE TRANSIENT 
386.33

396.53

406.73

416.93

427.13

437.33

447.53

457.73

467.93

478.13

488.33

498.53

508.73

518.93

529.13

539.33

549.53

559.73

569.93

580.13

590.33

600.23
$9.97549 \mathrm{E}-01$
9.97549E-01
$9.99434 \mathrm{E}-01$
9. $97360 \mathrm{E}-01$
9.97549E-01
$9.97549 E-01$
9.97549 $\mathrm{E}-01$
9.97360E-01
$9.97549 \mathrm{E}-01$
1. $00094 \mathrm{E}+00$
$9.97549 E-01$
9.97549E-01
$9.97549 \mathrm{E}-01$
9.97549E-01
9.97549E-01
9.97549E-01
9.97549E-01
9.97549E-01
9.97549E-01
9.97549E-01
9.97549E-01
9.97549E-01

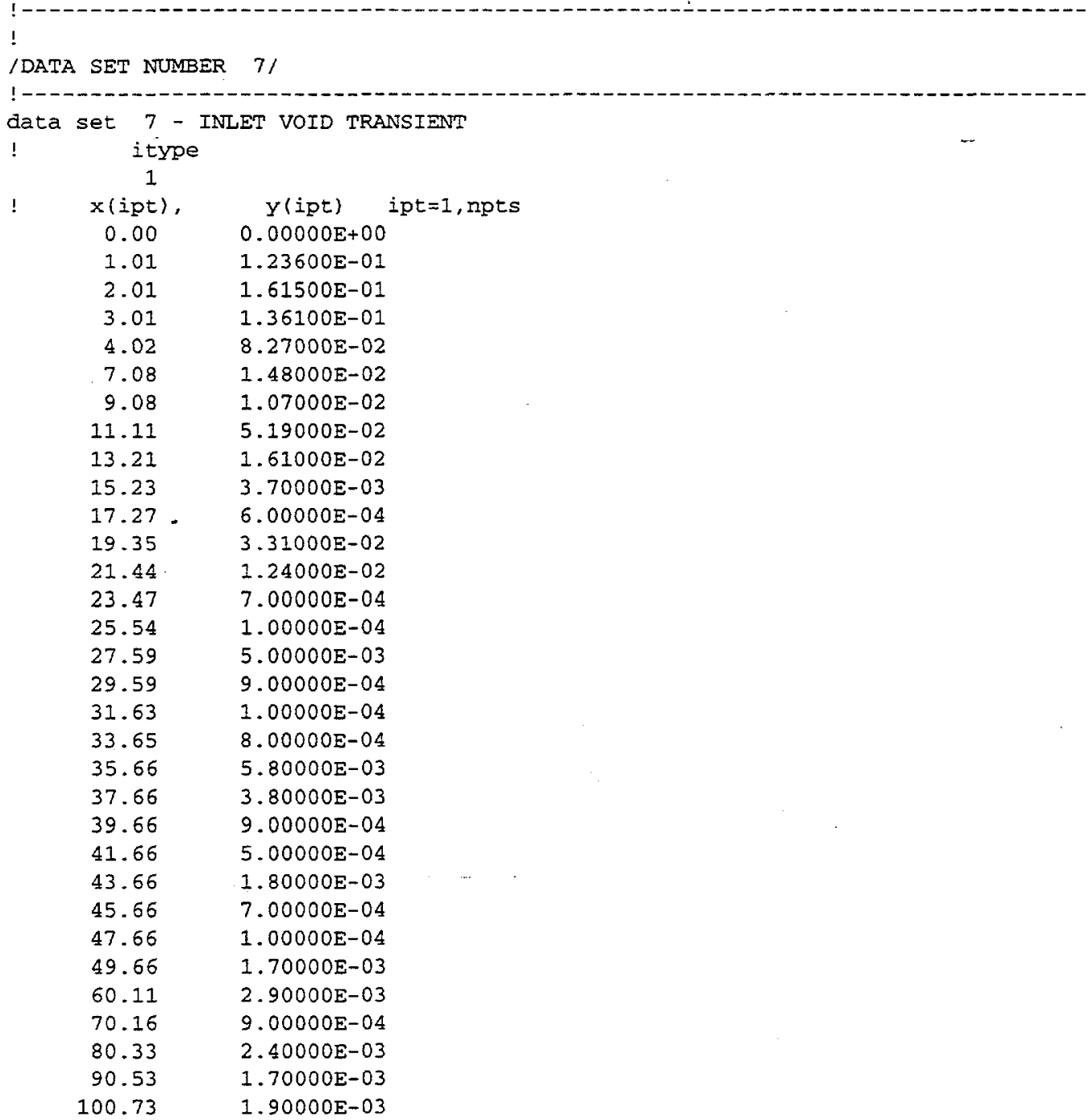


110.93

121.13

131.33

141.53

151.73

161.93

172.13

182.33

192.53

202.73

212.93

223.13

233.33

243.53

253.73

263.93

274.13

284.33

294.53

304.73

314.93

325.13

335.33

345.53

355.73

365.93

376.13

386.33

396.53

406.73

416.93

427.13

437.33

447.53

457.73

467.93

478.13

488.33

498.53

508.73

.518 .93

529.13

539.33

549.53

559.73

569.93

580.13

590.33

600.23
1. $70000 \mathrm{E}-03$

$1.80000 E-03$

$1.80000 \mathrm{E}-03$

1. $80000 \mathrm{E}-03$

$1.80000 \mathrm{E}-03$

1. $80000 E-03$

1. $80000 \mathrm{E}-03$

1. $80000 \mathrm{E}-03$

I. $80000 \mathrm{E}-03$

1. $80000 \mathrm{E}-03$

1. $80000 \mathrm{E}-03$

$1.80000 \mathrm{E}-03$

$1.80000 \mathrm{E}-03$

1. $80000 \mathrm{E}-03$

$1.80000 \mathrm{E}-03$

$2.00000 \mathrm{E}-03$

$2.00000 E-03$

1. $80000 \mathrm{E}-03$

1. $70000 \mathrm{E}-03$

1. $70000 \mathrm{E}-03$

1. $70000 \mathrm{E}-03$

1. $70000 \mathrm{E}-03$

1. $70000 \mathrm{E}-03$

1.70000E-03

1. $70000 \mathrm{E}-03$

$1.70000 \mathrm{E}-03$

$1.70000 \mathrm{E}-03$

$1.70000 \mathrm{E}-03$

$1.70000 \mathrm{E}-03$

$1.70000 \mathrm{E}-03$

$1.70000 \mathrm{E}-03$

$1.70000 \mathrm{E}-03$

$1.70000 \mathrm{E}-03$

$1.70000 \mathrm{E}-03$

$1.70000 E-03$

1. $60000 \mathrm{E}-03$

1. $70000 \mathrm{E}-03$

$1.60000 \mathrm{E}-03$

1. 50000E-03

1. 50000E-03

$8.00000 \mathrm{E}-04$

$2.00000 \mathrm{E}-04$

1. $00000 \mathrm{E}-04$

1. $00000 \mathrm{E}-04$

$0.00000 E+00$

$0.00000 \mathrm{E}+00$

$0.00000 \mathrm{E}+00$

$0.00000 \mathrm{E}+00$

$0.00000 \mathrm{E}+00$

data set 8 - OUTLET PRESSURE TRANSIENT

$$
\text { itype }
$$

$$
1
$$$$
x \text { (ipt), }
$$$$
0.00
$$

$y(i p t) \quad$ ipt $=1$, npts

1.01

1. $00000 \mathrm{E}+00$

2.01

1. $77830 \mathrm{E}+00$

$1.50819 \mathrm{E}+00$

1. $29845 \mathrm{E}+00$

4.02

$1.18550 \mathrm{E}+00$ 
7.08

9.08

11.11

13.21

15.23

17.27

19.35

21.44

23.47

25.54

27.59

29.59

31.63

33.65

35.66

37.66

39.66

41.66

43.66

45.66

47.66

49.66

60.11

70.16

80.33

90.53

100.73

110.93

121.13

131.33

141.53

151.73

161.93

172.13

182.33

192.53

202.73

212.93

223.13

233.33

243.53

253.73

263.93

274.13

284.33

294.53

304.73

314.93

325.13

335.33

345.53

355.73

365.93

376.13

386.33

396.53

406.73

416.93

427.13

437.33

447.53

457.73
$1.08128 \mathrm{E}+00$

$1.05487 E+00$

$9.75299 \mathrm{E}-01$

$9.03303 \mathrm{E}-01$

$1.04034 E+00$

$1.06088 \mathrm{E}+00$

$1.02095 \mathrm{E}+00$

$9.84029 \mathrm{E}-02$

$1.00170 \mathrm{E}+00$

$1.00282 \mathrm{E}+00$

9.84981E-01

$9.93816 \mathrm{E}-01$

$1.02251 E+00$

$1.01975 \mathrm{E}+00$

$1.00694 \mathrm{E}+00$

9.95908E-01

$9.96000 \mathrm{E}-01$

$9.98926 \mathrm{E}-01$

$9.98906 \mathrm{E}-01$

$1.00189 \mathrm{E}+00$

$1.00682 \mathrm{E}+00$

$1.00640 E+00$

$9.99211 \mathrm{E}-01$

$1.00145 \mathrm{E}+00$

1. $00222 \mathrm{E} \div 00$

$1.00203 \mathrm{E}+00$

$1.00177 \mathrm{E}+00$

$1.00204 \mathrm{E}+00$

$1.00181 \mathrm{E}+00$

$1.00194 \mathrm{E}+00$

$1.00185 \mathrm{E}+00$

$1.00191 \mathrm{E}+00$

$1.00187 \mathrm{E}+00$

$1.00188 \mathrm{E}+00$

$1.00187 \mathrm{E}+00$

$1.00187 \mathrm{E}+00$

$1.00186 \mathrm{E}+00$

$1.00186 \mathrm{E}+00$

$1.00186 \mathrm{E}+00$

$1.00186 \mathrm{E}+00$

$1.00186 \mathrm{E}+00$

$1.00200 \mathrm{E}+00$

$1.00194 \mathrm{E}+00$

$1.00193 \mathrm{E}+00$

1. $00184 \mathrm{E}+00$

$1.00185 \mathrm{E}+00$

$1.00180 \mathrm{E}+00$

$1.00181 \mathrm{E}+00$

$1.00180 \mathrm{E}+00$

1. $00180 \mathrm{E}+00$

$1.00182 \mathrm{E}+00$

$1.00185 \mathrm{E}+00$

$1.00178 \mathrm{E}+00$

$1.00179 E+00$

$1.00178 \mathrm{E}+00$

$1.00178 \mathrm{E}+00$

$1.00182 \mathrm{E}+00$

1. $00173 \mathrm{E}+00$

$1.00178 \mathrm{E}+00$

$1.00175 E+00$

$1.00175 E+00$

$1.00170 \mathrm{E}+00$ 


$\begin{array}{ll}467.93 & 1.00176 E+00 \\ 478.13 & 1.00175 E+00 \\ 488.33 & 1.00172 E+00 \\ 498.53 & 1.00171 E+00 \\ 508.73 & 1.00166 E+00 \\ 518.93 & 1.00151 E+00 \\ 529.13 & 1.00145 E+00 \\ 539.33 & 1.00134 E+00 \\ 549.53 & 1.00125 E+00 \\ 559.73 & 1.00124 E+00 \\ 569.93 & 1.00124 E+00 \\ 580.13 & 1.00124 E+00 \\ 590.33 & 1.00124 E+00 \\ 600.23 & 1.00124 E+00\end{array}$

/DATA SET NUMBER $9 /$

data set 9 - OUTLET TEMPERATURE TRANSIENT 
WESTINGHOUSE SAVANNAH RIVER COMPANY

APT BLANKET SYSTEM FOR LOHGA

(HELIUM SUPPLY RUPTURE INTO BLANKET MODULE)
Report: WSRC-TR-98-00177

Section: Appendix F

Date:

Page:
192.53

202.73

212.93

223.13

233.33

243.53

253.73

263.93

274.13

284.33

294.53

304.73

314.93

325.13

335.33

345.53

355.73

365.93

376.13

386.33

396.53

406.73

416.93

427.13

437.33

447.53

457.73

467.93

478.13

488.33

498.53

508.73

518.93

529.13

539.33

549.53

559.73

569.93

580.13

590.33

600.23 .
$9.97995 E-01$

9. $97995 E-01$

9. $97995 E-01$

9. $97828 \mathrm{E}-01$

$9.97828 \mathrm{E}-01$

$9.97828 \mathrm{E}-01$

$9.97828 \mathrm{E}-01$

$9.97828 \mathrm{E}-01$

$9.97828 E-01$

$9.97828 E-01$

$9.97828 \mathrm{E}-01$

9. $97828 \mathrm{E}-01$

9.97828 $\mathrm{E}-01$

9.97828E-01

9.97828E-01

$9.98663 \mathrm{E}-01$

$9.98162 \mathrm{E}-01$

9.98162 $\mathrm{x}-01$

$9.97828 \mathrm{E}-01$

$9.97828 \mathrm{E}-01$

$9.97828 \mathrm{E}-01$

$9.98496 \mathrm{E}-01$

9.97995E-01

$9.97828 \mathrm{E}-01$

$9.97828 \mathrm{E}-01$

$9.97828 \mathrm{E}-01$

$9.97995 \mathrm{E}-01$

$9.97828 \mathrm{E}-01$

$9.97995 \mathrm{E}-01$

$9.97828 \mathrm{E}-01$

$9.97828 \mathrm{E}-01$

$9.97828 \mathrm{E}-01$

9.97660E-0I

$9.97660 \mathrm{E}-01$

9.97660E-0I

$9.97660 \mathrm{E}-01$

9. $97660 \mathrm{E}-0 \mathrm{I}$

$9.97660 \mathrm{E}-01$

$9.97660 \mathrm{E}-01$

$9.97660 E^{\circ}-01$

9.97660E-01

/DATA SET NUMBER 10/

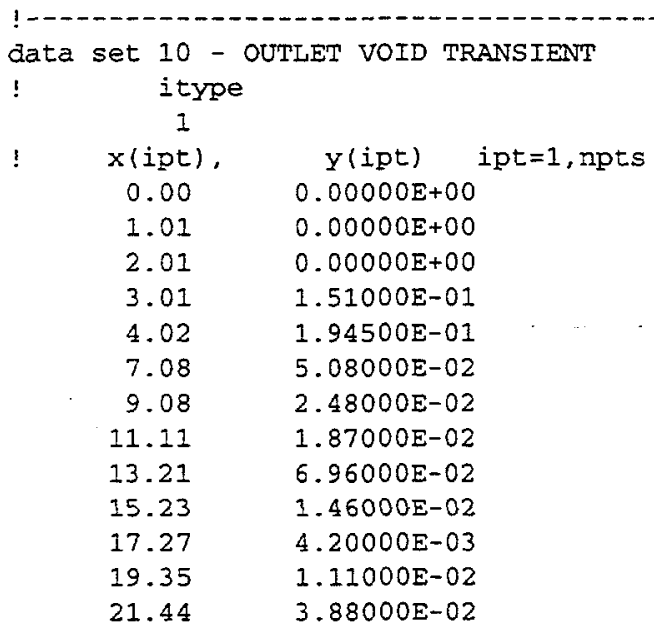




\begin{tabular}{|c|c|}
\hline 23.47 & $1.09000 \mathrm{E}-02$ \\
\hline 25.54 & $1.40000 \mathrm{E}-03$ \\
\hline 27.59 & $2.60000 \mathrm{E}-03$ \\
\hline 29.59 & $4.70000 E-03$ \\
\hline 31.63 & $1.20000 \mathrm{E}-03$ \\
\hline 33.65 & $2.00000 \mathrm{E}-04$ \\
\hline 35.66 & $4.00000 \mathrm{E}-03$ \\
\hline 37.66 & $7.80000 \mathrm{E}-03$ \\
\hline 39.66 & $4.00000 \mathrm{E}-03$ \\
\hline 41.66 & $1.20000 \mathrm{E}-03$ \\
\hline 43.66 & $1.30000 \mathrm{E}-03$ \\
\hline 45.66 & $1.90000 \mathrm{E}-03$ \\
\hline 47.66 & $7.00000 \mathrm{E}-04$ \\
\hline 49.66 & $5.00000 E-04$ \\
\hline 60.11 & $3.50000 \mathrm{E}-03$ \\
\hline 70.16 & $2.30000 \mathrm{E}-03$ \\
\hline 80.33 & $2.80000 \mathrm{E}-03$ \\
\hline 90.53 & $2.30000 \mathrm{E}-03$ \\
\hline 100.73 & $2.60000 \mathrm{E}-03$ \\
\hline 110.93 & $2.30000 \mathrm{E}-03$ \\
\hline 121.13 & $2.40000 \mathrm{E}-03$ \\
\hline 131.33 & $2.40000 \mathrm{E}-03$ \\
\hline 141.53 & $2.40000 \mathrm{E}-03$ \\
\hline 151.73 & $2.40000 \mathrm{E}-03$ \\
\hline 161.93 & $2.40000 \mathrm{E}-0$ \\
\hline 172.13 & $2.40000 \mathrm{E}-03$ \\
\hline 182.33 & $2.40000 E-03$ \\
\hline 192.53 & $2.40000 \mathrm{E}-0$ \\
\hline 202.73 & $2.40000 E-03$ \\
\hline 212.93 & $2.40000 \mathrm{E}-03$ \\
\hline 223.13 & $2.40000 E-03$ \\
\hline 233.33 & $2.40000 \mathrm{E}-03$ \\
\hline 243.53 & $2.40000 \mathrm{E}-03$ \\
\hline 253.73 & $2.40000 E-03$ \\
\hline 263.93 & $2.70000 \mathrm{E}-03$ \\
\hline 274.13 & $2.60000 \mathrm{E}-03$ \\
\hline 284.33 & $2.40000 E-03$ \\
\hline 294.53 & $2.30000 \mathrm{E}-03$ \\
\hline 304.73 & $2.30000 \mathrm{E}-03$ \\
\hline 314.93 & $2.20000 E-03$ \\
\hline 325.13 & $2.30000 \mathrm{E}-03$ \\
\hline 335.33 & $2.20000 \mathrm{E}-0$ \\
\hline 345.53 & $2.20000 \mathrm{E}-03$ \\
\hline 355.73 & $2.20000 \mathrm{E}-0$ \\
\hline 365.93 & $2.20000 E-03$ \\
\hline 376.13 & $2.20000 \mathrm{E}-03$ \\
\hline 386.33 & $2.20000 \mathrm{E}-03$ \\
\hline 396.53 & $2.20000 \mathrm{E}-03$ \\
\hline 406.73 & $2.20000 \mathrm{E}-0$ \\
\hline 416.93 & $2.20000 \mathrm{E}-03$ \\
\hline 427.13 & $2.20000 E-.0$ \\
\hline 437.33 & $2.20000 \mathrm{E}-0$ \\
\hline 447.53 & $2.20000 \mathrm{E}-0$ \\
\hline 457.73 & $2.20000 E-03$ \\
\hline 467.93 & $2.20000 \mathrm{E}-03$ \\
\hline 478.13 & $2.10000 \mathrm{E}-03$ \\
\hline 488.33 & $2.10000 \mathrm{E}-0$ \\
\hline 498.53 & $2.00000 \mathrm{E}-0$ \\
\hline 508.73 & $1.90000 \mathrm{E}-\mathrm{C}$ \\
\hline 518.93 & $1.20000 \mathrm{E}-0$ \\
\hline 529.13 & $4.00000 E-0$ \\
\hline 539.33 & $1.00000 \mathrm{E}-\mathrm{C}$ \\
\hline
\end{tabular}


549.53

559.73

569.93

580.13

590.33

600.23
$1.00000 \mathrm{E}-04$

$1.00000 \mathrm{E}-04$

$1.00000 \mathrm{E}-04$

$0.00000 \mathrm{E}+00$

$0.00000 E+00$

$0.00000 E+00$ 